In 1993 the World Health Organization (WHO) undertook a comprehensive review of the uses and interpretation of anthropometric references. The review concluded that the NCHS/WHO growth reference, which had been recommended for international use since the late 1970s, did not adequately represent early childhood growth and that new growth curves were necessary. The World Health Assembly endorsed this recommendation in 1994. The WHO Multicentre Growth Reference Study (MGRS) was undertaken in response to that endorsement and implemented between 1997 and 2003 to generate new curves for assessing the growth and development of children the world over. The MGRS collected primary growth data and related information from 8440 healthy breastfed infants and young children from diverse ethnic backgrounds and cultural settings (Brazil, Ghana, India, Norway, Oman and USA). The growth standards developed based on these data and presented in this report provide a technically robust tool that represents the best description of physiological growth for children under five years of age. The standards depict normal early childhood growth under optimal environmental conditions and can be used to assess children everywhere, regardless of ethnicity, socioeconomic status and type of feeding.

\section{WHO Child Growth Standards}

Head circumference-for-age, arm circumference-for-age, triceps skinfold-for-age and subscapular skinfold-for-age

Methods and development

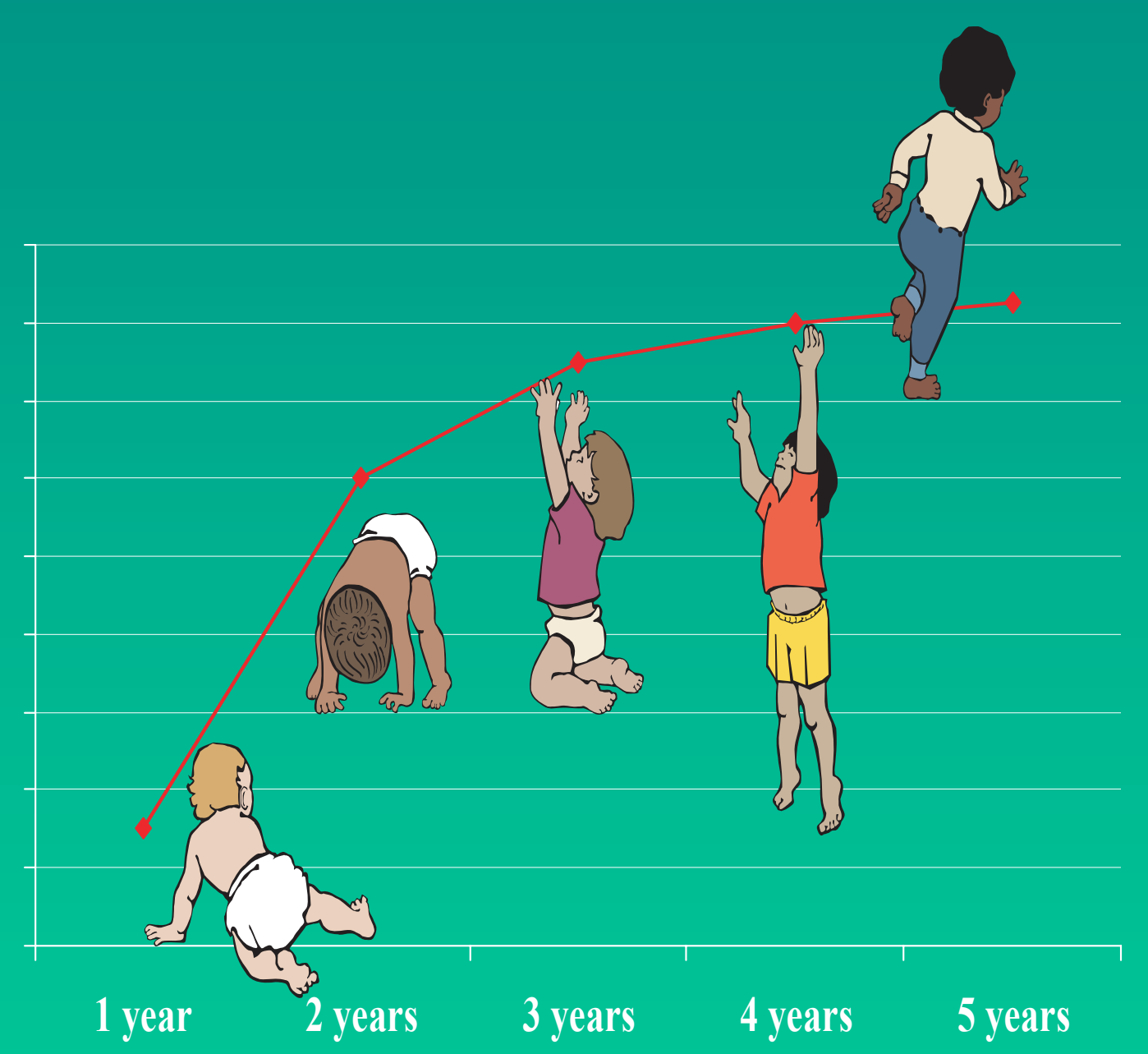

ISBN 9789241547185

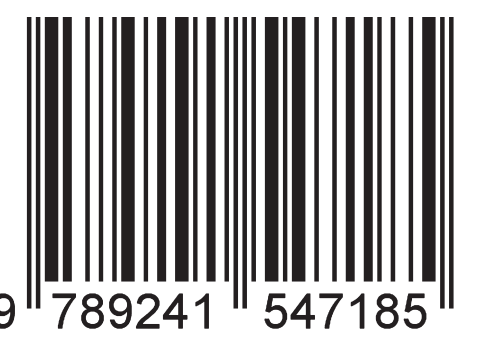

World Health

Organization 


\section{WHO Child Growth Standards}

Head circumference-for-age, arm circumference-for-age, triceps skinfold-for-age and subscapular skinfold-for-age

Methods and development 


\section{WHO Library Cataloguing-in-Publication Data}

WHO child growth standards : head circumference-for-age, arm circumference-forage, triceps skinfold-for-age and subscapular skinfold-for-age : methods and development.

Coordinating team: Mercedes de Onis ... [et al.].

1.Anthropometry. 2.Anthropometry - methods. 3.Body size - standards. 4.Child development. 5.Growth. 6.Reference standards. 7.Nutrition assessment. I.de Onis, Mercedes. II.World Health Organization. III.Title: World Health Organization child growth standards.

\section{c) World Health Organization 2007}

All rights reserved. Publications of the World Health Organization can be obtained from WHO Press, World Health Organization, 20 Avenue Appia, 1211 Geneva 27, Switzerland (tel.: +41 22791 3264; fax: +41 22791 4857; e-mail: bookorders@who.int). Requests for permission to reproduce or translate WHO publications - whether for sale or for noncommercial distribution should be addressed to WHO Press, at the above address (fax: +41 22791 4806; e-mail: permissions@who.int).

The designations employed and the presentation of the material in this publication do not imply the expression of any opinion whatsoever on the part of the World Health Organization concerning the legal status of any country, territory, city or area or of its authorities, or concerning the delimitation of its frontiers or boundaries. Dotted lines on maps represent approximate border lines for which there may not yet be full agreement.

The mention of specific companies or of certain manufacturers' products does not imply that they are endorsed or recommended by the World Health Organization in preference to others of a similar nature that are not mentioned. Errors and omissions excepted, the names of proprietary products are distinguished by initial capital letters.

All reasonable precautions have been taken by the World Health Organization to verify the information contained in this publication. However, the published material is being distributed without warranty of any kind, either expressed or implied. The responsibility for the interpretation and use of the material lies with the reader. In no event shall the World Health Organization be liable for damages arising from its use.

Printed in China, Hong Kong Special Administrative Region. 


\section{Members of the WHO Multicentre Growth Reference Study Group}

\section{Coordinating Team}

Mercedes de Onis [Study Coordinator], Adelheid Onyango, Elaine Borghi, Amani Siyam, Alain Pinol (Department of Nutrition for Health and Development, World Health Organization).

\section{Executive Committee}

Cutberto Garza [Chair], Mercedes de Onis, Jose Martines, Reynaldo Martorell, Cesar G. Victora (up to October 2002), Maharaj K. Bhan (from November 2002).

\section{Steering Committee}

Coordinating Centre (WHO, Geneva): Mercedes de Onis, Jose Martines, Adelheid Onyango, Alain Pinol.

Investigators (by country): Cesar G. Victora and Cora Luiza Araújo (Brazil), Anna Lartey and William B. Owusu (Ghana), Maharaj K. Bhan and Nita Bhandari (India), Kaare R. Norum and GunnElin Aa. Bjoerneboe (Norway), Ali Jaffer Mohamed (Oman), Kathryn G. Dewey (USA).

United Nations Agency Representatives: Cutberto Garza (UNU), Krishna Belbase (UNICEF).

\section{Advisory Group}

Maureen Black, Wm. Cameron Chumlea, Tim Cole, Edward Frongillo, Laurence Grummer-Strawn, Reynaldo Martorell, Roger Shrimpton, Jan Van den Broeck. For the work presented in this document, Huiqi Pan, Robert Rigby, Mikis Stasinopoulos and Stef van Buuren, participated in an advisory capacity.

\section{Participating countries and investigators}

Brazil: Cora Luiza Araújo, Cesar G. Victora, Elaine Albernaz, Elaine Tomasi, Rita de Cássia Fossati da Silveira, Gisele Nader (Departamento de Nutrição and Departamento de Medicina Social, Universidade Federal de Pelotas; and Núcleo de Pediatria and Escola de Psicologia, Universidade Católica de Pelotas).

Ghana: Anna Lartey, William B. Owusu, Isabella Sagoe-Moses, Veronica Gomez, Charles SagoeMoses (Department of Nutrition and Food Science, University of Ghana; and Ghana Health Service).

India: Nita Bhandari, Maharaj K. Bhan, Sunita Taneja, Temsunaro Rongsen, Jyotsna Chetia, Pooja Sharma, Rajiv Bahl (All India Institute of Medical Sciences).

Norway: Gunn-Elin Aa. Bjoerneboe, Anne Baerug, Elisabeth Tufte, Kaare R. Norum, Karin Rudvin, Hilde Nysaether (Directorate of Health and Social Affairs; National Breastfeeding Centre, Rikshospitalet University Hospital; and Institute for Nutrition Research, University of Oslo).

Oman: Ali Jaffer Mohamed, Deena Alasfoor, Nitya S. Prakash, Ruth M. Mabry, Hanadi Jamaan Al Rajab, Sahar Abdou Helmi (Ministry of Health).

USA: Kathryn G. Dewey, Laurie A. Nommsen-Rivers, Roberta J. Cohen, M. Jane Heinig (University of California, Davis). 


\section{Acknowledgements}

The WHO Child Growth Standards were constructed by the Coordinating Team in the Department of Nutrition for Health and Development of the World Health Organization.

The Study Group is indebted to the parents, children and more than 200 field staff that participated in the WHO Multicentre Growth Reference Study. The generous contribution of many individuals that provided expertise and advice was also crucial to the development of the growth standards.

The project has received funding from the Bill \& Melinda Gates Foundation, the Netherlands Minister for Development Cooperation, the Norwegian Royal Ministry of Foreign Affairs, and the United States Department of Agriculture (USDA). Financial support was also provided by the Ministry of Health of Oman, the United States National Institutes of Health, the Brazilian Ministry of Health and Ministry of Science and Technology, the Canadian International Development Agency, the United Nations University, the Arab Gulf Fund for United Nations Development, the Office of the WHO Representative to India, and the Department of Child and Adolescent Health and Development. 


\section{Contents}

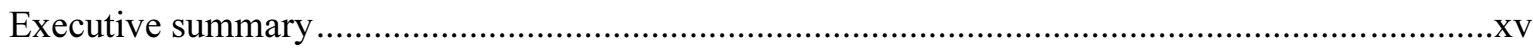

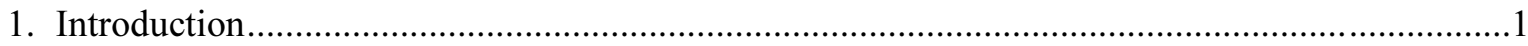

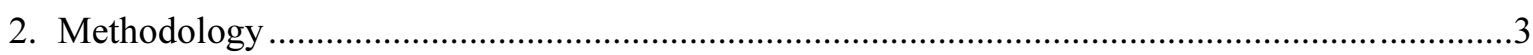

2.1 Design of the WHO Multicentre Growth Reference Study ....................................................

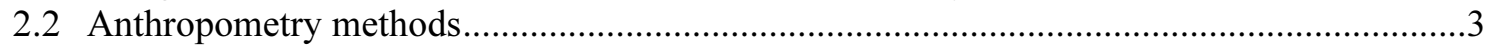

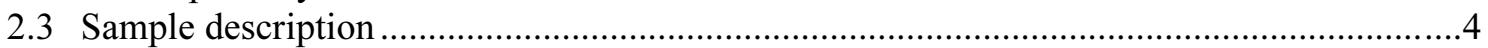

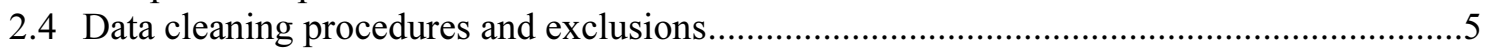

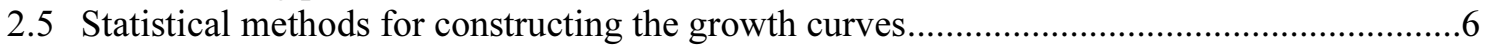

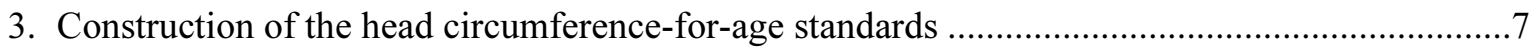

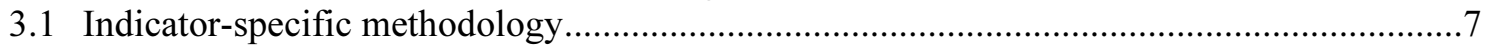

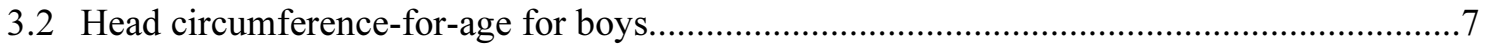

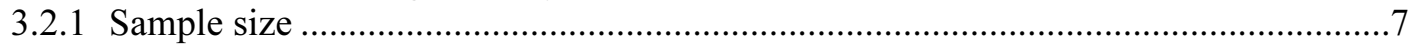

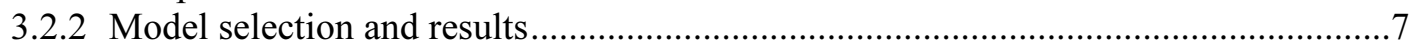

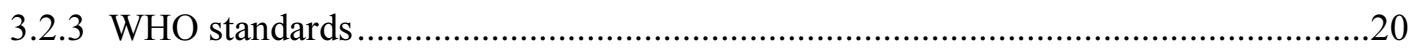

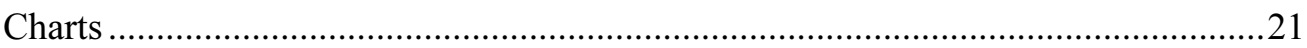

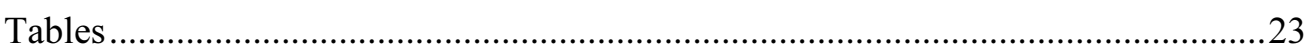

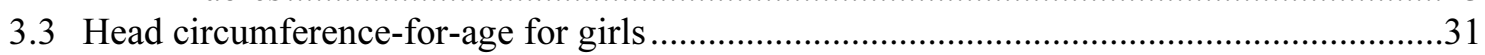

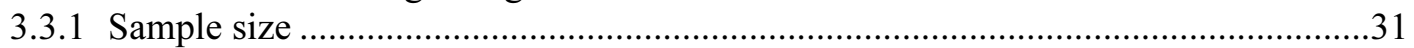

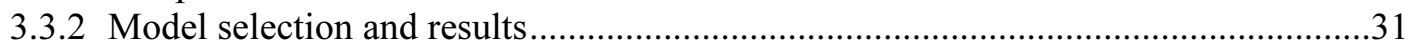

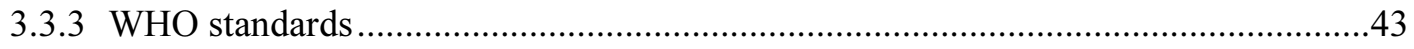

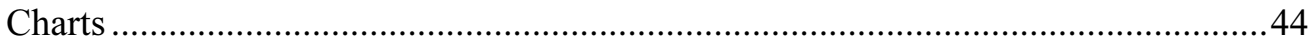

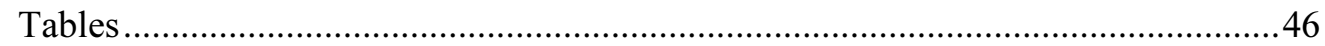

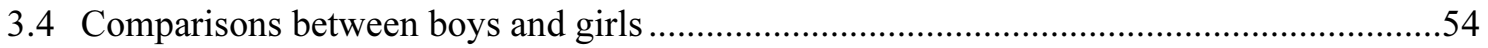

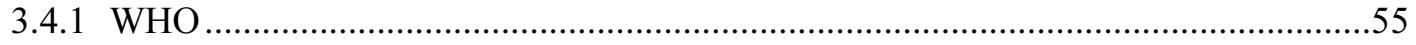

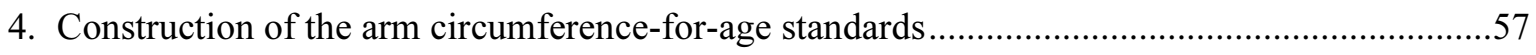

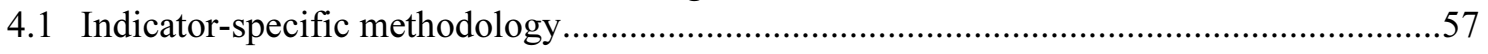

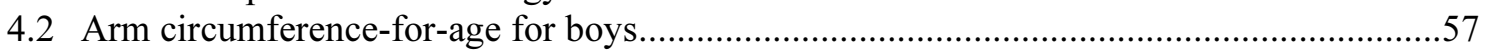

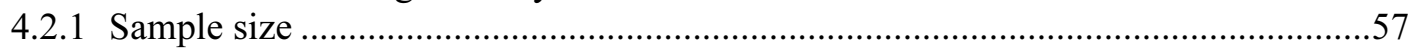

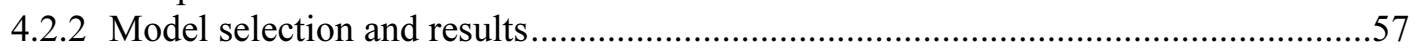

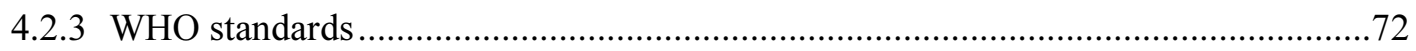

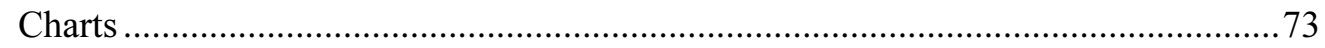

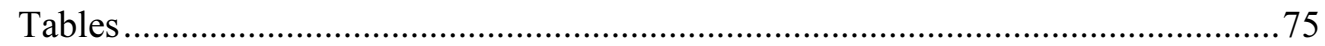

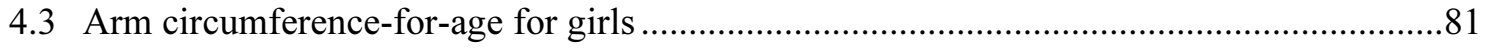

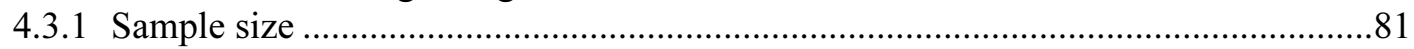

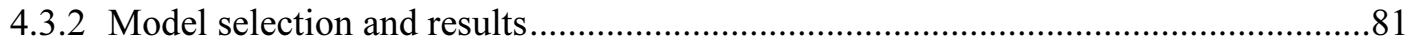

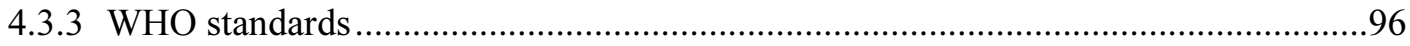

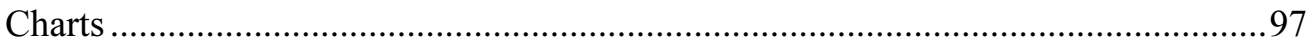

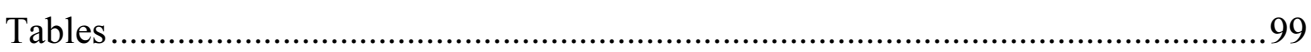

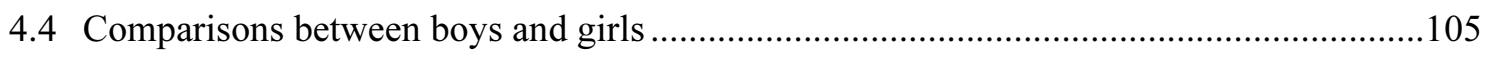

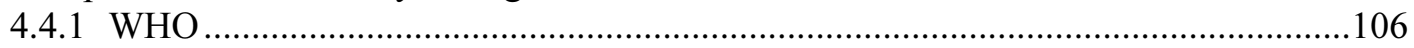

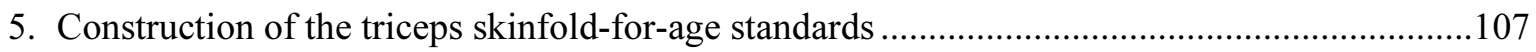

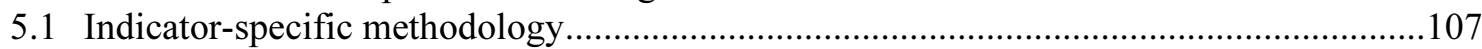

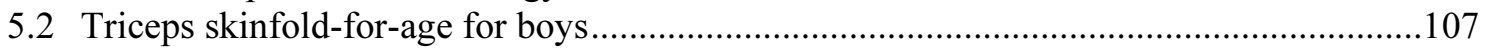

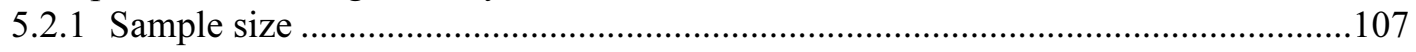

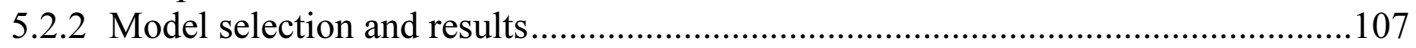

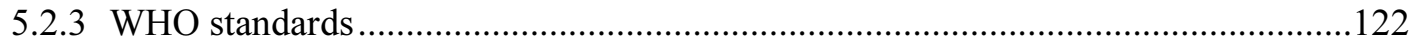

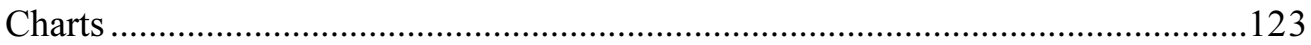

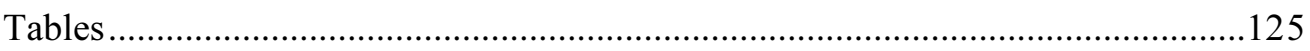

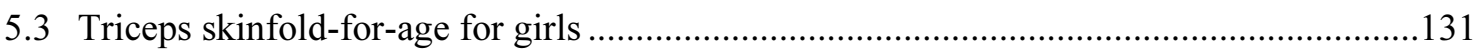

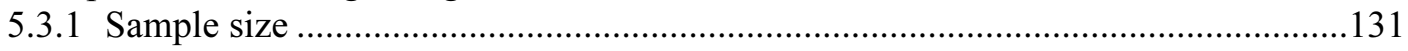




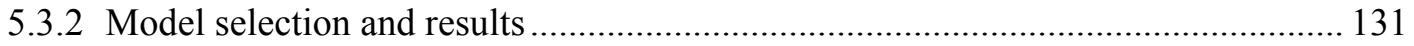

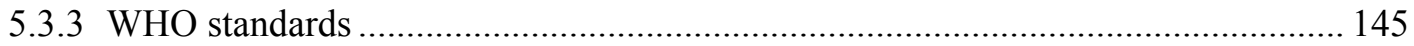

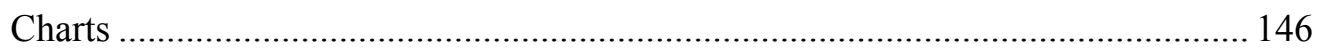

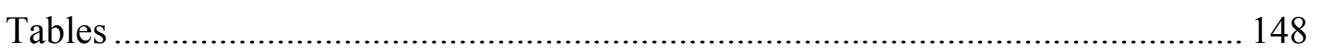

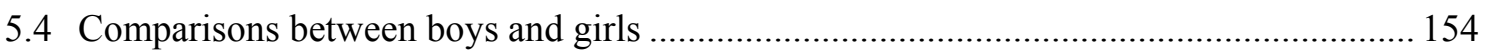

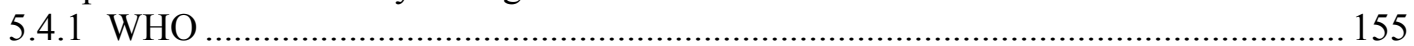

6. Construction of the subscapular skinfold-for-age standards ............................................... 157

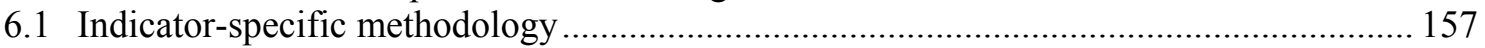

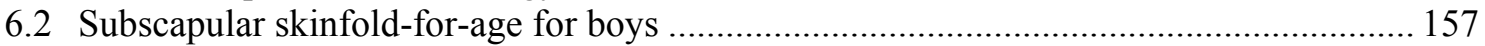

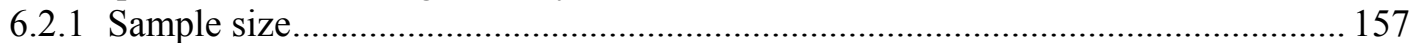

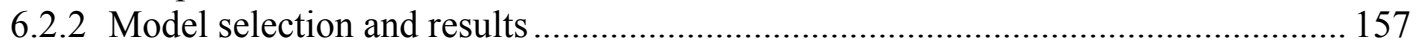

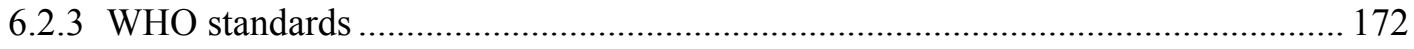

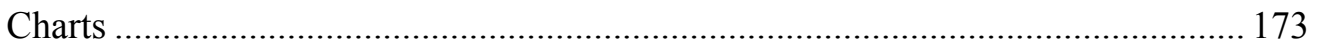

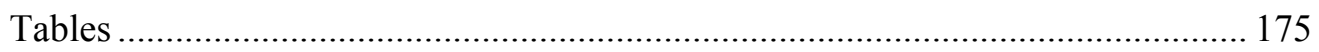

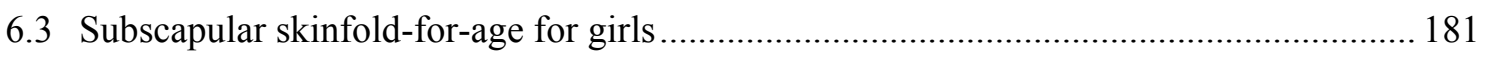

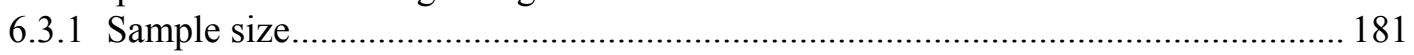

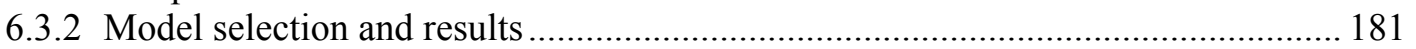

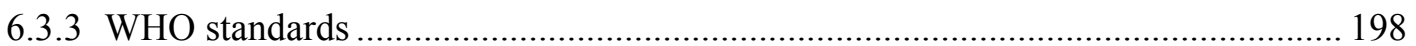

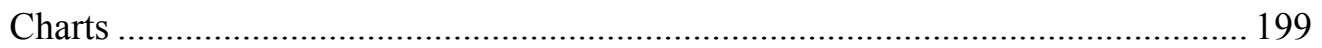

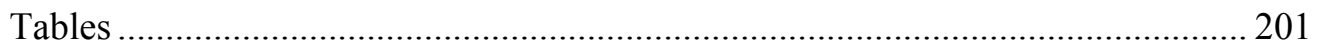

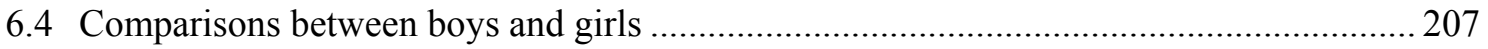

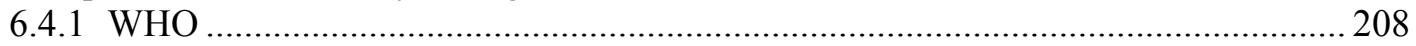

7. Computation of centiles and z-scores for head circumference-for-age, arm circumference -for-age, triceps skinfold-for-age, subscapular skinfold-for-age ...............................................2 209

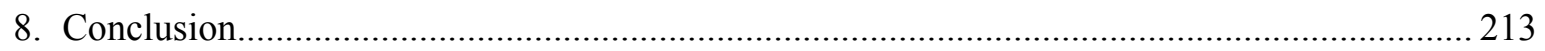

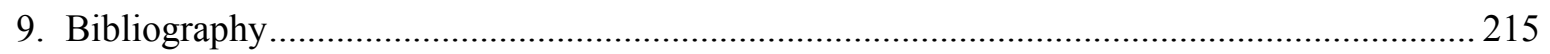

Appendix A. Model specifications of the WHO child growth standards ...................................... 217 


\section{Figures}

Figure $1 \quad$ Fitting of $\mu$ and $\sigma$ curves of Model 1 for head circumference-for-age for boys (dotted line) and their respective sample estimates (points with solid line)

Figure 2 Centile residuals from fitting Model 1 for head circumference-for-age from 0 to 24 months for boys....

Figure 3 Centile residuals from fitting Model 1 for head circumference-for-age from 24 to 71 months for boys.

Figure 4 Worm plots of z-scores for Model 1 for head circumference-for-age for boys

Figure 53 rd, 10th, 50th, 90th, 97th smoothed centile curves and empirical values: head circumference-for-age for boys from birth to 24 months

Figure 6 5th, 25th, 50th, 75th, 95th smoothed centile curves and empirical values: head circumference-for-age for boys from birth to 24 months

Figure 73 rd, 10th, 50th, 90th, 97th smoothed centile curves and empirical values: head circumference-for-age for boys from 24 to 71 months

Figure $8 \quad$ 5th, 25th, 50th, 75th, 95th smoothed centile curves and empirical values: head circumference-for-age for boys from 24 to 71 months

Figure 9 WHO head circumference-for-age z-scores for boys from birth to 60 months.

Figure 10

WHO head circumference -for-age percentiles for boys from birth to 60 months

Figure 11 Fitting of the $\mu$ and $\sigma$ curves of Model 1 for head circumference-for-age for girls (dotted line) and their respective sample estimates (points with solid line)

Figure 12 Centile residuals from fitting Model 1 for head circumference-for-age from 0 to 24 months for girls.

Figure 13 Centile residuals from fitting Model 1 for head circumference-for-age from 24 to 71 months for girls.

Figure 14 Worm plots of z-scores for Model 1 for head circumference-for-age for girls

Figure 153 rd, 10th, 50th, 90th, 97th smoothed centile curves and empirical values: head circumference-for-age for girls from birth to 24 months

Figure 16 5th, 25th, 50th, 75th, 95th smoothed centile curves and empirical values: head circumference-for-age for girls from birth to 24 months

Figure 17 3rd, 10th, 50th, 90th, 97th smoothed centile curves and empirical values: head circumference-for-age for girls from 24 to 71 months

Figure 18 5th, 25th, 50th, 75th, 95th smoothed centile curves and empirical values: head circumference-for-age for girls from 24 to 71 months.

Figure 19 WHO head circumference-for-age z-scores for girls from birth to 60 months ...............44

Figure 20 WHO head circumference-for-age percentiles for girls from birth to 60 months...........45

Figure 21 Comparison of boys' and girls' head circumference-for-age z-scores..............................55

Figure 22 Worm plots of $\mathrm{z}$-scores for Model 1 for arm circumference-for-age for boys.................59

Figure 23 Fitting of the $\mu, \sigma$, and $\nu$ curves of Model 2 for arm circumference-for-age for boys from 3 to 71 months (dotted line) and their respective sample estimates (points with solid line)

Figure 24 Centile residuals from fitting Model 2 for arm circumference-for-age from 3 to 24 months for boys. 
Figure 25 Centile residuals from fitting Model 2 for arm circumference-for-age from 24 to 71

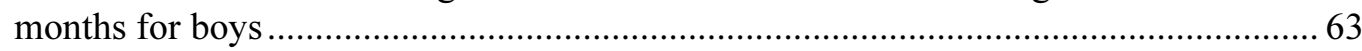

Figure 26 Worm plots of z-scores for Model 2 for arm circumference-for-age for boys ................ 64

Figure 27 3rd, 10th, 50th, 90th, 97th smoothed centile curves and empirical values: arm circumference-for-age for boys from 3 to 24 months...

Figure $28 \quad 5$ th, 25th, 50th, 75th, 95th smoothed centile curves and empirical values: arm circumference-for-age for boys from 3 to 24 months.....

Figure 293 rd, 10th, 50th, 90th, 97th smoothed centile curves and empirical values: arm circumference-for-age for boys from 24 to 71 months..

Figure $30 \quad 5$ th, 25th, 50th, 75th, 95th smoothed centile curves and empirical values: arm circumference-for-age for boys from 24 to 71 months.................................................. 71

Figure 31 WHO arm circumference-for-age z-scores for boys from 3 to 60 months..................... 73

Figure 32 WHO arm circumference-for-age percentiles for boys from 3 to 60 months................. 74

Figure 33 Worm plots of z-scores for Model 1 for arm circumference-for-age for girls

Figure 34 Fitting of the $\mu, \sigma$, and $v$ curves of Model 2 for arm circumference-for-age for girls from 3 to 71 months (dotted line) and their respective sample estimates (points with solid line).

Figure 35 Centile residuals from fitting Model 2 for arm circumference-for-age from 3 to 24 months for girls

Figure 36 Centile residuals from fitting Model 2 for arm circumference-for-age from 24 to 71 months for girls

Figure 37 Worm plots of z-scores for Model 2 for arm circumference-for-age for girls

Figure 38 3rd, 10th, 50th, 90th, 97th smoothed centile curves and empirical values: arm circumference-for-age for girls from 3 to 24 months

Figure 395 th, 25th, 50th, 75th, 95th smoothed centile curves and empirical values: arm circumference-for-age for girls from 3 to 24 months

Figure 403 rd, 10th, 50th, 90th, 97th smoothed centile curves and empirical values: arm circumference-for-age for girls from 24 to 71 months.

Figure $41 \quad 5$ th, 25th, 50th, 75th, 95th smoothed centile curves and empirical values: arm circumference-for-age for girls from 24 to 71 months.

Figure 42 WHO arm circumference-for-age z-scores for girls from 3 to 60 months......

Figure 43

WHO arm circumference-for-age percentiles for girls from 3 to 60 months

Figure 44

Comparison of boys' and girls' arm circumference-for-age z-scores

Figure 45

Worm plots of z-scores for Model 1 for triceps skinfold-for-age for boys

Figure 46

Fitting of the $\mu, \sigma$, and $\nu$ curves of Model 2 for triceps skinfold-for-age for boys from 3 to 71 months (dotted line) and their respective sample estimates (points with solid line).

Figure 47 Centile residuals from fitting Model 2 for triceps skinfold-for-age from 3 to 24 months for boys

Figure 48 Centile residuals from fitting Model 2 for triceps skinfold-for-age from 24 to 71 months for boys

Figure 49 Worm plots of z-scores for Model 2 for triceps skinfold-for-age for boys 
Figure 503 3rd, 10th, 50th, 90th, 97th smoothed centile curves and empirical values: triceps skinfold-for-age for boys from 3 to 24 months

Figure 515 th, 25th, 50th, 75th, 95th smoothed centile curves and empirical values: triceps skinfold-for-age for boys from 3 to 24 months.

Figure 523 rd, 10th, 50th, 90th, 97th smoothed centile curves and empirical values: triceps skinfold-for-age for boys from 24 to 71 months.

Figure 535 th, 25th, 50th, 75th, 95th smoothed centile curves and empirical values: triceps skinfold-for-age for boys from 24 to 71 months.

Figure 54 WHO triceps skinfold-for-age z-scores for boys from 3 to 60 months.

Figure 55 WHO triceps skinfold-for-age percentiles for boys from 3 to 60 months.

Figure 56 Worm plots of z-scores for Model 1 for triceps skinfold-for-age for girls

Figure $57 \quad$ Fitting of the $\mu, \sigma$, and $v$ curves of Model 2 for triceps skinfold-for-age for girls from 3 to 71 months (dotted line) and their respective sample estimates (points with solid line)

Figure 58 Centile residuals from fitting Model 2 for triceps skinfold-for-age from 3 to 24

months for girls 136

Figure 59 Centile residuals from fitting Model 2 for triceps skinfold-for-age from 24 to 71 months for girls

Figure 60 Worm plots of z-scores for Model 2 for triceps skinfold-for-age for girls

Figure 613 rd, 10th, 50th, 90th, 97th smoothed centile curves and empirical values: triceps skinfold-for-age for girls from 3 to 24 months .

Figure 625 th, 25th, 50th, 75th, 95th smoothed centile curves and empirical values: triceps skinfold-for-age for girls from 3 to 24 months

Figure 633 rd, 10th, 50th, 90th, 97th smoothed centile curves and empirical values: triceps skinfold-for-age for girls from 24 to 71 months

Figure 645 th, 25th, 50th, 75th, 95th smoothed centile curves and empirical values: triceps skinfold-for-age for girls from 24 to 71 months

Figure 65

WHO triceps skinfold-for-age z-scores for girls from 3 to 60 months 146

Figure 66

WHO triceps skinfold-for-age percentiles for girls from 3 to 60 months......

Figure 67

Comparison of boys' and girls' triceps skinfold-for-age z-scores

Figure 68

Worm plots of z-scores for Model 1 for subscapular skinfold-for-age for boys.

Figure 69 Fitting of the $\mu, \sigma$, and $v$ curves of Model 2 for subscapular skinfold-for-age for boys from 3 to 71 months (dotted line) and their respective sample estimates (points with solid line)

Figure 70 Centile residuals from fitting Model 2 for subscapular skinfold-for-age from 3 to 24 months for boys.

Figure 71 Centile residuals from fitting Model 2 for subscapular skinfold-for-age from 24 to 71 months for boys.

Figure 72 Worm plots of z-scores for Model 2 for subscapular skinfold-for-age for boys 164

Figure 73 3rd, 10th, 50th, 90th, 97th smoothed centile curves and empirical values: subscapular skinfold-for-age for boys from 3 to 24 months

Figure 74 5th, 25th, 50th, 75th, 95th smoothed centile curves and empirical values: subscapular skinfold-for-age for boys from 3 to 24 months. 
Figure 753 rd, 10th, 50th, 90th, 97th smoothed centile curves and empirical values: subscapular skinfold-for-age for boys from 24 to 71 months

Figure 76 5th, 25th, 50th, 75th, 95th smoothed centile curves and empirical values: subscapular skinfold-for-age for boys from 24 to 71 months ...................................................... 171

Figure 77 WHO subscapular skinfold-for-age z-scores for boys from 3 to 60 months ................ 173

Figure 78 WHO subscapular skinfold-for-age percentiles for boys from 3 to 60 months............ 174

Figure 79 Worm plots of z-scores for Model 1 for subscapular skinfold-for-age for girls ........... 183

Figure $80 \quad$ Fitting of the $\mu, \sigma$, and $v$ curves of Model 2 for subscapular skinfold-for-age for girls from 3 to 71 months (dotted line) and their respective sample estimates (points with solid line) (............................................................................. 186

Figure 81 Centile residuals from fitting Model 2 for subscapular skinfold-for-age from 3 to 24 months for girls

Figure 82 Centile residuals from fitting Model 2 for subscapular skinfold-for-age from 24 to 71

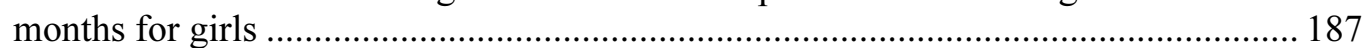

Figure 83 Worm plots of z-scores for Model 2 for subscapular skinfold-for-age for girls .......... 188

Figure 84 Comparison of the fitted growth curves using Model 2 (solid line) with the model adjusting for kurtosis (dashed line) .......................................................................... 191

Figure 853 rd, 10th, 50th, 90th, 97th smoothed centile curves and empirical values: subscapular skinfold-for-age for girls from 3 to 24 months.

Figure 86 5th, 25th, 50th, 75th, 95th smoothed centile curves and empirical values: subscapular skinfold-for-age for girls from 3 to 24 months.............................................................. 195

Figure 87 3rd, 10th, 50th, 90th, 97th smoothed centile curves and empirical values: subscapular skinfold-for-age for girls from 24 to 71 months........................................................ 196

Figure 88 5th, 25th, 50th, 75th, 95th smoothed centile curves and empirical values: subscapular skinfold-for-age for girls from 24 to 71 months......................................................... 197

Figure 89 WHO subscapular skinfold-for-age z-scores for girls from 3 to 60 months ................ 199

Figure 90 WHO subscapular skinfold-for-age percentiles for girls from 3 to 60 months ............ 200

Figure 91 Comparison of boys' and girls' subscapular skinfold-for-age z-score .......................... 208

Figure 92 Examples of children ranked according to the WHO arm circumference-for-age standards 
Tables

Table 1 Number of observations used in the construction of the WHO child growth standards by sex and anthropometric indicator. .5

Table 2 Longitudinal sample sizes for head circumference-for-age for boys ...............................7

Table 3 Cross-sectional sample sizes for head circumference-for-age for boys ............................7

Table 4 Global deviance (GD) for models within the class BCPE $\left(x=\operatorname{age}^{\lambda}, \operatorname{df}(\mu)=9, \operatorname{df}(\sigma)=4\right.$, $v=1, \tau=2$ ) for head circumference-for-age for boys

Table 5 Goodness-of-fit summary for models using the BCPE distribution with fixed $v=1$ and $\tau=2$ for head circumference-for-age for boys.

Table 6 Observed proportions of children with measurements below the fitted centiles from Model 1, head circumference-for-age for boys.

Table 7 Q-test for z-scores from Model $1\left[\operatorname{BCPE}\left(x=\operatorname{age}^{0.20}, \operatorname{df}(\mu)=9, \operatorname{df}(\sigma)=5, v=1, \tau=2\right)\right]$ for

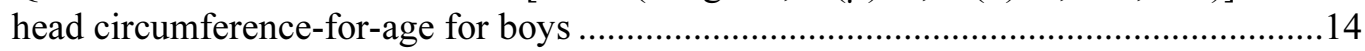

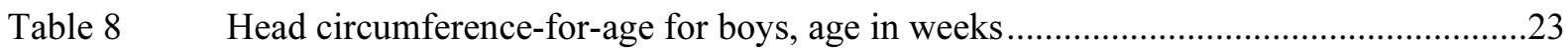

Table 9 Head circumference-for-age for boys, age in years and months ...................................25

Table 10 Longitudinal sample sizes for head circumference-for-age for girls .............................31

Table 11 Cross-sectional sample sizes for head circumference-for-age for girls ..........................31

Table 12 Global deviance (GD) for models within the class BCPE $\left(x=\operatorname{age}^{\lambda}, \operatorname{df}(\mu)=9, \operatorname{df}(\sigma)=4\right.$, $\nu=1, \tau=2$ ) for head circumference-for-age for girls

Table 13 Goodness-of-fit summary for models using the BCPE distribution with fixed $v=1$ and $\tau=2$ for head circumference-for-age for girls

Table 14 Observed proportions of children with measurements below the fitted centiles from Model 1, head circumference-for-age for girls

Table 15 Q-test for z-scores from Model $1\left[\operatorname{BCPE}\left(x=\operatorname{age}^{0.20}, \operatorname{df}(\mu)=9, \operatorname{df}(\sigma)=2, v=1, \tau=2\right)\right]$ for head circumference-for-age for girls

Table 16 Head circumference-for-age for girls, age in weeks ....................................................46

Table 17 Head circumference-for-age for girls, age in years and months .....................................48

Table 18 Longitudinal sample sizes for arm circumference-for-age for boys ................................57

Table 19 Cross-sectional sample sizes for arm circumference-for-age for boys ...........................57

Table 20 Global deviance (GD) for models within the class BCPE $\left(x=\operatorname{age}^{\lambda}, \operatorname{df}(\mu)=9, \operatorname{df}(\sigma)=4\right.$, $\operatorname{df}(v)=4, \tau=2)$ for arm circumference-for-age for boys.

Table 21 Goodness-of-fit summary for models using the BCPE distribution with fixed $v=1$ and $\tau=2$ for arm circumference-for-age for boys . .

Table 22 Q-test for z-scores from Model $1\left[\operatorname{BCPE}\left(x=\operatorname{age}^{0.35}, \operatorname{df}(\mu)=8, \operatorname{df}(\sigma)=4, \nu=1, \tau=2\right)\right]$ for arm circumference-for-age for boys.

Table 23 Goodness-of-fit summary for models BCPE $\left(x=\operatorname{age}^{0.35}, \operatorname{df}(\mu)=8, \operatorname{df}(\sigma)=4, \operatorname{df}(v)=\right.$, $\left.\tau=2\right)$ for arm circumference-for-age for boys....

Table 24 Q-test for z-scores from Model $2\left[\operatorname{BCPE}\left(x=\operatorname{age}^{0.35}, \operatorname{df}(\mu)=7, \operatorname{df}(\sigma)=4, \operatorname{df}(v)=2, \tau=2\right)\right]$

for arm circumference-for-age for boys....

Table 25 Observed proportions of children with measurements below the fitted centiles from

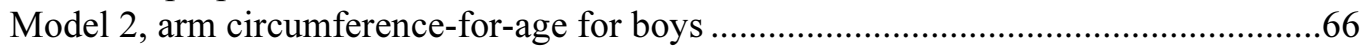

Table 26 Arm circumference-for-age for boys, age in years and months .....................................75 
Table 27 Longitudinal sample sizes for arm circumference-for-age for girls ....

Table 28 Cross-sectional sample sizes for arm circumference-for-age for girls

Table 29 Global deviance (GD) for models within the class $\operatorname{BCPE}\left(x=\operatorname{age}^{\lambda}, \operatorname{df}(\mu)=9, \operatorname{df}(\sigma)=4\right.$, $\operatorname{df}(v)=4, \tau=2)$ for arm circumference-for-age for girls

Table 30 Goodness-of-fit summary for models using the BCPE distribution with fixed $v=1$ and $\tau=2$ for arm circumference-for-age for girls.

Table 31 Q-test for z-scores from Model $1\left[\operatorname{BCPE}\left(x=\operatorname{age}^{0.35}, \operatorname{df}(\mu)=8, \operatorname{df}(\sigma)=4, \nu=1, \tau=2\right)\right]$ for arm circumference-for-age for girls

Table 32 Goodness-of-fit summary for models $\operatorname{BCPE}\left(x=\operatorname{age}^{0.35}, \operatorname{df}(\mu)=8, \operatorname{df}(\sigma)=4, \operatorname{df}(v)=?, \tau=2\right)$

for arm circumference-for-age for girls..

Table 33 Q-test for z-scores from Model $2\left[\operatorname{BCPE}\left(x=\operatorname{age}^{0.35}, \operatorname{df}(\mu)=8, \operatorname{df}(\sigma)=4, \operatorname{df}(v)=1, \tau=2\right)\right]$ for arm circumference-for-age for girls.

Table 34 Observed proportions of children with measurements below the fitted centiles from Model 2, arm circumference-for-age for girls

Table 35 Arm circumference-for-age for girls, age in years and months.

Table 36 Longitudinal sample sizes for triceps skinfold-for-age for boys.....

Table 37 Cross-sectional sample sizes for triceps skinfold-for-age for boys

Table 38 Global deviance (GD) for models within the class BCPE $\left(x=\operatorname{age}^{\lambda}, \operatorname{df}(\mu)=9, \operatorname{df}(\sigma)=4\right.$, $\mathrm{df}(v)=4, \tau=2)$ for triceps skinfold-for-age for boys

Table 39 Goodness-of-fit summary for models using the BCPE distribution with fixed $v=1$ and $\tau=2$ for triceps skinfold-for-age for boys...

Table 40 Q-test for $z$-scores from Model $1\left[\operatorname{BCPE}\left(x=\operatorname{age}^{0.30}, \operatorname{df}(\mu)=7, \operatorname{df}(\sigma)=5, \nu=1, \tau=2\right)\right]$ for triceps skinfold-for-age for boys

Table 41 Goodness-of-fit summary for models $\operatorname{BCPE}\left(x=\operatorname{age}^{0.30}, \operatorname{df}(\mu)=7, \operatorname{df}(\sigma)=5, \operatorname{df}(v)=?, \tau=2\right)$ for triceps skinfold-for-age for boys.

Table 42 Q-test for z-scores from Model $2\left[\operatorname{BCPE}\left(x=\operatorname{age}^{0.30}, \operatorname{df}(\mu)=7, \operatorname{df}(\sigma)=5, \operatorname{df}(v)=2, \tau=2\right)\right]$ for triceps skinfold-for-age for boys.

Table 43 Observed proportions of children with measurements below the fitted centiles from Model 2, triceps skinfold-for-age for boys.

Table $44 \quad$ Triceps skinfold-for-age for boys, age in years and months ...................................... 125

Table 45 Longitudinal sample sizes for triceps skinfold-for-age for girls .................................. 131

Table 46 Cross-sectional sample sizes for triceps skinfold-for-age for girls .............................. 131

Table 47 Global deviance (GD) for models within the class $B C P E\left(x=\operatorname{age}^{\lambda}, \operatorname{df}(\mu)=9, \operatorname{df}(\sigma)=4\right.$, $\operatorname{df}(v)=4, \tau=2)$ for triceps skinfold-for-age for girls

Table 48 Goodness-of-fit summary for models using the BCPE distribution with fixed $v=1$ and $\tau=2$ for triceps skinfold-for-age for girls

Table 49 Q-test for z-scores from Model $1\left[\operatorname{BCPE}\left(x=\operatorname{age}^{0.15}, \operatorname{df}(\mu)=7, \operatorname{df}(\sigma)=5, \nu=1, \tau=2\right)\right]$ for triceps skinfold-for-age for girls.

Table 50 Goodness-of-fit summary for models BCPE $\left(x=\operatorname{age}^{0.15}, \operatorname{df}(\mu)=7, \operatorname{df}(\sigma)=5 \operatorname{df}(v)=\right.$ ?, $\left.\tau=2\right)$ for triceps skinfold-for-age for girls.....

Table 51 Q-test for z-scores from Model $2\left[\operatorname{BCPE}\left(x=\operatorname{age}^{0.15}, \operatorname{df}(\mu)=7, \operatorname{df}(\sigma)=5, \operatorname{df}(v)=3, \tau=2\right)\right]$ for triceps skinfold-for-age for girls. 
Table 52 Observed proportions of children with measurements below the fitted centiles from Model 2, triceps skinfold-for-age for girls

Table 53 Triceps skinfold-for-age for girls, age in years and months.

Table 54 Longitudinal sample sizes for subscapular skinfold-for-age for boys

Table 55 Cross-sectional sample sizes for subscapular skinfold-for-age for boys

Table 56 Global deviance (GD) for models within the class BCPE $\left(x=\operatorname{age}^{\lambda}, \operatorname{df}(\mu)=9, \operatorname{df}(\sigma)=4\right.$, $\operatorname{df}(v)=4, \tau=2)$ for subscapular skinfold-for-age for boys.

Table 57 Goodness-of-fit summary for models using the BCPE distribution with fixed $v=1$ and $\tau=2$ for subscapular skinfold-for-age for boys

Table 58 Q-test for z-scores from Model $1\left[\operatorname{BCPE}\left(x=\operatorname{age}^{0.65}, \operatorname{df}(\mu)=6, \operatorname{df}(\sigma)=3, v=1, \tau=2\right)\right]$ for subscapular skinfold-for-age for boys

Table 59 Goodness-of-fit summary for models BCPE $\left(x=a g e^{0.65}, \operatorname{df}(\mu)=6, \operatorname{df}(\sigma)=3, \operatorname{df}(v)=\right.$, $\tau=2$ ) for subscapular skinfold-for-age for boys

Table 60 Goodness-of-fit summary for models using the BCPE distribution with $\mathrm{df}(v)=2$ and $\tau=2$ for subscapular skinfold-for-age for boys

Table 61 Q-test for z-scores from Model $2\left[\operatorname{BCPE}\left(x=\operatorname{age}^{0.65}, \operatorname{df}(\mu)=6, \operatorname{df}(\sigma)=2, \operatorname{df}(v)=2, \tau=2\right)\right]$ for subscapular skinfold-for-age for boys

Table 62 Observed proportions of children with measurements below the fitted centiles from Model 2, subscapular skinfold-for-age for boys .

Table 63 Subscapular skinfold-for-age for boys, age in years and months. 175

Table 64 Longitudinal sample sizes for subscapular skinfold-for-age for girls.....

Table 65 Cross-sectional sample sizes for subscapular skinfold-for-age for girls

Table 66

Global deviance (GD) for models within the class BCPE $\left(x=\operatorname{age}^{\lambda}, \operatorname{df}(\mu)=9, \operatorname{df}(\sigma)=4\right.$, $\mathrm{df}(v)=4, \tau=2)$ for subscapular skinfold-for-age for girls .

Table 67 Goodness-of-fit summary for models using the BCPE distribution with fixed $v=1$ and $\tau=2$ for subscapular skinfold-for-age for girls

Table 68 Q-test for z-scores from Model $1\left[\operatorname{BCPE}\left(x=\operatorname{age}^{0.50}, \operatorname{df}(\mu)=5, \operatorname{df}(\sigma)=4, v=1, \tau=2\right)\right]$ for subscapular skinfold-for-age for girls

Table 69 Goodness-of-fit summary for models BCPE $\left(x=\operatorname{age}^{0.50}, \operatorname{df}(\mu)=5, \operatorname{df}(\sigma)=4, \operatorname{df}(v)=\right.$ ?, $\tau=2$ ) for subscapular skinfold-for-age for girls

Table 70 Goodness-of-fit summary for models using the BCPE distribution with $\lambda=0.15$, $\operatorname{df}(v)=2$ and $\tau=2$ for subscapular skinfold-for-age for girls

Table 71 Q-test for z-scores from Model $2\left[\operatorname{BCPE}\left(x=\operatorname{age}^{0.15}, \operatorname{df}(\mu)=5 \operatorname{df}(\sigma)=4, \operatorname{df}(v)=2, \tau=2\right)\right]$ for subscapular skinfold-for-age for girls

Table 72 Goodness-of-fit summary for models BCPE $\left(x=a g e^{0.15}, \operatorname{df}(\mu)=5, \operatorname{df}(\sigma)=4, \operatorname{df}(v)=2\right.$, $\operatorname{df}(\tau)=$ ?) for subscapular skinfold-for-age for girls.

Table 73 Observed proportions of children with measurements below the fitted centiles from Model 2, subscapular skinfold-for-age for girls

Table 74 Subscapular skinfold-for-age for girls, age in years and months 


\section{Glossary}

\section{BCPE}

$\mu$

$\sigma$

$\tau$

$\lambda$

Body mass index (BMI)

Box-Cox transformation

Coefficient of variation

Cubic spline

Cut-off

Degrees of freedom (df)

Kurtosis

P-value

Q-test

Skewness

Standard deviation score (SD)

Worm plots

Z-score
The Box-Cox power exponential distribution.

The median of the Box-Cox power exponential distribution.

The approximate coefficient of variation of the Box-Cox power exponential distribution - related to the variance.

The power of the Box-Cox transformation (to the normal distribution) of the Box-Cox power exponential distribution related to the skewness.

The power exponential parameter of the Box-Cox power exponential distribution - related to the kurtosis.

The power of the age (or length/height) transformation.

The ratio weight (in $\mathrm{kg}$ ) / recumbent length or standing height (in $\mathrm{m}^{2}$ ).

A power transformation to the normal distribution.

The ratio of the standard deviation to the mean.

A piecewise third-order polynomial function that passes through a set of $m$ (or degrees of freedom) control points; it can have a very simple form locally, yet be globally flexible and smooth.

A designated limit beyond which a subject or observation is classified according to a pre-set condition.

The number of control points used to fit the cubic splines.

An attribute of a distribution describing "peakedness". A high kurtosis portrays a distribution with fat tails in contrast to a low kurtosis, which portrays a distribution with skinny tails.

The probability of falsely rejecting the hypothesis being tested. In this report all $p$-values were compared to a level of significance set to 0.05 .

A statistical test which combines overall and local tests assessing departures from the normal distribution with respect to median, variance, skewness and kurtosis.

A statistical term used to describe a distribution's asymmetry in relation to a normal distribution.

See z-score.

A set of detrended Q-Q plots - plots that compare the distribution of a given set of observations to the normal distribution.

The deviation of an individual's value from the median value of a reference population, divided by the standard deviation of the reference population (or transformed to normal distribution). 


\section{Executive summary}

In 1993 the World Health Organization (WHO) undertook a comprehensive review of the uses and interpretation of anthropometric references. The review concluded that the NCHS/WHO growth reference, which had been recommended for international use since the late 1970s, did not adequately represent early childhood growth and that new growth curves were necessary. The World Health Assembly endorsed this recommendation in 1994. In response WHO undertook the Multicentre Growth Reference Study (MGRS) between 1997 and 2003 to generate new curves for assessing the growth and development of children the world over.

The MGRS combined a longitudinal follow-up from birth to 24 months and a cross-sectional survey of children aged 18 to 71 months. Primary growth data and related information were gathered from 8440 healthy breastfed infants and young children from widely diverse ethnic backgrounds and cultural settings (Brazil, Ghana, India, Norway, Oman and USA). The MGRS is unique in that it was purposely designed to produce a standard by selecting healthy children living under conditions likely to favour the achievement of their full genetic growth potential. Furthermore, the mothers of the children selected for the construction of the standards engaged in fundamental health-promoting practices, namely breastfeeding and not smoking.

This report presents the second set of WHO Child Growth Standards (i.e. head circumference-for-age, arm circumference-for-age, triceps skinfold-for-age and subscapular skinfold-for-age) and describes the methodical process followed in their development. The first step in this process was a consultative expert review of some 30 growth curve construction methods, including types of distributions and smoothing techniques to identify the best approach to constructing the standards. Next was the selection of a software package flexible enough to allow the comparative testing of the alternative methods used to generate the growth curves. Then the selected approach was applied systematically to search for the best models to fit the data for each indicator.

The Box-Cox-power-exponential (BCPE) method, with curve smoothing by cubic splines was selected for constructing the WHO child growth curves. The BCPE accommodates various kinds of distributions, from normal to skewed or kurtotic. The age-based indicators originating at birth required a power-transformation to stretch the age scale (x-axis) as a preliminary step to fitting the curves. For each set of curves, the search for the best model specification began by examining various combinations of degrees of freedom to fit the median and variance estimator curves. When data had a non-normal distribution, degrees of freedom for parameters to model skewness and kurtosis were added to the initial model and adequacy of fit evaluated. Apart from head circumference-for-age, which followed a normal distribution, the other standards in the second set required the modelling of skewness, but not kurtosis. The diagnostic tools used iteratively to detect possible model misfits and biases in the fitted curves included various tests of local and global goodness of fit, worm plots and residual plots. Patterns of differences between empirical and fitted percentiles were also examined, as were proportions of observed versus expected percentages of children with measurements below selected percentiles.

The sample used for the construction of the second set of growth standards was the same one used for the construction of the first set of standards. The methodology described above was followed to generate - for boys and girls - percentile and z-score curves for head circumference-for-age ( 0 to 60 months), arm circumference-for-age (3 to 60 months), triceps skinfold-for-age (3 to 60 months) and subscapular skinfold-for-age (3 to 60 months).

The data of the longitudinal and cross-sectional samples were merged without any adjustments and a single model was fitted to generate one continuous set of curves constituting each sex-specific standard. 
Head circumference followed a normal distribution. The data for arm circumference and skinfold thicknesses were skewed, so in specifying the model, the parameter related to skewness was fitted in addition to the median and the coefficient of variation. Results from the final model for girls' subscapular skinfold suggested the need to investigate potential improvements in the curves by modelling kurtosis. Adjustment for kurtosis, however had a negligible impact on the final centiles. Therefore, considering that modelling the fourth parameter would increase complexity in application of the standards and create inconsistency between the sexes, the final curves were generated without adjusting for kurtosis.

The power transformation of age was applied to stretch the age scale for each of the sexes before fitting cubic splines to generate the growth curves. The same power transformation of age was applied to both boys' and girls' head and arm circumferences. For the skinfold thicknesses, boys required a higher power transformation than did girls.

Cubic spline fitting was achieved with variable degrees of freedom for each indicator and sex. For the median curves, different degrees of freedom were required for boys and girls for arm circumference and subscapular skinfold. For the coefficient of variation curves, the degrees of freedom varied between sexes for head circumference and subscapular skinfold. For the indicators that required fitting skewness, all but the subscapular skinfold required different degrees of freedom for the parameter modelling skewness.

Overall, concordance between smoothed curves and empirical centiles was free of bias in both the median range and the tails, indicating that the resulting curves provide an adequate description of the true growth of healthy children.

The method used to construct the WHO standards generally relied on the Box-Cox power exponential distribution and the final selected models simplified to the LMS model. As a result, the computation of percentiles and z-scores for these standards uses formulae based on the LMS method. However, as was done for the construction of the first set of growth standards, a restriction was imposed on all indicators to enable the derivation of percentiles only within the interval corresponding to z-scores between -3 and 3 . The underlying reasoning is that percentiles beyond $\pm 3 \mathrm{SD}$ are invariant to changes in equivalent z-scores. The loss accruing to this restriction is small since the inclusion range corresponds to the 0.135 th to 99.865 th percentiles.

The arm circumference and skinfold thicknesses presented right-skewed distributions. When modelled correctly, right skewness has the effect of making distances between positive z-scores increase progressively the farther away they are from the median, while distances between negative z-scores decrease progressively. The LMS method fits skewed data adequately by using a Box-Cox normal distribution, which follows the empirical data closely. The drawback, however, is that the outer tails of the distribution are highly affected by extreme data points even if only very few. A restricted application of the LMS method was thus used for the construction of the indicators with skewed distributions, limiting the Box-Cox normal distribution to the interval corresponding to z-scores where empirical data were available (i.e. between -3 SD and 3 SD). Beyond these limits, the standard deviation at each age was fixed to the distance between $\pm 2 \mathrm{SD}$ and $\pm 3 \mathrm{SD}$, respectively. This approach avoids making assumptions about the distribution of data beyond the limits of the observed values.

All four indicators presented in this report are a new addition to the previously available set of indicators in the NCHS/WHO reference. Head circumference-for-age is often used in clinical settings as part of health screening for potential developmental or neurological disabilities in infants and young children. Very small and very large circumferences are both indicative of health or developmental risk. Arm circumference-for-age is used as an alternative indicator of nutritional status when the collection of length/height and weight measurements is difficult, as happens in emergency humanitarian situations due to famine or refugee crises. Triceps and subscapular skinfold measurements assess the 
thickness of subcutaneous tissue and reflect fatness primarily. The skinfold indicators are thus a useful addition to the battery of growth standards for assessing childhood obesity.

The WHO Child Growth Standards provide a technically robust set of tool that represents the best description of physiological growth for children under five years of age. The standards depict normal early childhood growth under optimal environmental conditions and can be used to assess children everywhere, regardless of ethnicity, socioeconomic status and type of feeding. 



\section{INTRODUCTION}

Growth charts are an essential component of the paediatric toolkit. Their value resides in helping to determine the degree to which physiological needs for growth and development are met during the important childhood period. Beyond their usefulness in assessing children's nutritional status, many governmental and United Nations agencies rely on growth charts to measure the general well-being of populations, formulate health and related policies, and plan interventions and monitor their effectiveness.

The origin of the WHO Child Growth Standards dates back to the early 1990s when a group of experts was appointed to conduct a meticulous evaluation of the National Center for Health Statistics/World Health Organization (NCHS/WHO) growth reference that had been recommended for international use since the late 1970s (WHO, 1995). The limitations of the NCHS/WHO reference have been documented (WHO Working Group on Infant Growth, 1994; de Onis and Habicht, 1996; de Onis and Yip, 1996). The data used to construct the reference covering birth to three years of age came from a longitudinal study of children of European ancestry from a single community in the USA. These children were measured every three months, which is inadequate to describe the rapid and changing rate of growth in early infancy. Also, the statistical methods available at the time the NCHS/WHO growth curves were constructed were too limited to correctly model the pattern and variability of growth. As a result, the NCHS/WHO curves do not adequately represent early childhood growth.

The initial phase of the expert group's work documented the deficiencies of the reference and led to a plan for developing new growth charts that would show how children should grow in all countries rather than merely describing how they grew at a particular time and place. The experts underscored the importance of ensuring that the new growth charts were consistent with "best" health practices (Garza and de Onis, 2004).

A logical outcome of this plan was the WHO Multicentre Growth Reference Study (MGRS), which was implemented between 1997 and 2003 (de Onis et al., 2004a). The MGRS is unique in that it was purposely designed to produce a standard rather than a reference. Although standards and references both serve as a basis for comparison, each enables a different interpretation. Since a standard defines how children should grow, deviations from the pattern it describes are evidence of abnormal growth. A reference, on the other hand, does not provide as sound a basis for such value judgments, although in practice references often are mistakenly used as standards.

The MGRS data provide a solid foundation for developing a standard because they are based on healthy children living under conditions likely to favour achievement of their full genetic growth potential. Furthermore, the mothers of the children selected for the construction of the standards engaged in fundamental health-promoting practices, namely breastfeeding and not smoking (de Onis et al., 2004b).

A second feature of the study that makes it attractive as a basis for an internationally applicable standard is that it included children from a diverse set of countries: Brazil, Ghana, India, Norway, Oman and the USA. By selecting privileged, healthy populations the study reduced the impact of environmental variation (WHO Multicentre Growth Reference Study Group, 2006a). Another key characteristic of the new standards is that they explicitly identify breastfeeding as the biological norm and establish the breastfed child as the normative model for growth and development (WHO Multicentre Growth Reference Study Group, 2006b). In addition, the new standards include windows of achievement for six gross motor developmental milestones which are presented elsewhere (WHO Multicentre Growth Reference Study Group, 2006c). Although WHO in the past issued recommendations concerning attained physical growth, it had not previously made any recommendations for assessing motor development. 
This report presents the second set of WHO Child Growth Standards and describes the methods used to construct the standards for head circumference-for-age, arm circumference-for-age, triceps skinfoldfor-age and subscapular skinfold-for-age. The standards based on length or height, weight and age are presented in an earlier publication (WHO Multicentre Growth Reference Study Group, 2006d; Web site www.who.int/childgrowth/publications/technical report pub/en/index.html). Electronic copies of the WHO growth charts and tables together with tools developed to facilitate their use are available on the Web: www.who.int/childgrowth/en. 


\section{METHODOLOGY}

\subsection{Design of the WHO Multicentre Growth Reference Study}

The Multicentre Growth Reference Study (MGRS) (July 1997-December 2003) was a populationbased study that took place in the cities of Davis, California, USA; Muscat, Oman; Oslo, Norway; and Pelotas, Brazil; and in selected affluent neighbourhoods of Accra, Ghana and South Delhi, India. The MGRS protocol and its implementation in the six sites are described in detail elsewhere (de Onis et al., 2004a). Briefly, the MGRS combined a longitudinal component from birth to 24 months with a crosssectional component of children aged 18-71 months. In the longitudinal component, mothers and newborns were screened and enrolled at birth and visited at home a total of 21 times on weeks 1, 2, 4 and 6; monthly from 2-12 months; and bimonthly in the second year. In the cross-sectional component, children aged 18-71 months were measured once, except in the two sites (Brazil and USA) that used a mixed-longitudinal design in which some children were measured two or three times at three-month intervals. Both recumbent length and standing height were measured for all children aged 18-30 months. Data were collected on anthropometry, motor development, feeding practices, child morbidity, perinatal factors, and socioeconomic, demographic and environmental characteristics (de Onis et al., 2004b).

The study populations lived in socioeconomic conditions favourable to growth and where mobility was low, $\geq 20 \%$ of mothers followed WHO feeding recommendations and breastfeeding support was available (de Onis et al., 2004b). Individual inclusion criteria were: no known health or environmental constraints to growth, mothers willing to follow MGRS feeding recommendations (i.e. exclusive or predominant breastfeeding for at least 4 months, introduction of complementary foods by the age of 6 months, and continued partial breastfeeding up to at least 12 months), no maternal smoking before and after delivery, single term birth, and absence of significant morbidity (de Onis et al., 2004b).

As part of the site-selection process in Ghana, India and Oman, surveys were conducted to identify socioeconomic characteristics that could be used to select groups whose growth was not environmentally constrained (Owusu et al., 2004; Bhandari et al., 2002; Mohamed et al., 2004). Local criteria for screening newborns, based on parental education and/or income levels, were developed from those surveys. Pre-existing survey data for this purpose were available from Brazil, Norway and the USA. Of the 13741 mother-infant pairs screened for the longitudinal component, about $83 \%$ were ineligible (WHO Multicentre Growth Reference Study Group, 2006e). Families' low socioeconomic status was the most common reason for ineligibility in Brazil, Ghana, India and Oman, whereas parental refusal was the main reason for non-participation in Norway and the USA (WHO Multicentre Growth Reference Study Group, 2006e). For the cross-sectional component, 69\% of the 21510 subjects screened were excluded for reasons similar to those observed in the longitudinal component.

Term low-birth-weight $(<2500 \mathrm{~g})$ infants $(2.3 \%)$ were not excluded. Since it is likely that in well-off populations such infants represent small but normal children, their exclusion would have artificially distorted the standards' lower percentiles. Eligibility criteria for the cross-sectional component were the same as those for the longitudinal component with the exception of infant feeding practices. A minimum of three months of any breastfeeding was required for participants in the study's crosssectional component.

\subsection{Anthropometry methods}

Data collection teams were trained at each site during the study's preparatory phase, at which time measurement techniques were standardized against one of two MGRS anthropometry experts. During the study, bimonthly standardization sessions were conducted at each site. Once a year the anthropometry expert visited each site to participate in these sessions (de Onis et al., 2004c). Results from the anthropometry standardization sessions have been reported elsewhere (WHO Multicentre 
Growth Reference Study Group, 2006f). For the longitudinal component of the study, screening teams measured newborns within 24 hours of delivery, and follow-up teams conducted home visits until 24 months of age. The follow-up teams were also responsible for taking measurements in the crosssectional component involving children aged 18-71 months (de Onis et al., 2004b). The MGRS data included weight and head circumference at all ages, recumbent length (longitudinal component), height (cross-sectional component), and arm circumference, triceps and subscapular skinfolds (all children aged $\geq 3$ months). This report presents only the standards based on head circumference, arm circumference and skinfolds. The standards based on length or height and weight are presented in an earlier publication (WHO Multicentre Growth Reference Study Group, 2006d; web site http://www.who.int/childgrowth/publications/technical_report_pub/en/index.html).

Observers working in pairs collected anthropometric data. Each observer independently measured and recorded a complete set of measurements, after which the two compared their readings. If any pair of readings exceeded the maximum allowable difference for a given variable (e.g. head circumference, $5 \mathrm{~mm}$; arm circumference, $5 \mathrm{~mm}$; skinfold thickness, $2 \mathrm{~mm}$ ), both observers once again independently measured and recorded a second and, if necessary, a third set of readings for the variable(s) in question (de Onis et al., 2004c).

All study sites used identical measuring equipment. Instruments needed to be highly accurate and precise. A self-retracting, $0.7 \mathrm{~cm}$-wide, flat metal tape with blank lead-in strip (range, 0-200 cm, calibrated to $1 \mathrm{~mm}$ ), was used to measure circumferences. Metal tapes were chosen because they are more robust and accurate, and stay in a single plane around the head. They were replaced on a regular basis when the grading marks faded. The Holtain/Tanner-Whitehouse skinfold caliper (jaw face area, $35 \mathrm{~mm}^{2}$; pressure between the jaws, $10 \pm 2 \mathrm{~g} / \mathrm{mm}^{2}$; range, $0-40 \mathrm{~mm}$; calibrated to $0.2 \mathrm{~mm}$ ) was used to measure skinfolds. The skinfold calipers, being particularly fragile, were checked before each use with calibration blocks of various widths for accuracy and to ensure that the needle moved smoothly and continuously with the opening of the caliper jaws. Full details of the instruments used and how measurements were taken are provided elsewhere (de Onis et al., 2004c).

\subsection{Sample description}

The total sample size for the longitudinal and cross-sectional components from all six sites was 8440 children. A total of 1743 children were enrolled in the longitudinal sample, six of whom were excluded for morbidities affecting growth (4 cases of repeated episodes of diarrhoea, 1 case of repeated episodes of malaria, and 1 case of protein-energy malnutrition) leaving a sample of 1737 children ( 894 boys and 843 girls). Of these, the mothers of 882 children ( 428 boys and 454 girls) complied fully with the MGRS infant-feeding and no-smoking criteria and completed the follow-up period of 24 months (96\% of compliant children completed the 24-month follow-up). The other 855 either failed to comply with the study's infant-feeding and no-smoking criteria or dropped out before 24 months. These children, whose size at birth was similar to that of the compliant sample, contributed only birth measurements. The increased sample size at birth served to minimize the left-edge effect in the head circumference-for-age curves. For arm circumference and the skinfolds, which were measured starting at age 3 months, the data did not allow for this correction in the corresponding standards. The total number of records for the longitudinal component was 19900.

The cross-sectional sample comprised 6697 children. Of these, 28 were excluded for medical conditions affecting growth (20 cases of protein-energy malnutrition, five cases of haemolytic anaemia G6PD deficiency, two cases of renal tubulo-interstitial disease, and one case of Crohn disease) leaving a final sample of 6669 children ( 3450 boys and 3219 girls). The total number of records in the crosssectional component was 8306 as some children in Brazil and the USA were measured two or three times at three-month intervals. A full description of the MGRS sample with regard to screening, 
recruitment, sample attrition and compliance, as well as the baseline characteristics of the study sample is provided elsewhere (WHO Multicentre Growth Reference Study Group, 2006e).

\subsection{Data cleaning procedures and exclusions}

\section{Data cleaning}

The MGRS data management protocol (Onyango et al., 2004) was designed to create and manage a large databank of information collected from multiple sites over a period of several years. Data collection and processing instruments were prepared centrally and used in a standardized fashion across sites. The data management system contained internal validation features for timely detection of data errors and its standard operating procedures stipulated a method of master file updating and correction that maintained a clear trail for data-auditing purposes. Each site was responsible for collecting, entering, verifying and validating data, and for creating site-level master files. Data from the sites were sent to $\mathrm{WHO} / \mathrm{HQ}$ every month for master file consolidation and more extensive quality control checking. All errors identified were communicated to the site for correction at source.

After data collection was completed at a given site, a period of about 6 months was dedicated to indepth data quality checking and master file cleaning. Detailed validation reports, descriptive statistics and plots were produced from the site's master files. For the longitudinal component, each anthropometric measurement was plotted for every child from birth to the end of his/her participation. These plots were examined individually for any questionable patterns. Query lists from these analyses were sent to the site for investigation and correction, or confirmation, as required. As with the data collection process, the site data manager prepared correction batches to update the master files. The updated master files were then sent to $\mathrm{WHO} / \mathrm{HQ}$ and this iterative quality assurance process continued until all identifiable problems had been detected and corrected. The rigorous implementation of what was a highly demanding protocol yielded very high-quality data.

\section{Data exclusions}

In addition to exclusions of data based on weight-for-height (WHO Multicentre Growth Reference Study Group, 2006d), a few influential observations outside \pm 4 SD were excluded when constructing the individual standards included in this report. These were, for head circumference-for-age: boys, all 21 observations belonging to one boy in the longitudinal sample $(0.15 \%)$ and one single observation $(0.01 \%)$ among girls; for triceps skinfold-for-age: boys, $7(0.06 \%)$ and girls, $9(0.08 \%)$; for subscapular skinfold-for-age: boys, $17(0.16 \%)$ and girls, $19(0.17 \%)$ observations. These observations were set to missing in the final data set and therefore did not contribute to the construction of the corresponding standards. The final number of observations used in the construction of the WHO child growth standards is shown in Table 1.

Table 1 Number of observations used in the construction of the WHO child growth standards by sex and anthropometric indicator

\begin{tabular}{lccc}
\hline Indicator & Girls & Boys & Total \\
\hline Head circumference-for-age & 13798 & 13541 & 27339 \\
Arm circumference-for-age & 10970 & 10770 & 21740 \\
Triceps skinfold-for-age & 10943 & 10762 & 21705 \\
Subscapular skinfold-for-age & 10934 & 10757 & 21691 \\
\hline
\end{tabular}




\subsection{Statistical methods for constructing the growth curves}

The underlying methodology used for constructing the head circumference-for-age, arm circumference -for-age, triceps skinfold-for-age and subscapular skinfold-for-age standards was the same used to construct the standards for length/height-for-age, weight-for-age, weight-for-length, weight-for-height and body mass index-for-age. The growth curve fitting method and diagnostic tools used to select the best models for each of the indicators are described in detail in the report of the first set of standards (WHO Multicentre Growth Reference Study Group, 2006d, 2006g).

The Box-Cox-power-exponential (BCPE) method (Rigby and Stasinopoulos, 2004), with curve smoothing by cubic splines was selected for constructing the WHO child growth curves. The BCPE accommodates various kinds of distributions, from normal to skewed or kurtotic. All the indicators in this second set standards required a power-transformation to stretch the age scale (x-axis) as a preliminary step to fitting the curves. For each set of curves, the search for the best model specification began by examining various combinations of degrees of freedom to fit the median and variance estimator curves. When data had a non-normal distribution, degrees of freedom for parameters to model skewness and kurtosis were added to the initial model and adequacy of fit was evaluated. Apart from head circumference-for-age, which followed a normal distribution, the other standards required the modelling of skewness, but not kurtosis. The diagnostic tools used iteratively to detect possible model misfits and biases in the fitted curves included various tests of local and global goodness of fit, like Q-tests (Royston and Wright, 2000), worm plots (van Buuren and Fredriks, 2001) and residual plots. Patterns of differences between empirical and fitted percentiles were also examined, as were proportions of observed versus expected percentages of children with measurements below selected percentiles. The curves were constructed using all available data (i.e. from birth to 71 months) but the final standards were truncated at 60 completed months to avoid the right-edge effect (Borghi et al., 2006).

The GAMLSS package (Stasinopoulos et al., 2004) was used for the construction of the growth curves. 


\section{CONSTRUCTION OF THE HEAD CIRCUMFERENCE-FOR-AGE STANDARDS}

\subsection{Indicator-specific methodology}

The same approach as that described to select the best model for the length/height-for-age growth curves (WHO Multicentre Growth Reference Study Group, 2006d) was followed to select the best model to construct the head circumference-for-age growth standards. The BCPE method was used and, starting from the simplest model (i.e. with the normal distribution), the best model was sought and its goodness of fit evaluated. The diagnostic tools applied to evaluate and compare candidate models were the same as those used for constructing the first set of indicators. All data up to 71 months were used for modelling the head circumference-for-age growth curves and the standards afterwards truncated at 60 completed months to correct for the right-edge effect (Borghi et al., 2006).

\subsection{Head circumference-for-age for boys}

\subsubsection{Sample size}

There were 13541 head circumference observations for boys. The longitudinal and cross-sectional sample sizes by visit and age are shown in Tables 2 and 3 .

Table 2 Longitudinal sample sizes for head circumference-for-age for boys

\begin{tabular}{lccccccc}
\hline Visit & Birth & $\mathbf{1}$ & $\mathbf{2}$ & $\mathbf{3}$ & $\mathbf{4}$ & $\mathbf{5}$ & $\mathbf{6}$ \\
Age & $\mathbf{0}$ & $\mathbf{2} \mathbf{~ w k}$ & $\mathbf{4} \mathbf{w k}$ & $\mathbf{6} \mathbf{~ w k}$ & $\mathbf{2} \mathbf{~ m o}$ & $\mathbf{3} \mathbf{~ m o}$ & $\mathbf{4} \mathbf{~ m o}$ \\
$\mathrm{N}$ & 893 & 424 & 423 & 423 & 422 & 418 & 417 \\
\hline Visit & $\mathbf{7}$ & $\mathbf{8}$ & $\mathbf{9}$ & $\mathbf{1 0}$ & $\mathbf{1 1}$ & $\mathbf{1 2}$ & $\mathbf{1 3}$ \\
Age & $\mathbf{5} \mathbf{~ m o}$ & $\mathbf{6} \mathbf{~ m o}$ & $\mathbf{7} \mathbf{~ m o}$ & $\mathbf{8} \mathbf{~ m o}$ & $\mathbf{9} \mathbf{~ m o}$ & $\mathbf{1 0} \mathbf{~ m o}$ & $\mathbf{1 1} \mathbf{~ m o}$ \\
$\mathrm{N}$ & 419 & 422 & 418 & 418 & 413 & 409 & 420 \\
\hline Visit & $\mathbf{1 4}$ & $\mathbf{1 5}$ & $\mathbf{1 6}$ & $\mathbf{1 7}$ & $\mathbf{1 8}$ & $\mathbf{1 9}$ & $\mathbf{2 0}$ \\
Age & $\mathbf{1 2} \mathbf{~ m o}$ & $\mathbf{1 4} \mathbf{~ m o}$ & $\mathbf{1 6} \mathbf{~ m o}$ & $\mathbf{1 8} \mathbf{~ m o}$ & $\mathbf{2 0} \mathbf{~ m o}$ & $\mathbf{2 2} \mathbf{~ m o}$ & $\mathbf{2 4} \mathbf{~ m o}$ \\
$\mathrm{N}$ & 420 & 418 & 417 & 416 & 422 & 416 & 421 \\
\hline
\end{tabular}

Table 3 Cross-sectional sample sizes for head circumference-for-age for boys

\begin{tabular}{lccccccc}
\hline Age (mo) & $<\mathbf{1 8}$ & $\mathbf{1 8}-\mathbf{2 0}$ & $\mathbf{2 1 - 2 3}$ & $\mathbf{2 4 - 2 6}$ & $\mathbf{2 7 - 2 9}$ & $\mathbf{3 0 - 3 2}$ & $\mathbf{3 3 - 3 5}$ \\
$\mathrm{N}$ & 3 & 184 & 191 & 244 & 267 & 229 & 261 \\
\hline Age (mo) & $\mathbf{3 6 - 3 8}$ & $\mathbf{3 9 - 4 1}$ & $\mathbf{4 2 - 4 4}$ & $\mathbf{4 5 - 4 7}$ & $\mathbf{4 8}-\mathbf{5 0}$ & $\mathbf{5 1 - 5 3}$ & $\mathbf{5 4 - 5 6}$ \\
$\mathrm{N}$ & 274 & 254 & 263 & 244 & 245 & 229 & 233 \\
\hline Age (mo) & $\mathbf{5 7 - 5 9}$ & $\mathbf{6 0 - 6 2}$ & $\mathbf{6 3 - 6 5}$ & $\mathbf{6 6 - 6 8}$ & $\mathbf{6 9 - 7 1}$ & $>\mathbf{7 1}$ & \\
$\mathrm{N}$ & 245 & 236 & 221 & 224 & 221 & 4 & \\
\hline
\end{tabular}

\subsubsection{Model selection and results}

The model BCPE $\left(x=\operatorname{age}^{\lambda}, \operatorname{df}(\mu)=9, \operatorname{df}(\sigma)=4, v=1, \tau=2\right)$ served as a starting point to construct the head circumference-for-age growth curves. Improvement of the model's fit was investigated by studying changes in global deviance at varying levels of the age-transformation power $\lambda$. Table 4 shows the global deviance for a grid of $\lambda$ values. The smallest global deviance corresponded to agetransformation power $\lambda=0.20$. 
Table 4 Global deviance (GD) for models within the class $\operatorname{BCPE}\left(x=\operatorname{age}^{\lambda}, \operatorname{df}(\mu)=9, \operatorname{df}(\sigma)=4, v=1\right.$, $\tau=2$ ) for head circumference-for-age for boys

\begin{tabular}{lcccccccccc}
\hline $\boldsymbol{\lambda}$ & $\mathbf{0 . 0 5}$ & $\mathbf{0 . 1 0}$ & $\mathbf{0 . 1 5}$ & $\mathbf{0 . 2 0}$ & $\mathbf{0 . 2 5}$ & $\mathbf{0 . 3 0}$ & $\mathbf{0 . 3 5}$ & $\mathbf{0 . 4 0}$ & $\mathbf{0 . 4 5}$ & $\mathbf{0 . 5 0}$ \\
$\mathrm{GD}^{\mathrm{a}}$ & 735.7 & 735.9 & 735.8 & 735.5 & 735.9 & 738.6 & 744.9 & 755.6 & 769.6 & 783.6 \\
\hline $\boldsymbol{\lambda}$ & $\mathbf{0 . 5 5}$ & $\mathbf{0 . 6 0}$ & $\mathbf{0 . 6 5}$ & $\mathbf{0 . 7 0}$ & $\mathbf{0 . 7 5}$ & $\mathbf{0 . 8 0}$ & $\mathbf{0 . 8 5}$ & $\mathbf{0 . 9 0}$ & $\mathbf{0 . 9 5}$ & $\mathbf{1 . 0 0}$ \\
$\mathrm{GD}^{\mathrm{a}}$ & 793.4 & 795.7 & 791.3 & 786.9 & 795.4 & 835.5 & 929.0 & $\mathrm{NA}$ & $\mathrm{NA}$ & $\mathrm{NA}$ \\
\hline
\end{tabular}

${ }^{a}$ In excess of 45000 ; NA: not available (method did not converge with this power).

Having chosen the age-transformation power $\lambda=0.20$, the search for the best $\operatorname{df}(\mu)$ and $\operatorname{df}(\sigma)$ followed, comparing models in which the parameters $v$ and $\tau$ had the fixed values 1 and 2, respectively, i.e. adjusting neither for skewness nor for kurtosis. For this, all possible combinations of $\mathrm{df}(\mu)$ ranging from 5 to 15 and $\operatorname{df}(\sigma)$ from 2 to 10 were considered. Partial results are presented in Table 5.

Table 5 Goodness-of-fit summary for models using the BCPE distribution with fixed $v=1$ and $\tau=2$ for head circumference-for-age for boys

\begin{tabular}{cccccc}
\hline $\mathbf{d f}(\boldsymbol{\mu})$ & $\mathbf{d f}(\boldsymbol{\sigma})$ & $\mathbf{G D}^{\mathbf{a}}$ & $\mathbf{A I C}^{\mathbf{a}}$ & GAIC(3) $^{\mathbf{a}}$ & Total df \\
\hline \multirow{4}{*}{$\mathbf{7}$} & $\mathbf{3}$ & 758.7 & 778.7 & 788.7 & 10 \\
& $\mathbf{4}$ & 747.4 & 769.4 & 780.4 & 11 \\
& $\mathbf{5}$ & 743.6 & 767.6 & 779.6 & 12 \\
& $\mathbf{6}$ & 741.9 & 767.9 & 780.9 & 13 \\
& $\mathbf{7}$ & 741.1 & 769.1 & 783.1 & 14 \\
\hline \multirow{8}{*}{$\mathbf{3}$} & $\mathbf{3}$ & 750.3 & 772.3 & 783.3 & 11 \\
& $\mathbf{5}$ & 739.0 & 763.0 & 775.0 & 12 \\
& $\mathbf{6}$ & 733.6 & 761.2 & 774.2 & 13 \\
& $\mathbf{7}$ & 732.7 & 762.7 & 775.6 & 14 \\
& $\mathbf{3}$ & 746.9 & 770.9 & 782.9 & 15 \\
$\mathbf{9}$ & $\mathbf{4}$ & 735.5 & 761.5 & 774.5 & 13 \\
& $\mathbf{5}$ & 731.8 & 759.8 & 773.8 & 14 \\
& $\mathbf{6}$ & 730.1 & 760.1 & 775.1 & 15 \\
& $\mathbf{7}$ & 729.3 & 761.3 & 777.3 & 16 \\
\hline & $\mathbf{3}$ & 745.2 & 771.2 & 784.2 & 13 \\
$\mathbf{1 0}$ & $\mathbf{4}$ & 733.8 & 761.8 & 775.8 & 14 \\
& $\mathbf{5}$ & 730.1 & 760.1 & 775.1 & 15 \\
& $\mathbf{6}$ & 728.5 & 760.5 & 776.5 & 16 \\
& $\mathbf{7}$ & 727.7 & 761.7 & 778.7 & 17 \\
\hline & $\mathbf{3}$ & 744.2 & 772.2 & 786.2 & 14 \\
& $\mathbf{4}$ & 732.9 & 762.9 & 777.9 & 15 \\
$\mathbf{1 1}$ & $\mathbf{5}$ & 729.2 & 761.2 & 777.2 & 16 \\
& $\mathbf{6}$ & 727.6 & 761.6 & 778.6 & 17 \\
& $\mathbf{7}$ & 726.7 & 762.7 & 780.7 & 18 \\
\hline
\end{tabular}

GD, Global Deviance; AIC, Akaike Information Criterion;

GAIC(3), Generalized AIC with penalty equal to 3;

${ }^{\text {a }}$ In excess of 45000.

The best combination of $A I C$ and $\operatorname{GAIC}(3)$ corresponds to $\operatorname{df}(\mu)=9$ and $\operatorname{df}(\sigma)=5$. Further evaluations of this model were carried out by examining the fit of the $\mu$ and $\sigma$ curves and the patterns of the centile residuals (the empirical minus the fitted centiles) across age. 
Model 1: $\operatorname{BCPE}\left(x=\operatorname{age}^{0.20}, \operatorname{df}(\mu)=9, \operatorname{df}(\sigma)=5, v=1, \tau=2\right)$

The fitted parameter curves showed adequate smoothing despite an erratic coefficient of variation in the cross-sectional sample (Figure 1). The residual plots of the fitted centiles for the period 0 to 24 months (Figure 2) showed some bias at the upper centiles $\left(90^{\text {th }}, 95^{\text {th }}\right.$ and $\left.97^{\text {th }}\right)$, but the underestimation represents only $1 \mathrm{~mm}$ on average, which was considered negligible in practical terms. The lower centiles $\left(3^{\text {rd }}, 5^{\text {th }}, 10^{\text {th }}\right)$ showed no biased pattern. For the age range 24 to 71 months, residuals of the fitted centiles showed a non-random pattern only for the $3^{\text {rd }}$ centile, the average bias was about $2 \mathrm{~mm}$ (Figure 3).

Table 6 shows the proportions of children with head circumference below the fitted centiles. Age group labels correspond to the same age intervals provided in Table 7. There was indication of underestimation at the upper percentiles $\left(90^{\text {th }}\right.$ and above) as well as the $1^{\text {st }}$ percentile for some age groups. For the other percentiles, no biases were observed.
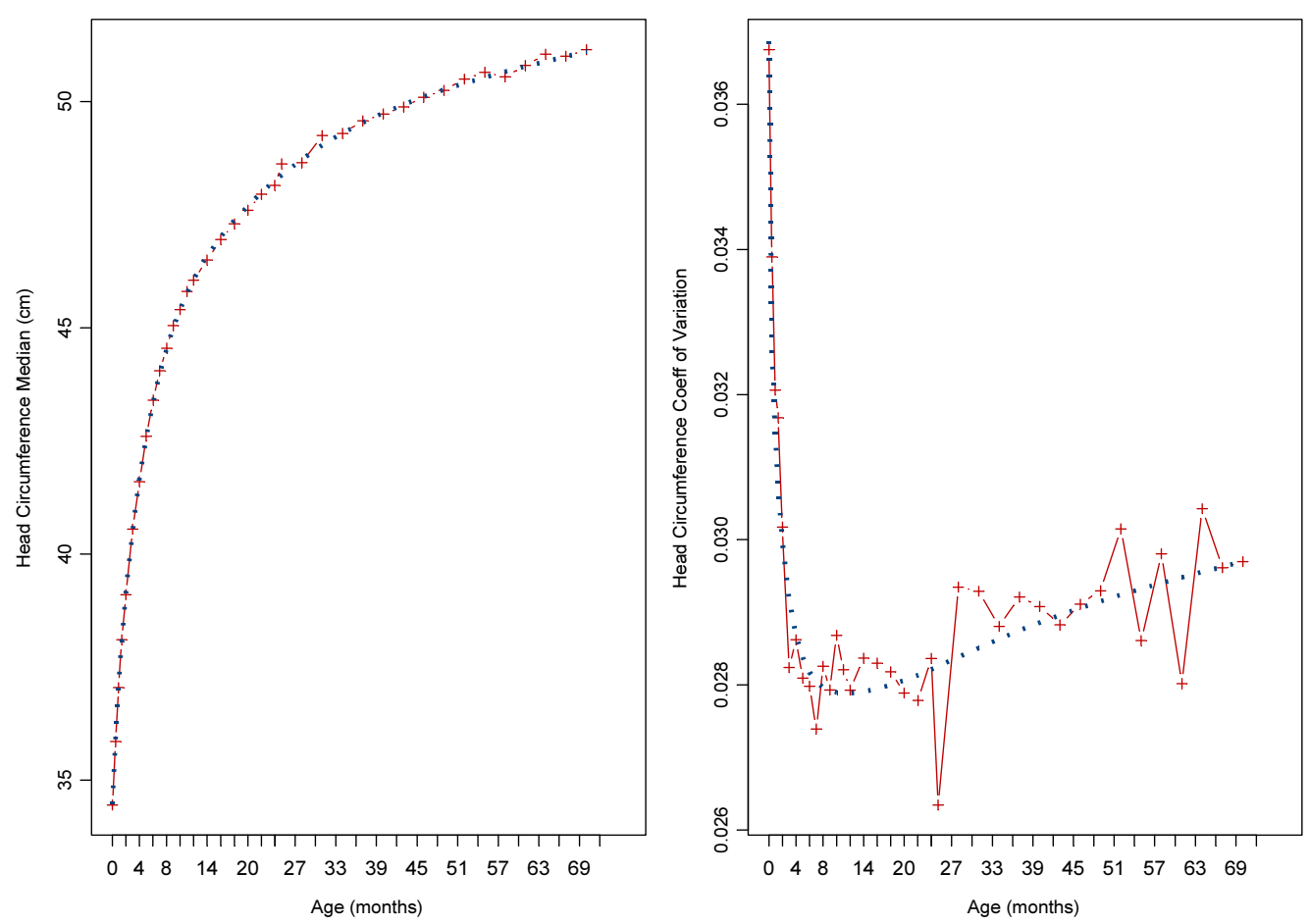

Figure 1 Fitting of $\mu$ and $\sigma$ curves of Model 1 for head circumference-for-age for boys (dotted line) and their respective sample estimates (points with solid line) 

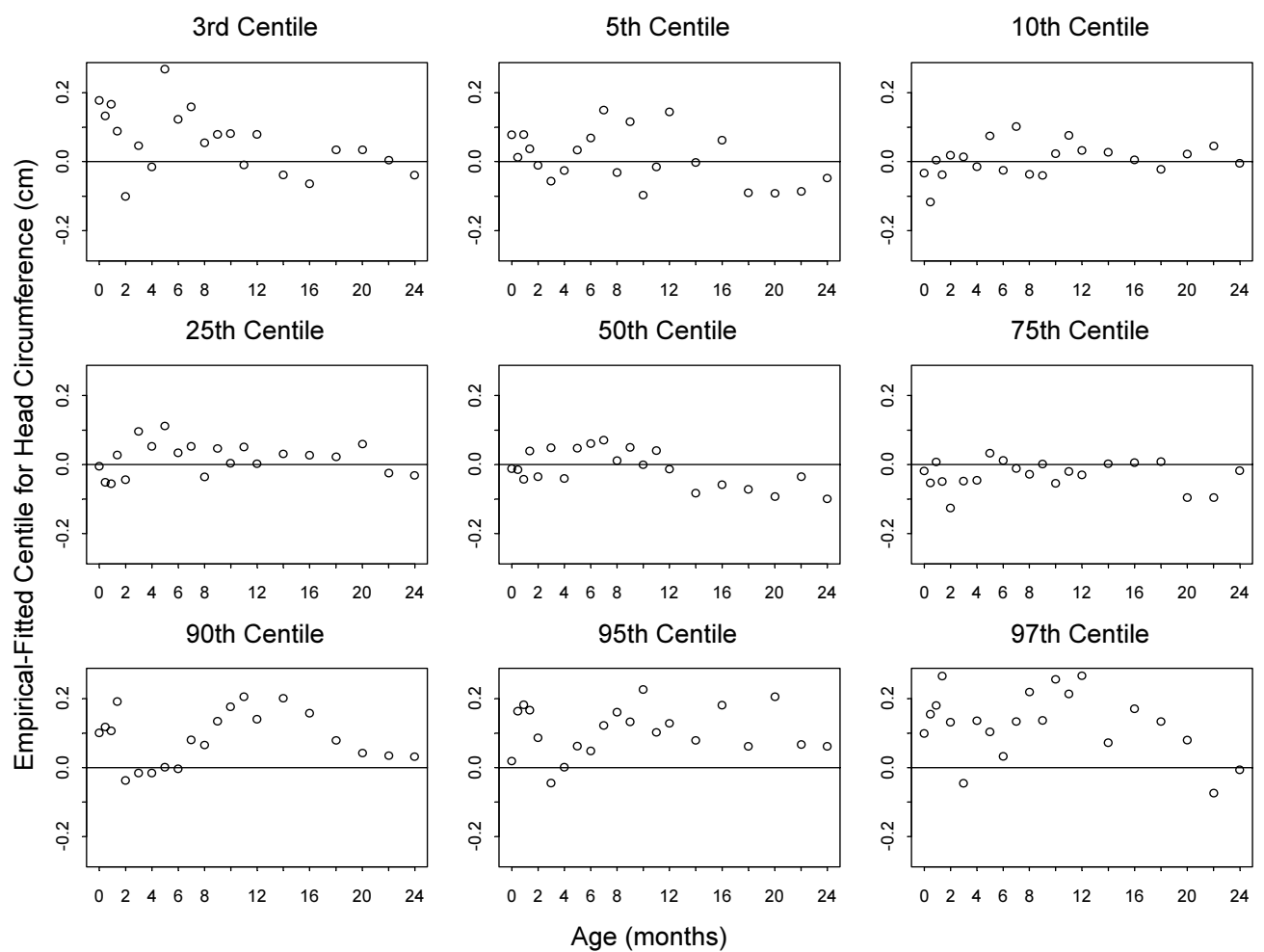

Figure 2 Centile residuals from fitting Model 1 for head circumference-for-age from 0 to 24 months for boys
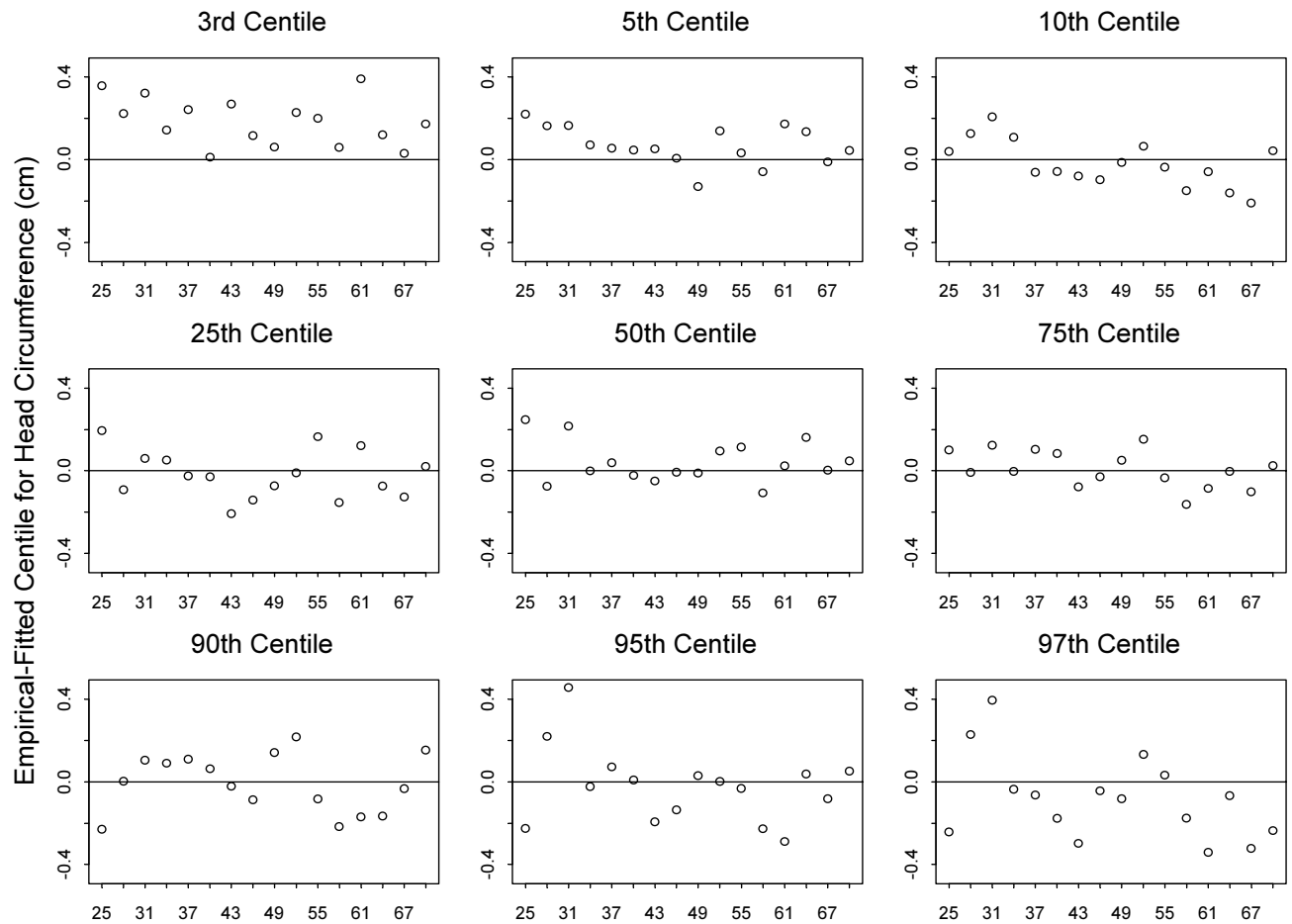

75th Centile

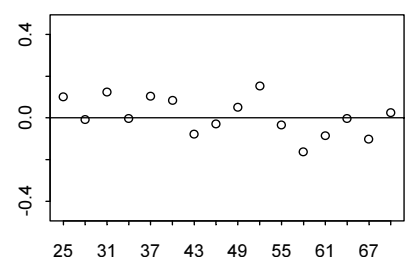

97th Centile
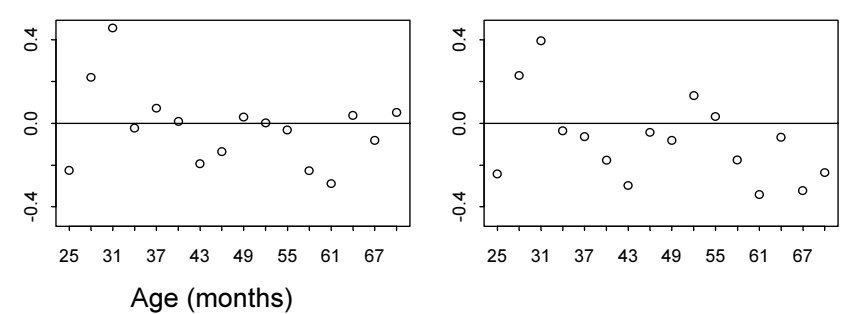

Figure 3 Centile residuals from fitting Model 1 for head circumference-for-age from 24 to 71 months for boys 
The worm plots for Model 1 are shown in Figure 4. There are only three groups for which worms present slight upward or downward shifts ( $40 \mathrm{mo}, 52 \mathrm{mo}$ and $58 \mathrm{mo}$ ), but overall, the fit of the median is adequate. Groups at birth and $42 \mathrm{~d}$ present $\mathrm{U}$-shaped worms, indicating residual skewness to the left. There are no worms with a slope, which would indicate misfit in the variance curve. S-shaped worms indicate a misfit in the curve of the parameter related to kurtosis as is the case in the 40 mo age group only. Despite the slight deviances present in a few groups, overall, the worms fitted to the points (solid red line) are all contained within the $95 \%$ confidence interval (dotted curves).
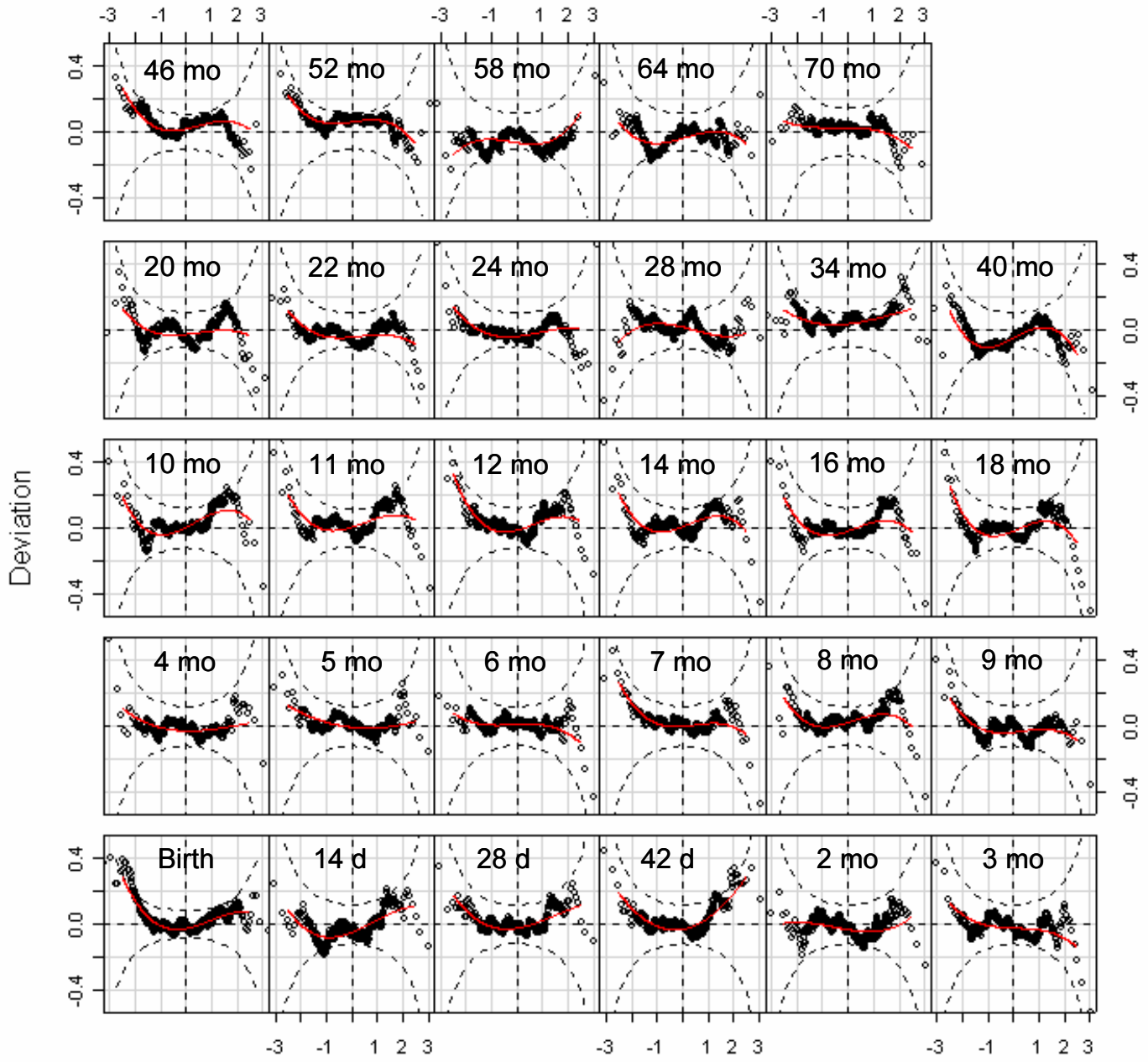

Unit normal quantile

Figure 4 Worm plots of z-scores for Model 1 for head circumference-for-age for boys 


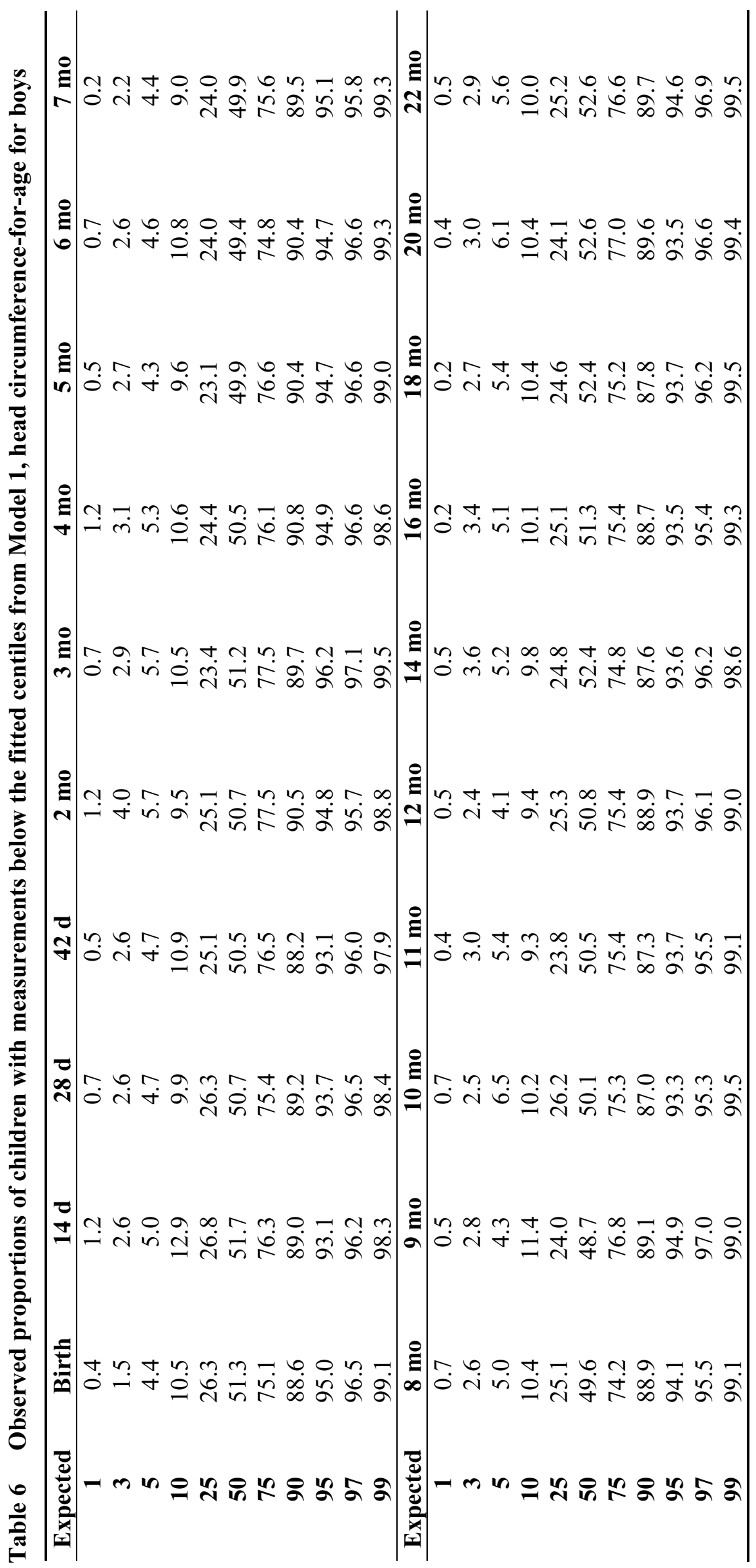




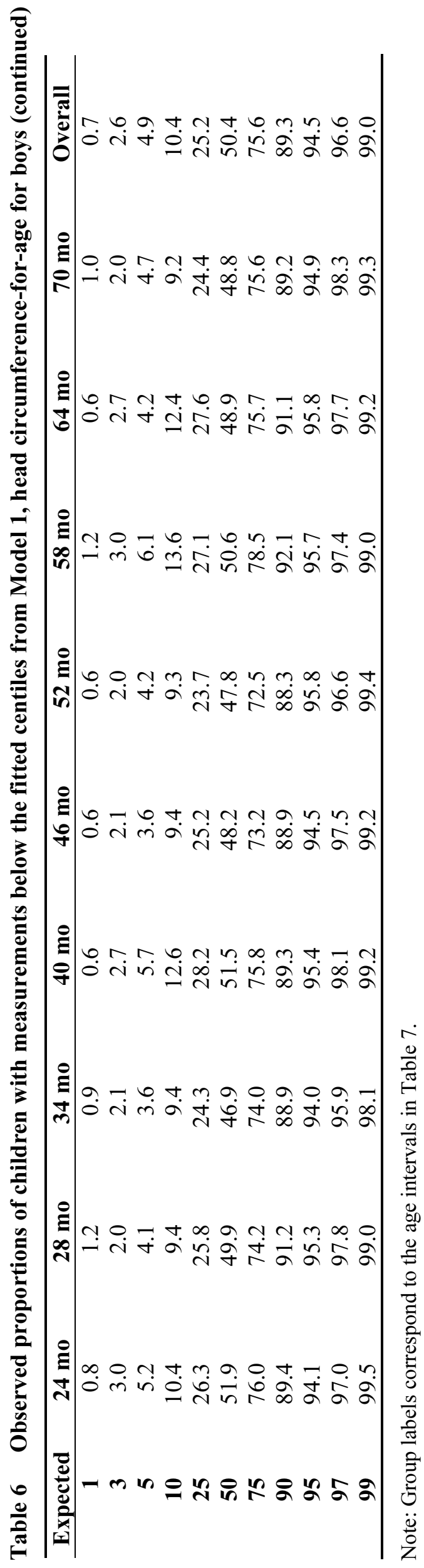


Head circumference-for-age, boys

The Q-test was performed to assess the overall significance of deviations that were noted based on the worm plots (Table 7). Absolute values of z3 larger than 2 were observed only in the age groups birth and $42 \mathrm{~d}$, and for $\mathrm{z} 4$ in $40 \mathrm{mo}$. The overall tests (p-values shown for each statistic in the last row of the table) did not suggest any significant departures of the fitted model z-scores from normality at the $5 \%$ level of significance.

Table 7 Q-test for $z$-scores from Model $1\left[\operatorname{BCPE}\left(x=\operatorname{age}^{0.20}, \operatorname{df}(\mu)=9, \operatorname{df}(\sigma)=5, v=1, \tau=2\right)\right]$ for head circumference-for-age for boys

\begin{tabular}{|c|c|c|c|c|c|c|}
\hline Age (days) & Group & $\mathbf{N}$ & $\mathrm{z} 1$ & $\mathbf{z 2}$ & $\mathbf{z 3}$ & $\mathrm{z4}$ \\
\hline 0 & Birth & 893 & 0.2 & -0.1 & 2.3 & -1.4 \\
\hline 1 to 16 & $14 \mathrm{~d}$ & 418 & -0.6 & 1.0 & 1.1 & -0.7 \\
\hline 17 to 34 & $28 d$ & 426 & 0.1 & 0.2 & 1.3 & -0.4 \\
\hline 35 to 49 & $42 \mathrm{~d}$ & 422 & 0.2 & 0.7 & 2.1 & 0.2 \\
\hline 50 to 69 & $2 \mathrm{mo}$ & 422 & -0.5 & -0.2 & 0.5 & 0.5 \\
\hline 70 to 99 & $3 \mathrm{mo}$ & 418 & -0.4 & -0.8 & 0.1 & -0.6 \\
\hline 100 to 129 & $4 \mathrm{mo}$ & 414 & -0.3 & -0.2 & 0.7 & -0.1 \\
\hline 130 to 159 & $5 \mathrm{mo}$ & 415 & 0.2 & -0.6 & 0.7 & 0.1 \\
\hline 160 to 189 & $6 \mathrm{mo}$ & 417 & 0.1 & -0.3 & -0.2 & -0.6 \\
\hline 190 to 219 & $7 \mathrm{mo}$ & 409 & 0.2 & -0.8 & 0.9 & -0.8 \\
\hline 220 to 249 & $8 \mathrm{mo}$ & 423 & 0.7 & 0.4 & 0.6 & -1.2 \\
\hline 250 to 279 & 9 mo & 396 & -0.4 & -0.5 & 0.6 & -0.7 \\
\hline 280 to 309 & $10 \mathrm{mo}$ & 401 & 0.5 & 0.8 & 0.7 & -1.6 \\
\hline 310 to 349 & $11 \mathrm{mo}$ & 463 & 0.5 & 0.4 & 1.0 & -1.0 \\
\hline 350 to 379 & $12 \mathrm{mo}$ & 415 & 0.3 & -0.1 & 1.5 & -1.3 \\
\hline 380 to 439 & $14 \mathrm{mo}$ & 420 & 0.4 & 0.4 & 0.7 & -1.4 \\
\hline 440 to 499 & $16 \mathrm{mo}$ & 415 & -0.1 & 0.3 & 0.8 & -1.2 \\
\hline 500 to 559 & $18 \mathrm{mo}$ & 443 & -0.1 & 0.1 & 0.8 & -1.8 \\
\hline 560 to 619 & $20 \mathrm{mo}$ & 527 & -0.6 & -0.2 & 0.7 & -0.6 \\
\hline 620 to 679 & $22 \mathrm{mo}$ & 551 & -0.8 & -0.3 & 0.5 & -0.8 \\
\hline 680 to 749 & $24 \mathrm{mo}$ & 597 & -0.6 & -0.1 & 1.1 & -0.4 \\
\hline 750 to 929 & $28 \mathrm{mo}$ & 489 & 0.2 & -0.5 & -0.6 & 0.9 \\
\hline 930 to 1119 & $34 \mathrm{mo}$ & 531 & 1.3 & 0.3 & 0.8 & -0.2 \\
\hline 1120 to 1309 & $40 \mathrm{mo}$ & 524 & -1.1 & 0.6 & 0.3 & -2.5 \\
\hline 1310 to 1499 & $46 \mathrm{mo}$ & 523 & 0.8 & -0.1 & 1.1 & -0.9 \\
\hline 1500 to 1689 & $52 \mathrm{mo}$ & 506 & 1.4 & -0.5 & 0.1 & -1.4 \\
\hline 1690 to 1879 & $58 \mathrm{mo}$ & 494 & -1.3 & 0.5 & 0.6 & 1.5 \\
\hline 1880 to 2069 & $64 \mathrm{mo}$ & 474 & -0.7 & 0.3 & 0.2 & -1.1 \\
\hline 2070 to 2249 & $70 \mathrm{mo}$ & 295 & 0.3 & -0.4 & -0.3 & -0.4 \\
\hline Overall Q stats & & 13541 & 11.6 & 6.7 & 25.6 & 32.4 \\
\hline degrees of freedom & & & 20.0 & 26.0 & 29.0 & 29.0 \\
\hline p-value & & & 0.9301 & $>0.99$ & 0.6447 & 0.3015 \\
\hline
\end{tabular}

Note: Absolute values of z1, z2, z3 or z4 larger than 2 indicate misfit of, respectively, mean, variance, skewness or kurtosis.

Considering local goodness of fit, the Q-test and worm plots based on Model 1 indicated minor departures from normality in very few age groups, but overall deviations were not significant. Thus, there was no reason to fit a more complex model, since there was neither remaining skewness nor kurtosis. 
Model 1 was selected and a new iteration was done using the values $\operatorname{df}(\mu)=9$ and $\operatorname{df}(\sigma)=5$ to re-search for the best age-transformation power $\lambda$. The smallest global deviance in this case was for $\lambda=0.05$ $(\mathrm{GD}=45731.0)$, but with only a very minor difference from the model using $\lambda=0.20(\mathrm{GD}=45$ 731.8). There was thus no need to update $\lambda$, and the selected model for constructing the head circumferencefor-age growth curves for boys remained $\operatorname{BCPE}\left(x=\operatorname{age}^{0.20}, \operatorname{df}(\mu)=9, \operatorname{df}(\sigma)=5, \nu=1, \tau=2\right)$. Figures 5 to 8 show the fitted centile curves derived from the selected model against empirical head circumferencefor-age values. 


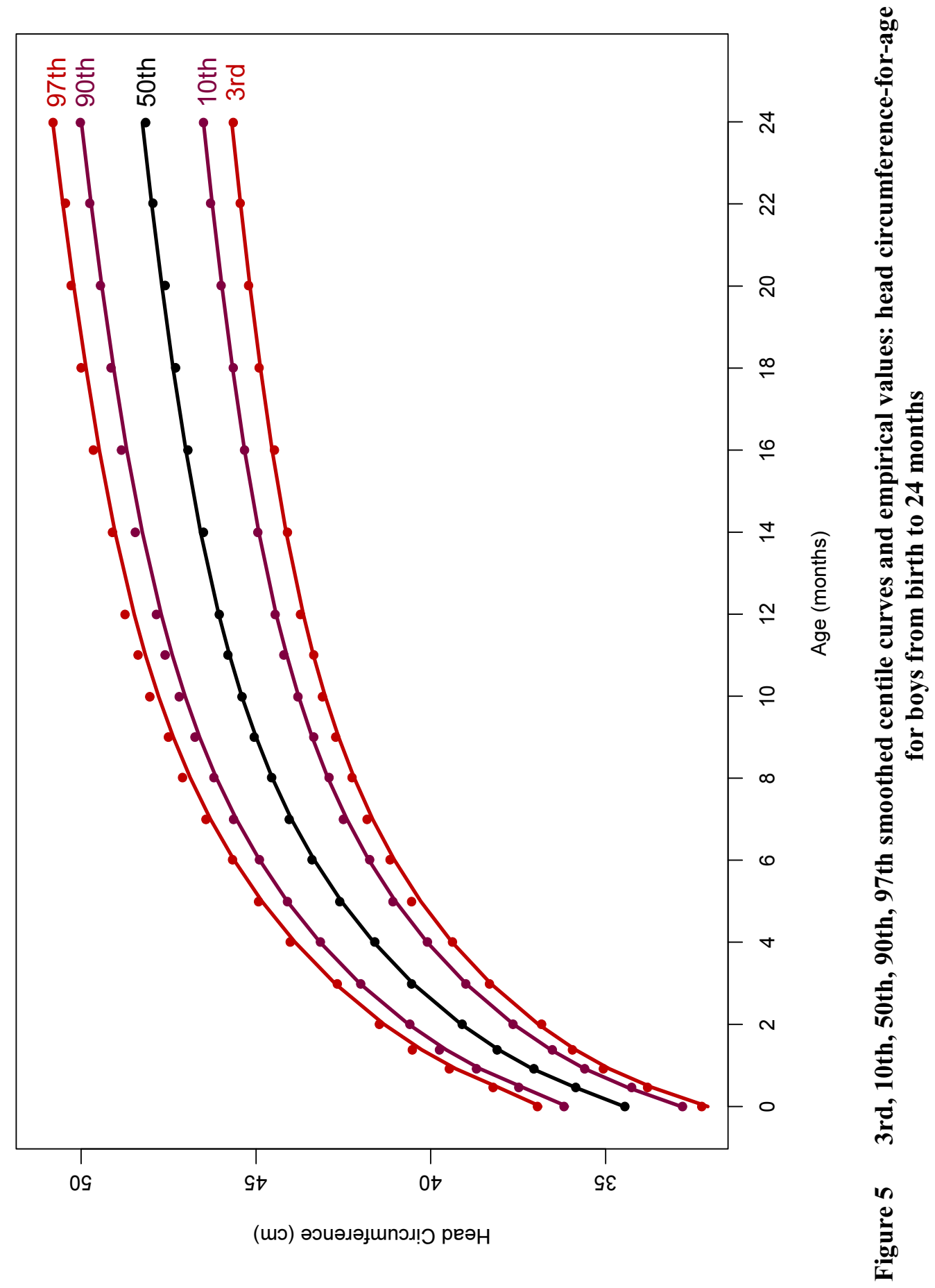




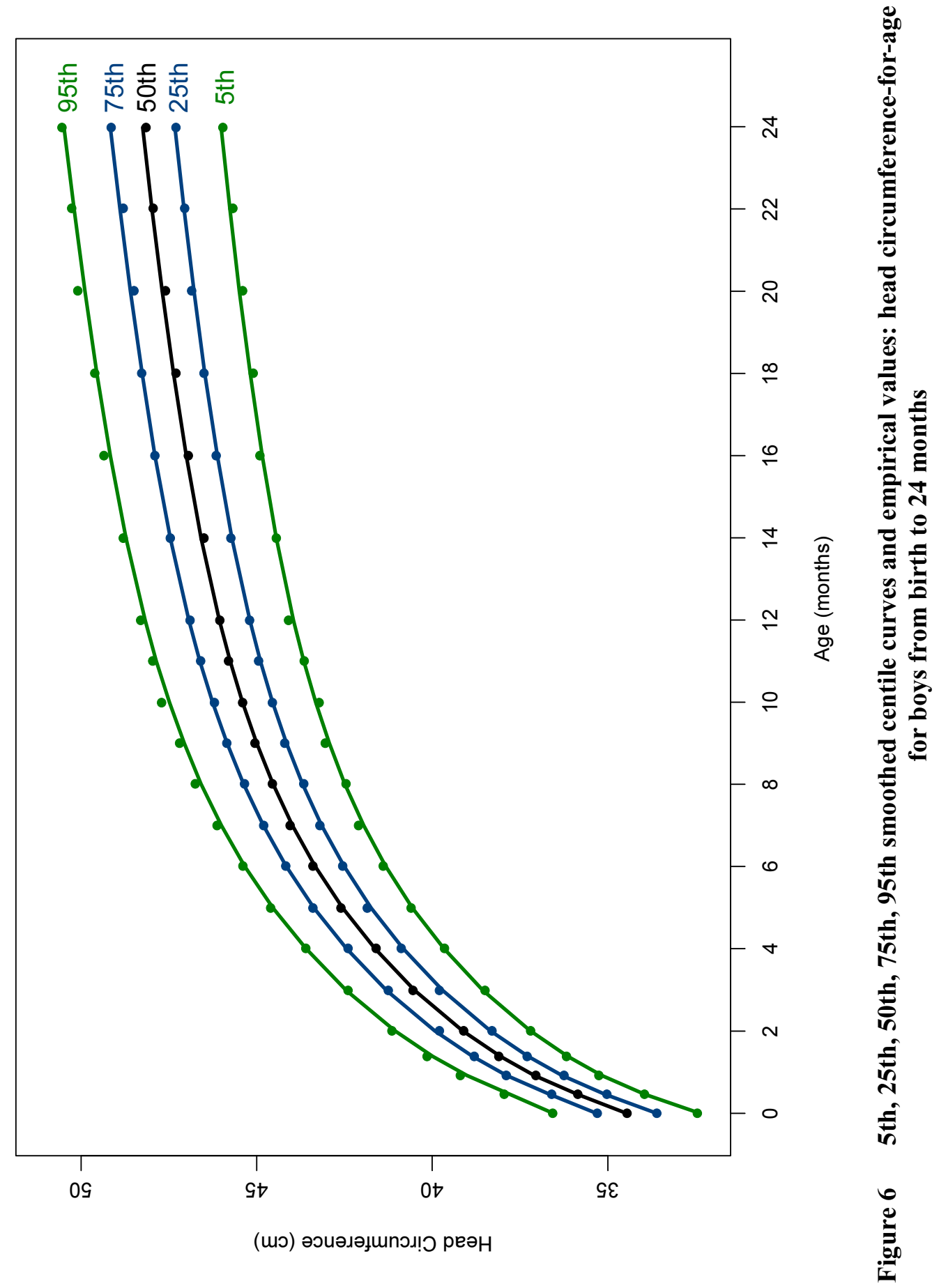




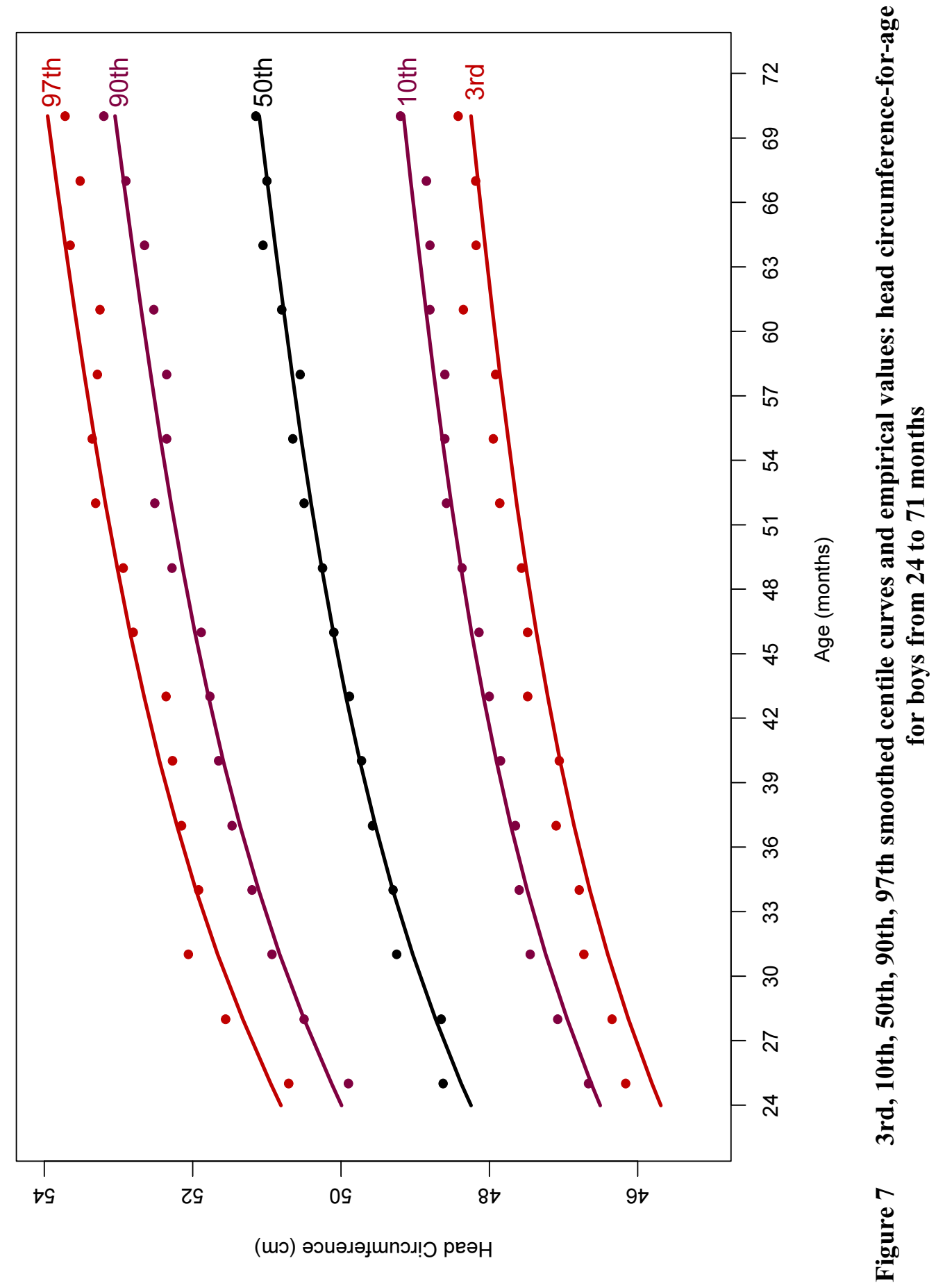




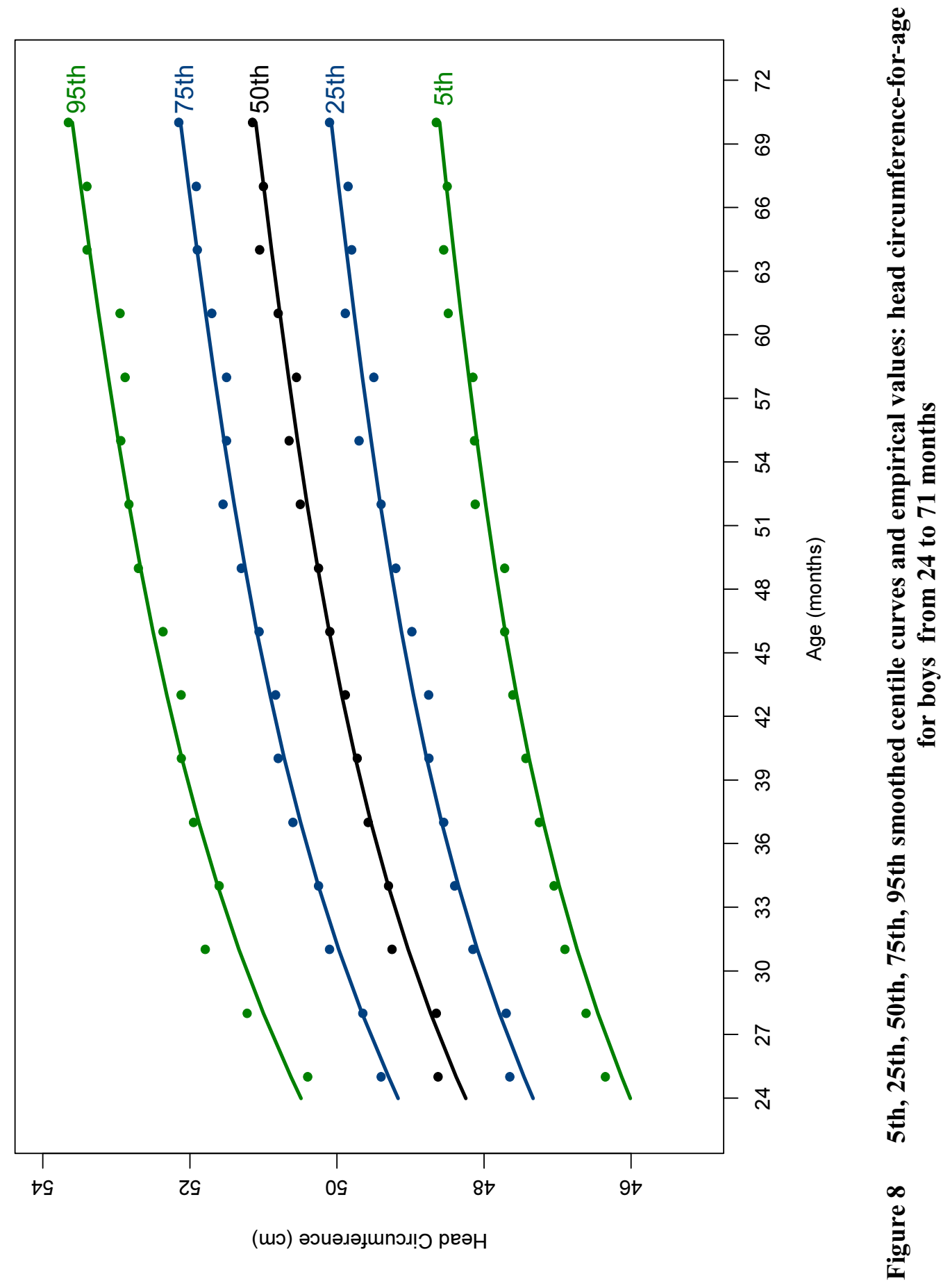




\subsubsection{WHO standards}

This section presents the final WHO head circumference-for-age z-score and percentile charts (Figures 9 and 10) and tables (Tables 8 and 9) for boys. 


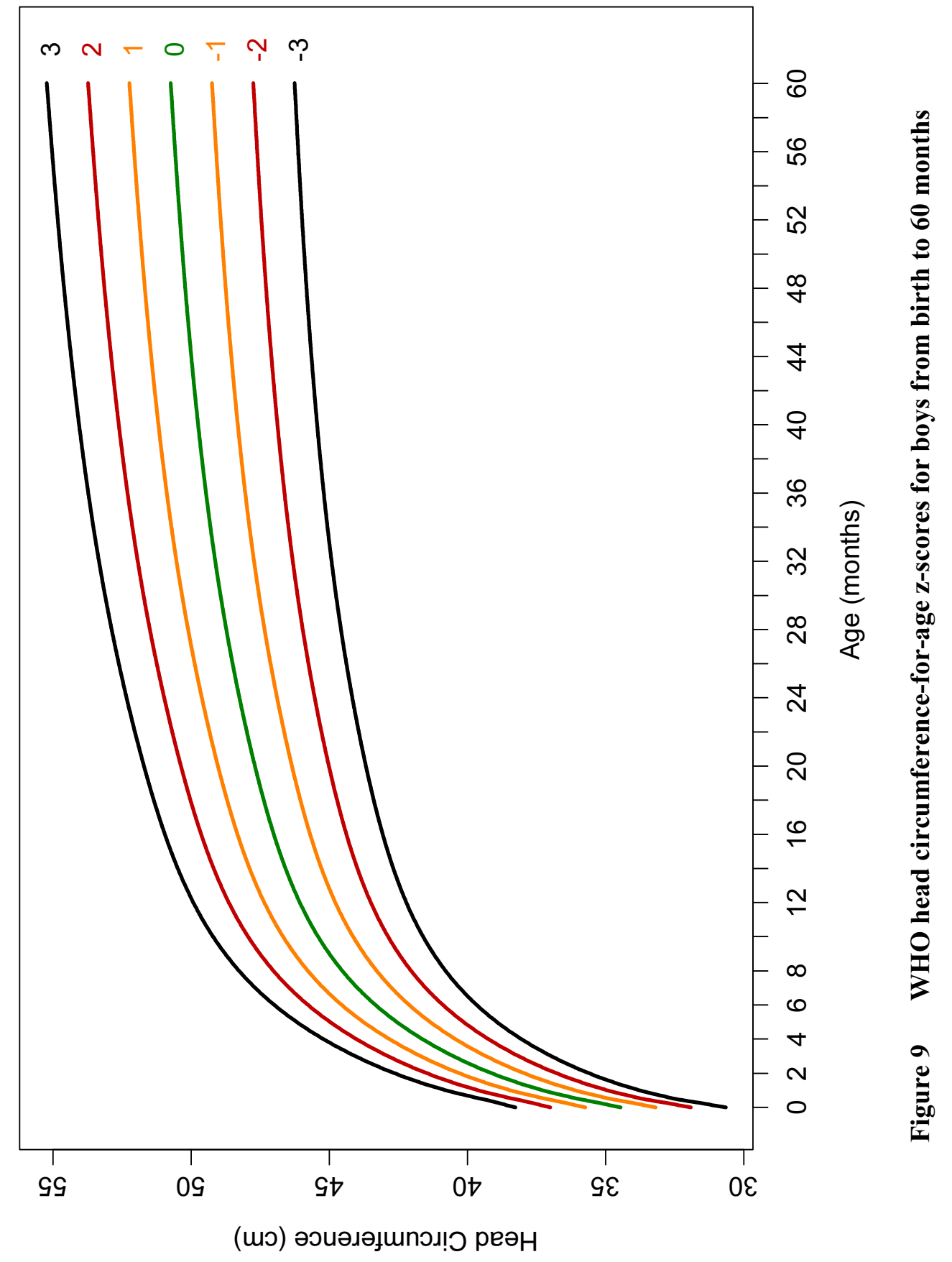




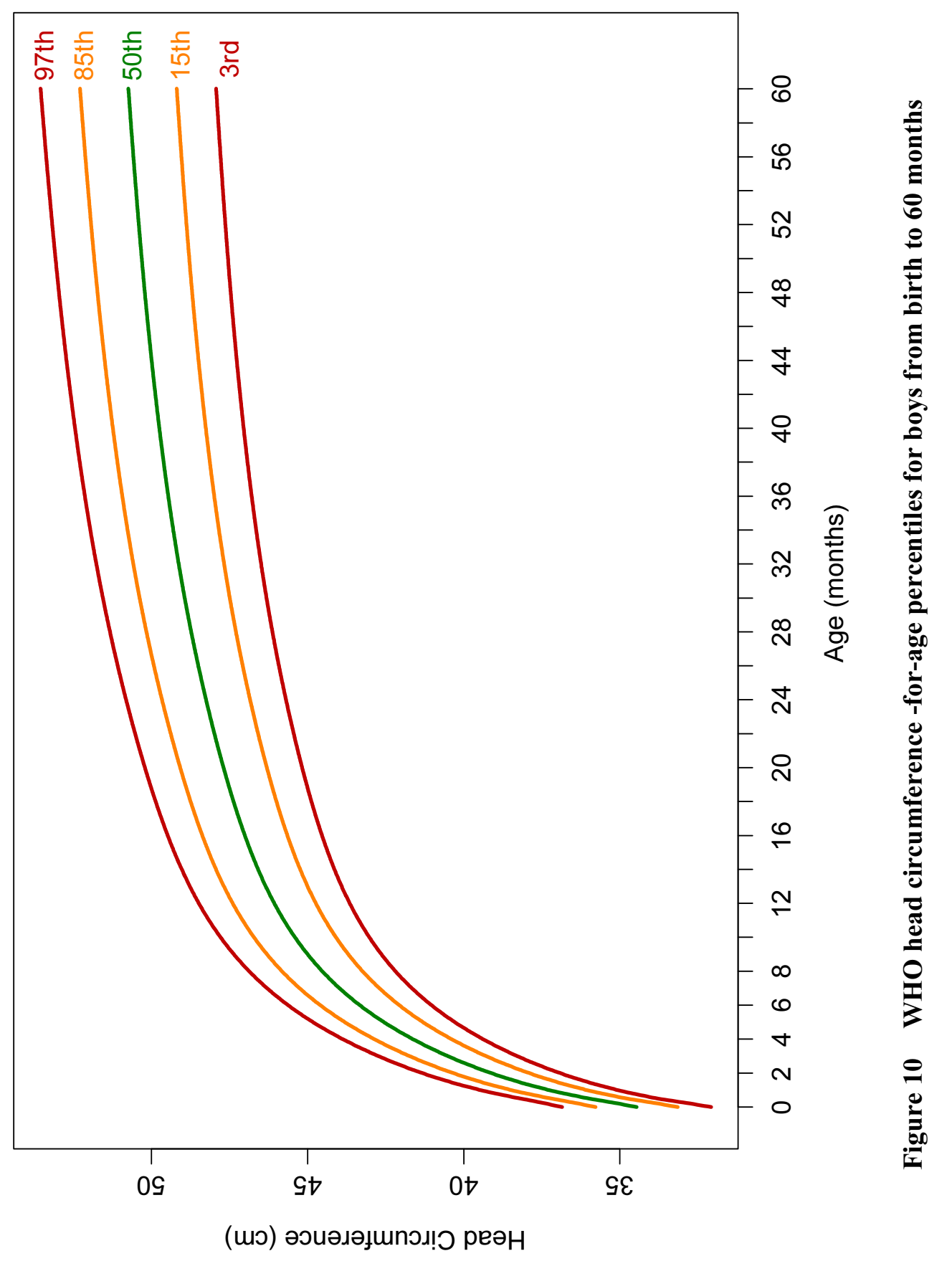




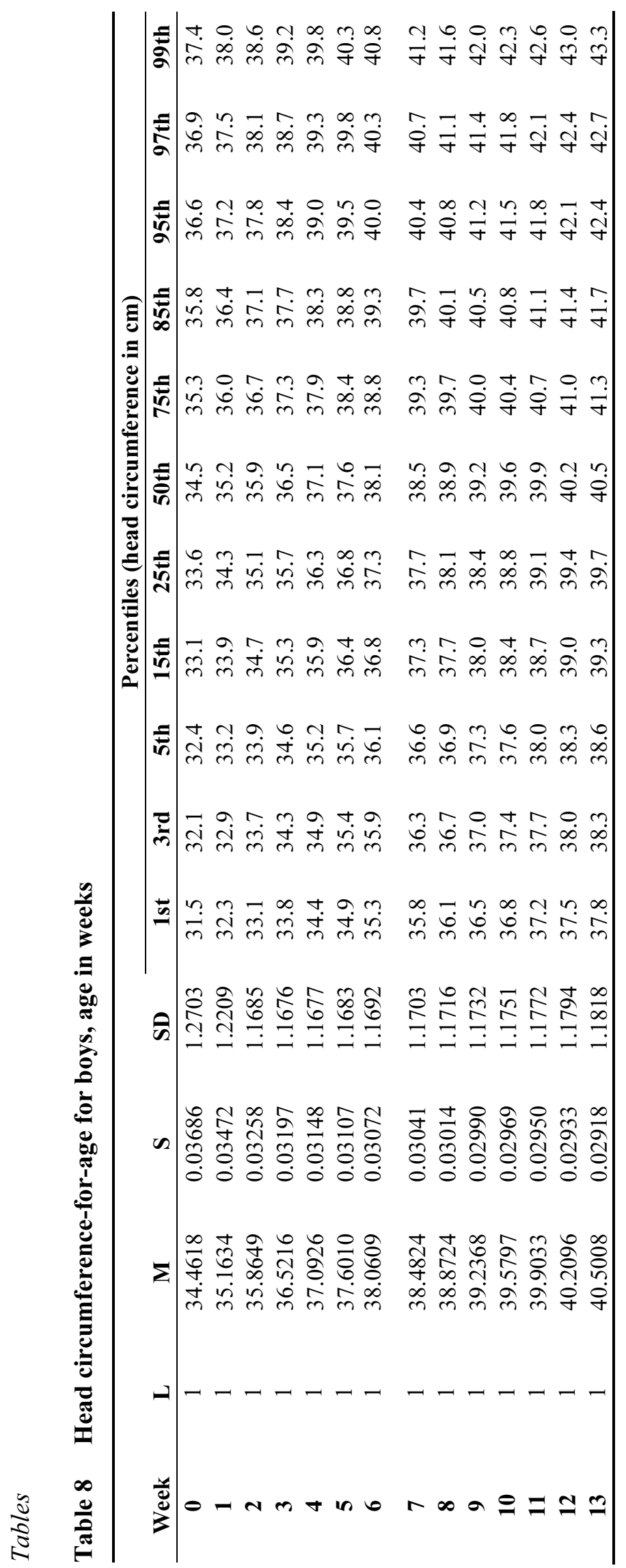




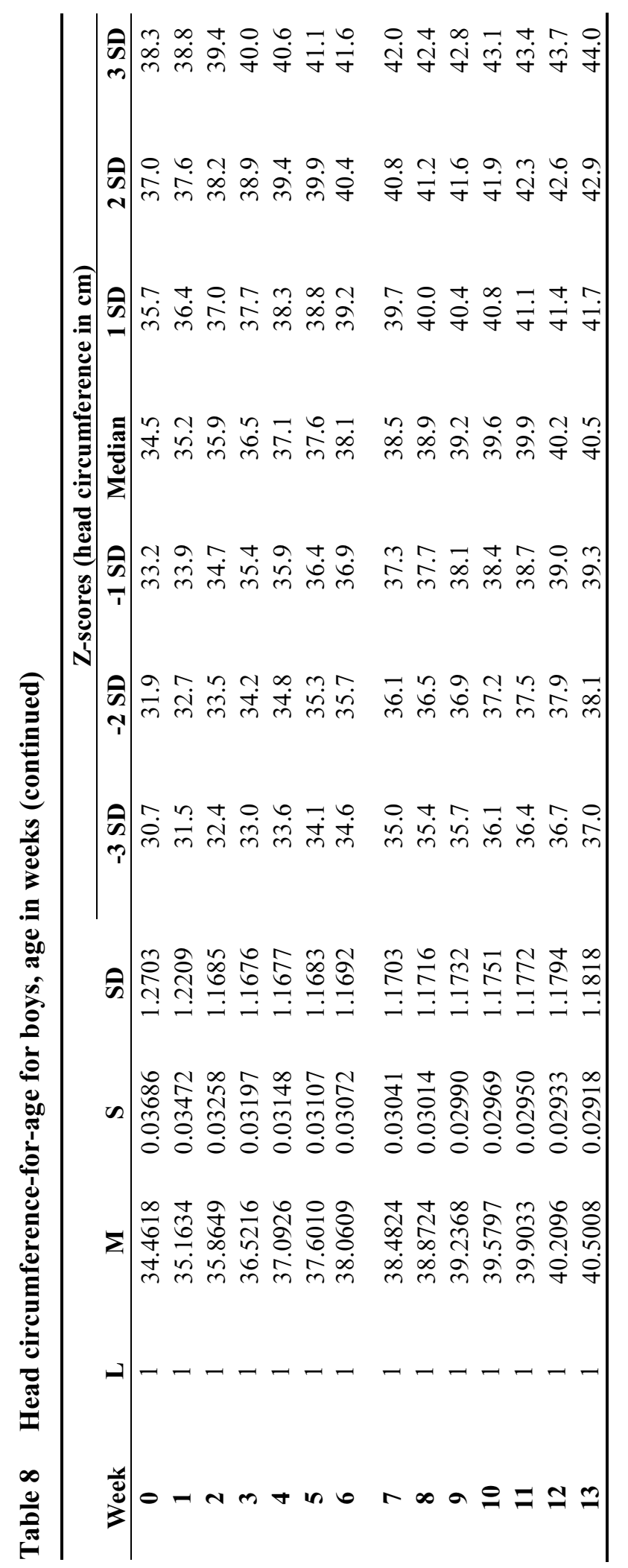




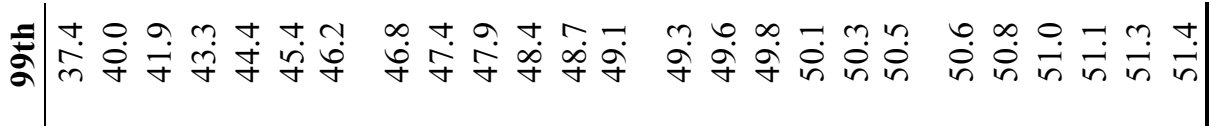

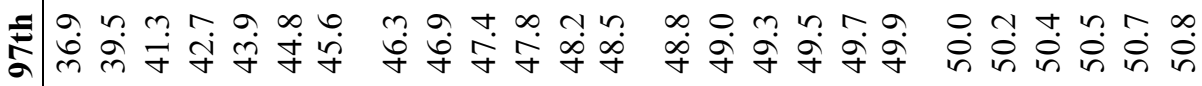

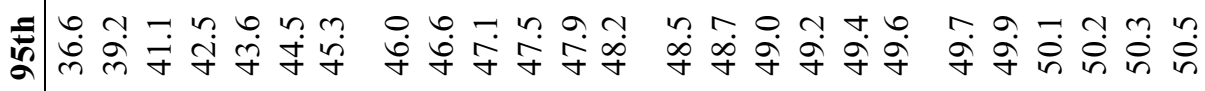

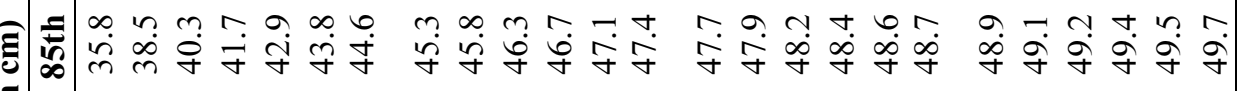

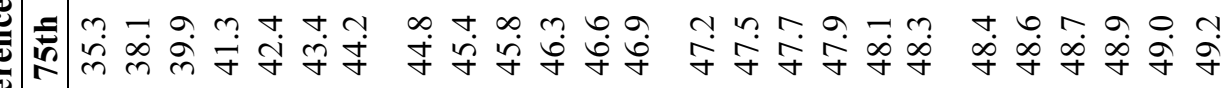

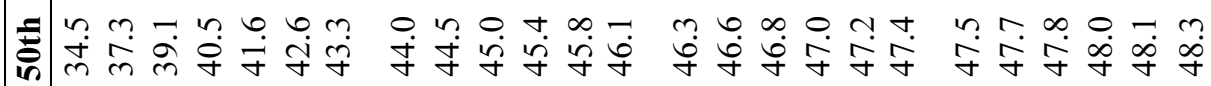

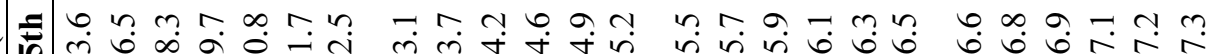

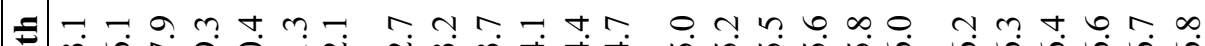

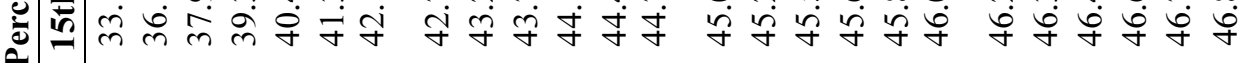

$\Rightarrow$ t ம்

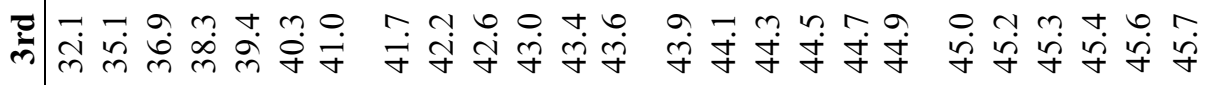

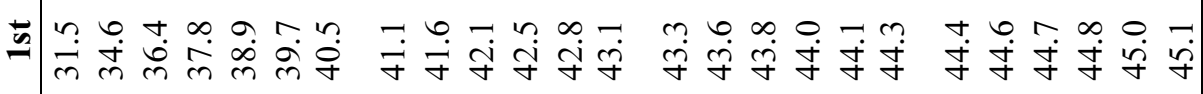

ڤิ)

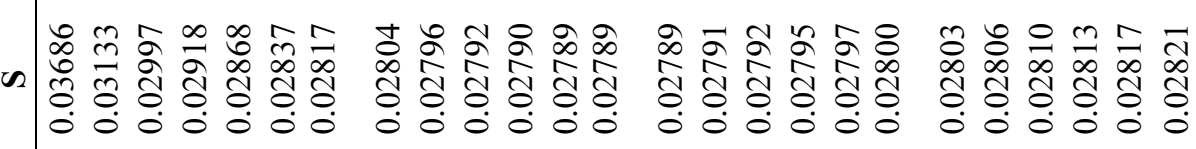

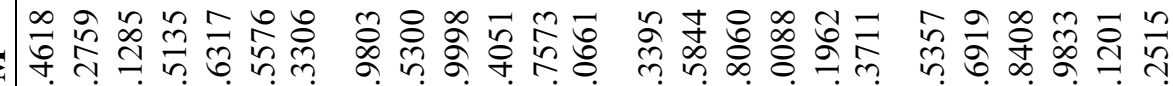

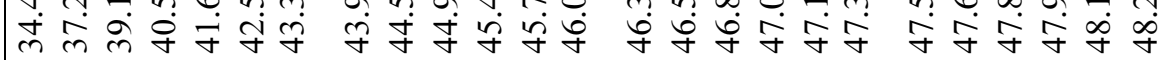

$-$

를

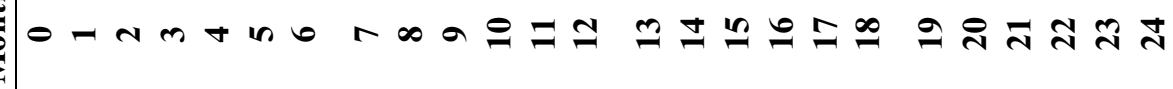

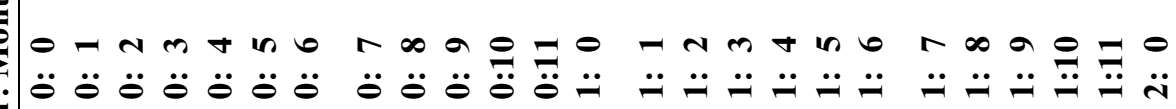




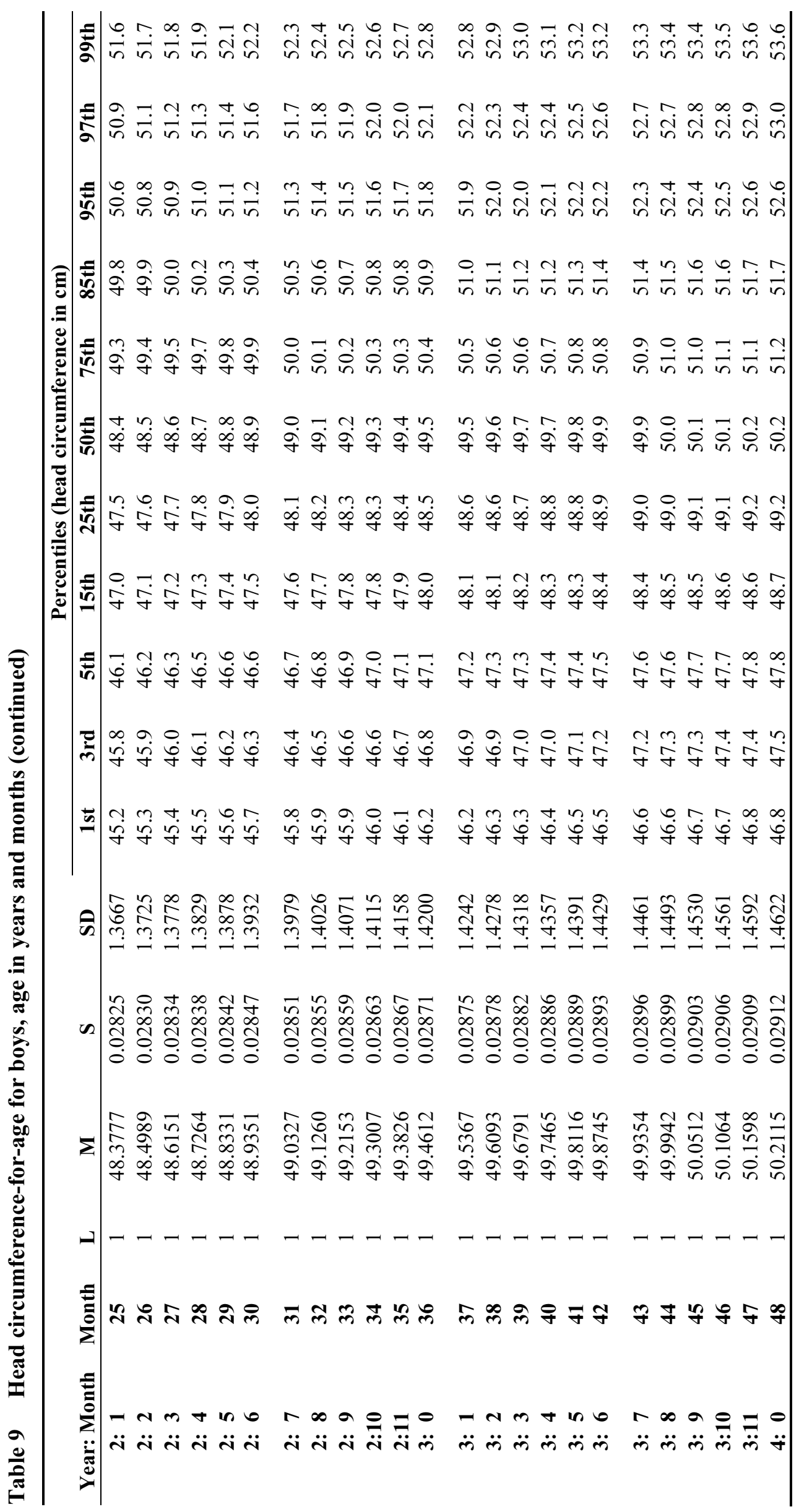




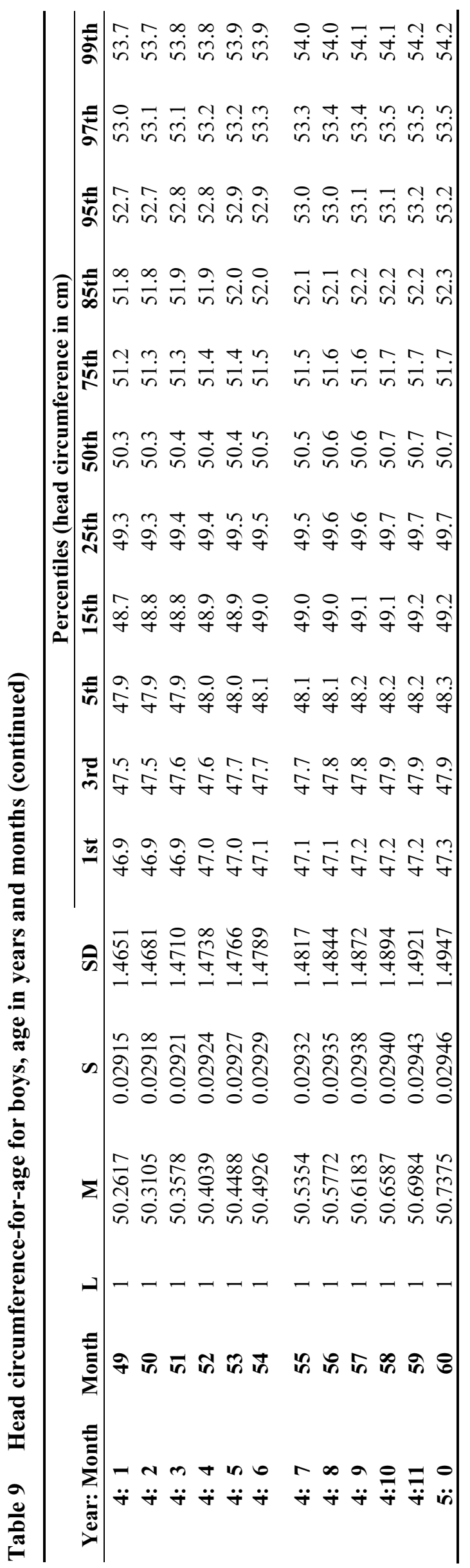




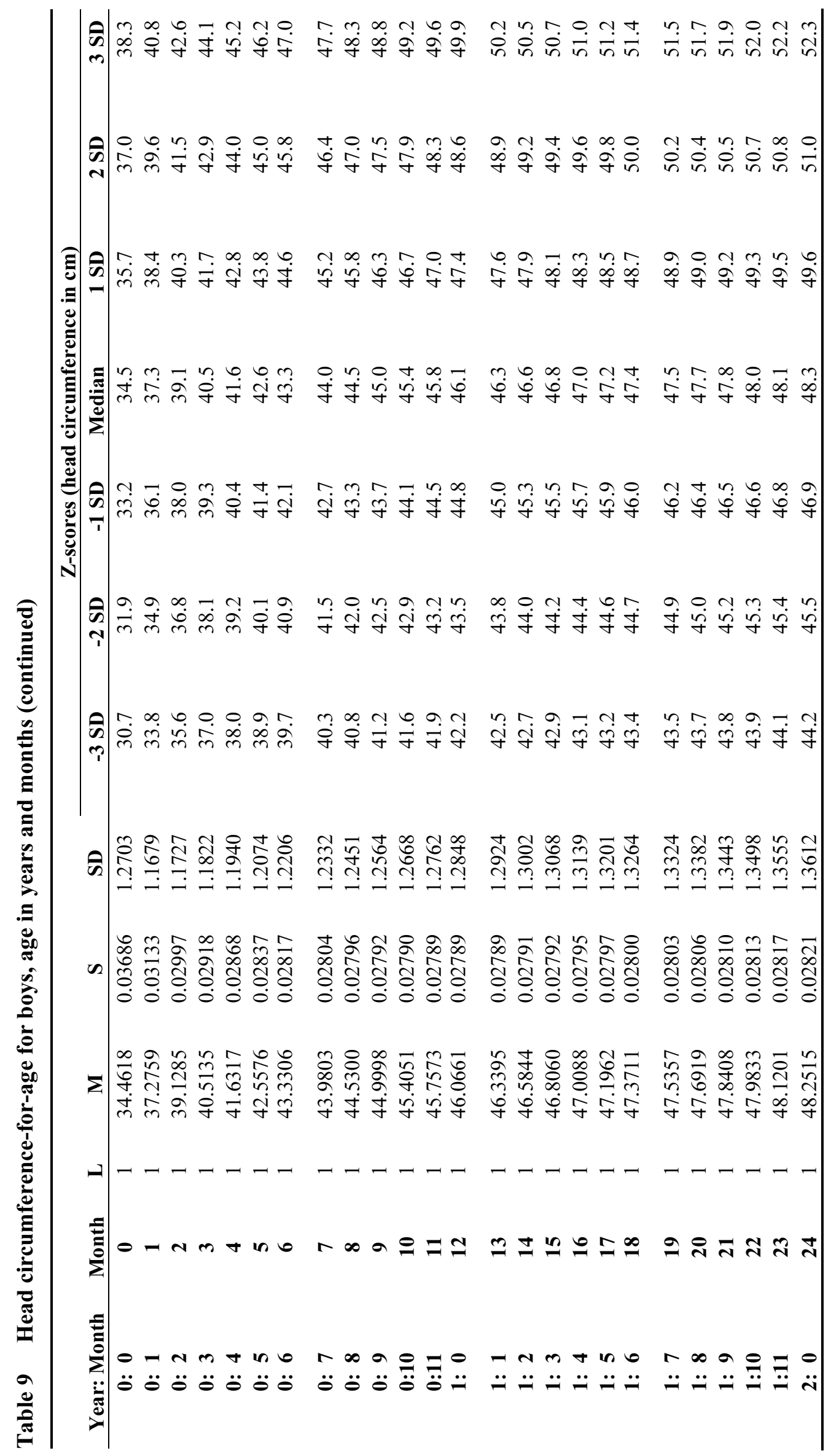




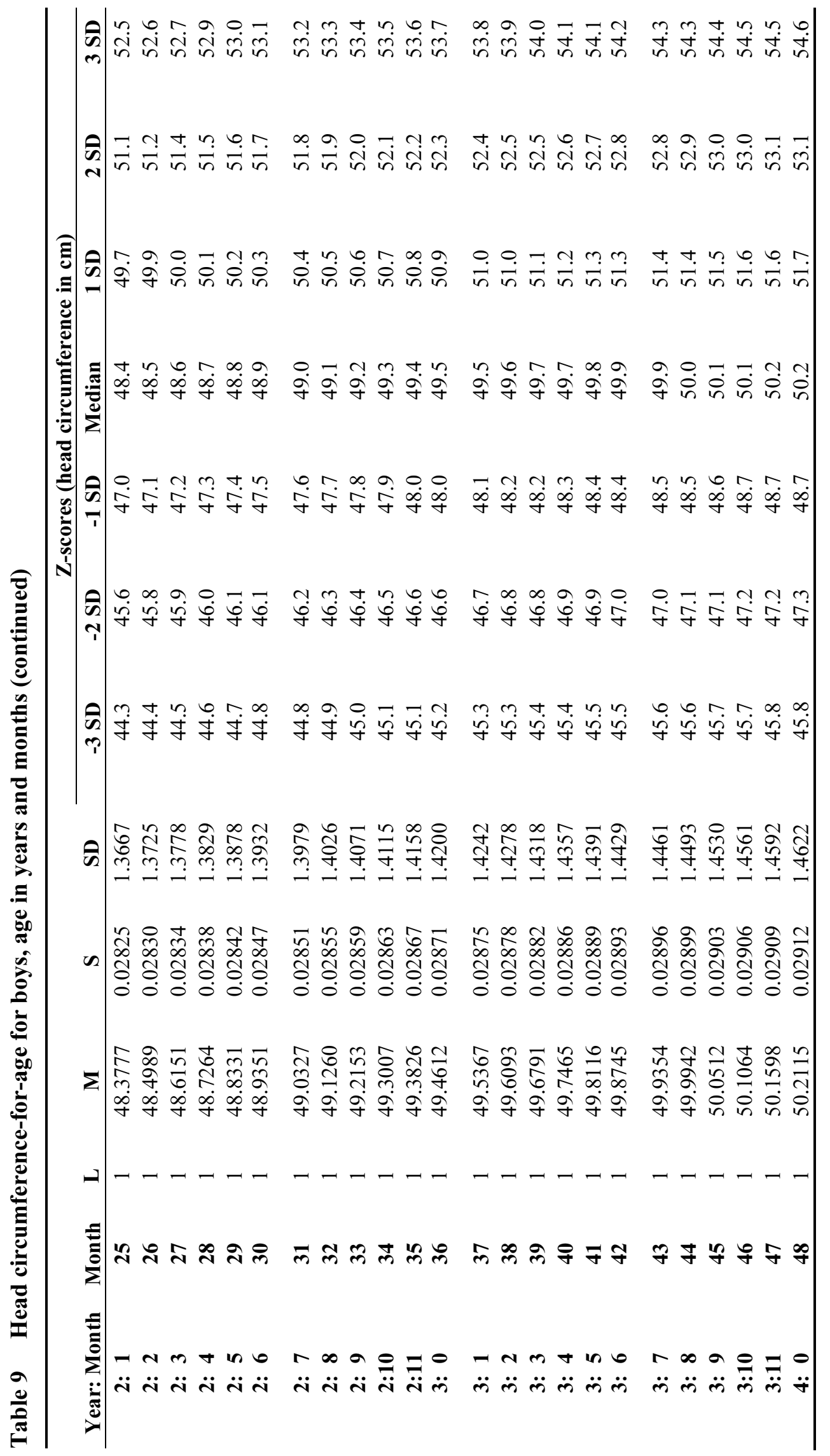




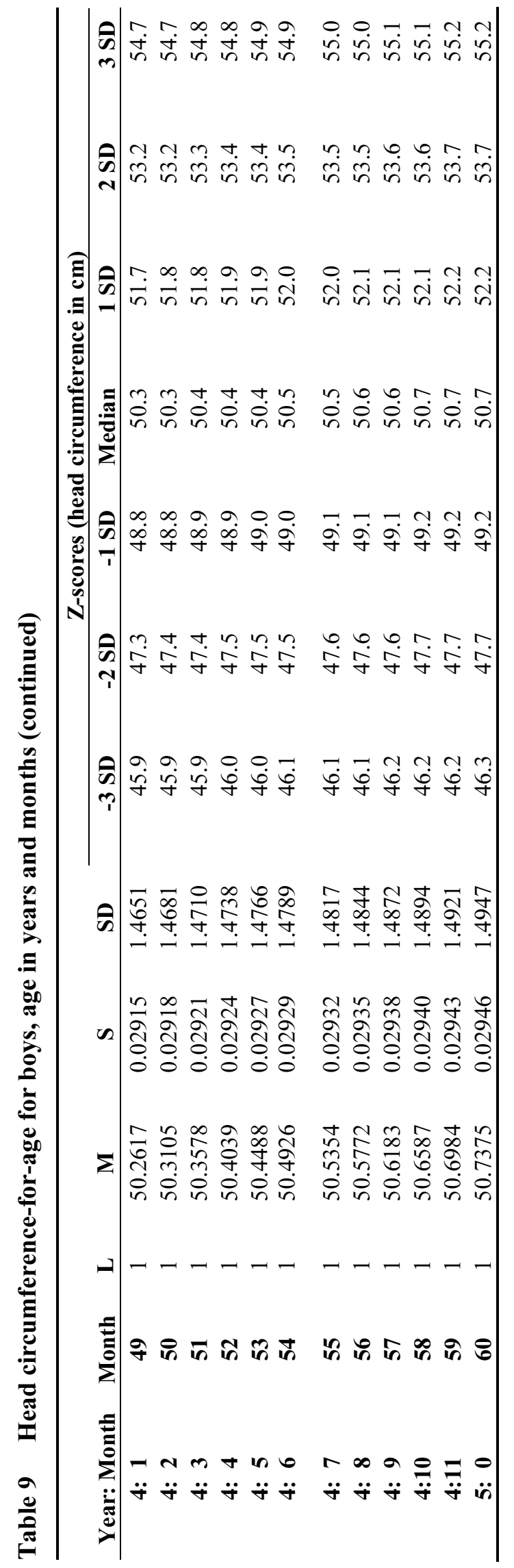




\subsection{Head circumference-for-age for girls}

The choice of the model to construct the head circumference-for-age standard for girls followed the steps described for the corresponding standard for boys. In principle, unless a clear inadequacy is detected, models used for constructing the standards for both sexes should belong to the same class.

\subsubsection{Sample size}

There were 13798 head circumference observations for girls. The longitudinal and cross-sectional sample sizes by visit and age are presented in Tables 10 and 11 .

Table 10 Longitudinal sample sizes for head circumference-for-age for girls

\begin{tabular}{lccccccc}
\hline Visit & Birth & $\mathbf{1}$ & $\mathbf{2}$ & $\mathbf{3}$ & $\mathbf{4}$ & $\mathbf{5}$ & $\mathbf{6}$ \\
Age & $\mathbf{0}$ & $\mathbf{2} \mathbf{~ w k}$ & $\mathbf{4} \mathbf{~ w k}$ & $\mathbf{6} \mathbf{w k}$ & $\mathbf{2} \mathbf{~ m o}$ & $\mathbf{3} \mathbf{~ m o}$ & $\mathbf{4} \mathbf{~ m o}$ \\
$\mathrm{N}$ & 841 & 449 & 449 & 447 & 447 & 448 & 447 \\
\hline Visit & $\mathbf{7}$ & $\mathbf{8}$ & $\mathbf{9}$ & $\mathbf{1 0}$ & $\mathbf{1 1}$ & $\mathbf{1 2}$ & $\mathbf{1 3}$ \\
Age & $\mathbf{5} \mathbf{~ m o}$ & $\mathbf{6} \mathbf{~ m o}$ & $\mathbf{7} \mathbf{~ m o}$ & $\mathbf{8} \mathbf{~ m o}$ & $\mathbf{9} \mathbf{~ m o}$ & $\mathbf{1 0} \mathbf{~ m o}$ & $\mathbf{1 1} \mathbf{~ m o}$ \\
$\mathrm{N}$ & 450 & 448 & 448 & 445 & 449 & 446 & 446 \\
\hline Visit & $\mathbf{1 4}$ & $\mathbf{1 5}$ & $\mathbf{1 6}$ & $\mathbf{1 7}$ & $\mathbf{1 8}$ & $\mathbf{1 9}$ & $\mathbf{2 0}$ \\
Age & $\mathbf{1 2} \mathbf{~ m o}$ & $\mathbf{1 4} \mathbf{~ m o}$ & $\mathbf{1 6} \mathbf{~ m o}$ & $\mathbf{1 8} \mathbf{~ m o}$ & $\mathbf{2 0} \mathbf{~ m o}$ & $\mathbf{2 2} \mathbf{~ m o}$ & $\mathbf{2 4} \mathbf{~ m o}$ \\
$\mathrm{N}$ & 452 & 452 & 445 & 449 & 445 & 439 & 449 \\
\hline
\end{tabular}

Table 11 Cross-sectional sample sizes for head circumference-for-age for girls

\begin{tabular}{lccccccc}
\hline Age (mo) & $<\mathbf{1 8}$ & $\mathbf{1 8 - 2 0}$ & $\mathbf{2 1 - 2 3}$ & $\mathbf{2 4 - 2 6}$ & $\mathbf{2 7 - 2 9}$ & $\mathbf{3 0 - 3 2}$ & $\mathbf{3 3 - 3 5}$ \\
$\mathrm{N}$ & 2 & 164 & 177 & 249 & 220 & 237 & 230 \\
\hline Age (mo) & $\mathbf{3 6 - 3 8}$ & $\mathbf{3 9 - 4 1}$ & $\mathbf{4 2 - 4 4}$ & $\mathbf{4 5}-\mathbf{4 7}$ & $\mathbf{4 8}-\mathbf{5 0}$ & $\mathbf{5 1 - 5 3}$ & $\mathbf{5 4 - 5 6}$ \\
$\mathrm{N}$ & 226 & 242 & 254 & 218 & 232 & 208 & 239 \\
\hline Age (mo) & $\mathbf{5 7 - 5 9}$ & $\mathbf{6 0 - 6 2}$ & $\mathbf{6 3 - 6 5}$ & $\mathbf{6 6 - 6 8}$ & $\mathbf{6 9 - 7 1}$ & $>\mathbf{7 1}$ & \\
$\mathrm{N}$ & 241 & 223 & 205 & 230 & 210 & 0 & \\
\hline
\end{tabular}

\subsubsection{Model selection and results}

Using the model $\operatorname{BCPE}\left(x=\operatorname{age}^{\lambda}, \operatorname{df}(\mu)=9, \operatorname{df}(\sigma)=4, v=1, \tau=2\right)$ as the starting point, the best agetransformation power $(\lambda)$ was sought. As for boys, the same value $\lambda=0.20$ corresponded to the smallest value of global deviance and was selected as the age-transformation power (Table 12). A search followed for the best combination of degrees of freedom for the cubic splines to fit the BCPE distribution parameter curves. 
Table 12 Global deviance (GD) for models within the class $\operatorname{BCPE}\left(x=\operatorname{age}^{\lambda}, \operatorname{df}(\mu)=9, \operatorname{df}(\sigma)=4, v=1\right.$, $\tau=2$ ) for head circumference-for-age for girls

\begin{tabular}{llllllllllc}
\hline $\boldsymbol{\lambda}$ & $\mathbf{0 . 0 5}$ & $\mathbf{0 . 1 0}$ & $\mathbf{0 . 1 5}$ & $\mathbf{0 . 2 0}$ & $\mathbf{0 . 2 5}$ & $\mathbf{0 . 3 0}$ & $\mathbf{0 . 3 5}$ & $\mathbf{0 . 4 0}$ & $\mathbf{0 . 4 5}$ & $\mathbf{0 . 5 0}$ \\
$\mathrm{GD}^{\mathrm{a}}$ & 989.1 & 988.7 & 988.3 & 988.1 & 988.9 & 991.7 & 997.4 & 1006.3 & 1017.3 & 1027.3 \\
\hline $\boldsymbol{\lambda}$ & $\mathbf{0 . 5 5}$ & $\mathbf{0 . 6 0}$ & $\mathbf{0 . 6 5}$ & $\mathbf{0 . 7 0}$ & $\mathbf{0 . 7 5}$ & $\mathbf{0 . 8 0}$ & $\mathbf{0 . 8 5}$ & $\mathbf{0 . 9 0}$ & $\mathbf{0 . 9 5}$ & $\mathbf{1 . 0 0}$ \\
$\mathrm{GD}^{\mathrm{a}}$ & 1033.0 & 1031.8 & 1024.8 & 1018.4 & 1024.3 & 1058.8 & 1140.1 & $\mathrm{~N} A$ & $\mathrm{NA}$ & $\mathrm{NA}$ \\
\hline
\end{tabular}

${ }^{a}$ In excess of 46000 ; NA: not available (method did not converge with this power).

The search for the best combination of degrees of freedom for the cubic splines to fit the $\mu$ and $\sigma$ parameter curves started from the simplest class of models using the BCPE distribution and fixing $v=1$, $\tau=2$, and $\lambda=0.20$. Table 13 shows various combinations that were considered. The best combination of $A I C$ and $\operatorname{GAIC}(3)$ supported the model with $\operatorname{df}(\mu)=9$ and $\mathrm{df}(\sigma)=2$. The properties of this model were evaluated using the same set of diagnostic tools as those used for boys' head circumference-for-age.

Table 13 Goodness-of-fit summary for models using the BCPE distribution with fixed $v=1$ and $\tau=\mathbf{2}$ for head circumference-for-age for girls

\begin{tabular}{ccrrrr}
\hline $\mathbf{d f}(\boldsymbol{\mu})$ & $\mathbf{d f}(\boldsymbol{\sigma})$ & \multicolumn{1}{c}{$\mathbf{G D}^{\mathbf{a}}$} & \multicolumn{1}{c}{ AIC $^{\mathbf{a}}$} & GAIC(3) $^{\mathbf{a}}$ & Total df \\
\hline & $\mathbf{1}$ & 1077.7 & 1093.7 & 1101.7 & 8 \\
$\mathbf{7}$ & $\mathbf{2}$ & 1002.9 & 1020.9 & 1029.9 & 9 \\
& $\mathbf{3}$ & 1000.9 & 1020.9 & 1030.9 & 10 \\
& $\mathbf{4}$ & 1000.2 & 1022.2 & 1033.2 & 11 \\
& $\mathbf{5}$ & 998.9 & 1022.9 & 1034.9 & 12 \\
\hline & $\mathbf{1}$ & 1069.9 & 1087.9 & 1096.9 & 9 \\
$\mathbf{8}$ & $\mathbf{2}$ & 994.8 & 1014.8 & 1024.8 & 10 \\
& $\mathbf{3}$ & 992.9 & 1014.9 & 1025.9 & 11 \\
& $\mathbf{4}$ & 992.1 & 1016.1 & 1028.1 & 12 \\
& $\mathbf{5}$ & 990.8 & 1016.8 & 1029.8 & 13 \\
\hline & $\mathbf{1}$ & 1066.0 & 1086.0 & 1096.0 & 10 \\
$\mathbf{9}$ & $\mathbf{2}$ & 990.9 & 1012.9 & 1023.9 & 11 \\
& $\mathbf{3}$ & 988.9 & 1012.9 & 1024.9 & 12 \\
& $\mathbf{4}$ & 988.1 & 1014.1 & 1027.1 & 13 \\
& $\mathbf{5}$ & 986.8 & 1014.8 & 1028.8 & 14 \\
\hline & $\mathbf{1}$ & 1063.8 & 1085.8 & 1096.8 & 11 \\
& $\mathbf{2}$ & 988.7 & 1012.7 & 1024.7 & 12 \\
$\mathbf{1 0}$ & $\mathbf{3}$ & 986.6 & 1012.6 & 1025.6 & 13 \\
& $\mathbf{4}$ & 985.9 & 1013.9 & 1027.9 & 14 \\
& $\mathbf{5}$ & 984.6 & 1014.6 & 1029.6 & 15 \\
\hline & $\mathbf{1}$ & 1062.4 & 1086.4 & 1098.4 & 12 \\
& $\mathbf{2}$ & 987.2 & 1013.2 & 1026.2 & 13 \\
$\mathbf{1 1}$ & $\mathbf{3}$ & 985.2 & 1013.2 & 1027.2 & 14 \\
& $\mathbf{4}$ & 984.4 & 1014.4 & 1029.4 & 15 \\
& $\mathbf{5}$ & 983.1 & 1015.1 & 1031.1 & 16 \\
\hline
\end{tabular}

GD, Global Deviance; AIC, Akaike Information Criterion;

GAIC(3), Generalized AIC with penalty equal to 3;

${ }^{\mathrm{a}}$ In excess of 46000 . 


\section{Model 1: $\operatorname{BCPE}\left(x=\operatorname{age}^{0.20}, \operatorname{df}(\mu)=9, \operatorname{df}(\sigma)=2, v=1, \tau=2\right)$}

The two fitted parameter curves, i.e. the median and coefficient of variation appeared to be adequately smoothed, considering the substantial variation observed in the empirical coefficient of variation pattern in the cross-sectional sample (Figure 11). With respect to the differences between empirical values and fitted centiles (Figures 12 and 13), there was some evidence of bias for a few of the considered percentiles. There seemed to be underestimation around the $3^{\text {rd }}$ centile and overestimation around the $10^{\text {th }}$ centile between birth and 24 months. The average bias was approximately $1 \mathrm{~mm}$. Fitted centile residuals for the age interval 24 to 71 months showed a mild systematic underestimation, only for the $25^{\text {th }}$ centile curve, of less than $2 \mathrm{~mm}$ on average (Figure 13). These biases were very small when compared to the SD values that varied from $1.2 \mathrm{~cm}$ to $1.4 \mathrm{~cm}$ in the entire age range. Examining Table 14, the proportions of children in the sample that were below the $1^{\text {st }}$ and $3^{\text {rd }}$ fitted centiles confirmed some underestimation in the lower tail. For the other centiles, the distribution of the proportions below each of the fitted centiles did not indicate any systematic bias.
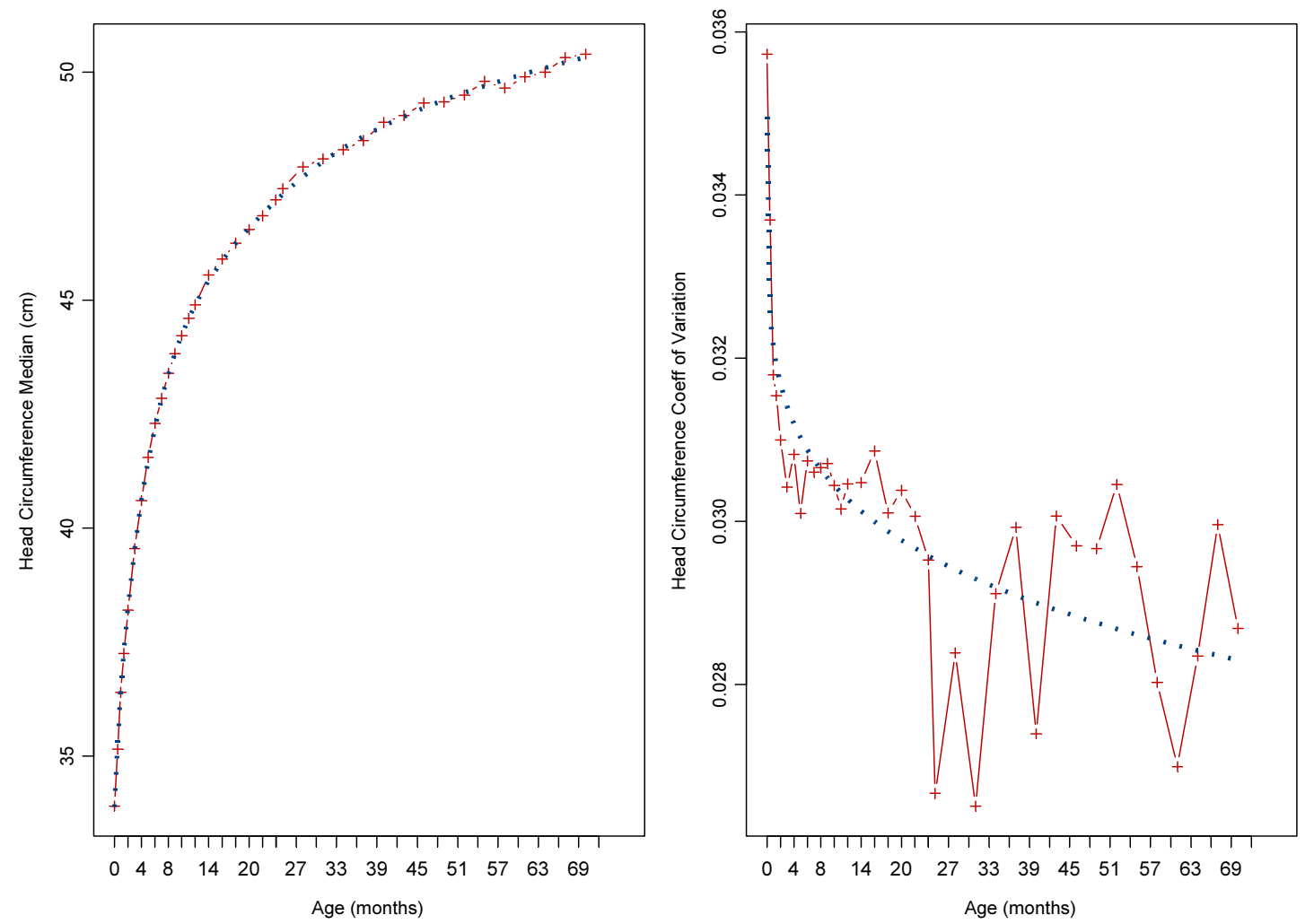

Figure 11 Fitting of the $\mu$ and $\sigma$ curves of Model 1 for head circumference-for-age for girls (dotted line) and their respective sample estimates (points with solid line)

Figure 14 showed the worm plots for Model 1. For only two age groups, the shapes of the worms departed from the flat shape. The group 28 mo presented a slope, indicating misfit of the variance, and the worm corresponding to group 52 mo was slightly S-shaped, suggesting remaining kurtosis. Group 70 mo presented a worm that was U-shaped which would indicate skewness, yet the worm lay within the $95 \%$ confidence interval. 

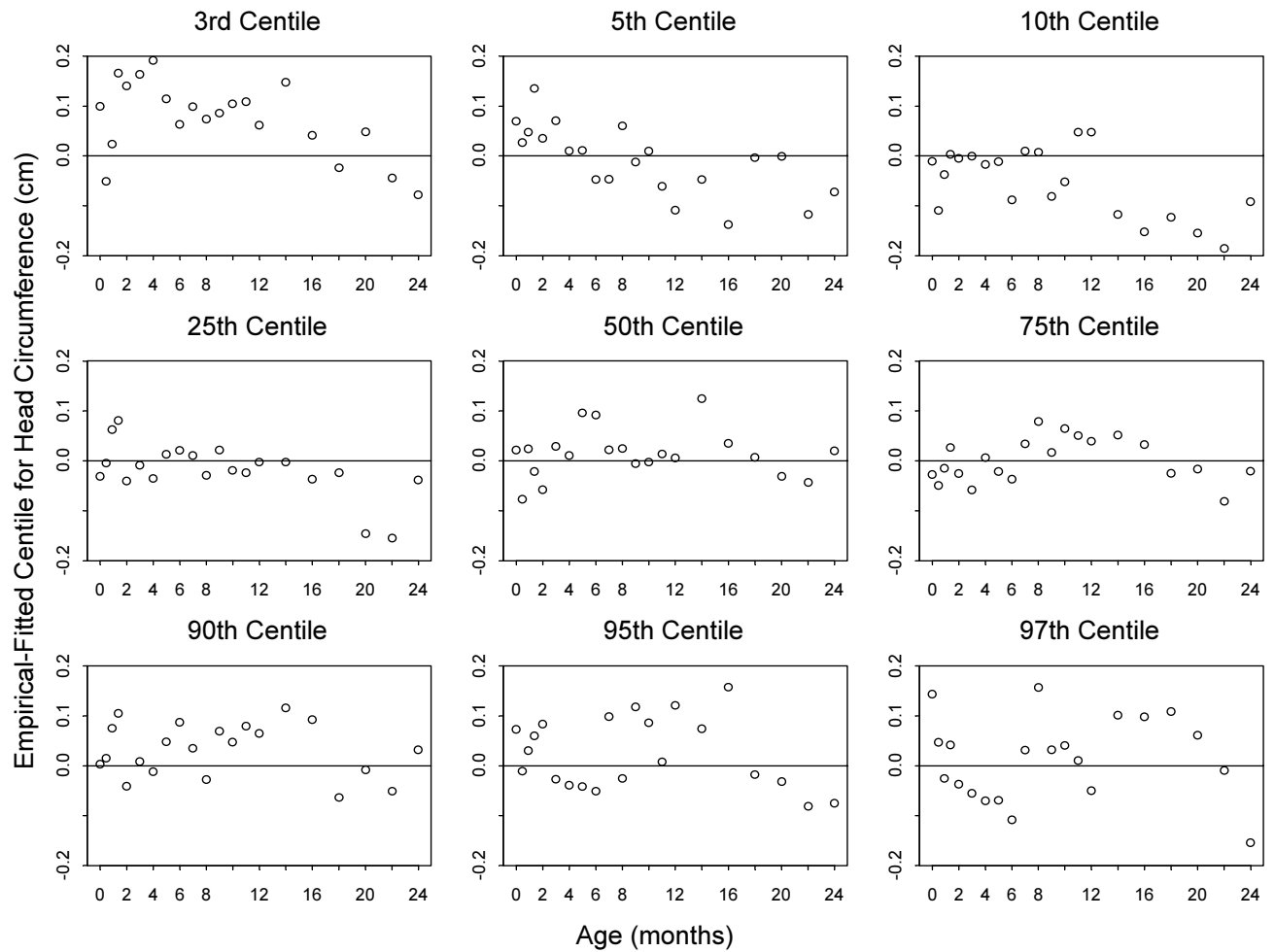

Figure 12 Centile residuals from fitting Model 1 for head circumference-for-age from 0 to 24 months for girls
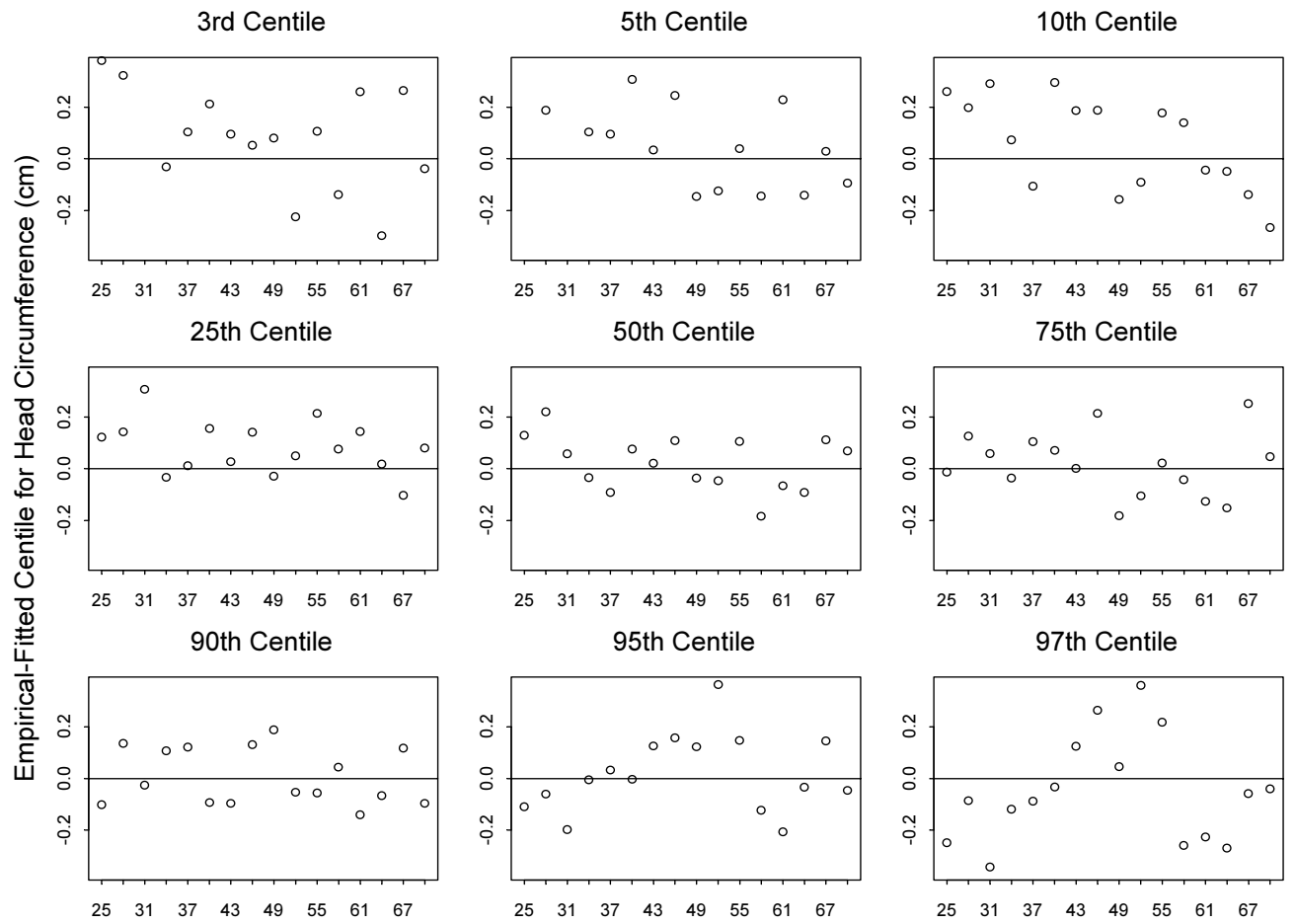

50th Centile

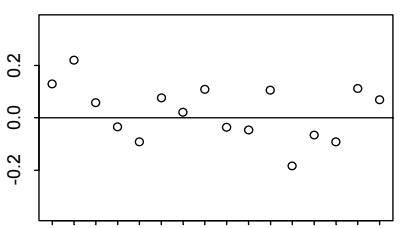

75th Centile

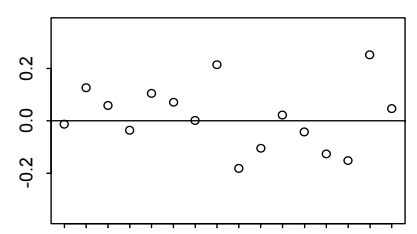

$\begin{array}{llllllll}25 & 31 & 37 & 43 & 49 & 55 & 61 & 67\end{array}$

$\begin{array}{llllllll}25 & 31 & 37 & 43 & 49 & 55 & 61 & 67\end{array}$

95th Centile
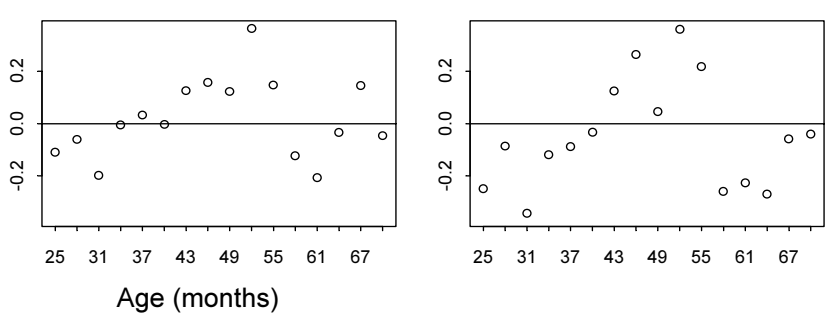

Figure 13 Centile residuals from fitting Model 1 for head circumference-for-age from 24 to 71 months for girls 


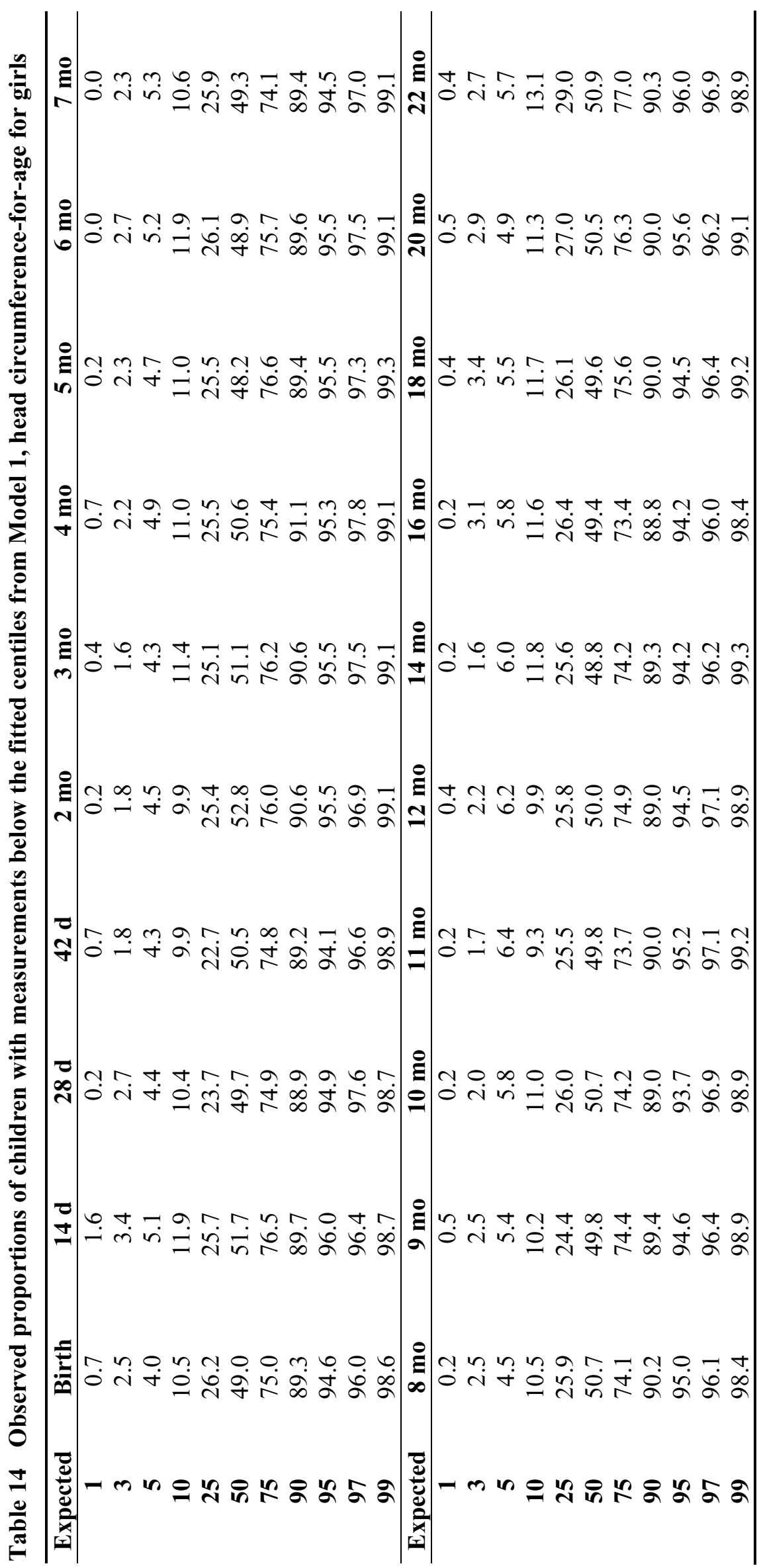




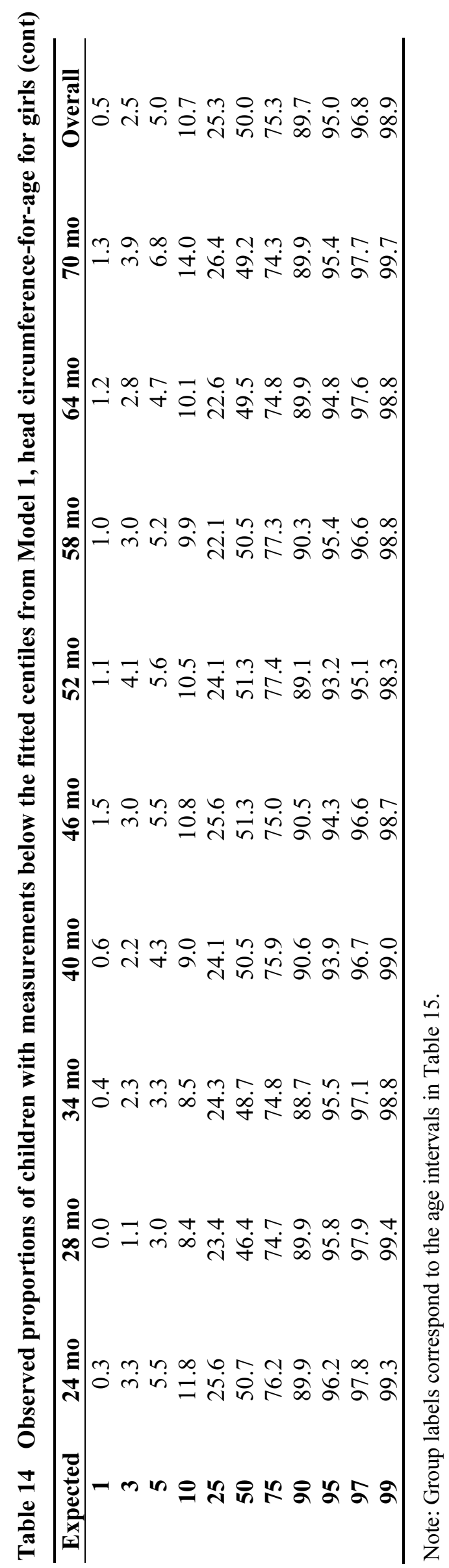



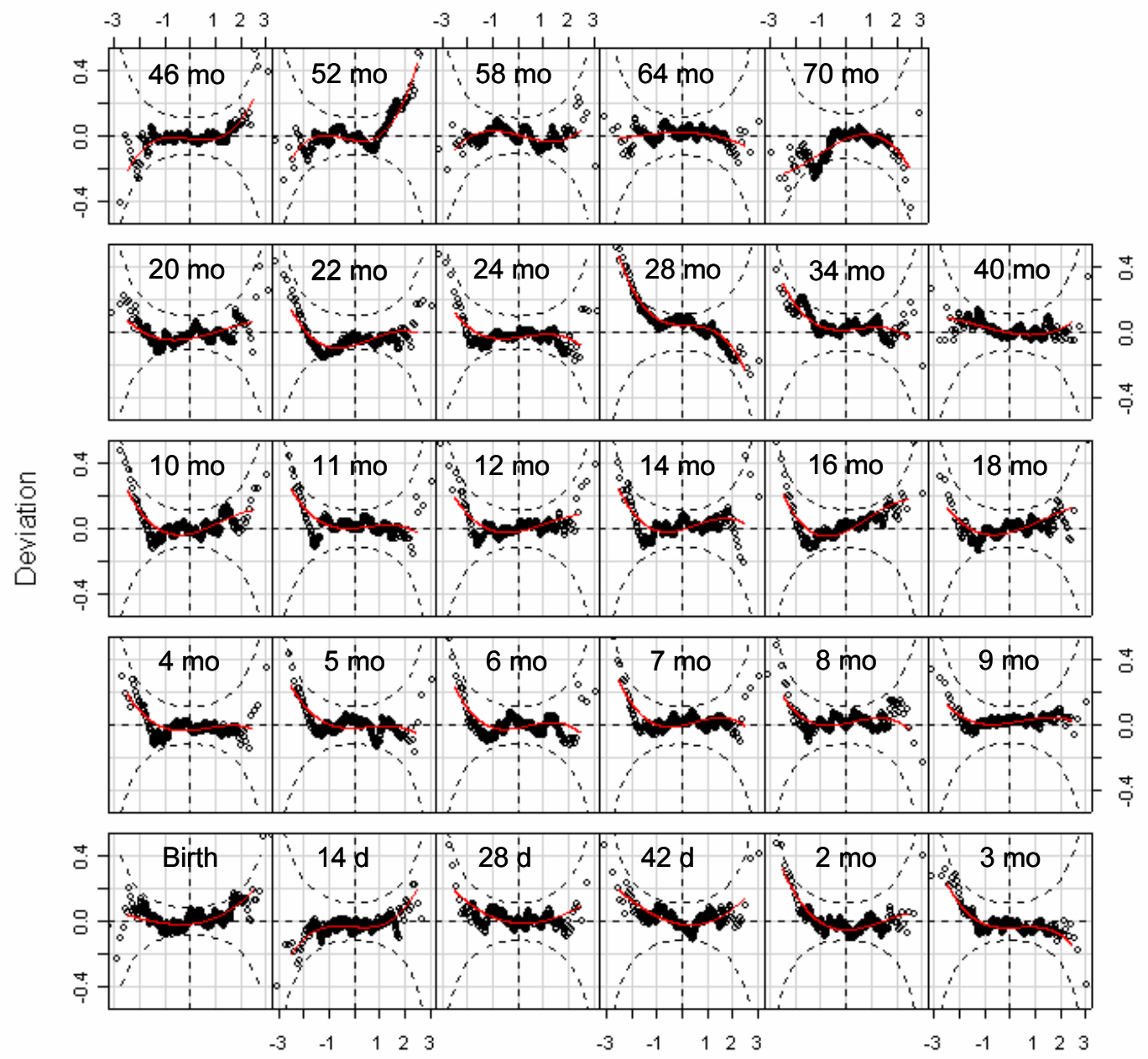

Unit normal quantile

Figure 14 Worm plots of z-scores for Model 1 for head circumference-for-age for girls

Results of the Q-test presented in Table 15 showed that only the misfit of the variance (group 28 mo) was significant, i.e. the corresponding $\mathrm{z} 2$ absolute value was above 2. For the same age group, the Qtest suggested remaining kurtosis ( $\mathrm{z} 4$ absolute value greater than 2), likely a consequence of the misfit of the variance. However, Q-test results for the z-scores derived from the selected model when applied across all age groups bore evidence of the model's overall adequacy ( $p$-values corresponding to overall statistics are all greater than 0.30 ). More complex modelling (e.g. fitting parameter $v$ ) was therefore not pursued.

A new iteration was done to re-search for the best $\lambda$ with $\operatorname{df}(\mu)=9$ and $\operatorname{df}(\sigma)=2$. This exercise did not result in any notable changes to the previous findings. The model $\operatorname{BCPE}\left(x=\operatorname{age}^{0.20}, \operatorname{df}(\mu)=9, \operatorname{df}(\sigma)=2\right.$, $v=1, \tau=2$ ) was thus chosen to construct the head circumference-for-age growth curves for girls. Figures 15 to 18 show the empirical and fitted centiles derived from the selected model for the head circumference-for-age ( 0 to 60 months) growth standard. 
Table 15 Q-test for $z$-scores from Model 1 [BCPE $\left.\left(x=a g e^{0.20}, \operatorname{df}(\mu)=9, \operatorname{df}(\sigma)=2, v=1, \tau=2\right)\right]$ for head circumference-for-age for girls

\begin{tabular}{|c|c|c|c|c|c|c|}
\hline Age (days) & Group & $\mathbf{N}$ & $\mathrm{z1}$ & $\mathrm{z2}$ & $\mathbf{z 3}$ & $\mathrm{z4}$ \\
\hline 0 & Birth & 841 & 0.1 & 0.9 & 1.4 & 0.5 \\
\hline 1 to 16 & $14 \mathrm{~d}$ & 447 & -0.7 & 1.0 & 0.3 & 1.4 \\
\hline 17 to 34 & $28 \mathrm{~d}$ & 451 & 0.3 & -0.4 & 1.3 & 0.4 \\
\hline 35 to 49 & $42 \mathrm{~d}$ & 444 & 0.3 & -0.5 & 1.6 & 0.5 \\
\hline 50 to 69 & $2 \mathrm{mo}$ & 445 & -0.4 & -0.7 & 1.9 & -0.4 \\
\hline 70 to 99 & $3 \mathrm{mo}$ & 446 & -0.5 & -1.0 & 0.6 & -1.3 \\
\hline 100 to 129 & $4 \mathrm{mo}$ & 447 & -0.4 & -0.3 & 0.9 & -0.5 \\
\hline 130 to 159 & $5 \mathrm{mo}$ & 444 & 0.1 & -0.7 & 0.7 & -0.6 \\
\hline 160 to 189 & $6 \mathrm{mo}$ & 444 & 0.1 & -0.2 & 0.6 & -1.0 \\
\hline 190 to 219 & $7 \mathrm{mo}$ & 436 & 0.4 & -0.1 & 1.0 & -1.0 \\
\hline 220 to 249 & $8 \mathrm{mo}$ & 440 & 0.3 & 0.0 & 0.6 & -0.9 \\
\hline 250 to 279 & 9 mo & 442 & 0.5 & 0.1 & 0.6 & -0.6 \\
\hline 280 to 309 & $10 \mathrm{mo}$ & 446 & -0.1 & 0.2 & 1.7 & -0.4 \\
\hline 310 to 349 & $11 \mathrm{mo}$ & 482 & 0.4 & -0.5 & 0.7 & -0.7 \\
\hline 350 to 379 & $12 \mathrm{mo}$ & 454 & 0.1 & 0.2 & 1.2 & -0.4 \\
\hline 380 to 439 & $14 \mathrm{mo}$ & 449 & 0.3 & 0.1 & 1.0 & -0.8 \\
\hline 440 to 499 & $16 \mathrm{mo}$ & 447 & 0.3 & 1.1 & 1.5 & -0.6 \\
\hline 500 to 559 & $18 \mathrm{mo}$ & 472 & 0.0 & 0.7 & 1.3 & -0.1 \\
\hline 560 to 619 & $20 \mathrm{mo}$ & 549 & -0.4 & 0.5 & 0.9 & -0.3 \\
\hline 620 to 679 & $22 \mathrm{mo}$ & 548 & -1.3 & 0.4 & 1.3 & -1.1 \\
\hline 680 to 749 & $24 \mathrm{mo}$ & 602 & -0.7 & -0.2 & 0.4 & -0.9 \\
\hline 750 to 929 & $28 \mathrm{mo}$ & 474 & 1.2 & -2.2 & 0.5 & -2.3 \\
\hline 930 to 1119 & $34 \mathrm{mo}$ & 485 & 0.8 & -0.9 & 0.9 & -1.1 \\
\hline 1120 to 1309 & $40 \mathrm{mo}$ & 489 & 0.1 & -0.4 & 0.8 & 0.4 \\
\hline 1310 to 1499 & $46 \mathrm{mo}$ & 472 & -0.4 & 1.0 & 0.2 & 1.6 \\
\hline 1500 to 1689 & $52 \mathrm{mo}$ & 468 & 0.0 & 1.5 & 1.6 & 2.0 \\
\hline 1690 to 1879 & $58 \mathrm{mo}$ & 503 & 0.1 & -0.4 & -0.3 & 1.1 \\
\hline 1880 to 2069 & $64 \mathrm{mo}$ & 424 & 0.2 & -0.2 & -0.5 & -0.1 \\
\hline 2070 to 2191 & $70 \mathrm{mo}$ & 307 & -0.9 & 1.0 & -1.2 & -0.6 \\
\hline Overall Q stats & & 13798 & 7.5 & 16.8 & 32.3 & 27.3 \\
\hline degrees of freedom & & & 20.0 & 27.5 & 29.0 & 29.0 \\
\hline p-value & & & 0.9947 & 0.9440 & 0.3074 & 0.5551 \\
\hline
\end{tabular}

Note: Absolute values of z1, z2, z3 or z4 larger than 2 indicate misfit of, respectively, mean, variance, skewness or kurtosis. 


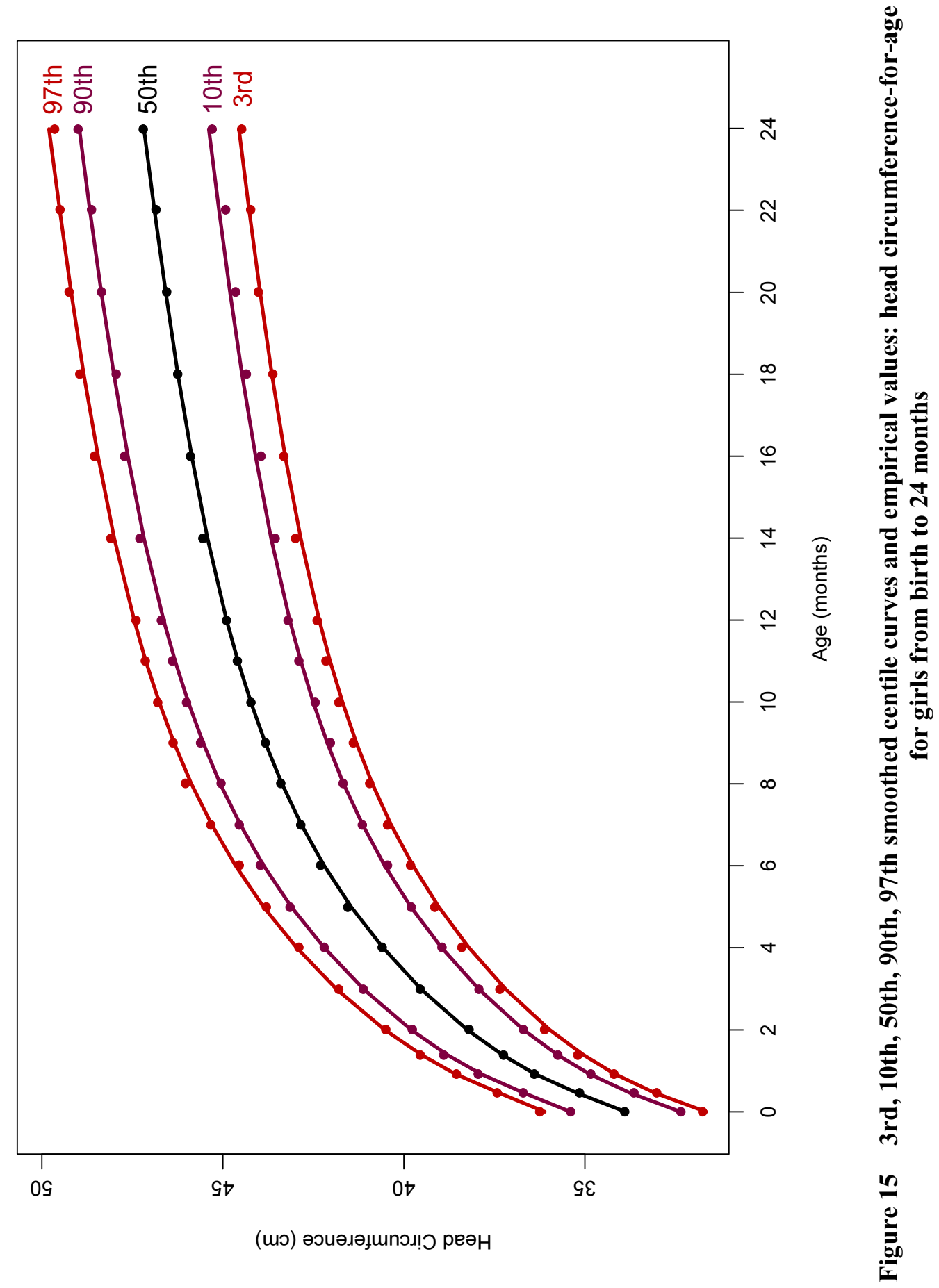




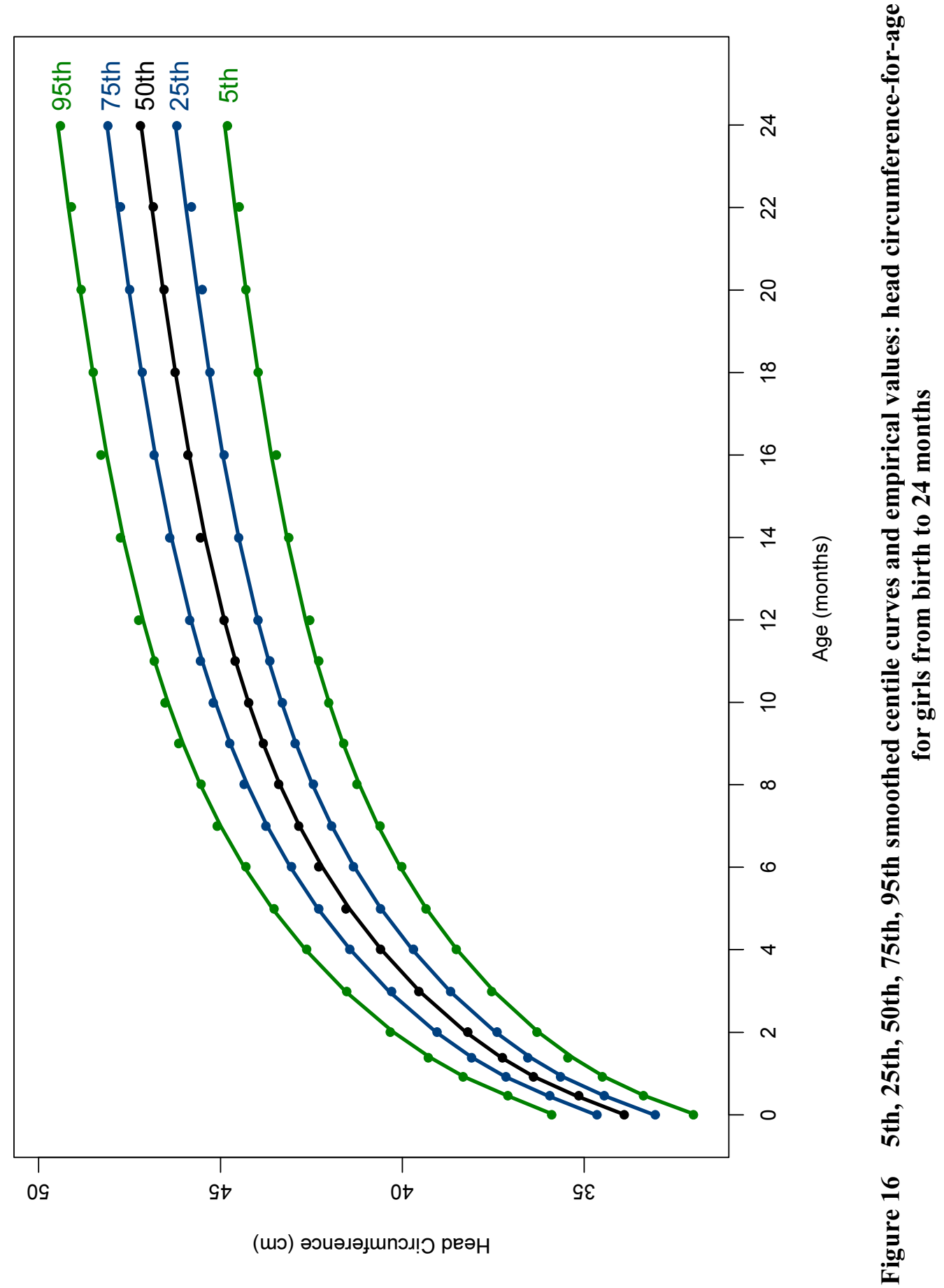




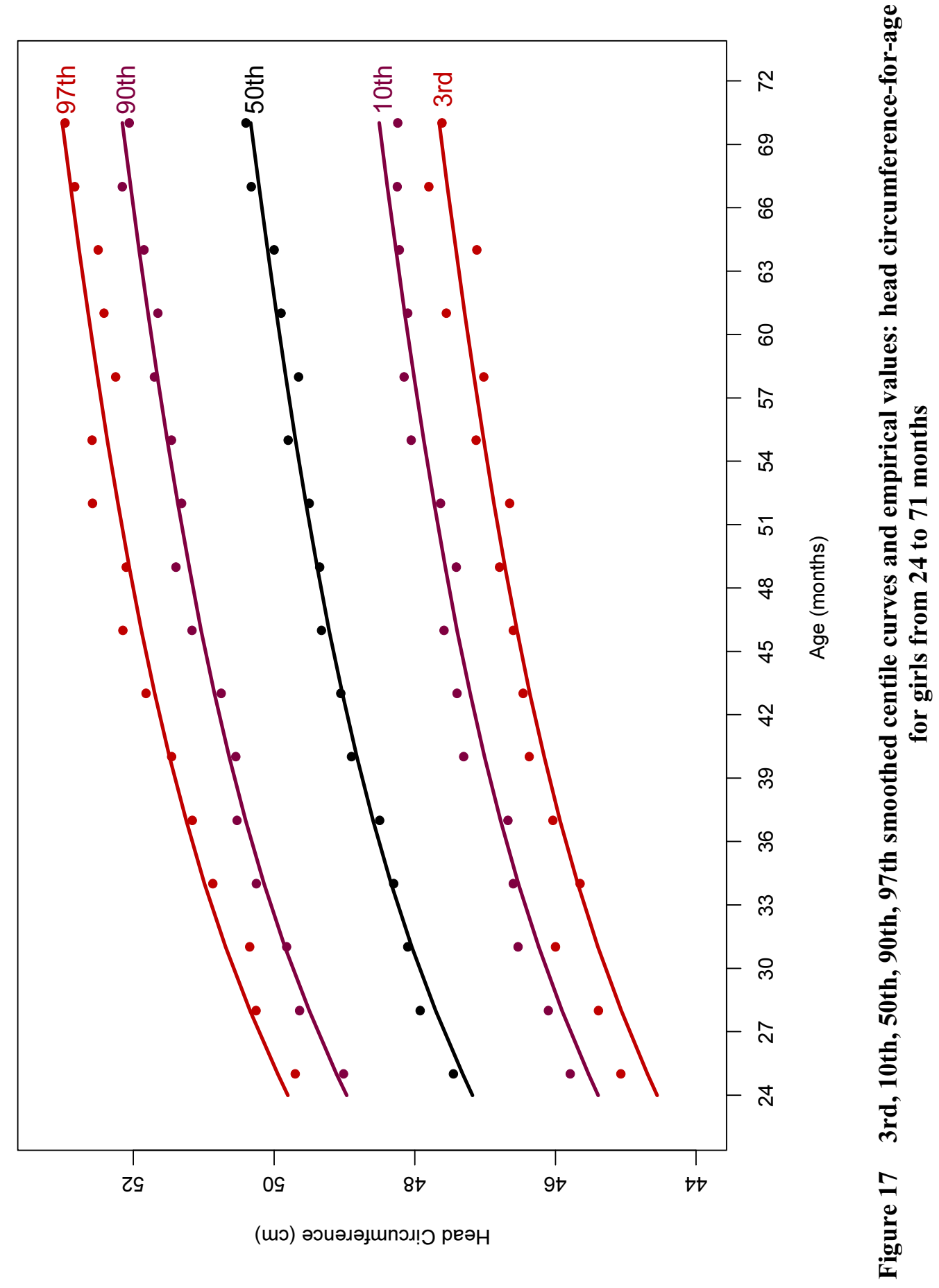




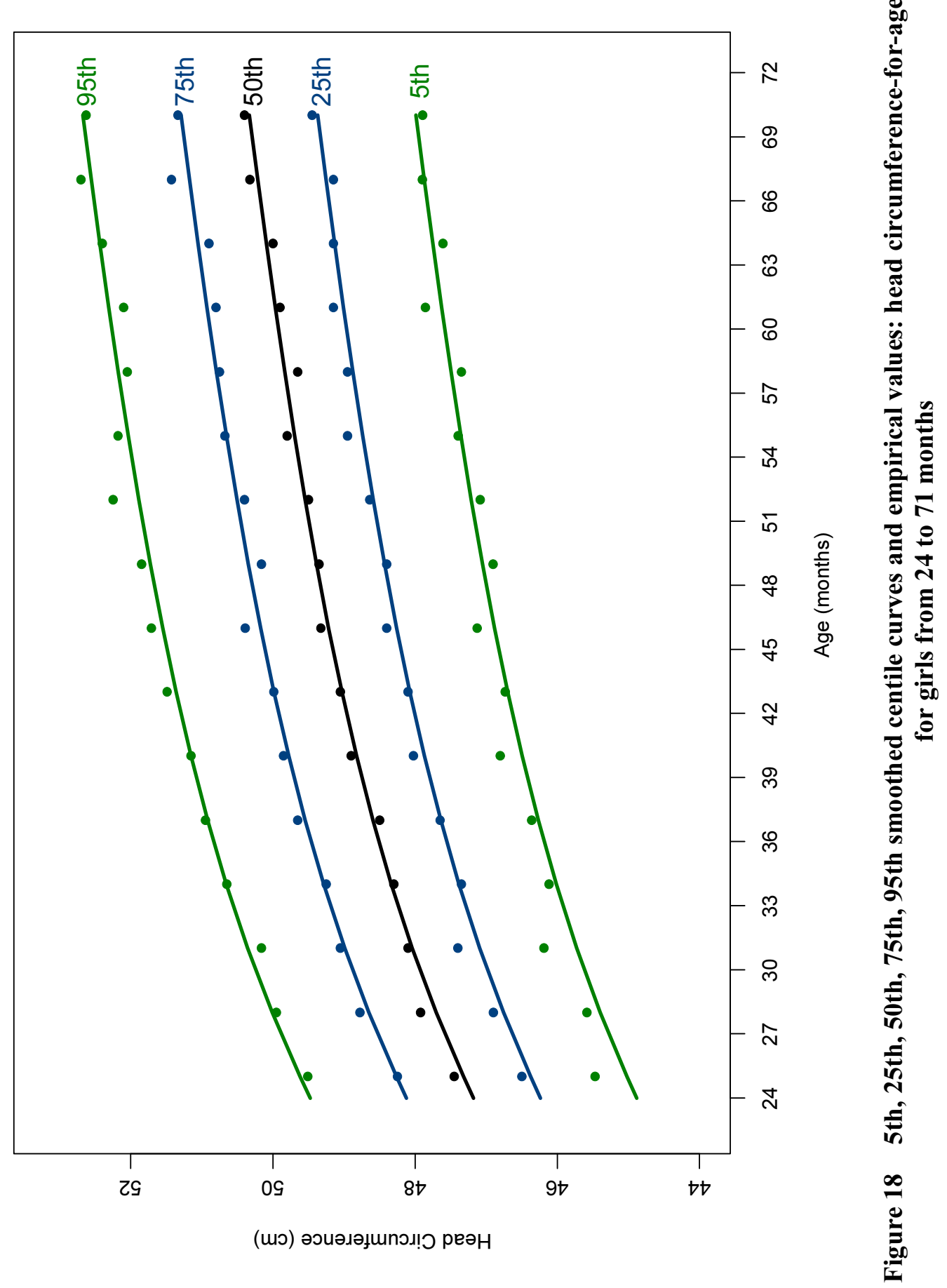




\subsubsection{WHO standards}

This section presents the final WHO head circumference-for-age z-score and percentile charts (Figures 19 and 20) and tables (Tables 16 and 17) for girls. 


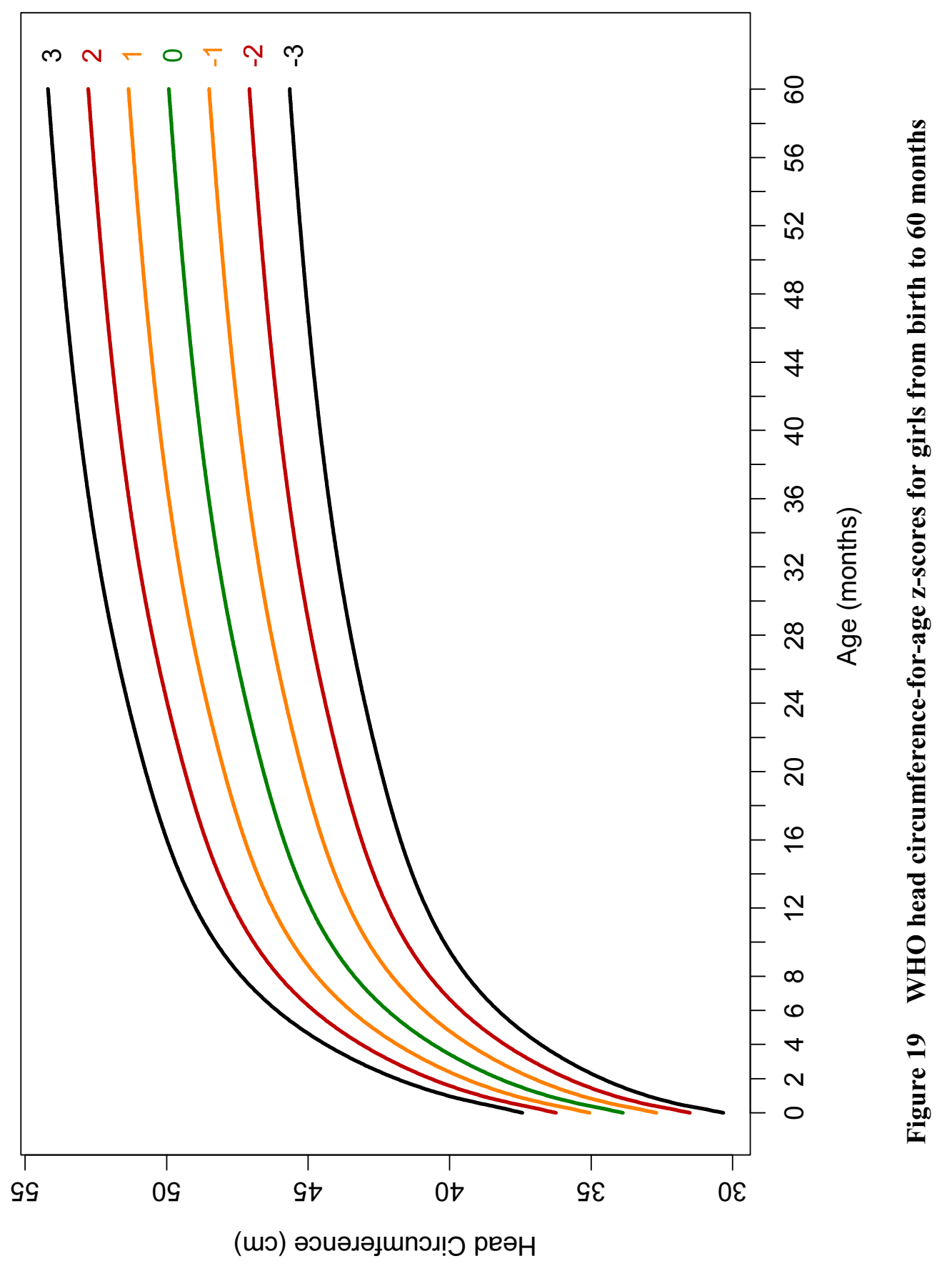




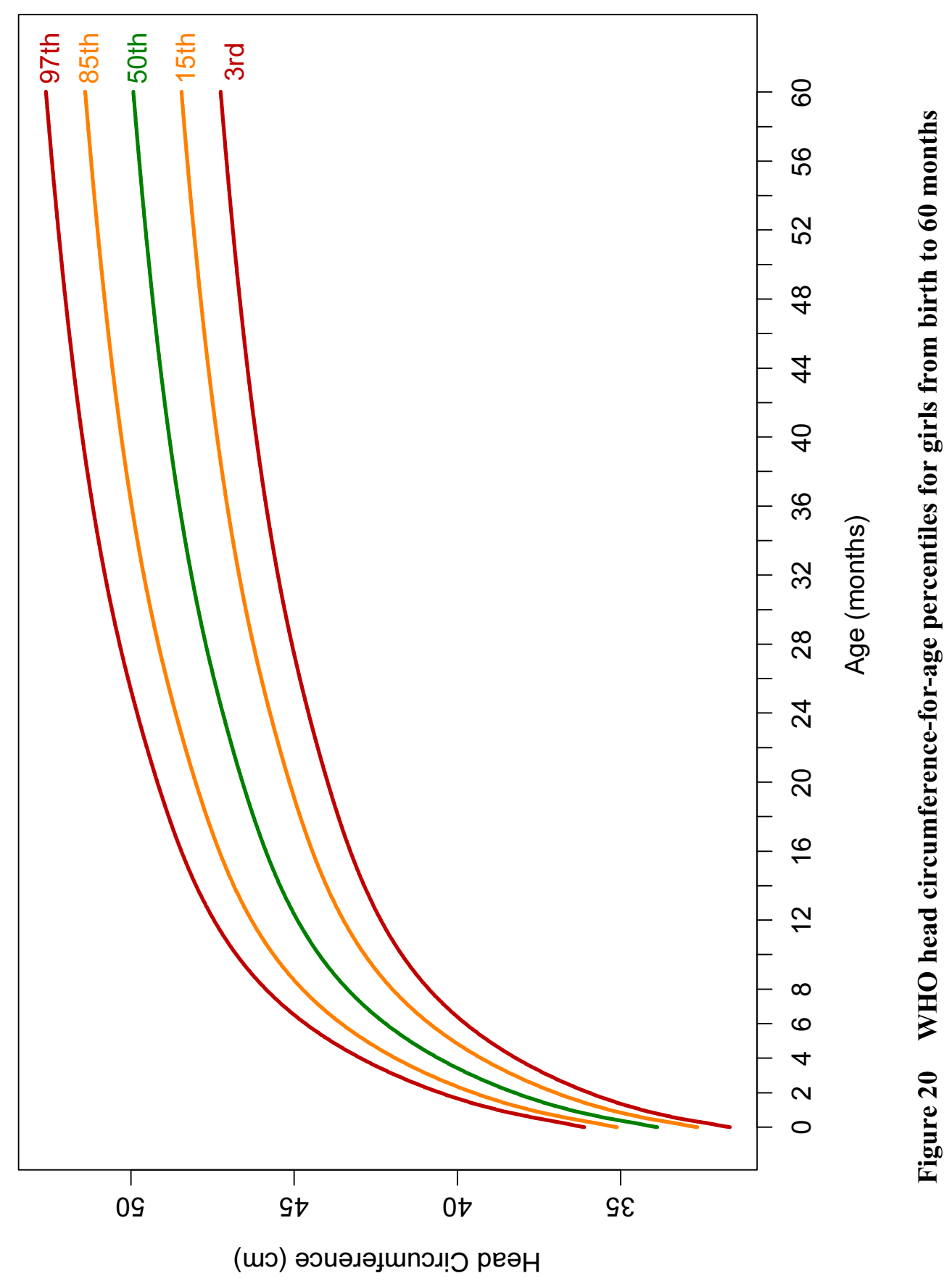




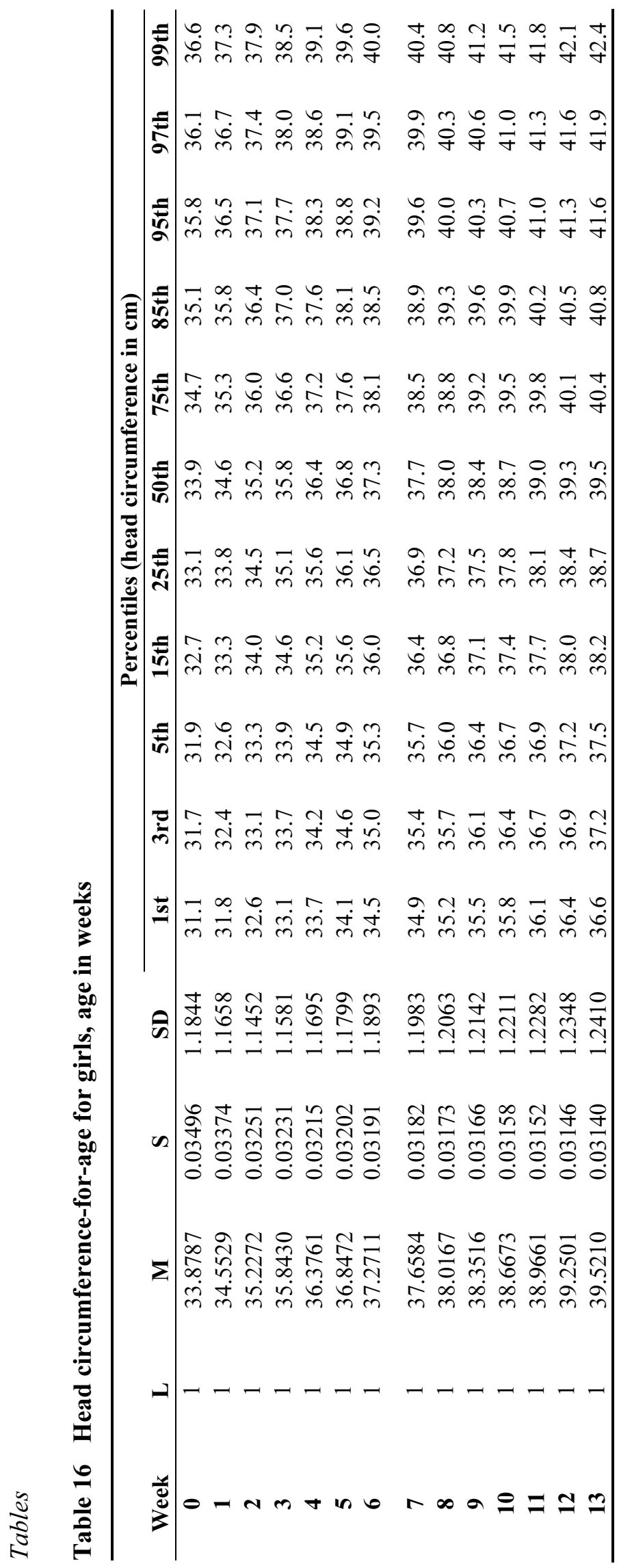




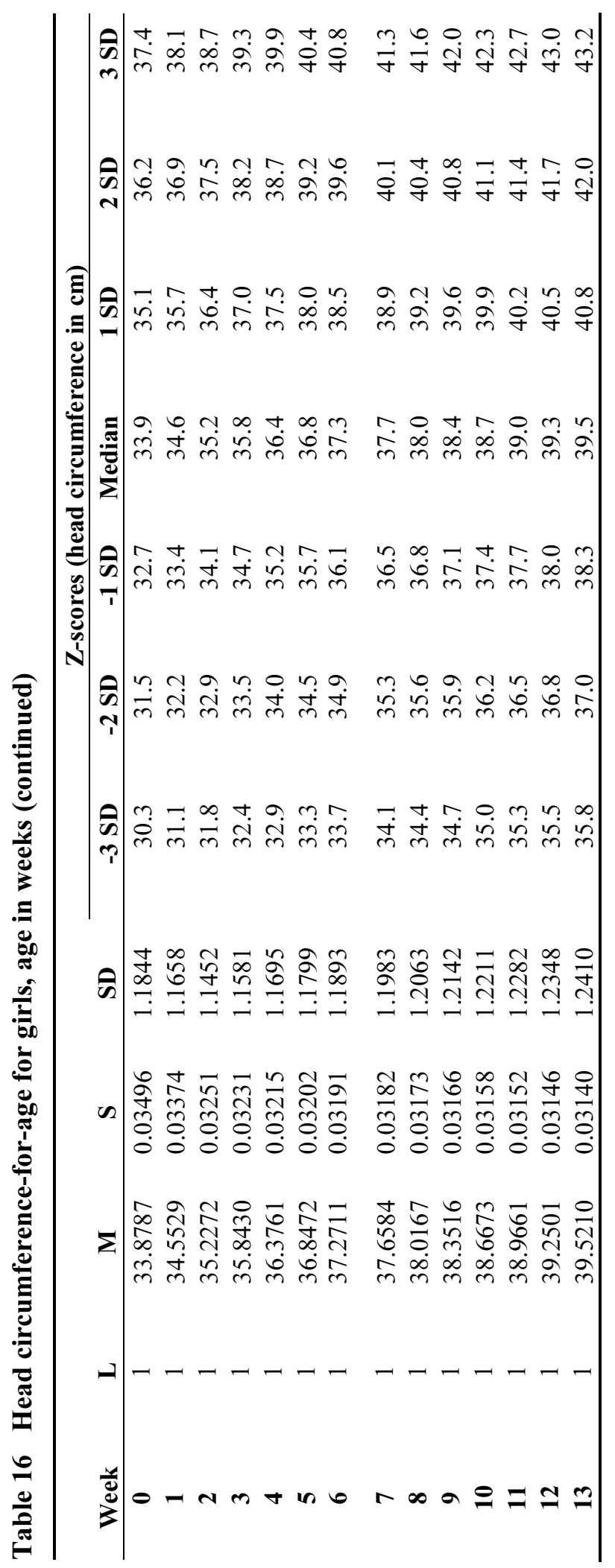




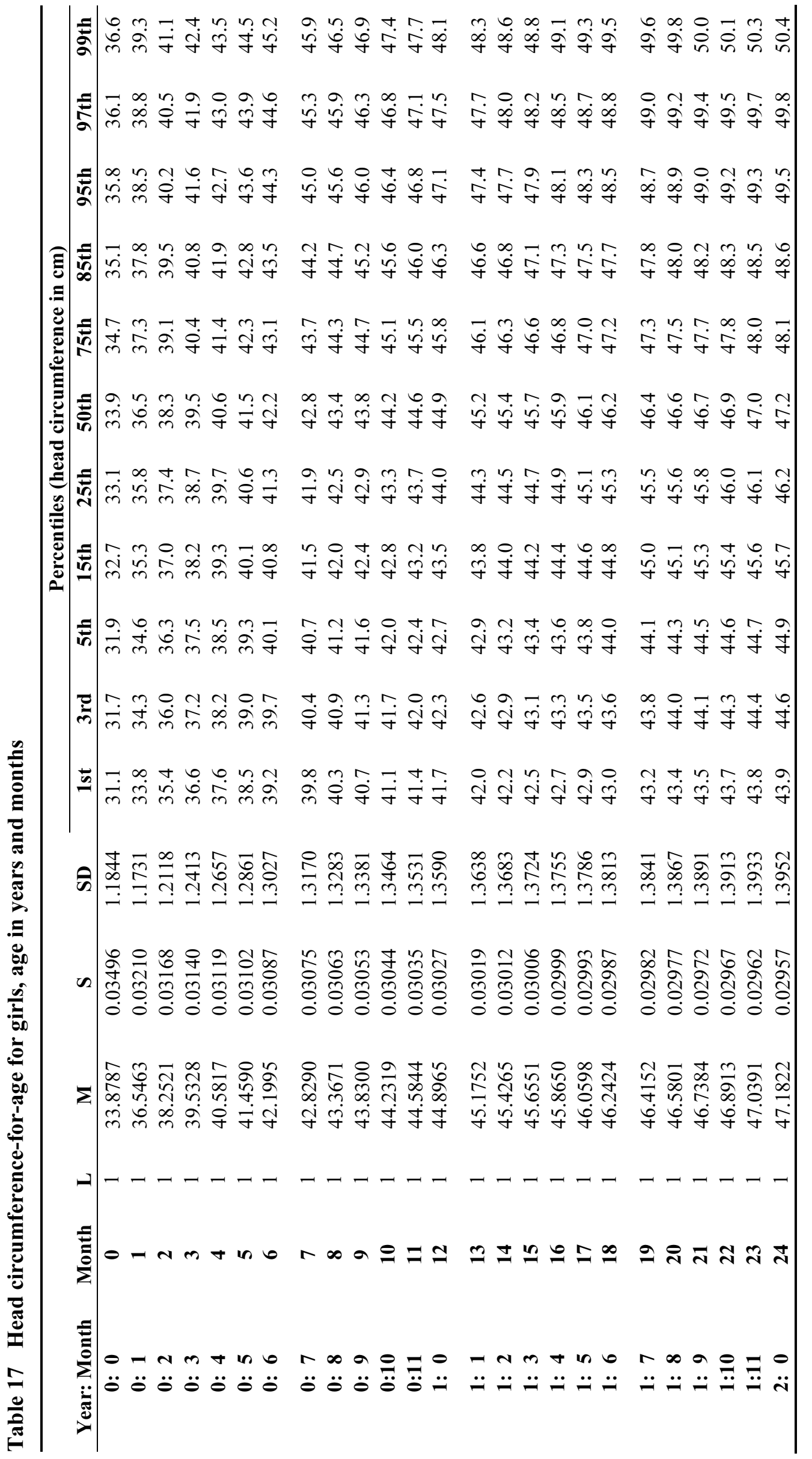




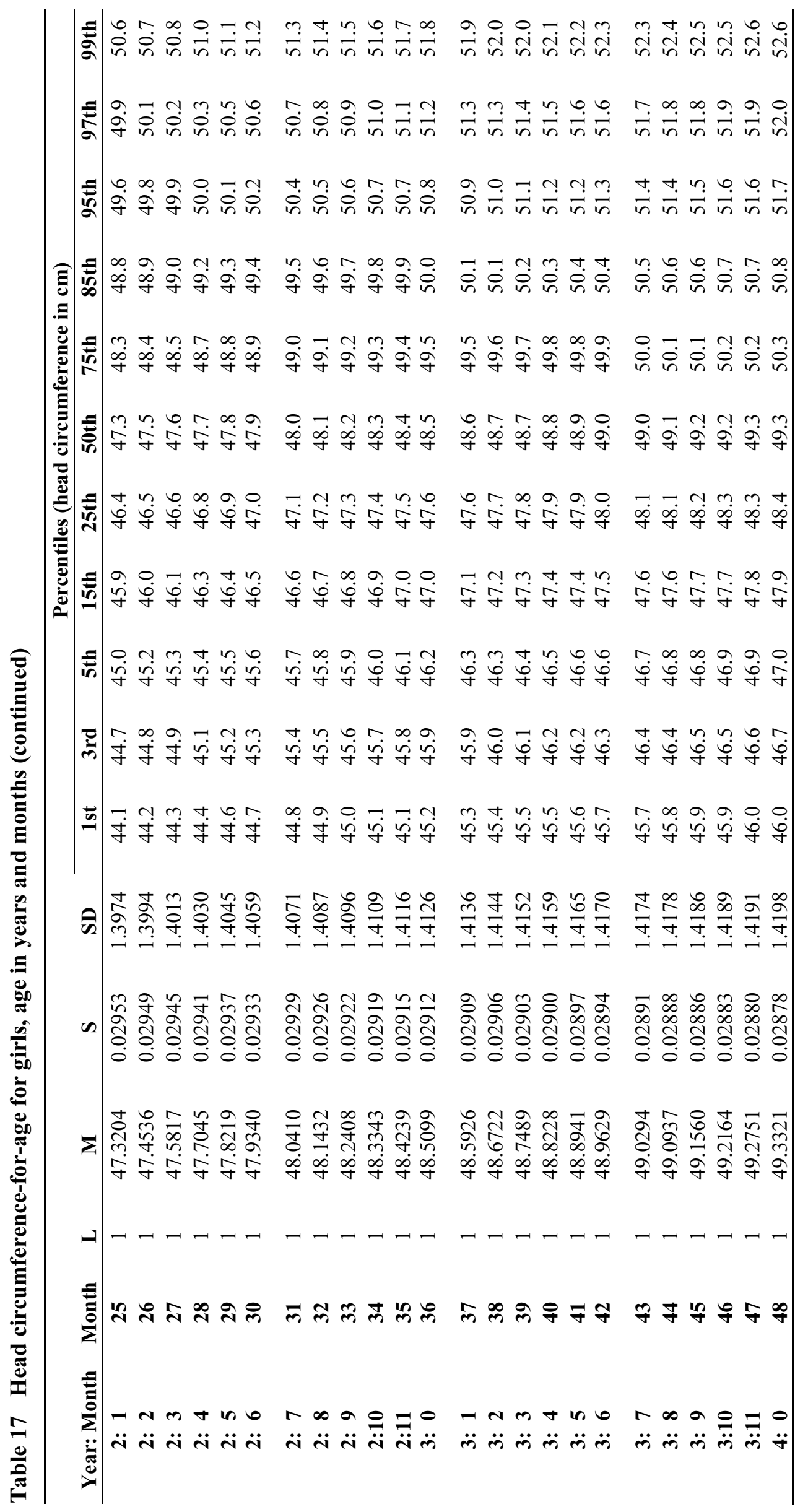




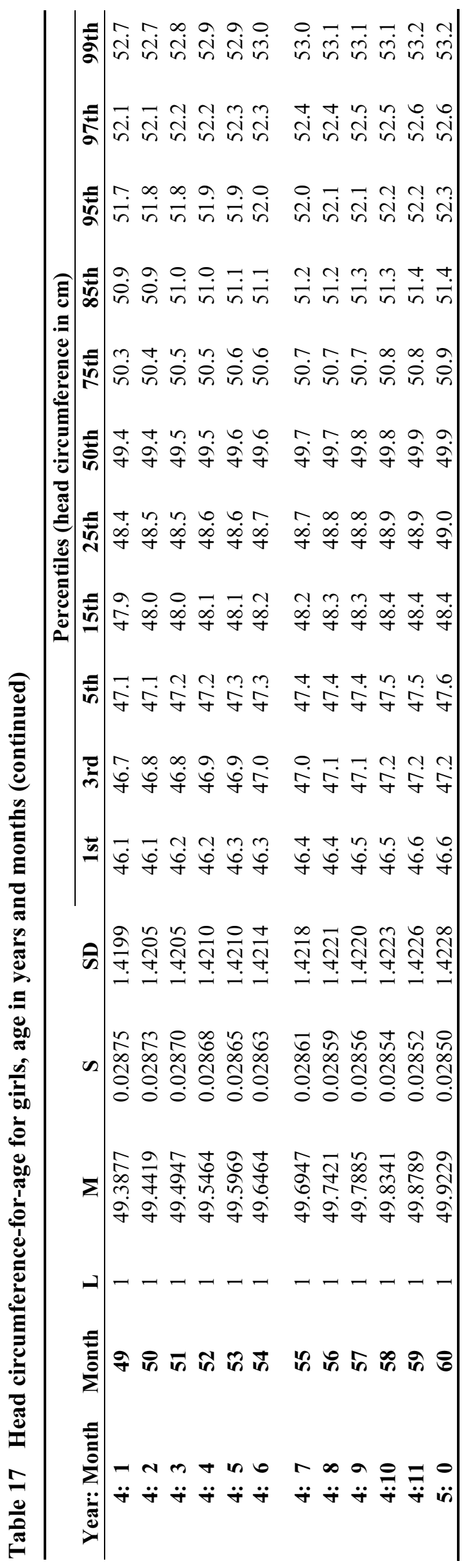




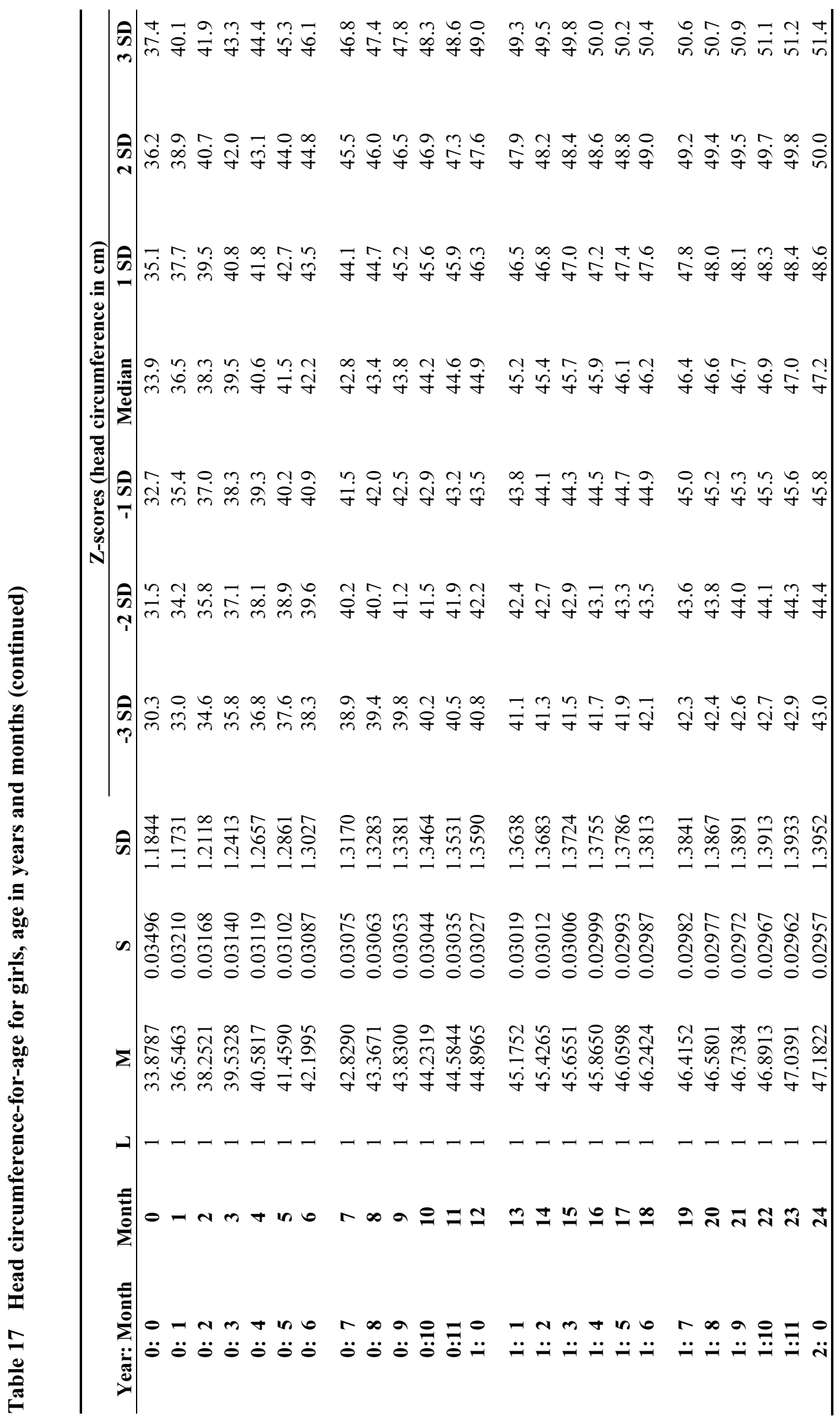




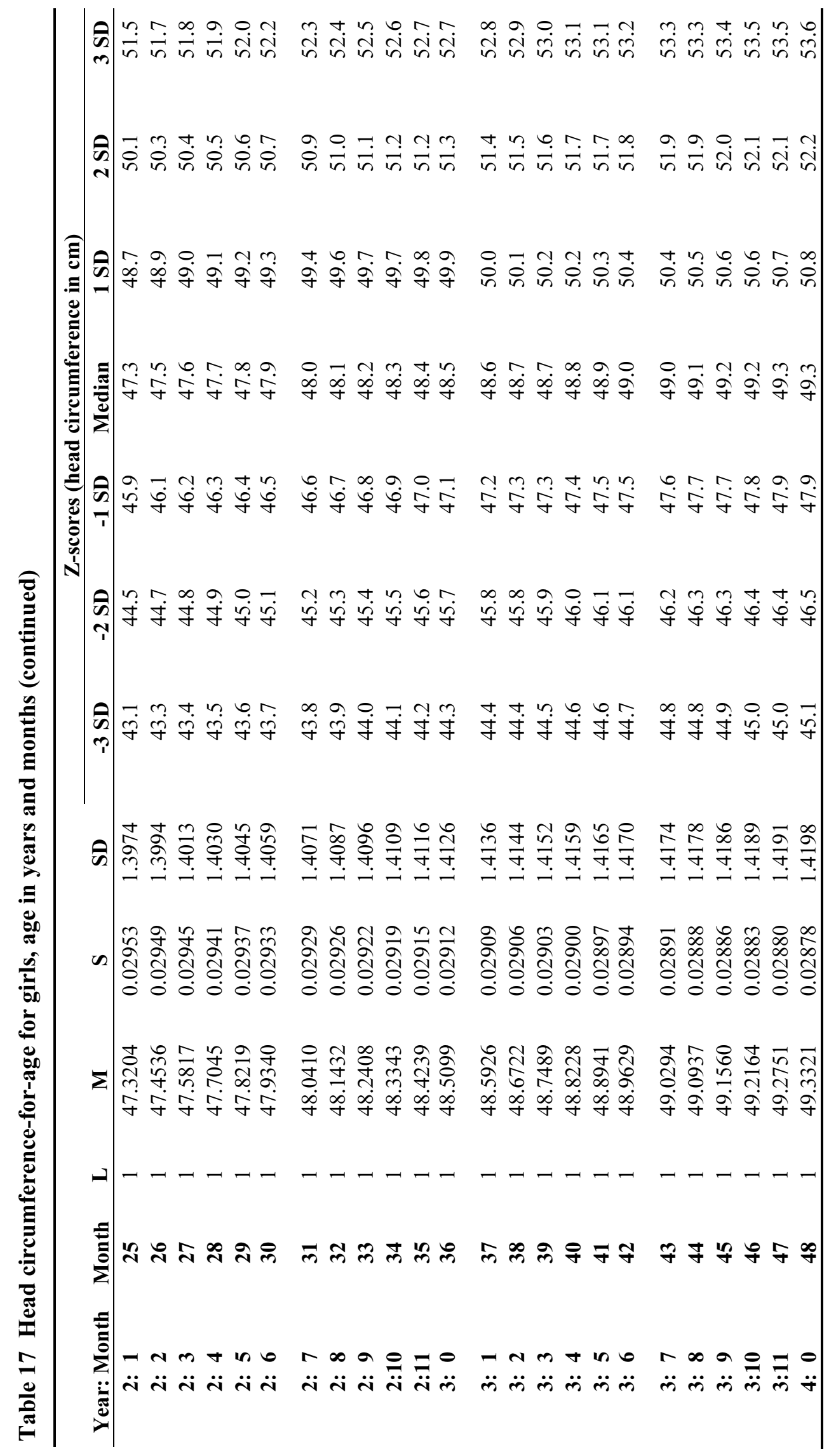




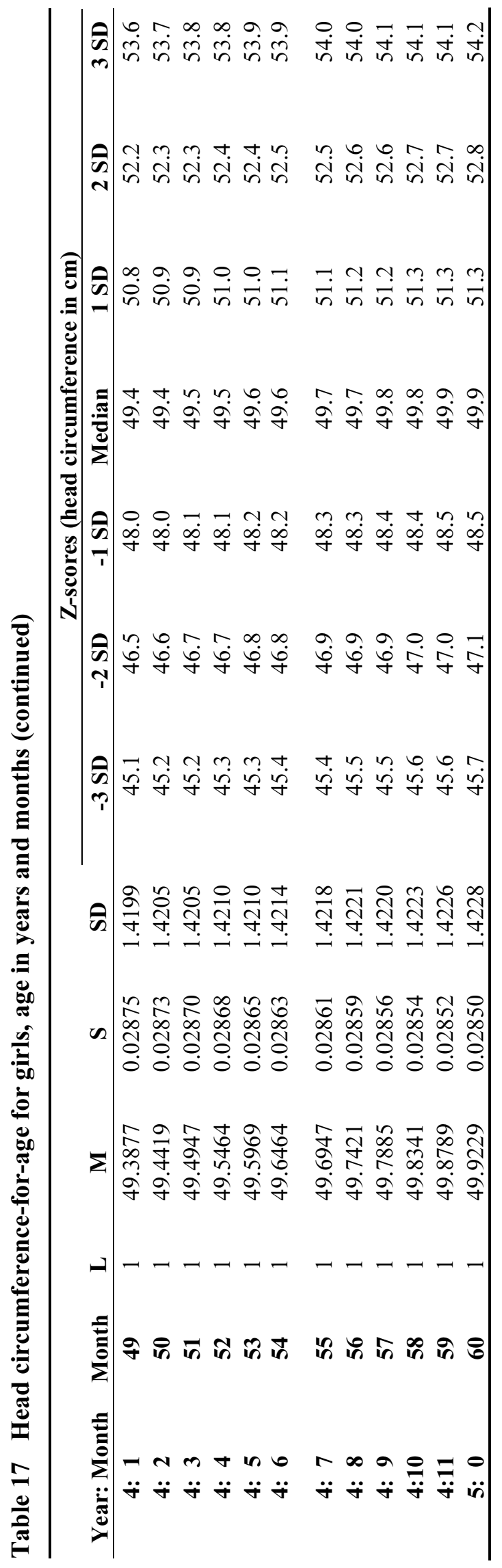




\subsection{Comparisons between boys and girls}

This section presents the head circumference-for-age z-score comparisons between boys and girls for the WHO standards (Figure 21). 


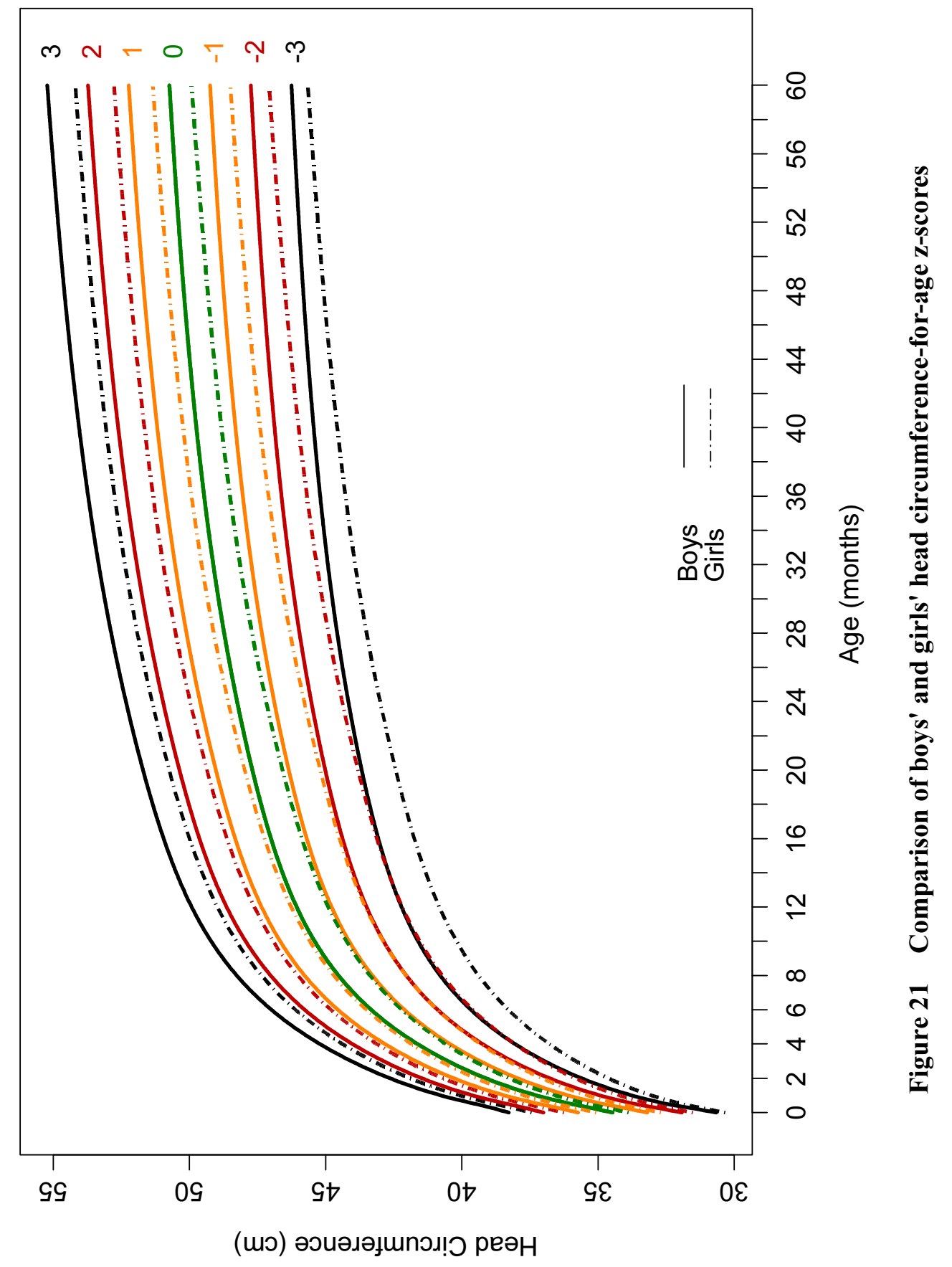

i. 



\section{CONSTRUCTION OF THE ARM CIRCUMFERENCE-FOR-AGE STANDARDS}

\subsection{Indicator-specific methodology}

Similar steps to those described for the head circumference-for-age growth curves were followed to construct the arm circumference-for-age growth curves. The diagnostic tools applied to evaluate and compare candidate models were also similar. All data up to 71 months were used for modelling the arm circumference-for-age growth curves and the standards afterwards truncated at 60 completed months to correct for the right-edge effect (Borghi et al., 2006).

\subsection{Arm circumference-for-age for boys}

\subsubsection{Sample size}

There were a total of 10770 arm circumference observations for boys. The longitudinal and crosssectional sample sizes by visit and age are presented in Tables 18 and 19. The measurement of arm circumference started at 3 months of age (de Onis et al., 2004b).

Table 18 Longitudinal sample sizes for arm circumference-for-age for boys

\begin{tabular}{|c|c|c|c|c|c|c|}
\hline Visit & 5 & 6 & 7 & 8 & 9 & 10 \\
\hline Age & $3 \mathrm{mo}$ & $4 \mathrm{mo}$ & 5 mo & $6 \mathrm{mo}$ & $7 \mathrm{mo}$ & $8 \mathrm{mo}$ \\
\hline $\mathrm{N}$ & 413 & 407 & 412 & 417 & 415 & 415 \\
\hline Visit & 11 & 12 & 13 & 14 & 15 & 16 \\
\hline Age & $9 \mathrm{mo}$ & $10 \mathrm{mo}$ & $11 \mathrm{mo}$ & $12 \mathrm{mo}$ & $14 \mathrm{mo}$ & $16 \mathrm{mo}$ \\
\hline $\mathrm{N}$ & 409 & 407 & 418 & 414 & 416 & 417 \\
\hline Visit & 17 & 18 & 19 & 20 & & \\
\hline Age & $18 \mathrm{mo}$ & $20 \mathrm{mo}$ & $22 \mathrm{mo}$ & $24 \mathrm{mo}$ & & \\
\hline $\mathrm{N}$ & 416 & 422 & 415 & 421 & & \\
\hline
\end{tabular}

Table 19 Cross-sectional sample sizes for arm circumference-for-age for boys

\begin{tabular}{lccccccc}
\hline Age (mo) & $<\mathbf{1 8}$ & $\mathbf{1 8 - 2 0}$ & $\mathbf{2 1 - 2 3}$ & $\mathbf{2 4 - 2 6}$ & $\mathbf{2 7 - 2 9}$ & $\mathbf{3 0 - 3 2}$ & $\mathbf{3 3 - 3 5}$ \\
$\mathrm{N}$ & 3 & 177 & 183 & 236 & 257 & 218 & 252 \\
\hline Age (mo) & $\mathbf{3 6 - 3 8}$ & $\mathbf{3 9 - 4 1}$ & $\mathbf{4 2 - 4 4}$ & $\mathbf{4 5}-\mathbf{4 7}$ & $\mathbf{4 8}-\mathbf{5 0}$ & $\mathbf{5 1 - 5 3}$ & $\mathbf{5 4 - 5 6}$ \\
$\mathrm{N}$ & 266 & 249 & 255 & 237 & 237 & 221 & 225 \\
\hline Age (mo) & $\mathbf{5 7 - 5 9}$ & $\mathbf{6 0 - 6 2}$ & $\mathbf{6 3 - 6 5}$ & $\mathbf{6 6 - 6 8}$ & $\mathbf{6 9 - 7 1}$ & $>\mathbf{7 1}$ & \\
$\mathrm{N}$ & 241 & 228 & 216 & 217 & 214 & 4 & \\
\hline
\end{tabular}

\subsubsection{Model selection and results}

To construct the arm circumference-for-age growth curves, the model $\mathrm{BCPE}\left(\mathrm{x}=\operatorname{age}^{\lambda}, \operatorname{df}(\mu)=9, \operatorname{df}(\sigma)=4\right.$, $\operatorname{df}(v)=4, \tau=2)$ was used as a starting point to search for the best value of the age-transformation power $\lambda$ by examining changes in global deviance. Note that, the empirical distribution clearly indicated right-skewness, thus the starting arbitrary model included degrees of freedom for $v$. Table 20 shows the global deviance for values of $\lambda$ from 0.05 to 1 . The smallest value of global deviance was associated with $\lambda=0.35$ and thus this age transformation power was selected. 
Table 20 Global deviance (GD) for models within the class $\operatorname{BCPE}\left(x=\operatorname{age}^{\lambda}, \operatorname{df}(\mu)=9, \operatorname{df}(\sigma)=4\right.$, $\mathrm{df}(v)=4, \tau=2)$ for arm circumference-for-age for boys

\begin{tabular}{lcccccccccc}
\hline $\boldsymbol{\lambda}$ & $\mathbf{0 . 0 5}$ & $\mathbf{0 . 1 0}$ & $\mathbf{0 . 1 5}$ & $\mathbf{0 . 2 0}$ & $\mathbf{0 . 2 5}$ & $\mathbf{0 . 3 0}$ & $\mathbf{0 . 3 5}$ & $\mathbf{0 . 4 0}$ & $\mathbf{0 . 4 5}$ & $\mathbf{0 . 5 0}$ \\
$\mathrm{GD}^{\mathrm{a}}$ & 230.1 & 229.6 & 229.1 & 228.7 & 228.4 & 228.2 & 228.1 & 228.2 & 228.4 & 228.8 \\
\hline $\boldsymbol{\lambda}$ & $\mathbf{0 . 5 5}$ & $\mathbf{0 . 6 0}$ & $\mathbf{0 . 6 5}$ & $\mathbf{0 . 7 0}$ & $\mathbf{0 . 7 5}$ & $\mathbf{0 . 8 0}$ & $\mathbf{0 . 8 5}$ & $\mathbf{0 . 9 0}$ & $\mathbf{0 . 9 5}$ & $\mathbf{1 . 0 0}$ \\
$\mathrm{GD}^{\mathrm{a}}$ & 229.4 & 230.2 & 231.4 & 232.9 & 234.9 & 237.3 & 240.2 & 243.8 & 248.0 & 252.9 \\
\hline
\end{tabular}

${ }^{\mathrm{a}}$ In excess of 34000 .

The search for the best $\operatorname{df}(\mu)$ and $\operatorname{df}(\sigma)$ followed, using $\lambda=0.35$, and fixing $\nu=1$ and $\tau=2$. All possible combinations with $\operatorname{df}(\mu)$ values ranging from 7 to 15 and $\operatorname{df}(\sigma)$ from 2 to 10 were considered and partial results are presented in Table 21. No fitted model minimized both AIC and GAIC(3) simultaneously. Overall it was noticed that for data with a higher dispersion such as arm circumference and skinfolds, the criterion GAIC(3) provided more parsimonious models, and therefore only this criterion was considered in selecting the final models for the construction of the standards for these indicators. For boys' the arm circumference-for-age GAIC(3) was most minimized by the combination $\operatorname{df}(\mu)=8$ and $\operatorname{df}(\sigma)=4$. This model was thus selected for further evaluation.

Table 21 Goodness-of-fit summary for models using the BCPE distribution with fixed $v=1$ and $\tau=2$ for arm circumference-for-age for boys

\begin{tabular}{cccccc}
\hline $\mathbf{d f}(\boldsymbol{\mu})$ & $\mathbf{d f}(\boldsymbol{\sigma})$ & $\mathbf{G D}^{\mathbf{a}}$ & AIC $^{\mathbf{a}}$ & GAIC(3) $^{\mathbf{a}}$ & Total df \\
\hline & $\mathbf{2}$ & 360.9 & 378.9 & 387.9 & 9 \\
$\mathbf{7}$ & $\mathbf{3}$ & 351.9 & 371.9 & 381.9 & 10 \\
& $\mathbf{4}$ & 348.6 & 370.6 & 381.6 & 11 \\
& $\mathbf{5}$ & 347.2 & 371.2 & 383.2 & 12 \\
& $\mathbf{6}$ & 346.1 & 372.1 & 385.1 & 13 \\
\hline \multirow{4}{*}{$\mathbf{8}$} & $\mathbf{2}$ & 357.5 & 377.5 & 387.5 & 10 \\
& $\mathbf{3}$ & 348.5 & 370.5 & 381.5 & 11 \\
& $\mathbf{4}$ & 345.2 & 369.2 & 381.2 & 12 \\
& $\mathbf{5}$ & 343.7 & 369.7 & 382.7 & 13 \\
& $\mathbf{6}$ & 342.6 & 370.6 & 384.6 & 14 \\
$\mathbf{9}$ & $\mathbf{2}$ & 355.5 & 377.5 & 388.5 & 11 \\
& $\mathbf{3}$ & 346.5 & 370.5 & 382.5 & 12 \\
& $\mathbf{4}$ & 343.2 & 369.2 & 382.2 & 13 \\
& $\mathbf{5}$ & 341.8 & 369.8 & 383.8 & 14 \\
& $\mathbf{6}$ & 340.6 & 370.6 & 385.6 & 15 \\
\hline \multirow{4}{*}{$\mathbf{0}$} & $\mathbf{2}$ & 354.2 & 378.2 & 390.2 & 12 \\
& $\mathbf{3}$ & 345.2 & 371.2 & 384.2 & 13 \\
& $\mathbf{4}$ & 341.8 & 369.8 & 383.8 & 14 \\
& $\mathbf{5}$ & 340.4 & 370.4 & 385.4 & 15 \\
& $\mathbf{6}$ & 339.3 & 371.3 & 387.3 & 16 \\
\hline \multirow{4}{*}{$\mathbf{1 1}$} & $\mathbf{2}$ & 353.2 & 379.2 & 392.2 & 13 \\
& $\mathbf{3}$ & 344.2 & 372.2 & 386.2 & 14 \\
& $\mathbf{4}$ & 340.8 & 370.8 & 385.8 & 15 \\
& $\mathbf{5}$ & 339.4 & 371.4 & 387.4 & 16 \\
& $\mathbf{6}$ & 338.2 & 372.2 & 389.2 & 17 \\
\hline
\end{tabular}

GD, Global Deviance; AIC, Akaike Information Criterion;

GAIC(3), Generalized AIC with penalty equal to 3;

${ }^{\mathrm{a}}$ In excess of 34000 . 
Model 1: $\operatorname{BCPE}\left(x=\operatorname{age}^{0.35}, \operatorname{df}(\mu)=8, \operatorname{df}(\sigma)=4, v=1, \tau=2\right)$

For this model, both the worm plots (Figure 22) and the Q-test results (Table 22) indicated good model fit of the $\mu$ and $\sigma$ curves but pointed to the need to adjust for skewness. The worms had a U-shape in most age groups after 12 months, indicating residual skewness. This finding was confirmed by the Qtest results (Table 22), which showed various groups in the same age range with absolute values of $\mathrm{z} 3$ higher than 2 . The overall test for skewness was significant ( $p$-value $<0.01$ ). Only two age groups presented absolute values of $\mathrm{z} 4$ higher than 2 , indicating residual kurtosis.
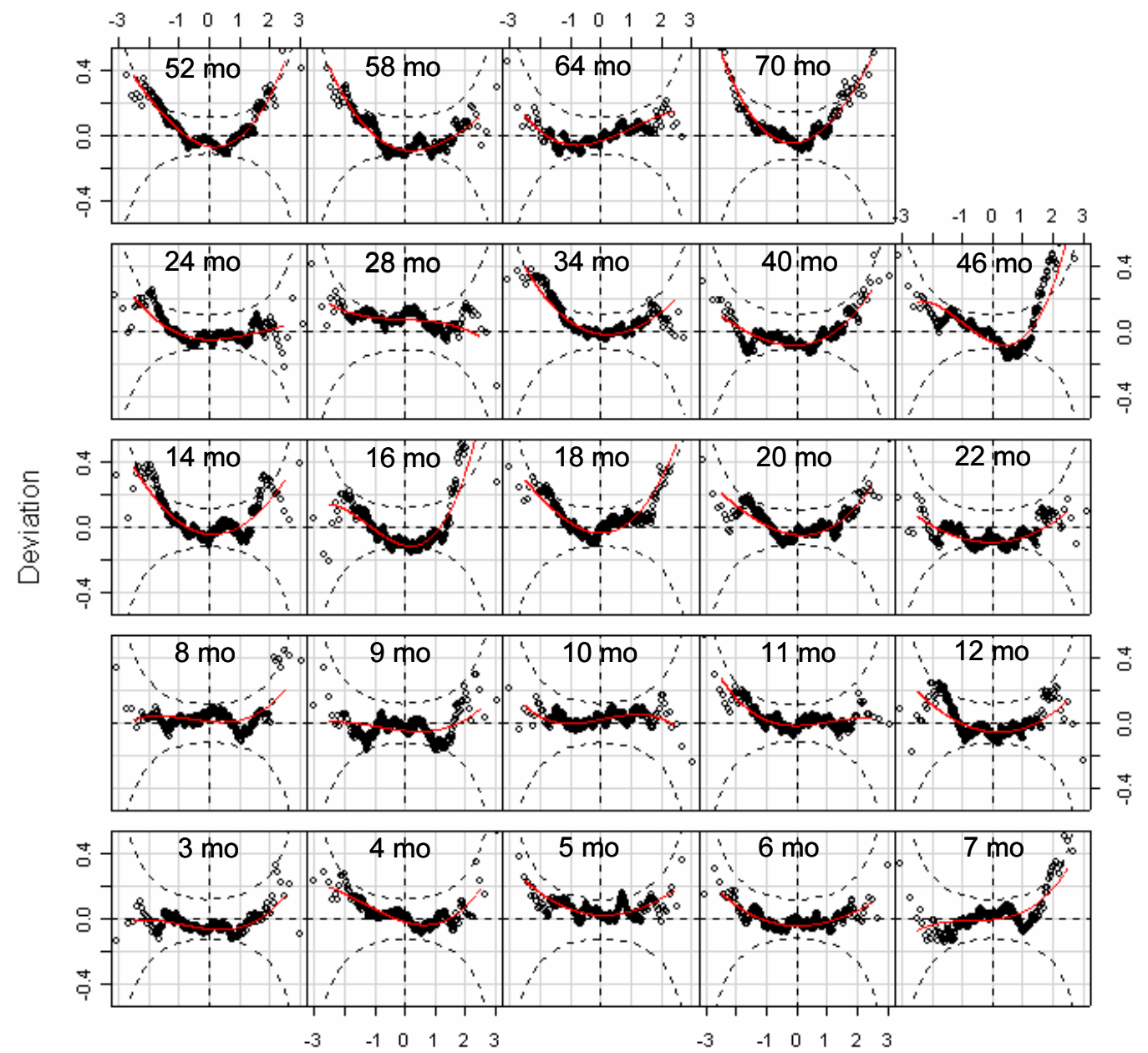

Unit normal quantile

Figure 22 Worm plots of z-scores for Model 1 for arm circumference-for-age for boys 
Table 22 Q-test for $z$-scores from Model $1\left[\operatorname{BCPE}\left(x=a g e^{0.35}, \operatorname{df}(\mu)=8, \operatorname{df}(\sigma)=4, v=1, \tau=2\right)\right]$ for arm circumference-for-age for boys

\begin{tabular}{|c|c|c|c|c|c|c|}
\hline Age (days) & Group & $\mathbf{N}$ & $\mathrm{z1}$ & $\mathrm{z2}$ & $\mathbf{z 3}$ & $\mathrm{z4}$ \\
\hline 79 to 99 & 3 mo & 409 & -0.85 & 0.15 & 1.02 & 0.70 \\
\hline 100 to 129 & $4 \mathrm{mo}$ & 404 & 0.23 & -0.76 & 1.79 & 0.99 \\
\hline 130 to 159 & $5 \mathrm{mo}$ & 409 & 1.15 & -0.28 & 1.51 & 0.35 \\
\hline 160 to 189 & $6 \mathrm{mo}$ & 411 & -0.32 & -0.38 & 1.33 & -0.03 \\
\hline 190 to 219 & $7 \mathrm{mo}$ & 406 & 0.25 & 1.44 & 1.03 & 1.08 \\
\hline 220 to 249 & $8 \mathrm{mo}$ & 420 & 0.37 & 0.30 & 1.08 & 1.06 \\
\hline 250 to 279 & 9 mo & 392 & -0.83 & 0.11 & 1.01 & 0.73 \\
\hline 280 to 309 & $10 \mathrm{mo}$ & 399 & 0.41 & 0.53 & 0.19 & -1.14 \\
\hline 310 to 349 & $11 \mathrm{mo}$ & 461 & 0.24 & -0.65 & 1.37 & -0.67 \\
\hline 350 to 379 & $12 \mathrm{mo}$ & 409 & -0.39 & -0.32 & 1.69 & 0.26 \\
\hline 380 to 439 & $14 \mathrm{mo}$ & 418 & 0.33 & -0.37 & 2.87 & 0.49 \\
\hline 440 to 499 & $16 \mathrm{mo}$ & 415 & -0.60 & 1.15 & 4.15 & 2.48 \\
\hline 500 to 559 & $18 \mathrm{mo}$ & 442 & 0.66 & 0.80 & 3.52 & 1.59 \\
\hline 560 to 619 & $20 \mathrm{mo}$ & 523 & -0.04 & 0.05 & 2.43 & 0.65 \\
\hline 620 to 679 & $22 \mathrm{mo}$ & 545 & -1.38 & -0.13 & 1.83 & 0.16 \\
\hline 680 to 749 & $24 \mathrm{mo}$ & 591 & -0.70 & -0.69 & 1.69 & -0.33 \\
\hline 750 to 929 & $28 \mathrm{mo}$ & 471 & 1.58 & -0.74 & 0.00 & -0.42 \\
\hline 930 to 1119 & $34 \mathrm{mo}$ & 510 & 1.01 & -1.24 & 3.29 & -0.07 \\
\hline 1120 to 1309 & $40 \mathrm{mo}$ & 511 & -1.01 & 0.64 & 2.24 & 0.73 \\
\hline 1310 to 1499 & $46 \mathrm{mo}$ & 508 & 0.12 & 0.36 & 4.32 & 2.81 \\
\hline 1500 to 1689 & $52 \mathrm{mo}$ & 487 & 0.21 & -0.02 & 4.03 & 1.59 \\
\hline 1690 to 1879 & $58 \mathrm{mo}$ & 482 & -0.64 & -1.15 & 2.91 & 0.00 \\
\hline 1880 to 2069 & $64 \mathrm{mo}$ & 461 & -0.14 & 1.06 & 1.31 & -0.48 \\
\hline 2070 to 2249 & $70 \mathrm{mo}$ & 286 & 0.67 & 0.44 & 3.18 & 0.64 \\
\hline Overall Q stats & & 10770 & 12.22 & 11.66 & 137.37 & 27.45 \\
\hline degrees of freedom & & & 16.0 & 21.5 & 24.0 & 24.0 \\
\hline p-value & & & 0.7284 & 0.9569 & $<0.01$ & 0.2841 \\
\hline
\end{tabular}

Note: Absolute values of z1, z2, z3 or z4 larger than 2 indicate misfit of, respectively, mean, variance, skewness or kurtosis.

The next step involved fitting the parameter $v$ for skewness using the BCPE distribution with fixed parameter $\tau=2$, keeping the degrees of freedom for the $\mu$ and $\sigma$ curves selected for Model 1 . Table 23 shows the GAIC(3) values for various degrees of freedom for the $v$ curve. 
Table 23 Goodness-of-fit summary for models $B C P E\left(x=a g e^{0.35}, \operatorname{df}(\mu)=8, \operatorname{df}(\sigma)=4, \operatorname{df}(v)=?, \tau=2\right)$ for arm circumference-for-age for boys

\begin{tabular}{cccc}
\hline $\mathbf{d f}(\mathbf{v})$ & $\mathbf{G D}^{\mathbf{a}}$ & GAIC(3) $^{\mathbf{a}}$ & Total df \\
\hline $\mathbf{1}$ & 238.3 & 277.3 & 13 \\
$\mathbf{2}$ & 232.0 & 274.0 & 14 \\
$\mathbf{3}$ & 231.4 & 276.4 & 15 \\
$\mathbf{4}$ & 230.2 & 278.3 & 16 \\
$\mathbf{5}$ & 228.1 & 279.1 & 17 \\
$\mathbf{6}$ & 225.1 & 279.1 & 18 \\
$\mathbf{7}$ & 222.2 & 279.2 & 19 \\
\hline
\end{tabular}

GD, Global Deviance; GAIC(3), Generalized Akaike Information Criterion with penalty equal to 3 ;

${ }^{\mathrm{a}}$ In excess of 34000 .

The smallest $G A I C(3)$ value corresponded to $\mathrm{df}(v)=2$. A new iteration was performed using $\operatorname{df}(v)=2$ to re-search for $\operatorname{df}(\mu)$ and $\operatorname{df}(\sigma)$. This search led to similar results as before, i.e. $\operatorname{df}(\mu)=8$ and $\operatorname{df}(\sigma)=4$ was still the best combination $(\operatorname{GAIC}(3)=34274.0)$, but the combination $\operatorname{df}(\mu)=7$ and $\operatorname{df}(\sigma)=4$ yielded a very similar GAIC(3) value (34274.5) and thus it was selected in the interest of further smoothing the median curve. A new search for the best $\mathrm{df}(v)$ and, subsequently, age-transformation power $\lambda$ using this model was carried out, but results indicated no need to update the selected model. Thus, the model $\operatorname{BCPE}\left(x=\operatorname{age}^{0.35}, \operatorname{df}(\mu)=7, \operatorname{df}(\sigma)=4, \operatorname{df}(v)=2, \tau=2\right)$ was further evaluated.

Model 2: $\operatorname{BCPE}\left(x=\operatorname{age}^{0.35}, \operatorname{df}(\mu)=7, \operatorname{df}(\sigma)=4, \operatorname{df}(v)=2, \tau=2\right)$

Figure 23 shows the fitting of the parameters $\mu, \sigma$ and $v$ for Model 2 with their respective sample estimates, that is, the median for $\mu$ and Box-Cox transform power for $v$. It should be noted that the sample estimate for the parameter $\sigma$ is no longer the coefficient of variation when the distribution departs significantly from the normal distribution. In this case, the best estimator of $\sigma$ is the sample standard deviation of the Box-Cox transformed data (Cole and Green, 1992).

Figures 24 and 25 show the distribution of differences between empirical values and fitted centiles for the longitudinal and cross-sectional samples, respectively. There was indication of systematic bias between 3 and 24 months only for the $90^{\text {th }}$ centile (Figure 24), and between 24 and 71 months only for the $10^{\text {th }}$ centile (Figure 25).

The worm plots for this model (Figure 26) were significantly improved compared to those of Model 1 (Figure 22). The U-shaped worms flattened out significantly (Figure 26), indicating that the residual skewness associated with Model 1 had been corrected. There was indication of remaining skewness only for one age group $(28 \mathrm{mo})$. Another two groups presented slightly S-shaped worm (16 mo and $46 \mathrm{mo}$ ) suggesting residual kurtosis but it was contained within the $95 \%$ confidence interval. 

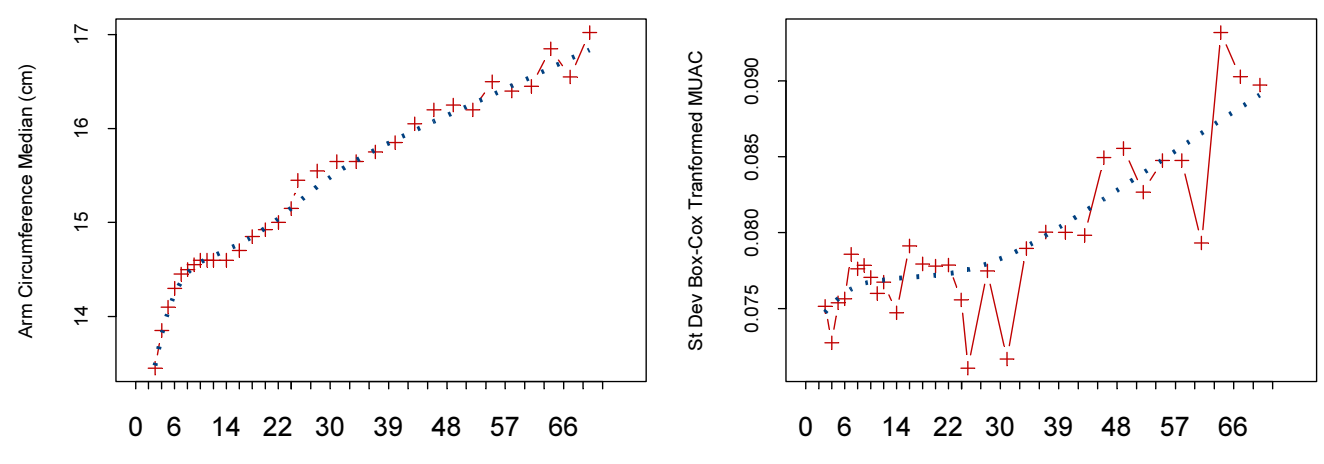

Age (months)

$\begin{array}{lllllllll}0 & 6 & 14 & 22 & 30 & 39 & 48 & 57 & 66\end{array}$

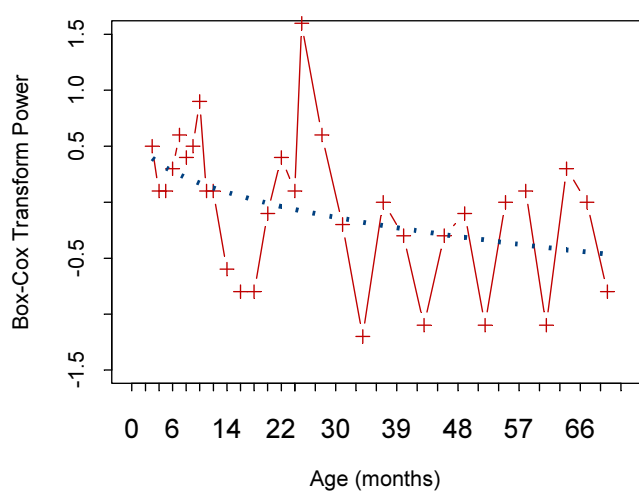

Figure 23 Fitting of the $\mu, \sigma$, and $v$ curves of Model 2 for arm circumference-for-age for boys from 3 to 71 months (dotted line) and their respective sample estimates (points with solid line) 

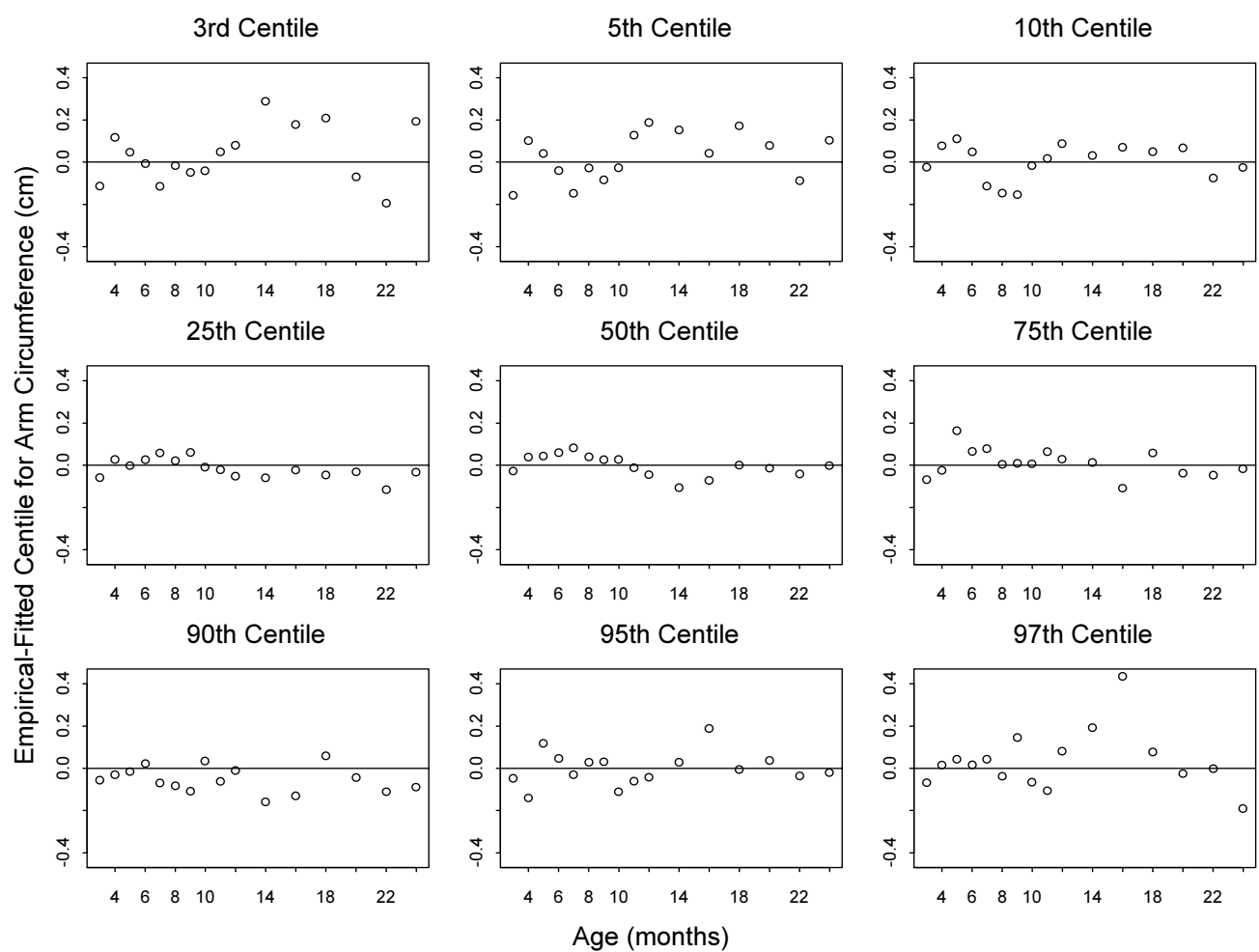

Figure 24 Centile residuals from fitting Model 2 for arm circumference-for-age from 3 to 24 months for boys
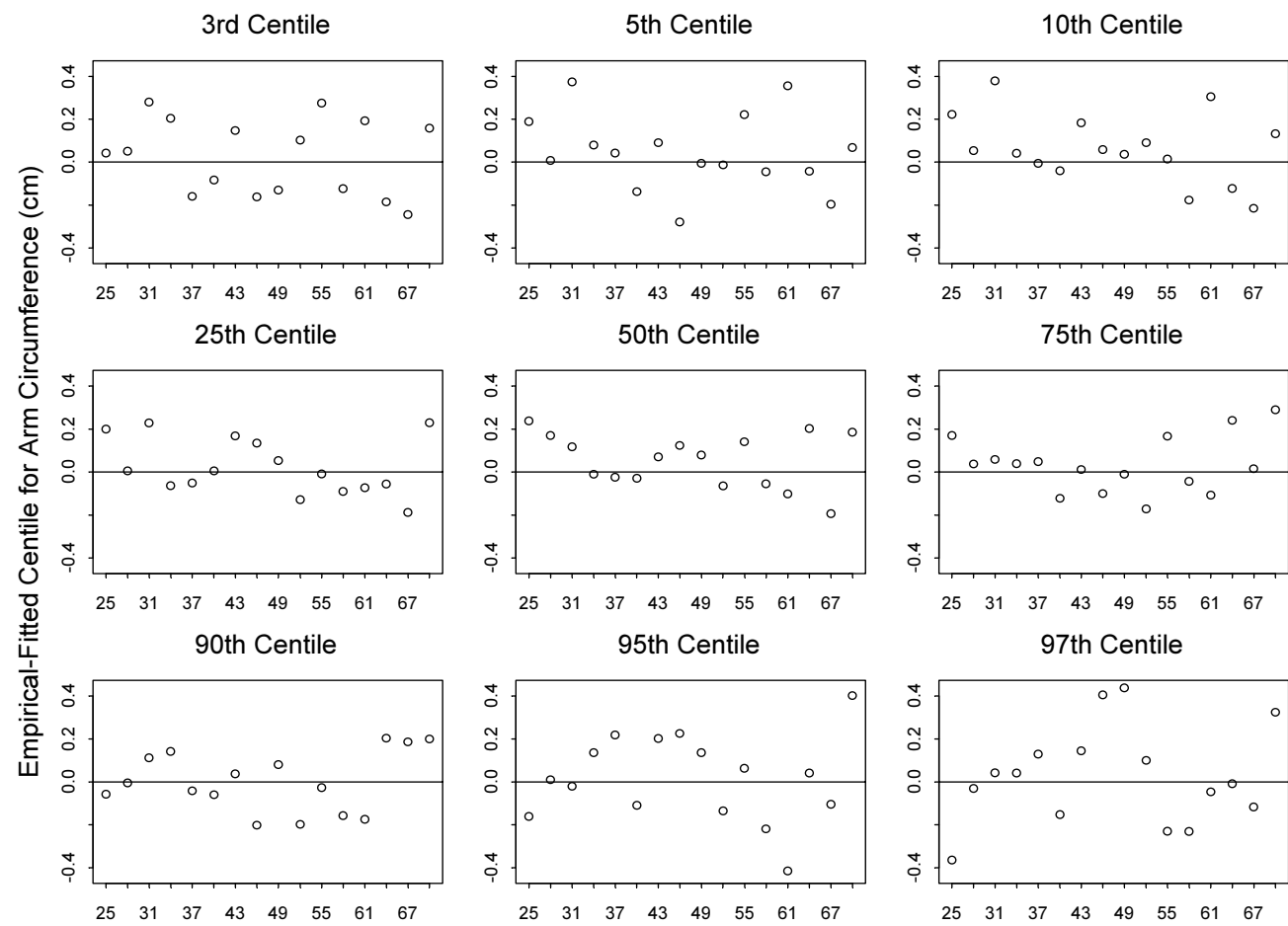

50th Centile
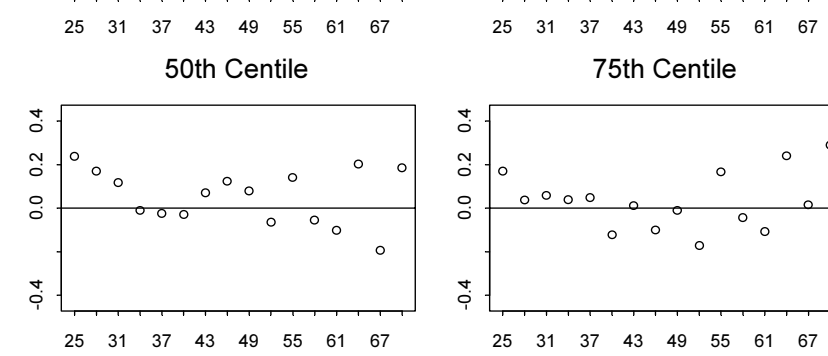

75th Centile

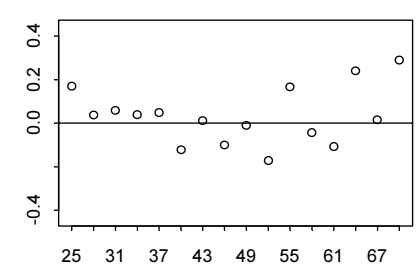

95th Centile
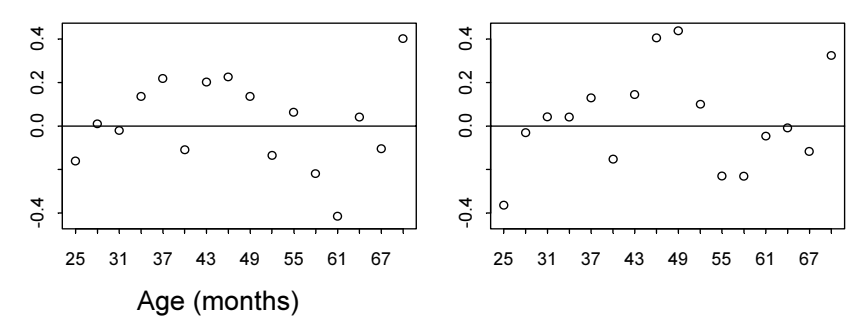

Figure 25 Centile residuals from fitting Model 2 for arm circumference-for-age from 24 to 71 months for boys 

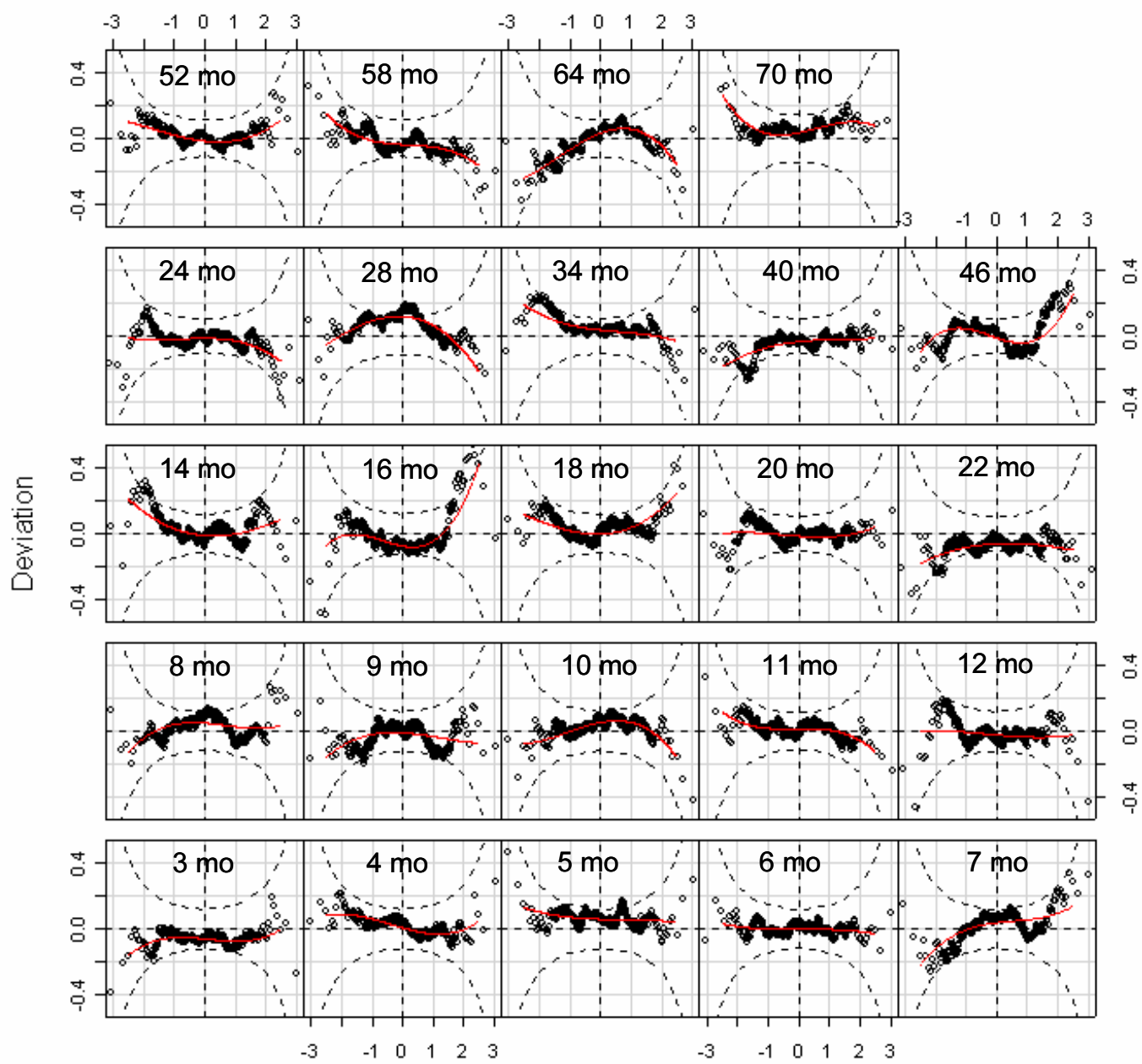

Unit normal quantile

Figure 26 Worm plots of z-scores for Model 2 for arm circumference-for-age for boys

The Q-test results from Model 2 are shown in Table 24. There were two age groups with an absolute value larger than 2 for the statistic z3 (16 mo and $28 \mathrm{mo}$ ) suggesting remaining skewness. No group presented an absolute value of $\mathrm{z} 4$ greater than 2, indicating no remaining kurtosis. The overall Q-test p-values were all non-significant, indicating an adequate fit of the boys' arm circumference-for-age curves and presenting no justification for increasing the complexity of the model by adjusting for kurtosis (modelling $\tau$ ).

Table 25 presents observed percentages with arm circumferences below the fitted centiles. Age groups above 24 months presented larger differences compared to the age range younger than 24 months, yet there was no detectable pattern suggesting systematic bias. 
Table 24 Q-test for z-scores from Model $2\left[\operatorname{BCPE}\left(x=\operatorname{age}^{0.35}, \operatorname{df}(\mu)=7, \operatorname{df}(\sigma)=4, \operatorname{df}(v)=2, \tau=2\right)\right]$ for arm circumference-for-age for boys

\begin{tabular}{|c|c|c|c|c|c|c|}
\hline Age (days) & Group & $\mathbf{N}$ & $\mathrm{z1}$ & $\mathbf{z 2}$ & $\mathbf{z 3}$ & $\mathrm{z4}$ \\
\hline 79 to 99 & $3 \mathrm{mo}$ & 409 & -1.30 & 0.17 & -0.21 & 0.71 \\
\hline 100 to 129 & $4 \mathrm{mo}$ & 404 & 0.24 & -0.85 & 0.54 & 0.65 \\
\hline 130 to 159 & $5 \mathrm{mo}$ & 409 & 1.36 & -0.40 & 0.25 & -0.02 \\
\hline 160 to 189 & $6 \mathrm{mo}$ & 411 & -0.05 & -0.37 & 0.02 & -0.25 \\
\hline 190 to 219 & $7 \mathrm{mo}$ & 406 & 0.43 & 1.47 & -0.59 & 0.69 \\
\hline 220 to 249 & $8 \mathrm{mo}$ & 420 & 0.54 & 0.32 & -0.58 & 0.73 \\
\hline 250 to 279 & $9 \mathrm{mo}$ & 392 & -0.73 & 0.24 & -0.60 & 0.49 \\
\hline 280 to 309 & $10 \mathrm{mo}$ & 399 & 0.43 & 0.64 & -1.21 & -0.82 \\
\hline 310 to 349 & $11 \mathrm{mo}$ & 461 & 0.26 & -0.64 & -0.16 & -0.86 \\
\hline 350 to 379 & $12 \mathrm{mo}$ & 409 & -0.42 & -0.28 & 0.02 & 0.41 \\
\hline 380 to 439 & $14 \mathrm{mo}$ & 418 & 0.28 & -0.53 & 1.25 & 0.02 \\
\hline 440 to 499 & $16 \mathrm{mo}$ & 415 & -0.73 & 0.94 & 2.03 & 1.96 \\
\hline 500 to 559 & $18 \mathrm{mo}$ & 442 & 0.53 & 0.54 & 1.53 & 0.52 \\
\hline 560 to 619 & $20 \mathrm{mo}$ & 523 & -0.20 & 0.04 & 0.24 & 0.25 \\
\hline 620 to 679 & $22 \mathrm{mo}$ & 545 & -1.56 & 0.10 & -0.44 & 0.06 \\
\hline 680 to 749 & $24 \mathrm{mo}$ & 591 & -0.77 & -0.51 & -0.61 & -0.31 \\
\hline 750 to 929 & $28 \mathrm{mo}$ & 471 & 1.72 & -0.61 & -2.12 & 0.00 \\
\hline 930 to 1119 & $34 \mathrm{mo}$ & 510 & 1.27 & -1.51 & 1.19 & -1.28 \\
\hline 1120 to 1309 & $40 \mathrm{mo}$ & 511 & -1.03 & 0.90 & -0.62 & 0.20 \\
\hline 1310 to 1499 & $46 \mathrm{mo}$ & 508 & 0.04 & 0.15 & 0.87 & 1.88 \\
\hline 1500 to 1689 & $52 \mathrm{mo}$ & 487 & 0.08 & -0.26 & 1.05 & 0.40 \\
\hline 1690 to 1879 & $58 \mathrm{mo}$ & 482 & -0.66 & -1.07 & 0.14 & -0.94 \\
\hline 1880 to 2069 & $64 \mathrm{mo}$ & 461 & -0.14 & 1.45 & -1.80 & -0.72 \\
\hline 2070 to 2249 & $70 \mathrm{mo}$ & 286 & 0.91 & 0.08 & 0.80 & -0.78 \\
\hline Overall Q stats & & 10770 & 15.93 & 12.75 & 23.65 & 15.46 \\
\hline degrees of freedom & & & 17.0 & 21.5 & 22.0 & 24.0 \\
\hline p-value & & & 0.5289 & 0.9291 & 0.3657 & 0.9064 \\
\hline
\end{tabular}

Note: Absolute values of z1, z2, z3 or z4 larger than 2 indicate misfit of, respectively, mean, variance, skewness or kurtosis.

In conclusion, the model selected for constructing the arm circumference-for-age growth curves for boys was $\operatorname{BCPE}\left(x=a g e^{0.35}, \operatorname{df}(\mu)=7, \operatorname{df}(\sigma)=4, \operatorname{df}(v)=2, \tau=2\right)$ that adjusts only for skewness, and thus reduces in essence to the LMS method (Cole and Green, 1992). The fitted centile curves and empirical centiles are shown in Figures 27 to 30. 


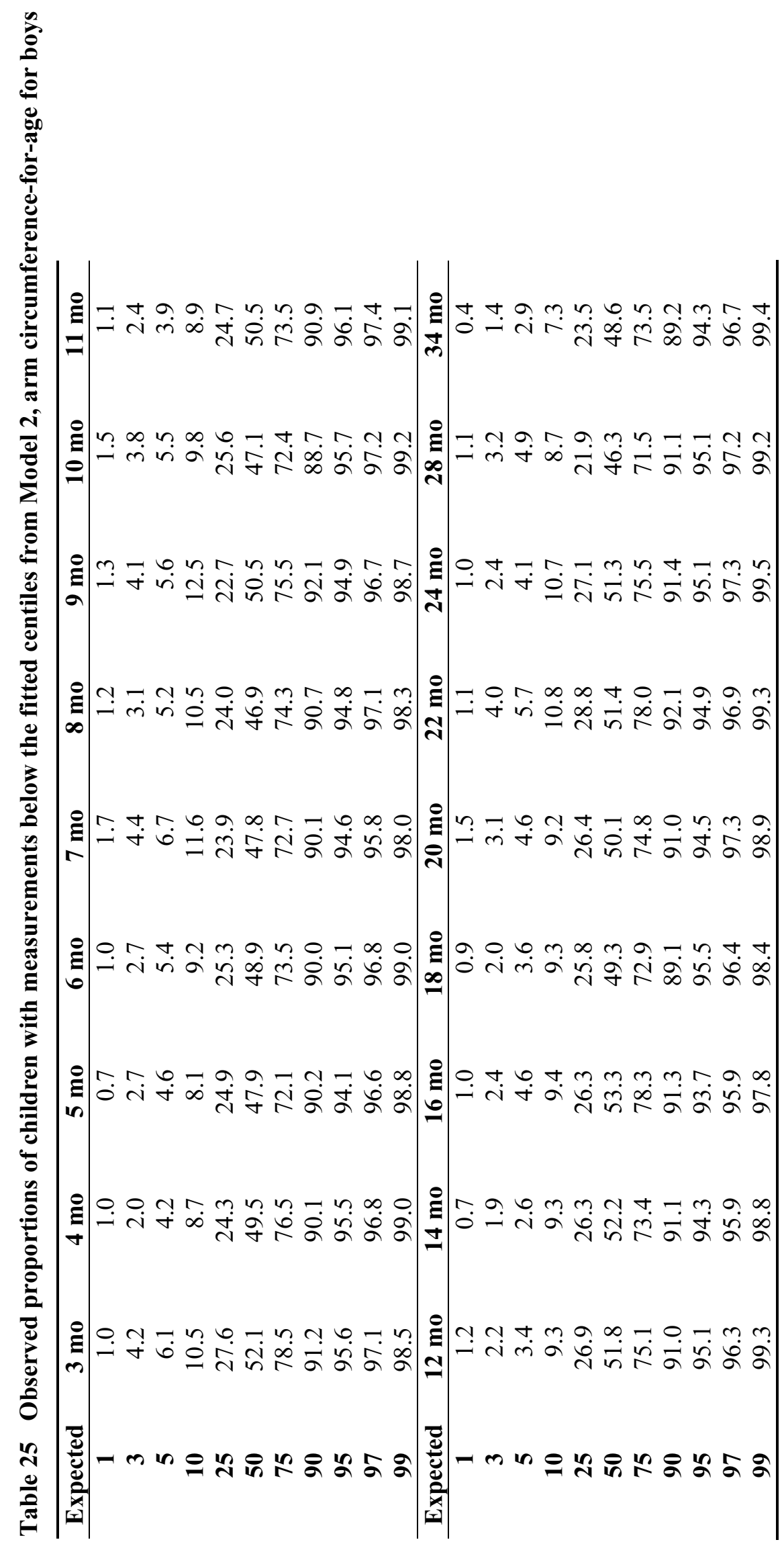




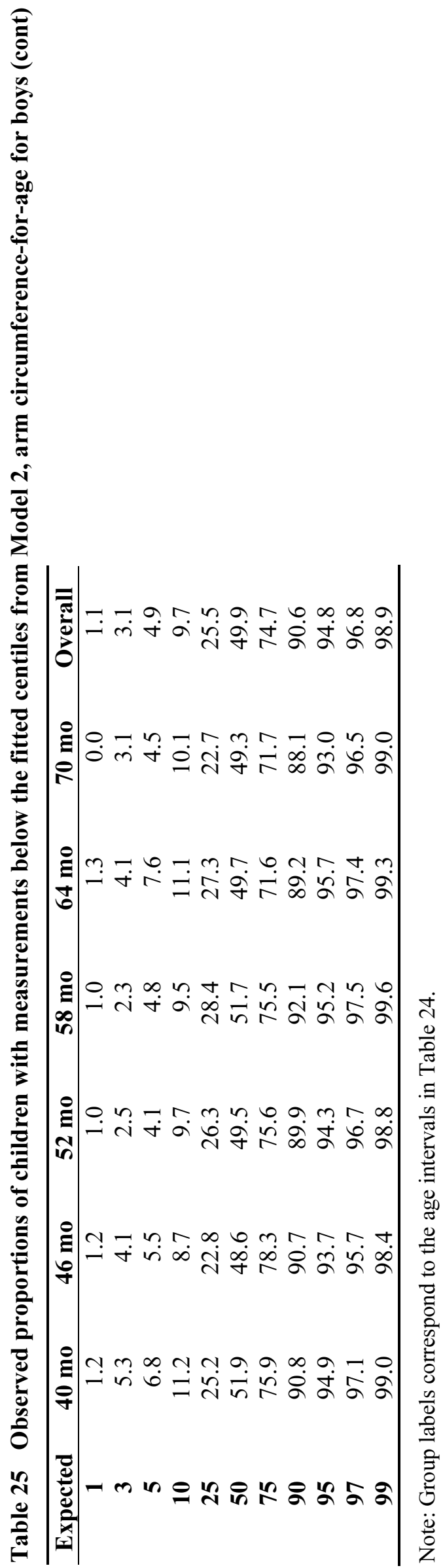




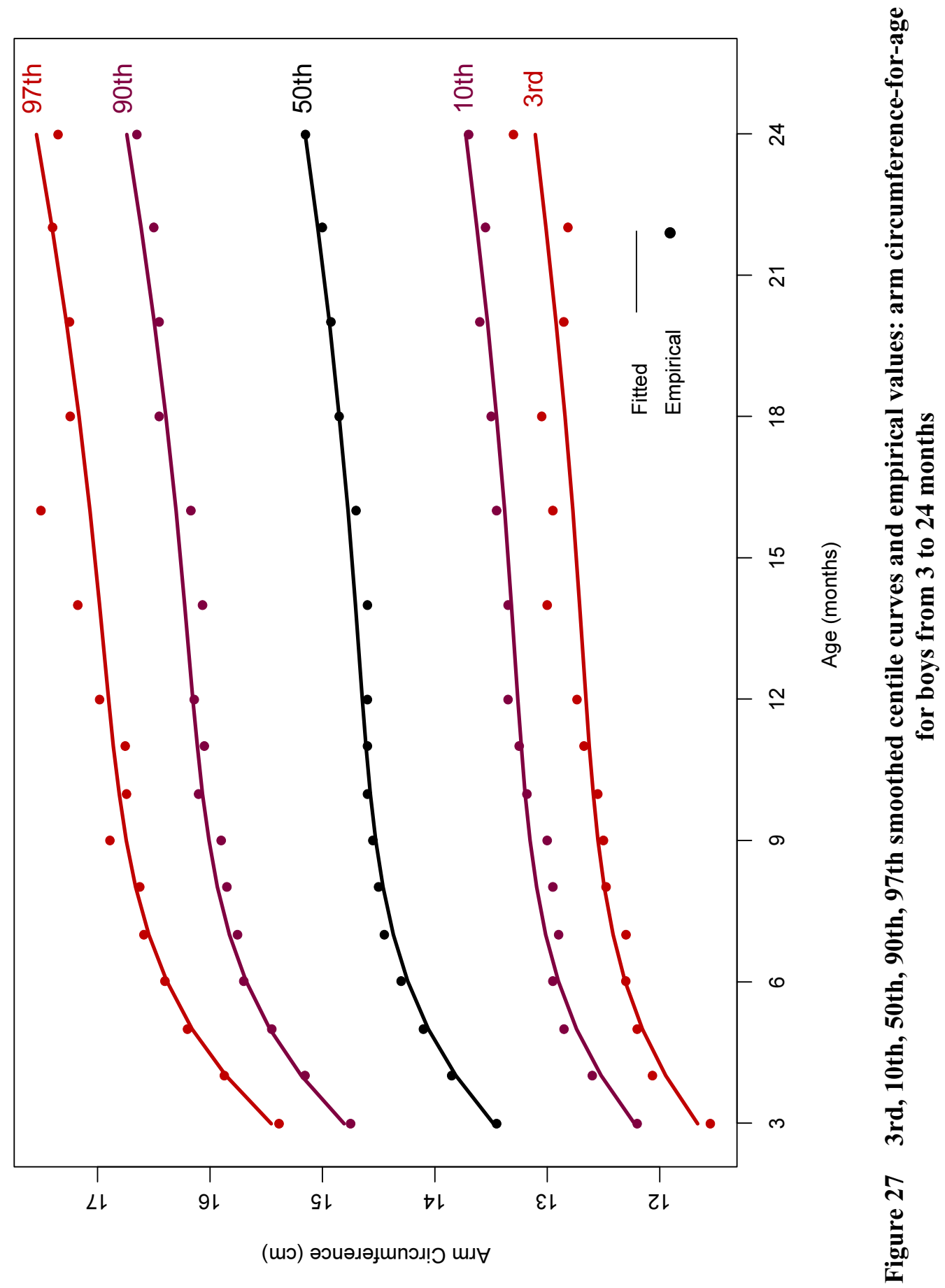




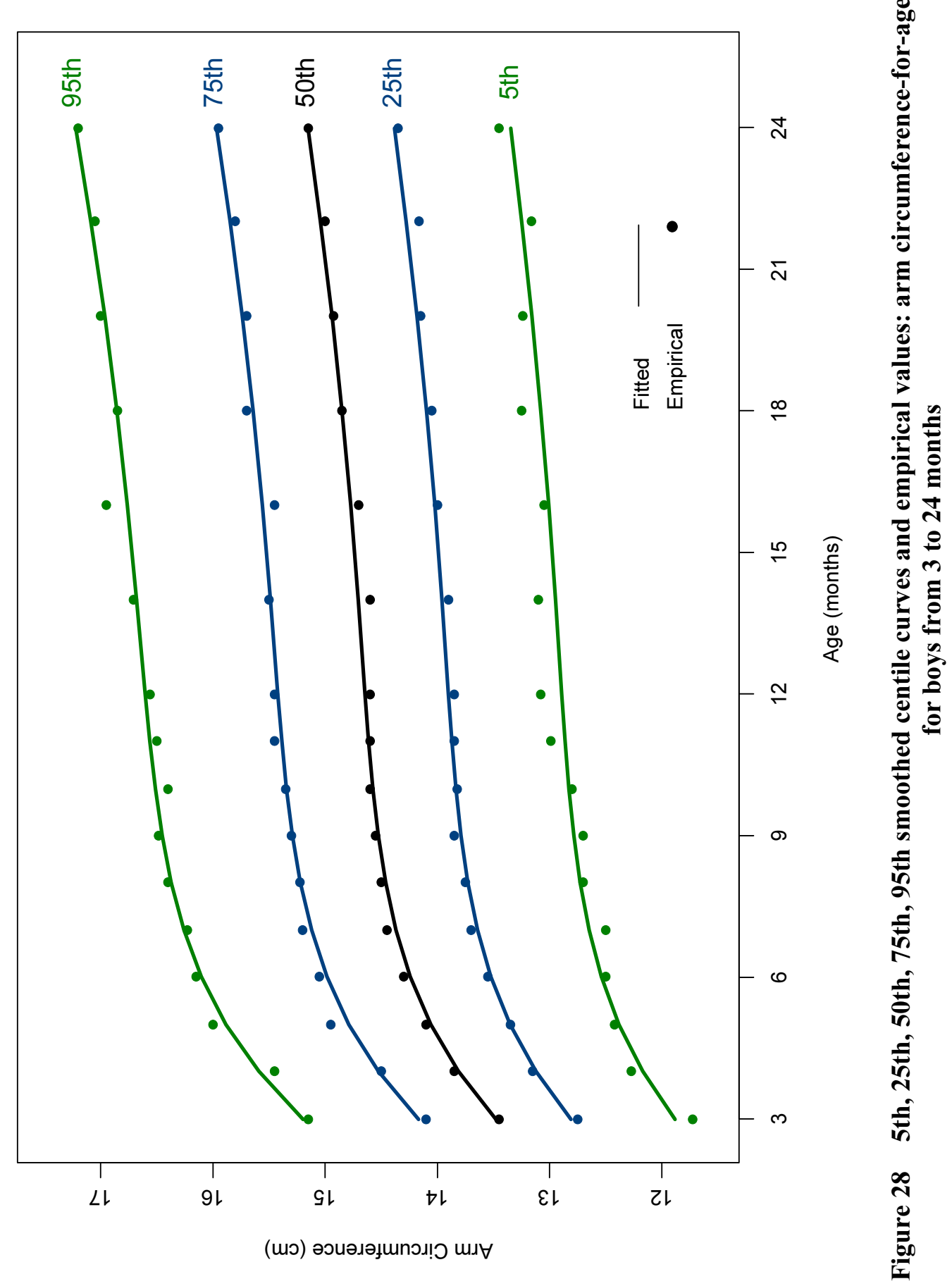




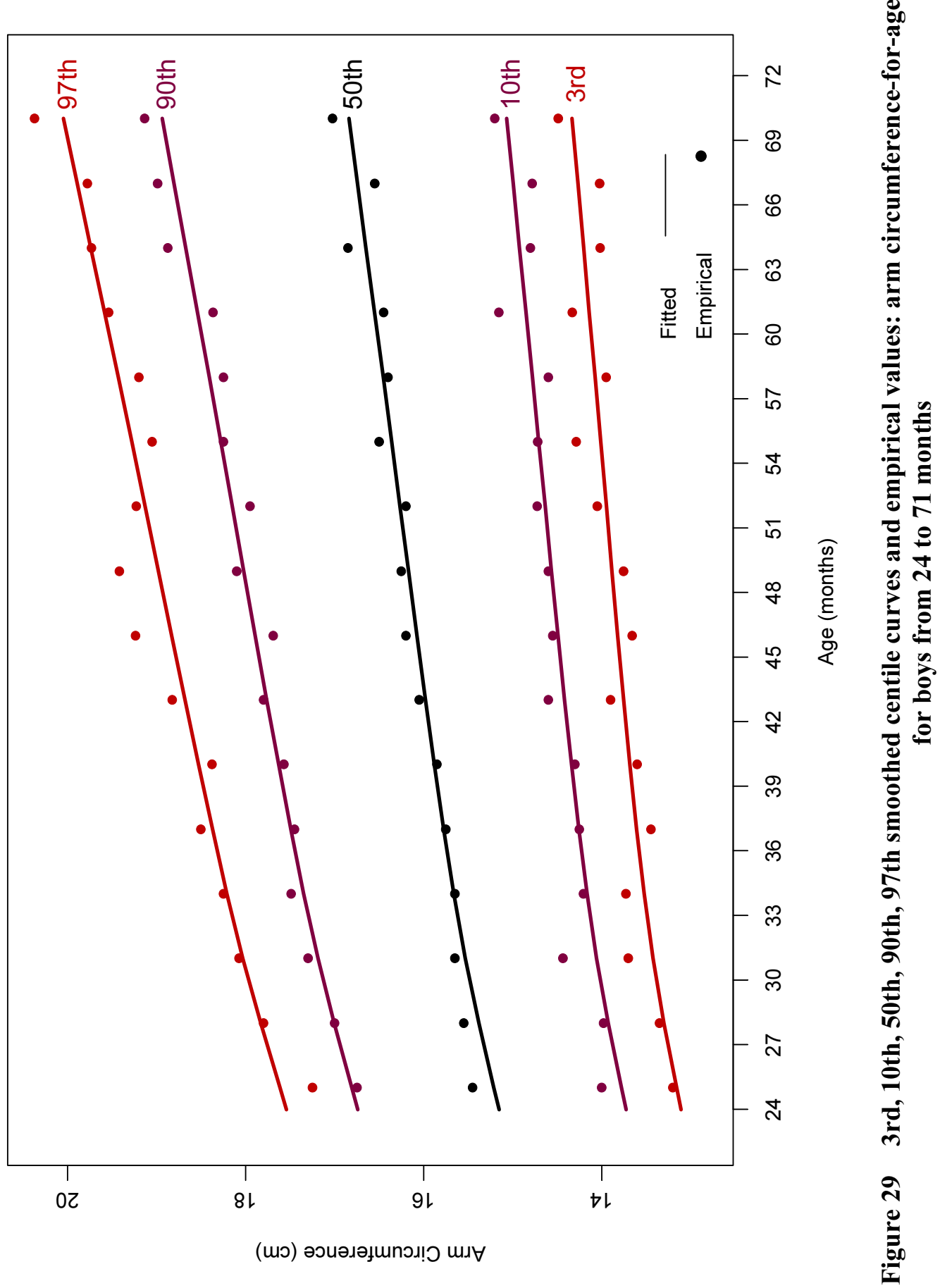




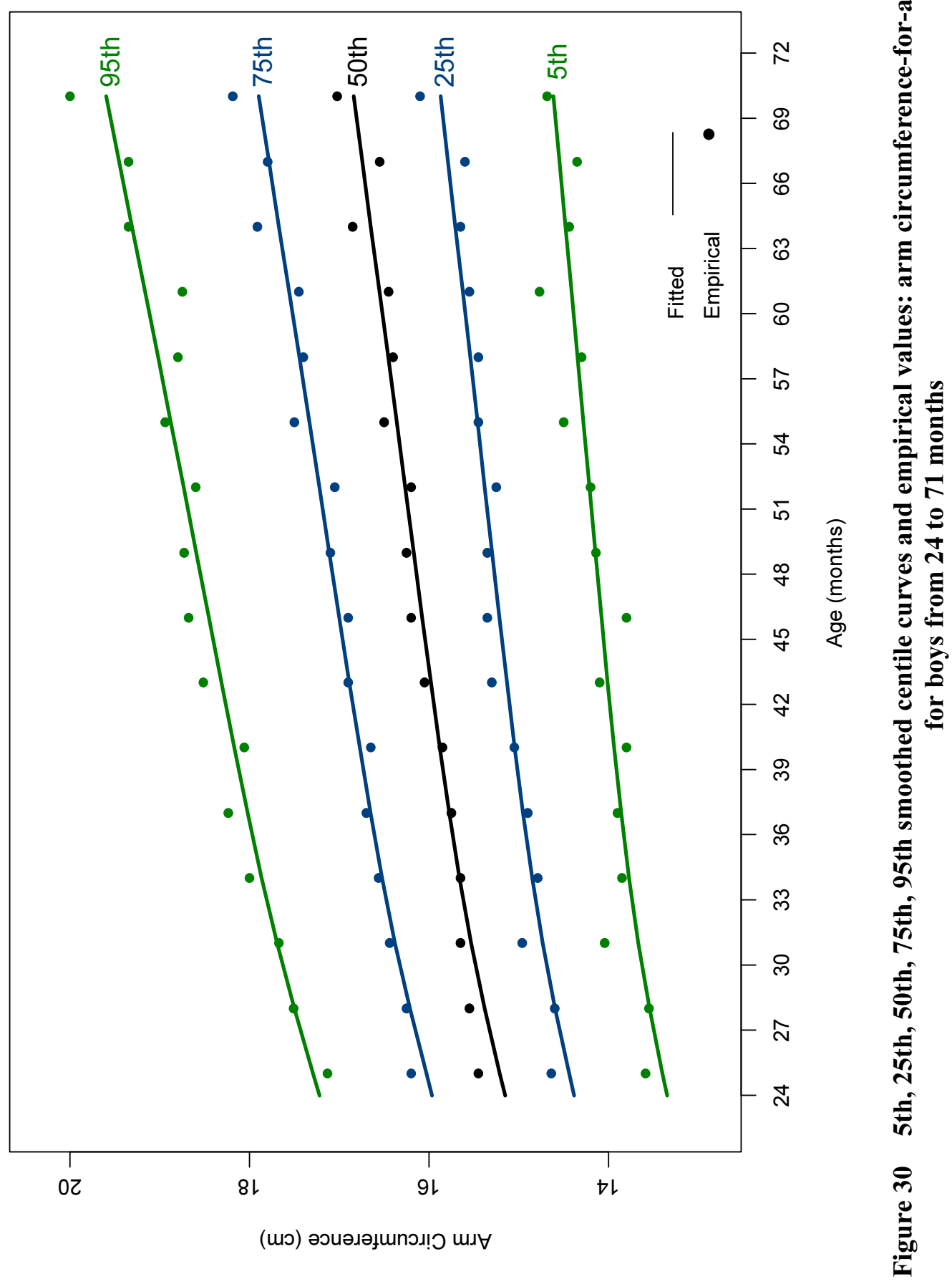




\subsubsection{WHO standards}

This section presents the final WHO arm circumference-for-age z-score and percentile charts (Figures 31 and 32) and table (Table 26) for boys. 


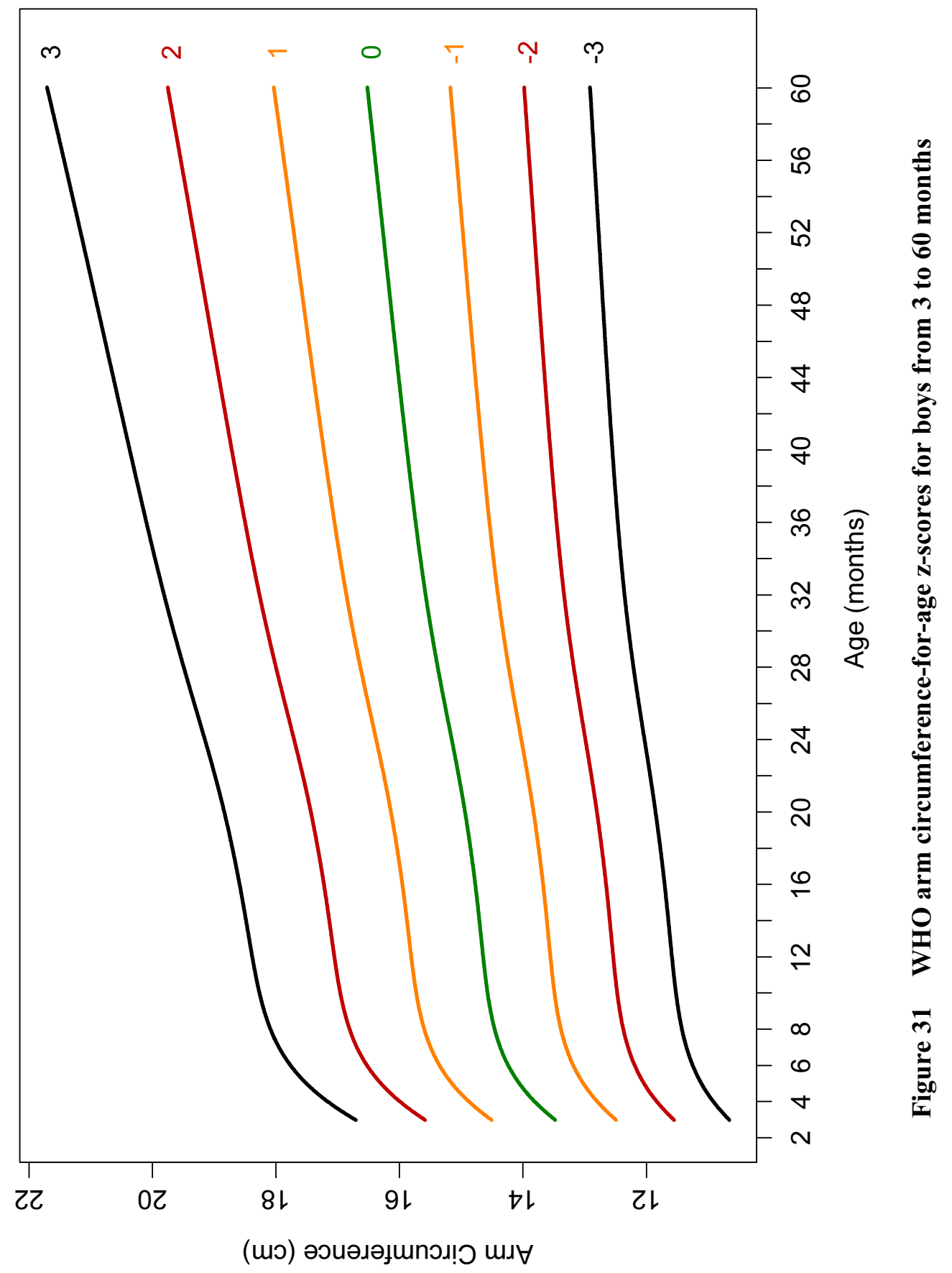




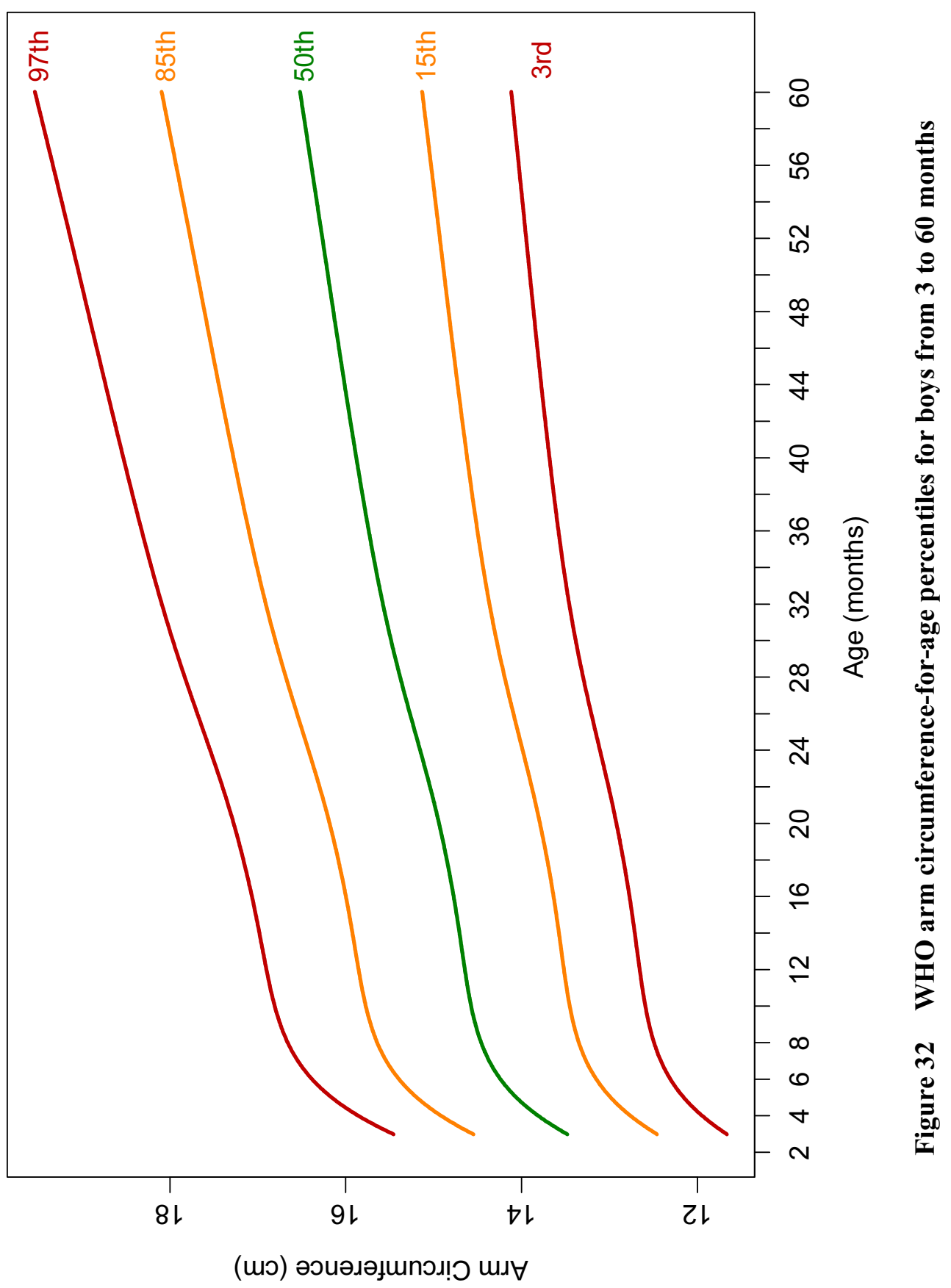




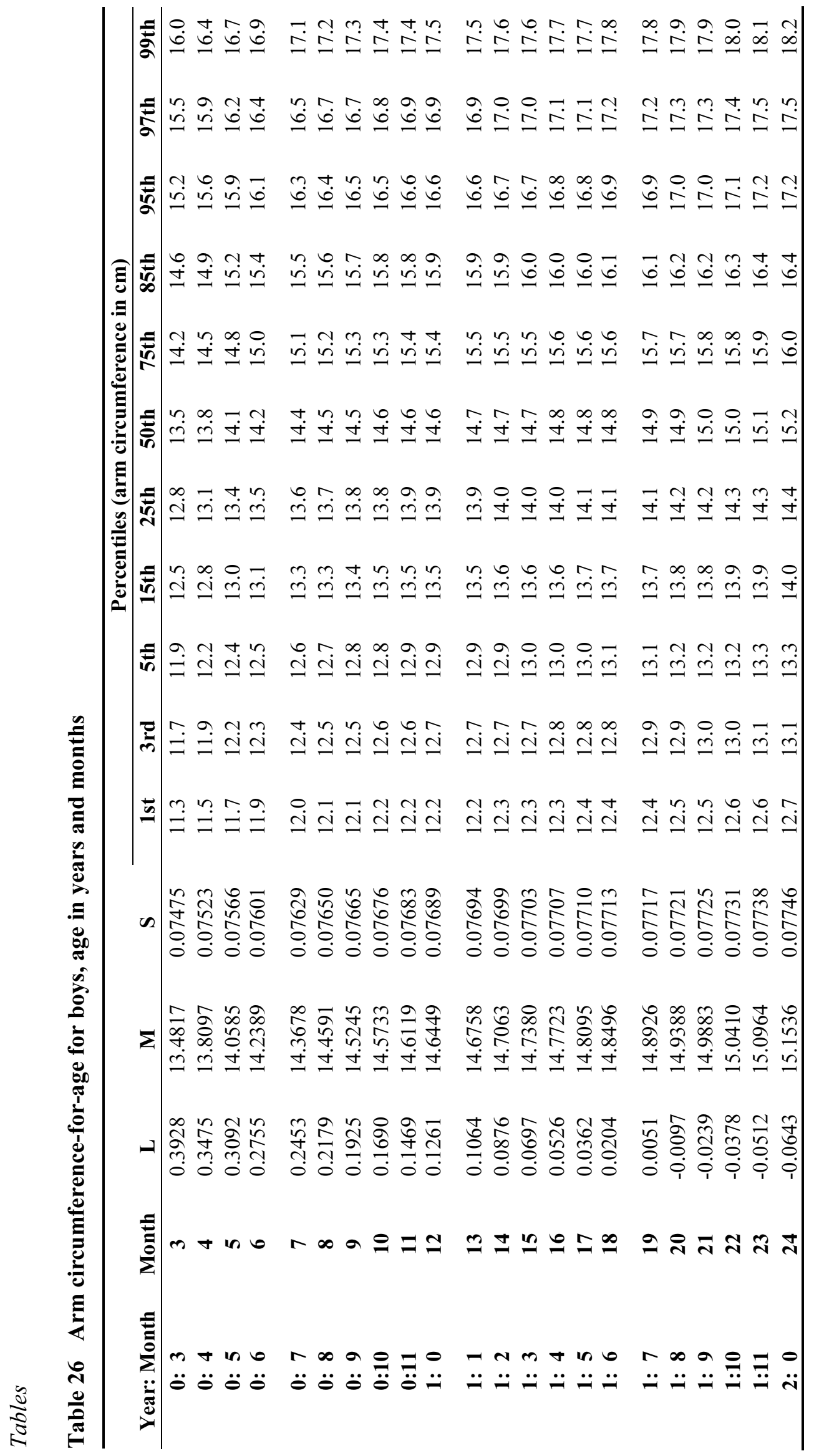




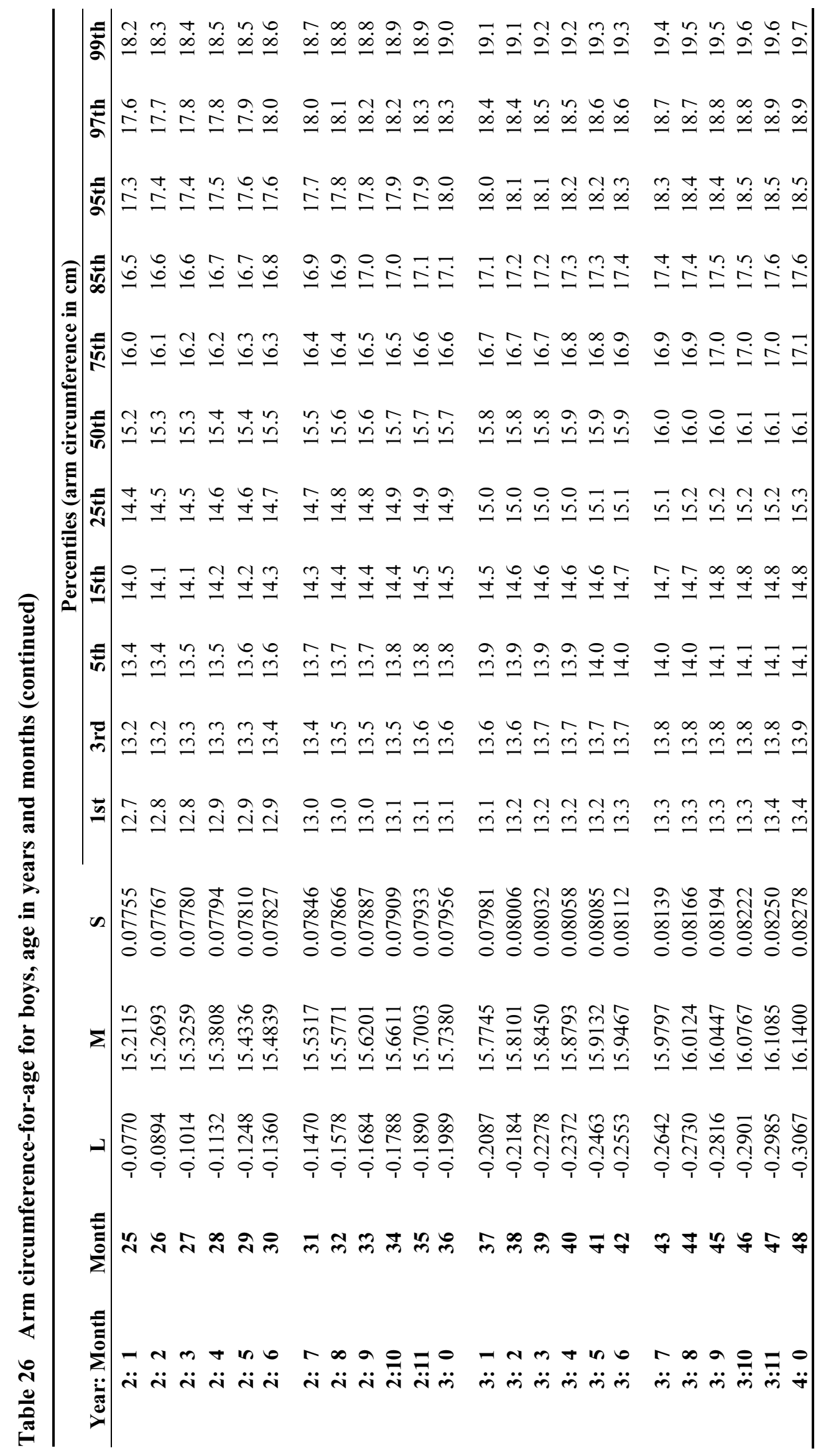




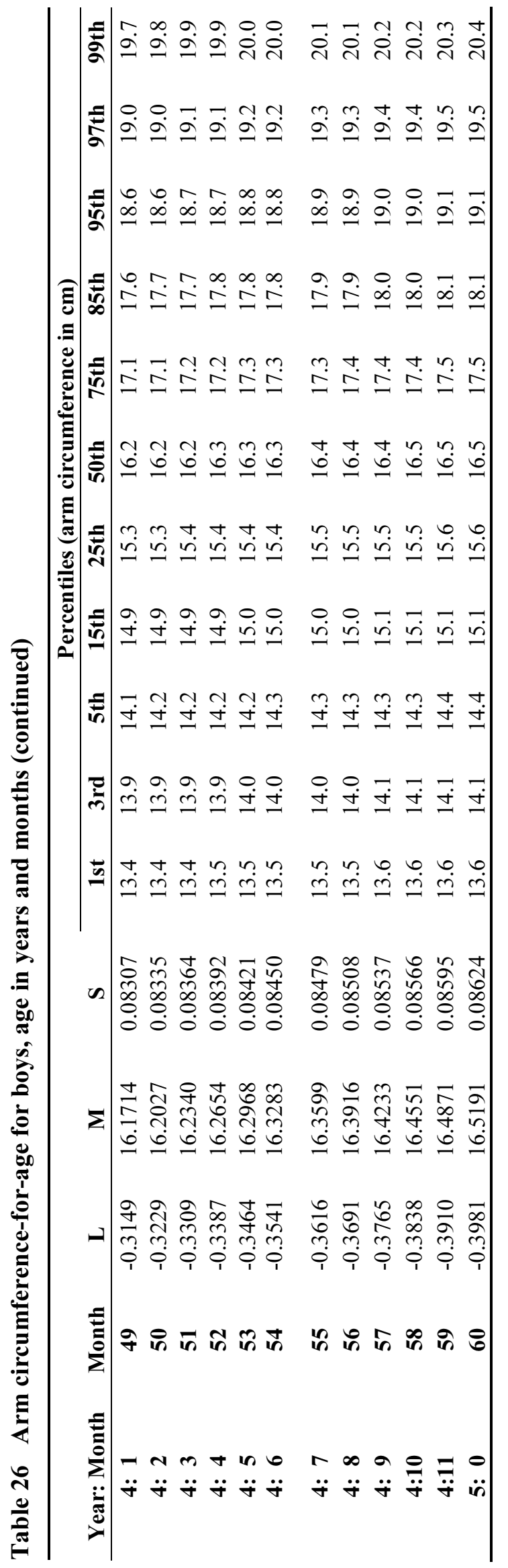




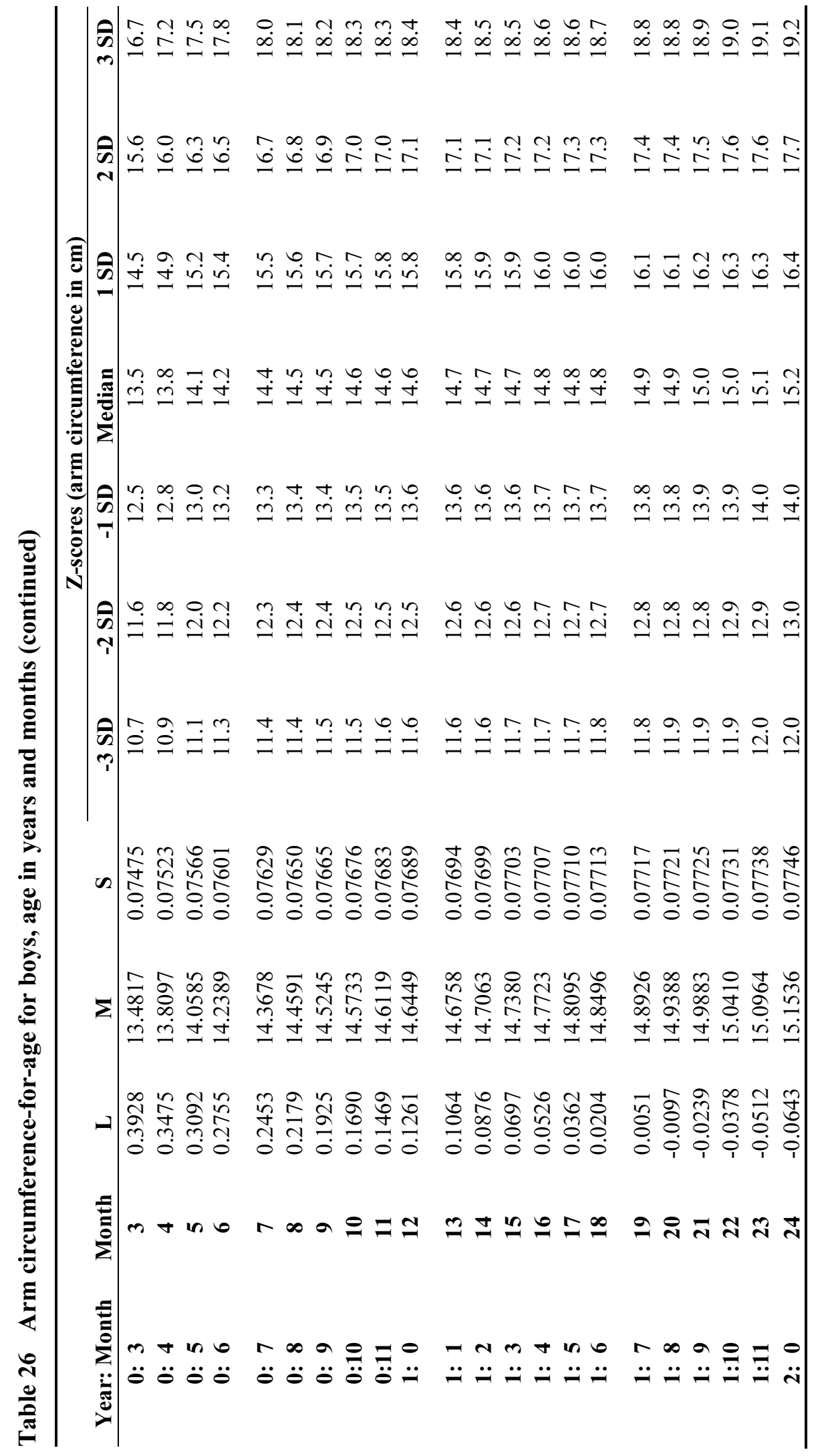




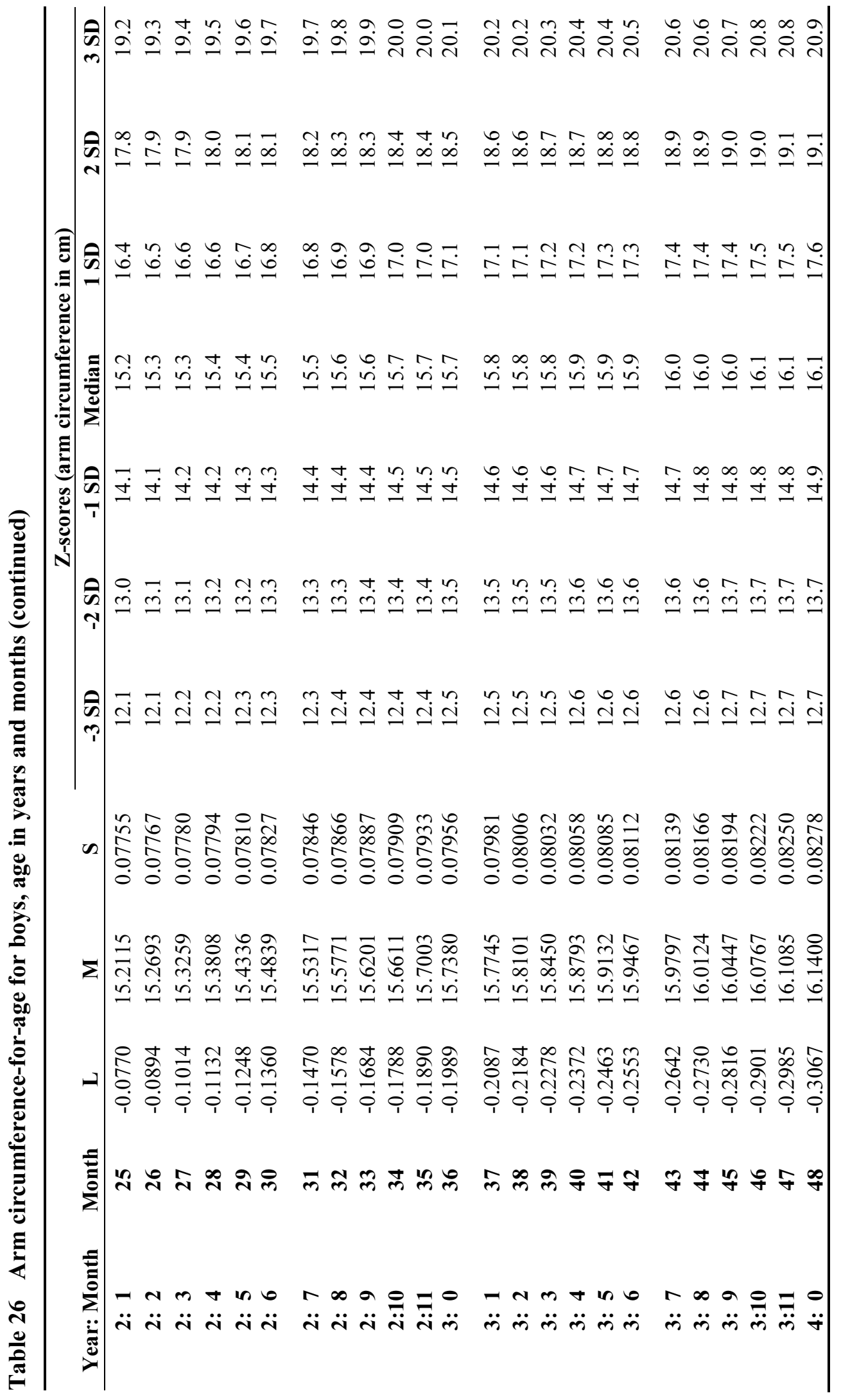




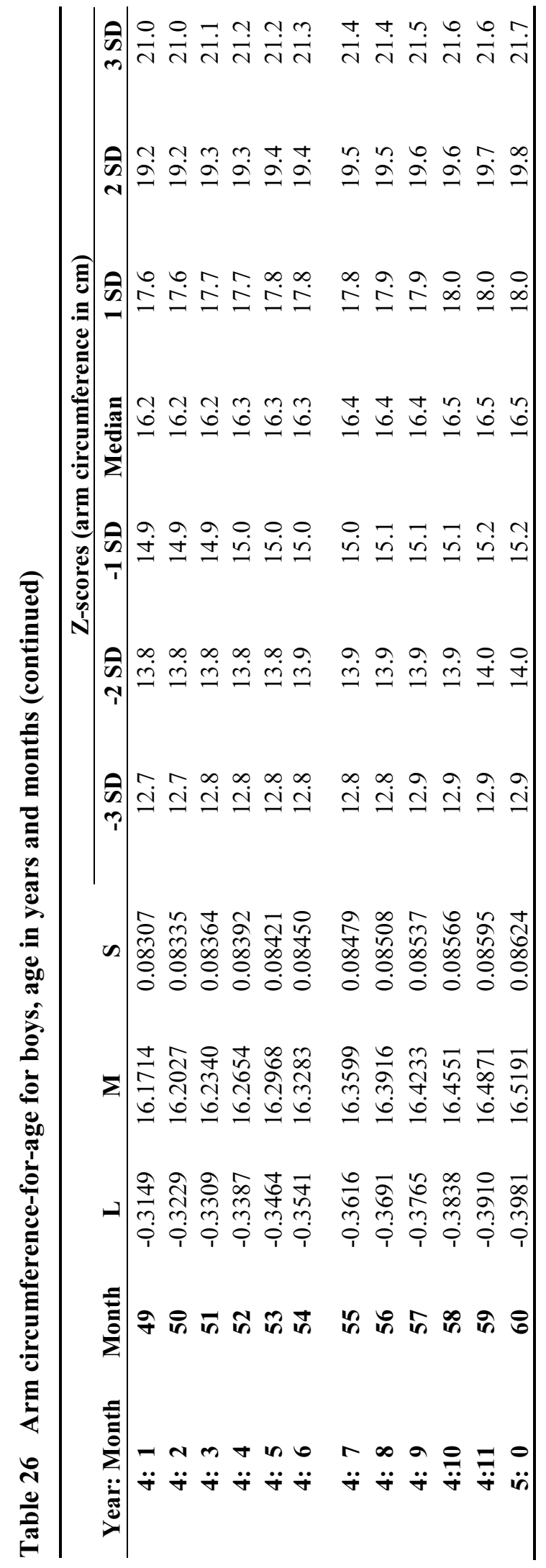




\subsection{Arm circumference-for-age for girls}

Steps similar to those described in the preceding sections were followed to select the best model to fit the arm circumference-for-age growth curves for girls.

\subsubsection{Sample size}

There were a total of 10970 arm circumference measurements for girls. The longitudinal and crosssectional sample sizes by visit and age are presented in Tables 27 and 28.

Table 27 Longitudinal sample sizes for arm circumference-for-age for girls

\begin{tabular}{|c|c|c|c|c|c|c|}
\hline Visit & 5 & 6 & 7 & 8 & 9 & 10 \\
\hline Age & $3 \mathrm{mo}$ & $4 \mathrm{mo}$ & $5 \mathrm{mo}$ & $6 \mathrm{mo}$ & $7 \mathrm{mo}$ & $8 \mathrm{mo}$ \\
\hline $\mathrm{N}$ & 437 & 440 & 447 & 445 & 444 & 440 \\
\hline Visit & 11 & 12 & 13 & 14 & 15 & 16 \\
\hline Age & 9 mo & $10 \mathrm{mo}$ & $11 \mathrm{mo}$ & $12 \mathrm{mo}$ & $14 \mathrm{mo}$ & $16 \mathrm{mo}$ \\
\hline $\mathrm{N}$ & 445 & 444 & 441 & 449 & 447 & 442 \\
\hline Visit & 17 & 18 & 19 & 20 & & \\
\hline Age & $18 \mathrm{mo}$ & $20 \mathrm{mo}$ & $22 \mathrm{mo}$ & $24 \mathrm{mo}$ & & \\
\hline $\mathrm{N}$ & 445 & 443 & 434 & 446 & & \\
\hline
\end{tabular}

Table 28 Cross-sectional sample sizes for arm circumference-for-age for girls

\begin{tabular}{lccccccc}
\hline Age (mo) & $<\mathbf{1 8}$ & $\mathbf{1 8 - 2 0}$ & $\mathbf{2 1 - 2 3}$ & $\mathbf{2 4 - 2 6}$ & $\mathbf{2 7 - 2 9}$ & $\mathbf{3 0 - 3 2}$ & $\mathbf{3 3 - 3 5}$ \\
$\mathrm{N}$ & 2 & 159 & 167 & 238 & 214 & 227 & 223 \\
\hline Age (mo) & $\mathbf{3 6 - 3 8}$ & $\mathbf{3 9 - 4 1}$ & $\mathbf{4 2 - 4 4}$ & $\mathbf{4 5}-\mathbf{4 7}$ & $\mathbf{4 8}-\mathbf{5 0}$ & $\mathbf{5 1 - 5 3}$ & $\mathbf{5 4 - 5 6}$ \\
$\mathrm{N}$ & 220 & 235 & 246 & 208 & 224 & 201 & 230 \\
\hline Age (mo) & $\mathbf{5 7 - 5 9}$ & $\mathbf{6 0 - 6 2}$ & $\mathbf{6 3 - 6 5}$ & $\mathbf{6 6 - 6 8}$ & $\mathbf{6 9 - 7 1}$ & $>\mathbf{7 1}$ & \\
$\mathrm{N}$ & 239 & 217 & 205 & 225 & 201 & 0 & \\
\hline
\end{tabular}

\subsubsection{Model selection and results}

The initial step in the modelling process was to search for the best age power transformation using the same starting model as that used for boys. Table 29 shows the values of global deviance for a grid of possible powers from 0.05 to 1 . The smallest global deviance value was associated with $\lambda=0.35$, and thus it was selected as the age-transformation power. 
Table 29 Global deviance (GD) for models within the class $\operatorname{BCPE}\left(x=\operatorname{age}^{\lambda}, \operatorname{df}(\mu)=9, \operatorname{df}(\sigma)=4\right.$, $\mathrm{df}(v)=4, \tau=2)$ for arm circumference-for-age for girls

\begin{tabular}{lcccccccccc}
\hline $\boldsymbol{\lambda}$ & $\mathbf{0 . 0 5}$ & $\mathbf{0 . 1 0}$ & $\mathbf{0 . 1 5}$ & $\mathbf{0 . 2 0}$ & $\mathbf{0 . 2 5}$ & $\mathbf{0 . 3 0}$ & $\mathbf{0 . 3 5}$ & $\mathbf{0 . 4 0}$ & $\mathbf{0 . 4 5}$ & $\mathbf{0 . 5 0}$ \\
$\mathrm{GD}^{\mathrm{a}}$ & 975.6 & 974.9 & 974.3 & 973.8 & 973.5 & 973.2 & 973.1 & 973.2 & 973.3 & 973.6 \\
\hline $\boldsymbol{\lambda}$ & $\mathbf{0 . 5 5}$ & $\mathbf{0 . 6 0}$ & $\mathbf{0 . 6 5}$ & $\mathbf{0 . 7 0}$ & $\mathbf{0 . 7 5}$ & $\mathbf{0 . 8 0}$ & $\mathbf{0 . 8 5}$ & $\mathbf{0 . 9 0}$ & $\mathbf{0 . 9 5}$ & $\mathbf{1 . 0 0}$ \\
$\mathrm{GD}^{\mathrm{a}}$ & 974.1 & 974.7 & 975.5 & 976.6 & 978.0 & 979.7 & 981.7 & 984.1 & 986.9 & 990.2 \\
\hline
\end{tabular}

${ }^{\mathrm{a}}$ In excess of 35000 .

Using $\lambda=0.35$, optimal values for $\operatorname{df}(\mu)$ and $\operatorname{df}(\sigma)$ were sought fixing $v=1$ and $\tau=2$. All possible combinations of $\operatorname{df}(\mu)$ values from 7 to 15 and $\operatorname{df}(\sigma)$ from 2 to 10 were considered. Partial results are presented in Table 30. The smallest value of GAIC(3) corresponded to $\operatorname{df}(\mu)=8$ and $\operatorname{df}(\sigma)=4$. Thus this model was selected and further evaluated.

Table 30 Goodness-of-fit summary for models using the BCPE distribution with fixed $v=1$ and $\tau=2$ for arm circumference-for-age for girls

\begin{tabular}{|c|c|c|c|c|c|}
\hline $\operatorname{df}(\mu)$ & $\operatorname{df}(\sigma)$ & GD $^{\mathbf{a}}$ & $\mathrm{AIC}^{\mathbf{a}}$ & GAIC(3) ${ }^{a}$ & Total df \\
\hline \multirow{5}{*}{7} & 2 & 1170.7 & 1188.7 & 1197.7 & 9 \\
\hline & 3 & 1143.0 & 1163.0 & 1173.0 & 10 \\
\hline & 4 & 1133.6 & 1155.6 & 1166.6 & 11 \\
\hline & 5 & 1130.6 & 1154.6 & 1166.6 & 12 \\
\hline & 6 & 1129.5 & 1155.5 & 1168.5 & 13 \\
\hline \multirow{5}{*}{8} & 2 & 1167.5 & 1187.5 & 1197.5 & 10 \\
\hline & 3 & 1139.6 & 1161.6 & 1172.6 & 11 \\
\hline & 4 & 1130.2 & 1154.2 & 1166.2 & 12 \\
\hline & 5 & 1127.2 & 1153.2 & 1166.2 & 13 \\
\hline & 6 & 1126.0 & 1154.0 & 1168.0 & 14 \\
\hline \multirow{5}{*}{9} & 2 & 1165.0 & 1187.0 & 1198.0 & 11 \\
\hline & 3 & 1137.2 & 1161.2 & 1173.2 & 12 \\
\hline & 4 & 1127.6 & 1153.6 & 1166.6 & 13 \\
\hline & 5 & 1124.7 & 1152.7 & 1166.7 & 14 \\
\hline & 6 & 1123.5 & 1153.5 & 1168.5 & 15 \\
\hline \multirow{5}{*}{10} & 2 & 1163.0 & 1187.0 & 1199.0 & 12 \\
\hline & 3 & 1135.1 & 1161.1 & 1174.1 & 13 \\
\hline & 4 & 1125.4 & 1153.4 & 1167.4 & 14 \\
\hline & 5 & 1122.4 & 1152.4 & 1167.4 & 15 \\
\hline & 6 & 1121.3 & 1153.3 & 1169.3 & 16 \\
\hline \multirow{5}{*}{11} & 2 & 1161.0 & 1187.0 & 1200.0 & 13 \\
\hline & 3 & 1133.0 & 1161.0 & 1175.0 & 14 \\
\hline & 4 & 1123.3 & 1153.3 & 1168.3 & 15 \\
\hline & 5 & 1120.2 & 1152.2 & 1168.2 & 16 \\
\hline & 6 & 1119.1 & 1153.1 & 1170.1 & 17 \\
\hline
\end{tabular}

GD, Global Deviance; AIC, Akaike Information Criterion;

GAIC(3), Generalized AIC with penalty equal to 3;

${ }^{\mathrm{a}}$ In excess of 35000 . 
Model 1: $\operatorname{BCPE}\left(x=\operatorname{age}^{0.35}, \operatorname{df}(\mu)=8, \operatorname{df}(\sigma)=4, v=1, \tau=2\right)$

The worm plots (Figure 33) pointed to the need to adjust for skewness, as the worms were U-shaped in most age groups. This finding was confirmed by the Q-test results (Table 31), which show various groups with absolute values of $\mathrm{z} 3$ higher than 2. Only one age group presented an absolute value of $\mathrm{z} 4$ higher than 2, indicating residual kurtosis. The overall test for skewness was significant $(\mathrm{p}$-value $<0.01)$.
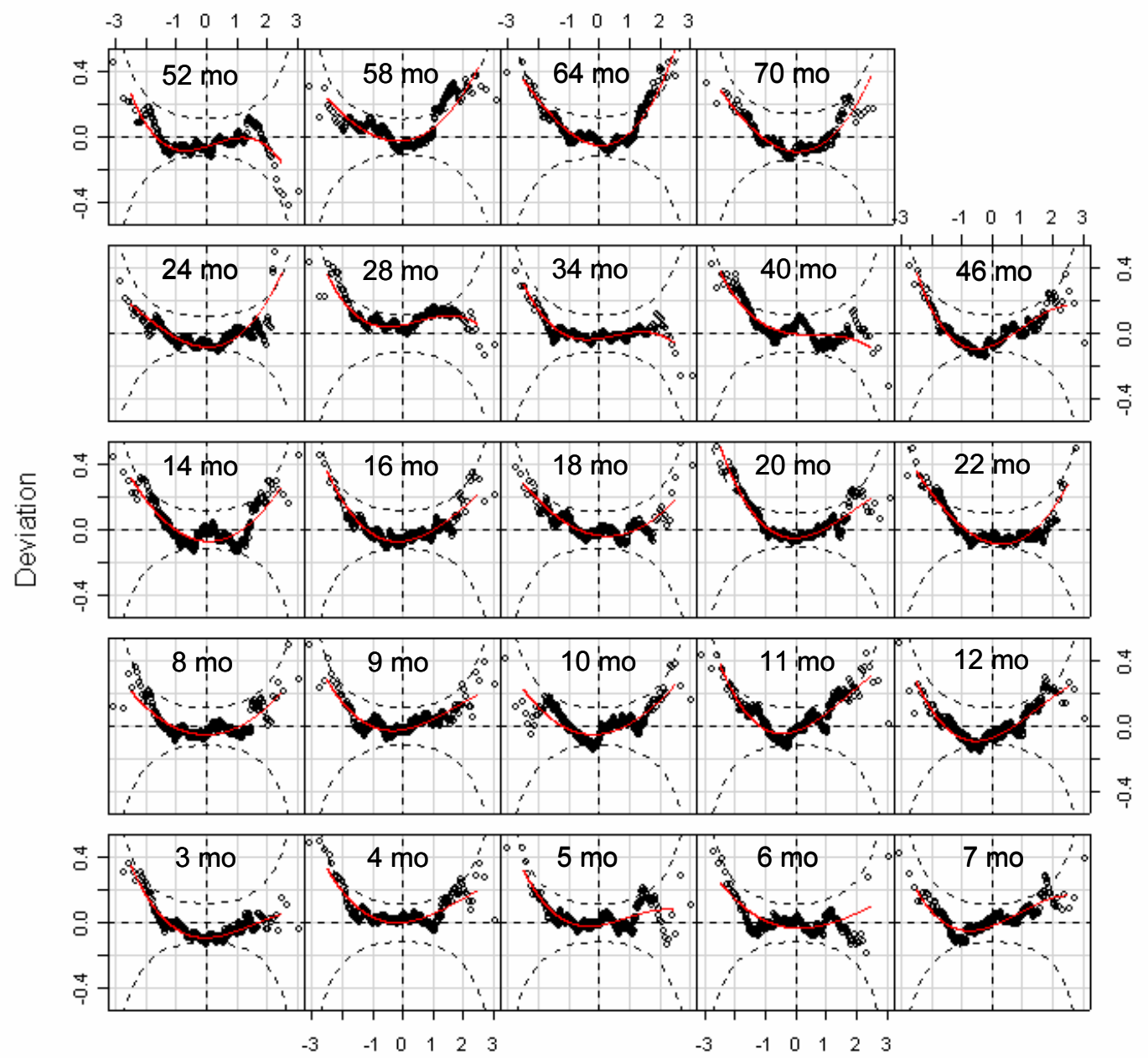

Unit normal quantile

Figure 33 Worm plots of z-scores for Model 1 for arm circumference-for-age for girls 
Table 31 Q-test for $z$-scores from Model $1\left[\operatorname{BCPE}\left(x=\operatorname{age} e^{0.35}, \operatorname{df}(\mu)=8, \operatorname{df}(\sigma)=4, v=1, \tau=2\right)\right]$ for $\operatorname{arm}$ circumference-for-age for girls

\begin{tabular}{|c|c|c|c|c|c|c|}
\hline Age (days) & Group & $\mathbf{N}$ & $\mathrm{z1}$ & $\mathbf{z 2}$ & $\mathbf{z 3}$ & $\mathbf{z 4}$ \\
\hline 83 to 99 & $3 \mathrm{mo}$ & 430 & -0.92 & -0.67 & 2.23 & -0.68 \\
\hline 100 to 129 & $4 \mathrm{mo}$ & 440 & 0.82 & -0.06 & 2.15 & -0.19 \\
\hline 130 to 159 & $5 \mathrm{mo}$ & 441 & 0.29 & -0.15 & 1.76 & -0.81 \\
\hline 160 to 189 & $6 \mathrm{mo}$ & 441 & -0.10 & -0.52 & 1.91 & 0.68 \\
\hline 190 to 219 & $7 \mathrm{mo}$ & 432 & 0.28 & 1.13 & 1.51 & -0.87 \\
\hline 220 to 249 & $8 \mathrm{mo}$ & 435 & -0.32 & 0.24 & 2.18 & 0.27 \\
\hline 250 to 279 & $9 \mathrm{mo}$ & 438 & 0.47 & 0.00 & 1.85 & -0.17 \\
\hline 280 to 309 & $10 \mathrm{mo}$ & 444 & -0.42 & 0.42 & 2.38 & 0.26 \\
\hline 310 to 349 & $11 \mathrm{mo}$ & 477 & 0.67 & 0.91 & 2.82 & -0.71 \\
\hline 350 to 379 & $12 \mathrm{mo}$ & 451 & -0.42 & 1.03 & 2.45 & -0.71 \\
\hline 380 to 439 & $14 \mathrm{mo}$ & 444 & -0.26 & -0.18 & 2.92 & 0.63 \\
\hline 440 to 499 & $16 \mathrm{mo}$ & 444 & -0.31 & -0.02 & 2.73 & -0.04 \\
\hline 500 to 559 & $18 \mathrm{mo}$ & 467 & 0.09 & -0.56 & 2.32 & 0.56 \\
\hline 560 to 619 & $20 \mathrm{mo}$ & 543 & 0.28 & -0.67 & 3.44 & -0.42 \\
\hline 620 to 679 & $22 \mathrm{mo}$ & 538 & -0.42 & -0.86 & 3.71 & 1.58 \\
\hline 680 to 749 & $24 \mathrm{mo}$ & 592 & -0.64 & 0.69 & 3.52 & 1.82 \\
\hline 750 to 929 & $28 \mathrm{mo}$ & 456 & 1.53 & -0.29 & 1.26 & -1.54 \\
\hline 930 to 1119 & $34 \mathrm{mo}$ & 470 & -0.15 & -0.58 & 1.16 & -1.50 \\
\hline 1120 to 1309 & $40 \mathrm{mo}$ & 475 & 0.27 & -1.67 & 1.23 & -1.12 \\
\hline 1310 to 1499 & $46 \mathrm{mo}$ & 453 & -0.44 & 0.66 & 2.54 & -1.16 \\
\hline 1500 to 1689 & $52 \mathrm{mo}$ & 451 & -1.09 & -0.31 & 0.94 & -2.38 \\
\hline 1690 to 1879 & $58 \mathrm{mo}$ & 494 & 0.67 & 1.10 & 2.98 & 0.48 \\
\hline 1880 to 2069 & $64 \mathrm{mo}$ & 418 & 0.52 & 0.48 & 3.78 & 1.49 \\
\hline 2070 to 2191 & $70 \mathrm{mo}$ & 296 & -0.42 & 0.16 & 2.77 & 0.90 \\
\hline Overall Q stats & & 10970 & 8.35 & 11.36 & 148.40 & 26.39 \\
\hline degrees of freedom & & & 16.0 & 21.5 & 24.0 & 24.0 \\
\hline p-value & & & 0.9376 & 0.9629 & $<0.01$ & 0.3338 \\
\hline
\end{tabular}

Note: Absolute values of z1, z2, z3 or z4 larger than 2 indicate misfit of, respectively, mean, variance, skewness or kurtosis.

The next step involved fitting the parameter $v$ for skewness using the BCPE distribution with fixed parameter $\tau=2$ and keeping the degrees of freedom for the $\mu$ and $\sigma$ curves selected for Model 1 . Table 32 shows the GAIC(3) values for various degrees of freedom for the $v$ curve. 
Table 32 Goodness-of-fit summary for models $\operatorname{BCPE}\left(x=\operatorname{age}^{0.35}, \operatorname{df}(\mu)=8, \operatorname{df}(\sigma)=4, \operatorname{df}(v)=?, \tau=2\right)$ for arm circumference-for-age for girls

\begin{tabular}{cccc}
\hline $\mathbf{d f}(\mathbf{v})$ & $\mathbf{G D}^{\mathbf{a}}$ & GAIC(3) $^{\mathbf{a}}$ & Total df \\
\hline $\mathbf{1}$ & 978.9 & 1018.0 & 13 \\
$\mathbf{2}$ & 978.9 & 1020.9 & 14 \\
$\mathbf{3}$ & 977.7 & 1022.8 & 15 \\
$\mathbf{4}$ & 975.6 & 1023.6 & 16 \\
$\mathbf{5}$ & 973.8 & 1024.8 & 17 \\
$\mathbf{6}$ & 972.8 & 1026.8 & 18 \\
$\mathbf{7}$ & 972.2 & 1029.2 & 19 \\
\hline
\end{tabular}

GD, Global Deviance; GAIC(3), Generalized Akaike Information Criterion with penalty equal to 3 ;

${ }^{\mathrm{a}}$ In excess of 35000 .

The smallest $\operatorname{GAIC}(3)$ value corresponded to $\mathrm{df}(\mathrm{v})=1$ (i.e. fitting a constant for the $v$ curve). A new iteration was performed using $\operatorname{df}(v)=1$ to re-search for $\operatorname{df}(\mu)$ and $\operatorname{df}(\sigma)$. The best combination remained $\operatorname{df}(\mu)=8$ and $\operatorname{df}(\sigma)=4$. A new search for the best age-transformation power $\lambda$ using this model was carried out but results indicated no need to update the model. The model with $\lambda=0.35, \operatorname{df}(\mu)=8$, $\operatorname{df}(\sigma)=4$ and $\operatorname{df}(v)=1$ was thus further evaluated.

\section{Model 2: $\operatorname{BCPE}\left(x=\operatorname{age}^{0.35}, \operatorname{df}(\mu)=8, \operatorname{df}(\sigma)=4, \operatorname{df}(v)=1, \tau=2\right)$}

Figure 34 shows the fitting of the parameters $\mu, \sigma$ and $v$ for Model 2 with their respective sample estimates, that is, the median for $\mu$ and Box-Cox transform power for $v$. The fitted curve for $\sigma$ ignores a dip in the sample between 30 and 39 months, but overall the degree of smoothness for this parameter seemed adequate. Similarly, the $v$ curve fitting smoothed out the zigzagging of empirical values. Figures 35 and 36 show the distribution of differences between empirical values and fitted centiles for the longitudinal and cross-sectional samples, respectively. There was some indication of bias in the $3^{\text {rd }}$ centile between 3 and 24 months (Figure 35) and also between 24 and 71 months, the average bias being between $1 \mathrm{~mm}$ and $2 \mathrm{~mm}$ (Figure 36).

The worm plots for this model (Figure 37) were better conformed to a flat shape than the significant deviations depicted by those from Model 1 (Figure 33). There was no indication of remaining skewness or kurtosis. 

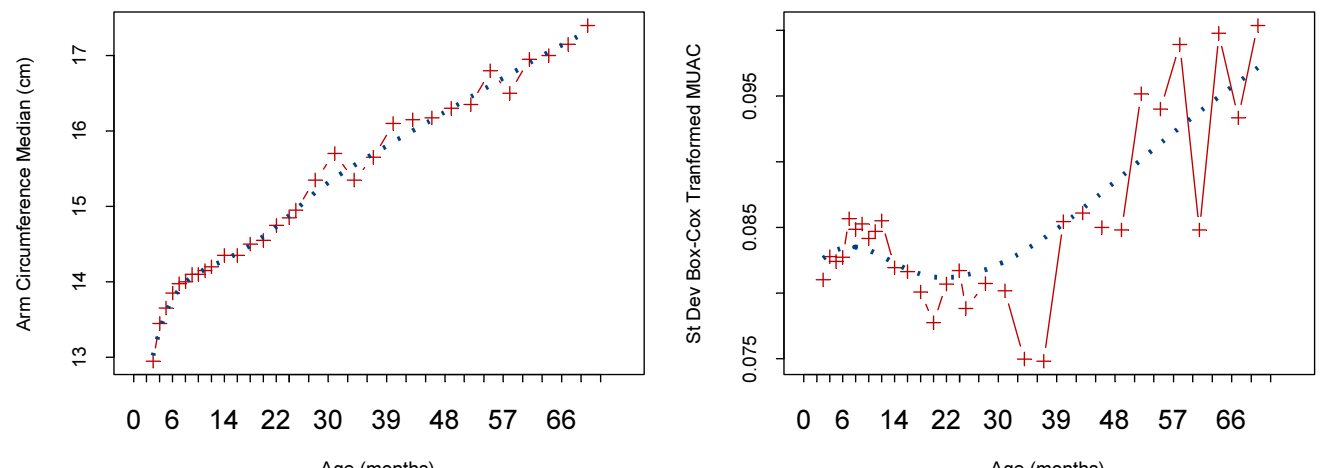

Age (months)

$\begin{array}{lllllllll}0 & 6 & 14 & 22 & 30 & 39 & 48 & 57 & 66\end{array}$

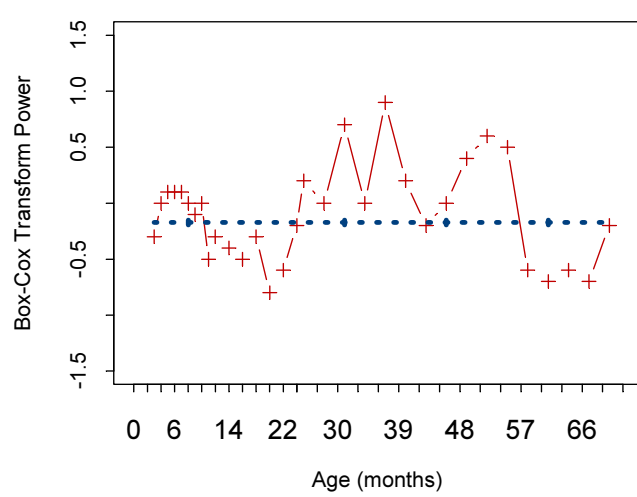

Figure 34 Fitting of the $\mu, \sigma$, and $v$ curves of Model 2 for arm circumference-for-age for girls from 3 to 71 months (dotted line) and their respective sample estimates (points with solid line) 

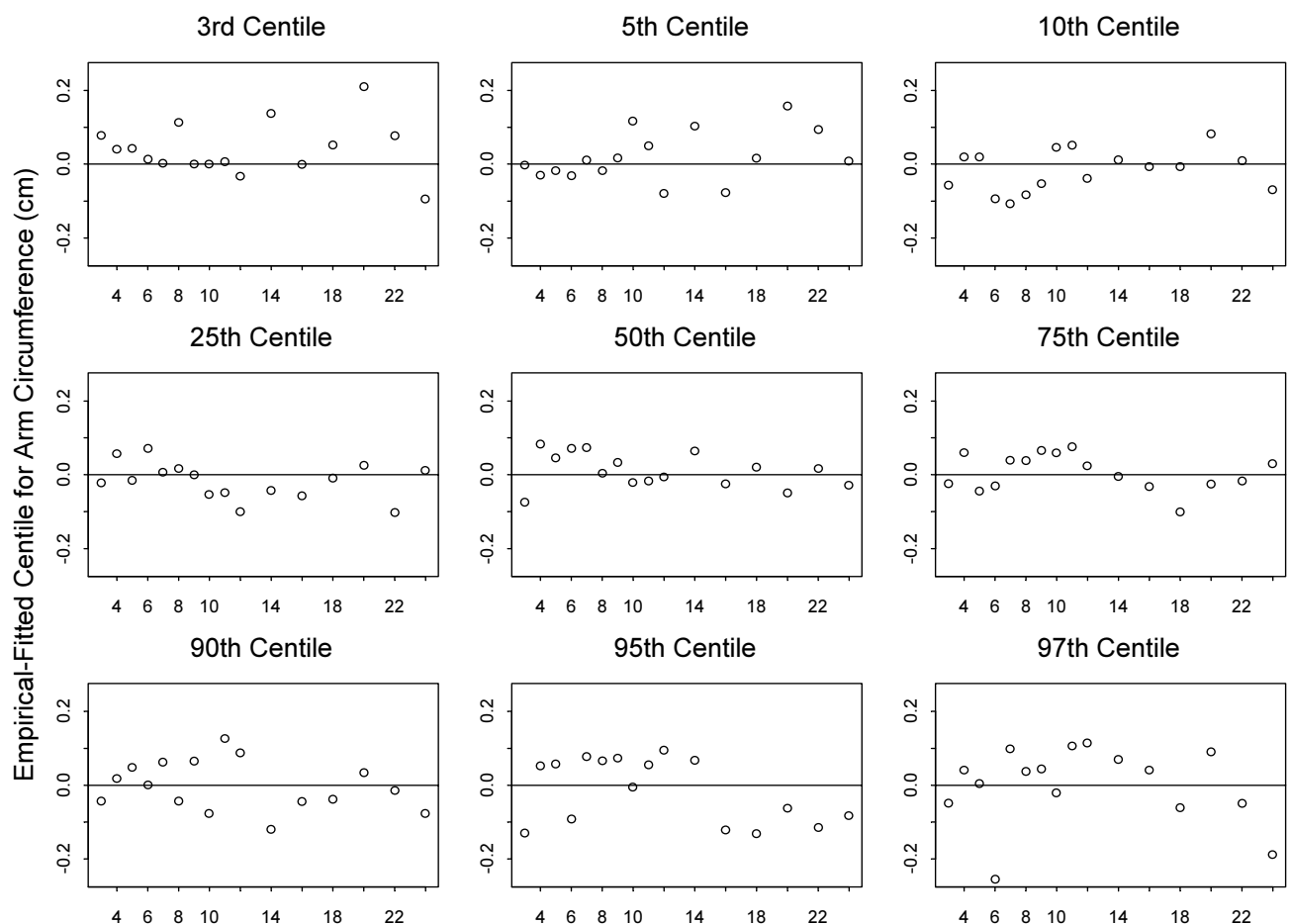

50th Centile

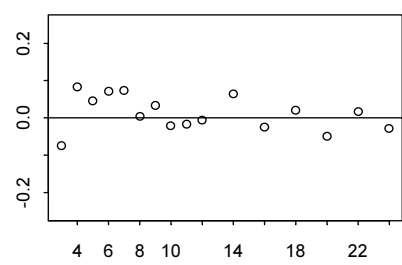

75th Centile

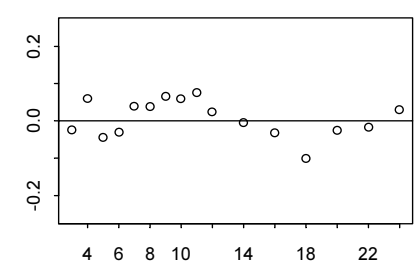

95th Centile

97th Centile
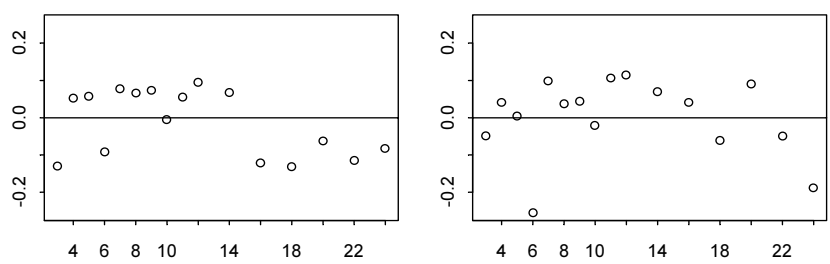

Age (months)

Figure 35 Centile residuals from fitting Model 2 for arm circumference-for-age from 3 to 24 months for girls
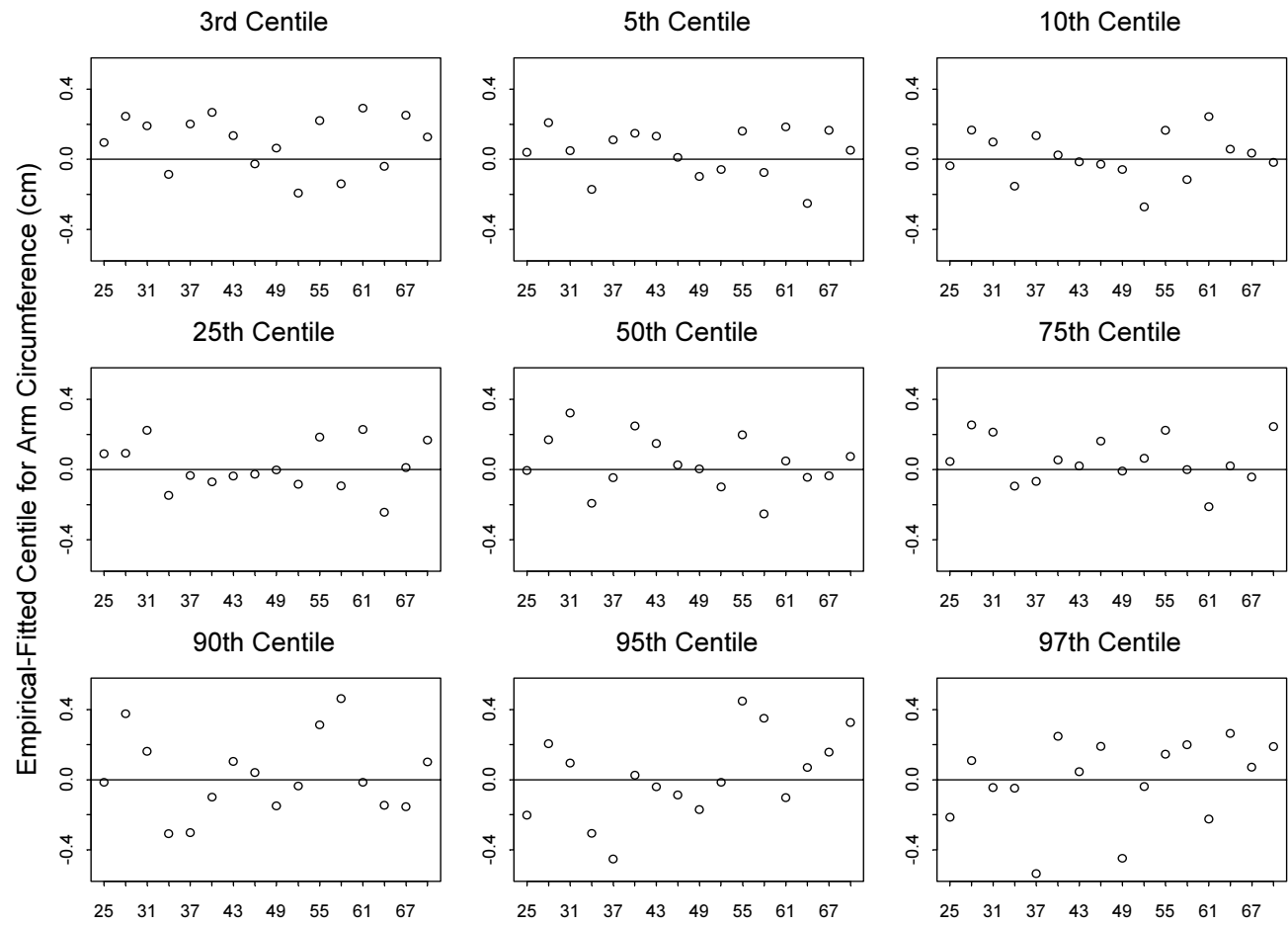

50th Centile

75th Centile
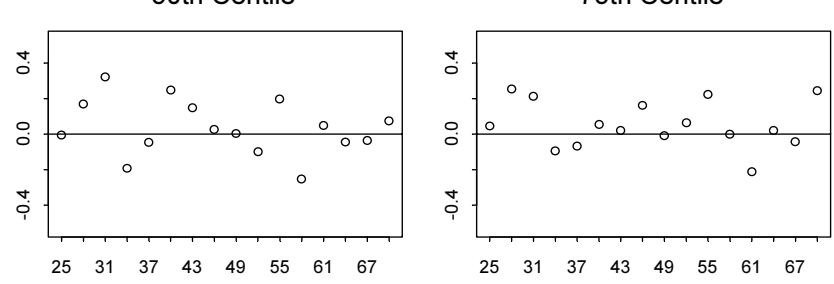

95th Centile

97th Centile
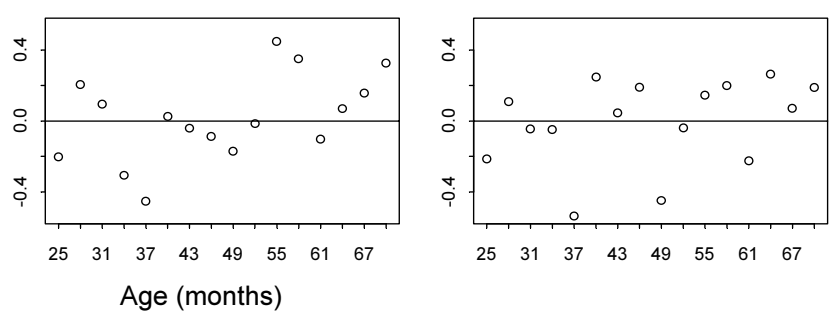

Figure 36 Centile residuals from fitting Model 2 for arm circumference-for-age from 24 to 71 months for girls 

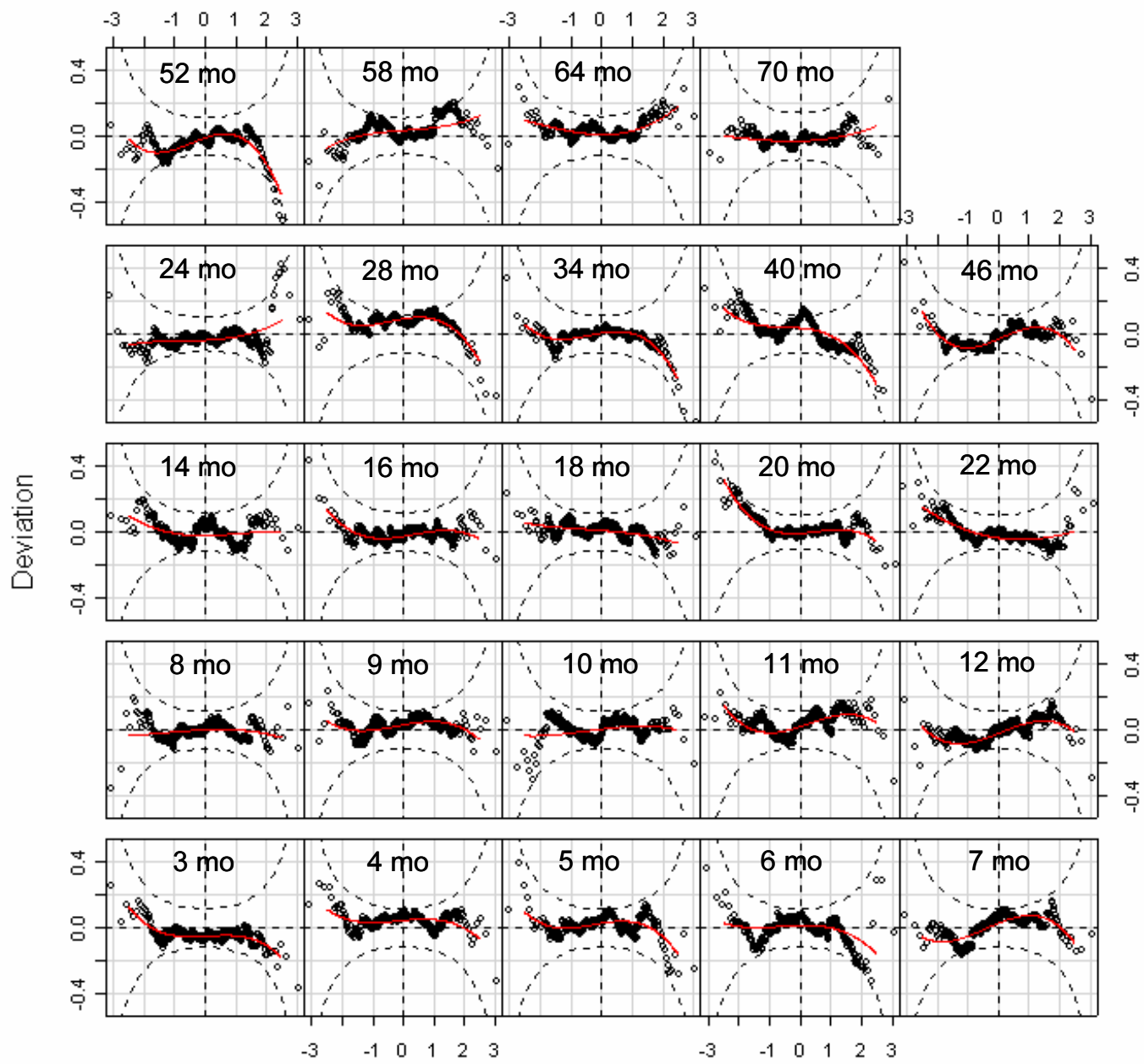

Unit normal quantile

Figure 37 Worm plots of z-scores for Model 2 for arm circumference-for-age for girls

The Q-test results from Model 2 are shown in Table 33. Only one age group (46 mo) had a $\mathrm{z} 4$ absolute value larger than 2 , suggesting remaining kurtosis. The overall Q-test $\mathrm{p}$-values were all non-significant, indicating an adequate fit of the girls' arm circumference-for-age curves. There was thus no need for modelling $\tau$ to adjust for kurtosis.

Table 34 presents observed percentages with arm circumferences below the fitted centiles. As was the case for the boys, no systematic bias was detected. 
Table 33 Q-test for $z$-scores from Model $2\left[\operatorname{BCPE}\left(x=\operatorname{age}^{0.35}, \operatorname{df}(\mu)=8, \operatorname{df}(\sigma)=4, \operatorname{df}(v)=1, \tau=2\right)\right]$ for arm circumference-for-age for girls

\begin{tabular}{|c|c|c|c|c|c|c|}
\hline Age (days) & Group & $\mathbf{N}$ & $\mathrm{z1}$ & $\mathbf{z 2}$ & $\mathbf{z 3}$ & $\mathrm{z4}$ \\
\hline 83 to 99 & $3 \mathrm{mo}$ & 430 & -0.98 & -0.61 & 0.15 & -1.25 \\
\hline 100 to 129 & $4 \mathrm{mo}$ & 440 & 0.77 & -0.20 & -0.12 & -0.79 \\
\hline 130 to 159 & $5 \mathrm{mo}$ & 441 & 0.28 & -0.15 & -0.41 & -1.25 \\
\hline 160 to 189 & $6 \mathrm{mo}$ & 441 & -0.05 & -0.46 & -0.52 & -0.38 \\
\hline 190 to 219 & $7 \mathrm{mo}$ & 432 & 0.25 & 1.20 & -0.74 & -1.34 \\
\hline 220 to 249 & $8 \mathrm{mo}$ & 435 & -0.25 & 0.31 & -0.26 & -0.17 \\
\hline 250 to 279 & $9 \mathrm{mo}$ & 438 & 0.57 & 0.01 & -0.46 & -0.74 \\
\hline 280 to 309 & $10 \mathrm{mo}$ & 444 & -0.34 & 0.49 & -0.09 & -0.08 \\
\hline 310 to 349 & $11 \mathrm{mo}$ & 477 & 0.73 & 0.74 & 0.57 & -1.37 \\
\hline 350 to 379 & $12 \mathrm{mo}$ & 451 & -0.40 & 1.08 & 0.21 & -1.25 \\
\hline 380 to 439 & $14 \mathrm{mo}$ & 444 & -0.22 & -0.21 & 0.58 & -0.33 \\
\hline 440 to 499 & $16 \mathrm{mo}$ & 444 & -0.34 & -0.02 & 0.54 & -1.00 \\
\hline 500 to 559 & $18 \mathrm{mo}$ & 467 & 0.06 & -0.51 & -0.11 & -0.22 \\
\hline 560 to 619 & $20 \mathrm{mo}$ & 543 & 0.25 & -0.85 & 1.25 & -1.55 \\
\hline 620 to 679 & $22 \mathrm{mo}$ & 538 & -0.43 & -0.95 & 1.09 & 0.19 \\
\hline 680 to 749 & $24 \mathrm{mo}$ & 592 & -0.73 & 0.69 & 0.48 & 0.55 \\
\hline 750 to 929 & $28 \mathrm{mo}$ & 456 & 1.51 & -0.35 & -0.84 & -1.35 \\
\hline 930 to 1119 & 34 mo & 470 & -0.25 & -0.41 & -0.98 & -1.54 \\
\hline 1120 to 1309 & $40 \mathrm{mo}$ & 475 & 0.27 & -1.59 & -0.95 & -1.38 \\
\hline 1310 to 1499 & $46 \mathrm{mo}$ & 453 & -0.52 & 0.63 & 0.35 & -2.09 \\
\hline 1500 to 1689 & $52 \mathrm{mo}$ & 451 & -1.03 & 0.08 & -1.24 & -1.95 \\
\hline 1690 to 1879 & $58 \mathrm{mo}$ & 494 & 0.70 & 0.98 & -0.03 & 0.31 \\
\hline 1880 to 2069 & $64 \mathrm{mo}$ & 418 & 0.59 & 0.21 & 1.01 & 0.32 \\
\hline 2070 to 2191 & $70 \mathrm{mo}$ & 296 & -0.35 & 0.19 & 0.41 & 0.08 \\
\hline Overall Q stats & & 10970 & 8.56 & 10.83 & 10.72 & 28.08 \\
\hline degrees of freedom & & & 16.0 & 21.5 & 23.0 & 24.0 \\
\hline p-value & & & 0.9306 & 0.9720 & 0.9859 & 0.2569 \\
\hline
\end{tabular}

Note: Absolute values of z1, z2, z3 or z4 larger than 2 indicate misfit of, respectively, mean, variance, skewness or kurtosis.

In conclusion, the model selected for constructing the arm circumference-for-age growth curves for girls was $\operatorname{BCPE}\left(x=a g e^{0.35}, \operatorname{df}(\mu)=8, \operatorname{df}(\sigma)=4, \operatorname{df}(v)=1, \tau=2\right)$ that adjusts only for skewness, and thus reduces to the LMS method. The fitted centile curves and empirical centiles are shown in Figures 38 to 41. 


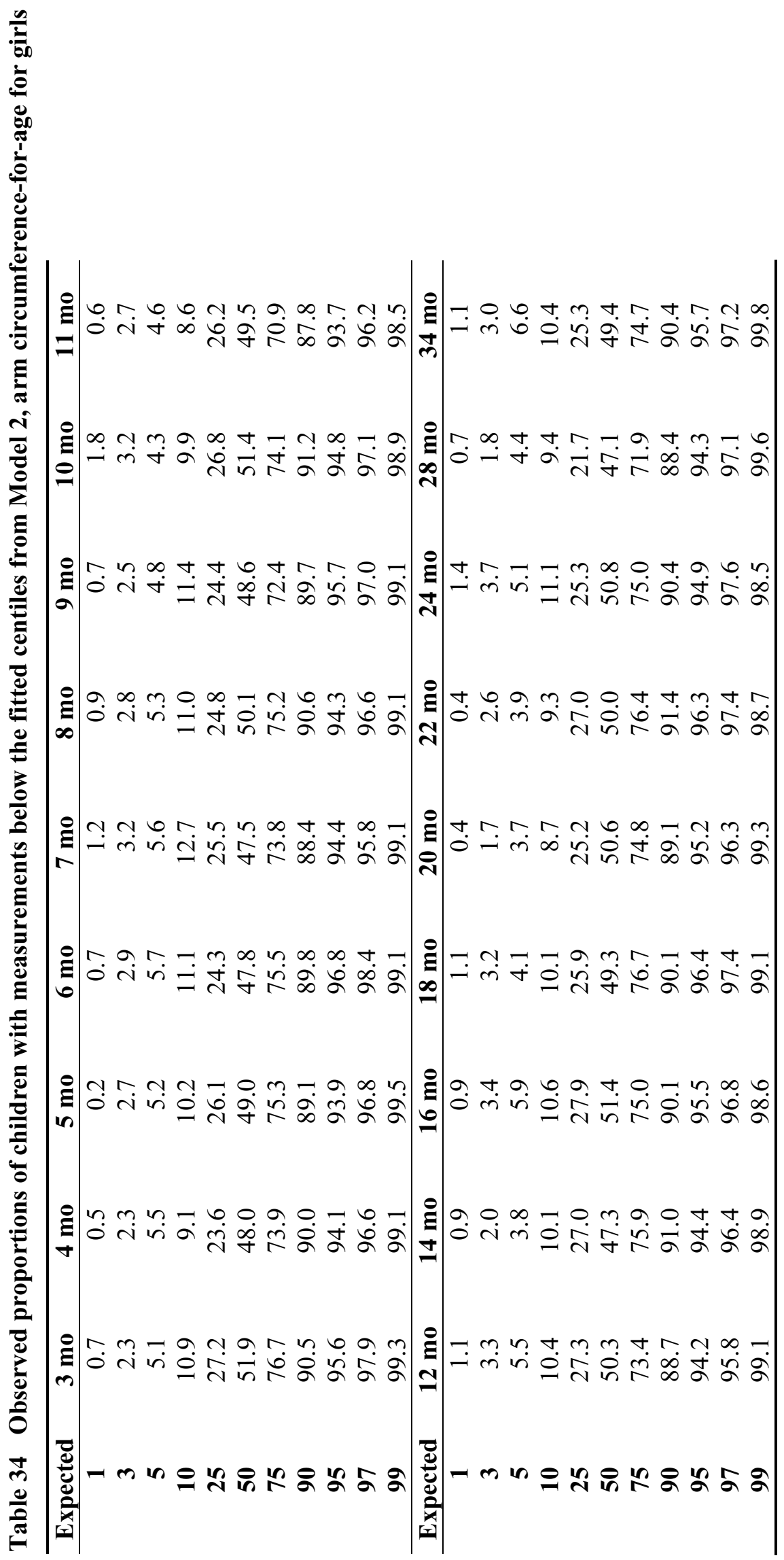




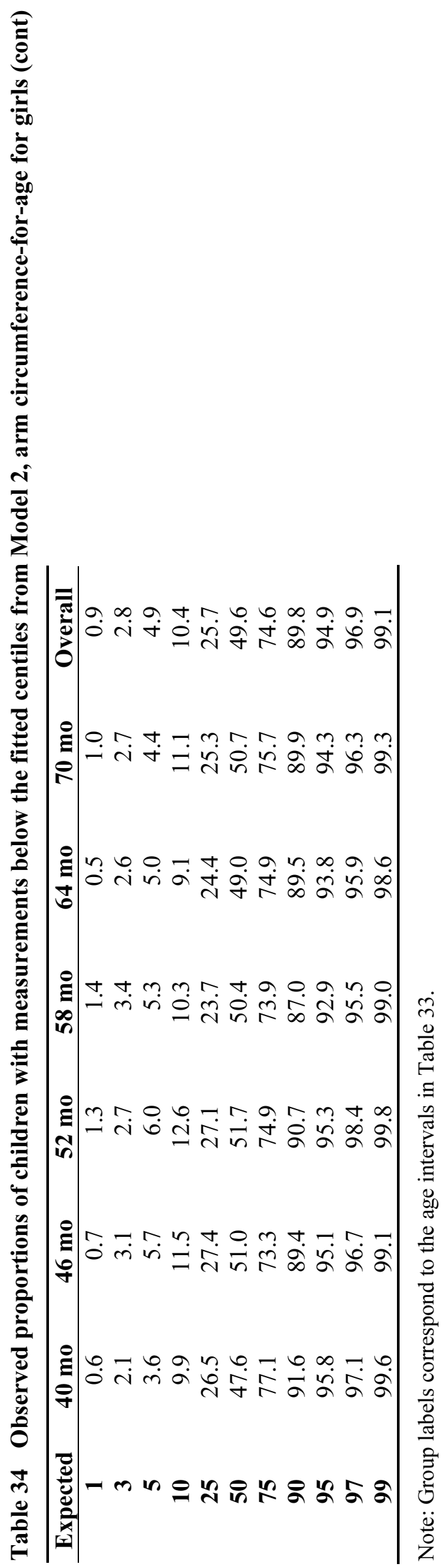




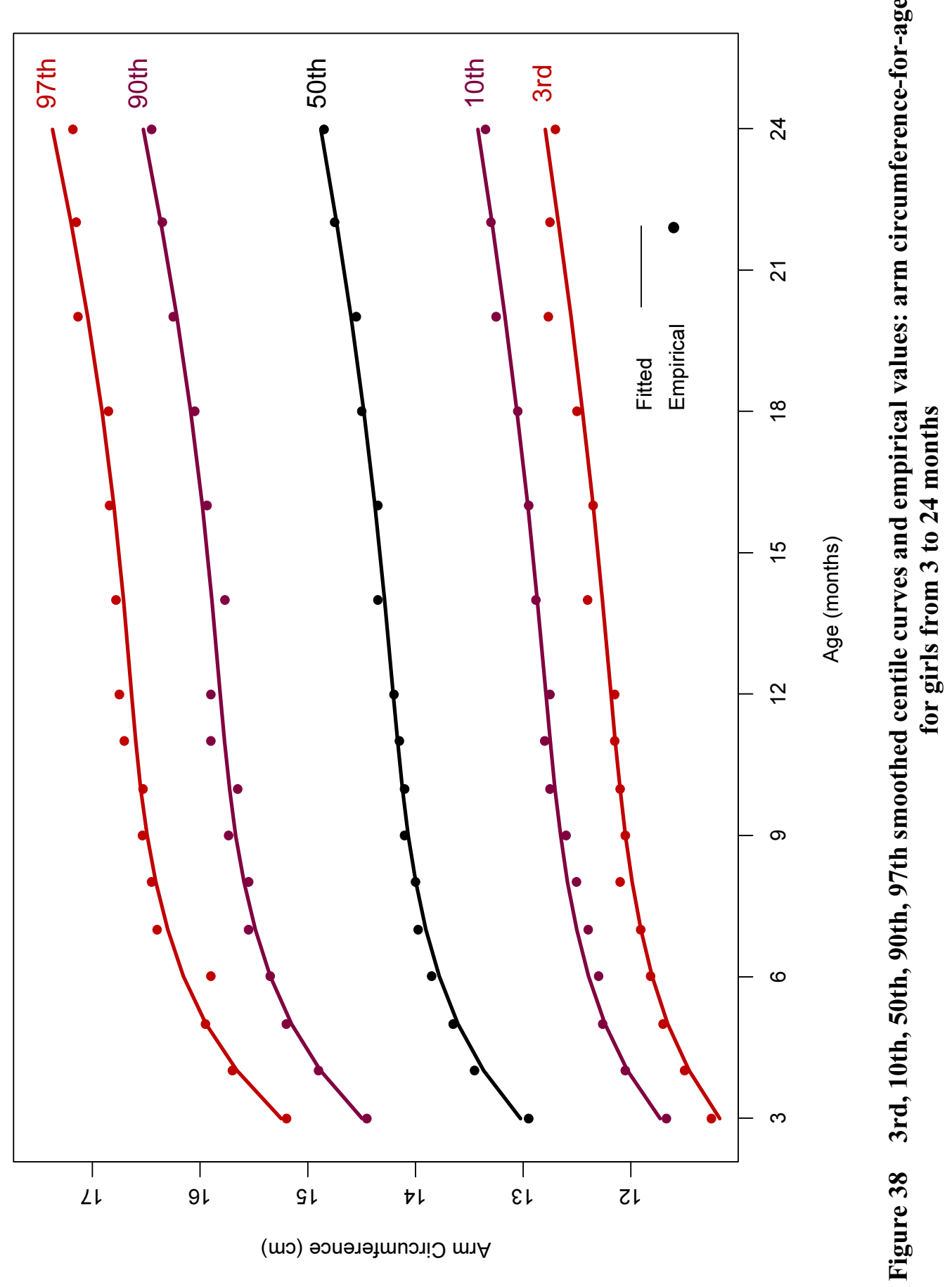




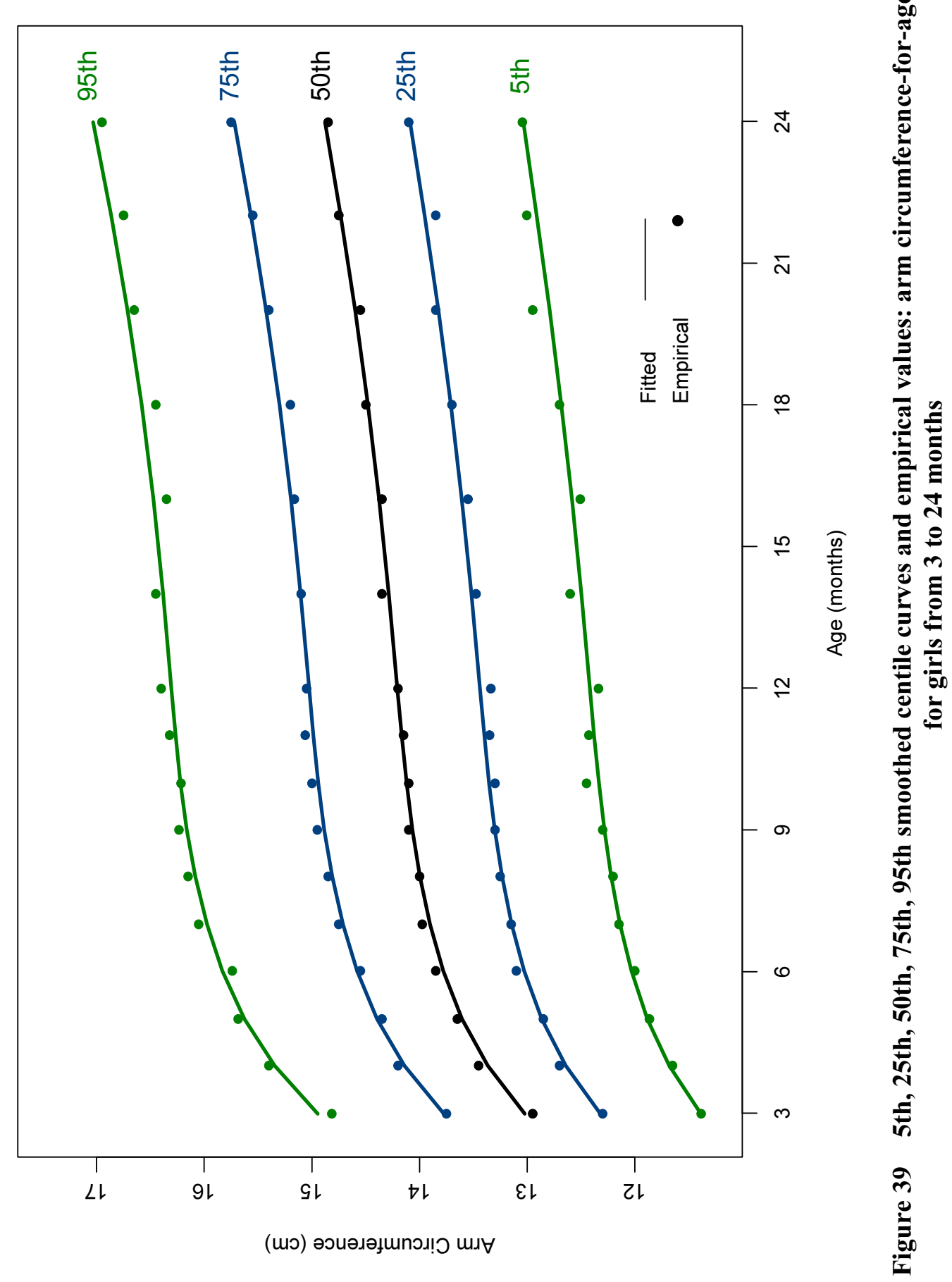




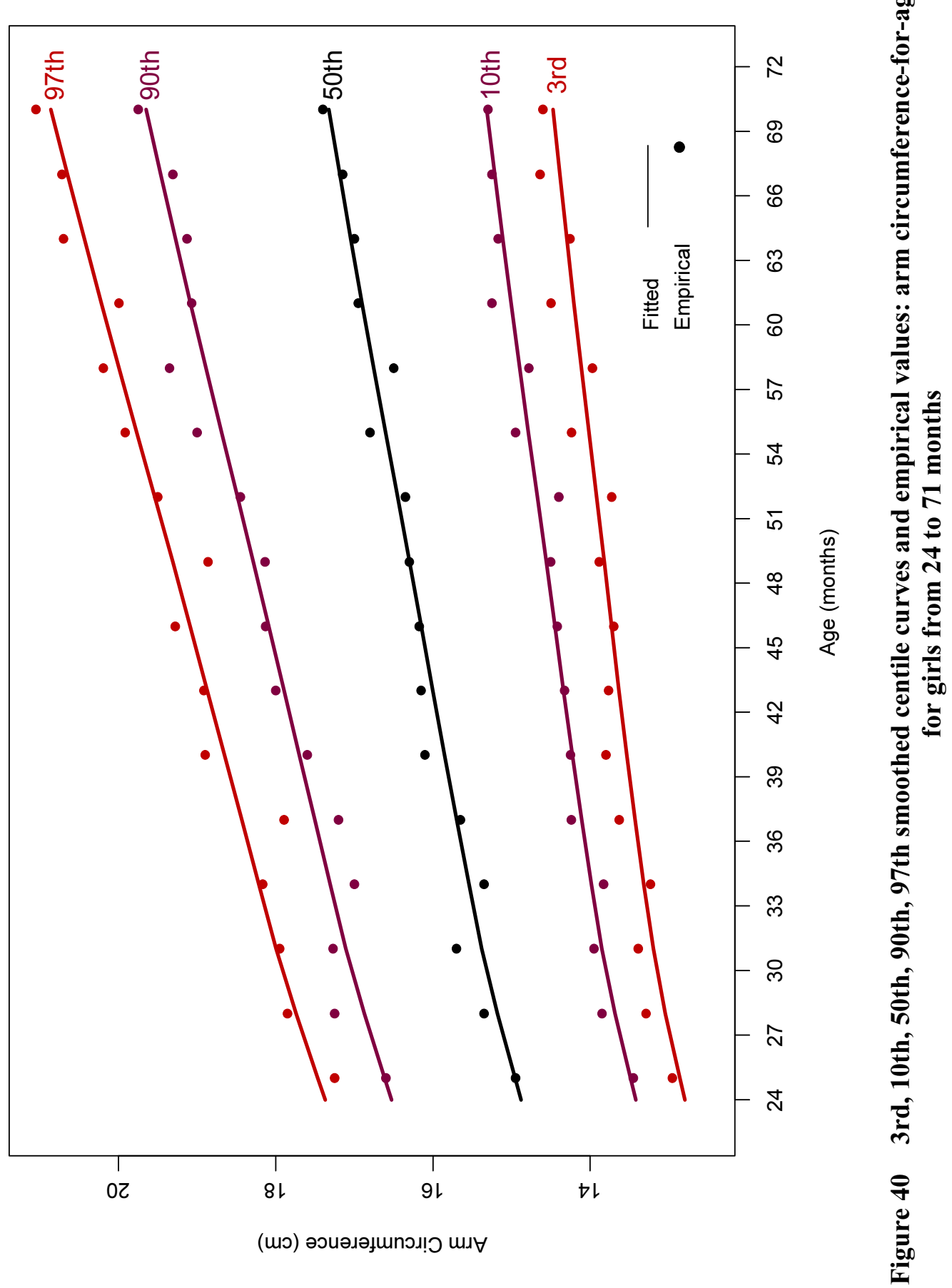




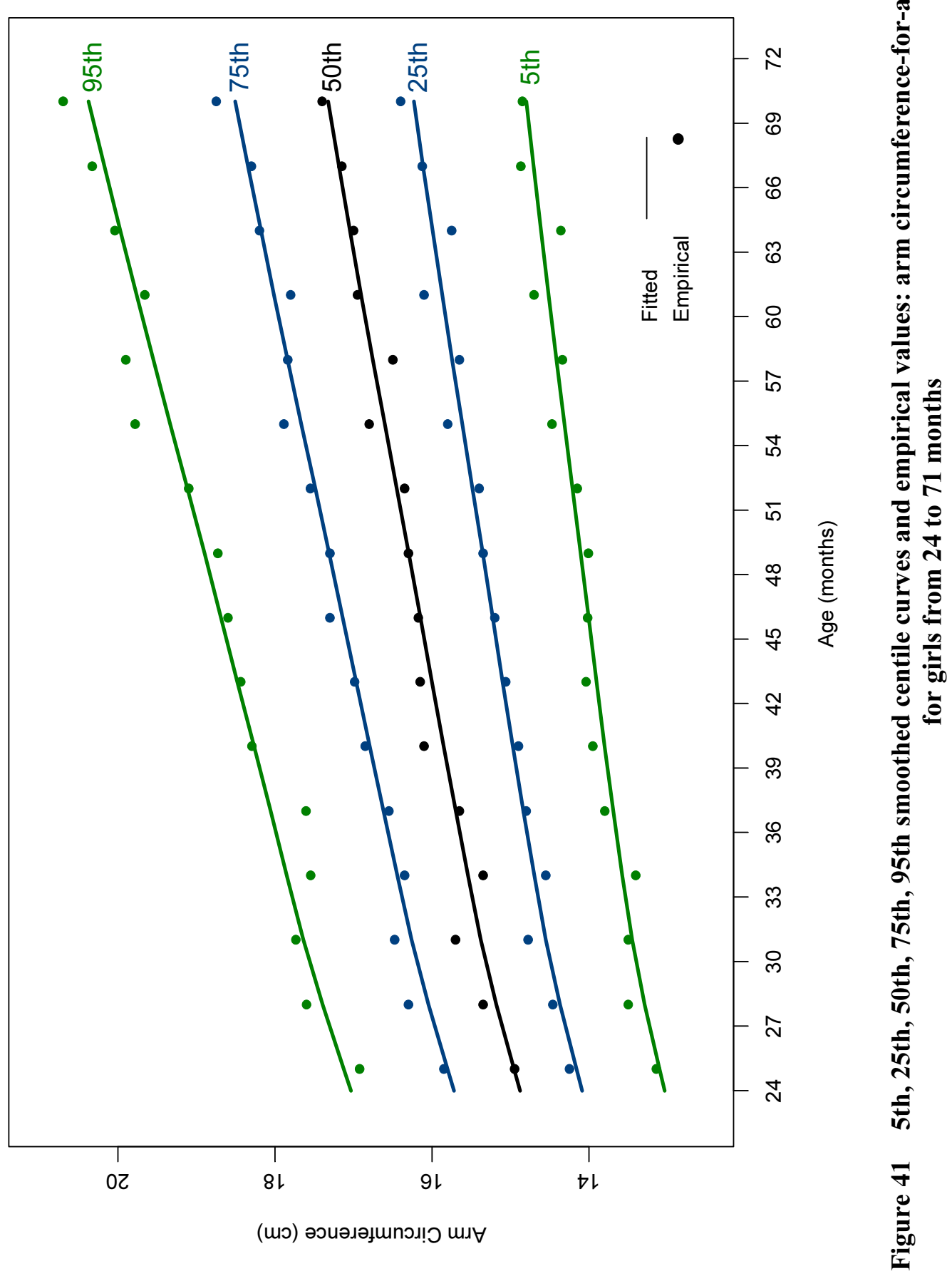




\subsubsection{WHO standards}

This section presents the final WHO arm circumference-for-age z-score and percentile charts (Figures 42 and 43) and table (Table 35) for girls. 


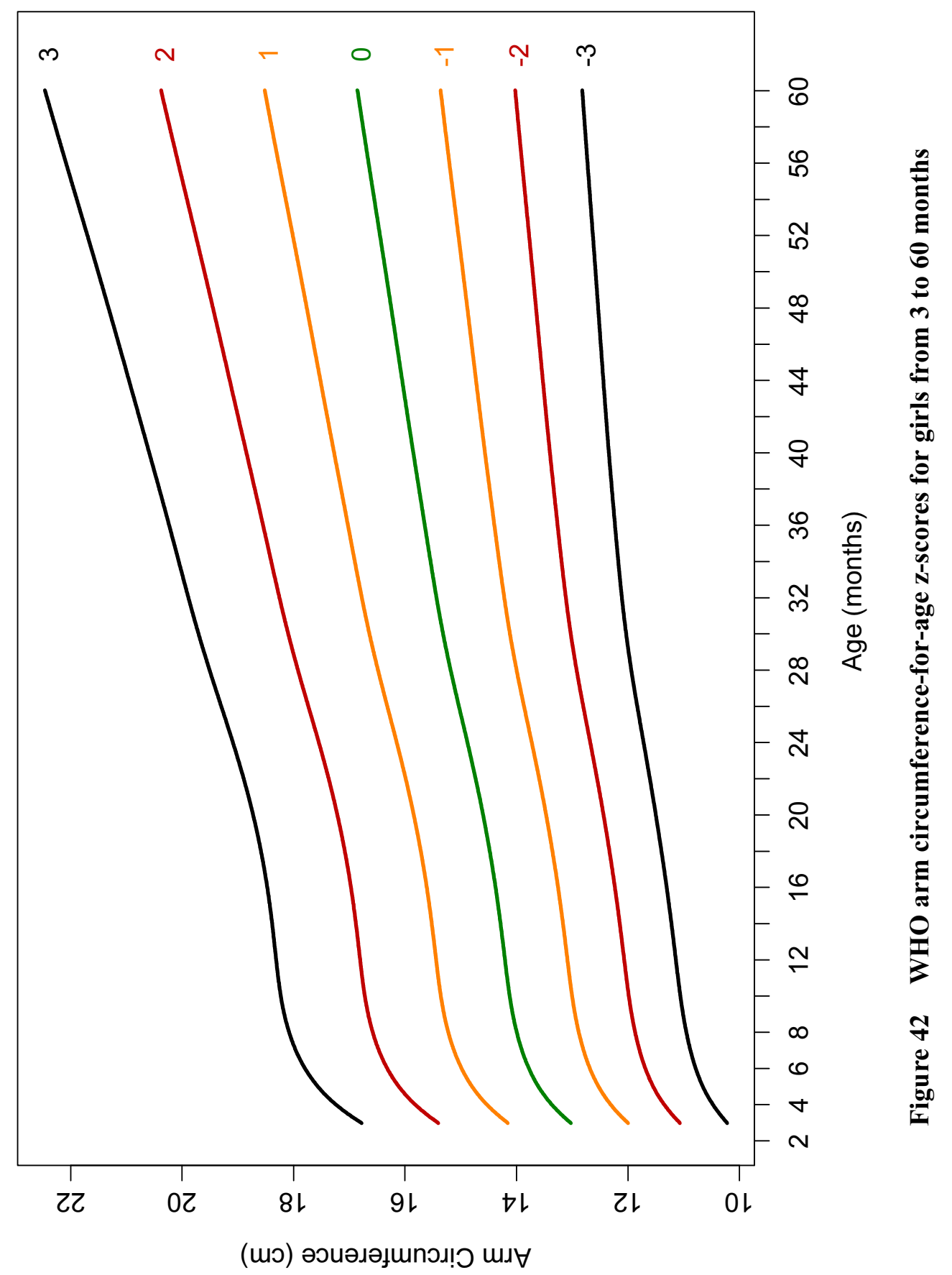




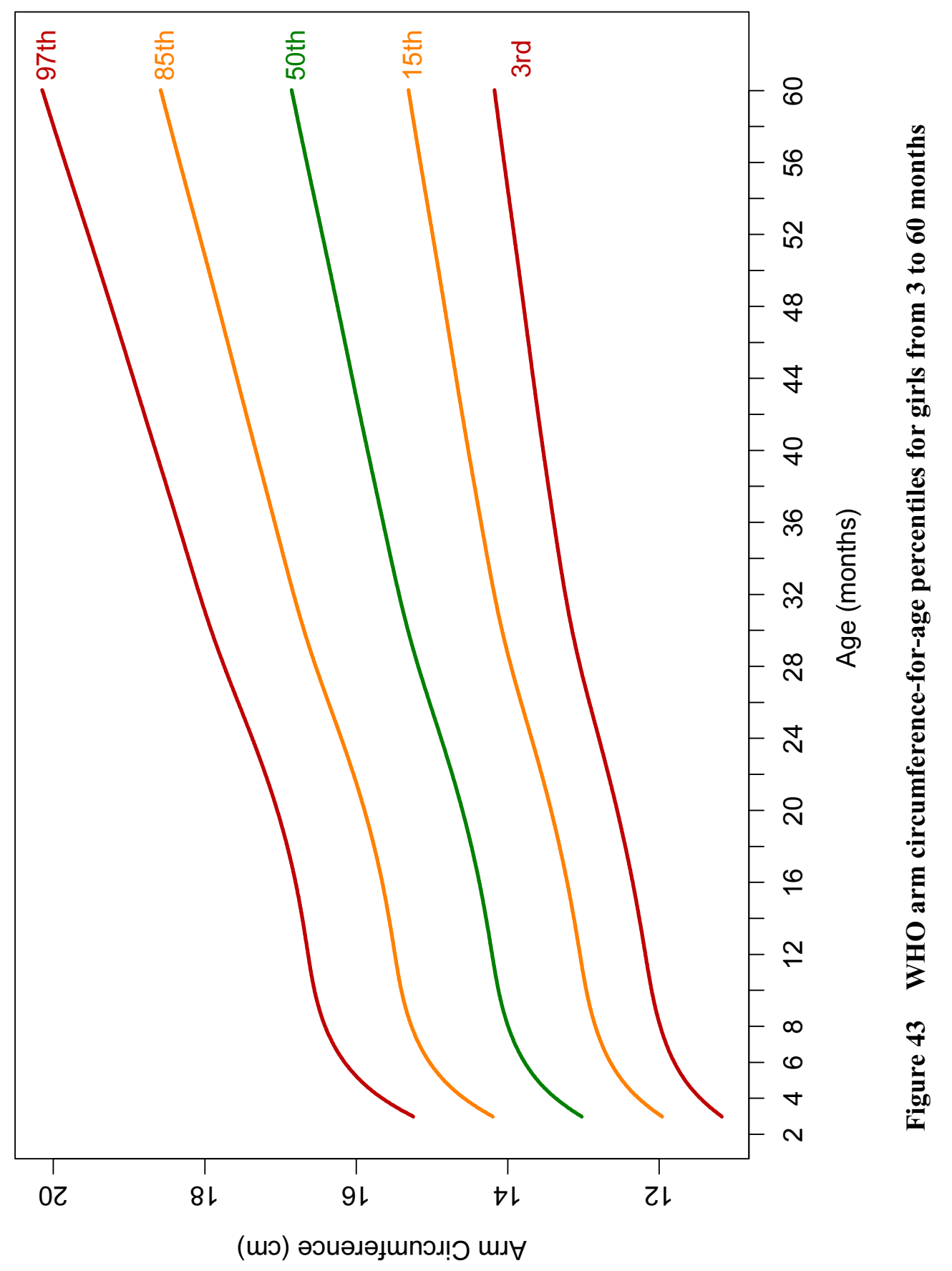




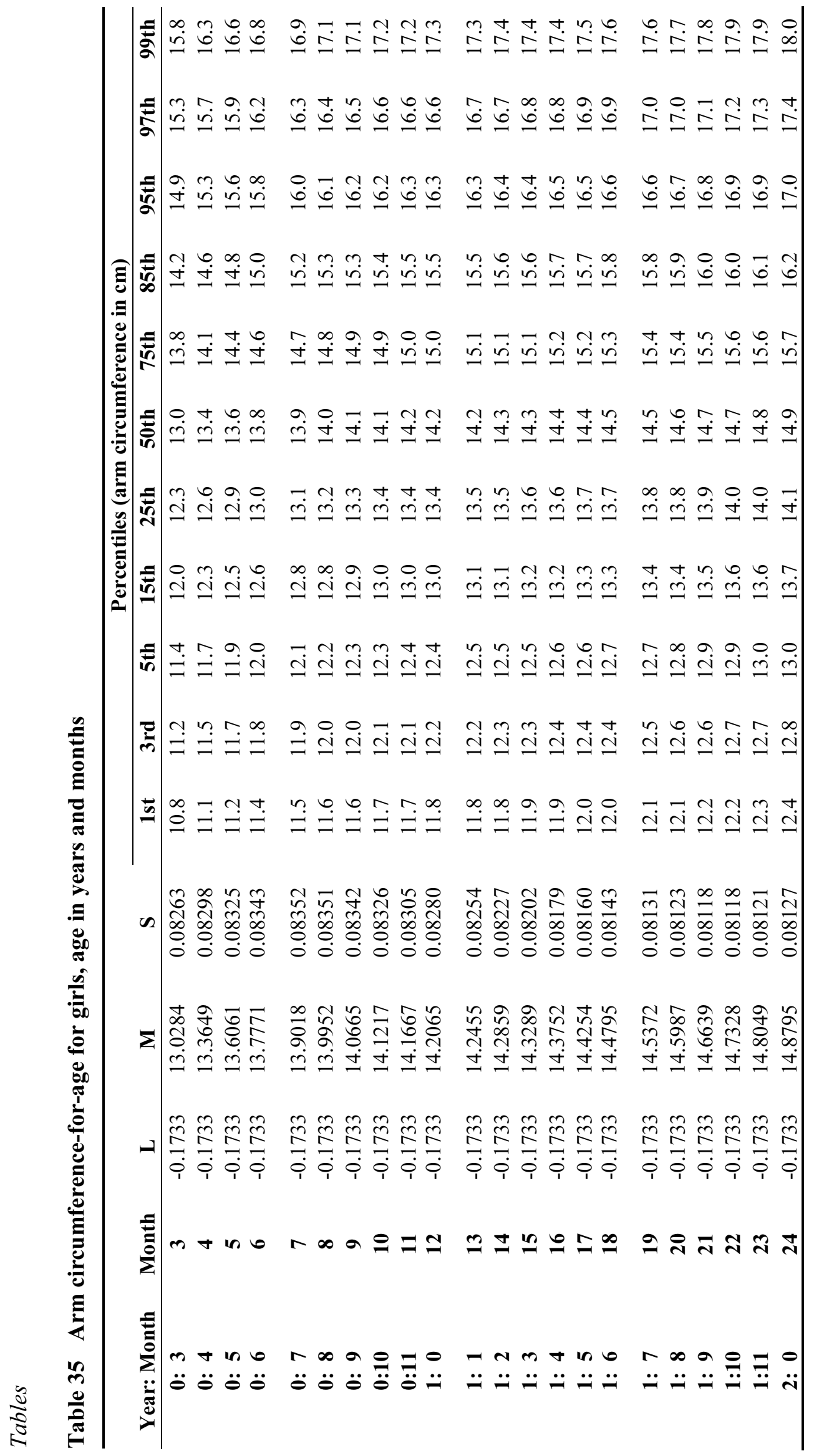




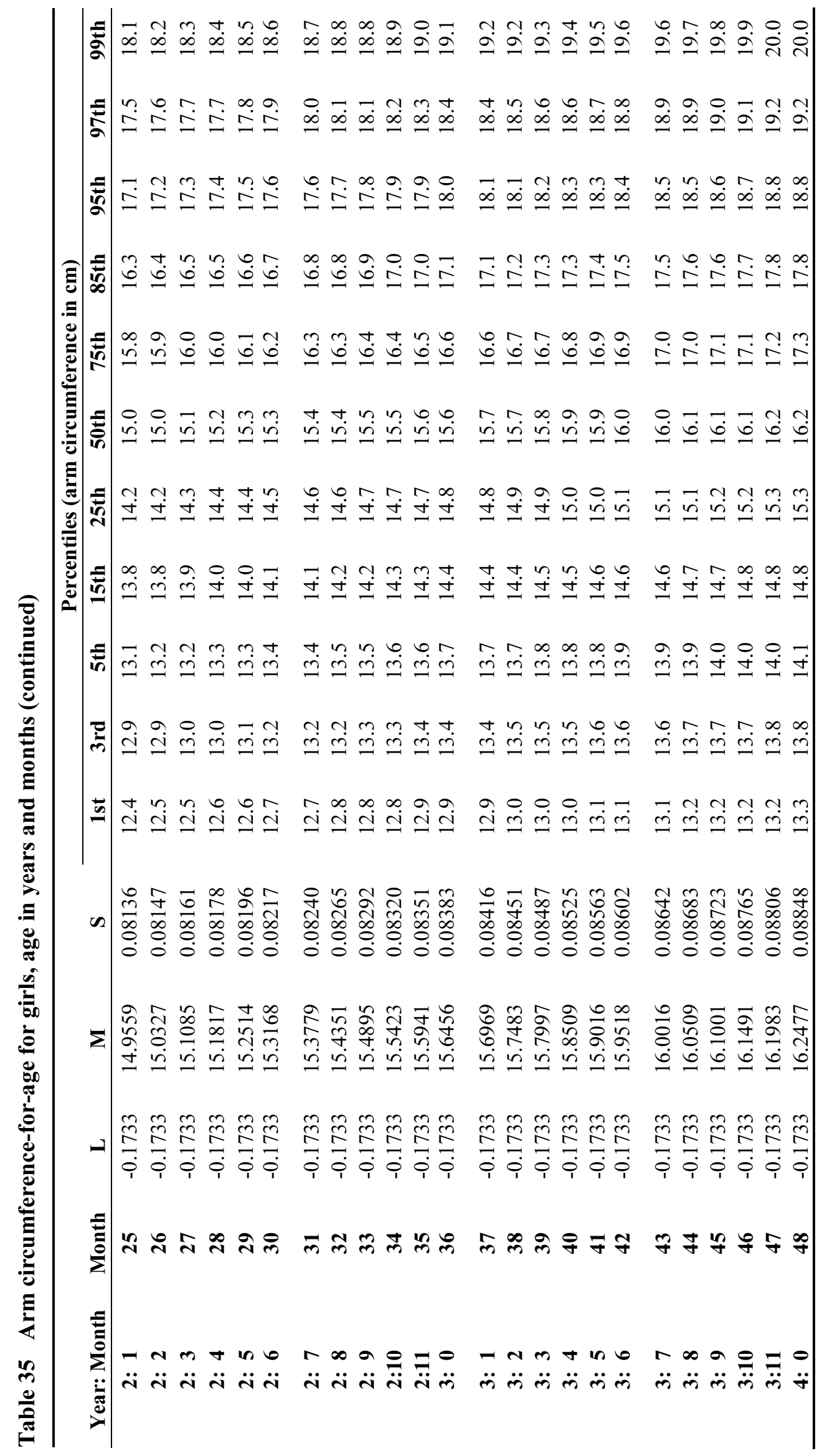




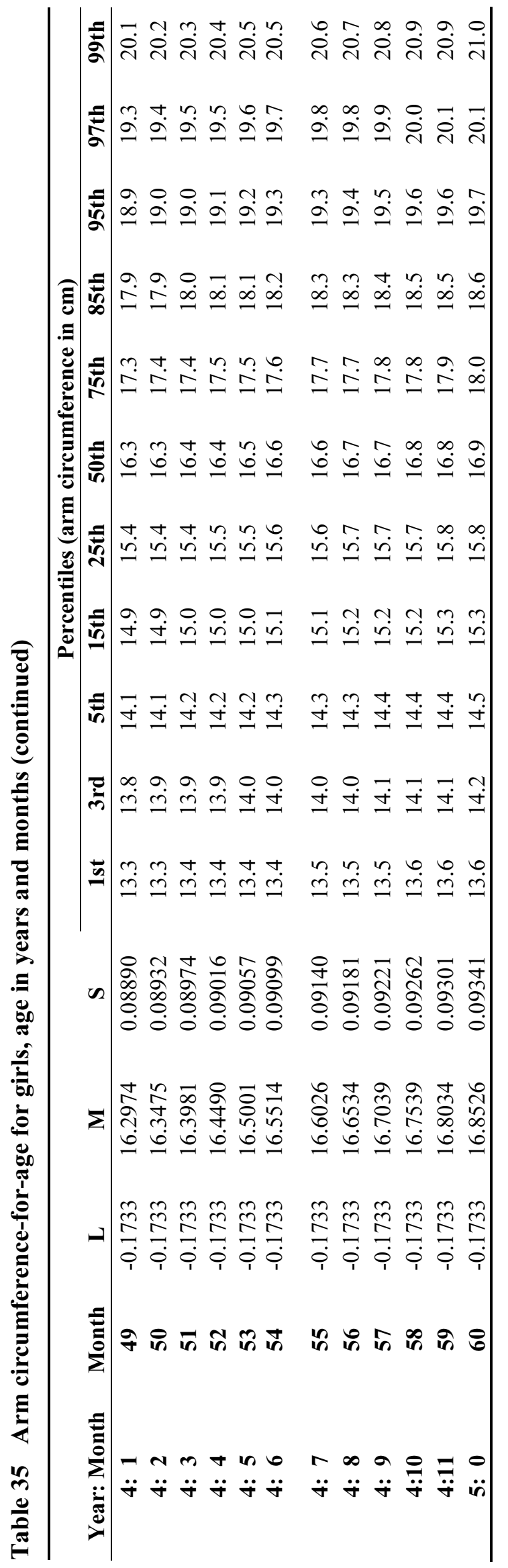




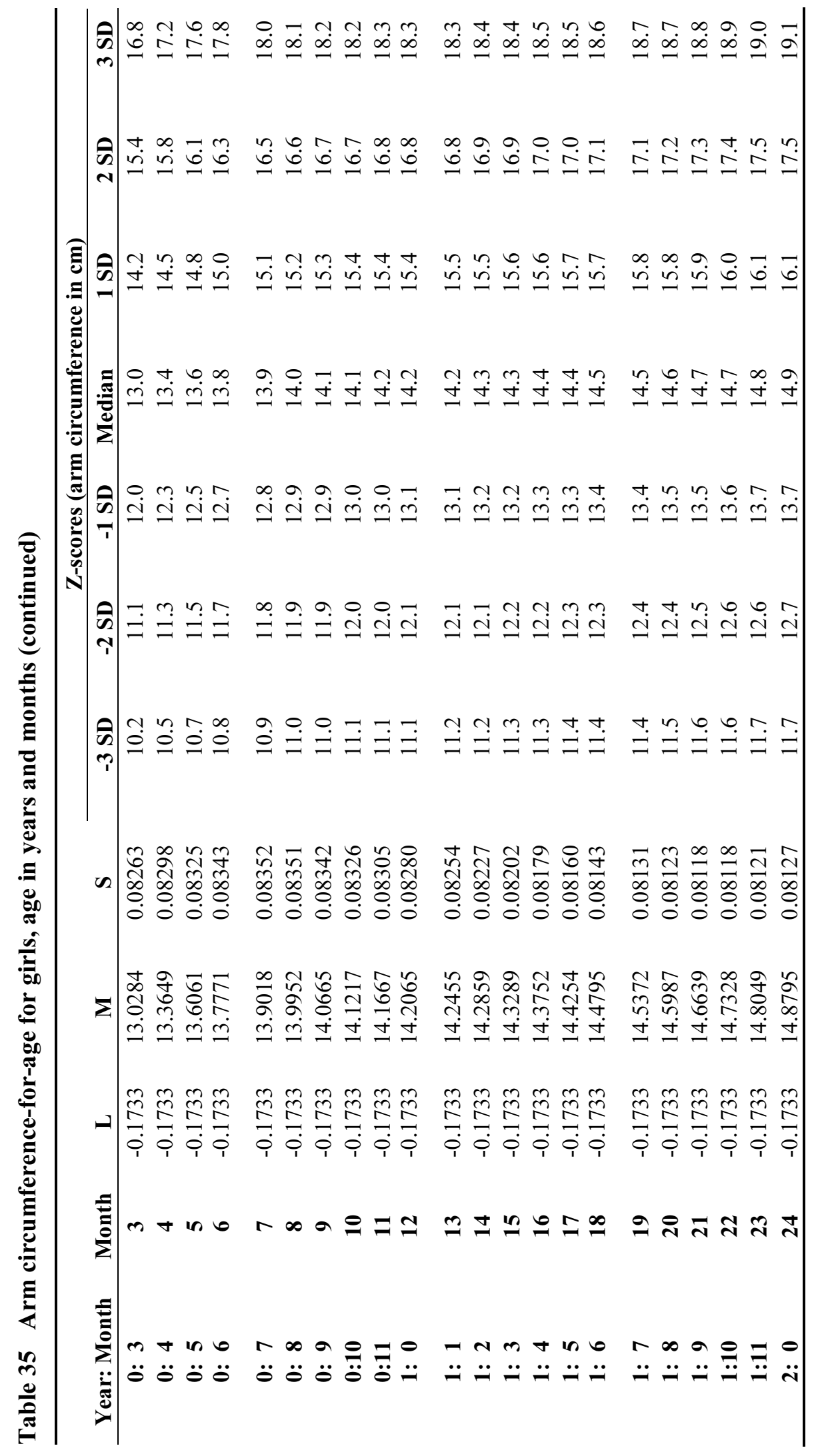




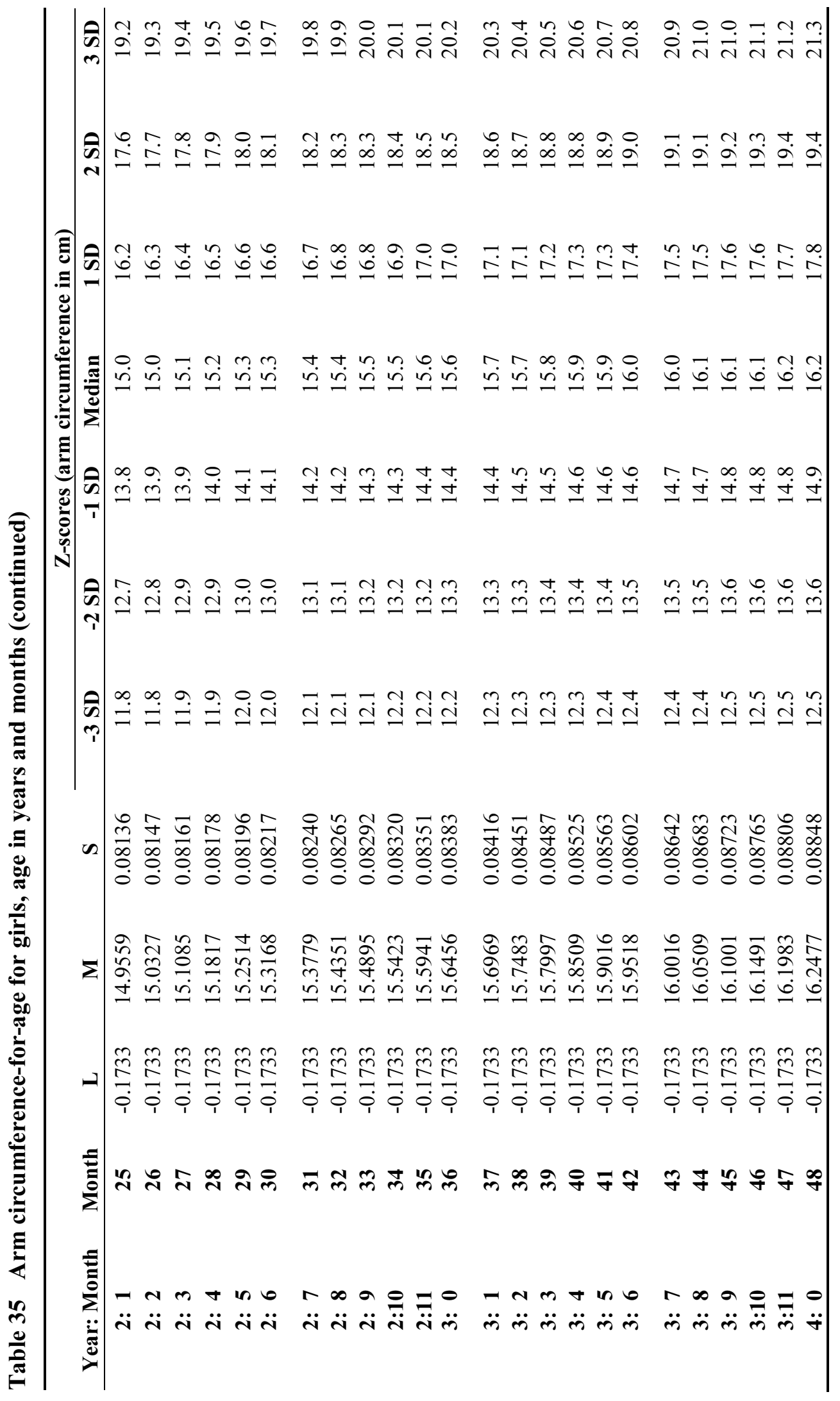




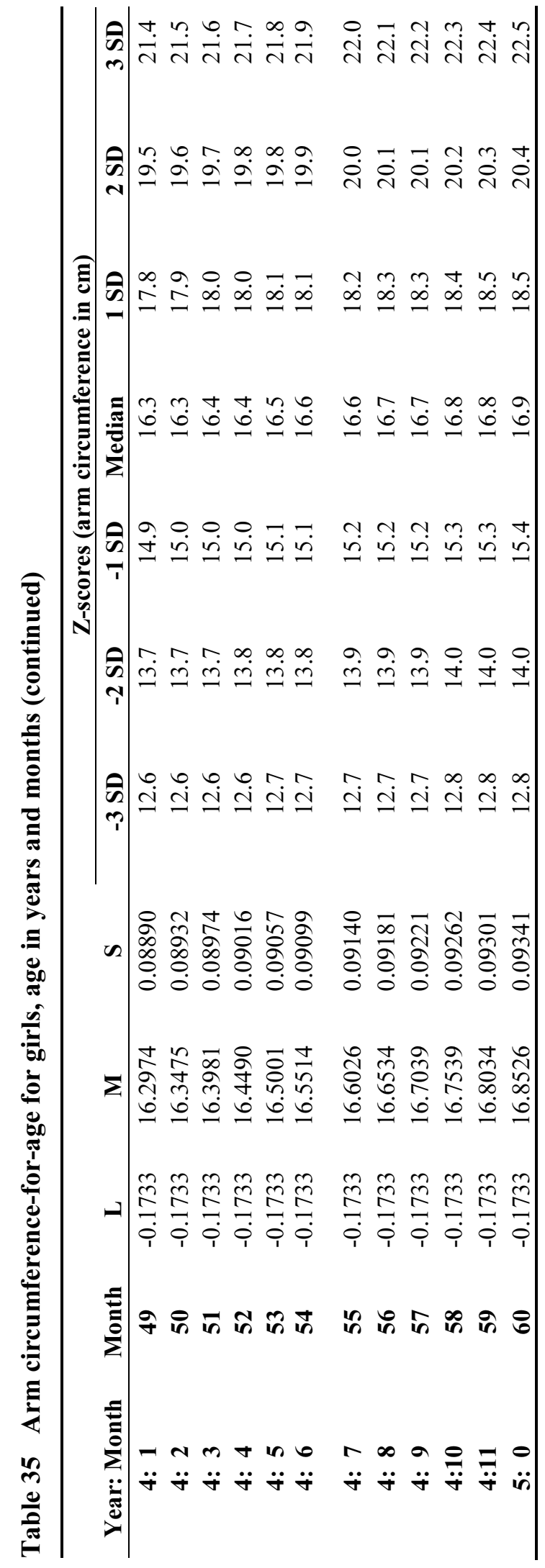




\subsection{Comparisons between boys and girls}

This section presents the arm circumference-for-age z-score comparisons between boys and girls for the WHO standards (Figure 44) 


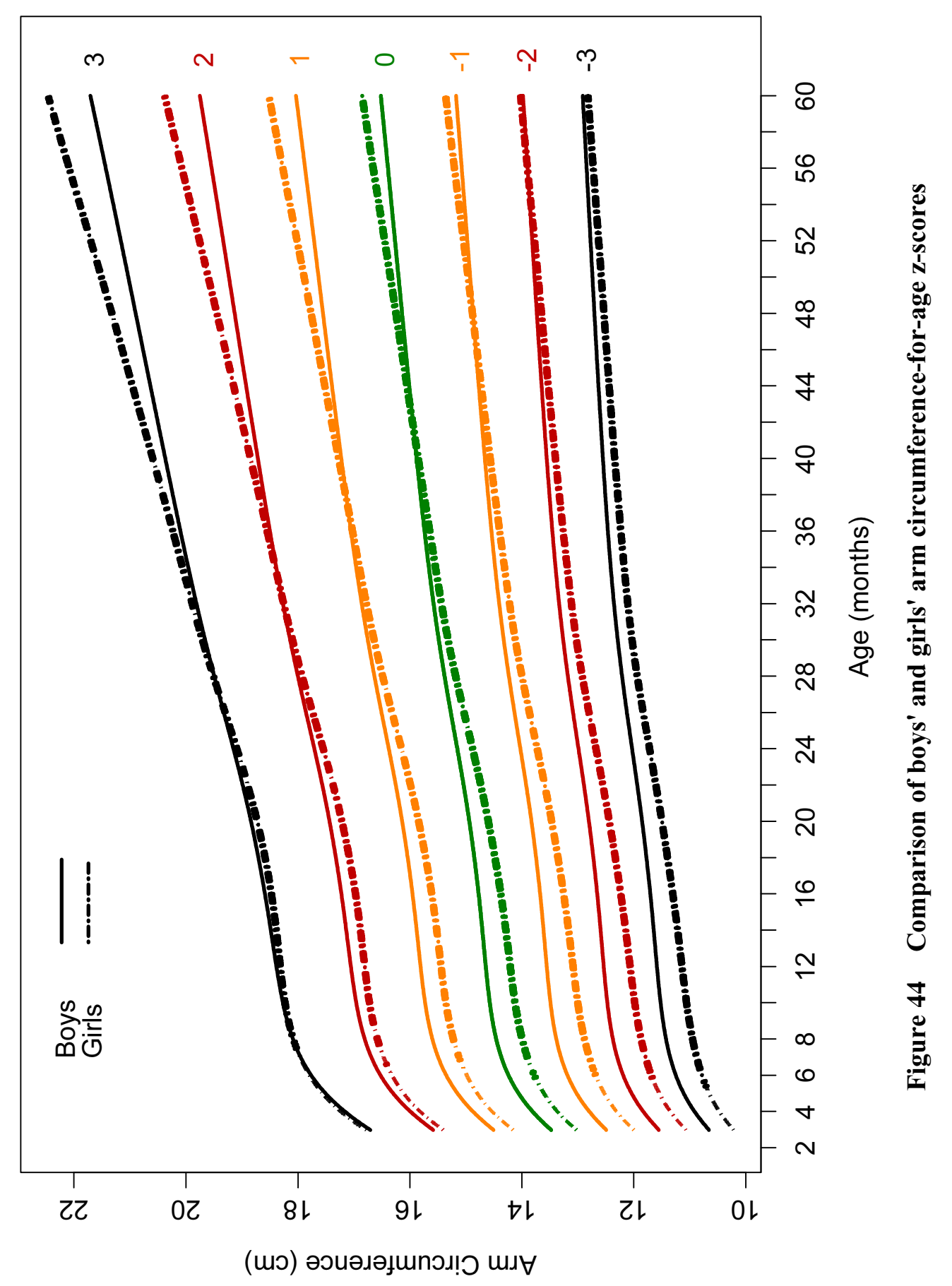

$\stackrel{0}{\stackrel{0}{7}}$ 


\section{CONSTRUCTION OF THE TRICEPS SKINFOLD-FOR-AGE STANDARDS}

\subsection{Indicator-specific methodology}

Similar steps to those described to select the best model for the head circumference-for-age and armcircumference-for-age curves were followed to select the best model to construct the triceps skinfoldfor-age standards. The diagnostic tools applied to evaluate and compare candidate models were the same. All data up to 71 months were used for modelling the triceps skinfold-for-age growth curves and the standards afterwards truncated at 60 completed months to correct for the right-edge effect (Borghi et al., 2006).

\subsection{Triceps skinfold-for-age for boys}

\subsubsection{Sample size}

There were a total of 10762 triceps skinfold observations for boys. The longitudinal and crosssectional sample sizes by visit and age are presented in Tables 36 and 37. The measurement of triceps skinfold started at 3 months of age (de Onis et al., 2004b).

Table 36 Longitudinal sample sizes for triceps skinfold-for-age for boys

\begin{tabular}{|c|c|c|c|c|c|c|}
\hline Visit & 5 & 6 & 7 & 8 & 9 & 10 \\
\hline Age & $3 \mathrm{mo}$ & $4 \mathrm{mo}$ & $5 \mathrm{mo}$ & $6 \mathrm{mo}$ & $7 \mathrm{mo}$ & $8 \mathrm{mo}$ \\
\hline $\mathrm{N}$ & 419 & 411 & 412 & 417 & 414 & 414 \\
\hline Visit & 11 & 12 & 13 & 14 & 15 & 16 \\
\hline Age & $9 \mathrm{mo}$ & $10 \mathrm{mo}$ & $11 \mathrm{mo}$ & $12 \mathrm{mo}$ & $14 \mathrm{mo}$ & $16 \mathrm{mo}$ \\
\hline $\mathrm{N}$ & 407 & 407 & 418 & 412 & 416 & 417 \\
\hline Visit & 17 & 18 & 19 & 20 & & \\
\hline Age & $18 \mathrm{mo}$ & $20 \mathrm{mo}$ & $22 \mathrm{mo}$ & $24 \mathrm{mo}$ & & \\
\hline $\mathrm{N}$ & 415 & 421 & 415 & 421 & & \\
\hline
\end{tabular}

Table 37 Cross-sectional sample sizes for triceps skinfold-for-age for boys

\begin{tabular}{lccccccc}
\hline Age (mo) & $<\mathbf{1 8}$ & $\mathbf{1 8}-\mathbf{2 0}$ & $\mathbf{2 1 - 2 3}$ & $\mathbf{2 4 - 2 6}$ & $\mathbf{2 7 - 2 9}$ & $\mathbf{3 0 - 3 2}$ & $\mathbf{3 3 - 3 5}$ \\
$\mathrm{N}$ & 3 & 175 & 183 & 236 & 255 & 217 & 252 \\
\hline Age (mo) & $\mathbf{3 6 - 3 8}$ & $\mathbf{3 9 - 4 1}$ & $\mathbf{4 2 - 4 4}$ & $\mathbf{4 5 - 4 7}$ & $\mathbf{4 8}-\mathbf{5 0}$ & $\mathbf{5 1 - 5 3}$ & $\mathbf{5 4 - 5 6}$ \\
$\mathrm{N}$ & 265 & 249 & 255 & 236 & 236 & 221 & 225 \\
\hline Age (mo) & $\mathbf{5 7 - 5 9}$ & $\mathbf{6 0 - 6 2}$ & $\mathbf{6 3 - 6 5}$ & $\mathbf{6 6 - 6 8}$ & $\mathbf{6 9 - 7 1}$ & $>\mathbf{7 1}$ & \\
$\mathrm{N}$ & 239 & 228 & 216 & 217 & 214 & 4 & \\
\hline
\end{tabular}

\subsubsection{Model selection and results}

To search for the best value of the age-transformation power $\lambda$, the model $\operatorname{BCPE}\left(x=\operatorname{age}^{\lambda}, \operatorname{df}(\mu)=9\right.$, $\operatorname{df}(\sigma)=4, \operatorname{df}(v)=4, \tau=2)$ was used as a starting point. Table 38 shows the global deviance for values of $\lambda$ from 0.05 to 1 . The global deviance value was smallest for $\lambda=0.30$ and thus this age-transformation power was selected. 
Table 38 Global deviance (GD) for models within the class $\operatorname{BCPE}\left(x=\operatorname{age}^{\lambda}, \operatorname{df}(\mu)=9, \operatorname{df}(\sigma)=4\right.$, $\operatorname{df}(v)=4, \tau=2)$ for triceps skinfold-for-age for boys

\begin{tabular}{rcccccccccc}
\hline $\boldsymbol{\lambda}$ & $\mathbf{0 . 0 5}$ & $\mathbf{0 . 1 0}$ & $\mathbf{0 . 1 5}$ & $\mathbf{0 . 2 0}$ & $\mathbf{0 . 2 5}$ & $\mathbf{0 . 3 0}$ & $\mathbf{0 . 3 5}$ & $\mathbf{0 . 4 0}$ & $\mathbf{0 . 4 5}$ & $\mathbf{0 . 5 0}$ \\
$\mathrm{GD}^{\mathrm{a}}$ & 628.4 & 627.7 & 627.1 & 626.8 & 626.6 & 626.5 & 626.6 & 626.8 & 627.2 & 627.6 \\
\hline $\boldsymbol{\lambda}$ & $\mathbf{0 . 5 5}$ & $\mathbf{0 . 6 0}$ & $\mathbf{0 . 6 5}$ & $\mathbf{0 . 7 0}$ & $\mathbf{0 . 7 5}$ & $\mathbf{0 . 8 0}$ & $\mathbf{0 . 8 5}$ & $\mathbf{0 . 9 0}$ & $\mathbf{0 . 9 5}$ & $\mathbf{1 . 0 0}$ \\
$\mathrm{GD}^{\mathrm{a}}$ & 628.2 & 628.9 & 629.6 & 630.3 & 631.0 & 631.7 & 632.4 & 633.1 & 633.8 & 634.5 \\
\hline
\end{tabular}

${ }^{\mathrm{a}}$ In excess of 41000 .

The search for the best $\operatorname{df}(\mu)$ and $\operatorname{df}(\sigma)$ followed, fixing $\lambda=0.30, v=1$ and $\tau=2$. All possible combinations with $\operatorname{df}(\mu)$ values ranging from 6 to 15 and $\operatorname{df}(\sigma)$ from 2 to 10 were considered and partial results are presented in Table 39. The criterion considered was the GAIC(3), as for the construction of arm circumference-for-age (see Section 4.2.2). The model with $\operatorname{df}(\mu)=8$ and $\operatorname{df}(\sigma)=5$ provided the smallest value of $\operatorname{GAIC}(3)$ but the model with $\operatorname{df}(\mu)=7$ and $\operatorname{df}(\sigma)=5$, with a smoother median curve, yielded a very similar $G A I C(3)$ value and thus the latter was selected for further evaluation.

Table 39 Goodness-of-fit summary for models using the BCPE distribution with fixed $v=1$ and $\tau=2$ for triceps skinfold-for-age for boys

\begin{tabular}{|c|c|c|c|c|c|}
\hline $\operatorname{df}(\mu)$ & $\operatorname{df}(\sigma)$ & GD $^{\mathbf{a}}$ & $\mathbf{A I C}^{\mathbf{a}}$ & ${\text { GAIC }(3)^{a}}^{a}$ & Total df \\
\hline \multirow{5}{*}{6} & 3 & 1597.8 & 1615.8 & 1624.8 & 9 \\
\hline & 4 & 1585.3 & 1605.3 & 1615.3 & 10 \\
\hline & 5 & 1579.8 & 1601.8 & 1612.8 & 11 \\
\hline & 6 & 1576.8 & 1600.8 & 1612.8 & 12 \\
\hline & 7 & 1574.2 & 1600.2 & 1613.2 & 13 \\
\hline \multirow{5}{*}{7} & 3 & 1591.7 & 1611.7 & 1621.7 & 10 \\
\hline & 4 & 1579.1 & 1601.1 & 1612.1 & 11 \\
\hline & 5 & 1573.7 & 1597.7 & 1609.7 & 12 \\
\hline & 6 & 1570.8 & 1596.8 & 1609.8 & 13 \\
\hline & 7 & 1568.3 & 1596.3 & 1610.3 & 14 \\
\hline \multirow{5}{*}{8} & 3 & 1588.3 & 1610.3 & 1621.3 & 11 \\
\hline & 4 & 1575.6 & 1599.6 & 1611.6 & 12 \\
\hline & 5 & 1570.3 & 1596.3 & 1609.3 & 13 \\
\hline & 6 & 1567.3 & 1595.4 & 1609.4 & 14 \\
\hline & 7 & 1564.9 & 1594.9 & 1609.9 & 15 \\
\hline \multirow{5}{*}{9} & 3 & 1585.7 & 1609.7 & 1621.7 & 12 \\
\hline & 4 & 1573.0 & 1599.0 & 1612.0 & 13 \\
\hline & 5 & 1567.7 & 1595.7 & 1609.7 & 14 \\
\hline & 6 & 1564.8 & 1594.8 & 1609.8 & 15 \\
\hline & 7 & 1562.4 & 1594.4 & 1610.4 & 16 \\
\hline \multirow{5}{*}{10} & 3 & 1583.5 & 1609.5 & 1622.5 & 13 \\
\hline & 4 & 1570.8 & 1598.8 & 1612.8 & 14 \\
\hline & 5 & 1565.4 & 1595.5 & 1610.5 & 15 \\
\hline & 6 & 1562.6 & 1594.6 & 1610.6 & 16 \\
\hline & 7 & 1560.2 & 1594.2 & 1611.2 & 17 \\
\hline
\end{tabular}

GD, Global Deviance; AIC, Akaike Information Criterion;

GAIC(3), Generalized AIC with penalty equal to 3;

${ }^{\mathrm{a}}$ In excess of 41000 . 
Model 1: $\operatorname{BCPE}\left(x=\operatorname{age}^{0.30}, \operatorname{df}(\mu)=7, \operatorname{df}(\sigma)=5, v=1, \tau=2\right)$

This model was inadequate as there was clear evidence of residual skewness in both the worm plots (Figure 45) and the Q-test results (Table 40). The worms depicted U-shapes for most age groups and Q-test results (Table 40) showed all groups with absolute values of z3 higher than 2. Most of the age groups also presented absolute values of $\mathrm{z} 4$ higher than 2 , indicating residual kurtosis. The overall tests for skewness and kurtosis were significant ( $p$-values $<0.01$ ). Only two groups presented absolute values of z2 larger than 2 , indicating misfit of the variance (40 mo and $58 \mathrm{mo}$ ).
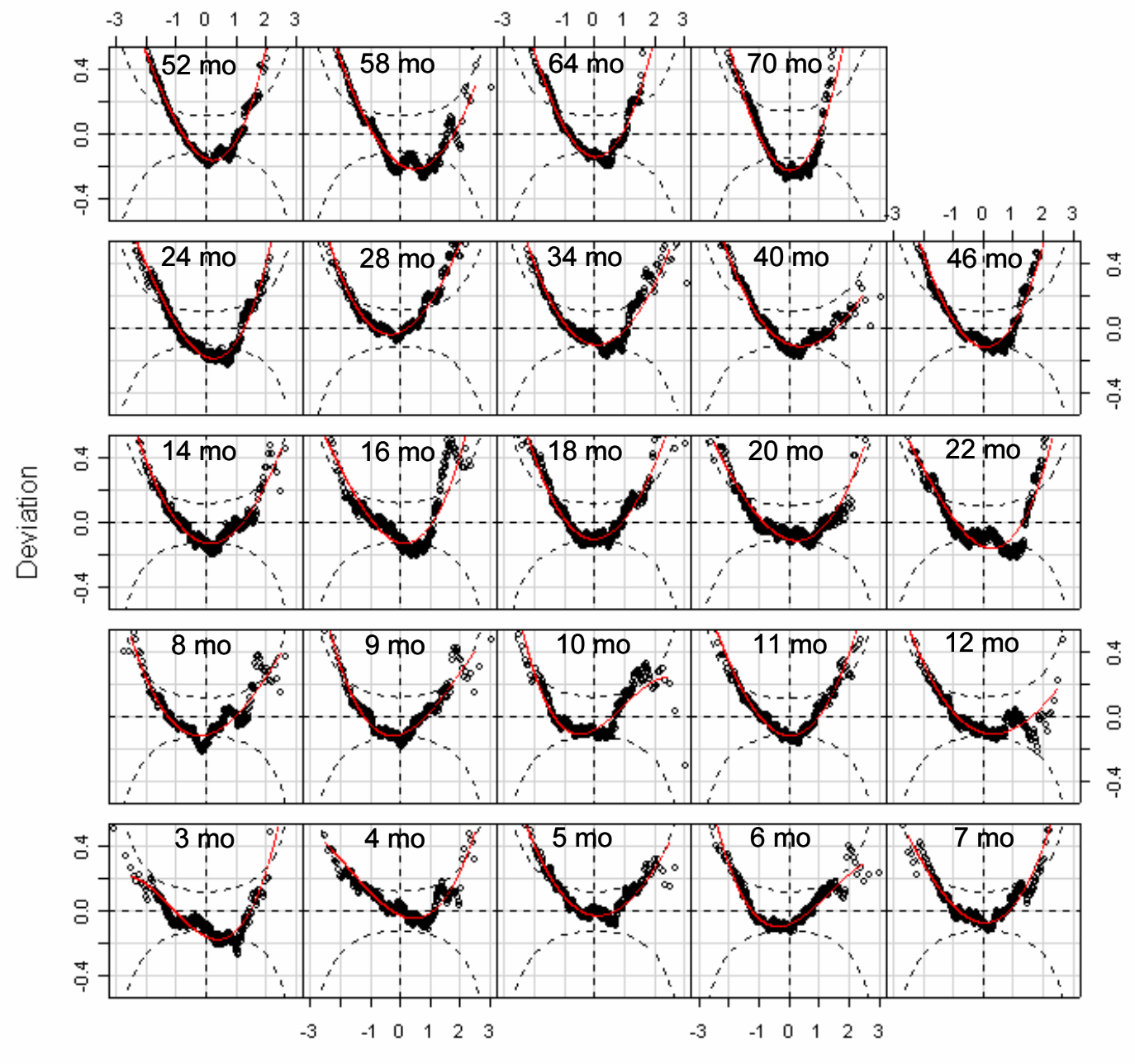

Unit normal quantile

Figure 45 Worm plots of z-scores for Model 1 for triceps skinfold-for-age for boys 
Table 40 Q-test for $z$-scores from Model $1\left[\operatorname{BCPE}\left(x=\operatorname{age}^{0.30}, \operatorname{df}(\mu)=7, \operatorname{df}(\sigma)=5, v=1, \tau=2\right)\right]$ for triceps skinfold-for-age for boys

\begin{tabular}{|c|c|c|c|c|c|c|}
\hline Age (days) & Group & $\mathbf{N}$ & $\mathrm{z1}$ & $\mathbf{z 2}$ & $\mathbf{z 3}$ & $\mathrm{z4}$ \\
\hline 79 to 99 & $3 \mathrm{mo}$ & 415 & -1.4 & 0.0 & 4.9 & 3.4 \\
\hline 100 to 129 & $4 \mathrm{mo}$ & 408 & 1.0 & -0.8 & 4.1 & 2.5 \\
\hline 130 to 159 & $5 \mathrm{mo}$ & 409 & 1.4 & -0.7 & 4.1 & 0.9 \\
\hline 160 to 189 & $6 \mathrm{mo}$ & 411 & 0.0 & 0.4 & 3.6 & -0.7 \\
\hline 190 to 219 & $7 \mathrm{mo}$ & 405 & 0.8 & 0.8 & 4.9 & 2.5 \\
\hline 220 to 249 & $8 \mathrm{mo}$ & 419 & -0.7 & 0.4 & 4.0 & 0.5 \\
\hline 250 to 279 & $9 \mathrm{mo}$ & 390 & -0.3 & 0.6 & 4.1 & 0.0 \\
\hline 280 to 309 & $10 \mathrm{mo}$ & 399 & 0.1 & 0.9 & 3.4 & -1.5 \\
\hline 310 to 349 & $11 \mathrm{mo}$ & 461 & 0.0 & 0.6 & 5.7 & 2.7 \\
\hline 350 to 379 & $12 \mathrm{mo}$ & 407 & -0.5 & -1.6 & 3.7 & 1.1 \\
\hline 380 to 439 & $14 \mathrm{mo}$ & 418 & -0.4 & -0.7 & 5.0 & 1.7 \\
\hline 440 to 499 & $16 \mathrm{mo}$ & 415 & 0.0 & 0.6 & 5.9 & 2.9 \\
\hline 500 to 559 & $18 \mathrm{mo}$ & 441 & 0.3 & 0.2 & 5.5 & 1.7 \\
\hline 560 to 619 & $20 \mathrm{mo}$ & 521 & -0.2 & -0.6 & 5.2 & 2.7 \\
\hline 620 to 679 & $22 \mathrm{mo}$ & 544 & -0.7 & -0.2 & 6.9 & 3.9 \\
\hline 680 to 749 & $24 \mathrm{mo}$ & 591 & -0.9 & 0.0 & 8.1 & 4.6 \\
\hline 750 to 929 & $28 \mathrm{mo}$ & 469 & 1.8 & 1.7 & 4.8 & 1.0 \\
\hline 930 to 1119 & $34 \mathrm{mo}$ & 509 & 0.4 & -0.5 & 5.6 & 1.5 \\
\hline 1120 to 1309 & $40 \mathrm{mo}$ & 510 & -0.2 & -2.4 & 4.9 & 0.7 \\
\hline 1310 to 1499 & $46 \mathrm{mo}$ & 506 & 0.8 & 0.9 & 7.2 & 3.5 \\
\hline 1500 to 1689 & $52 \mathrm{mo}$ & 487 & 0.3 & 0.4 & 8.2 & 4.8 \\
\hline 1690 to 1879 & $58 \mathrm{mo}$ & 480 & -1.6 & -2.7 & 6.1 & 2.5 \\
\hline 1880 to 2069 & $64 \mathrm{mo}$ & 461 & 0.7 & 1.3 & 7.5 & 3.5 \\
\hline 2070 to 2249 & $70 \mathrm{mo}$ & 286 & -0.2 & 1.8 & 6.9 & 3.4 \\
\hline Overall Q stats & & 10762 & 14.9 & 29.8 & 752.4 & 162.1 \\
\hline degrees of freedom & & & 17.0 & 21.0 & 24.0 & 24.0 \\
\hline p-value & & & 0.6013 & 0.0958 & $<0.01$ & $<0.01$ \\
\hline
\end{tabular}

Note: Absolute values of z1, z2, z3 or z4 larger than 2 indicate misfit of, respectively, mean, variance, skewness or kurtosis.

The next step involved fitting the parameter $v$ for skewness using the BCPE distribution with fixed parameter $\tau=2$ and keeping the degrees of freedom for the $\mu$ and $\sigma$ curves selected for Model 1. Table 41 shows the $G A I C(3)$ values for various degrees of freedom for the $v$ curve. 
Table 41 Goodness-of-fit summary for models $\operatorname{BCPE}\left(x=\operatorname{age}^{0.30}, \operatorname{df}(\mu)=7, \operatorname{df}(\sigma)=5, \operatorname{df}(v)=\right.$ ?, $\left.\tau=2\right)$ for triceps skinfold-for-age for boys

\begin{tabular}{cccc}
\hline $\mathbf{d f}(\mathbf{v})$ & $\mathbf{G D}^{\mathbf{a}}$ & $\mathbf{G A I C}^{\mathbf{3}} \mathbf{3}^{\mathbf{a}}$ & Total df \\
\hline $\mathbf{1}$ & 634.1 & 673.1 & 13 \\
$\mathbf{2}$ & 627.6 & 669.6 & 14 \\
$\mathbf{3}$ & 627.3 & 672.3 & 15 \\
$\mathbf{4}$ & 626.8 & 674.8 & 16 \\
$\mathbf{5}$ & 626.3 & 677.3 & 17 \\
$\mathbf{6}$ & 625.7 & 679.7 & 18 \\
$\mathbf{7}$ & 625.0 & 682.0 & 19 \\
\hline
\end{tabular}

GD, Global Deviance; GAIC(3), Generalized Akaike Information Criterion with penalty equal to 3 ;

${ }^{\mathrm{a}}$ In excess of 41000 .

The smallest $\operatorname{GAIC}(3)$ value corresponded to $\mathrm{df}(v)=2$. Fixing $\mathrm{df}(v)=2$, a re-search for the best $\mathrm{df}(\mu)$ and $\operatorname{df}(\sigma)$ was carried out but results indicated that there was no need for updating the model. A re-search for the best age-transformation power followed. Models using $\lambda$ from 0.05 to 0.30 were smallest and very similar and thus the value of 0.30 was kept unchanged.

Model 2: $\operatorname{BCPE}\left(x=\operatorname{age}^{0.30}, \operatorname{df}(\mu)=7, \operatorname{df}(\sigma)=5, \operatorname{df}(v)=2, \tau=2\right)$

Figure 46 shows the fitting of the parameters $\mu, \sigma$ and $v$ for Model 2 with their respective sample estimates, that is, the median for $\mu$, the sample standard deviation of the Box-Cox transformed data for $\sigma$ and Box-Cox transform power for $v$.

Figures 47 and 48 show the distribution of the empirical minus fitted centile differences for the longitudinal and cross-sectional samples, respectively. There was no indication of systematic biases either between 3 and 24 months (Figure 47), or between 24 and 71 months (Figure 48).

The worm plots for this model (Figure 49) were significantly improved compared to those of Model 1 (Figure 45). The U-shaped worms flattened out significantly (Figure 49), indicating that the residual skewness associated with Model 1 had been corrected. There was indication of residual kurtosis for only four age groups but their respective worms were contained within the $95 \%$ confidence interval. 

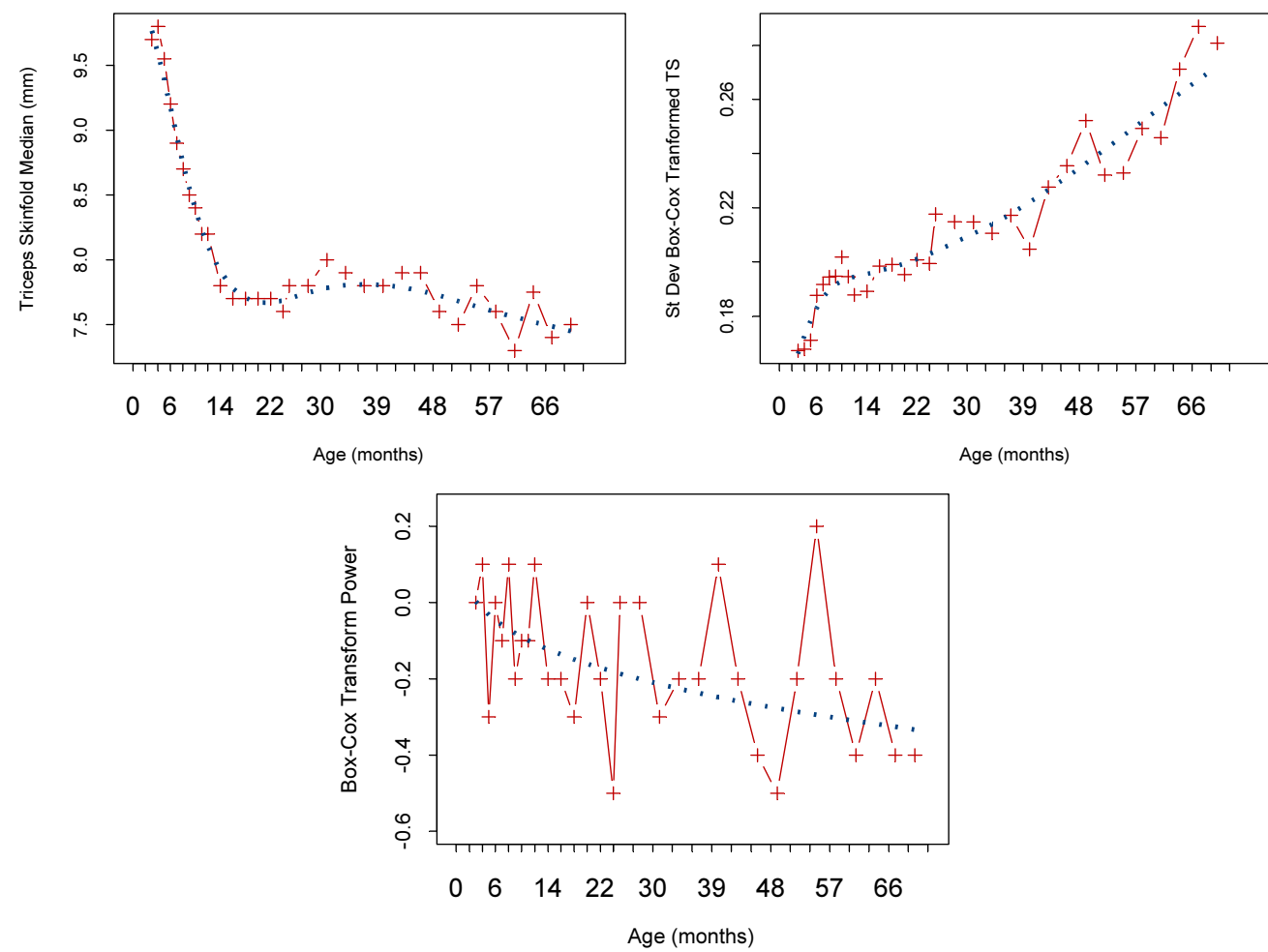

Figure 46 Fitting of the $\mu, \sigma$, and $v$ curves of Model 2 for triceps skinfold-for-age for boys from 3 to 71 months (dotted line) and their respective sample estimates (points with solid line) 

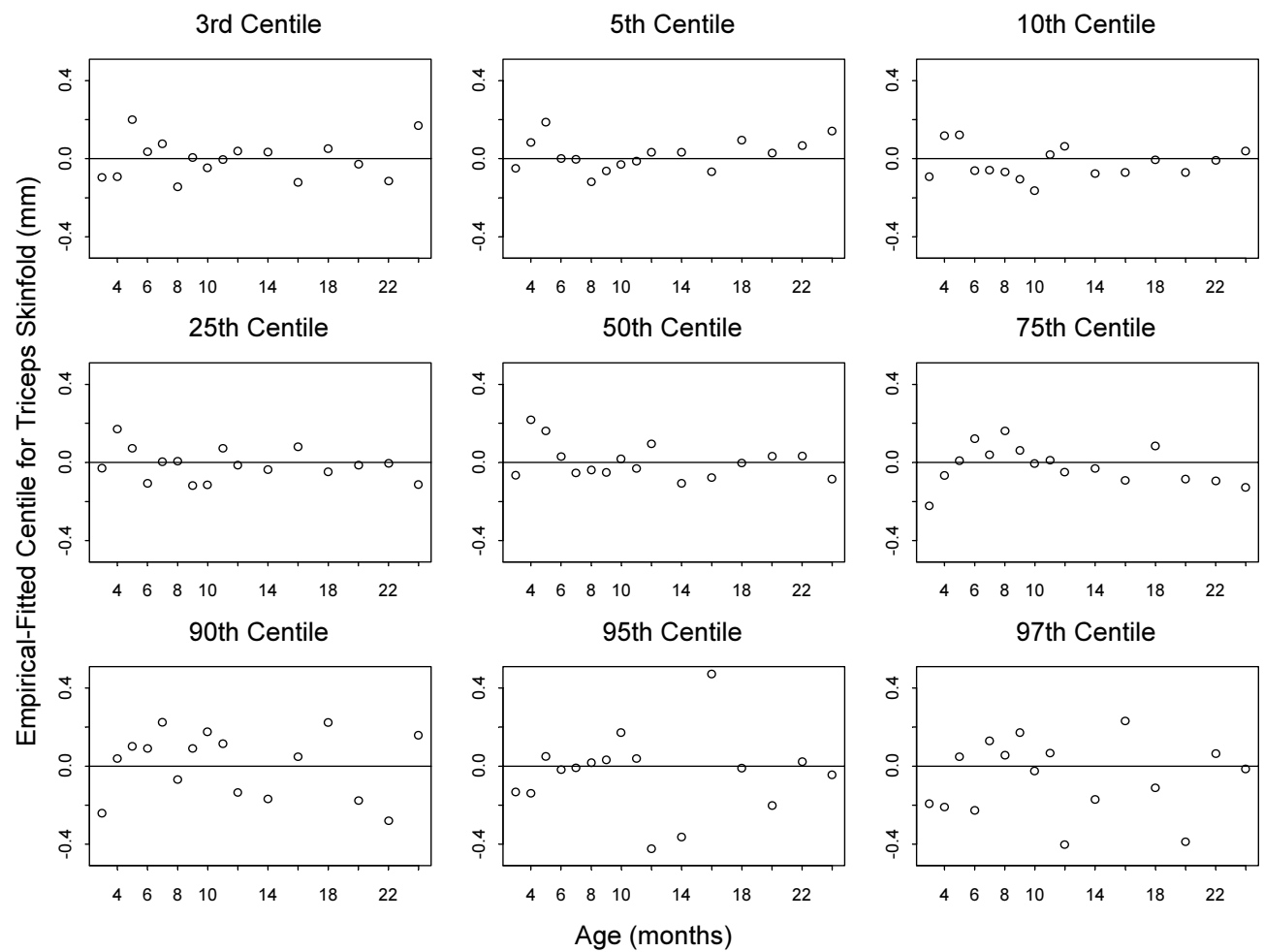

Figure 47 Centile residuals from fitting Model 2 for triceps skinfold-for-age from 3 to 24 months for boys
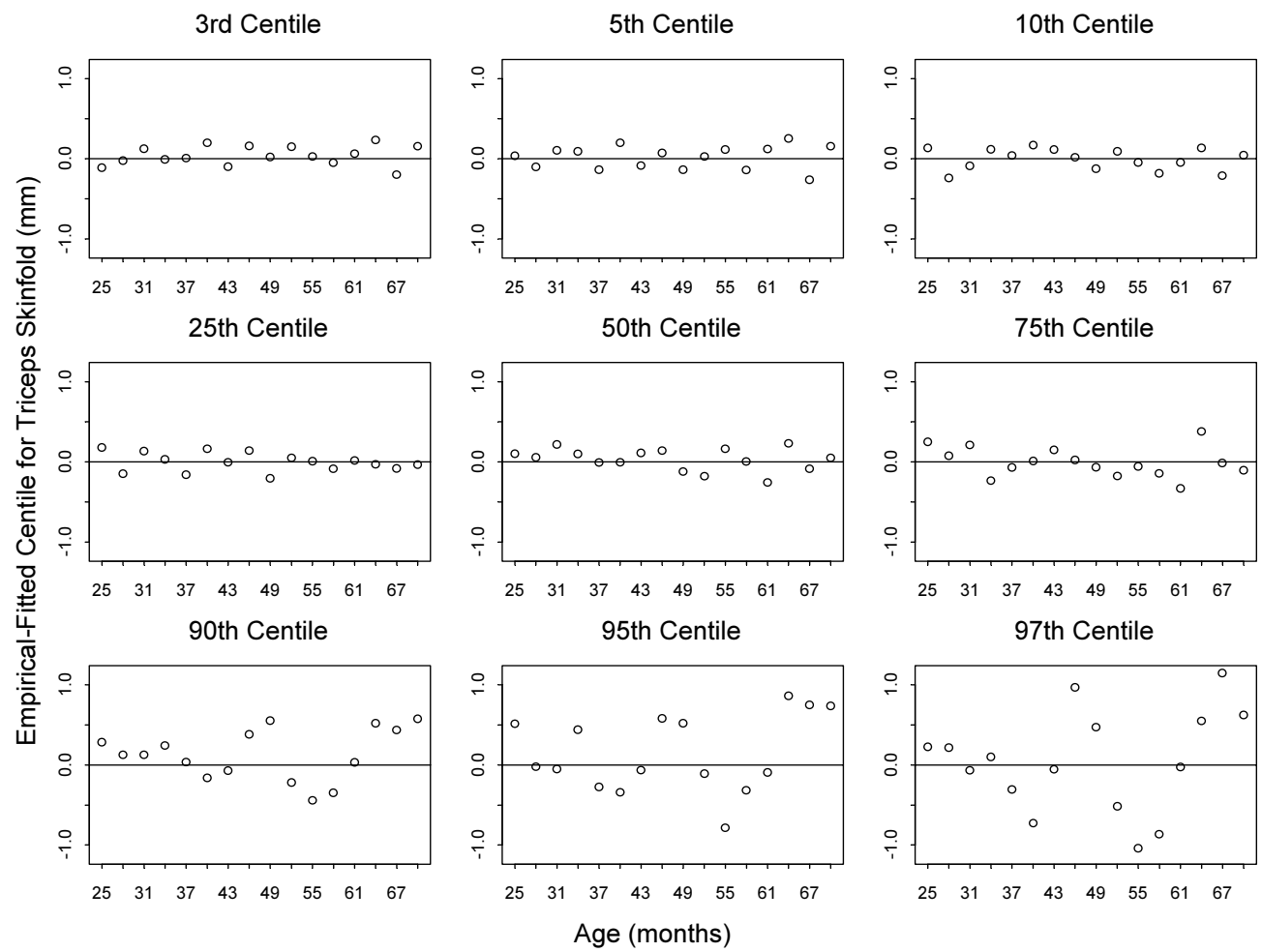

Figure 48 Centile residuals from fitting Model 2 for triceps skinfold-for-age from 24 to 71 months for boys 

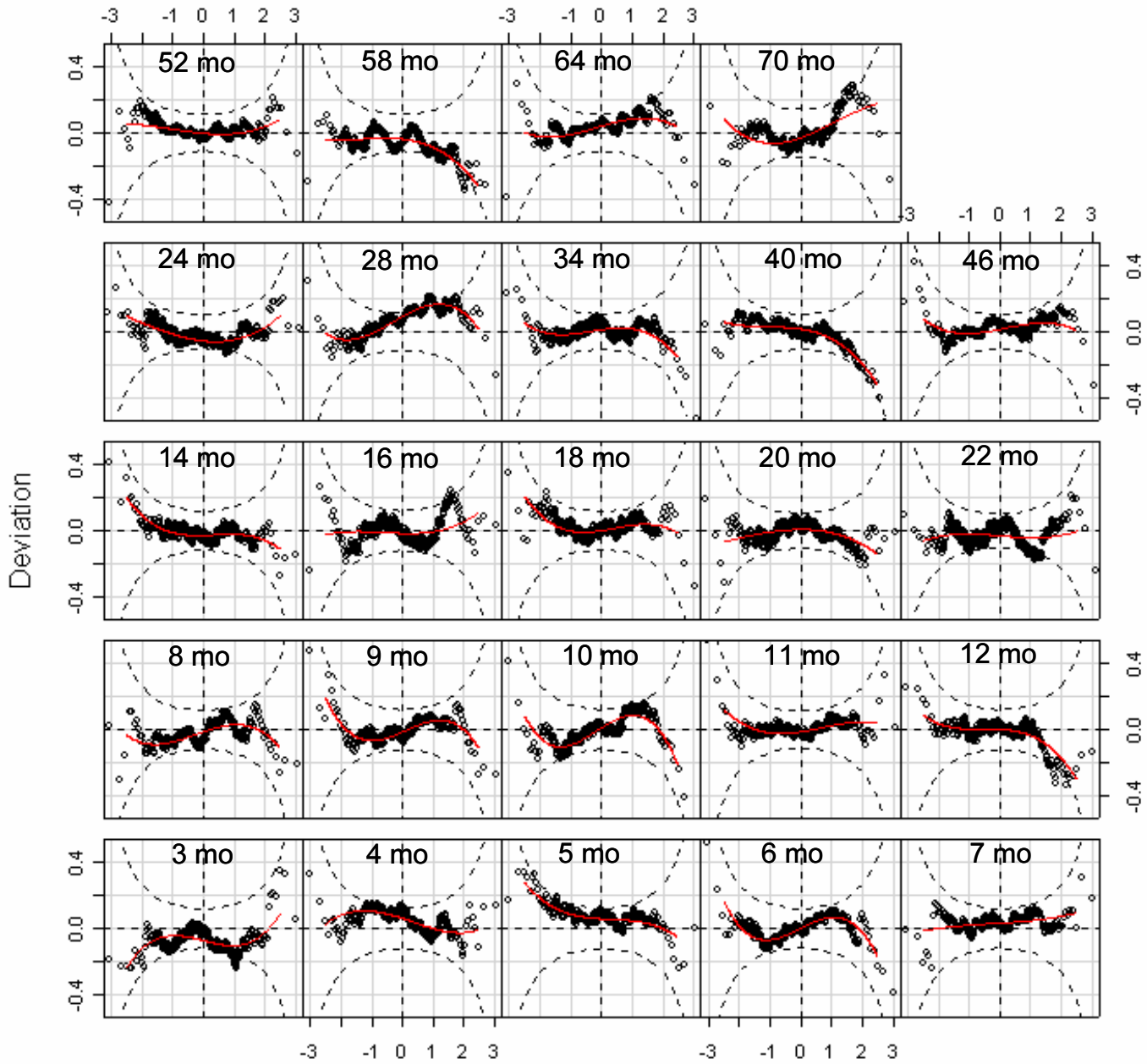

Unit normal quantile

Figure 49 Worm plots of z-scores for Model 2 for triceps skinfold-for-age for boys

The Q-test results from Model 2 are shown in Table 42. There were no longer indications of misfit of variance, as no $\mathrm{z} 2$ absolute values were larger than 2 . There were also no groups with absolute value larger than 2 for the statistic $\mathrm{z} 3$ that would suggest remaining skewness. Three age groups presented absolute values of $\mathrm{z} 4$ greater than 2 , indicating remaining kurtosis, but the overall test statistic for kurtosis had a p-value not significant at the 5\% level. The overall Q-test p-values were all nonsignificant, indicating an adequate fit of the boys' triceps skinfold-for-age curves and providing no justification for increasing the complexity of the model by adjusting for kurtosis (modelling $\tau$ ).

Table 43 presents observed percentages with triceps skinfolds below the fitted centiles. There was no detectable pattern suggesting a systematic bias. 
Table 42 Q-test for z-scores from Model $2\left[\operatorname{BCPE}\left(x=\operatorname{age}^{0.30}, \operatorname{df}(\mu)=7, \operatorname{df}(\sigma)=5, \operatorname{df}(v)=2, \tau=2\right)\right]$ for triceps skinfold-for-age for boys

\begin{tabular}{|c|c|c|c|c|c|c|}
\hline Age (days) & Group & $\mathbf{N}$ & $\mathrm{z} 1$ & $\mathrm{z2}$ & $\mathrm{z3}$ & $\mathrm{z} 4$ \\
\hline 79 to 99 & $3 \mathrm{mo}$ & 415 & -1.6 & 0.2 & 0.1 & 1.5 \\
\hline 100 to 129 & $4 \mathrm{mo}$ & 408 & 1.0 & -1.0 & -0.4 & 0.8 \\
\hline 130 to 159 & 5 mo & 409 & 1.4 & -1.0 & 0.3 & -0.9 \\
\hline 160 to 189 & $6 \mathrm{mo}$ & 411 & 0.0 & 0.5 & 0.0 & -2.4 \\
\hline 190 to 219 & $7 \mathrm{mo}$ & 405 & 0.8 & 0.4 & 0.1 & 0.3 \\
\hline 220 to 249 & 8 mo & 419 & -0.7 & 0.8 & -0.4 & -1.0 \\
\hline 250 to 279 & 9 mo & 390 & -0.3 & 0.6 & 0.3 & -2.3 \\
\hline 280 to 309 & $10 \mathrm{mo}$ & 399 & 0.1 & 1.1 & -0.6 & -2.4 \\
\hline 310 to 349 & $11 \mathrm{mo}$ & 461 & 0.0 & 0.2 & 0.7 & -0.5 \\
\hline 350 to 379 & $12 \mathrm{mo}$ & 407 & -0.3 & -1.1 & -0.8 & -1.1 \\
\hline 380 to 439 & $14 \mathrm{mo}$ & 418 & -0.4 & -0.8 & 0.6 & -1.1 \\
\hline 440 to 499 & $16 \mathrm{mo}$ & 415 & -0.2 & 0.3 & 0.5 & 0.5 \\
\hline 500 to 559 & $18 \mathrm{mo}$ & 441 & 0.3 & -0.1 & 0.8 & -1.0 \\
\hline 560 to 619 & $20 \mathrm{mo}$ & 521 & -0.2 & -0.1 & -1.0 & -0.2 \\
\hline 620 to 679 & $22 \mathrm{mo}$ & 544 & -0.6 & -0.2 & 0.4 & 0.2 \\
\hline 680 to 749 & $24 \mathrm{mo}$ & 591 & -0.9 & -0.4 & 1.5 & 0.5 \\
\hline 750 to 929 & $28 \mathrm{mo}$ & 469 & 1.6 & 1.6 & -0.8 & -1.5 \\
\hline 930 to 1119 & $34 \mathrm{mo}$ & 509 & 0.3 & -0.4 & -0.3 & -1.1 \\
\hline 1120 to 1309 & $40 \mathrm{mo}$ & 510 & -0.2 & -1.6 & -1.3 & -0.6 \\
\hline 1310 to 1499 & $46 \mathrm{mo}$ & 506 & 0.5 & 0.5 & 0.2 & -0.7 \\
\hline 1500 to 1689 & $52 \mathrm{mo}$ & 487 & 0.2 & -0.2 & 0.6 & 0.5 \\
\hline 1690 to 1879 & $58 \mathrm{mo}$ & 480 & -1.3 & -1.2 & -1.3 & -0.4 \\
\hline 1880 to 2069 & $64 \mathrm{mo}$ & 461 & 0.8 & 1.0 & -0.2 & -0.6 \\
\hline 2070 to 2249 & $70 \mathrm{mo}$ & 286 & -0.1 & 1.1 & 1.0 & -0.4 \\
\hline Overall Q stats & & 10762 & 13.3 & 16.0 & 12.2 & 31.0 \\
\hline degrees of freedom & & & 17.0 & 21.0 & 22.0 & 24.0 \\
\hline p-value & & & 0.7179 & 0.7701 & 0.9522 & 0.1551 \\
\hline
\end{tabular}

Note: Absolute values of z1, z2, z3 or z4 larger than 2 indicate misfit of, respectively, mean, variance, skewness or kurtosis.

In conclusion, the model selected for constructing the triceps skinfold-for-age growth curves for boys was $\operatorname{BCPE}\left(x=a g e^{0.30}, \operatorname{df}(\mu)=7, \operatorname{df}(\sigma)=5, \operatorname{df}(v)=2, \tau=2\right)$ that adjusts only for skewness, and thus reduces to the LMS method. The fitted centile curves and empirical centiles are shown in Figures 50 to 53. 


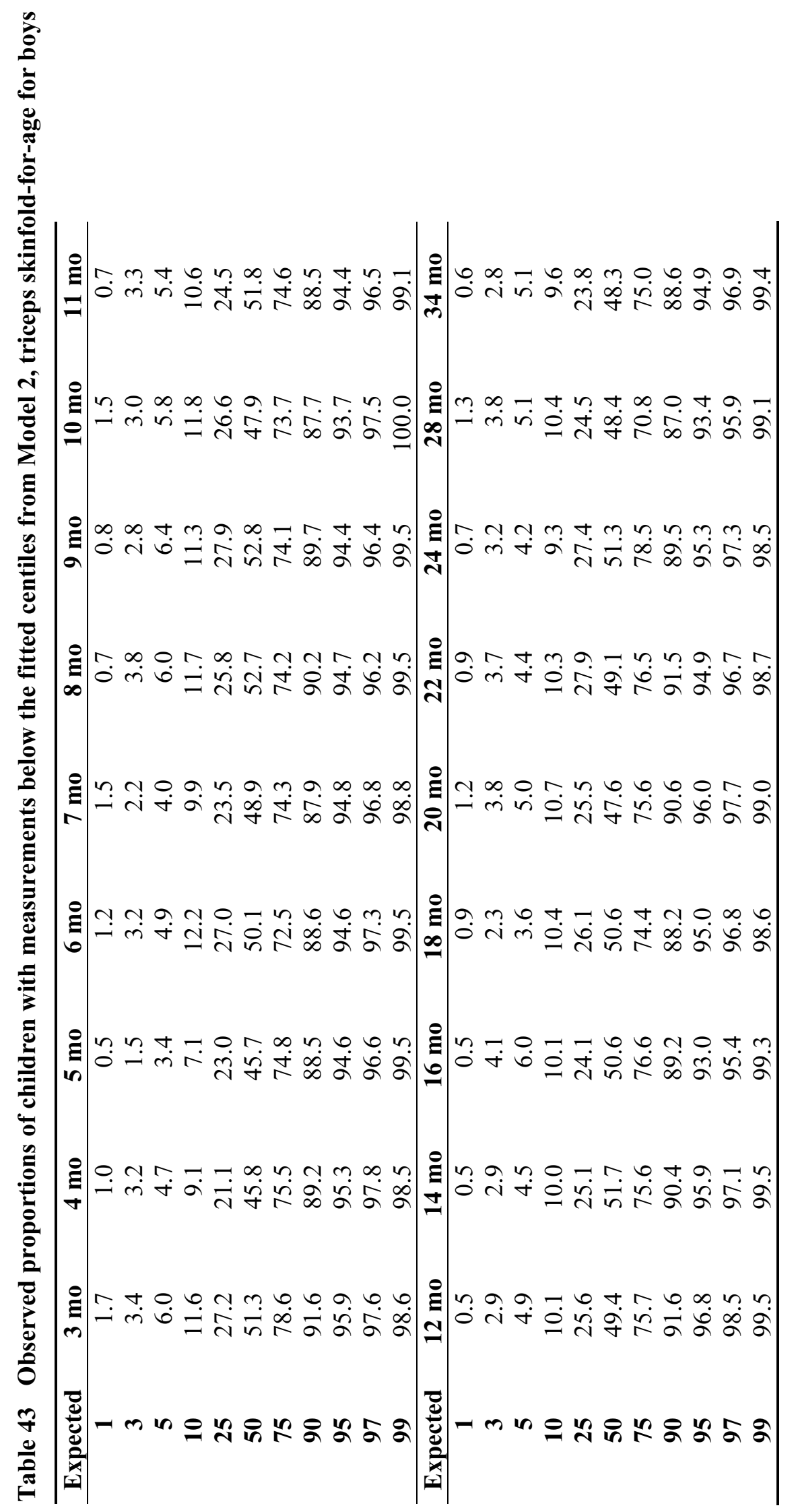




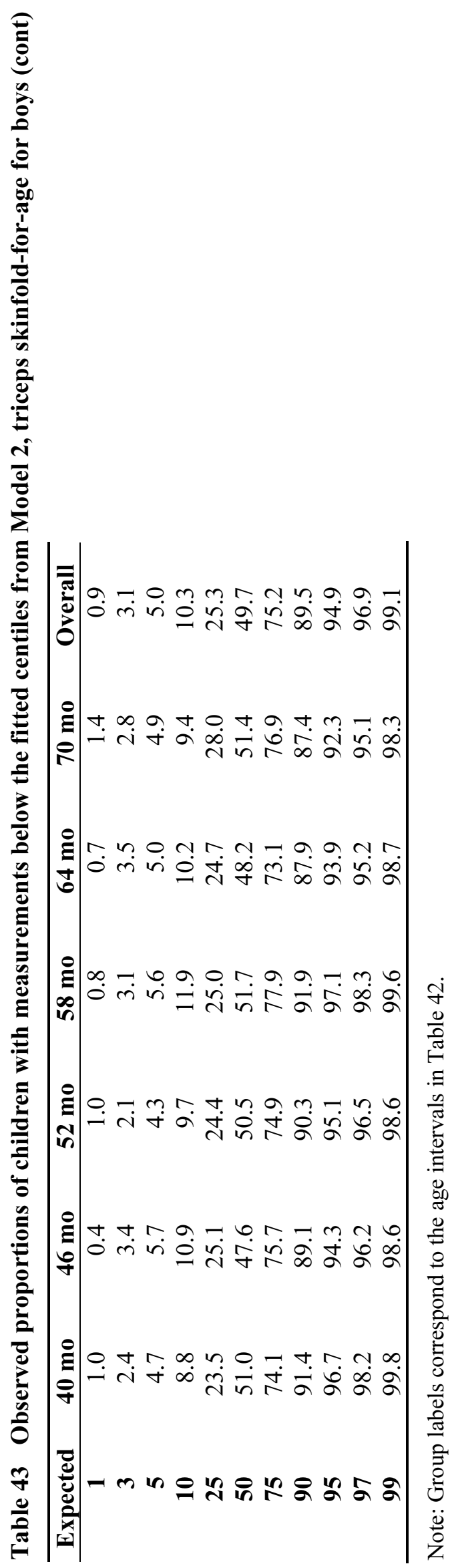




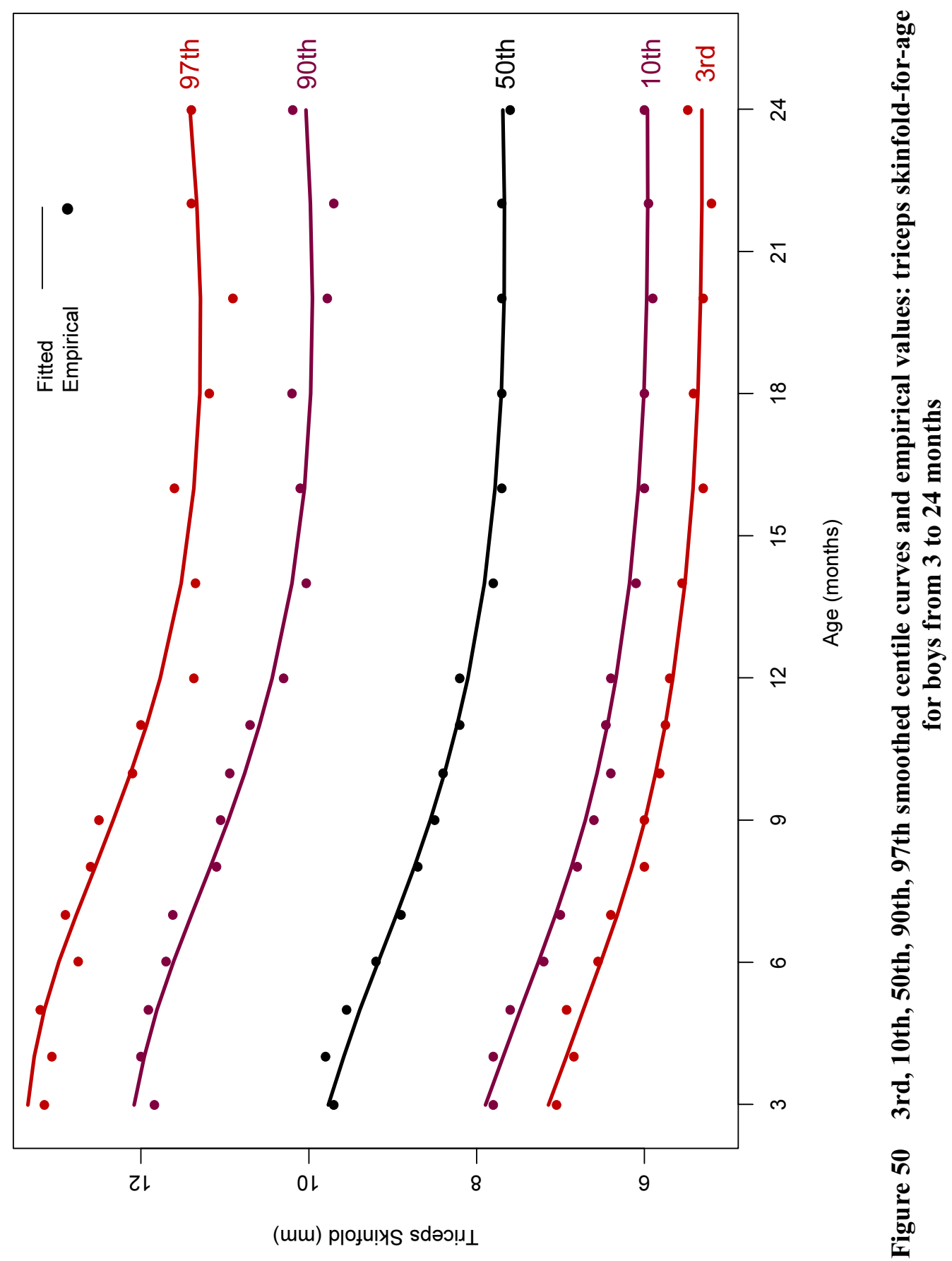




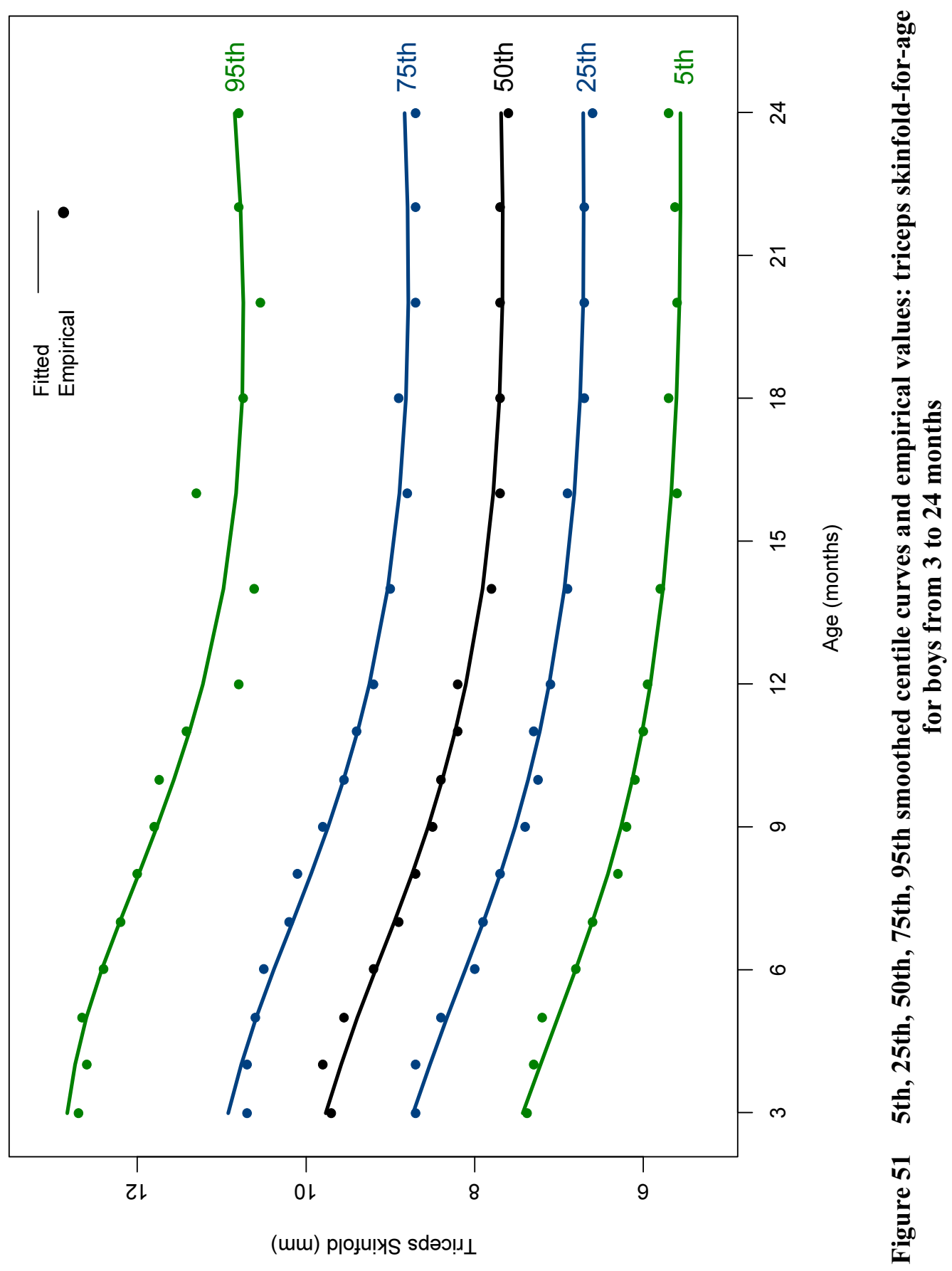




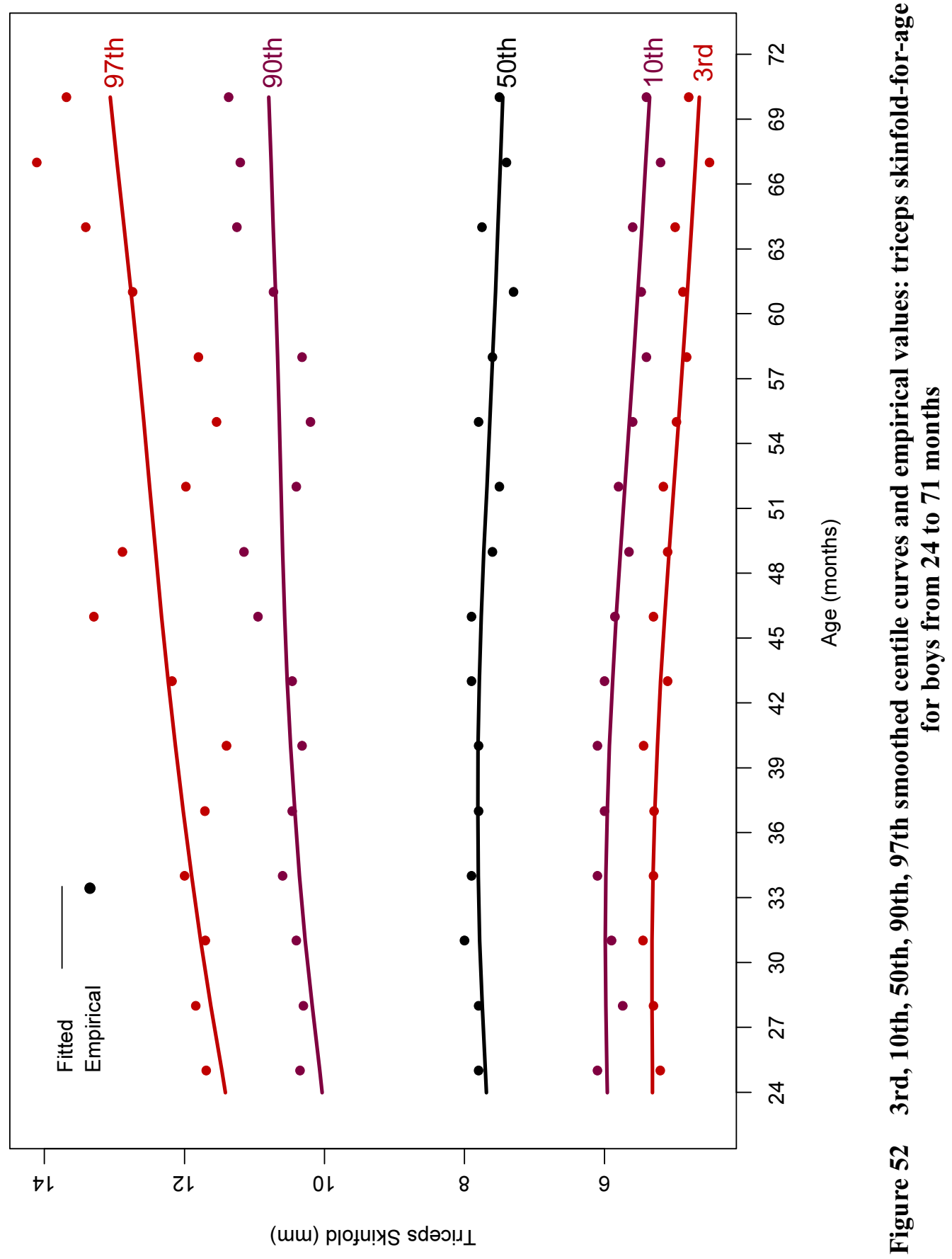




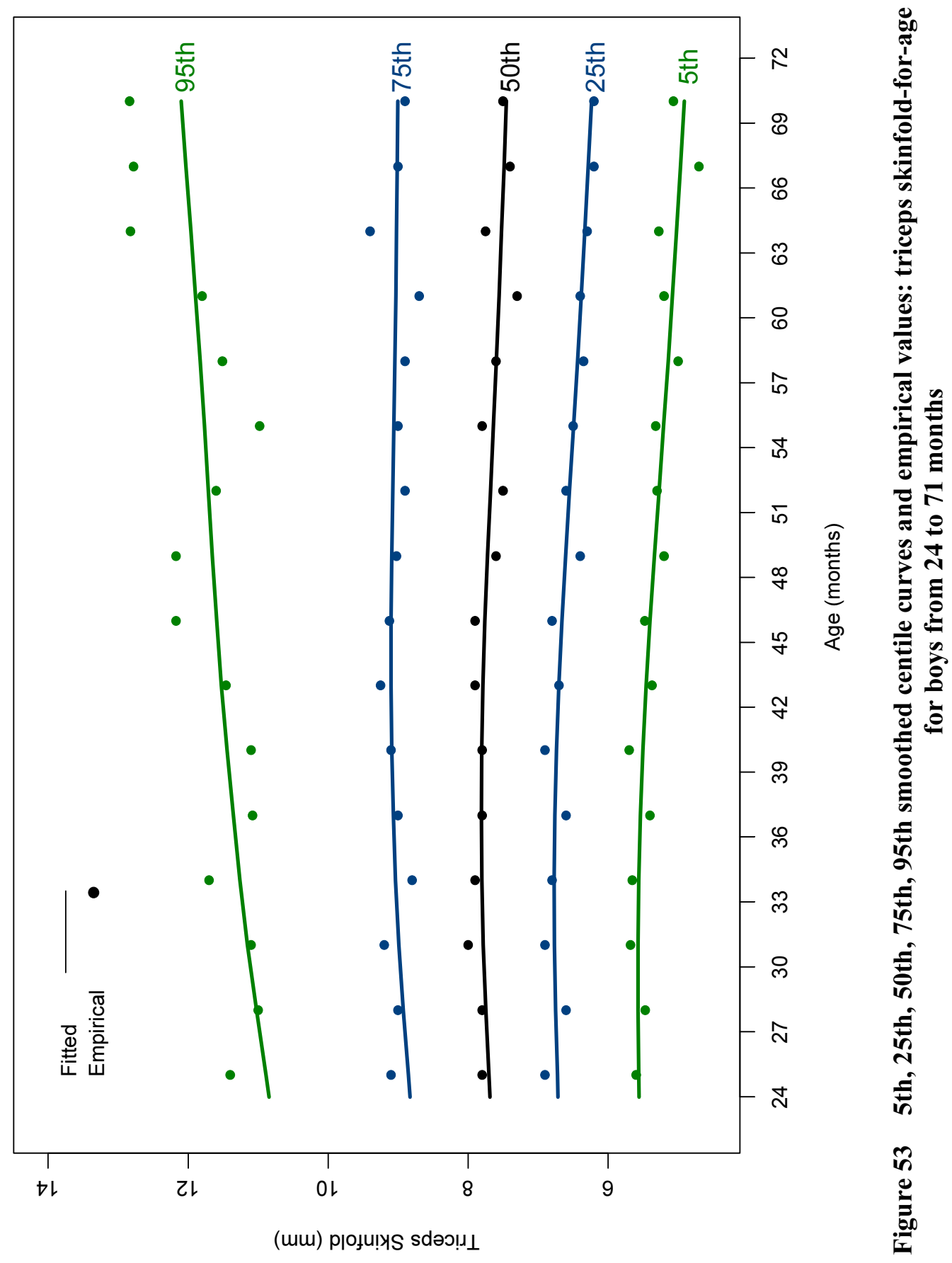




\subsubsection{WHO standards}

This section presents the final WHO triceps skinfold-for-age z-score and percentile charts (Figures 54 and 55) and table (Table 44) for boys. 


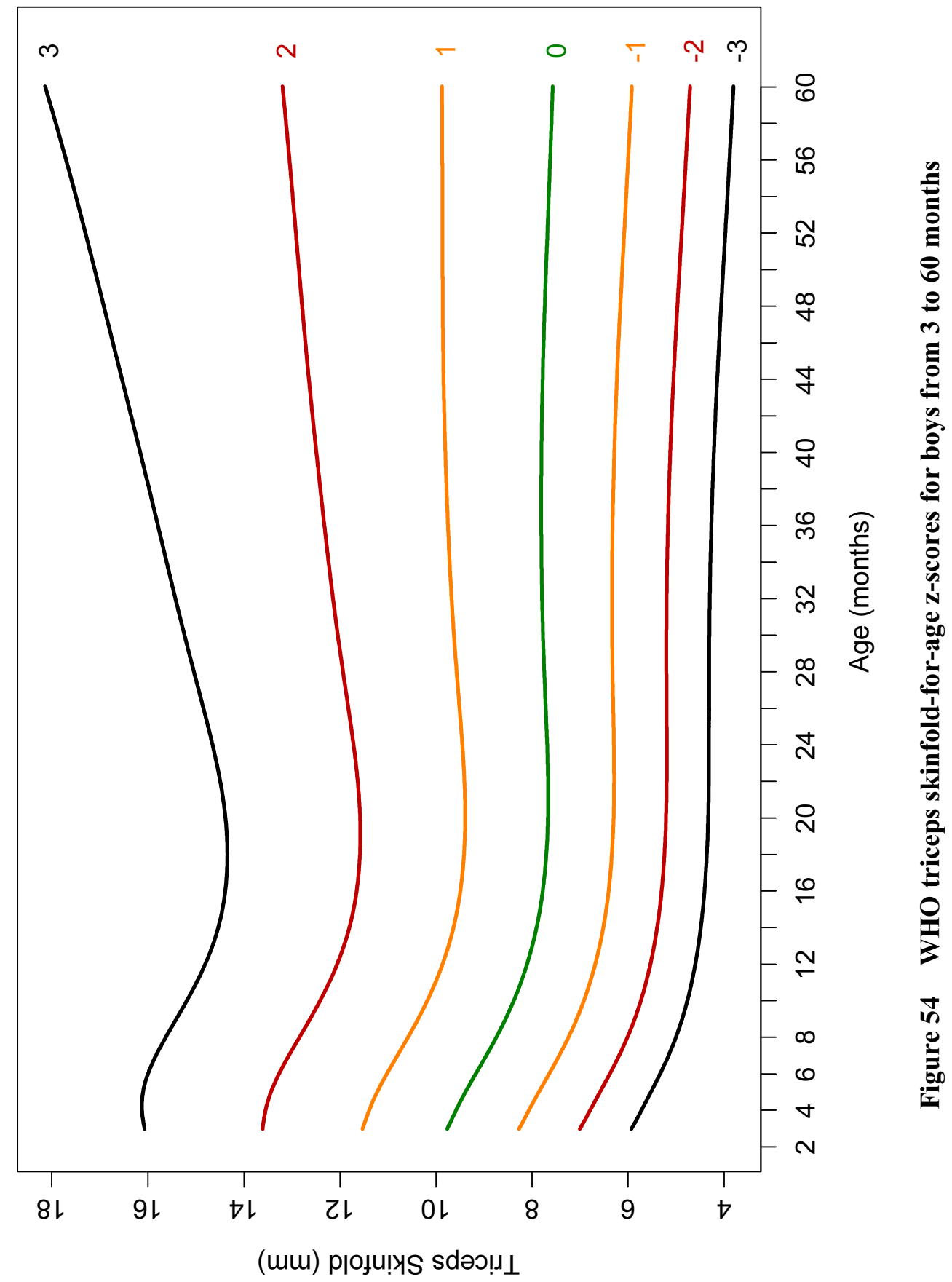




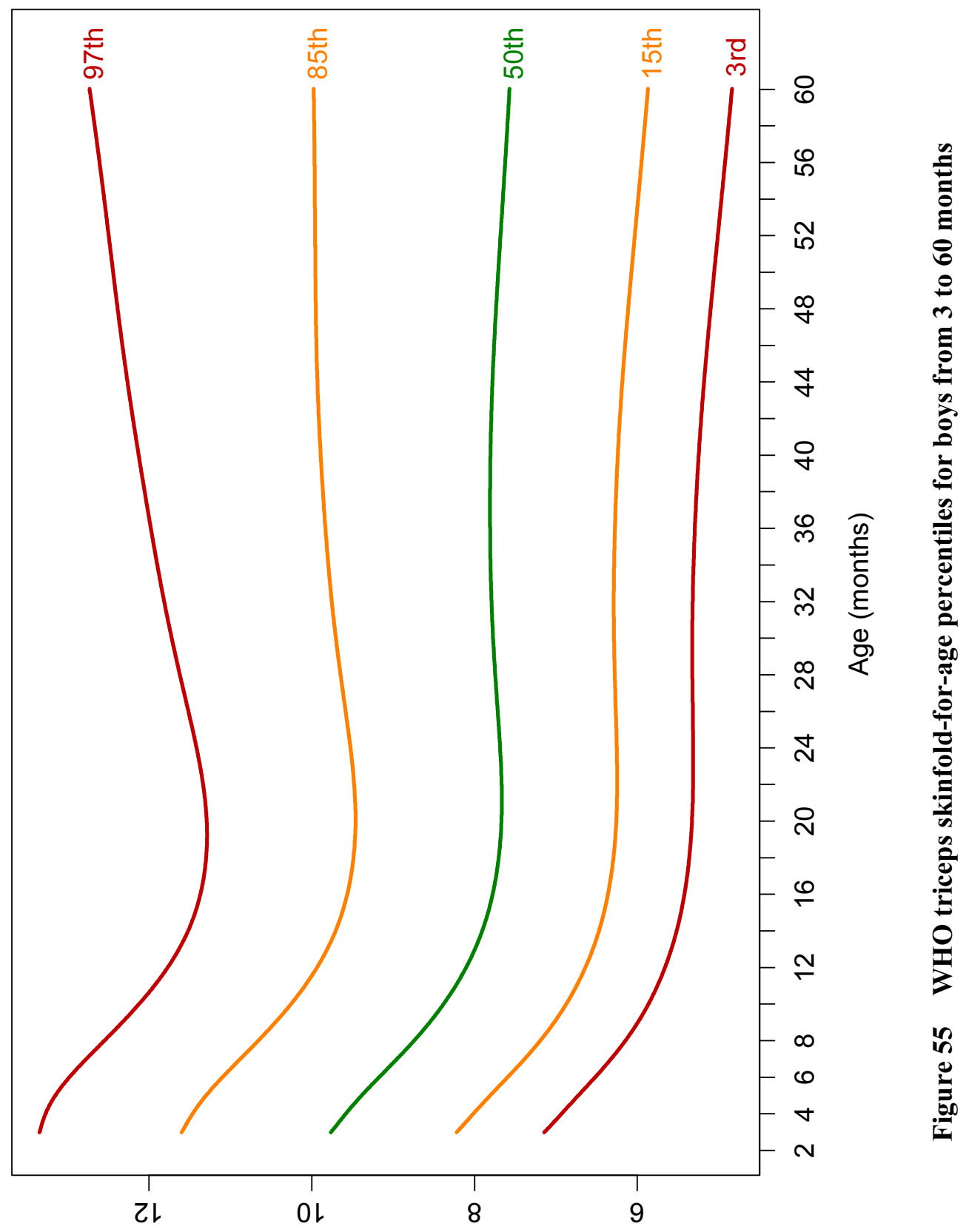

(mw) p|OJu!Ys sdəo!ı」 


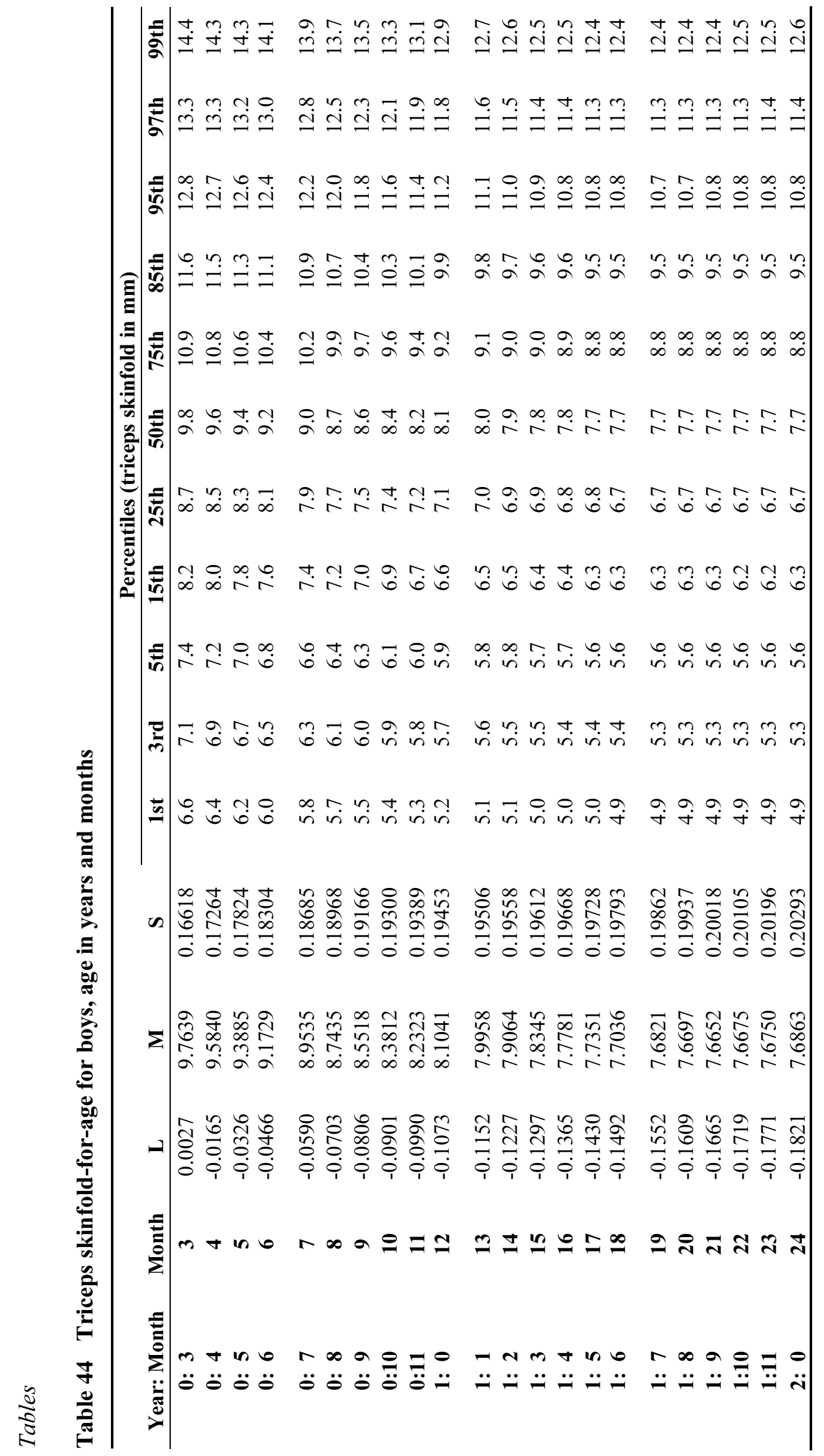




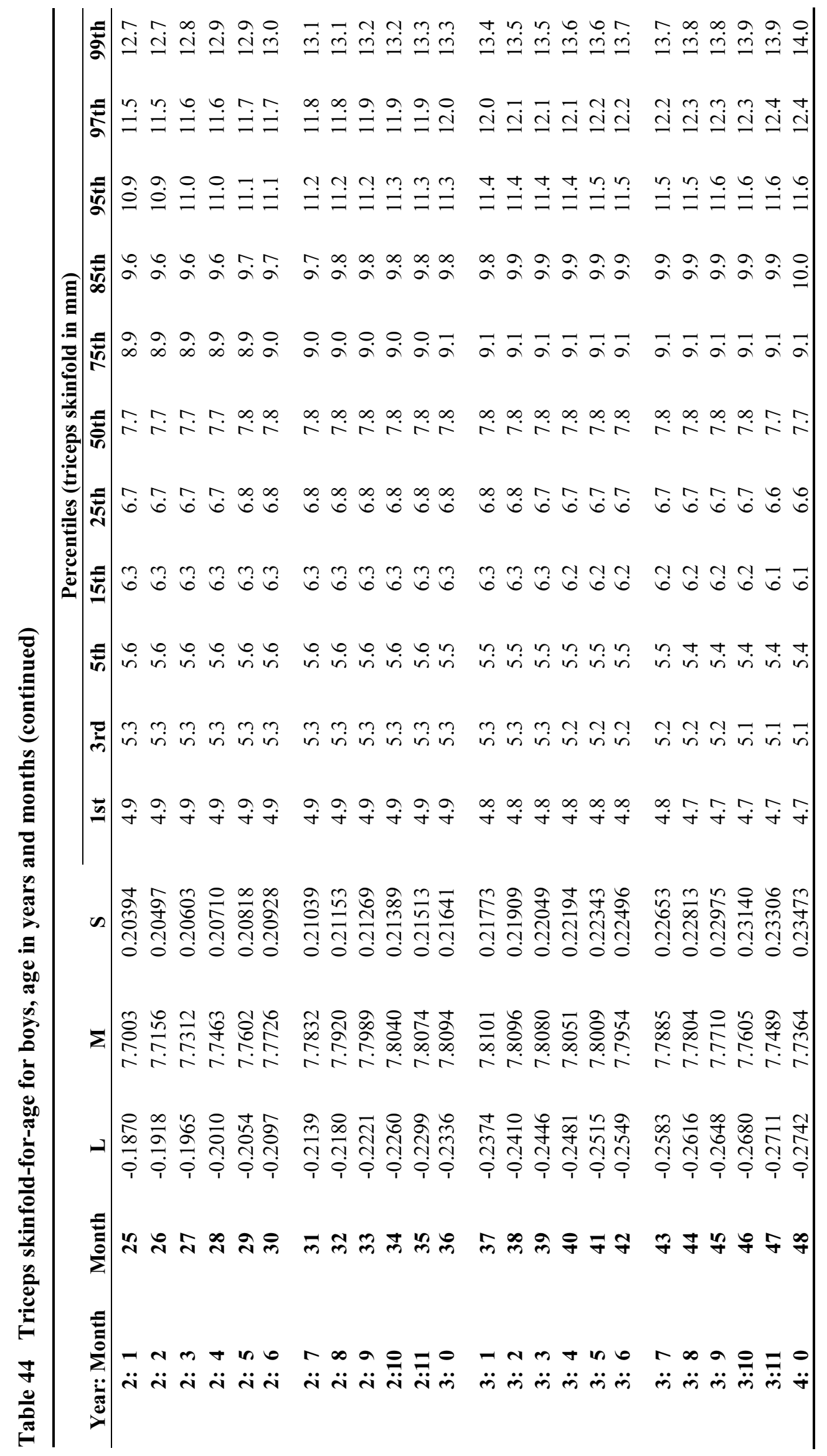




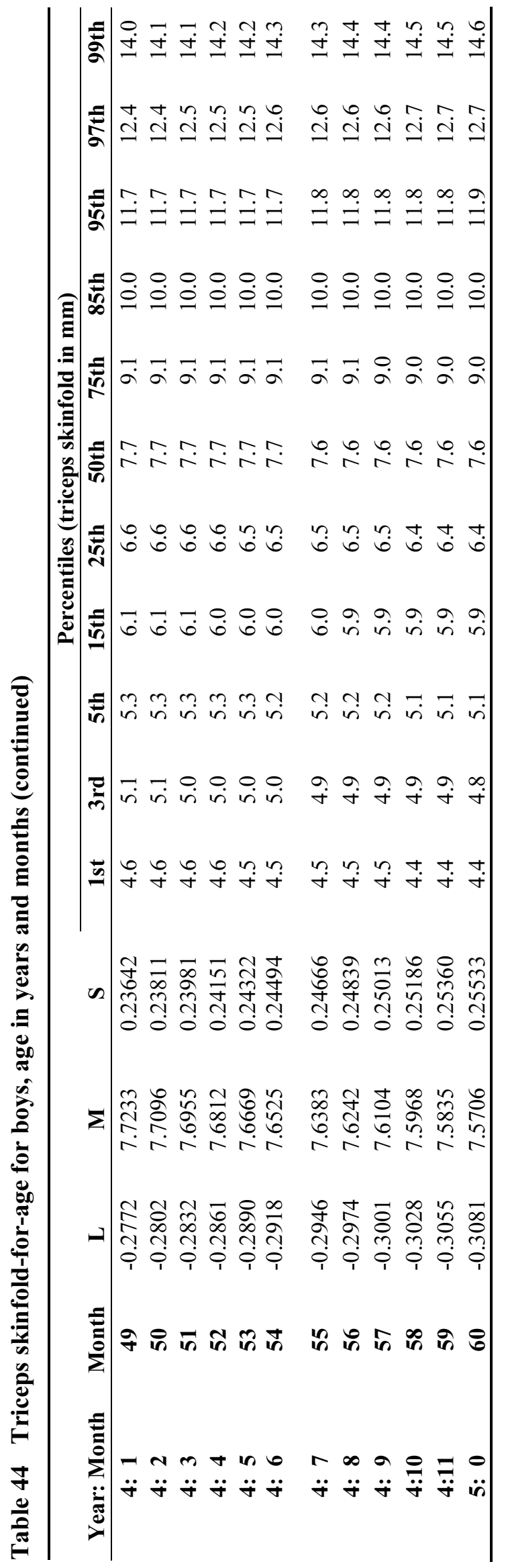




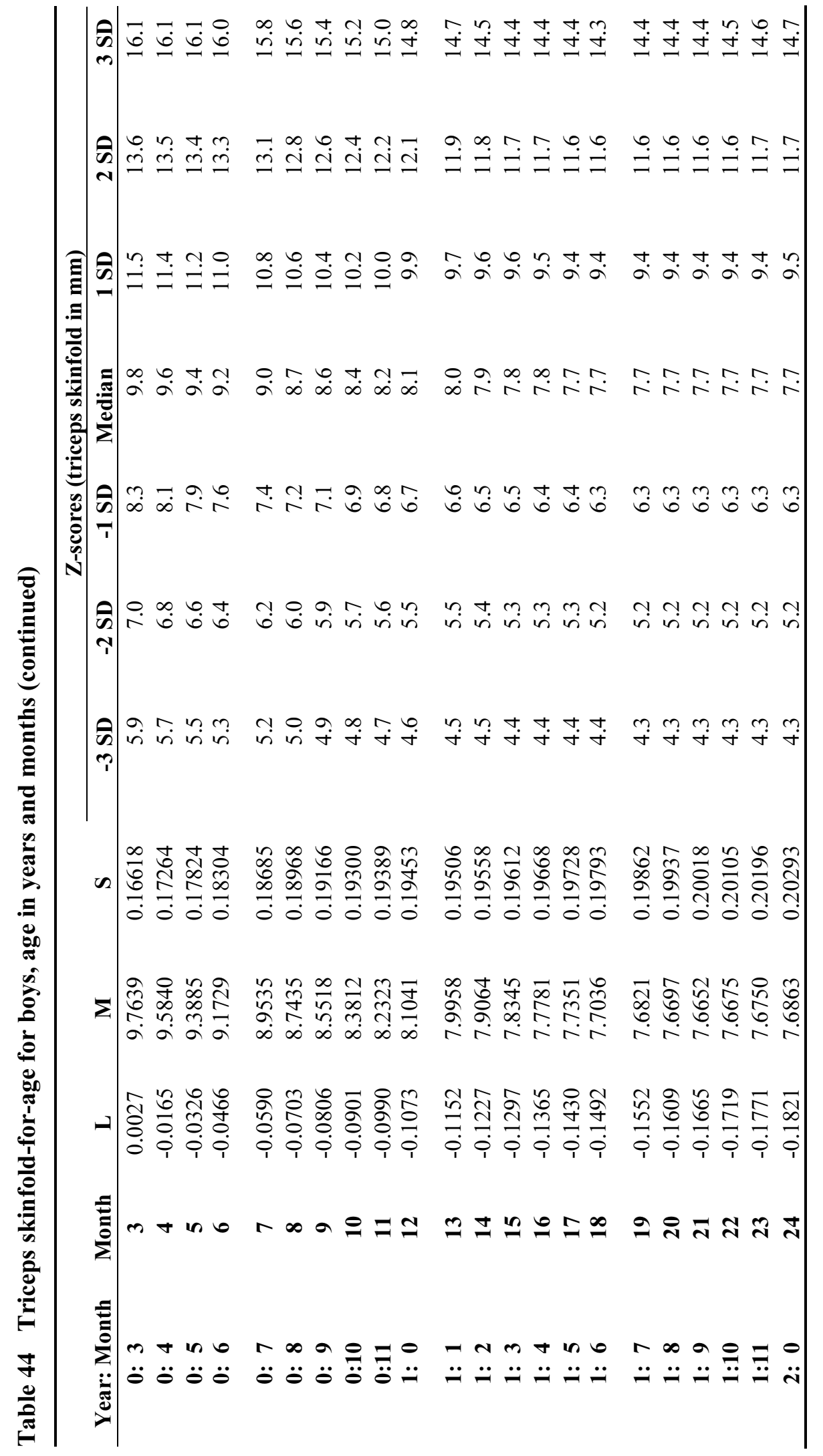




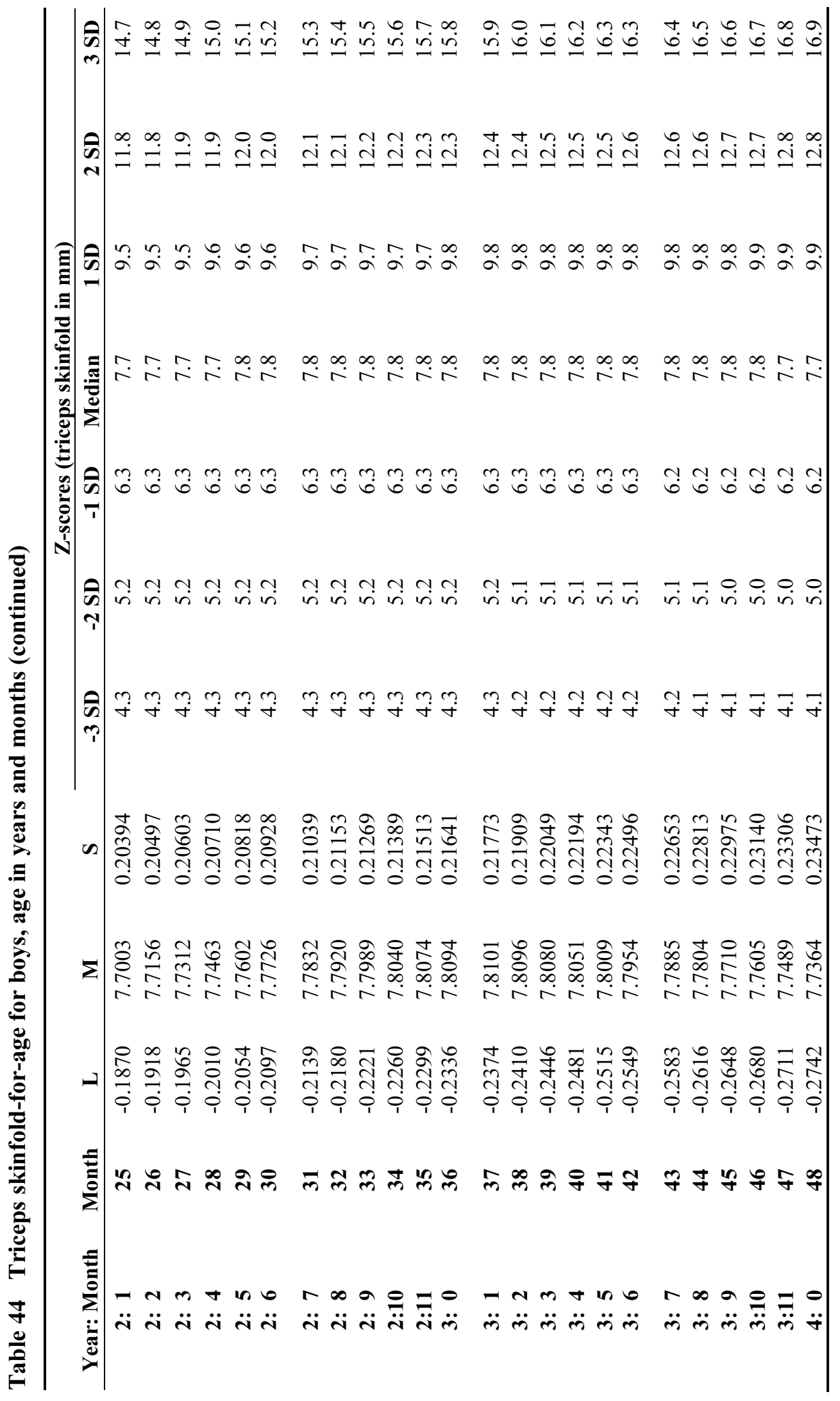




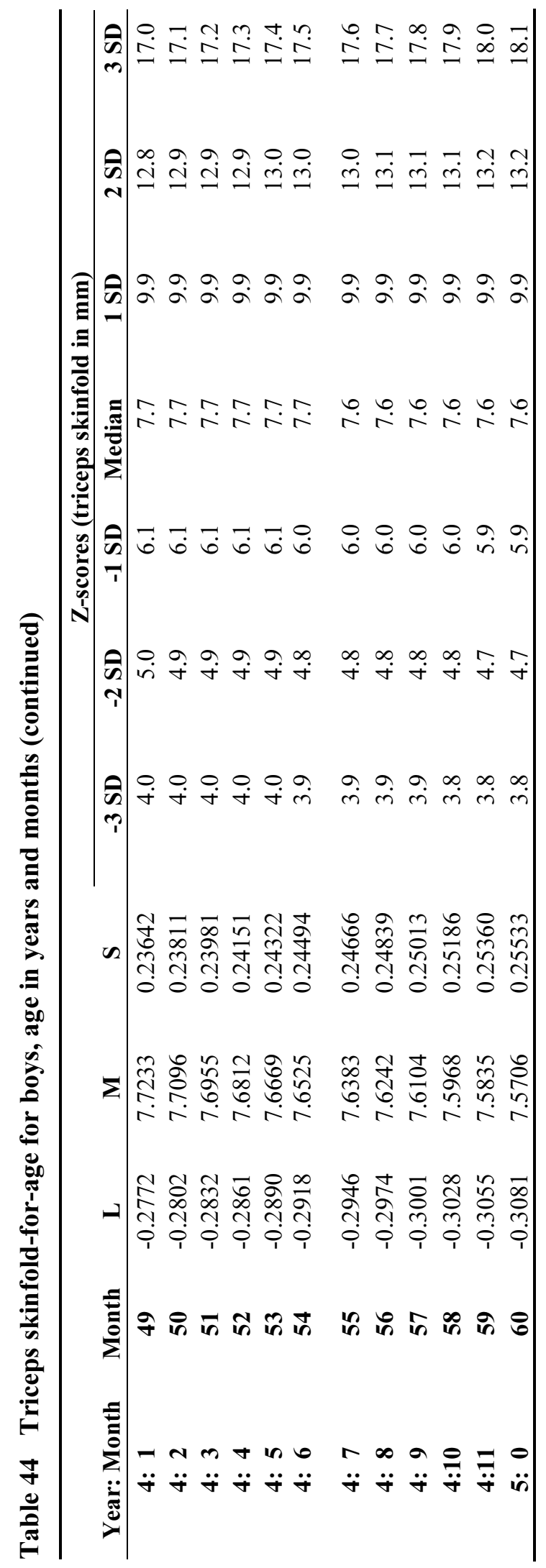




\subsection{Triceps skinfold-for-age for girls}

Steps similar to those described in the preceding sections were followed to select the best model to fit the triceps skinfold-for-age growth standard for girls.

\subsubsection{Sample size}

There were a total of 10943 triceps skinfold observations for girls. The longitudinal and crosssectional sample sizes by visit and age are presented in Tables 45 and 46 . The measurement of triceps skinfold started at 3 months of age (de Onis et al., 2004b).

Table 45 Longitudinal sample sizes for triceps skinfold-for-age for girls

\begin{tabular}{|c|c|c|c|c|c|c|}
\hline Visit & 5 & 6 & 7 & 8 & 9 & 10 \\
\hline Age & $3 \mathrm{mo}$ & $4 \mathrm{mo}$ & $5 \mathrm{mo}$ & $6 \mathrm{mo}$ & $7 \mathrm{mo}$ & $8 \mathrm{mo}$ \\
\hline $\mathrm{N}$ & 446 & 443 & 447 & 445 & 444 & 440 \\
\hline Visit & 11 & 12 & 13 & 14 & 15 & 16 \\
\hline Age & $9 \mathrm{mo}$ & $10 \mathrm{mo}$ & $11 \mathrm{mo}$ & $12 \mathrm{mo}$ & $14 \mathrm{mo}$ & $16 \mathrm{mo}$ \\
\hline $\mathrm{N}$ & 444 & 442 & 438 & 449 & 446 & 441 \\
\hline Visit & 17 & 18 & 19 & 20 & & \\
\hline Age & $18 \mathrm{mo}$ & $20 \mathrm{mo}$ & $22 \mathrm{mo}$ & $24 \mathrm{mo}$ & & \\
\hline $\mathrm{N}$ & 445 & 443 & 434 & 446 & & \\
\hline
\end{tabular}

Table 46 Cross-sectional sample sizes for triceps skinfold-for-age for girls

\begin{tabular}{lccccccc}
\hline Age (mo) & $<\mathbf{1 8}$ & $\mathbf{1 8 - 2 0}$ & $\mathbf{2 1 - 2 3}$ & $\mathbf{2 4 - 2 6}$ & $\mathbf{2 7 - 2 9}$ & $\mathbf{3 0 - 3 2}$ & $\mathbf{3 3 - 3 5}$ \\
$\mathrm{N}$ & 2 & 158 & 165 & 235 & 214 & 225 & 222 \\
\hline Age (mo) & $\mathbf{3 6}-\mathbf{3 8}$ & $\mathbf{3 9 - 4 1}$ & $\mathbf{4 2 - 4 4}$ & $\mathbf{4 5}-\mathbf{4 7}$ & $\mathbf{4 8}-\mathbf{5 0}$ & $\mathbf{5 1 - 5 3}$ & $\mathbf{5 4 - 5 6}$ \\
$\mathrm{N}$ & 216 & 232 & 244 & 207 & 220 & 199 & 229 \\
\hline Age (mo) & $\mathbf{5 7 - 5 9}$ & $\mathbf{6 0 - 6 2}$ & $\mathbf{6 3 - 6 5}$ & $\mathbf{6 6 - 6 8}$ & $\mathbf{6 9 - 7 1}$ & $>\mathbf{7 1}$ & \\
$\mathrm{N}$ & 238 & 216 & 204 & 224 & 200 & 0 & \\
\hline
\end{tabular}

\subsubsection{Model selection and results}

The model BCPE $\left(x=\operatorname{age}^{\lambda}, \operatorname{df}(\mu)=9, \operatorname{df}(\sigma)=4, \operatorname{df}(v)=4, \tau=2\right)$ was used as a starting point to search for the best value of the age-transformation power $\lambda$. Table 47 shows the global deviance for values of $\lambda$ from 0.05 to 1 . Global deviance values were smallest for $\lambda 0.15$ and 0.20 , and $\lambda=0.15$ was selected. 
Table 47 Global deviance (GD) for models within the class $\operatorname{BCPE}\left(x=\operatorname{age}^{\lambda}, \operatorname{df}(\mu)=9, \operatorname{df}(\sigma)=4\right.$, $\operatorname{df}(v)=4, \tau=2)$ for triceps skinfold-for-age for girls

\begin{tabular}{lcccccccccc}
\hline $\boldsymbol{\lambda}$ & $\mathbf{0 . 0 5}$ & $\mathbf{0 . 1 0}$ & $\mathbf{0 . 1 5}$ & $\mathbf{0 . 2 0}$ & $\mathbf{0 . 2 5}$ & $\mathbf{0 . 3 0}$ & $\mathbf{0 . 3 5}$ & $\mathbf{0 . 4 0}$ & $\mathbf{0 . 4 5}$ & $\mathbf{0 . 5 0}$ \\
$\mathrm{GD}^{\mathrm{a}}$ & 761.4 & 761.1 & 761.0 & 761.0 & 761.3 & 761.8 & 762.4 & 763.3 & 764.2 & 765.4 \\
\hline $\boldsymbol{\lambda}$ & $\mathbf{0 . 5 5}$ & $\mathbf{0 . 6 0}$ & $\mathbf{0 . 6 5}$ & $\mathbf{0 . 7 0}$ & $\mathbf{0 . 7 5}$ & $\mathbf{0 . 8 0}$ & $\mathbf{0 . 8 5}$ & $\mathbf{0 . 9 0}$ & $\mathbf{0 . 9 5}$ & $\mathbf{1 . 0 0}$ \\
$\mathrm{GD}^{\mathrm{a}}$ & 766.7 & 768.0 & 769.4 & 770.8 & 772.3 & 773.7 & 775.1 & 776.4 & 777.7 & 779.0 \\
\hline
\end{tabular}

${ }^{\mathrm{a}}$ In excess of 43000 .

The search for the best $\operatorname{df}(\mu)$ and $\operatorname{df}(\sigma)$ followed, fixing $\lambda=0.15, v=1$ and $\tau=2$. All possible combinations with $\operatorname{df}(\mu)$ values ranging from 6 to 15 and $\operatorname{df}(\sigma)$ from 2 to 10 were considered and partial results are presented in Table 48. Only GAIC(3) was considered for selecting the best combination. The model with $\operatorname{df}(\mu)=7$ and $\operatorname{df}(\sigma)=5$ provided the smallest value of $G A I C(3)$ and it was thus selected for further evaluation.

Table 48 Goodness-of-fit summary for models using the BCPE distribution with fixed $v=1$ and $\tau=\mathbf{2}$ for triceps skinfold-for-age for girls

\begin{tabular}{cccccc}
\hline $\mathbf{d f}(\boldsymbol{\mu})$ & $\mathbf{d f}(\boldsymbol{\sigma})$ & $\mathbf{G D}^{\mathbf{a}}$ & $\mathbf{A I C}^{\mathbf{a}}$ & GAIC(3) $^{\mathbf{a}}$ & Total df \\
\hline \multirow{6}{*}{$\mathbf{6}$} & $\mathbf{3}$ & 1775.3 & 1793.3 & 1802.3 & 9.0 \\
& $\mathbf{4}$ & 1759.0 & 1779.0 & 1789.0 & 10.0 \\
& $\mathbf{5}$ & 1753.2 & 1775.2 & 1786.2 & 11.0 \\
& $\mathbf{6}$ & 1751.2 & 1775.2 & 1787.2 & 12.0 \\
& $\mathbf{7}$ & 1749.9 & 1775.9 & 1788.9 & 13.0 \\
$\mathbf{7}$ & $\mathbf{3}$ & 1770.9 & 1790.9 & 1800.9 & 10.0 \\
& $\mathbf{4}$ & 1754.5 & 1776.5 & 1787.5 & 11.0 \\
& $\mathbf{5}$ & 1748.4 & 1772.4 & 1784.4 & 12.0 \\
& $\mathbf{6}$ & 1746.2 & 1772.2 & 1785.2 & 13.0 \\
& $\mathbf{7}$ & 1744.9 & 1772.9 & 1786.9 & 14.0 \\
$\mathbf{8}$ & $\mathbf{3}$ & 1768.3 & 1790.3 & 1801.3 & 11.0 \\
& $\mathbf{4}$ & 1751.7 & 1775.7 & 1787.7 & 12.0 \\
& $\mathbf{5}$ & 1745.5 & 1771.5 & 1784.5 & 13.0 \\
& $\mathbf{6}$ & 1743.2 & 1771.2 & 1785.2 & 14.0 \\
& $\mathbf{7}$ & 1741.8 & 1771.8 & 1786.8 & 15.0 \\
$\mathbf{9}$ & $\mathbf{3}$ & 1766.4 & 1790.4 & 1802.4 & 12.0 \\
& $\mathbf{4}$ & 1749.7 & 1775.7 & 1788.7 & 13.0 \\
& $\mathbf{5}$ & 1743.4 & 1771.4 & 1785.4 & 14.0 \\
& $\mathbf{6}$ & 1741.1 & 1771.1 & 1786.1 & 15.0 \\
& $\mathbf{7}$ & 1739.7 & 1771.7 & 1787.7 & 16.0 \\
\hline \multirow{3}{*}{$\mathbf{1 0}$} & $\mathbf{3}$ & 1764.9 & 1790.9 & 1803.9 & 13.0 \\
& $\mathbf{4}$ & 1748.2 & 1776.2 & 1790.2 & 14.0 \\
& $\mathbf{5}$ & 1741.8 & 1771.8 & 1786.8 & 15.0 \\
& $\mathbf{6}$ & 1739.5 & 1771.5 & 1787.5 & 16.0 \\
& $\mathbf{7}$ & 1738.0 & 1772.0 & 1789.0 & 17.0 \\
\hline
\end{tabular}

GD, Global Deviance; AIC, Akaike Information Criterion;

GAIC(3), Generalized AIC with penalty equal to 3;

${ }^{\text {a }}$ In excess of 43000. 
Model 1: $\operatorname{BCPE}\left(x=\operatorname{age}^{0.15}, \operatorname{df}(\mu)=7, \operatorname{df}(\sigma)=5, v=1, \tau=2\right)$

This model showed inadequacy as it clearly pointed to residual skewness in both the worm plots (Figure 56) and the Q-test results (Table 49). The worms presented U-shapes in all age groups and the Q-test results (Table 49) showed all groups with absolute values of z3 higher than 2. Most of the age groups also presented absolute values of $\mathrm{z} 4$ higher than 2 , indicating residual kurtosis. Both overall tests for skewness and kurtosis were significant ( $p$-value $<0.01$ ). No misfit could be detected in the mean and the variance.
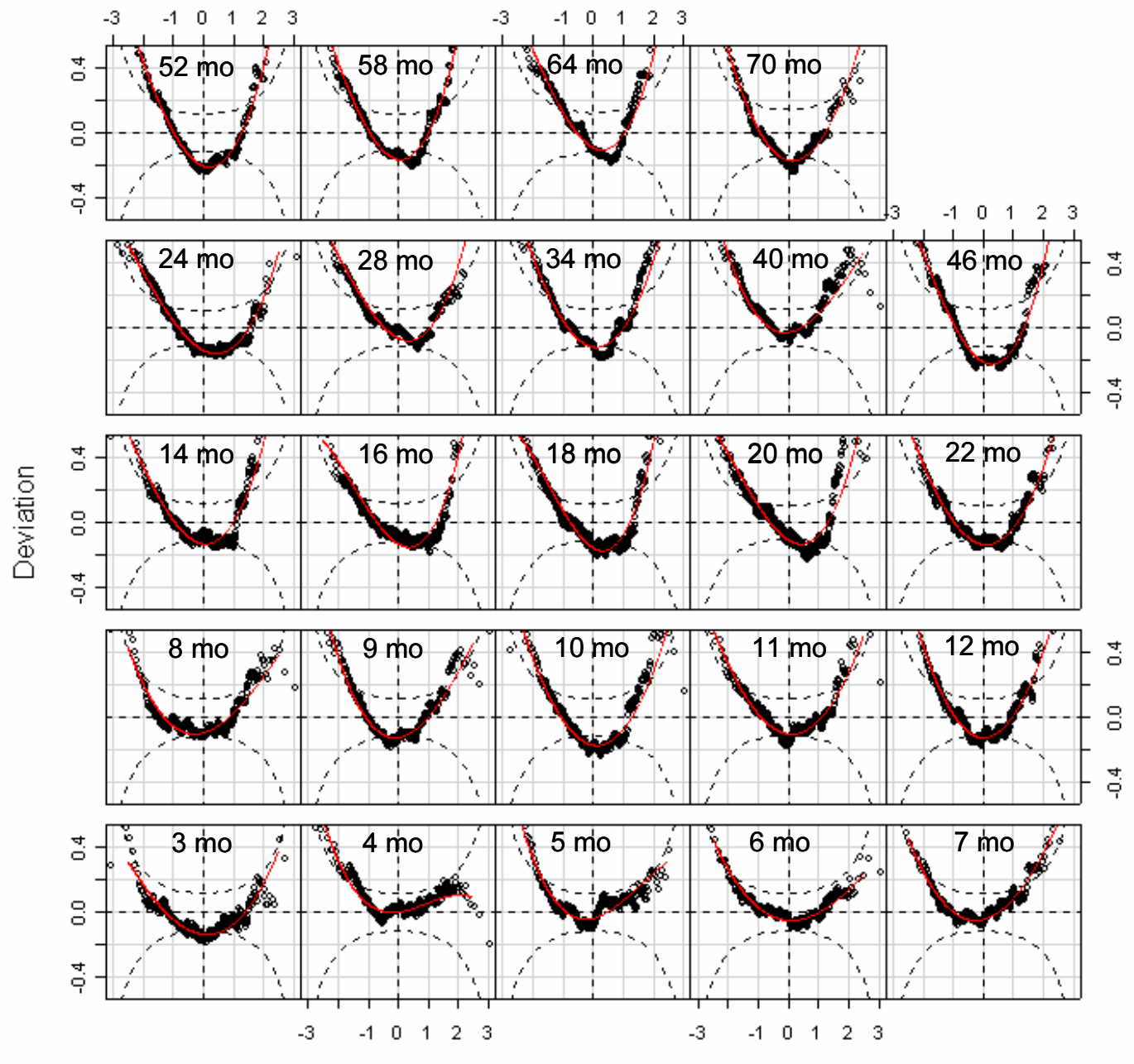

Unit normal quantile

Figure 56 Worm plots of z-scores for Model 1 for triceps skinfold-for-age for girls 
Table 49 Q-test for $z$-scores from Model $1\left[\operatorname{BCPE}\left(x=\operatorname{age}^{0.15}, \operatorname{df}(\mu)=7, \operatorname{df}(\sigma)=5, v=1, \tau=2\right)\right]$ for triceps skinfold-for-age for girls

\begin{tabular}{|c|c|c|c|c|c|c|}
\hline Age (days) & Group & $\mathbf{N}$ & $\mathrm{z} 1$ & $\mathbf{z 2}$ & $\mathbf{z 3}$ & $\mathrm{z4}$ \\
\hline 83 to 99 & $3 \mathrm{mo}$ & 439 & -1.3 & 0.1 & 3.9 & 1.8 \\
\hline 100 to 129 & $4 \mathrm{mo}$ & 443 & 1.1 & -0.8 & 2.5 & -1.5 \\
\hline 130 to 159 & $5 \mathrm{mo}$ & 441 & 0.9 & 0.1 & 3.5 & -0.2 \\
\hline 160 to 189 & $6 \mathrm{mo}$ & 441 & 0.2 & -0.7 & 3.0 & 0.3 \\
\hline 190 to 219 & $7 \mathrm{mo}$ & 432 & 0.9 & 1.1 & 4.0 & 1.2 \\
\hline 220 to 249 & $8 \mathrm{mo}$ & 435 & -0.5 & 0.9 & 3.6 & 0.1 \\
\hline 250 to 279 & $9 \mathrm{mo}$ & 437 & -0.2 & 0.1 & 5.1 & 0.7 \\
\hline 280 to 309 & $10 \mathrm{mo}$ & 442 & -1.4 & -0.3 & 5.9 & 2.2 \\
\hline 310 to 349 & $11 \mathrm{mo}$ & 474 & -0.1 & -0.2 & 5.1 & 1.7 \\
\hline 350 to 379 & $12 \mathrm{mo}$ & 451 & 0.2 & 0.3 & 6.1 & 2.2 \\
\hline 380 to 439 & $14 \mathrm{mo}$ & 443 & 0.1 & 1.2 & 6.4 & 3.2 \\
\hline 440 to 499 & $16 \mathrm{mo}$ & 443 & 0.0 & 0.0 & 7.0 & 4.9 \\
\hline 500 to 559 & $18 \mathrm{mo}$ & 467 & -0.1 & 0.6 & 7.8 & 5.1 \\
\hline 560 to 619 & $20 \mathrm{mo}$ & 542 & 0.3 & -1.0 & 6.9 & 3.8 \\
\hline 620 to 679 & $22 \mathrm{mo}$ & 538 & -0.3 & 0.2 & 6.3 & 2.7 \\
\hline 680 to 749 & $24 \mathrm{mo}$ & 589 & -0.9 & -1.5 & 6.1 & 3.2 \\
\hline 750 to 929 & $28 \mathrm{mo}$ & 454 & 1.1 & -0.6 & 6.4 & 3.9 \\
\hline 930 to 1119 & $34 \mathrm{mo}$ & 466 & 0.3 & 0.2 & 6.4 & 2.6 \\
\hline 1120 to 1309 & $40 \mathrm{mo}$ & 469 & 1.6 & -0.2 & 4.8 & 0.3 \\
\hline 1310 to 1499 & $46 \mathrm{mo}$ & 448 & -1.1 & -0.3 & 7.6 & 4.2 \\
\hline 1500 to 1689 & $52 \mathrm{mo}$ & 446 & -1.1 & 0.0 & 7.3 & 3.9 \\
\hline 1690 to 1879 & $58 \mathrm{mo}$ & 492 & 0.0 & 1.7 & 8.0 & 4.3 \\
\hline 1880 to 2069 & $64 \mathrm{mo}$ & 416 & 1.1 & -0.1 & 6.9 & 3.6 \\
\hline 2070 to 2191 & $70 \mathrm{mo}$ & 295 & -0.4 & -0.6 & 5.4 & 2.4 \\
\hline Overall Q stats & & 10943 & 15.6 & 12.3 & 829.4 & 203.5 \\
\hline degrees of freedom & & & 17.0 & 21.0 & 24.0 & 24.0 \\
\hline p-value & & & 0.5551 & 0.9307 & $<0.01$ & $<0.01$ \\
\hline
\end{tabular}

Note: Absolute values of z1, z2, z3 or z4 larger than 2 indicate misfit of, respectively, mean, variance, skewness or kurtosis.

The next step involved fitting the parameter $v$ for skewness using the BCPE distribution with fixed parameter $\tau=2$ and keeping the degrees of freedom for the $\mu$ and $\sigma$ curves selected for Model 1. Table 50 shows the $G A I C(3)$ values for various degrees of freedom for the $v$ curve.

Table 50 Goodness-of-fit summary for models $B C P E\left(x=a g e^{0.15}, \operatorname{df}(\mu)=7, \operatorname{df}(\sigma)=5 \operatorname{df}(v)=?, \tau=2\right)$ for triceps skinfold-for-age for girls

\begin{tabular}{cccc}
\hline $\mathbf{d f}(\mathbf{v})$ & $\mathbf{G D}^{\mathbf{a}}$ & GAIC(3) $^{\mathbf{a}}$ & Total df \\
\hline $\mathbf{1}$ & 773.6 & 812.6 & 13 \\
$\mathbf{2}$ & 766.1 & 808.1 & 14 \\
$\mathbf{3}$ & 762.3 & 807.3 & 15 \\
$\mathbf{4}$ & 760.8 & 808.8 & 16 \\
$\mathbf{5}$ & 759.6 & 810.6 & 17 \\
$\mathbf{6}$ & 758.4 & 812.4 & 18 \\
$\mathbf{7}$ & 757.3 & 814.3 & 19 \\
\hline
\end{tabular}

GD, Global Deviance; GAIC(3), Generalized Akaike Information Criterion with penalty equal to 3 ;

${ }^{a}$ In excess of 43000 . 
The smallest $\operatorname{GAIC}(3)$ value corresponded to $\operatorname{df}(v)=3$. Using $\operatorname{df}(v)=3$, a re-search for the best $\operatorname{df}(\mu)$ and $\operatorname{df}(\sigma)$ was carried out. The smallest value of $\operatorname{GAIC}(3)$ was associated with $\operatorname{df}(\mu)=8$ and $\operatorname{df}(\sigma)=5$ (43807.0), very similar to those of the previously selected model (43807.3, Table 50). A new search for best $\lambda$ was carried out but there were no changes in the previous results and therefore the model was not further updated.

Model 2: $\operatorname{BCPE}\left(x=\operatorname{age}^{0.15}, \operatorname{df}(\mu)=7, \operatorname{df}(\sigma)=5, \operatorname{df}(v)=3, \tau=2\right)$

Figure 57 shows the fitting of the parameters $\mu, \sigma$ and $v$ for Model 2 with their respective sample estimates, that is, the median for $\mu$, the sample standard deviation of the Box-Cox transformed data for $\sigma$ and Box-Cox transform power for $v$.

Figures 58 and 59 show the distribution of the empirical minus fitted centile differences for the longitudinal and cross-sectional samples, respectively. There was some indication of bias only for the $95^{\text {th }}$ and $97^{\text {th }}$ centiles for ages between 3 and 24 months (Figure 58), yet the maximum difference between the empirical and the fitted centile was only $0.4 \mathrm{~mm}$. There was no indication of systematic bias for ages between 24 and 71 months (Figure 59).

The worm plots for this model (Figure 60) show that the adjustment for skewness was effective (none of the age groups presented U-shaped worms with Model 2). There were few age groups with Sshaped worms, suggesting some remaining kurtosis (e.g. $5 \mathrm{mo}$ ), but those were contained within the $95 \%$ confidence intervals.
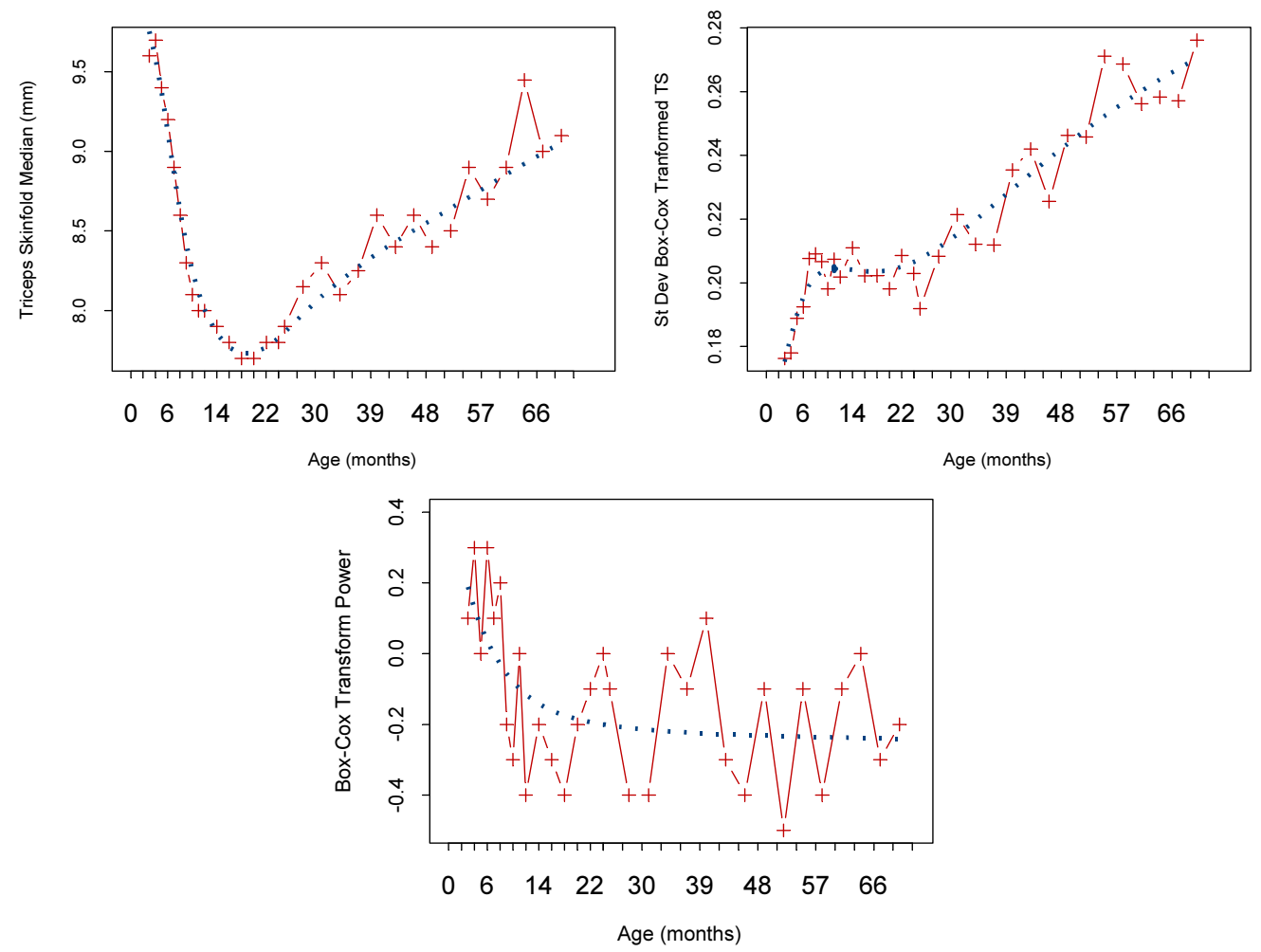

Figure 57 Fitting of the $\mu, \sigma$, and $v$ curves of Model 2 for triceps skinfold-for-age for girls from 3 to 71 months (dotted line) and their respective sample estimates (points with solid line) 

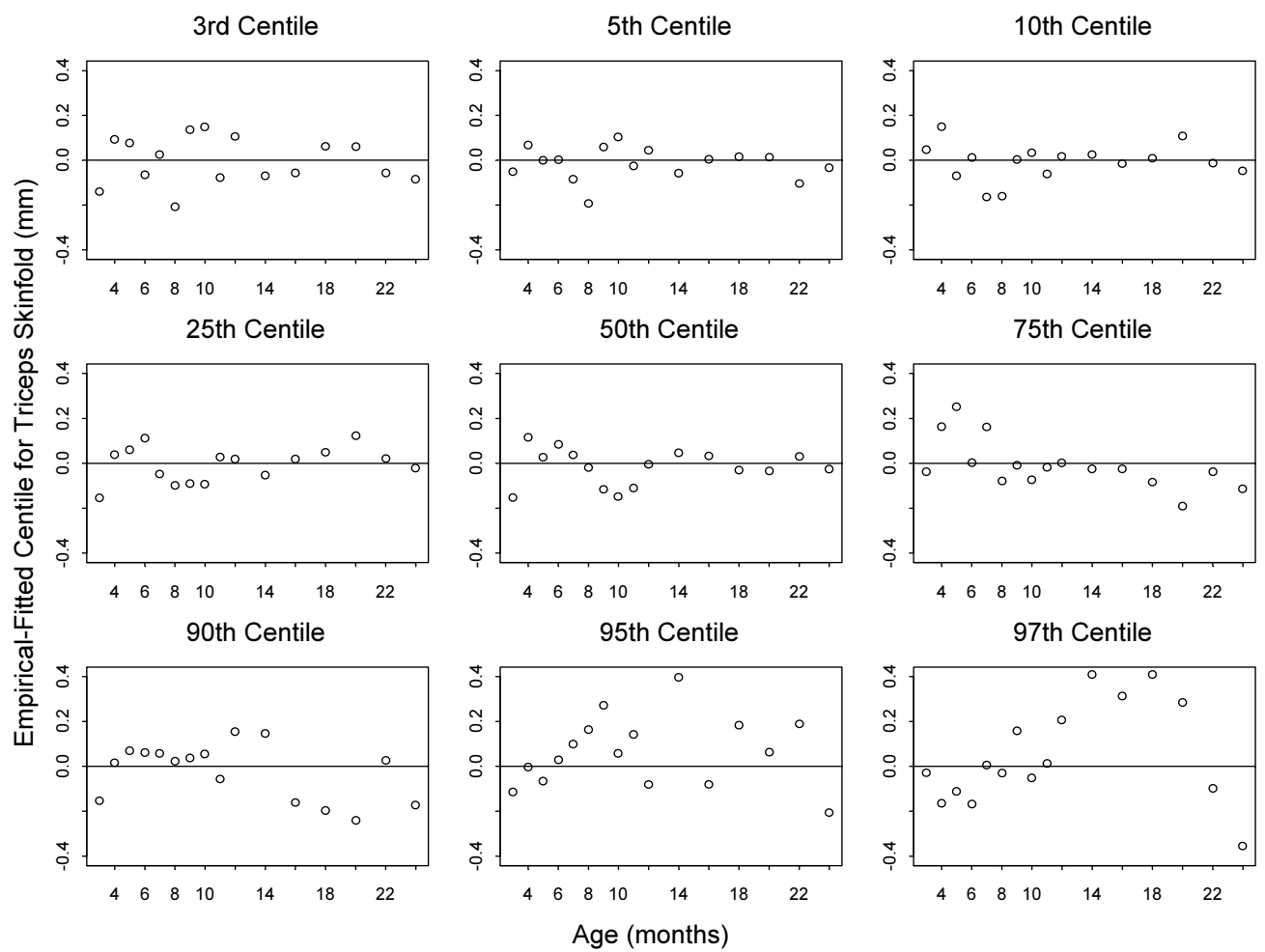

Figure 58 Centile residuals from fitting Model 2 for triceps skinfold-for-age from 3 to 24 months for girls
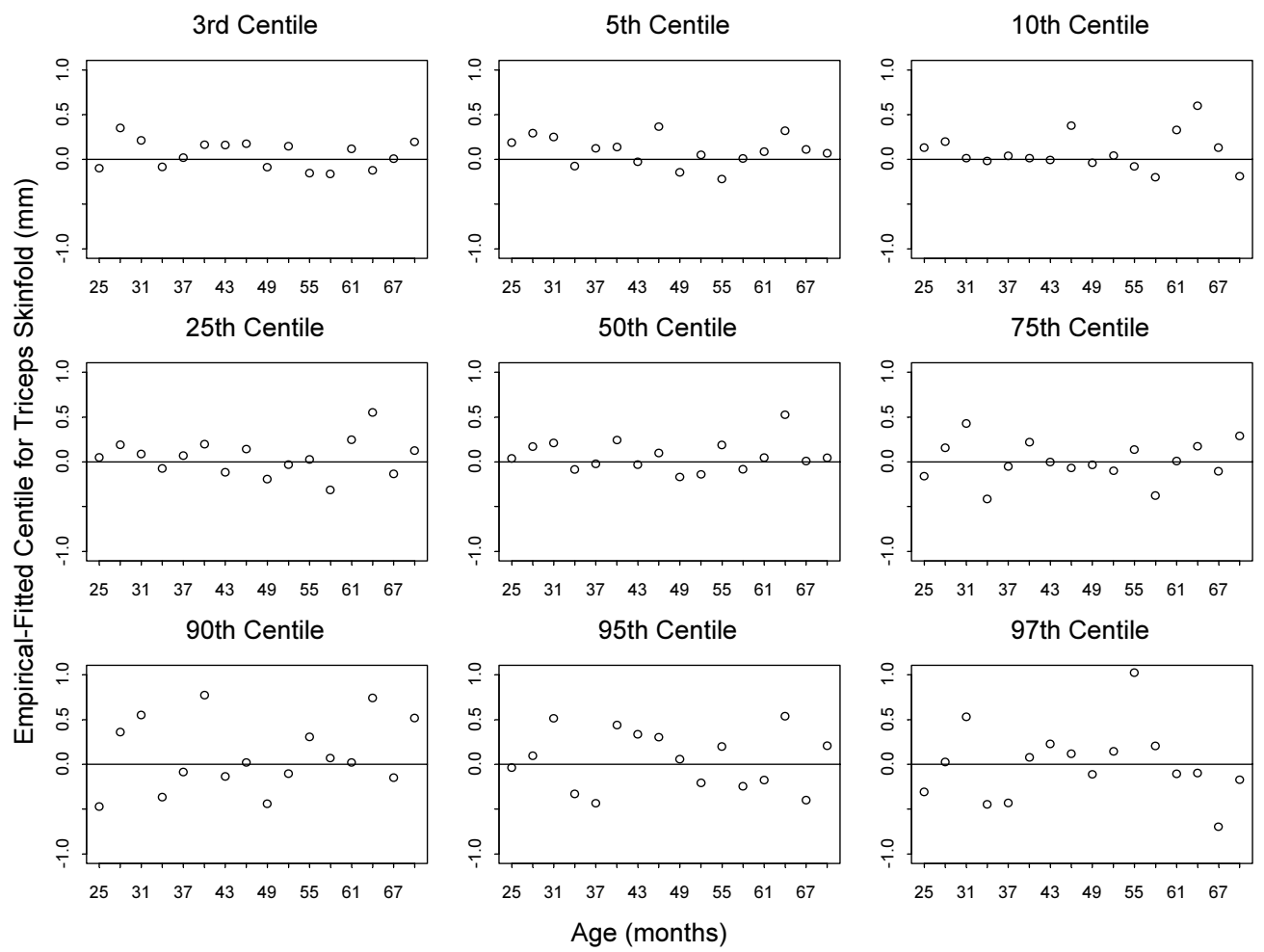

Figure 59 Centile residuals from fitting Model 2 for triceps skinfold-for-age from 24 to 71 months for girls 

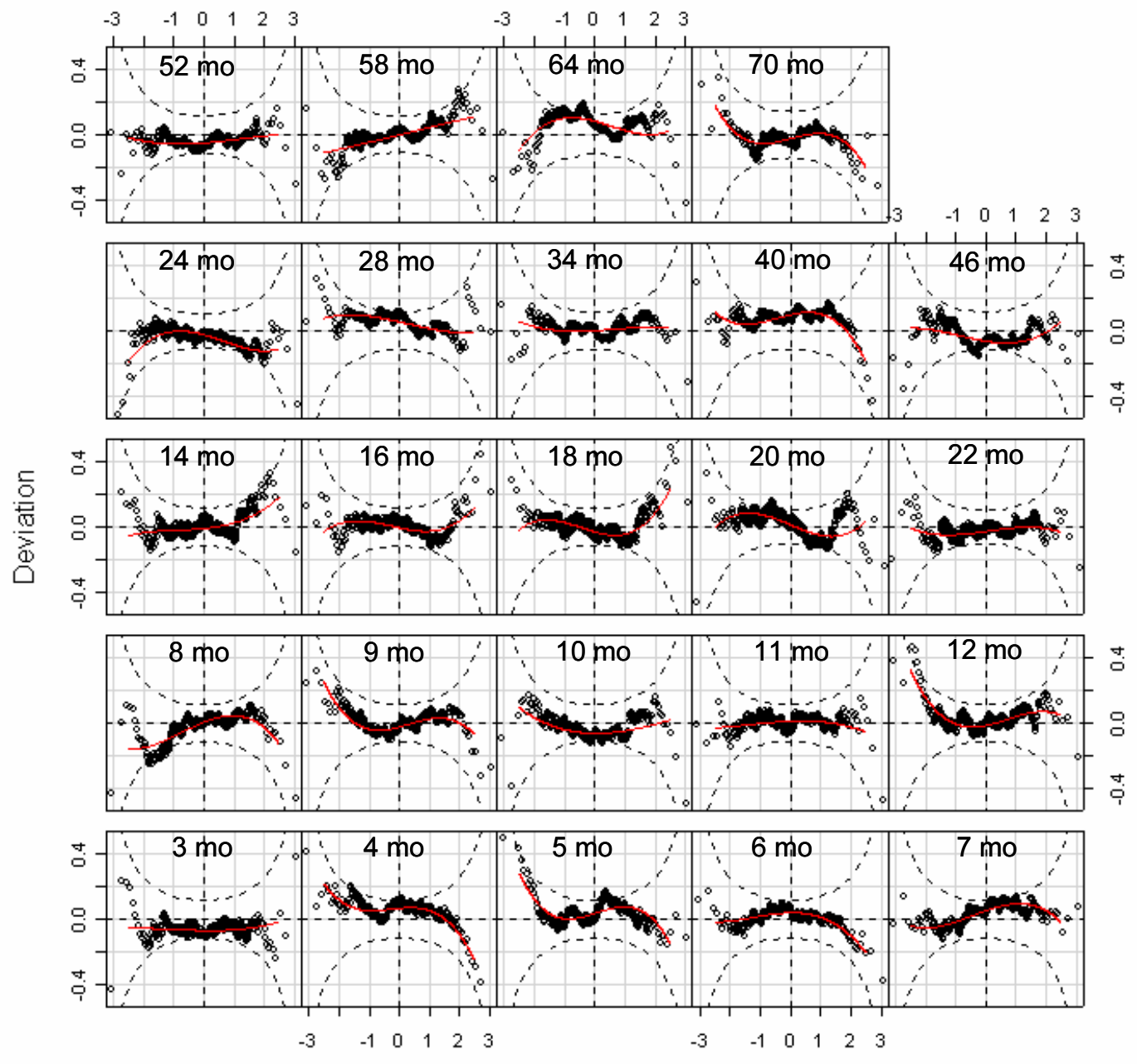

Unit normal quantile

Figure 60 Worm plots of z-scores for Model 2 for triceps skinfold-for-age for girls

The Q-test results from Model 2 are shown in Table 51. There was no indication of misfit of the mean or variance, as neither z1 nor z2 absolute values were larger than 2 . There were also no groups with z3 absolute value larger than 2 , indicating no remaining skewness. Only one age group presented an absolute value of $\mathrm{z} 4$ greater than 2 ( $5 \mathrm{mo}$ ), suggesting remaining kurtosis, but the overall test statistic for kurtosis had a p-value that was not significant at the 5\% level. The overall Q-test p-values were all non-significant, indicating an adequate fit of the girls' triceps skinfold-for-age curves and providing no justification for increasing the complexity of the model by adjusting for kurtosis (modelling $\tau$ ).

Table 52 presents observed percentages with triceps skinfolds below the fitted centiles. There was no detectable pattern suggesting systematic bias, even though some detectable deviations occur sporadically (e.g. only $0.9 \%$ of children have triceps skinfolds below the $3^{\text {rd }}$ centile at age 12 months). 
Table 51 Q-test for $z$-scores from Model $2\left[\operatorname{BCPE}\left(x=\operatorname{age}^{0.15}, \operatorname{df}(\mu)=7, \operatorname{df}(\sigma)=5, \operatorname{df}(v)=3, \tau=2\right)\right]$ for triceps skinfold-for-age for girls

\begin{tabular}{|c|c|c|c|c|c|c|}
\hline Age (days) & Group & $\mathbf{N}$ & $\mathrm{z1}$ & $\mathrm{z2}$ & $\mathbf{z 3}$ & $\mathrm{z} 4$ \\
\hline 83 to 99 & $3 \mathrm{mo}$ & 439 & -1.4 & 0.1 & 0.3 & 0.3 \\
\hline 100 to 129 & $4 \mathrm{mo}$ & 443 & 1.1 & -1.0 & -0.6 & -1.9 \\
\hline 130 to 159 & $5 \mathrm{mo}$ & 441 & 0.9 & -0.1 & 0.1 & -2.5 \\
\hline 160 to 189 & $6 \mathrm{mo}$ & 441 & 0.3 & -0.4 & -1.3 & -0.6 \\
\hline 190 to 219 & $7 \mathrm{mo}$ & 432 & 0.8 & 1.0 & -0.6 & -0.9 \\
\hline 220 to 249 & $8 \mathrm{mo}$ & 435 & -0.5 & 1.4 & -1.1 & -0.9 \\
\hline 250 to 279 & 9 mo & 437 & -0.1 & -0.1 & 1.0 & -1.9 \\
\hline 280 to 309 & $10 \mathrm{mo}$ & 442 & -1.3 & -0.2 & 1.1 & 0.1 \\
\hline 310 to 349 & $11 \mathrm{mo}$ & 474 & 0.1 & 0.1 & -0.4 & -0.1 \\
\hline 350 to 379 & $12 \mathrm{mo}$ & 451 & 0.3 & -0.1 & 1.5 & -1.3 \\
\hline 380 to 439 & $14 \mathrm{mo}$ & 443 & 0.0 & 1.0 & 0.5 & 0.7 \\
\hline 440 to 499 & $16 \mathrm{mo}$ & 443 & 0.1 & -0.2 & 0.5 & 1.1 \\
\hline 500 to 559 & $18 \mathrm{mo}$ & 467 & -0.2 & 0.1 & 1.2 & 1.6 \\
\hline 560 to 619 & $20 \mathrm{mo}$ & 542 & 0.3 & -1.1 & 0.0 & 1.5 \\
\hline 620 to 679 & $22 \mathrm{mo}$ & 538 & -0.6 & 0.5 & 0.0 & -0.5 \\
\hline 680 to 749 & $24 \mathrm{mo}$ & 589 & -1.1 & -0.6 & -1.4 & 1.6 \\
\hline 750 to 929 & $28 \mathrm{mo}$ & 454 & 1.0 & -0.9 & -0.1 & 0.4 \\
\hline 930 to 1119 & $34 \mathrm{mo}$ & 466 & 0.2 & 0.1 & 0.3 & -0.3 \\
\hline 1120 to 1309 & $40 \mathrm{mo}$ & 469 & 1.6 & -0.1 & -1.0 & -1.4 \\
\hline 1310 to 1499 & $46 \mathrm{mo}$ & 448 & -1.0 & -0.3 & 0.8 & 0.6 \\
\hline 1500 to 1689 & $52 \mathrm{mo}$ & 446 & -1.0 & 0.2 & 0.5 & -0.1 \\
\hline 1690 to 1879 & $58 \mathrm{mo}$ & 492 & -0.1 & 1.4 & 0.1 & 0.1 \\
\hline 1880 to 2069 & $64 \mathrm{mo}$ & 416 & 1.3 & -0.4 & -1.0 & 1.2 \\
\hline 2070 to 2191 & $70 \mathrm{mo}$ & 295 & -0.4 & -0.2 & 0.1 & -1.7 \\
\hline Overall Q stats & & 10943 & 16.0 & 10.4 & 15.6 & 33.7 \\
\hline degrees of freedom & & & 17.0 & 21.0 & 21.0 & 24.0 \\
\hline p-value & & & 0.5206 & 0.9736 & 0.7938 & 0.0899 \\
\hline
\end{tabular}

Note: Absolute values of z1, z2, z3 or z4 larger than 2 indicate misfit of, respectively, mean, variance, skewness or kurtosis.

The model $\operatorname{BCPE}\left(x=a g e^{0.15}, \operatorname{df}(\mu)=7, \operatorname{df}(\sigma)=5, \operatorname{df}(v)=3, \tau=2\right)$ was considered adequate and thus selected for constructing the triceps skinfold-for-age growth curves for girls. It adjusts only for skewness, since there was no evidence of significant kurtosis. The fitted centile curves and empirical centiles are shown in Figures 61 to 64. 


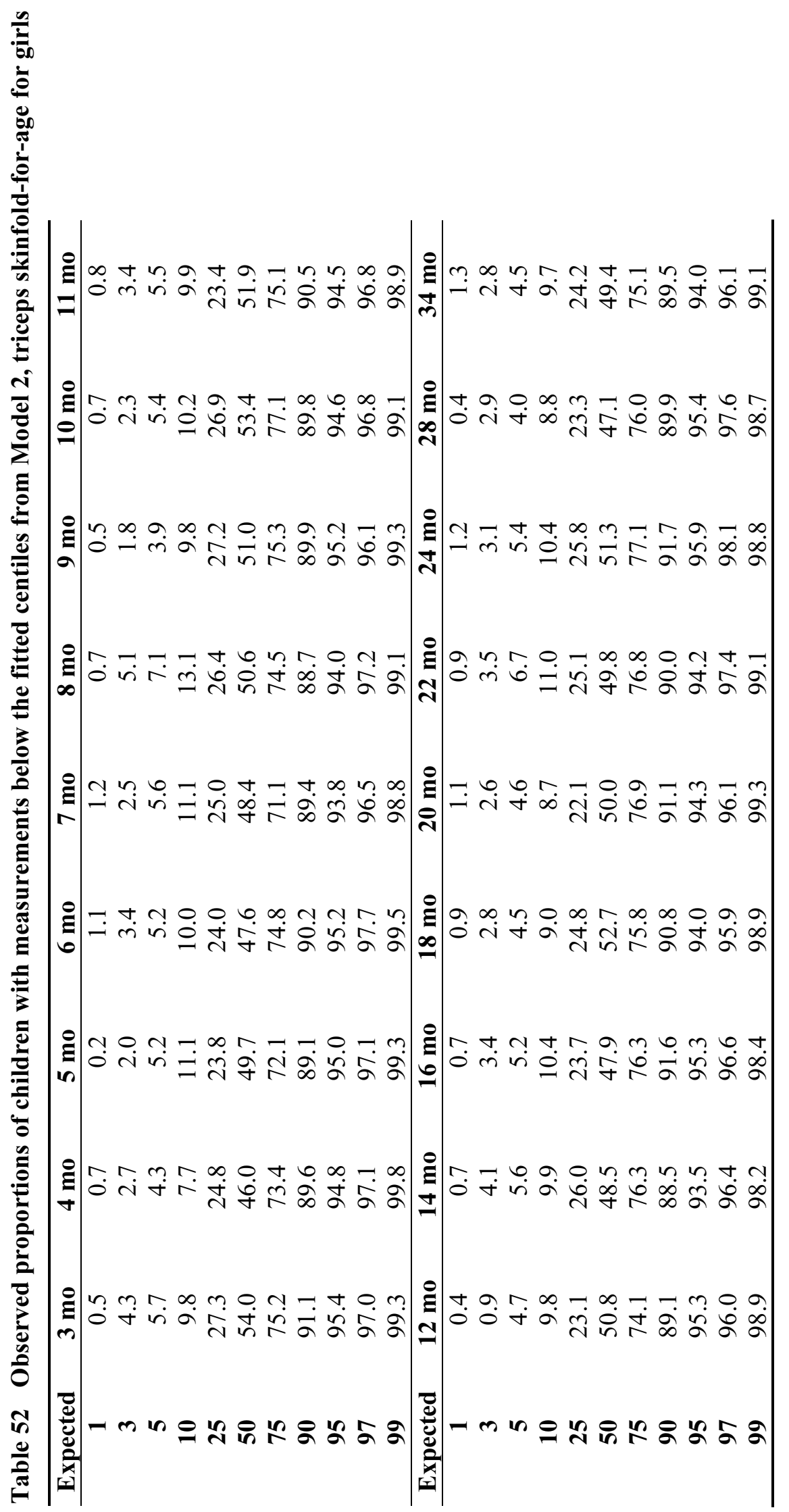




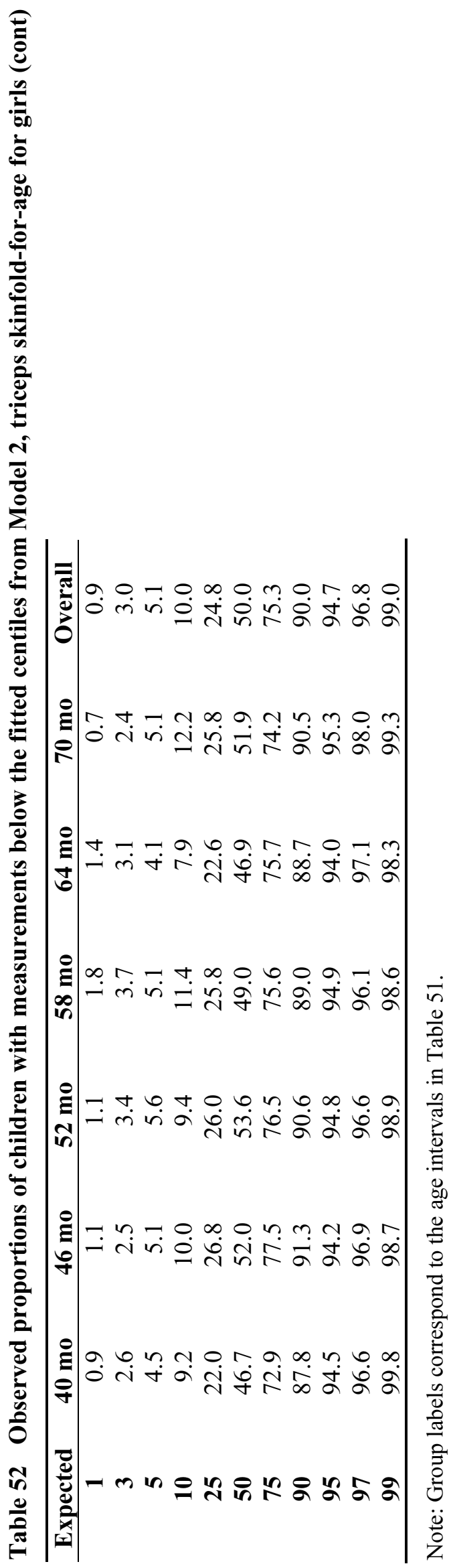




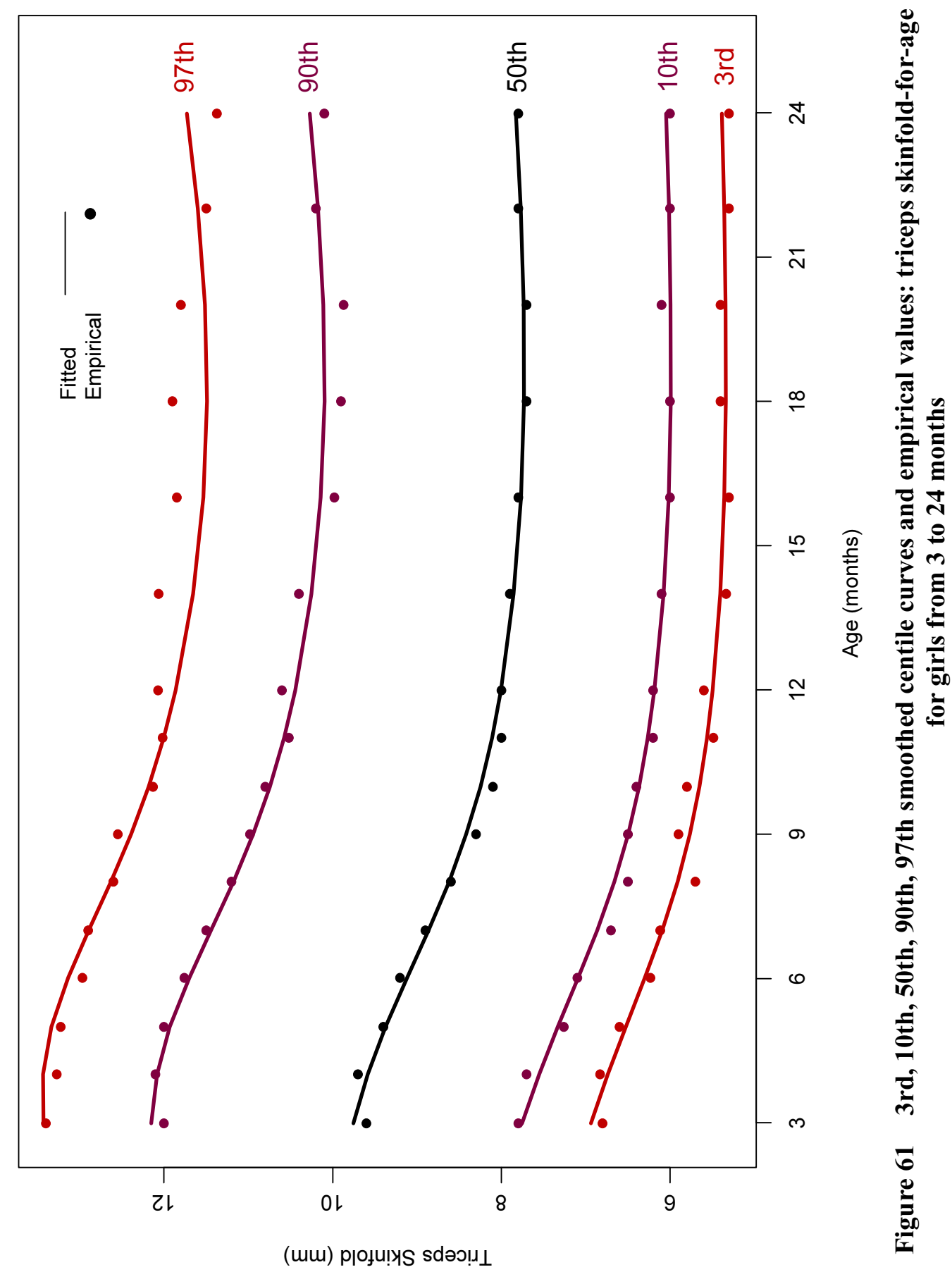




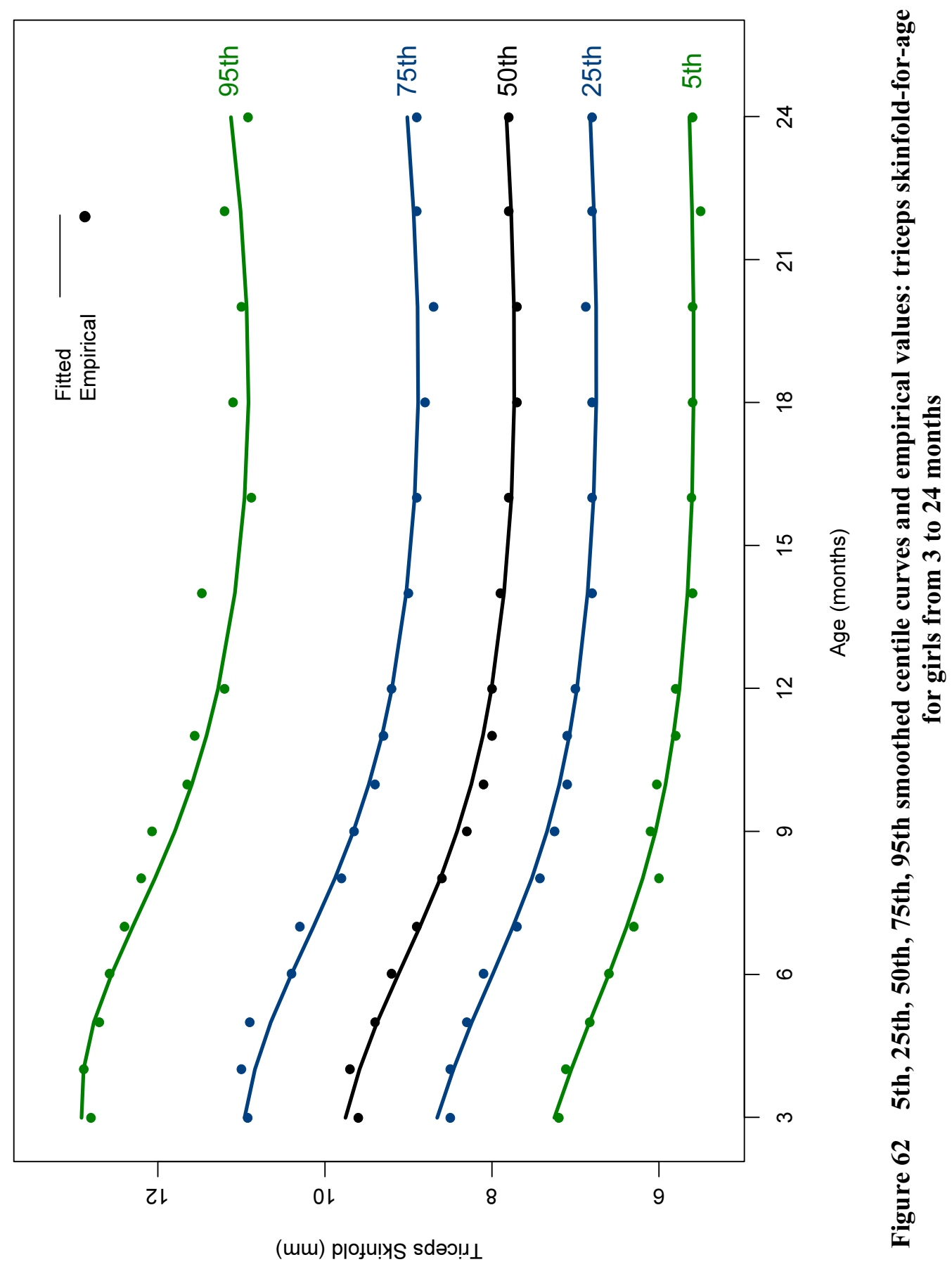




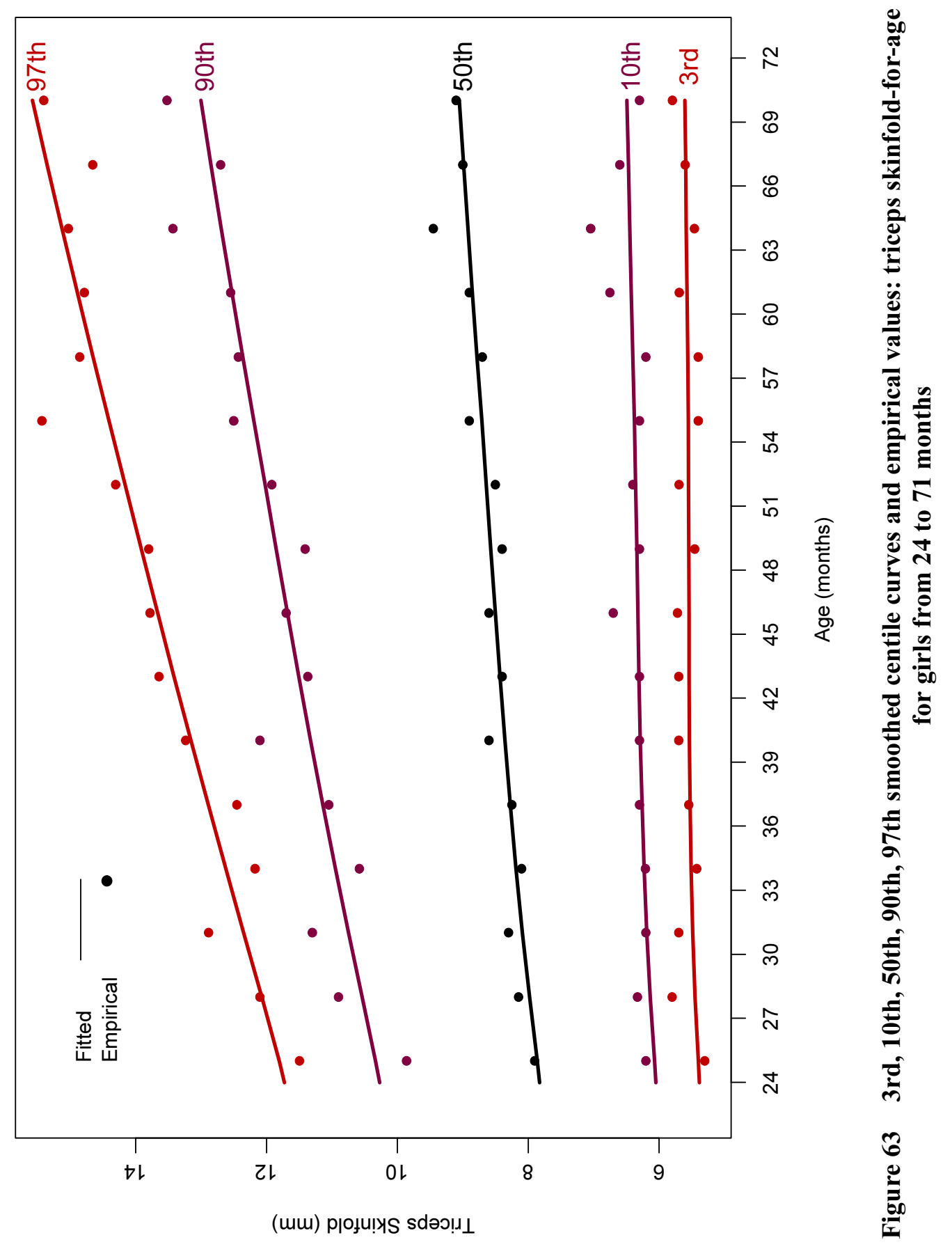




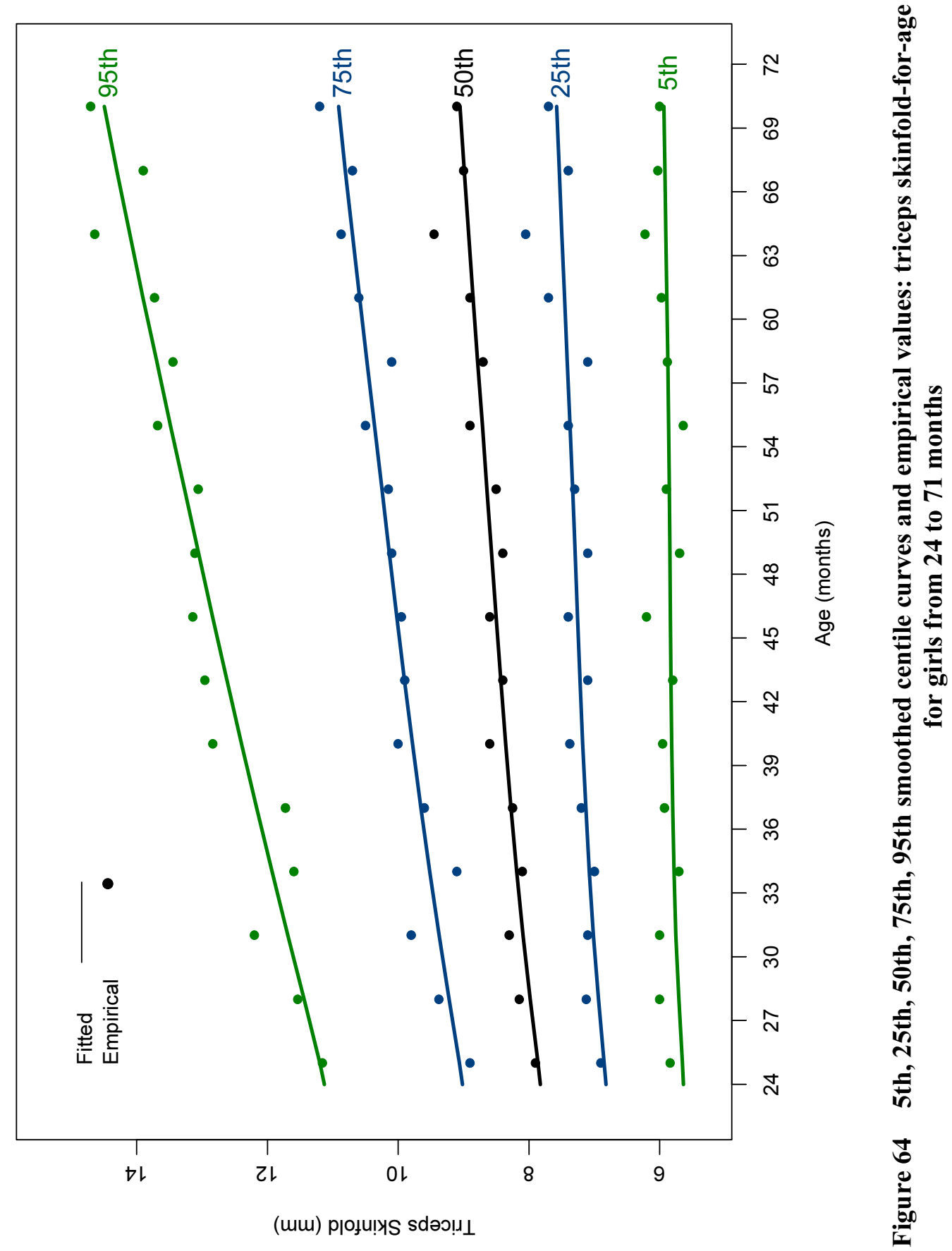




\subsubsection{WHO standards}

This section presents the final WHO triceps skinfold-for-age z-score and percentile charts (Figures 65 and 66) and table (Table 53) for girls. 


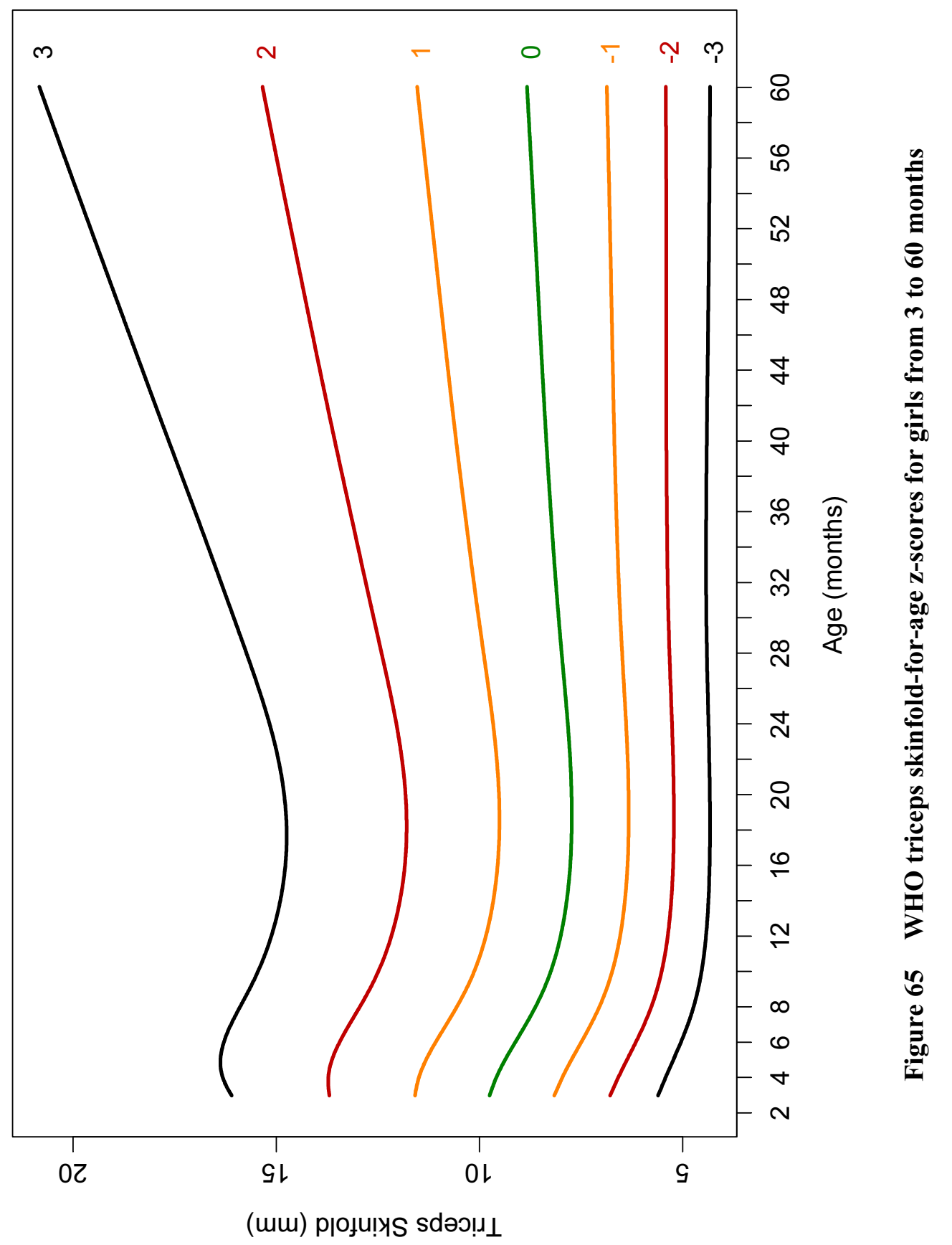




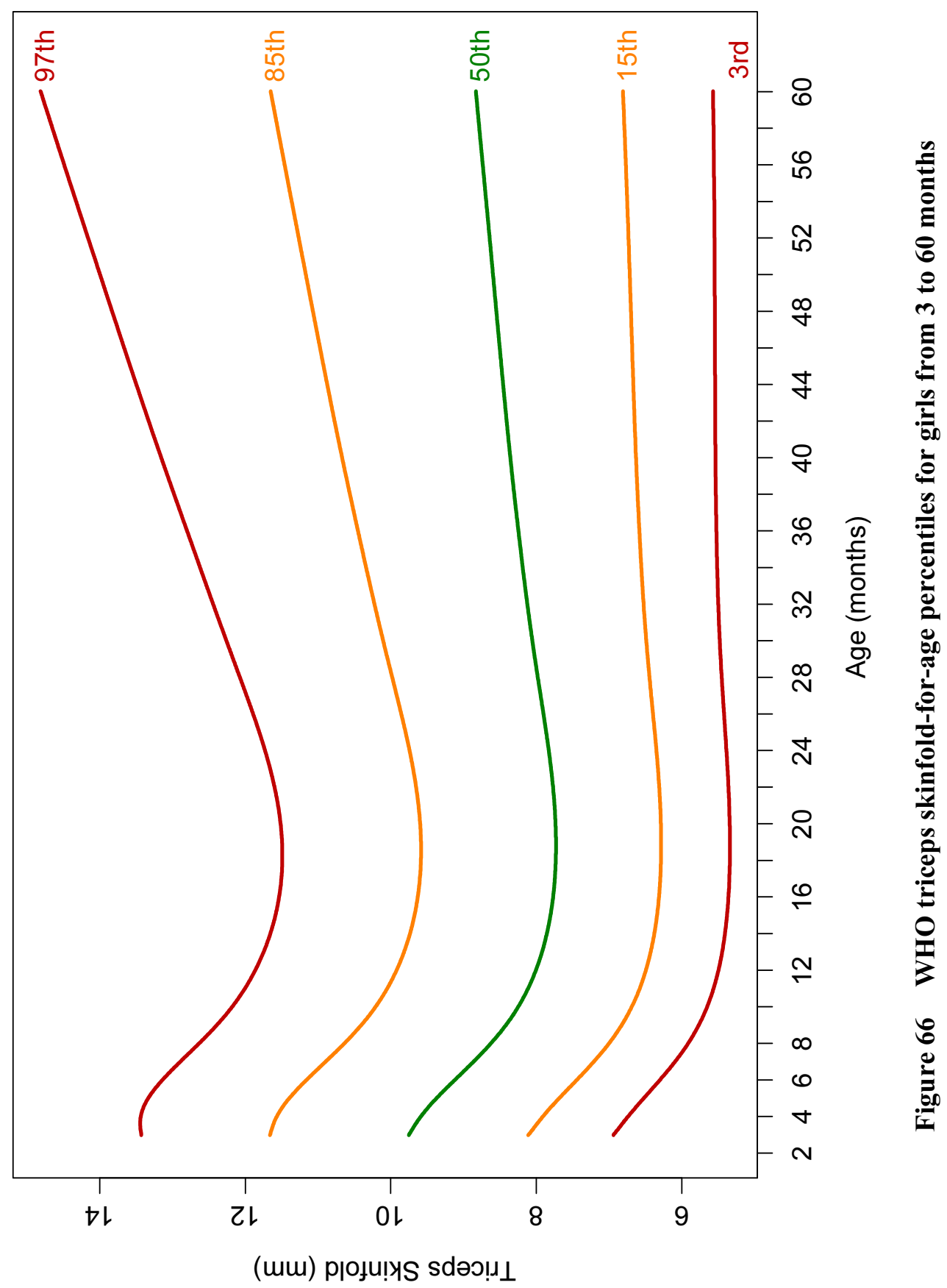




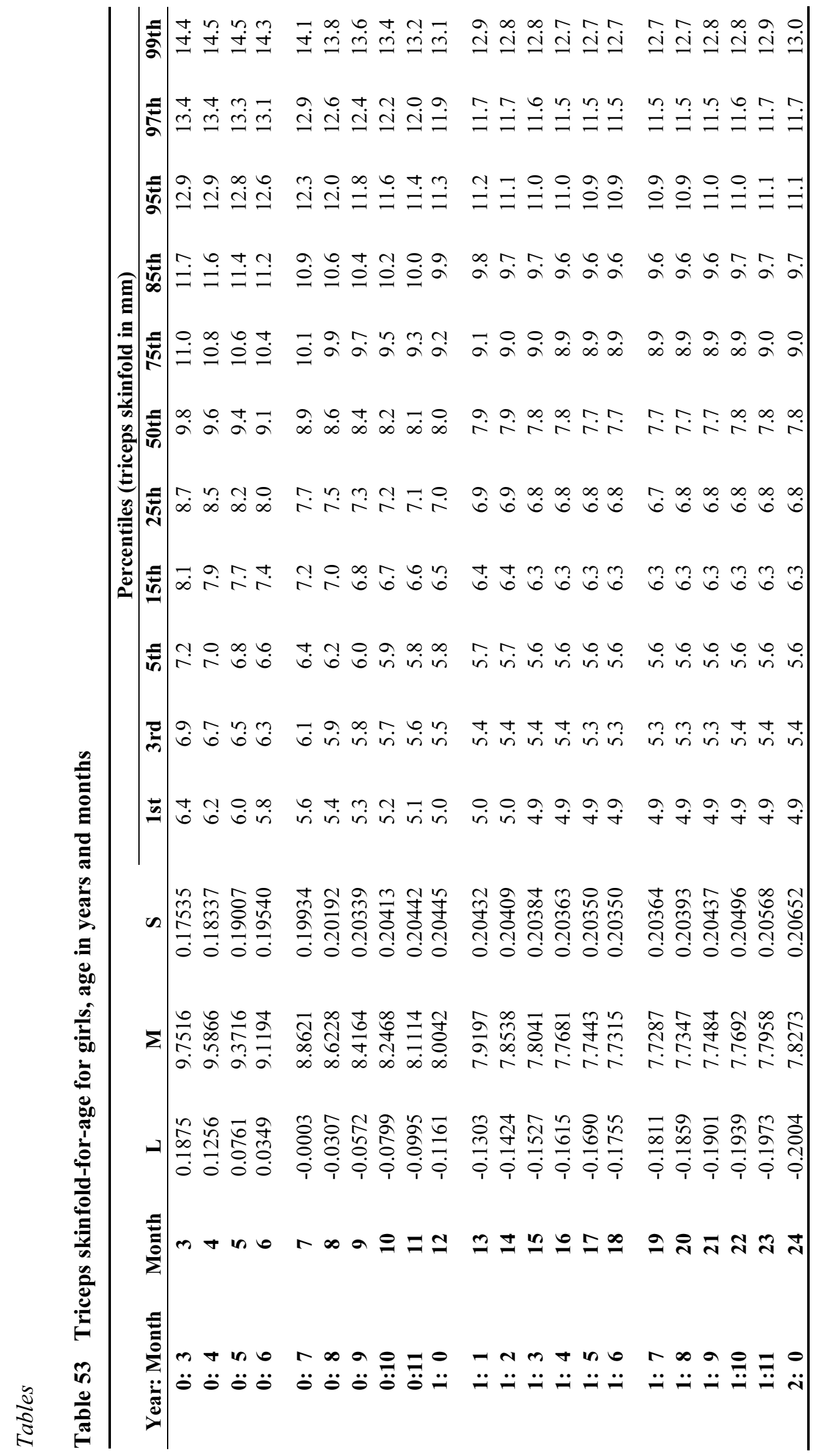




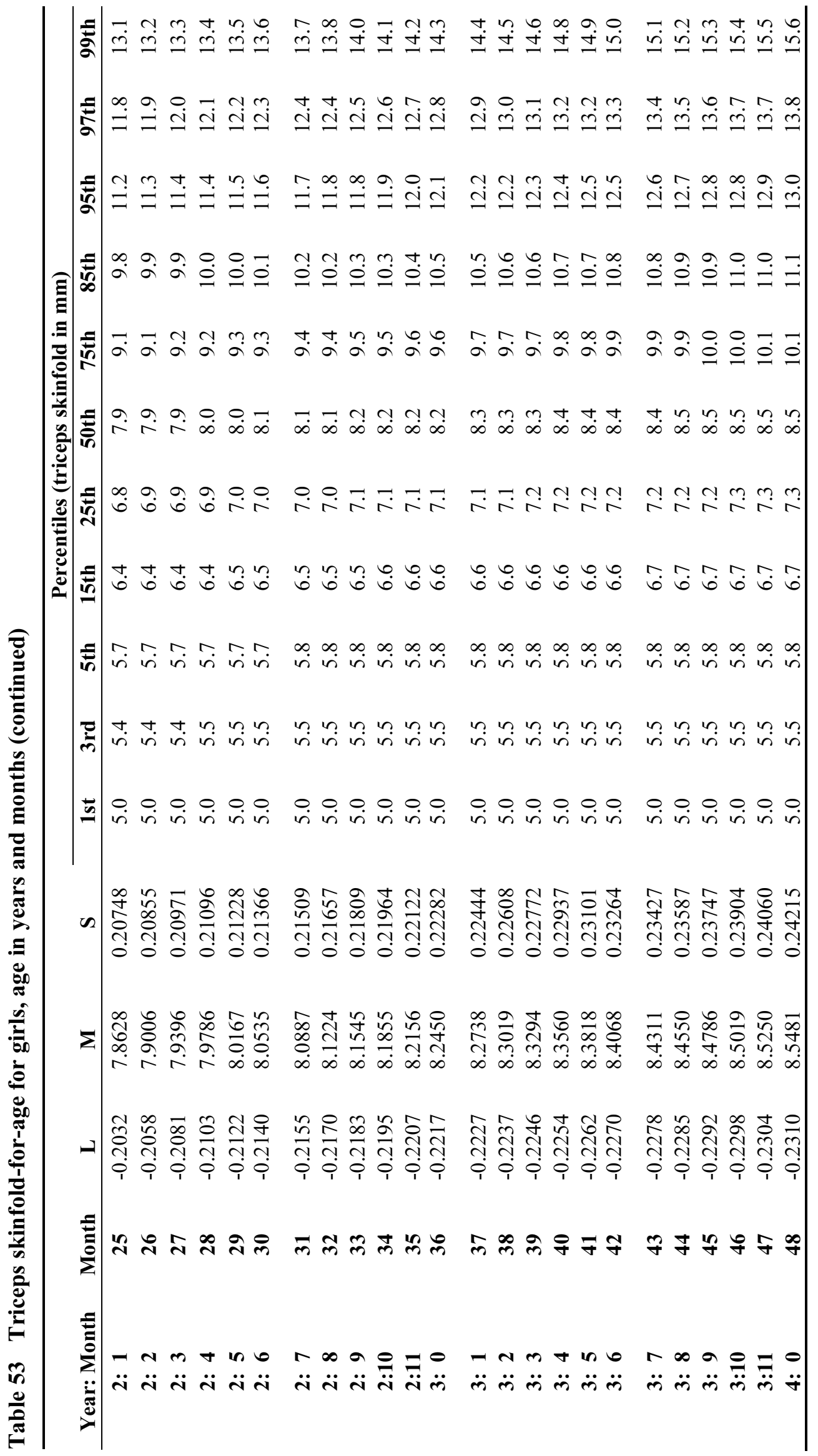




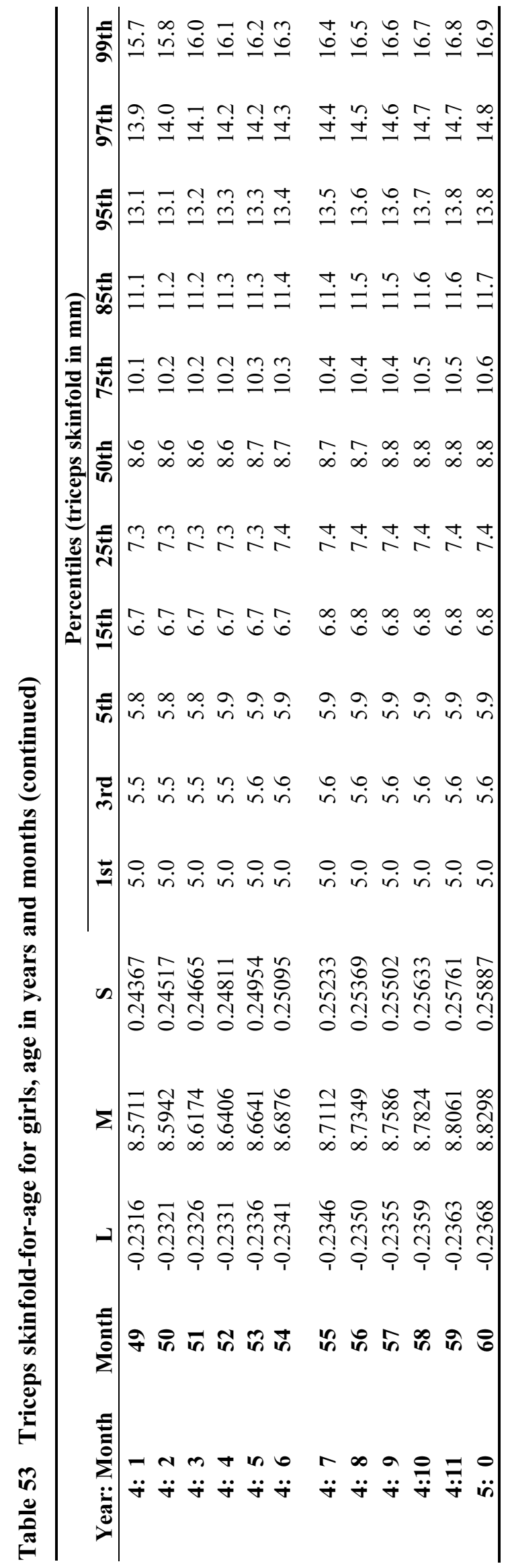




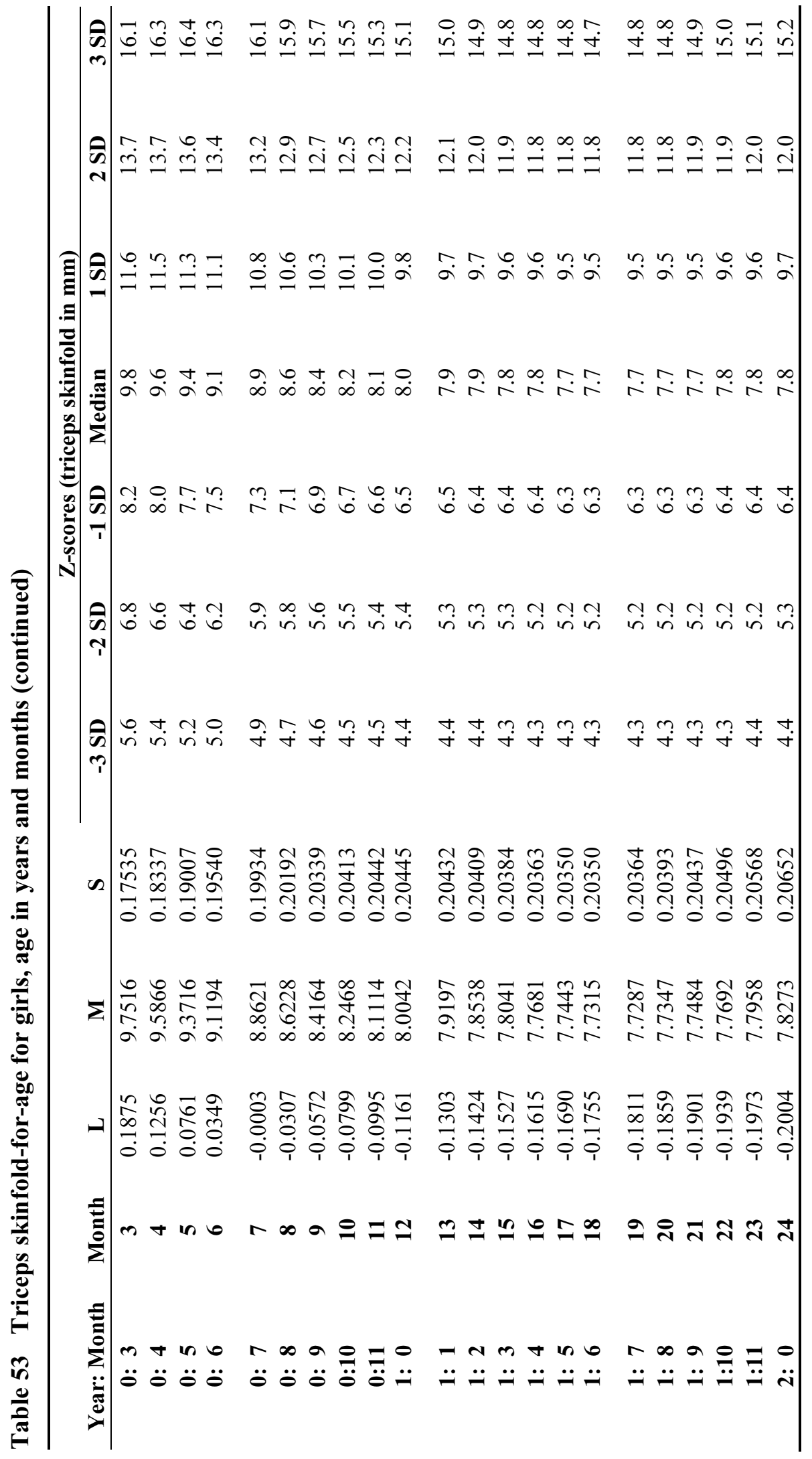




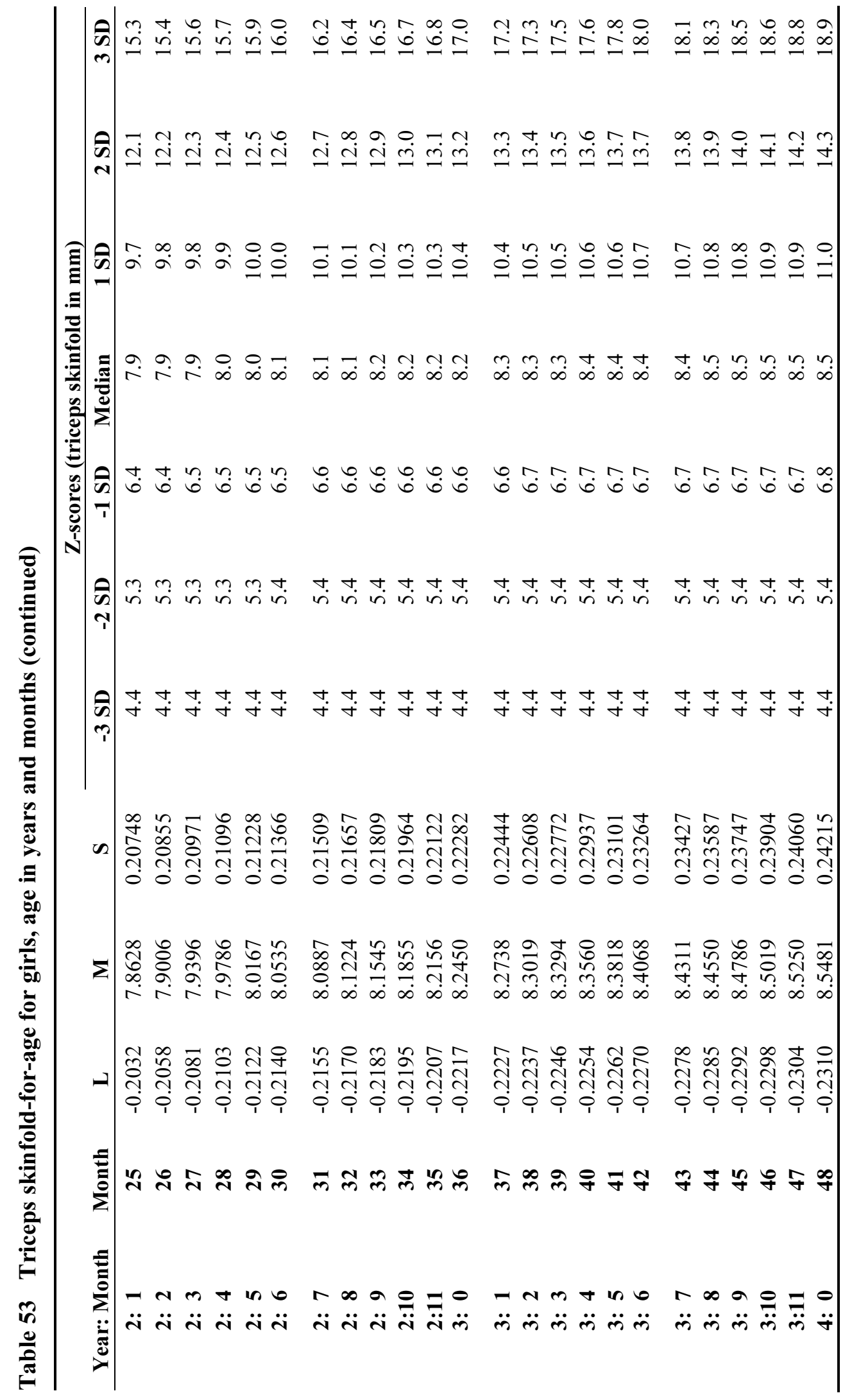




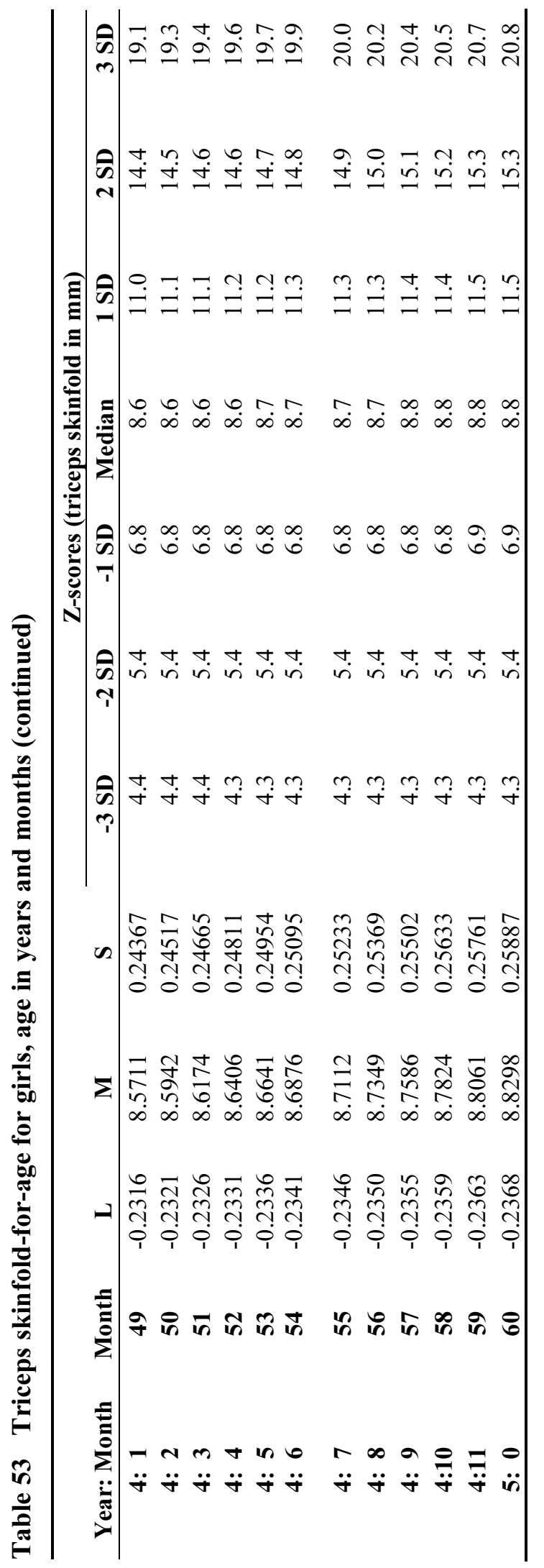




\subsection{Comparisons between boys and girls}

This section presents the triceps skinfold-for-age z-score comparisons between boys and girls for the WHO standards (Figure 67). 


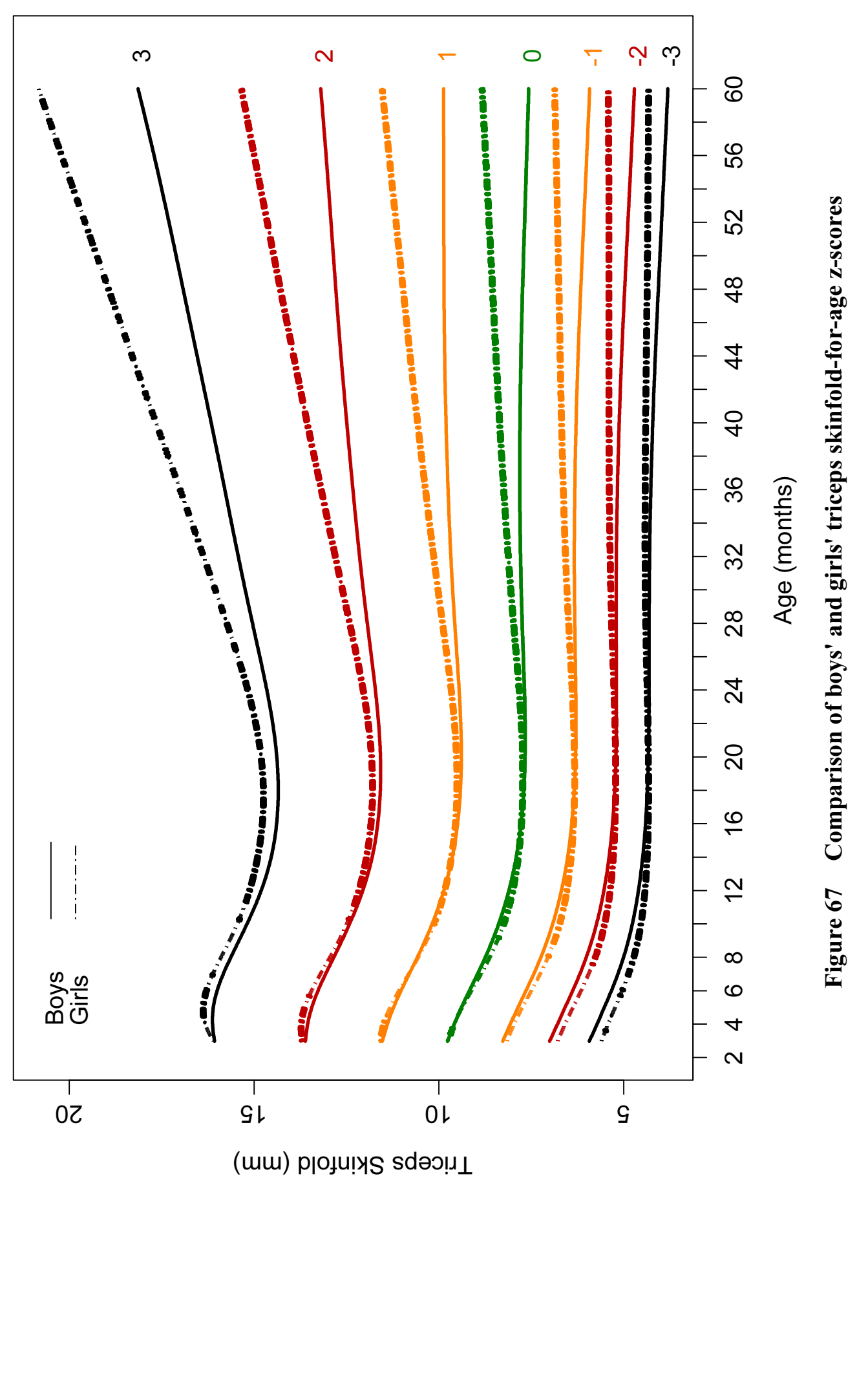





\section{CONSTRUCTION OF THE SUBSCAPULAR SKINFOLD-FOR-AGE STANDARDS}

\subsection{Indicator-specific methodology}

Similar steps to those described to select the best model for the head circumference-for-age, armcircumference-for-age and triceps skinfold-for-age growth curves were followed to select the best model to construct the subscapular skinfold-for-age growth standards. The diagnostic tools applied to evaluate and compare candidate models were the same. All data up to 71 months were used for modelling the subscapular skinfold-for-age growth curves and the standards afterwards truncated at 60 completed months to correct for the right-edge effect (Borghi et al., 2006).

\subsection{Subscapular skinfold-for-age for boys}

\subsubsection{Sample size}

There were a total of 10757 subscapular skinfold observations for boys. The longitudinal and crosssectional sample sizes by visit and age are presented in Tables 54 and 55. The measurement of subscapular skinfold started at 3 months of age (de Onis et al., 2004b).

Table 54 Longitudinal sample sizes for subscapular skinfold-for-age for boys

\begin{tabular}{|c|c|c|c|c|c|c|}
\hline Visit & 5 & 6 & 7 & 8 & 9 & 10 \\
\hline Age & $3 \mathrm{mo}$ & $4 \mathrm{mo}$ & $5 \mathrm{mo}$ & $6 \mathrm{mo}$ & $7 \mathrm{mo}$ & $8 \mathrm{mo}$ \\
\hline $\mathrm{N}$ & 418 & 410 & 413 & 417 & 415 & 415 \\
\hline Visit & 11 & 12 & 13 & 14 & 15 & 16 \\
\hline Age & $9 \mathrm{mo}$ & $10 \mathrm{mo}$ & $11 \mathrm{mo}$ & $12 \mathrm{mo}$ & $14 \mathrm{mo}$ & $16 \mathrm{mo}$ \\
\hline $\mathrm{N}$ & 408 & 407 & 417 & 415 & 416 & 417 \\
\hline Visit & 17 & 18 & 19 & 20 & & \\
\hline Age & $18 \mathrm{mo}$ & $20 \mathrm{mo}$ & $22 \mathrm{mo}$ & $24 \mathrm{mo}$ & & \\
\hline $\mathrm{N}$ & 415 & 422 & 417 & 421 & & \\
\hline
\end{tabular}

Table 55 Cross-sectional sample sizes for subscapular skinfold-for-age for boys

\begin{tabular}{lccccccc}
\hline Age (mo) & $<\mathbf{1 8}$ & $\mathbf{1 8}-\mathbf{2 0}$ & $\mathbf{2 1 - 2 3}$ & $\mathbf{2 4 - 2 6}$ & $\mathbf{2 7 - 2 9}$ & $\mathbf{3 0 - 3 2}$ & $\mathbf{3 3 - 3 5}$ \\
$\mathrm{N}$ & 3 & 176 & 183 & 236 & 255 & 215 & 251 \\
\hline Age (mo) & $\mathbf{3 6}-\mathbf{3 8}$ & $\mathbf{3 9 - 4 1}$ & $\mathbf{4 2 - 4 4}$ & $\mathbf{4 5 - 4 7}$ & $\mathbf{4 8}-\mathbf{5 0}$ & $\mathbf{5 1 - 5 3}$ & $\mathbf{5 4 - 5 6}$ \\
$\mathrm{N}$ & 264 & 248 & 254 & 235 & 235 & 218 & 225 \\
\hline Age (mo) & $\mathbf{5 7 - 5 9}$ & $\mathbf{6 0 - 6 2}$ & $\mathbf{6 3}-\mathbf{6 5}$ & $\mathbf{6 6 - 6 8}$ & $\mathbf{6 9 - 7 1}$ & $>\mathbf{7 1}$ & \\
$\mathrm{N}$ & 239 & 228 & 216 & 216 & 213 & 4 & \\
\hline
\end{tabular}

\subsubsection{Model selection and results}

To search for the best value of the age-transformation power $\lambda$, the model $B C P E\left(x=\operatorname{age}^{\lambda}, \operatorname{df}(\mu)=9\right.$, $\operatorname{df}(\sigma)=4, \operatorname{df}(v)=4, \tau=2)$ was used as a starting point when constructing the subscapular skinfold-for-age growth curves. Table 56 shows the global deviance for values of $\lambda$ from 0.05 to 1 . Global deviance values were smallest for $\lambda 0.55$ to 0.75 and we selected $\lambda=0.65$. 
Table 56 Global deviance (GD) for models within the class $\operatorname{BCPE}\left(x=\operatorname{age}^{\lambda}, \operatorname{df}(\mu)=9, \operatorname{df}(\sigma)=4\right.$, $\operatorname{df}(v)=4, \tau=2)$ for subscapular skinfold-for-age for boys

\begin{tabular}{lcccccccccc}
\hline $\boldsymbol{\lambda}$ & $\mathbf{0 . 0 5}$ & $\mathbf{0 . 1 0}$ & $\mathbf{0 . 1 5}$ & $\mathbf{0 . 2 0}$ & $\mathbf{0 . 2 5}$ & $\mathbf{0 . 3 0}$ & $\mathbf{0 . 3 5}$ & $\mathbf{0 . 4 0}$ & $\mathbf{0 . 4 5}$ & $\mathbf{0 . 5 0}$ \\
$\mathrm{GD}^{\mathrm{a}}$ & 323.1 & 323.0 & 322.8 & 322.7 & 322.6 & 322.5 & 322.4 & 322.4 & 322.3 & 322.3 \\
\hline $\boldsymbol{\lambda}$ & $\mathbf{0 . 5 5}$ & $\mathbf{0 . 6 0}$ & $\mathbf{0 . 6 5}$ & $\mathbf{0 . 7 0}$ & $\mathbf{0 . 7 5}$ & $\mathbf{0 . 8 0}$ & $\mathbf{0 . 8 5}$ & $\mathbf{0 . 9 0}$ & $\mathbf{0 . 9 5}$ & $\mathbf{1 . 0 0}$ \\
$\mathrm{GD}^{\mathrm{a}}$ & 322.2 & 322.2 & 322.2 & 322.2 & 322.2 & 322.3 & 322.5 & 322.8 & 323.3 & 324.1 \\
\hline
\end{tabular}

${ }^{\mathrm{a}}$ In excess of 33000 .

The search for the best $\operatorname{df}(\mu)$ and $\operatorname{df}(\sigma)$ followed, fixing $\lambda=0.65, \nu=1$ and $\tau=2$. All possible combinations with $\operatorname{df}(\mu)$ values ranging from 5 to 15 and $\operatorname{df}(\sigma)$ from 2 to 10 were considered and partial results are presented in Table 57. As for arm circumference-for-age and triceps skinfold-for-age, we considered only the criterion $\operatorname{GAIC}(3)$ to select the best combination. The model with $\operatorname{df}(\mu)=7$ and $\operatorname{df}(\sigma)=3$ provided the smallest value of $\operatorname{GAIC}(3)$, but the model with the smoother $\mu$ curve, with $\operatorname{df}(\mu)=6$ and $\operatorname{df}(\sigma)=3$, yielded similar $\operatorname{GAIC}(3)$ values. The latter model was thus selected for further evaluation.

Table 57 Goodness-of-fit summary for models using the BCPE distribution with fixed $v=1$ and $\tau=\mathbf{2}$ for subscapular skinfold-for-age for boys

\begin{tabular}{|c|c|c|c|c|c|}
\hline $\operatorname{df}(\mu)$ & $\operatorname{df}(\sigma)$ & GD $^{\mathbf{a}}$ & $\mathrm{AIC}^{\mathbf{a}}$ & $\operatorname{GAIC}(3)^{\mathrm{a}}$ & Total df \\
\hline \multirow{5}{*}{5} & 2 & 1895.9 & 1909.9 & 1916.9 & 7 \\
\hline & 3 & 1891.3 & 1907.3 & 1915.3 & 8 \\
\hline & 4 & 1888.9 & 1906.9 & 1915.9 & 9 \\
\hline & 5 & 1886.0 & 1906.0 & 1916.0 & 10 \\
\hline & 6 & 1882.7 & 1904.7 & 1915.7 & 11 \\
\hline \multirow{5}{*}{6} & 2 & 1888.3 & 1904.3 & 1912.3 & 8 \\
\hline & 3 & 1883.8 & 1901.8 & 1910.8 & 9 \\
\hline & 4 & 1881.4 & 1901.4 & 1911.4 & 10 \\
\hline & 5 & 1878.6 & 1900.6 & 1911.6 & 11 \\
\hline & 6 & 1875.5 & 1899.6 & 1911.6 & 12 \\
\hline \multirow{5}{*}{7} & 2 & 1885.0 & 1903.0 & 1912.0 & 9 \\
\hline & 3 & 1880.5 & 1900.5 & 1910.5 & 10 \\
\hline & 4 & 1878.1 & 1900.1 & 1911.1 & 11 \\
\hline & 5 & 1875.4 & 1899.4 & 1911.4 & 12 \\
\hline & 6 & 1872.5 & 1898.5 & 1911.5 & 13 \\
\hline \multirow{5}{*}{8} & 2 & 1882.8 & 1902.8 & 1912.8 & 10 \\
\hline & 3 & 1878.3 & 1900.3 & 1911.3 & 11 \\
\hline & 4 & 1875.9 & 1899.9 & 1911.9 & 12 \\
\hline & 5 & 1873.4 & 1899.4 & 1912.4 & 13 \\
\hline & 6 & 1870.5 & 1898.5 & 1912.5 & 14 \\
\hline \multirow{5}{*}{9} & 2 & 1881.0 & 1903.0 & 1914.0 & 11 \\
\hline & 3 & 1876.5 & 1900.5 & 1912.5 & 12 \\
\hline & 4 & 1874.2 & 1900.2 & 1913.2 & 13 \\
\hline & 5 & 1871.6 & 1899.6 & 1913.6 & 14 \\
\hline & 6 & 1868.9 & 1898.9 & 1913.9 & 15 \\
\hline
\end{tabular}

GD, Global Deviance; AIC, Akaike Information Criterion;

GAIC(3), Generalized AIC with penalty equal to 3;

${ }^{\mathrm{a}}$ In excess of 33000 . 
Model 1: $\operatorname{BCPE}\left(x=\operatorname{age}^{0.65}, \operatorname{df}(\mu)=6, \operatorname{df}(\sigma)=3, v=1, \tau=2\right)$

This model was inadequate with evidence of residual skewness in both the worm plots (Figure 68) and the Q-test results (Table 58). The worms presented U-shapes in all age groups and the Q-test results (Table 58) showed all groups with absolute values of $\mathrm{z} 3$ higher than 2. Most of the age groups also presented absolute values of $\mathrm{z} 4$ higher than 2 , indicating residual kurtosis. The overall tests for skewness and kurtosis also were significant ( $p$-values $<0.01$ ).
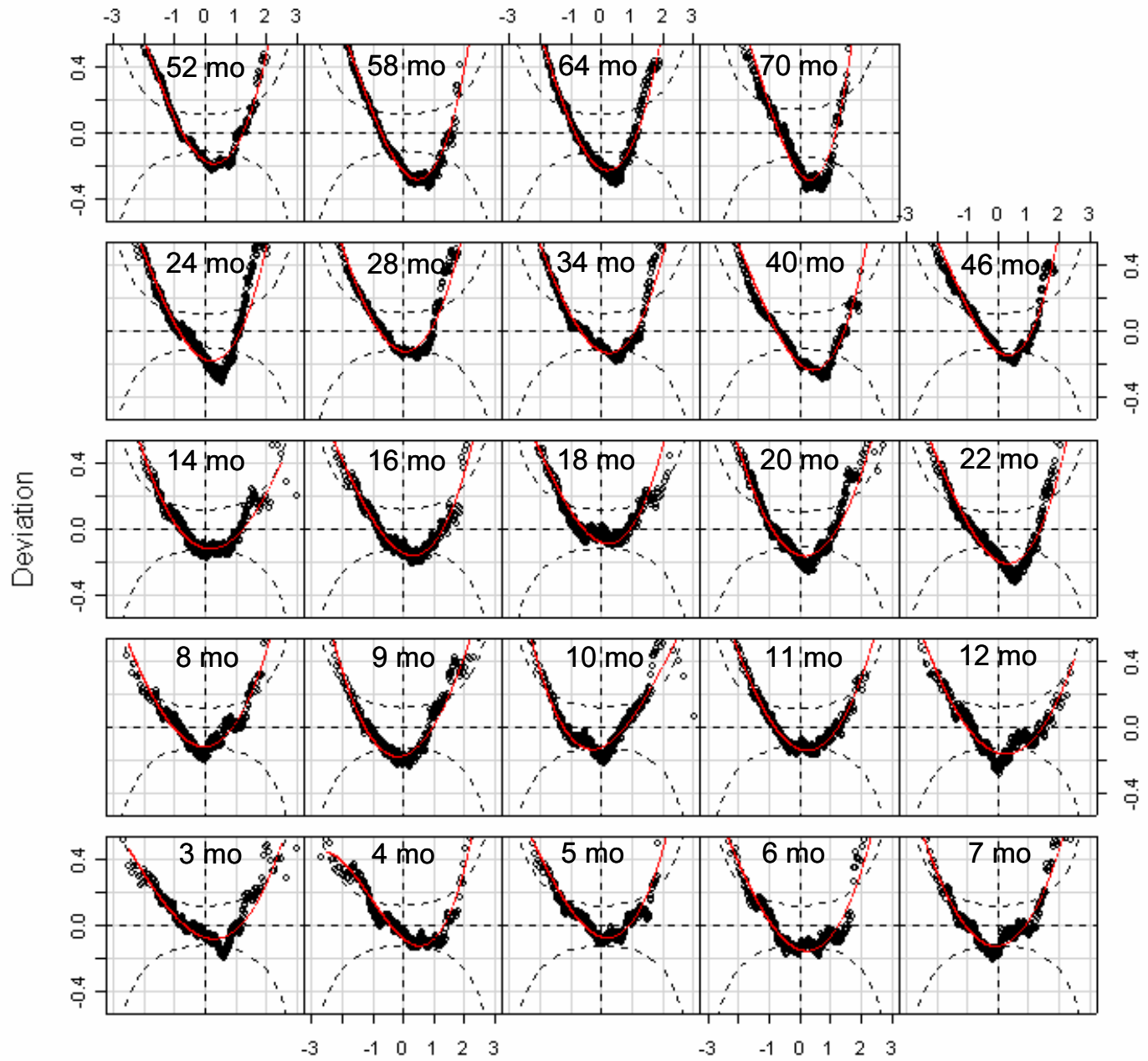

Unit normal quantile

Figure 68 Worm plots of z-scores for Model 1 for subscapular skinfold-for-age for boys 
Table 58 Q-test for $z$-scores from Model $1\left[\operatorname{BCPE}\left(x=a g e^{0.65}, \operatorname{df}(\mu)=6, \operatorname{df}(\sigma)=3, v=1, \tau=2\right)\right]$ for subscapular skinfold-for-age for boys

\begin{tabular}{|c|c|c|c|c|c|c|}
\hline Age (days) & Group & $\mathbf{N}$ & $\mathrm{z} 1$ & $\mathbf{z 2}$ & $\mathbf{z 3}$ & $\mathrm{z4}$ \\
\hline 79 to 99 & $3 \mathrm{mo}$ & 414 & 0.3 & -0.5 & 4.4 & 1.7 \\
\hline 100 to 129 & $4 \mathrm{mo}$ & 407 & 0.5 & -0.9 & 6.2 & 4.5 \\
\hline 130 to 159 & 5 mo & 410 & 1.2 & 0.3 & 6.0 & 3.2 \\
\hline 160 to 189 & $6 \mathrm{mo}$ & 411 & -0.7 & -0.3 & 6.0 & 3.2 \\
\hline 190 to 219 & $7 \mathrm{mo}$ & 406 & 0.1 & 1.2 & 5.6 & 2.4 \\
\hline 220 to 249 & $8 \mathrm{mo}$ & 420 & 0.0 & 1.2 & 5.5 & 2.6 \\
\hline 250 to 279 & 9 mo & 391 & -0.7 & 1.3 & 5.6 & 1.8 \\
\hline 280 to 309 & $10 \mathrm{mo}$ & 399 & -0.1 & 1.3 & 4.8 & 0.3 \\
\hline 310 to 349 & $11 \mathrm{mo}$ & 460 & -0.1 & -0.9 & 6.3 & 2.9 \\
\hline 350 to 379 & $12 \mathrm{mo}$ & 410 & -1.1 & -0.9 & 4.9 & 1.8 \\
\hline 380 to 439 & $14 \mathrm{mo}$ & 418 & -0.1 & -1.2 & 5.2 & 1.3 \\
\hline 440 to 499 & $16 \mathrm{mo}$ & 415 & -0.3 & -0.6 & 6.4 & 3.5 \\
\hline 500 to 559 & $18 \mathrm{mo}$ & 440 & 1.2 & 0.3 & 7.2 & 5.1 \\
\hline 560 to 619 & $20 \mathrm{mo}$ & 523 & -0.3 & -0.6 & 7.0 & 2.5 \\
\hline 620 to 679 & $22 \mathrm{mo}$ & 547 & -0.9 & -0.6 & 8.0 & 4.2 \\
\hline 680 to 749 & $24 \mathrm{mo}$ & 591 & -0.2 & 0.6 & 8.8 & 4.6 \\
\hline 750 to 929 & $28 \mathrm{mo}$ & 469 & 1.0 & 1.2 & 7.7 & 3.9 \\
\hline 930 to 1119 & $34 \mathrm{mo}$ & 505 & 1.0 & -0.2 & 7.7 & 4.1 \\
\hline 1120 to 1309 & $40 \mathrm{mo}$ & 509 & -0.9 & -1.3 & 8.8 & 5.8 \\
\hline 1310 to 1499 & $46 \mathrm{mo}$ & 505 & 1.1 & 0.4 & 9.1 & 5.8 \\
\hline 1500 to 1689 & $52 \mathrm{mo}$ & 482 & 0.0 & -0.4 & 8.4 & 4.7 \\
\hline 1690 to 1879 & $58 \mathrm{mo}$ & 480 & -1.0 & -1.7 & 9.6 & 6.0 \\
\hline 1880 to 2069 & $64 \mathrm{mo}$ & 460 & -0.2 & 0.8 & 9.1 & 5.3 \\
\hline 2070 to 2249 & $70 \mathrm{mo}$ & 285 & 0.5 & 2.0 & 9.3 & 6.2 \\
\hline Overall Q stats & & 10757 & 11.5 & 23.0 & 1227.4 & 378.9 \\
\hline degrees of freedom & & & 18.0 & 22.0 & 24.0 & 24.0 \\
\hline p-value & & & 0.8718 & 0.4023 & $<0.01$ & $<0.01$ \\
\hline
\end{tabular}

Note: Absolute values of z1, z2, z3 or z4 larger than 2 indicate misfit of, respectively, mean, variance, skewness or kurtosis.

The next step involved fitting the parameter $v$ for skewness using the BCPE distribution with fixed parameter $\tau=2$ and keeping the degrees of freedom for the $\mu$ and $\sigma$ curves identical to those selected for Model 1. Table 59 shows the GAIC(3) values for various degrees of freedom for the $v$ curve. 
Table 59 Goodness-of-fit summary for models $B C P E\left(x=a g e^{0.65}, \operatorname{df}(\mu)=6, \operatorname{df}(\sigma)=3, \operatorname{df}(v)=?, \tau=2\right)$ for subscapular skinfold-for-age for boys

\begin{tabular}{cccc}
\hline $\mathbf{d f}(\mathbf{v})$ & $\mathbf{G D}^{\mathbf{a}}$ & $\mathbf{G A I C}^{\mathbf{3}} \mathbf{a}^{\mathbf{a}}$ & Total df \\
\hline $\mathbf{1}$ & 361.9 & 391.9 & 10 \\
$\mathbf{2}$ & 330.8 & 363.8 & 11 \\
$\mathbf{3}$ & 330.0 & 366.0 & 12 \\
$\mathbf{4}$ & 328.6 & 367.6 & 13 \\
$\mathbf{5}$ & 327.5 & 369.5 & 14 \\
$\mathbf{6}$ & 326.5 & 371.6 & 15 \\
$\mathbf{7}$ & 325.7 & 373.7 & 16 \\
\hline
\end{tabular}

GD, Global Deviance; GAIC(3), Generalized Akaike Information Criterion with penalty equal to 3 ;

${ }^{\text {a }}$ In excess of 33000 .

The smallest $\operatorname{GAIC}(3)$ value corresponded to $\mathrm{df}(v)=2$. Fixing $\mathrm{df}(v)=2$, a re-search for the best $\operatorname{df}(\mu)$ and $\operatorname{df}(\sigma)$ was carried out. The summary of the results is shown in Table 60.

Table 60 Goodness-of-fit summary for models using the BCPE distribution with $\mathrm{df}(\mathrm{v})=2$ and $\tau=\mathbf{2}$ for subscapular skinfold-for-age for boys

\begin{tabular}{|c|c|c|c|c|c|}
\hline $\operatorname{df}(\mu)$ & $\operatorname{df}(\sigma)$ & GD $^{\mathbf{a}}$ & $\mathrm{AIC}^{\mathbf{a}}$ & GAIC(3) & Total df \\
\hline \multirow{5}{*}{5} & 1 & 372.5 & 388.5 & 396.5 & 8 \\
\hline & 2 & 339.4 & 357.4 & 366.4 & 9 \\
\hline & 3 & 338.7 & 358.7 & 368.7 & 10 \\
\hline & 4 & 337.7 & 359.7 & 370.7 & 11 \\
\hline & 5 & 336.3 & 360.3 & 372.3 & 12 \\
\hline \multirow{5}{*}{6} & 1 & 364.1 & 382.1 & 391.1 & 9 \\
\hline & 2 & 331.5 & 351.5 & 361.5 & 10 \\
\hline & 3 & 330.8 & 352.8 & 363.8 & 11 \\
\hline & 4 & 329.9 & 353.9 & 365.9 & 12 \\
\hline & 5 & 328.5 & 354.5 & 367.5 & 13 \\
\hline \multirow{5}{*}{7} & 1 & 361.1 & 381.1 & 391.1 & 10 \\
\hline & 2 & 328.7 & 350.7 & 361.7 & 11 \\
\hline & 3 & 328.0 & 352.0 & 364.0 & 12 \\
\hline & 4 & 327.1 & 353.1 & 366.1 & 13 \\
\hline & 5 & 325.8 & 353.8 & 367.8 & 14 \\
\hline \multirow{5}{*}{8} & 1 & 359.5 & 381.5 & 392.5 & 11 \\
\hline & 2 & 327.2 & 351.2 & 363.2 & 12 \\
\hline & 3 & 326.6 & 352.6 & 365.6 & 13 \\
\hline & 4 & 325.7 & 353.7 & 367.7 & 14 \\
\hline & 5 & 324.3 & 354.3 & 369.3 & 15 \\
\hline \multirow{5}{*}{9} & 1 & 358.4 & 382.4 & 394.4 & 12 \\
\hline & 2 & 326.1 & 352.2 & 365.2 & 13 \\
\hline & 3 & 325.5 & 353.5 & 367.5 & 14 \\
\hline & 4 & 324.6 & 354.6 & 369.6 & 15 \\
\hline & 5 & 323.2 & 355.2 & 371.2 & 16 \\
\hline
\end{tabular}

GD, Global Deviance; AIC, Akaike Information Criterion;

GAIC(3), Generalized AIC with penalty equal to 3;

${ }^{\text {a }}$ In excess of 33000 . 
The smallest value of $\operatorname{GAIC}(3)$ was associated with $\operatorname{df}(\mu)=6$ and $\operatorname{df}(\sigma)=2$ and thus this model was selected. A new search for the best $\lambda$ was carried out but global deviance values were similar for $\lambda$ from 0.45 to 0.65 , implying no need to update the model further.

Model 2: $\operatorname{BCPE}\left(x=\operatorname{age}^{0.65}, \operatorname{df}(\mu)=6, \operatorname{df}(\sigma)=2, \operatorname{df}(v)=2, \tau=2\right)$

Figure 69 shows the fitting of the parameters $\mu, \sigma$ and $v$ for Model 2 with their respective sample estimates, that is, the median for $\mu$, the sample standard deviation of the Box-Cox transformed data for $\sigma$, and the Box-Cox transform power for $v$. The selected model for the median curve smoothed the slightly wiggly pattern observed in the empirical values for ages above 24 months.

Figures 70 and 71 show the distribution of differences between empirical values and fitted centiles for the longitudinal and cross-sectional samples, respectively. There was no indication of systematic bias for ages between 3 and 24 months (Figure 70) or between 24 and 71 months (Figure 71).

The worm plots for this model (Figure 72) were significantly improved compared to those of Model 1 (Figure 68). The U-shaped worms flattened out significantly (Figure 72), indicating that the residual skewness associated with Model 1 had been corrected. There were three age groups with slight Sshaped worms that could suggest remaining kurtosis (4 mo, 9 mo and $10 \mathrm{mo}$ ) but the worms were contained within the $95 \%$ confidence interval.
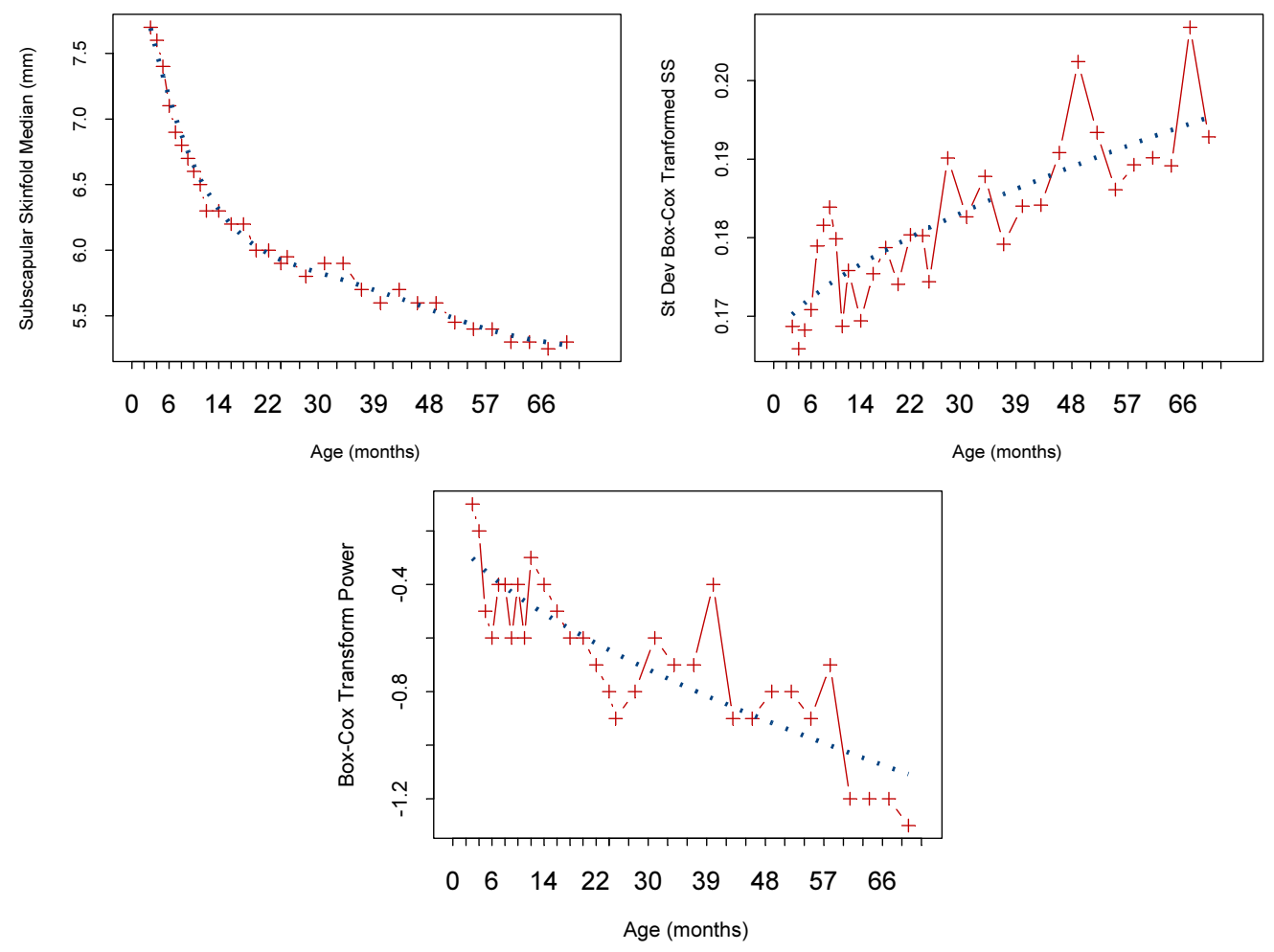

Figure 69 Fitting of the $\mu, \sigma$, and $v$ curves of Model 2 for subscapular skinfold-for-age for boys from 3 to 71 months (dotted line) and their respective sample estimates (points with solid line) 

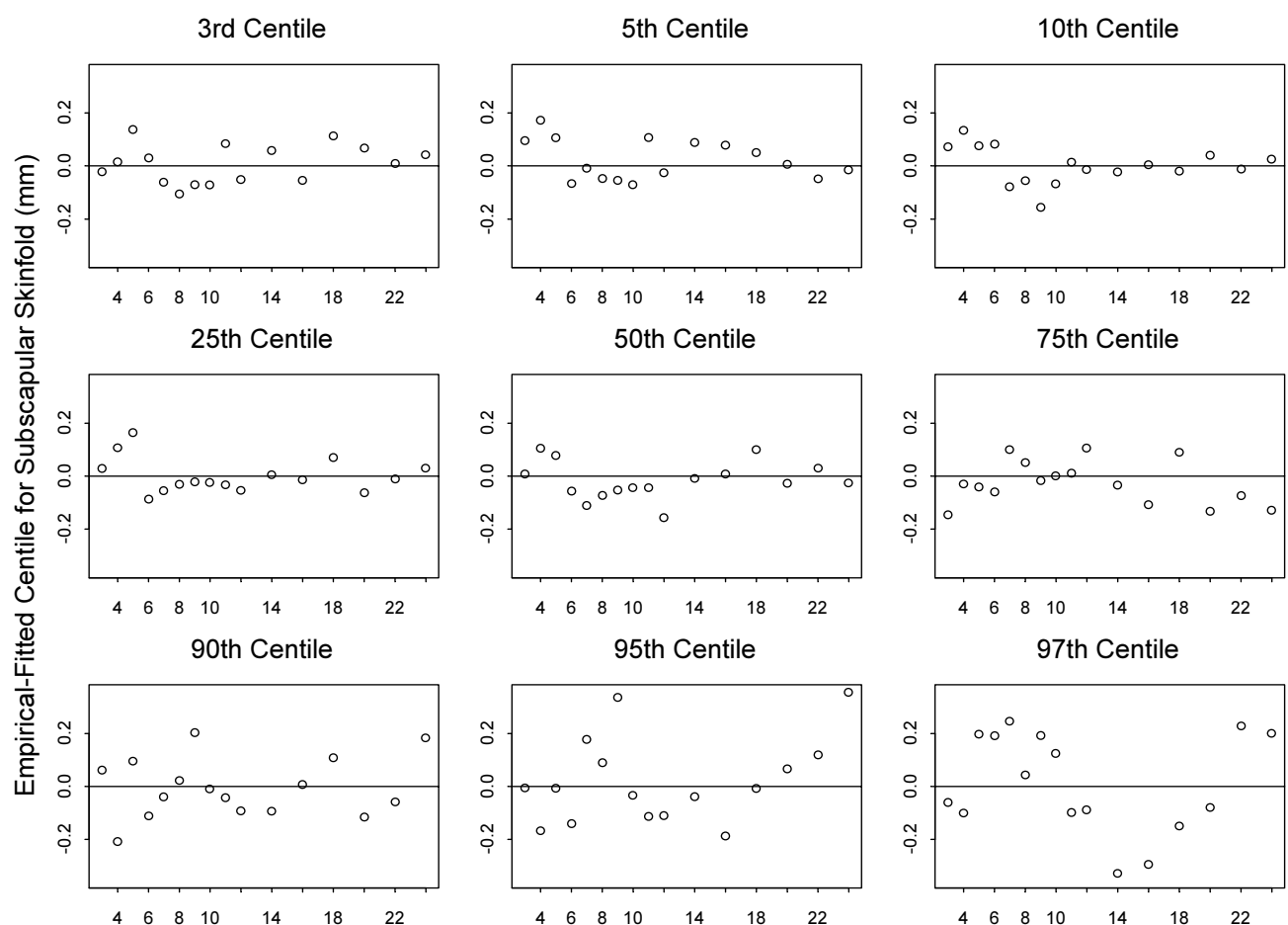

50th Centile

75th Centile
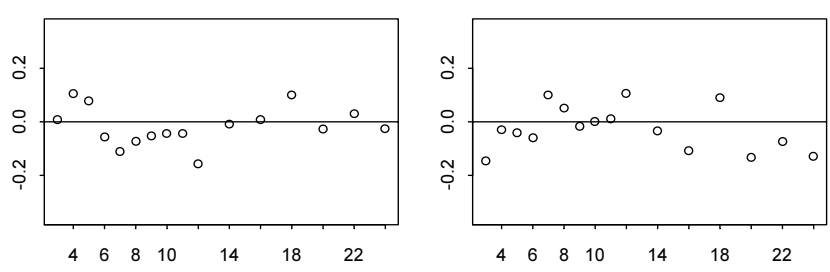

95th Centile

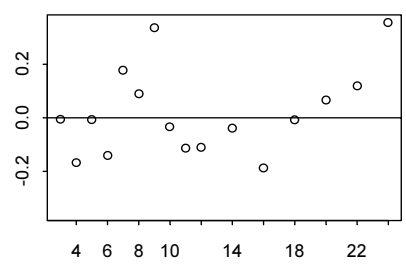

97th Centile

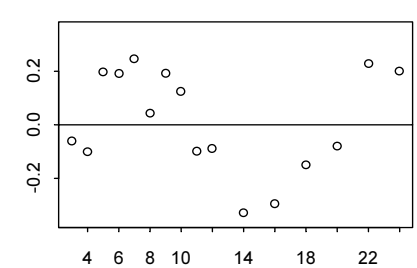

Age (months)

Figure 70 Centile residuals from fitting Model 2 for subscapular skinfold-for-age from 3 to 24 months for boys
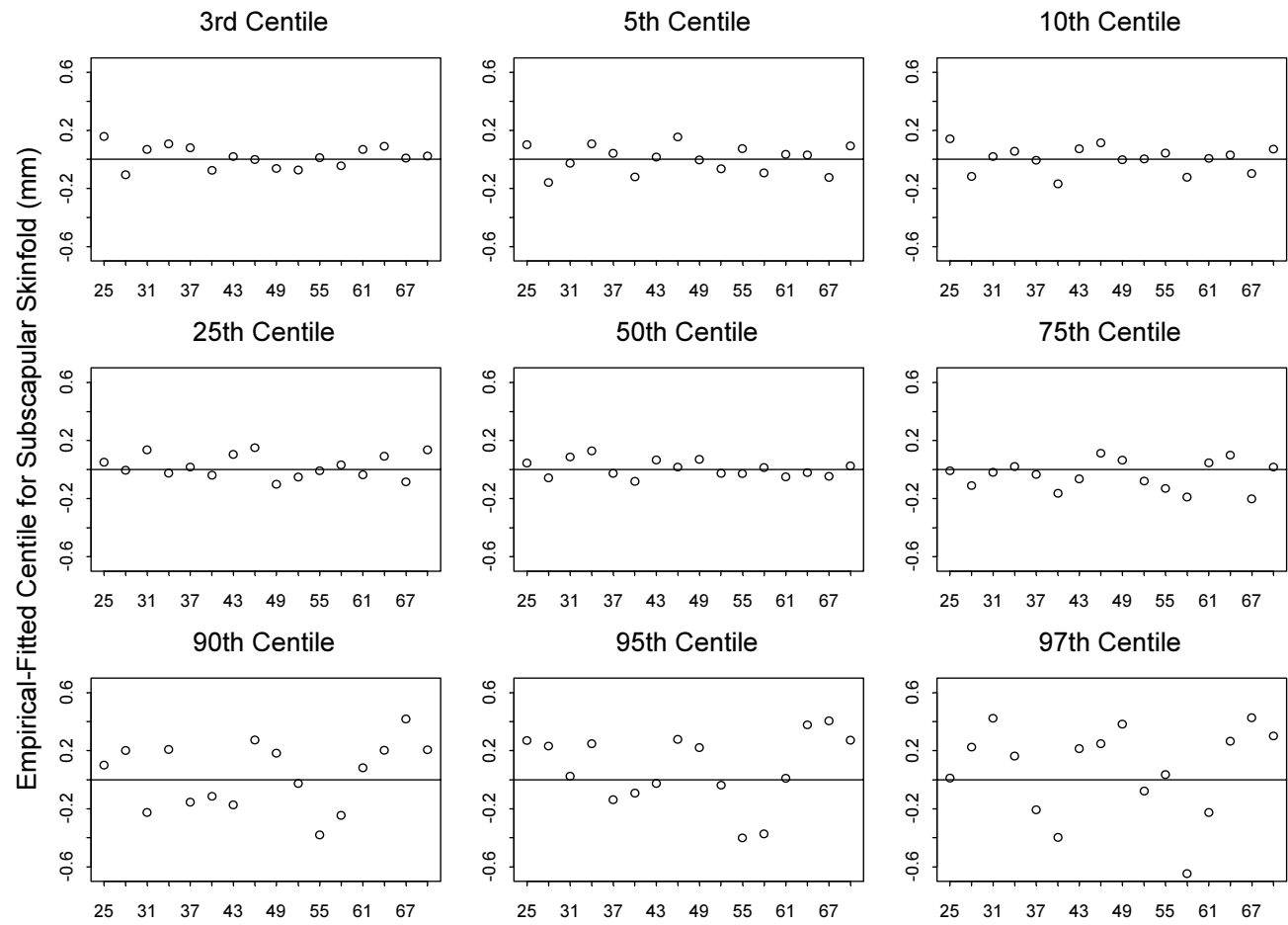

50th Centile

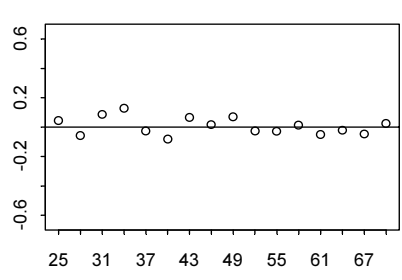

75th Centile
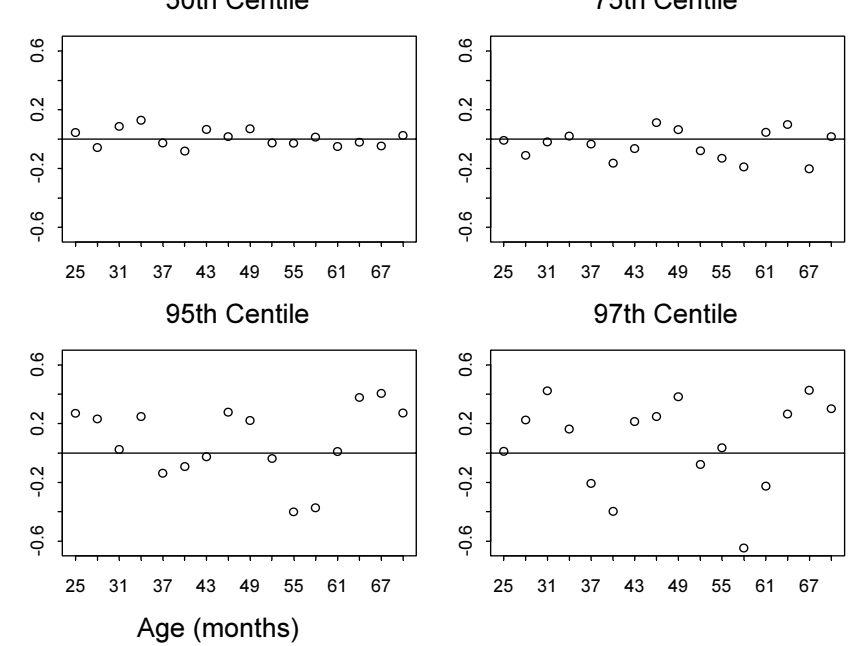

Figure 71 Centile residuals from fitting Model 2 for subscapular skinfold-for-age from 24 to 71 months for boys 

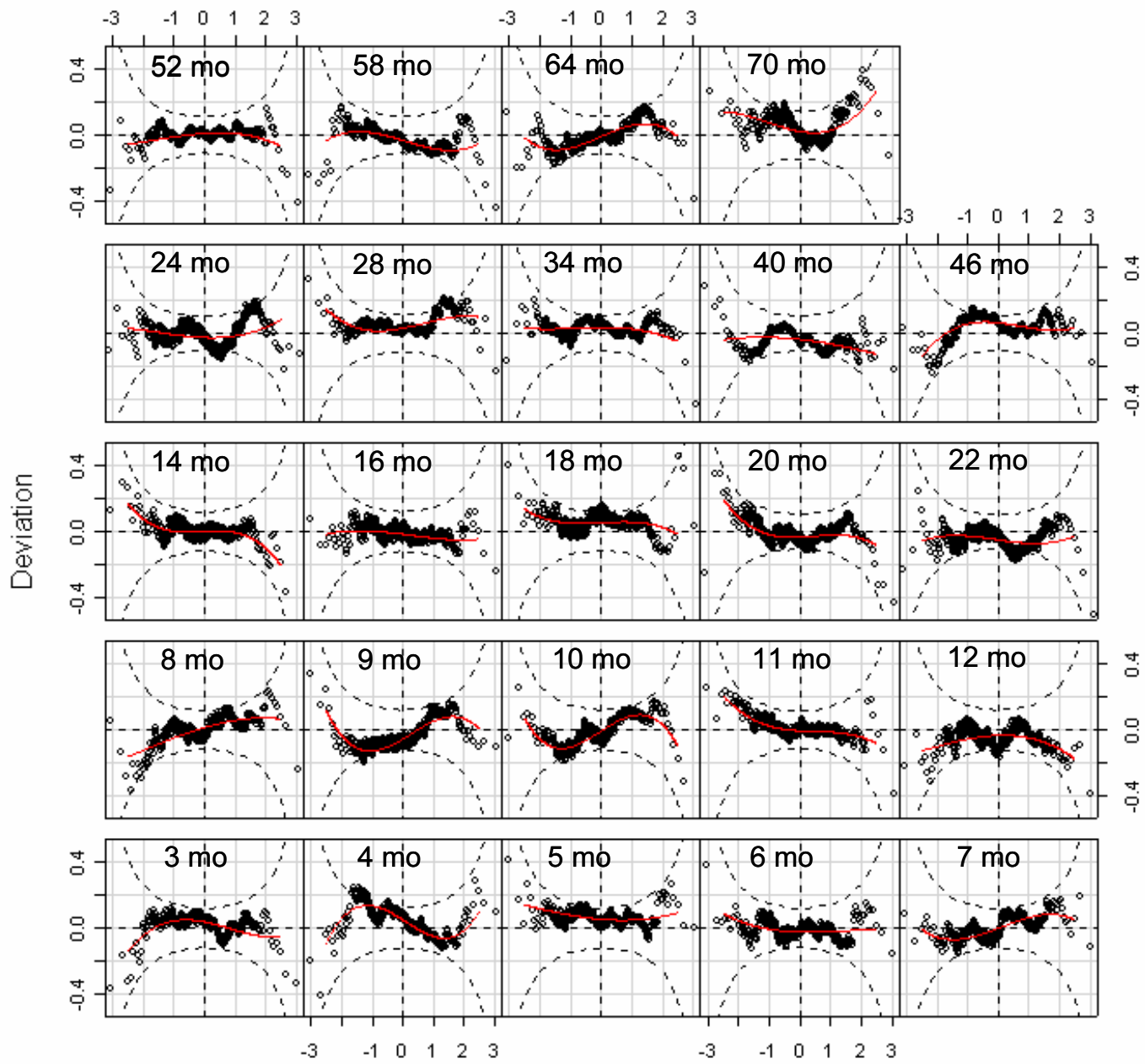

Unit normal quantile

Figure 72 Worm plots of z-scores for Model 2 for subscapular skinfold-for-age for boys

The Q-test results from Model 2 are shown in Table 61. There were no groups with absolute values larger than 2 for the statistic $\mathrm{z} 3$ that would suggest remaining skewness. Two age groups had absolute values of $\mathrm{z} 4$ greater than 2 (4 mo and $10 \mathrm{mo}$ ), indicating local remaining kurtosis, but the overall test statistic for kurtosis had a p-value that was not significant at the 5\% level. The overall Q-test p-values were all non-significant, indicating an adequate fit of the boys' subscapular skinfold-for-age curves and providing no justification for increasing the complexity of the model by adjusting for kurtosis (modelling $\tau$ ).

Table 62 presents observed percentages with subscapular skinfolds below the fitted centiles. There was no detectable pattern of a systematic bias. 
Table 61 Q-test for z-scores from Model $2\left[\operatorname{BCPE}\left(x=\operatorname{age}^{0.65}, \operatorname{df}(\mu)=6, \operatorname{df}(\sigma)=2, \operatorname{df}(v)=2, \tau=2\right)\right]$ for subscapular skinfold-for-age for boys

\begin{tabular}{|c|c|c|c|c|c|c|}
\hline Age (days) & Group & $\mathbf{N}$ & $\mathbf{z 1}$ & $\mathbf{z 2}$ & $\mathbf{z 3}$ & $\mathrm{z4}$ \\
\hline 79 to 99 & $3 \mathrm{mo}$ & 414 & 0.3 & -0.2 & -1.1 & 1.0 \\
\hline 100 to 129 & $4 \mathrm{mo}$ & 407 & 0.7 & -1.2 & -0.4 & 2.2 \\
\hline 130 to 159 & 5 mo & 410 & 1.3 & -0.3 & 0.5 & 0.1 \\
\hline 160 to 189 & $6 \mathrm{mo}$ & 411 & -0.5 & -0.4 & 0.6 & 0.0 \\
\hline 190 to 219 & $7 \mathrm{mo}$ & 406 & 0.1 & 1.0 & 0.4 & -1.0 \\
\hline 220 to 249 & $8 \mathrm{mo}$ & 420 & 0.0 & 1.4 & -0.6 & 0.2 \\
\hline 250 to 279 & $9 \mathrm{mo}$ & 391 & -0.8 & 1.4 & 0.8 & -1.7 \\
\hline 280 to 309 & $10 \mathrm{mo}$ & 399 & -0.3 & 1.6 & 0.0 & -2.3 \\
\hline 310 to 349 & $11 \mathrm{mo}$ & 460 & 0.1 & -1.0 & 0.6 & -0.7 \\
\hline 350 to 379 & $12 \mathrm{mo}$ & 410 & -1.1 & 0.0 & -0.9 & -0.2 \\
\hline 380 to 439 & $14 \mathrm{mo}$ & 418 & -0.1 & -0.8 & -0.1 & -1.3 \\
\hline 440 to 499 & $16 \mathrm{mo}$ & 415 & -0.4 & -0.5 & -0.1 & 0.3 \\
\hline 500 to 559 & $18 \mathrm{mo}$ & 440 & 1.1 & -0.3 & 0.1 & -0.4 \\
\hline 560 to 619 & $20 \mathrm{mo}$ & 523 & -0.3 & -0.6 & 0.7 & -0.9 \\
\hline 620 to 679 & $22 \mathrm{mo}$ & 547 & -0.9 & -0.4 & 0.1 & 0.7 \\
\hline 680 to 749 & $24 \mathrm{mo}$ & 591 & -0.4 & 0.2 & 0.9 & 0.3 \\
\hline 750 to 929 & $28 \mathrm{mo}$ & 469 & 0.9 & 0.4 & 0.7 & -0.6 \\
\hline 930 to 1119 & $34 \mathrm{mo}$ & 505 & 1.0 & -0.4 & -0.4 & -0.2 \\
\hline 1120 to 1309 & $40 \mathrm{mo}$ & 509 & -1.0 & -0.6 & -0.5 & 0.1 \\
\hline 1310 to 1499 & $46 \mathrm{mo}$ & 505 & 1.0 & 0.2 & -1.2 & 1.0 \\
\hline 1500 to 1689 & $52 \mathrm{mo}$ & 482 & -0.1 & 0.2 & -0.6 & -0.1 \\
\hline 1690 to 1879 & $58 \mathrm{mo}$ & 480 & -0.7 & -1.0 & -0.1 & 1.0 \\
\hline 1880 to 2069 & $64 \mathrm{mo}$ & 460 & -0.2 & 1.4 & 0.0 & -1.2 \\
\hline 2070 to 2249 & $70 \mathrm{mo}$ & 285 & 0.8 & -0.1 & 1.3 & 0.7 \\
\hline Overall Q stats & & 10757 & 11.8 & 15.3 & 9.9 & 22.9 \\
\hline degrees of freedom & & & 18.0 & 22.5 & 22.0 & 24.0 \\
\hline p-value & & & 0.8550 & 0.8681 & 0.9870 & 0.5273 \\
\hline
\end{tabular}

Note: Absolute values of z1, z2, z3 or z4 larger than 2 indicate misfit of, respectively, mean, variance, skewness or kurtosis.

In conclusion, the model selected for constructing the subscapular skinfold-for-age growth curves for boys was $\operatorname{BCPE}\left(x=a g e^{0.65}, \operatorname{df}(\mu)=6, \operatorname{df}(\sigma)=2, \operatorname{df}(v)=2, \tau=2\right)$ that adjusts only for skewness, and thus reduces to the LMS method. The fitted centile curves and empirical centiles are shown in Figures 73 to 76. 


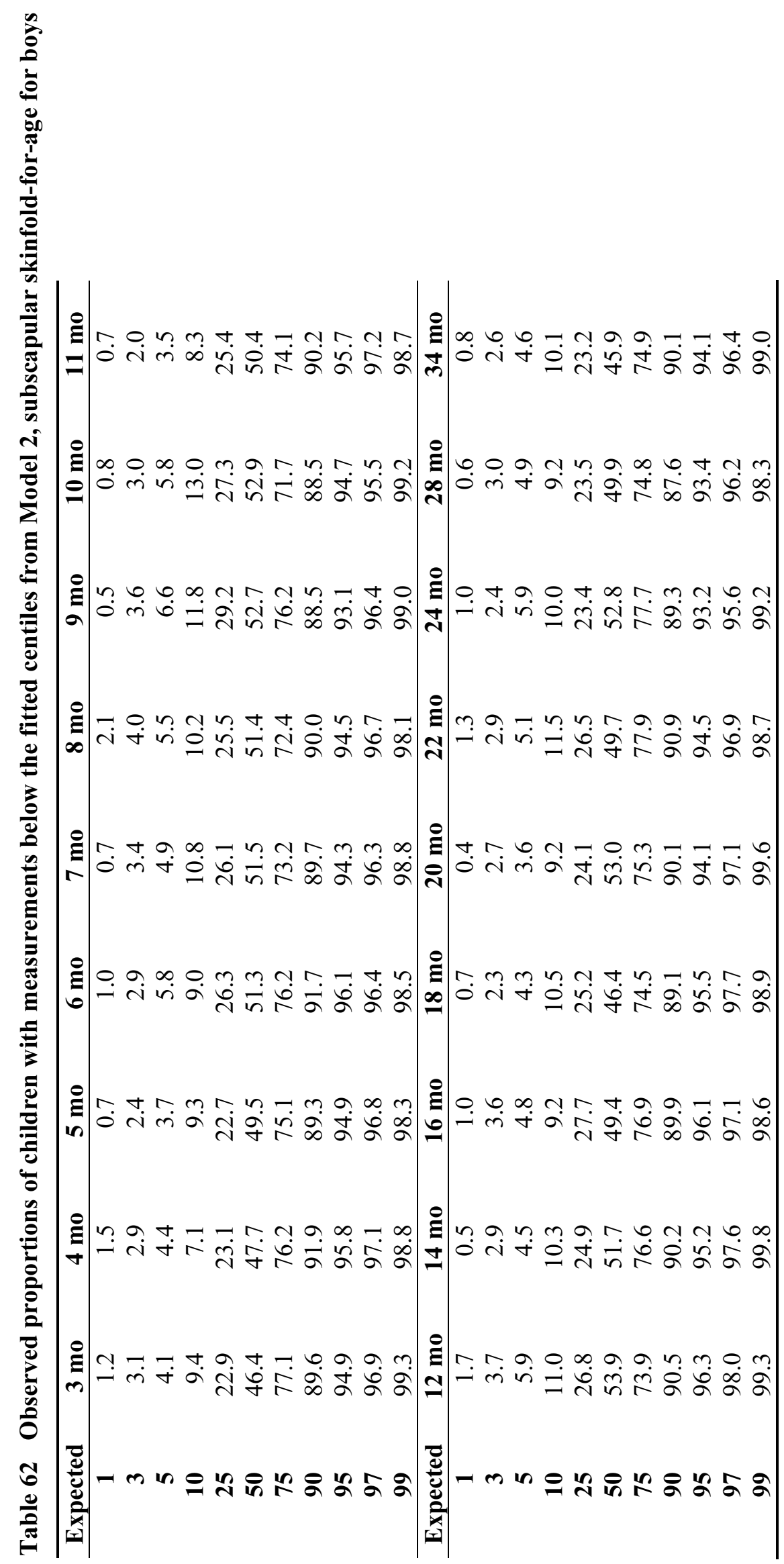




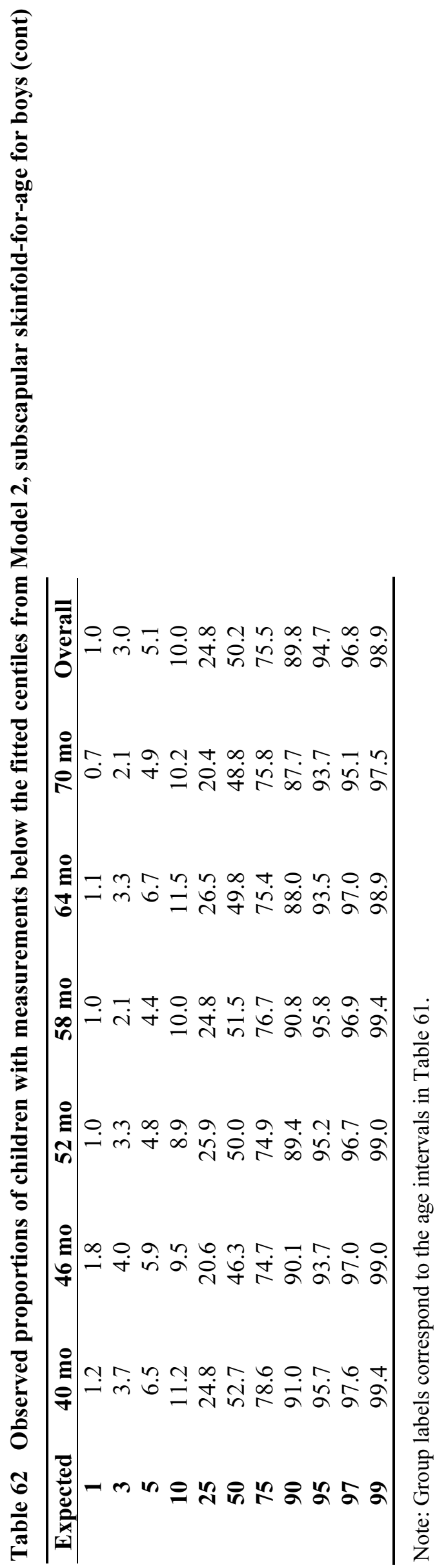




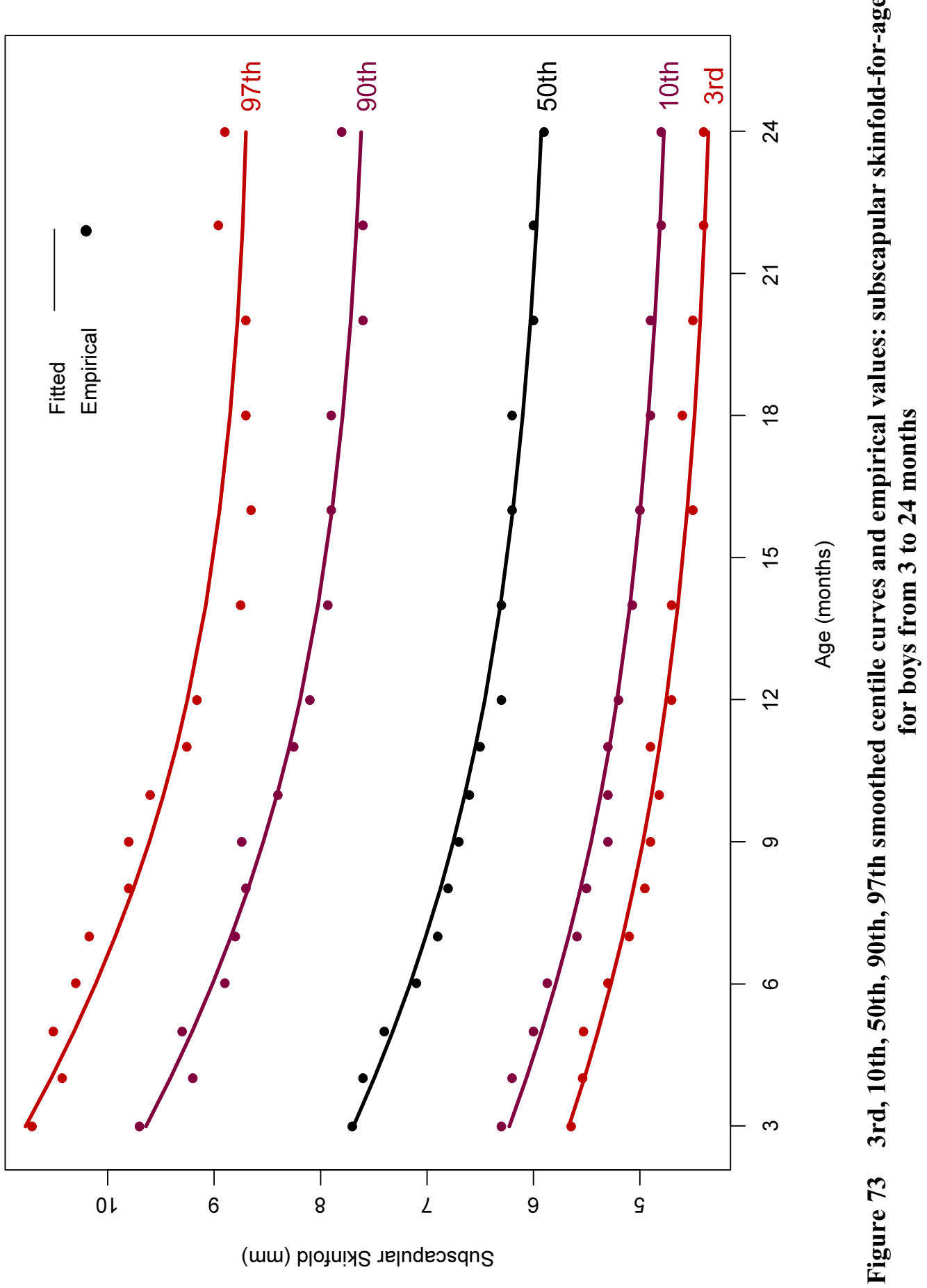




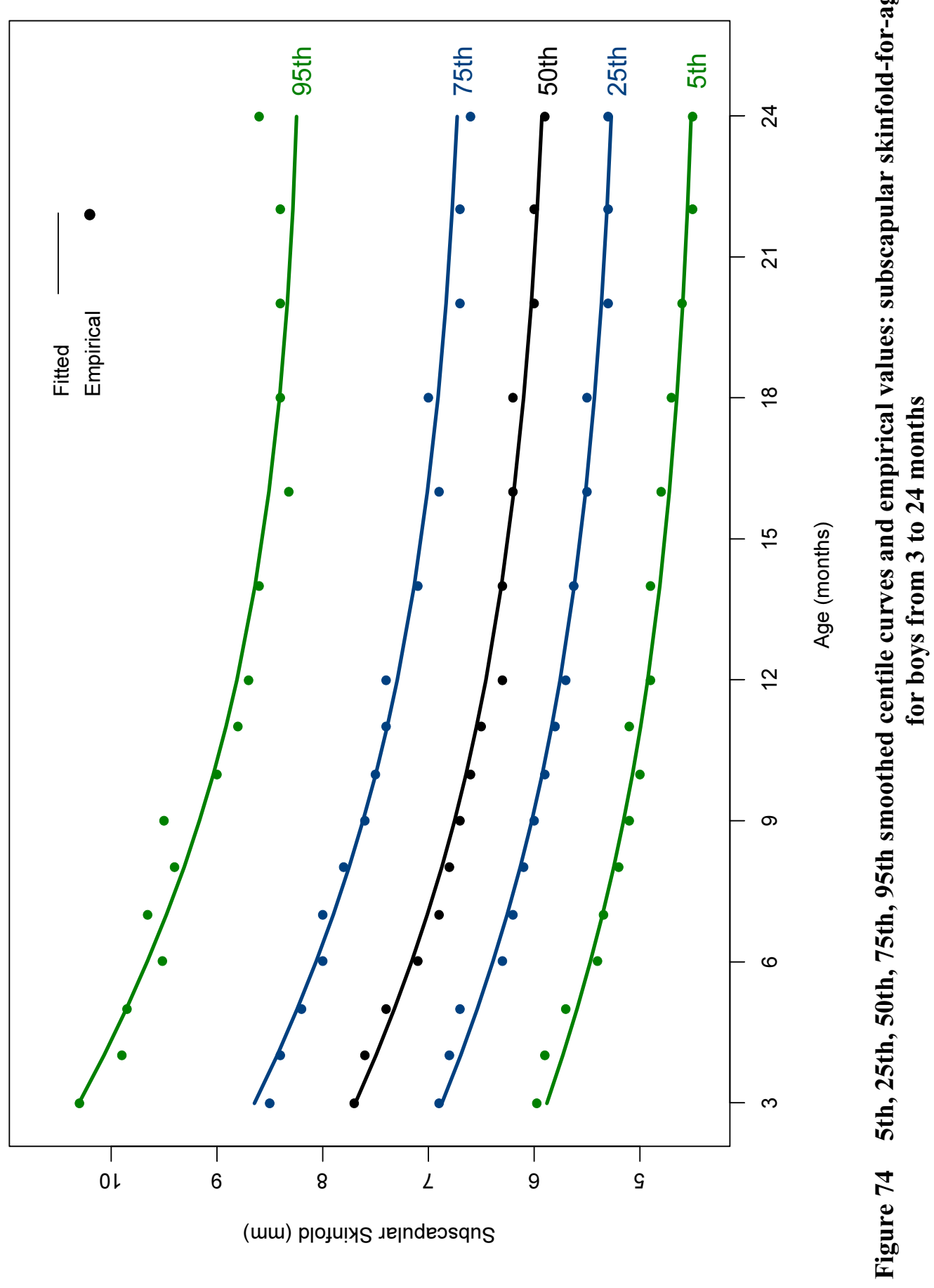




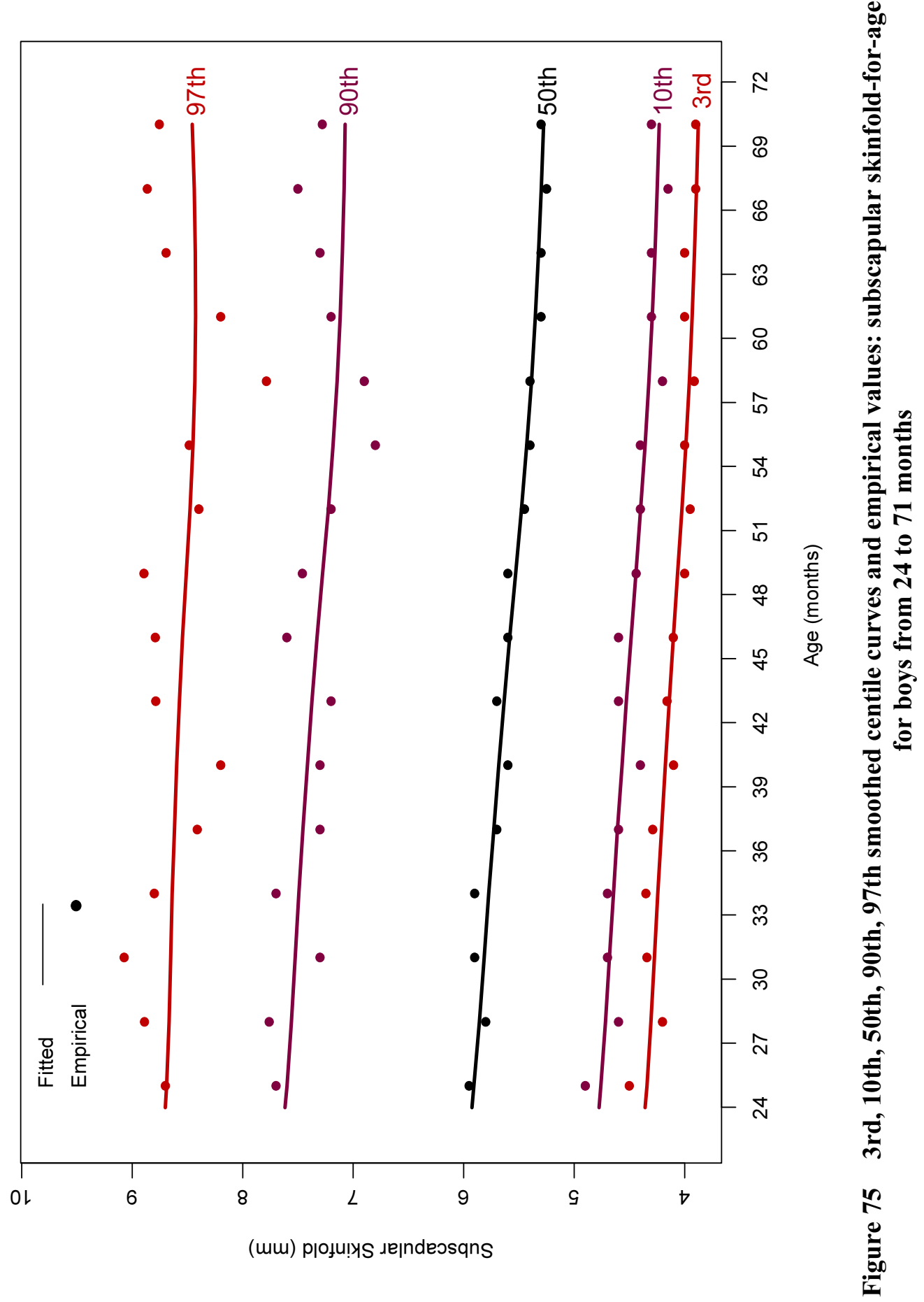




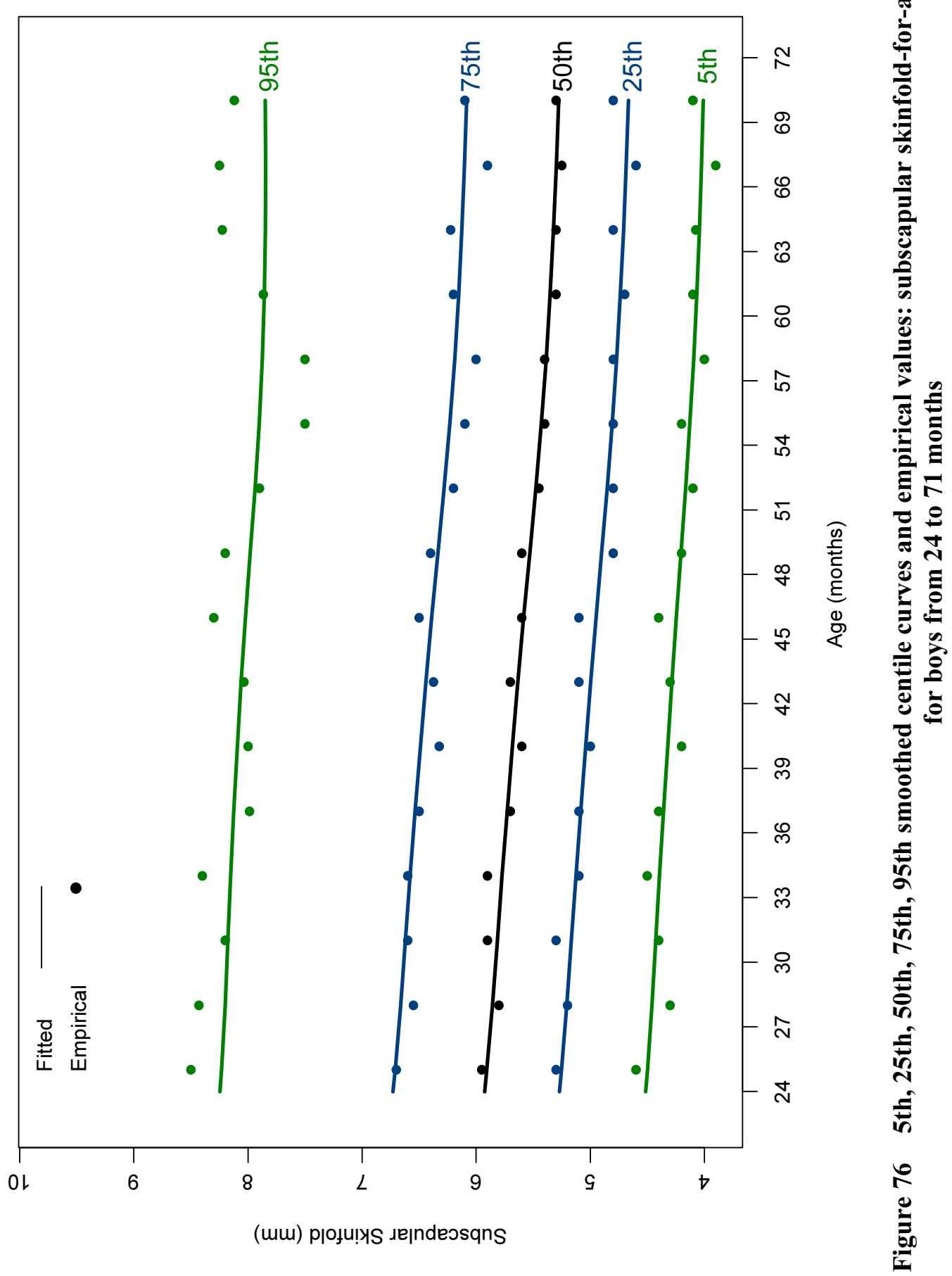




\subsubsection{WHO standards}

This section presents the final WHO subscapular skinfold-for-age z-score and percentile charts (Figures 77 and 78) and table (Table 63) for boys. 


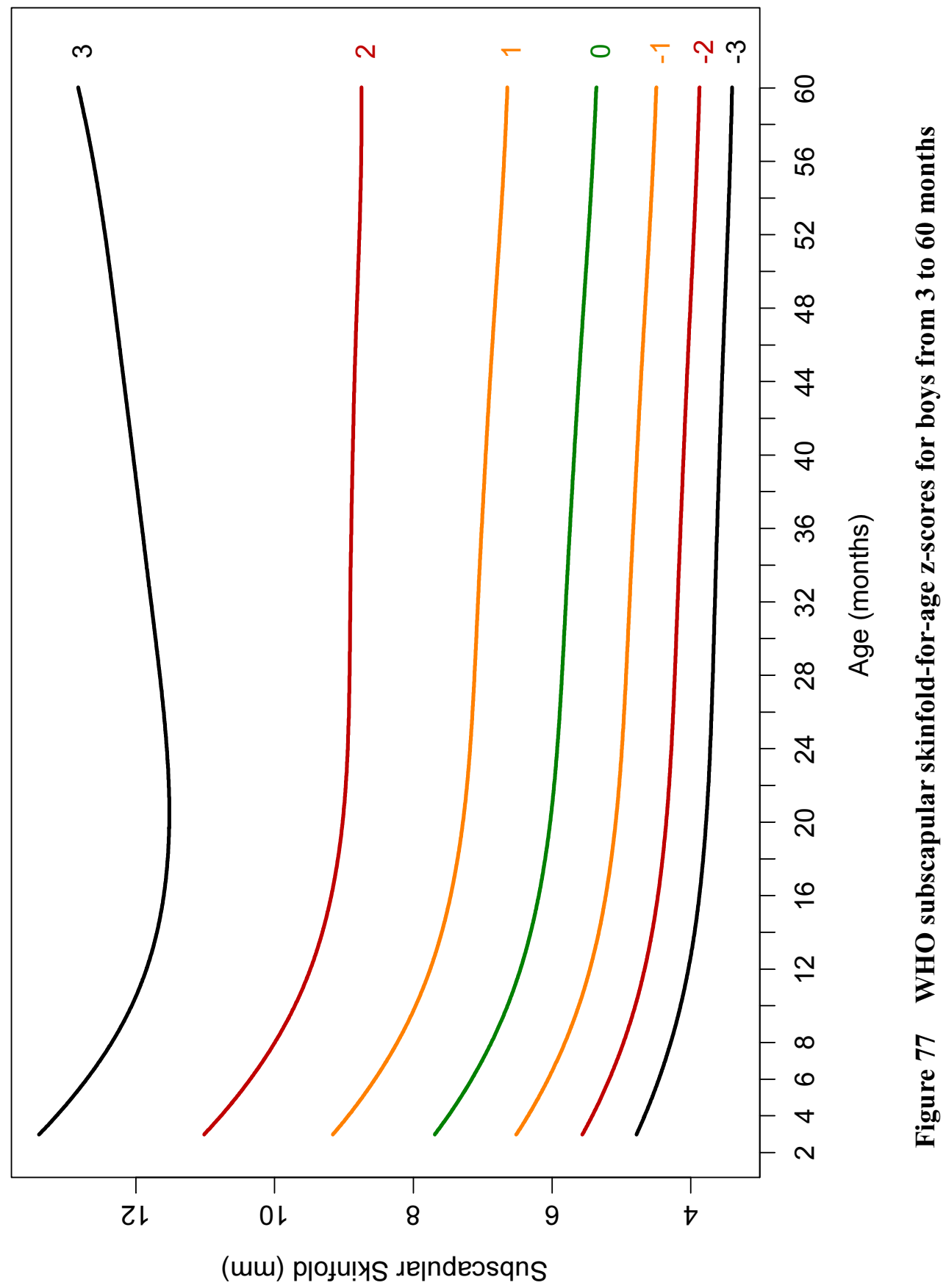




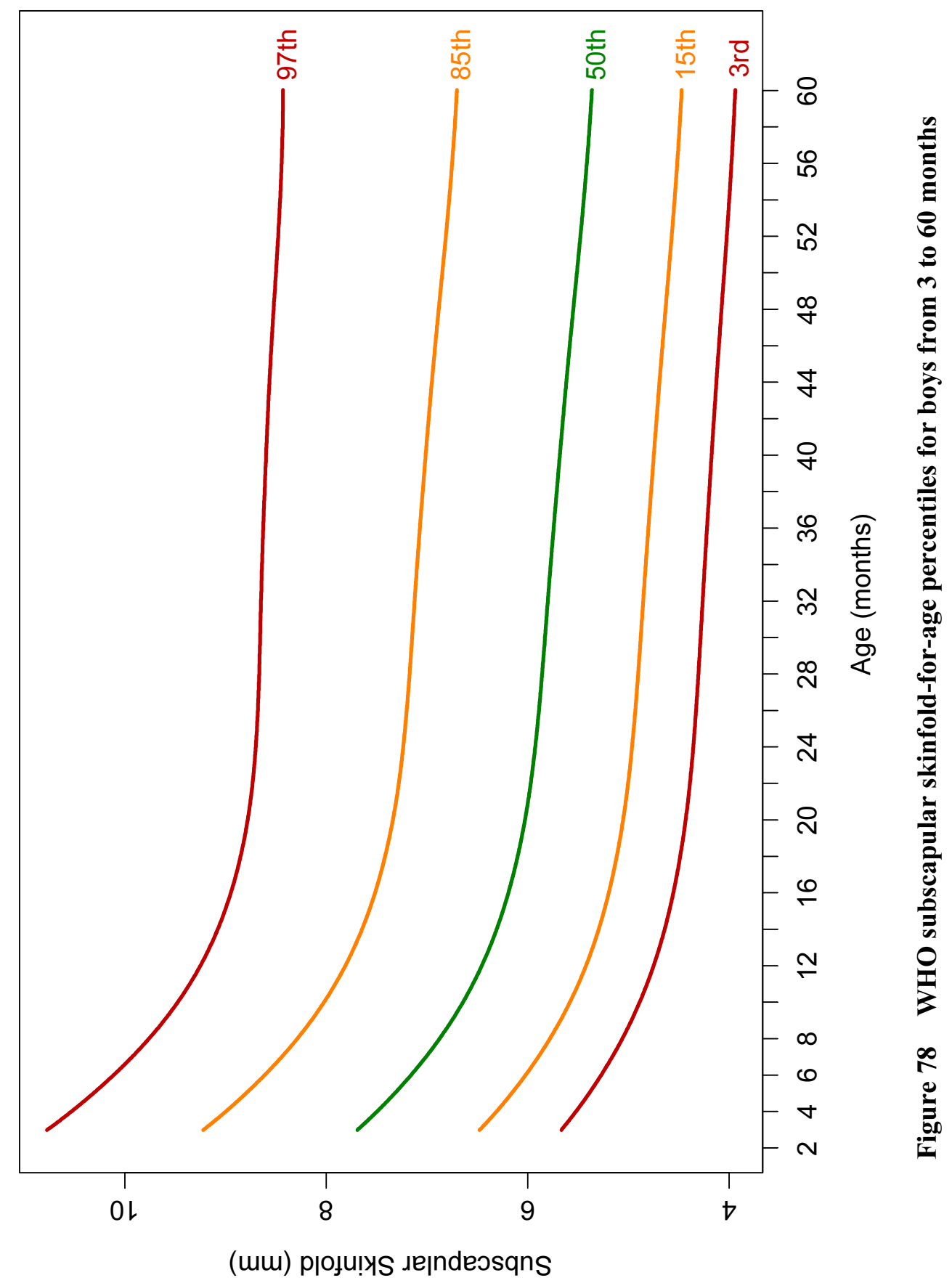




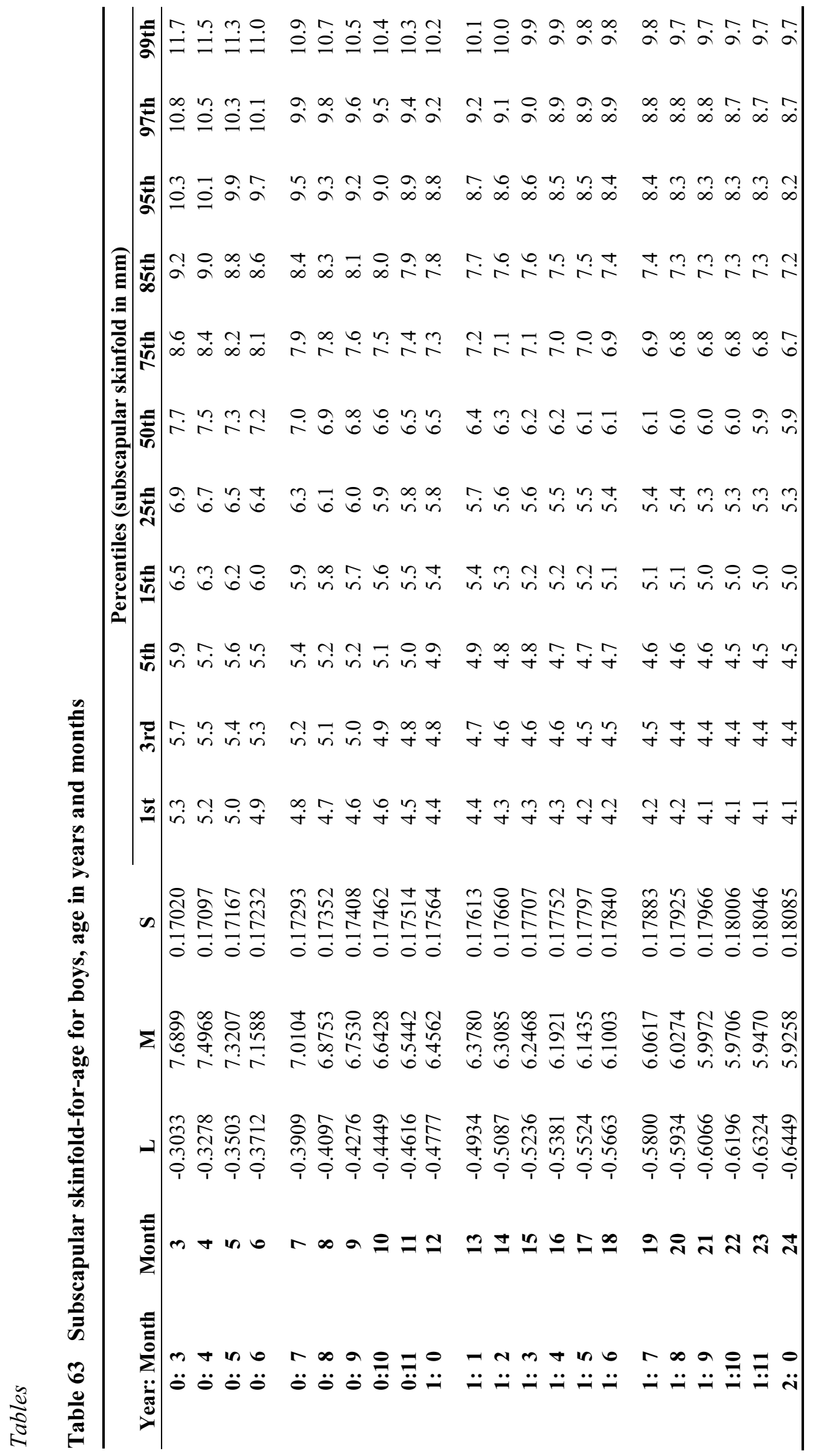




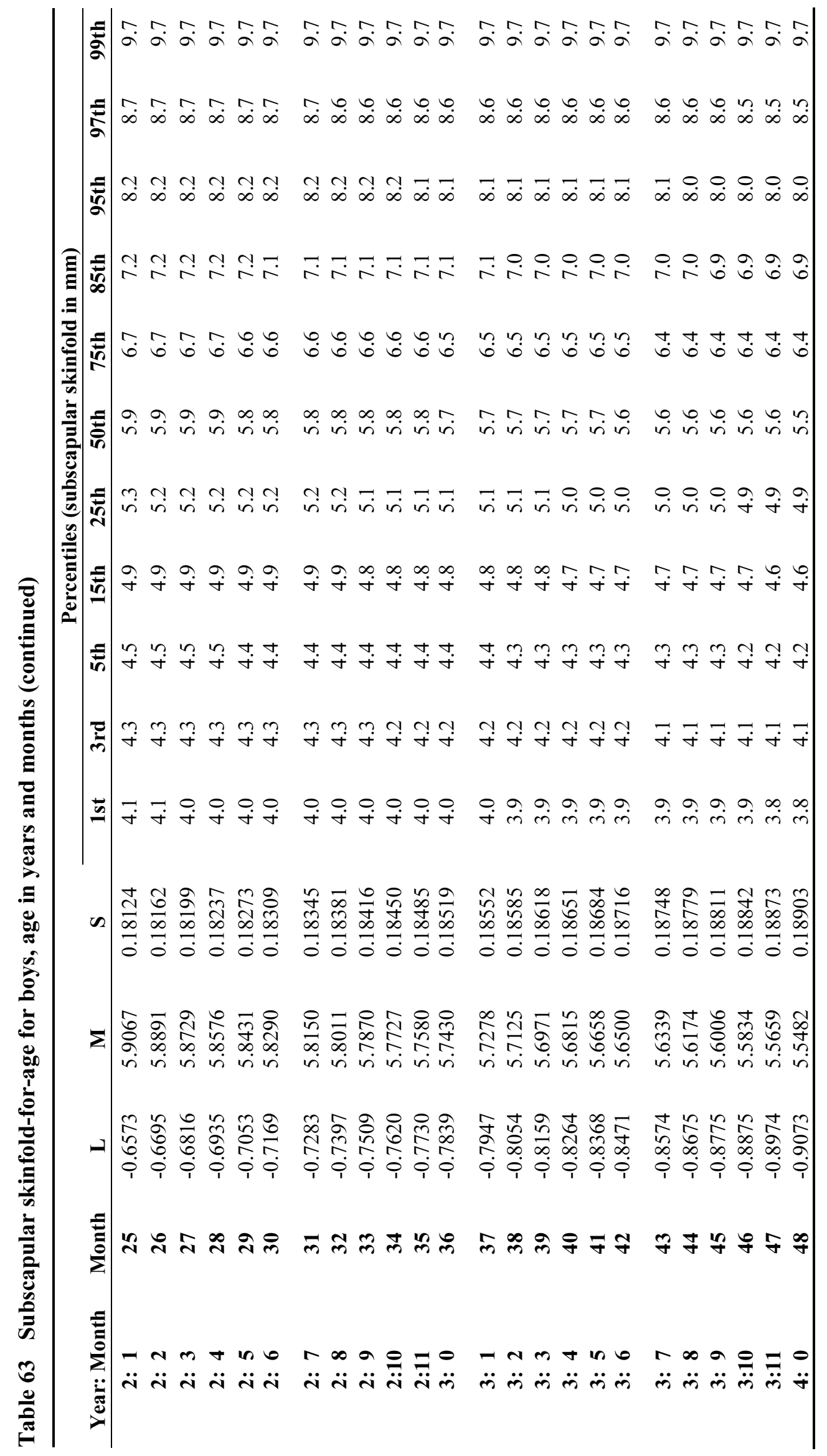




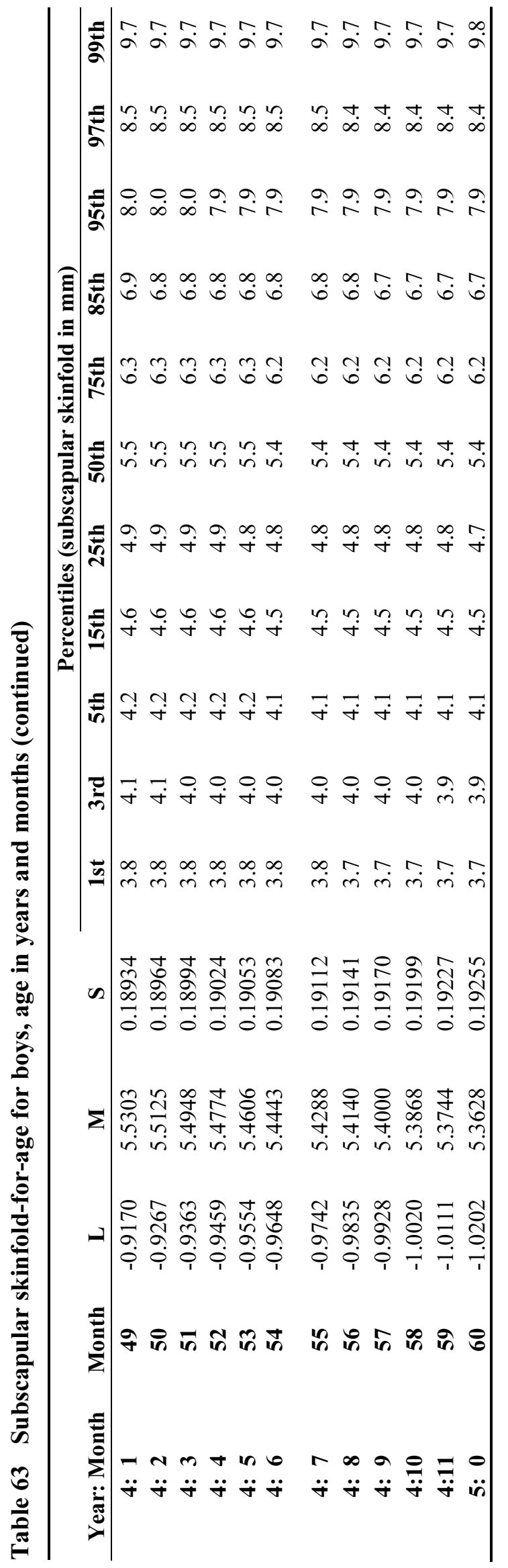




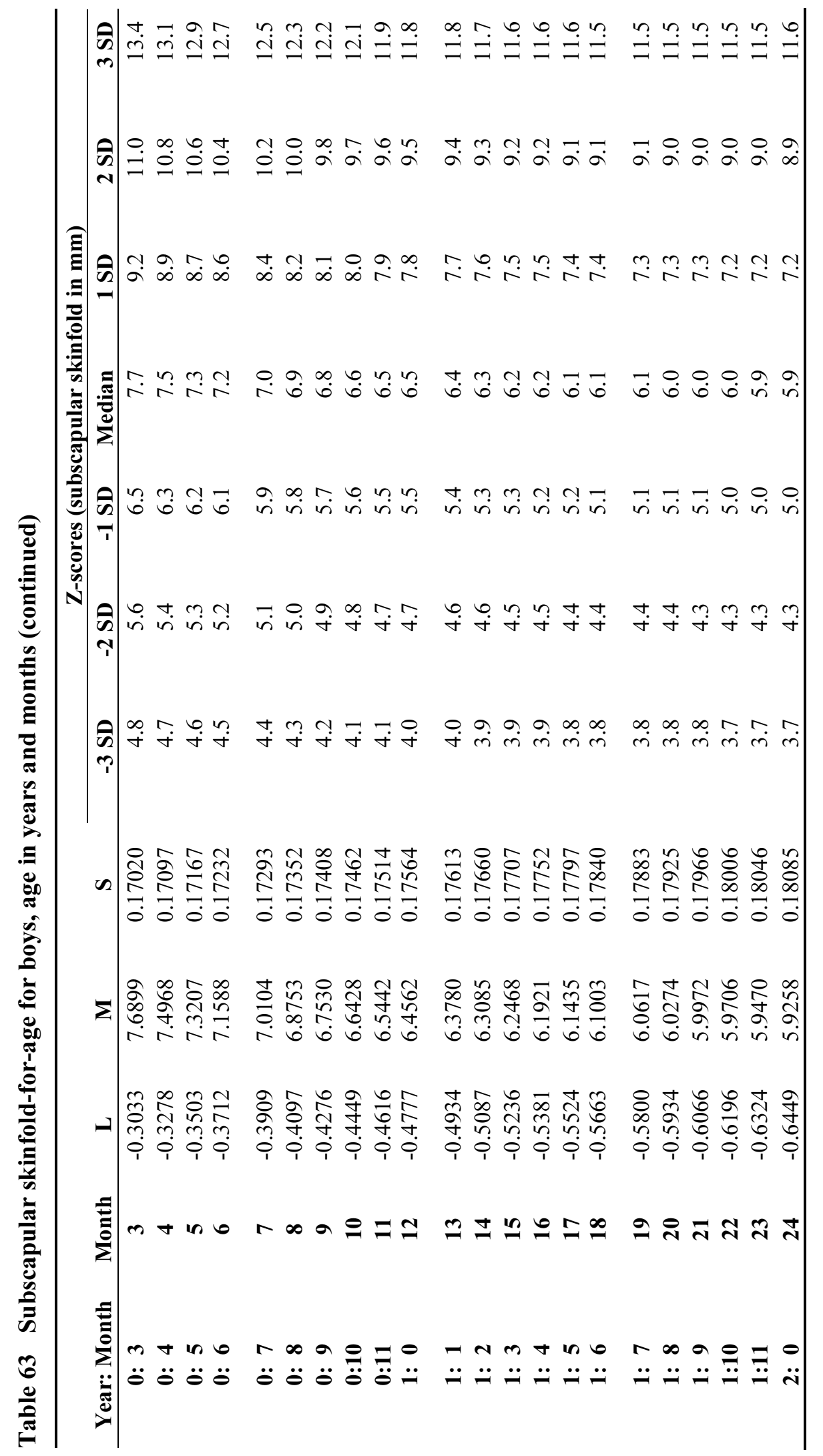




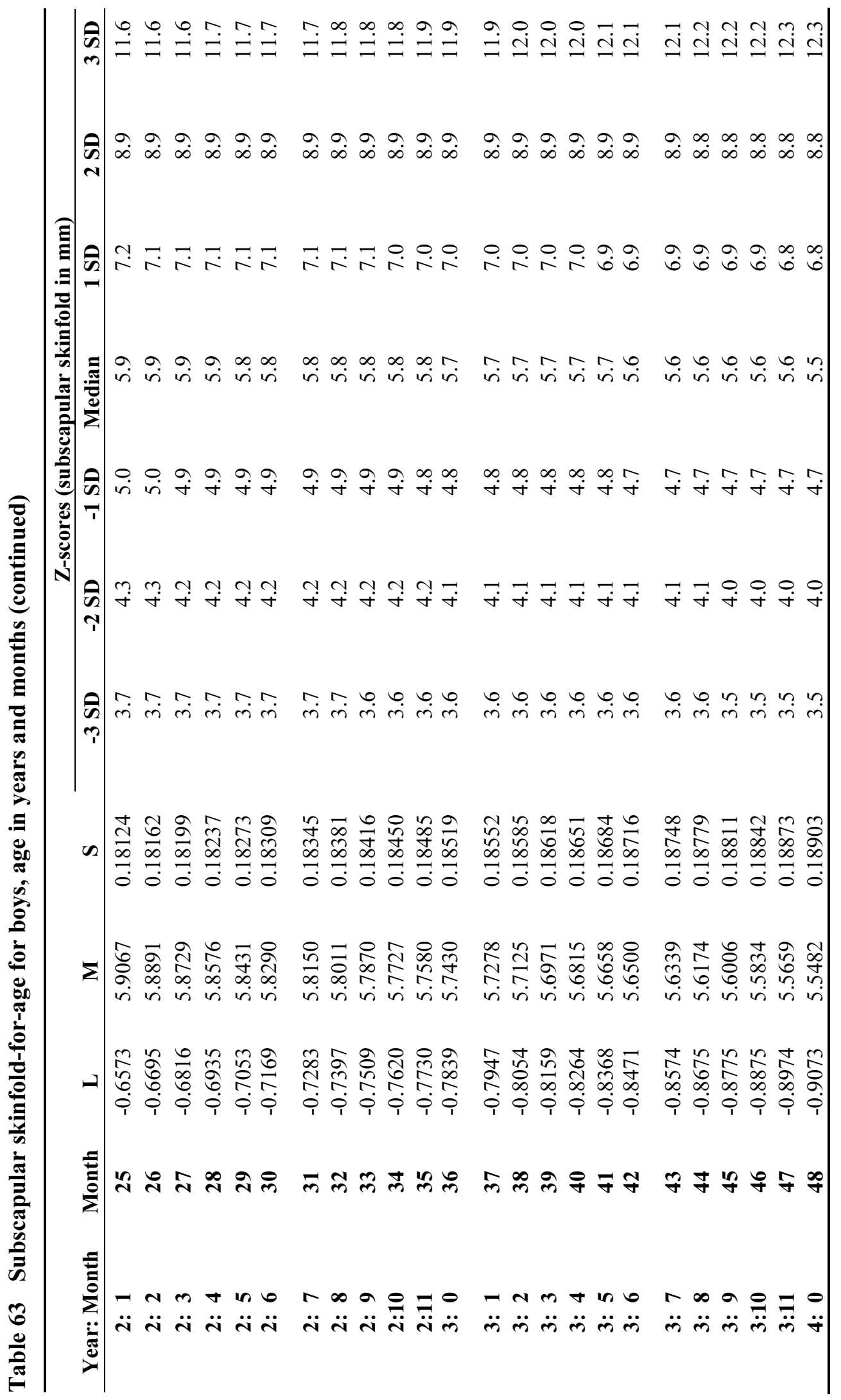




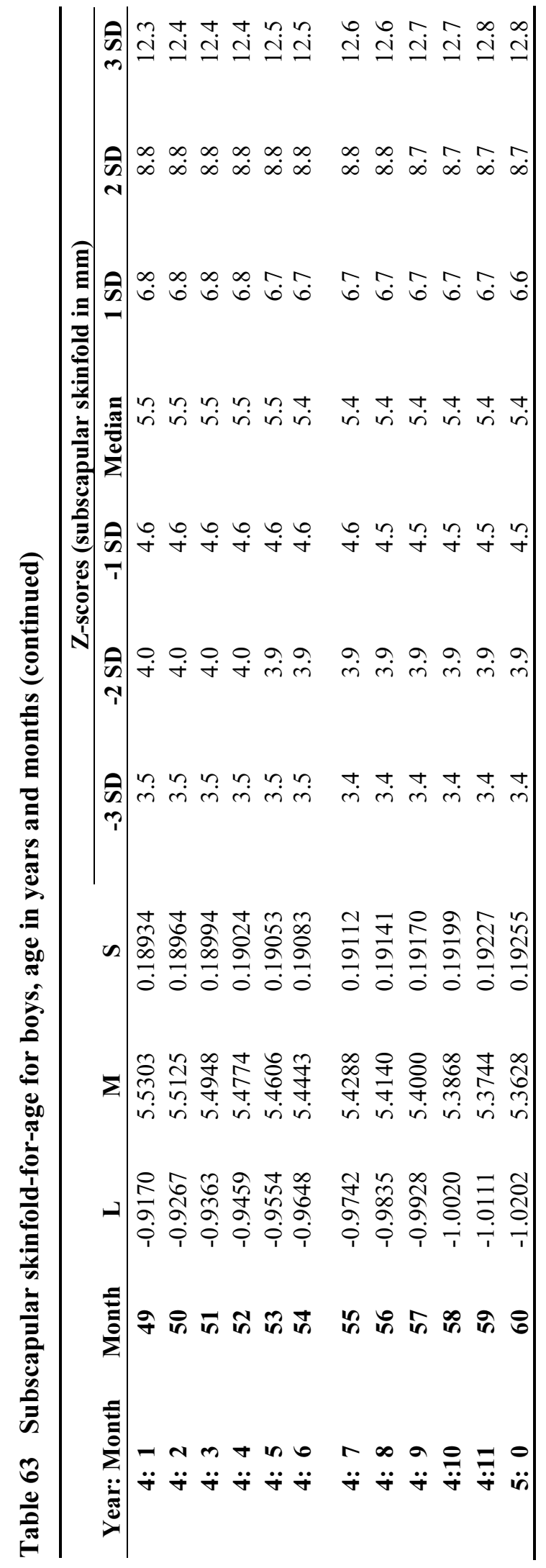




\subsection{Subscapular skinfold-for-age for girls}

Steps similar to those used to construct the subscapular skinfold-for-age growth curves for boys were followed to fit the subscapular skinfold-for-age growth curves for girls.

\subsubsection{Sample size}

There were a total of 10934 subscapular skinfold observations for girls. The longitudinal and crosssectional sample sizes by visit and age are presented in Tables 64 and 65 . The measurement of subscapular skinfold started at 3 months of age (de Onis et al., 2004b).

Table 64 Longitudinal sample sizes for subscapular skinfold-for-age for girls

\begin{tabular}{lcccccc}
\hline Visit & $\mathbf{5}$ & $\mathbf{6}$ & $\mathbf{7}$ & $\mathbf{8}$ & $\mathbf{9}$ & $\mathbf{1 0}$ \\
Age & $\mathbf{3} \mathbf{~ m o}$ & $\mathbf{4} \mathbf{~ m o}$ & $\mathbf{5} \mathbf{~ m o}$ & $\mathbf{6} \mathbf{~ m o}$ & $\mathbf{7} \mathbf{~ m o}$ & $\mathbf{8} \mathbf{~ m o}$ \\
$\mathrm{N}$ & 446 & 443 & 447 & 445 & 444 & 440 \\
\hline Visit & $\mathbf{1 1}$ & $\mathbf{1 2}$ & $\mathbf{1 3}$ & $\mathbf{1 4}$ & $\mathbf{1 5}$ & $\mathbf{1 6}$ \\
Age & $\mathbf{9} \mathbf{~ m o}$ & $\mathbf{1 0} \mathbf{~ m o}$ & $\mathbf{1 1} \mathbf{~ m o}$ & $\mathbf{1 2} \mathbf{~ m o}$ & $\mathbf{1 4} \mathbf{~ m o}$ & $\mathbf{1 6} \mathbf{~ m o}$ \\
$\mathrm{N}$ & 444 & 443 & 440 & 448 & 445 & 441 \\
\hline Visit & $\mathbf{1 7}$ & $\mathbf{1 8}$ & $\mathbf{1 9}$ & $\mathbf{2 0}$ & & \\
Age & $\mathbf{1 8} \mathbf{~ m o}$ & $\mathbf{2 0} \mathbf{~ m o}$ & $\mathbf{2 2} \mathbf{~ m o}$ & $\mathbf{2 4} \mathbf{~ m o}$ & & \\
$\mathrm{N}$ & 444 & 443 & 433 & 447 & & \\
\hline
\end{tabular}

Table 65 Cross-sectional sample sizes for subscapular skinfold-for-age for girls

\begin{tabular}{lccccccc}
\hline Age (mo) & $<\mathbf{1 8}$ & $\mathbf{1 8}-\mathbf{2 0}$ & $\mathbf{2 1 - 2 3}$ & $\mathbf{2 4 - 2 6}$ & $\mathbf{2 7 - 2 9}$ & $\mathbf{3 0 - 3 2}$ & $\mathbf{3 3 - 3 5}$ \\
$\mathrm{N}$ & 2 & 159 & 167 & 237 & 213 & 224 & 222 \\
\hline Age (mo) & $\mathbf{3 6}-\mathbf{3 8}$ & $\mathbf{3 9 - 4 1}$ & $\mathbf{4 2 - 4 4}$ & $\mathbf{4 5}-\mathbf{4 7}$ & $\mathbf{4 8}-\mathbf{5 0}$ & $\mathbf{5 1 - 5 3}$ & $\mathbf{5 4 - 5 6}$ \\
$\mathrm{N}$ & 217 & 233 & 246 & 205 & 219 & 196 & 229 \\
\hline Age (mo) & $\mathbf{5 7 - 5 9}$ & $\mathbf{6 0 - 6 2}$ & $\mathbf{6 3 - 6 5}$ & $\mathbf{6 6 - 6 8}$ & $\mathbf{6 9 - 7 1}$ & $>\mathbf{7 1}$ & \\
$\mathrm{N}$ & 236 & 213 & 203 & 222 & 198 & 0 & \\
\hline
\end{tabular}

\subsubsection{Model selection and results}

The model BCPE $\left(x=\operatorname{age}^{\lambda}, \operatorname{df}(\mu)=9, \operatorname{df}(\sigma)=4, \operatorname{df}(v)=4, \tau=2\right)$ was used as a starting point to search for the best value of the age-transformation power $\lambda$. Table 66 shows the global deviance for values of $\lambda$ from 0.05 to 1 . The minimum global deviance value was for $\lambda 0.45$ to 0.60 , and $\lambda=0.50$ was selected to continue the search for the best model. 
Table 66 Global deviance (GD) for models within the class $\operatorname{BCPE}\left(x=\operatorname{age}^{\lambda}, \operatorname{df}(\mu)=9, \operatorname{df}(\sigma)=4\right.$, $\operatorname{df}(v)=4, \tau=2)$ for subscapular skinfold-for-age for girls

\begin{tabular}{lcccccccccc}
\hline $\boldsymbol{\lambda}$ & $\mathbf{0 . 0 5}$ & $\mathbf{0 . 1 0}$ & $\mathbf{0 . 1 5}$ & $\mathbf{0 . 2 0}$ & $\mathbf{0 . 2 5}$ & $\mathbf{0 . 3 0}$ & $\mathbf{0 . 3 5}$ & $\mathbf{0 . 4 0}$ & $\mathbf{0 . 4 5}$ & $\mathbf{0 . 5 0}$ \\
$\mathrm{GD}^{\mathrm{a}}$ & 658.1 & 657.6 & 657.2 & 656.9 & 656.7 & 656.5 & 656.3 & 656.3 & 656.2 & 656.2 \\
\hline $\boldsymbol{\lambda}$ & $\mathbf{0 . 5 5}$ & $\mathbf{0 . 6 0}$ & $\mathbf{0 . 6 5}$ & $\mathbf{0 . 7 0}$ & $\mathbf{0 . 7 5}$ & $\mathbf{0 . 8 0}$ & $\mathbf{0 . 8 5}$ & $\mathbf{0 . 9 0}$ & $\mathbf{0 . 9 5}$ & $\mathbf{1 . 0 0}$ \\
$\mathrm{GD}^{\mathrm{a}}$ & 656.2 & 656.2 & 656.3 & 656.4 & 656.5 & 656.7 & 657.1 & 657.5 & 658.1 & 659.0 \\
\hline
\end{tabular}

${ }^{\mathrm{a}}$ In excess of 36000 .

The search for the best $\operatorname{df}(\mu)$ and $\operatorname{df}(\sigma)$ followed, fixing $\lambda=0.50, v=1$ and $\tau=2$. All possible combinations with $\operatorname{df}(\mu)$ values ranging from 4 to 15 and $\operatorname{df}(\sigma)$ from 2 to 10 were considered and partial results are presented in Table 67. The fitted model that minimized GAIC(3) was that with $\operatorname{df}(\mu)=5$ and $\operatorname{df}(\sigma)=4$ and it was thus selected for further evaluation.

Table 67 Goodness-of-fit summary for models using the BCPE distribution with fixed $v=1$ and $\tau=\mathbf{2}$ for subscapular skinfold-for-age for girls

\begin{tabular}{cccccc}
\hline $\mathbf{d f}(\boldsymbol{\mu})$ & $\mathbf{d f}(\boldsymbol{\sigma})$ & $\mathbf{G D}^{\mathbf{a}}$ & AIC $^{\mathbf{a}}$ & GAIC(3) $^{\mathbf{a}}$ & Total df \\
\hline \multirow{4}{*}{$\mathbf{4}$} & $\mathbf{2}$ & 2485.9 & 2497.9 & 2503.9 & 6 \\
& $\mathbf{3}$ & 2438.2 & 2452.2 & 2459.2 & 7 \\
& $\mathbf{4}$ & 2433.0 & 2449.0 & 2457.0 & 8 \\
& $\mathbf{5}$ & 2432.3 & 2450.3 & 2459.3 & 9 \\
& $\mathbf{6}$ & 2431.6 & 2451.6 & 2461.6 & 10 \\
\hline \multirow{5}{*}{$\mathbf{5}$} & $\mathbf{2}$ & 2477.0 & 2491.0 & 2498.0 & 7 \\
& $\mathbf{3}$ & 2430.9 & 2446.9 & 2454.9 & 8 \\
& $\mathbf{4}$ & 2424.4 & 2442.4 & 2451.4 & 9 \\
& $\mathbf{5}$ & 2423.2 & 2443.2 & 2453.2 & 10 \\
& $\mathbf{6}$ & 2422.4 & 2444.4 & 2455.4 & 11 \\
$\mathbf{6}$ & $\mathbf{2}$ & 2475.2 & 2491.2 & 2499.2 & 8 \\
& $\mathbf{3}$ & 2429.4 & 2447.4 & 2456.4 & 9 \\
& $\mathbf{4}$ & 2422.5 & 2442.5 & 2452.5 & 10 \\
& $\mathbf{5}$ & 2421.2 & 2443.2 & 2454.2 & 11 \\
& $\mathbf{6}$ & 2420.4 & 2444.4 & 2456.4 & 12 \\
\hline \multirow{7}{*}{7} & $\mathbf{2}$ & 2474.2 & 2492.2 & 2501.2 & 9 \\
& $\mathbf{3}$ & 2428.5 & 2448.5 & 2458.5 & 10 \\
& $\mathbf{4}$ & 2421.5 & 2443.5 & 2454.5 & 11 \\
& $\mathbf{5}$ & 2420.1 & 2444.1 & 2456.1 & 12 \\
& $\mathbf{6}$ & 2419.3 & 2445.3 & 2458.3 & 13 \\
\hline \multirow{6}{*}{$\mathbf{8}$} & $\mathbf{2}$ & 2473.0 & 2493.0 & 2503.0 & 10 \\
& $\mathbf{3}$ & 2427.5 & 2449.5 & 2460.5 & 11 \\
& $\mathbf{4}$ & 2420.4 & 2444.4 & 2456.4 & 12 \\
& $\mathbf{5}$ & 2419.1 & 2445.1 & 2458.1 & 13 \\
& $\mathbf{6}$ & 2418.2 & 2446.2 & 2460.2 & 14 \\
\hline
\end{tabular}

GD, Global Deviance; AIC, Akaike Information Criterion;

GAIC(3), Generalized AIC with penalty equal to 3;

${ }^{\text {a }}$ In excess of 36000 . 
Model 1: $\operatorname{BCPE}\left(x=\operatorname{age}^{0.50}, \operatorname{df}(\mu)=5, \operatorname{df}(\sigma)=4, v=1, \tau=2\right)$

This model was inadequate with evidence of residual skewness in both the worm plots (Figure 79) and the Q-test results (Table 68). The worms presented U-shapes in all age groups and the Q-test results (Table 68) showed that all groups had absolute values of z3 higher than 2. Most of the age groups also presented absolute values of $\mathrm{z} 4$ higher than 2 , indicating residual kurtosis. Both overall tests for skewness and kurtosis were significant $(p$-value $<0.01)$. No misfit was detected in the mean and the variance.
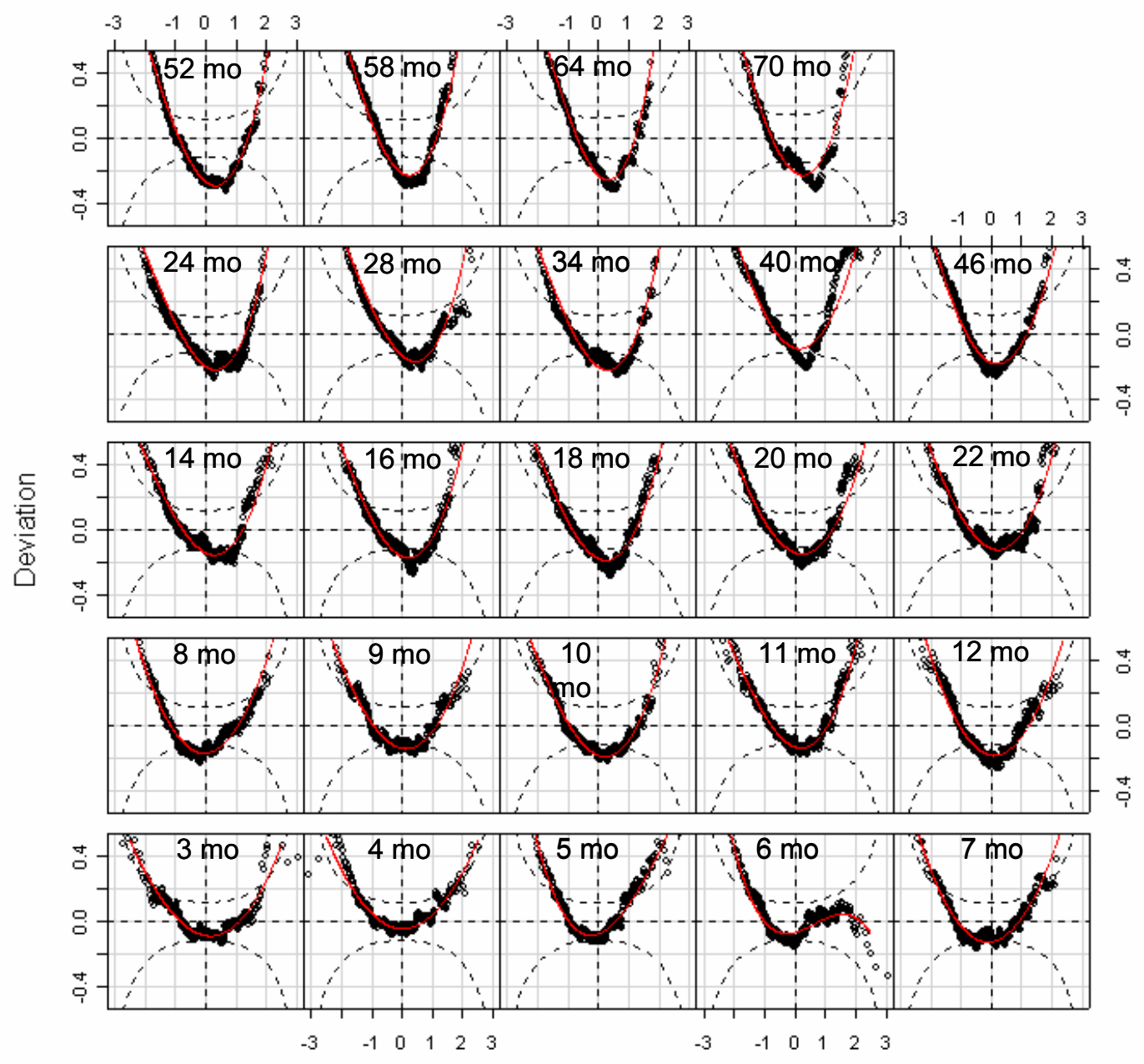

Unit normal quantile

Figure 79 Worm plots of z-scores for Model 1 for subscapular skinfold-for-age for girls 
Table 68 Q-test for z-scores from Model $1\left[\operatorname{BCPE}\left(x=\operatorname{age}^{0.50}, \operatorname{df}(\mu)=5, \operatorname{df}(\sigma)=4, v=1, \tau=2\right)\right]$ for subscapular skinfold-for-age for girls

\begin{tabular}{|c|c|c|c|c|c|c|}
\hline Age (days) & Group & $\mathbf{N}$ & $\mathrm{z1}$ & $\mathbf{z 2}$ & $\mathbf{z 3}$ & $\mathrm{z4}$ \\
\hline 83 to 99 & $3 \mathrm{mo}$ & 439 & 0.0 & -0.3 & 4.6 & 1.7 \\
\hline 100 to 129 & $4 \mathrm{mo}$ & 443 & 1.0 & 0.1 & 4.2 & 1.1 \\
\hline 130 to 159 & $5 \mathrm{mo}$ & 441 & 1.2 & 0.8 & 5.4 & 0.5 \\
\hline 160 to 189 & $6 \mathrm{mo}$ & 441 & 0.2 & -1.5 & 3.2 & -3.6 \\
\hline 190 to 219 & $7 \mathrm{mo}$ & 432 & 0.0 & 1.0 & 5.5 & 2.3 \\
\hline 220 to 249 & $8 \mathrm{mo}$ & 435 & -0.8 & 1.0 & 6.1 & 2.5 \\
\hline 250 to 279 & $9 \mathrm{mo}$ & 437 & -0.3 & -0.1 & 5.9 & 2.8 \\
\hline 280 to 309 & $10 \mathrm{mo}$ & 443 & -1.1 & -0.2 & 6.7 & 3.7 \\
\hline 310 to 349 & $11 \mathrm{mo}$ & 476 & 0.4 & 0.5 & 7.2 & 4.0 \\
\hline 350 to 379 & $12 \mathrm{mo}$ & 450 & -1.0 & -0.2 & 6.3 & 2.3 \\
\hline 380 to 439 & $14 \mathrm{mo}$ & 442 & 0.0 & -0.4 & 6.9 & 3.8 \\
\hline 440 to 499 & $16 \mathrm{mo}$ & 443 & 0.1 & 0.3 & 7.7 & 4.2 \\
\hline 500 to 559 & $18 \mathrm{mo}$ & 466 & -0.4 & 0.1 & 7.7 & 4.2 \\
\hline 560 to 619 & $20 \mathrm{mo}$ & 543 & -0.3 & -0.7 & 7.1 & 3.0 \\
\hline 620 to 679 & $22 \mathrm{mo}$ & 538 & 0.7 & 0.1 & 7.6 & 4.2 \\
\hline 680 to 749 & $24 \mathrm{mo}$ & 592 & -0.6 & 0.0 & 9.7 & 6.4 \\
\hline 750 to 929 & $28 \mathrm{mo}$ & 454 & 0.6 & -1.2 & 8.8 & 6.2 \\
\hline 930 to 1119 & $34 \mathrm{mo}$ & 466 & -0.5 & 0.1 & 9.0 & 6.0 \\
\hline 1120 to 1309 & $40 \mathrm{mo}$ & 470 & 1.7 & 0.0 & 7.5 & 3.2 \\
\hline 1310 to 1499 & $46 \mathrm{mo}$ & 448 & 0.0 & -0.5 & 7.9 & 3.6 \\
\hline 1500 to 1689 & $52 \mathrm{mo}$ & 443 & -1.5 & -0.7 & 9.4 & 5.9 \\
\hline 1690 to 1879 & $58 \mathrm{mo}$ & 487 & 0.3 & 1.1 & 9.9 & 5.6 \\
\hline 1880 to 2069 & $64 \mathrm{mo}$ & 413 & 0.3 & 0.9 & 10.3 & 6.6 \\
\hline 2070 to 2191 & $70 \mathrm{mo}$ & 292 & 0.1 & -0.2 & 7.7 & 4.5 \\
\hline Overall Q stats & & 10934 & 12.1 & 10.5 & 1308.7 & 416.5 \\
\hline degrees of freedom & & & 19.0 & 21.5 & 24.0 & 24.0 \\
\hline p-value & & & 0.8814 & 0.9773 & $<0.01$ & $<0.01$ \\
\hline
\end{tabular}

Note: Absolute values of z1, z2, z3 or z4 larger than 2 indicate misfit of, respectively, mean, variance, skewness or kurtosis.

The next step involved fitting the parameter $v$ for skewness using the BCPE distribution with fixed parameter $\tau=2$ and keeping the degrees of freedom for the $\mu$ and $\sigma$ curves selected for Model 1. Table 69 shows the $G A I C(3)$ values for various degrees of freedom for the $v$ curve.

Table 69 Goodness-of-fit summary for models $\operatorname{BCPE}\left(x=\operatorname{age}^{0.50}, \operatorname{df}(\mu)=5, \operatorname{df}(\sigma)=4, \operatorname{df}(v)=\right.$ ?, $\left.\tau=2\right)$ for subscapular skinfold-for-age for girls

\begin{tabular}{cccc}
\hline $\mathbf{d f}(\mathbf{v})$ & $\mathbf{G D}^{\mathbf{a}}$ & GAIC(3) $^{\mathbf{a}}$ & Total df \\
\hline $\mathbf{1}$ & 694.6 & 724.6 & 10 \\
$\mathbf{2}$ & 668.3 & 701.3 & 11 \\
$\mathbf{3}$ & 665.3 & 701.3 & 12 \\
$\mathbf{4}$ & 664.0 & 703.0 & 13 \\
$\mathbf{5}$ & 663.2 & 705.3 & 14 \\
$\mathbf{6}$ & 662.6 & 707.6 & 15 \\
$\mathbf{7}$ & 661.9 & 709.9 & 16 \\
\hline
\end{tabular}

GD, Global Deviance; GAIC(3), Generalized Akaike Information Criterion with penalty equal to 3 ;

${ }^{a}$ In excess of 36000. 
The smallest $\operatorname{GAIC}(3)$ value corresponded to $\mathrm{df}(v)=2$ or 3 . Selecting $\operatorname{df}(v)=2$, a re-search for the best $\operatorname{df}(\mu)$ and $\operatorname{df}(\sigma)$ was carried out and $\operatorname{df}(\mu)=5$ and $\operatorname{df}(\sigma)=4$ was still the best choice. A re-search for the age transformation power $\lambda$ using the model $\operatorname{BCPE}\left(x=\operatorname{age}^{0.50}, \operatorname{df}(\mu)=5, \operatorname{df}(\sigma)=4, \operatorname{df}(v)=2, \tau=2\right)$ was carried out and it pointed to the value 0.15 as the best choice of $\lambda(G D=36665.5)$. Using $\lambda=0.15$ and $\operatorname{df}(v)=2$, the best combination of $\operatorname{df}(\mu)$ and $\operatorname{df}(\sigma)$ was sought. Partial results of this re-search are presented in Table 70.

Table 70 Goodness-of-fit summary for models using the BCPE distribution with $\lambda=0.15$, $\operatorname{df}(v)=2$ and $\tau=2$ for subscapular skinfold-for-age for girls

\begin{tabular}{cccccc}
\hline $\mathbf{d f}(\boldsymbol{\mu})$ & $\mathbf{d f}(\boldsymbol{\sigma})$ & $\mathbf{G D}^{\mathbf{a}}$ & $\mathbf{A I C}^{\mathbf{a}}$ & $\mathbf{G A I C} \mathbf{3})^{\mathbf{a}}$ & Total df \\
\hline & $\mathbf{2}$ & 726.4 & 742.4 & 750.5 & 8 \\
$\mathbf{4}$ & $\mathbf{3}$ & 678.7 & 696.7 & 705.7 & 9 \\
& $\mathbf{4}$ & 669.7 & 689.7 & 699.7 & 10 \\
& $\mathbf{5}$ & 667.8 & 689.8 & 700.8 & 11 \\
& $\mathbf{6}$ & 667.1 & 691.1 & 703.1 & 12 \\
\hline & $\mathbf{2}$ & 722.7 & 740.7 & 749.7 & 9 \\
$\mathbf{5}$ & $\mathbf{3}$ & 674.3 & 694.3 & 704.3 & 10 \\
& $\mathbf{4}$ & 665.5 & 687.5 & 698.5 & 11 \\
& $\mathbf{5}$ & 663.8 & 687.8 & 699.8 & 12 \\
& $\mathbf{6}$ & 663.2 & 689.2 & 702.2 & 13 \\
\hline & $\mathbf{2}$ & 720.5 & 740.5 & 750.5 & 10 \\
$\mathbf{6}$ & $\mathbf{3}$ & 672.5 & 694.5 & 705.5 & 11 \\
& $\mathbf{4}$ & 663.9 & 687.9 & 699.9 & 12 \\
& $\mathbf{5}$ & 662.3 & 688.3 & 701.3 & 13 \\
& $\mathbf{6}$ & 661.6 & 689.6 & 703.6 & 14 \\
\hline & $\mathbf{2}$ & 719.6 & 741.6 & 752.6 & 11 \\
$\mathbf{7}$ & $\mathbf{3}$ & 671.1 & 695.1 & 707.1 & 12 \\
& $\mathbf{4}$ & 662.5 & 688.5 & 701.5 & 13 \\
& $\mathbf{5}$ & 660.9 & 688.9 & 702.9 & 14 \\
& $\mathbf{6}$ & 660.3 & 690.3 & 705.4 & 15 \\
\hline & $\mathbf{2}$ & 718.3 & 742.3 & 754.3 & 12 \\
& $\mathbf{3}$ & 669.8 & 695.8 & 708.8 & 13 \\
$\mathbf{8}$ & $\mathbf{4}$ & 661.3 & 689.3 & 703.3 & 14 \\
& $\mathbf{5}$ & 659.7 & 689.7 & 704.7 & 15 \\
& $\mathbf{6}$ & 659.2 & 691.2 & 707.2 & 16 \\
\hline
\end{tabular}

GD, Global Deviance; AIC, Akaike Information Criterion;

GAIC(3), Generalized AIC with penalty equal to 3;

${ }^{\mathrm{a}}$ In excess of 36000.

The smallest $\operatorname{GAIC}(3)$ value was associated with $\operatorname{df}(\mu)=5$ and $\operatorname{df}(\sigma)=4$ indicating no need for further updating the model.

Model 2: $\operatorname{BCPE}\left(x=\operatorname{age}^{0.15}, \operatorname{df}(\mu)=5, \operatorname{df}(\sigma)=4, \operatorname{df}(v)=2, \tau=2\right)$

Figure 80 shows the fit of parameters $\mu, \sigma$ and $v$ for Model 2 with their respective sample estimates, that is, the median for $\mu$, the sample standard deviation of the Box-Cox transformed data for $\sigma$ and Box-Cox transform power for $v$.

Figures 81 and 82 show the distribution of differences between empirical values and fitted centiles for the longitudinal and cross-sectional samples, respectively. There was a non-random pattern only for 
the $75^{\text {th }}$ centile for ages between 3 and 24 months (Figure 81), the maximum difference between the empirical and the fitted centiles being less than $0.2 \mathrm{~mm}$. There was no indication of systematic bias for ages between 24 and 71 months (Figure 82).

The worm plots for this model (Figure 83) show that adjustment for skewness was effective (no age groups presented U-shaped worms using Model 2). There were a few age groups with S-shaped worms but these were contained within the 95\% confidence intervals, except for age groups 5 mo and 6 mo, which indicated remaining kurtosis. There was also a worm with slope at age group 28 mo, suggesting misfit of the variance but it was contained within the $95 \%$ confidence interval.
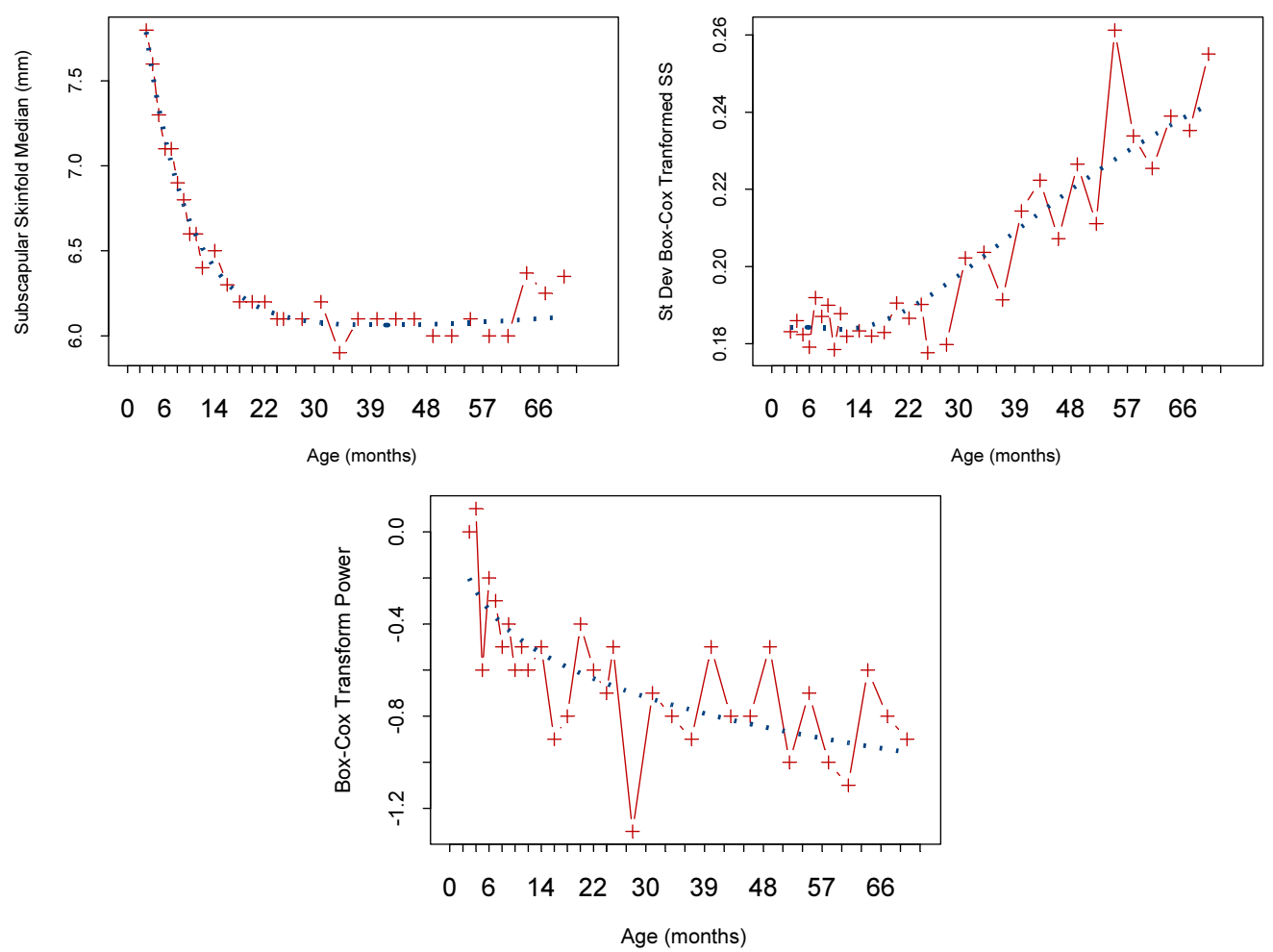

Figure 80 Fitting of the $\mu, \sigma$, and $v$ curves of Model 2 for subscapular skinfold-for-age for girls from 3 to 71 months (dotted line) and their respective sample estimates (points with solid line) 

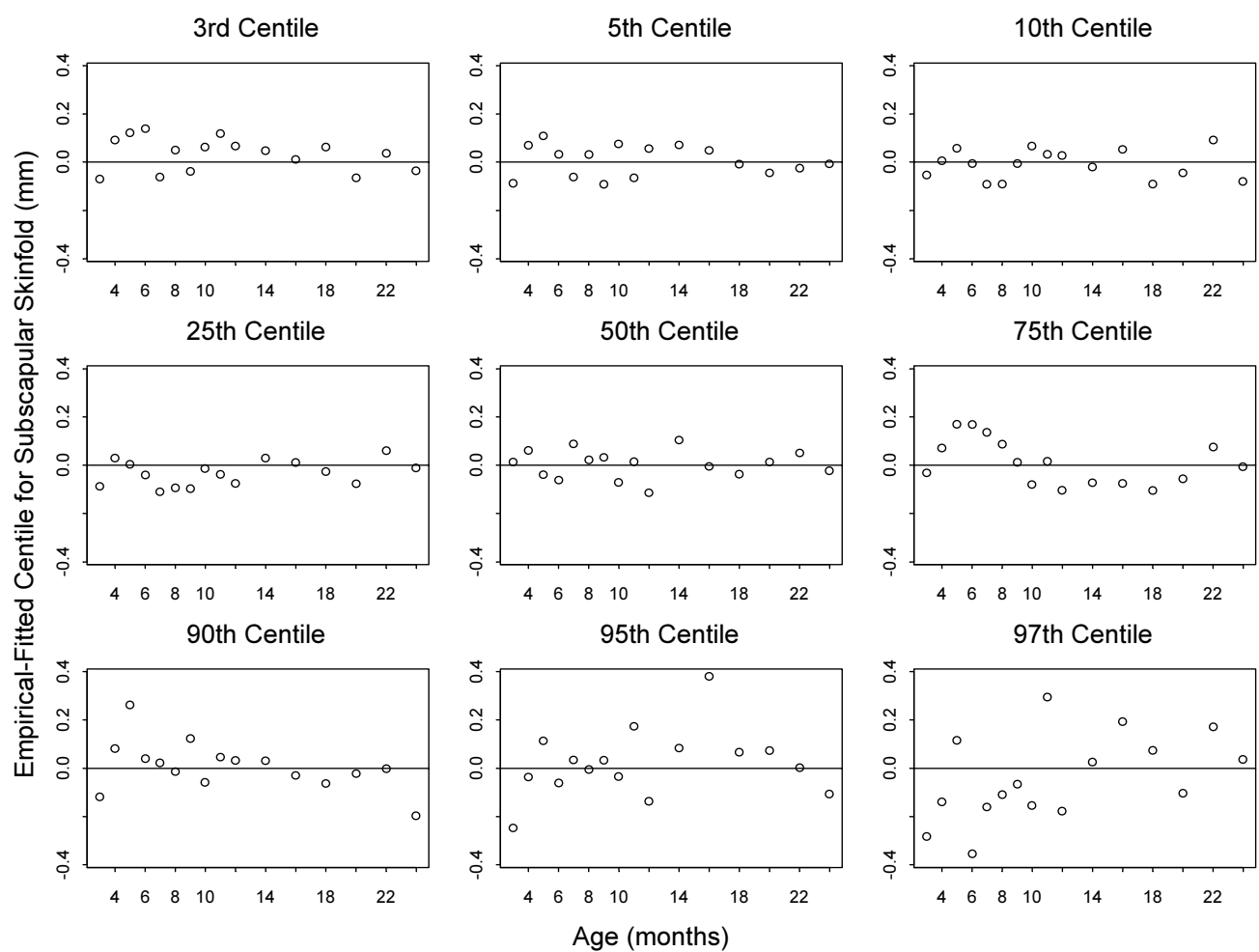

Figure 81 Centile residuals from fitting Model 2 for subscapular skinfold-for-age from 3 to 24 months for girls
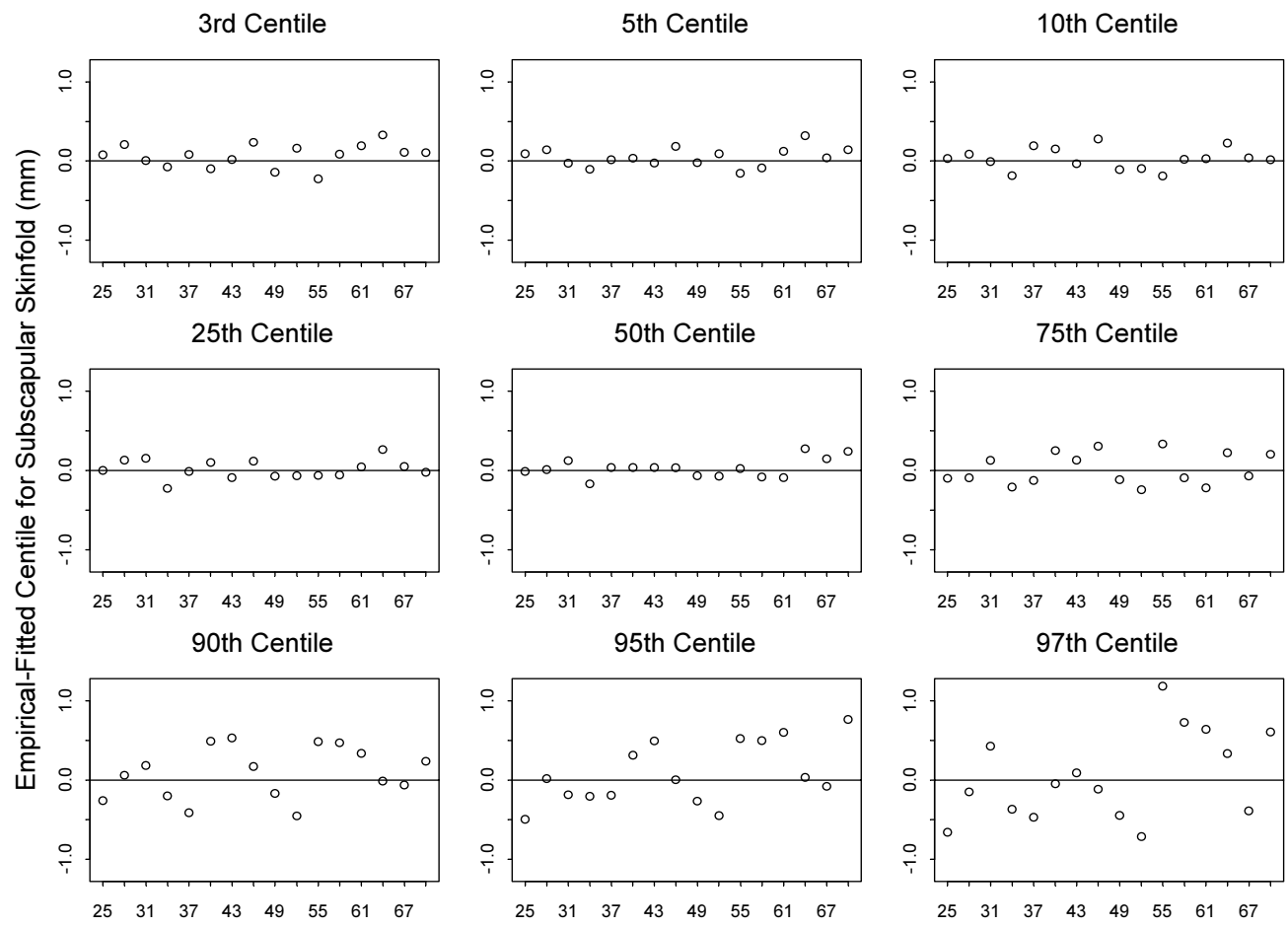

50th Centile

75th Centile
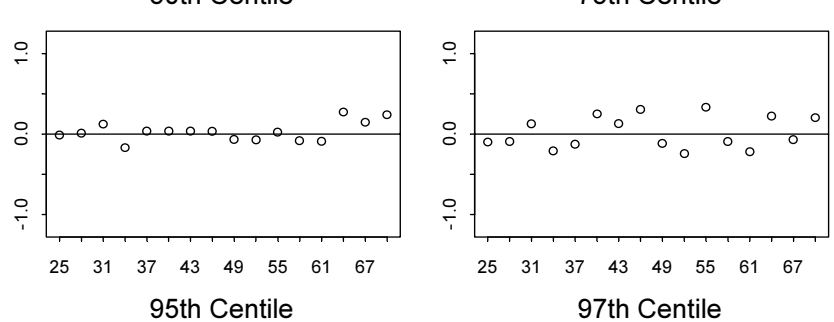

95th Centile
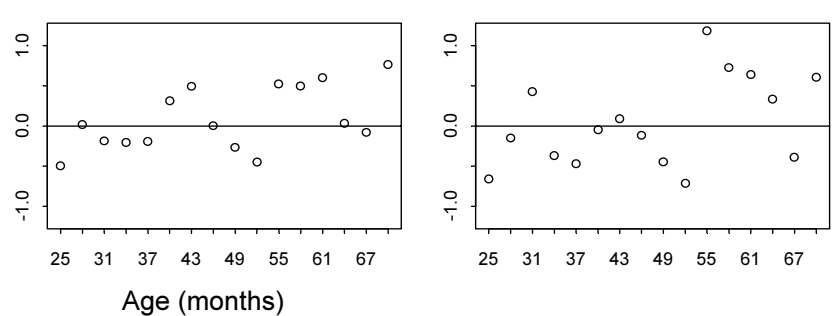

Figure 82 Centile residuals from fitting Model 2 for subscapular skinfold-for-age from 24 to 71 months for girls 

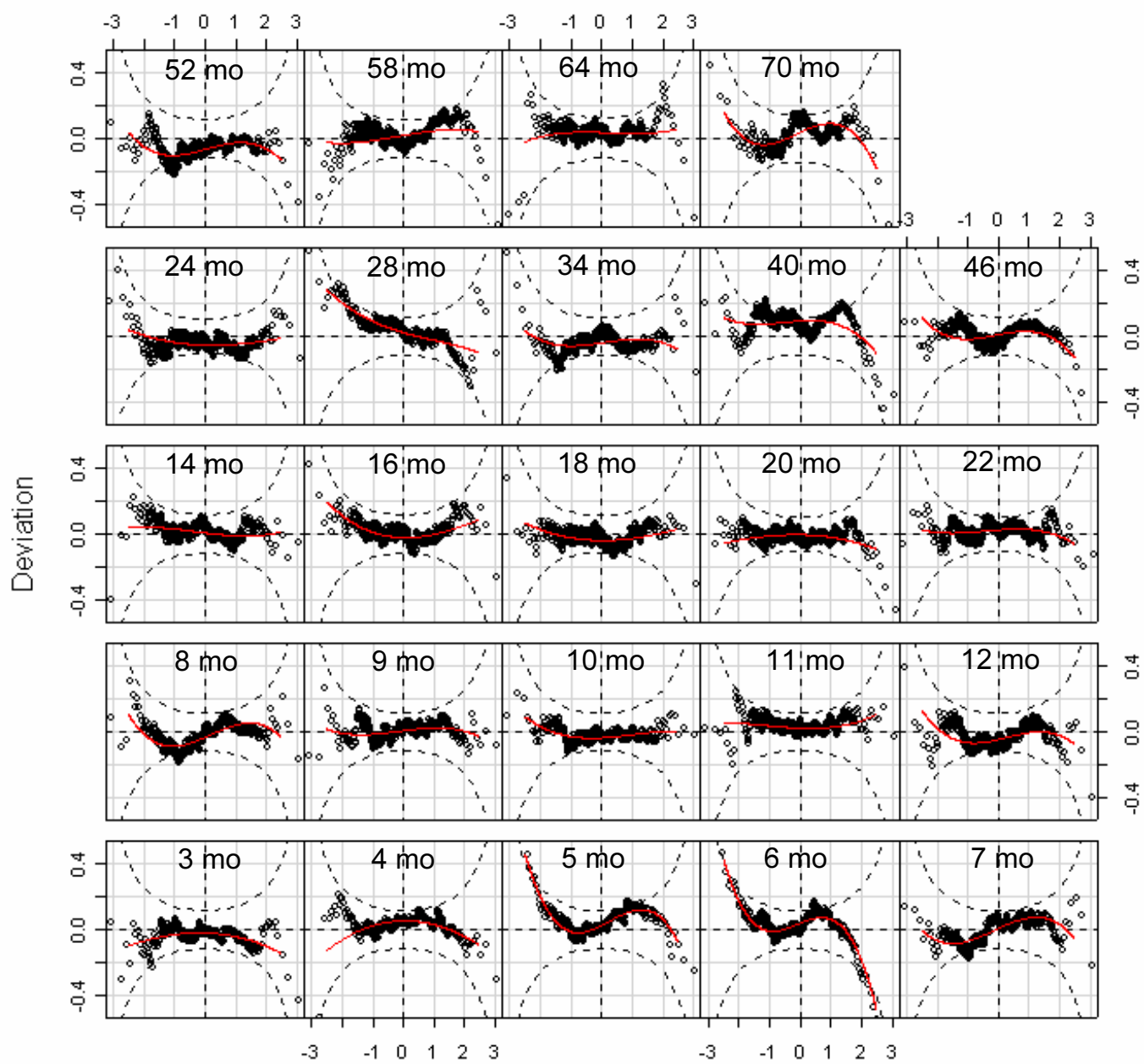

Unit normal quantile

Figure 83 Worm plots of z-scores for Model 2 for subscapular skinfold-for-age for girls

The Q-test results from Model 2 are shown in Table 71. There were no indications of misfit of the mean or variance, as neither $\mathrm{z} 1$ nor $\mathrm{z} 2$ absolute values were larger than 2 . There were also no age groups with $\mathrm{z} 3$ absolute values larger than 2 to indicate remaining skewness. Although only two age groups presented absolute values of $\mathrm{z} 4$ greater than 2, the overall test statistic for kurtosis had a significant p-value at the $5 \%$ level $(<0.01)$. Based on these results, a model adjusting for kurtosis (modelling $\tau$ ) was sought to assess if increasing the model's complexity and compromising the consistency between the sexes and across indicators would be justifiable.

A summary of results from the search for the best $\operatorname{df}(\tau)$ is found in Table 72 . The model with $\operatorname{df}(\tau)=6$ corresponded to the smallest values of $G A I C(3)$ and thus this model was selected for comparison with Model 2.

Figure 84 compares curves from Model 2 and the model BCPE $\left(x=a g e^{0.15}, \operatorname{df}(\mu)=5, \operatorname{df}(\sigma)=4, \operatorname{df}(v)=2\right.$, $\operatorname{df}(\tau)=6)$. The curves for the two models overlap even at the outer tails of the distribution providing no justification for using a more the complex model. Thus, Model 2 was selected as the final one for constructing the subscapular skinfold-for-age standards for girls. 
Table 71 Q-test for $\mathrm{z}$-scores from Model $2\left[\mathrm{BCPE}\left(\mathrm{x}=\operatorname{age}^{0.15}, \mathrm{df}(\mu)=5 \mathrm{df}(\sigma)=4, \mathrm{df}(v)=2, \tau=2\right)\right]$ for subscapular skinfold-for-age for girls

\begin{tabular}{|c|c|c|c|c|c|c|}
\hline Age (days) & Group & $\mathbf{N}$ & $\mathrm{z1}$ & $\mathbf{z 2}$ & $\mathbf{z 3}$ & $\mathrm{z4}$ \\
\hline 83 to 99 & $3 \mathrm{mo}$ & 439 & -0.8 & -0.2 & -0.8 & 0.1 \\
\hline 100 to 129 & $4 \mathrm{mo}$ & 443 & 0.6 & 0.2 & -1.6 & 1.1 \\
\hline 130 to 159 & $5 \mathrm{mo}$ & 441 & 1.1 & 0.1 & 1.2 & -3.5 \\
\hline 160 to 189 & $6 \mathrm{mo}$ & 441 & 0.6 & -1.0 & -0.5 & -5.5 \\
\hline 190 to 219 & $7 \mathrm{mo}$ & 432 & 0.1 & 1.1 & -0.2 & -1.5 \\
\hline 220 to 249 & $8 \mathrm{mo}$ & 435 & -0.6 & 1.1 & 0.7 & -1.8 \\
\hline 250 to 279 & $9 \mathrm{mo}$ & 437 & 0.1 & 0.0 & -0.2 & -0.4 \\
\hline 280 to 309 & $10 \mathrm{mo}$ & 443 & -0.7 & 0.0 & 0.6 & -0.7 \\
\hline 310 to 349 & $11 \mathrm{mo}$ & 476 & 0.7 & 0.1 & 0.5 & 0.2 \\
\hline 350 to 379 & $12 \mathrm{mo}$ & 450 & -0.8 & 0.2 & 0.6 & -1.3 \\
\hline 380 to 439 & $14 \mathrm{mo}$ & 442 & 0.2 & -0.4 & 0.1 & 0.4 \\
\hline 440 to 499 & $16 \mathrm{mo}$ & 443 & 0.1 & -0.3 & 1.4 & -0.1 \\
\hline 500 to 559 & $18 \mathrm{mo}$ & 466 & -0.6 & 0.0 & 0.8 & 0.0 \\
\hline 560 to 619 & $20 \mathrm{mo}$ & 543 & -0.5 & -0.1 & -0.6 & 0.3 \\
\hline 620 to 679 & $22 \mathrm{mo}$ & 538 & 0.4 & 0.1 & -0.4 & -0.6 \\
\hline 680 to 749 & $24 \mathrm{mo}$ & 592 & -1.0 & -0.1 & 0.6 & 0.0 \\
\hline 750 to 929 & $28 \mathrm{mo}$ & 454 & 0.7 & -1.9 & 0.7 & -0.2 \\
\hline 930 to 1119 & $34 \mathrm{mo}$ & 466 & -0.9 & 0.2 & 0.1 & -0.6 \\
\hline 1120 to 1309 & $40 \mathrm{mo}$ & 470 & 1.7 & -0.3 & -0.6 & -0.6 \\
\hline 1310 to 1499 & $46 \mathrm{mo}$ & 448 & 0.1 & -0.1 & -0.1 & -1.3 \\
\hline 1500 to 1689 & $52 \mathrm{mo}$ & 443 & -1.5 & 0.3 & 0.2 & -1.2 \\
\hline 1690 to 1879 & $58 \mathrm{mo}$ & 487 & 0.3 & 0.9 & 0.0 & -0.1 \\
\hline 1880 to 2069 & $64 \mathrm{mo}$ & 413 & 0.7 & 0.2 & -0.2 & 0.7 \\
\hline 2070 to 2191 & $70 \mathrm{mo}$ & 292 & 0.5 & 0.4 & -0.3 & -1.9 \\
\hline Overall Q stats & & 10934 & 13.6 & 8.7 & 10.7 & 60.2 \\
\hline degrees of freedom & & & 19.0 & 21.5 & 22.0 & 24.0 \\
\hline p-value & & & 0.8093 & 0.9934 & 0.9790 & $<0.01$ \\
\hline
\end{tabular}

Note: Absolute values of z1, z2, z3 or z4 larger than 2 indicate misfit of, respectively, mean, variance, skewness or kurtosis.

Table 72 Goodness-of-fit summary for models $\operatorname{BCPE}\left(x=a g e^{0.15}, \operatorname{df}(\mu)=5, \operatorname{df}(\sigma)=4, \operatorname{df}(v)=2\right.$, $\operatorname{df}(\tau)=$ ?) for subscapular skinfold-for-age for girls

\begin{tabular}{cccc}
\hline $\mathbf{d f}(\boldsymbol{\tau})$ & $\mathbf{G D}^{\mathbf{a}}$ & GAIC(3) $^{\mathbf{a}}$ & Total df \\
\hline $\mathbf{1}$ & 655.3 & 691.3 & 12 \\
$\mathbf{2}$ & 652.6 & 691.7 & 13 \\
$\mathbf{3}$ & 648.3 & 690.3 & 14 \\
$\mathbf{4}$ & 643.1 & 688.2 & 15 \\
$\mathbf{5}$ & 638.5 & 686.5 & 16 \\
$\mathbf{6}$ & 635.2 & 686.2 & 17 \\
$\mathbf{7}$ & 632.8 & 686.8 & 18 \\
\hline
\end{tabular}

GD, Global Deviance; GAIC(3), Generalized Akaike Information Criterion with penalty equal to 3 ;

${ }^{\mathrm{a}}$ In excess of 36000 . 
Table 73 presents observed percentages with subscapular skinfolds below the fitted centiles. There was no detectable pattern of a systematic bias.

The model BCPE $\left(x=a g e^{0.15}, \operatorname{df}(\mu)=5, \operatorname{df}(\sigma)=4, \operatorname{df}(v)=2, \tau=2\right)$ was considered adequate and selected for constructing the subscapular skinfold-for-age growth curves for girls. It adjusts only for skewness, since there was no difference in the final curves when adjusting for kurtosis. The fitted centile curves and empirical centiles are shown in Figures 85 to 88 . 


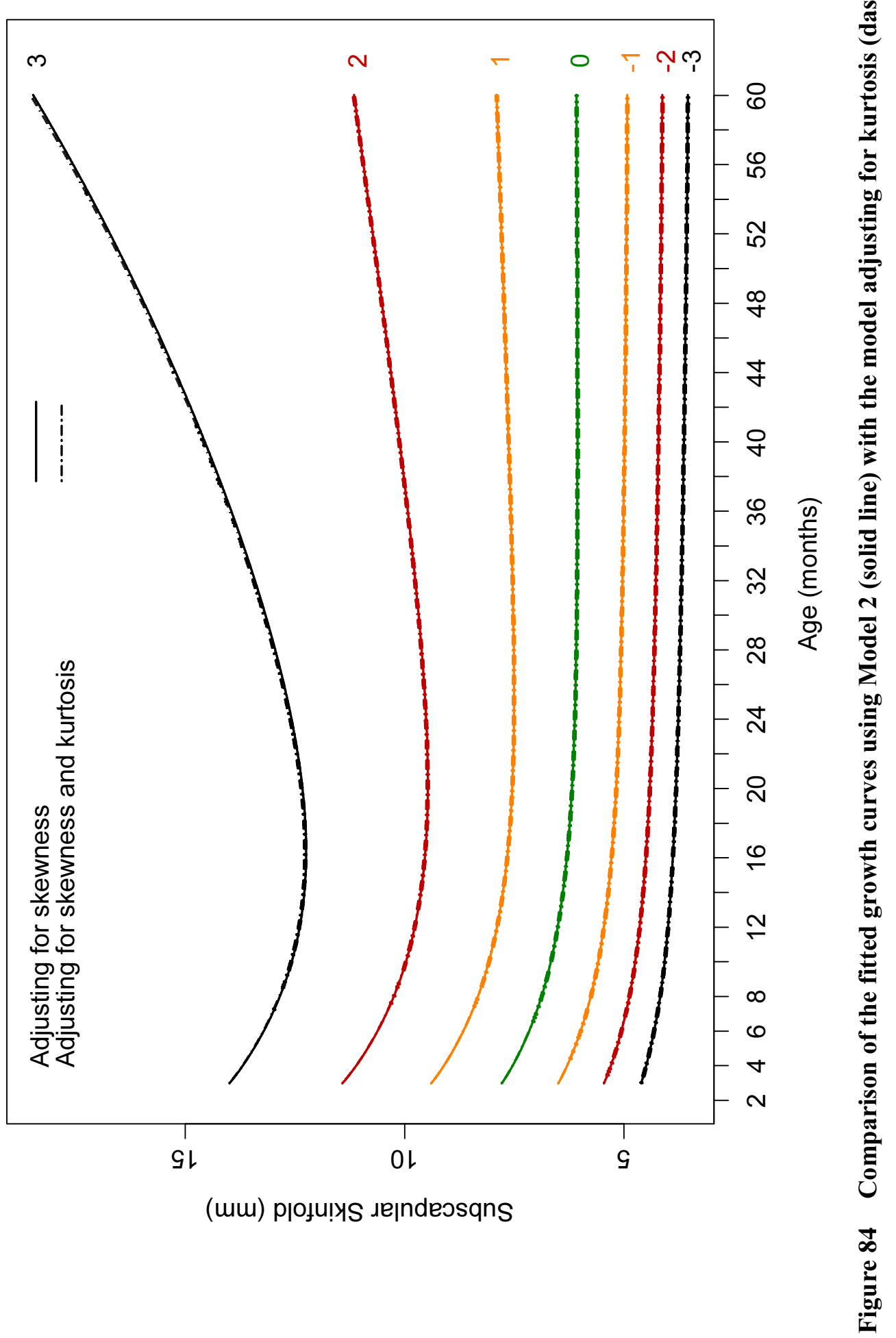




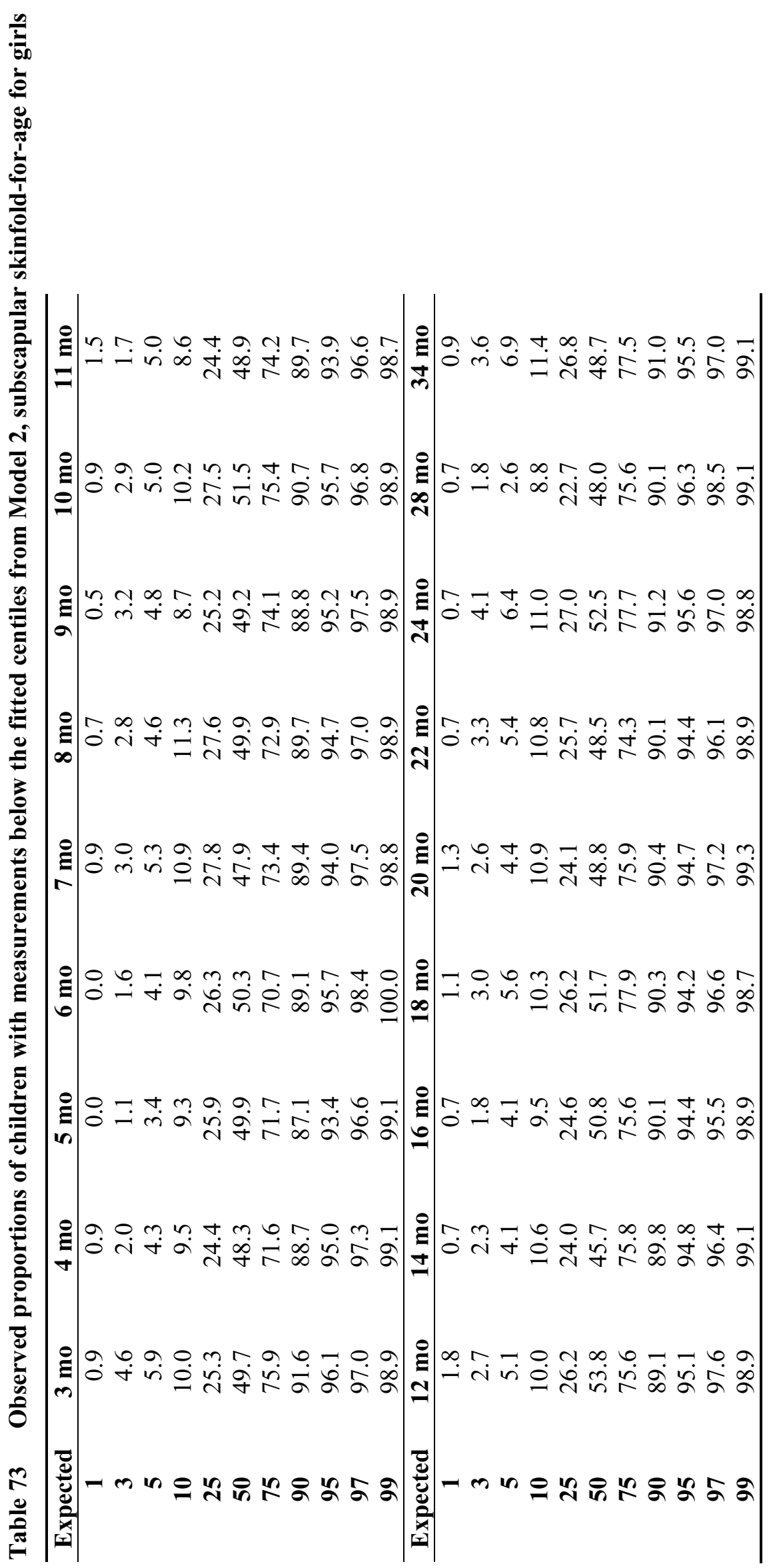




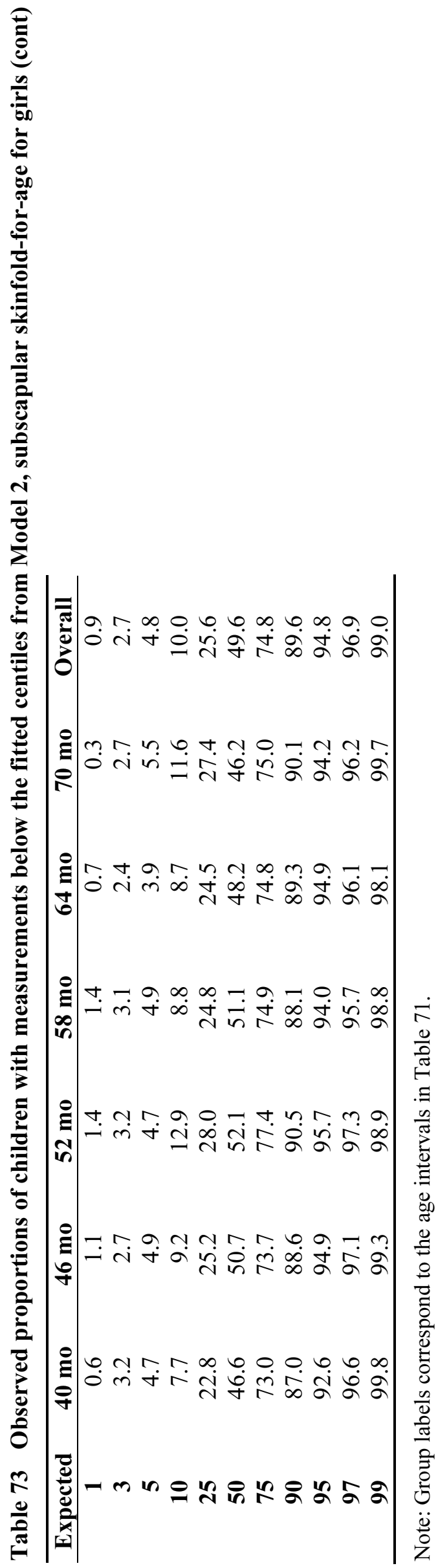




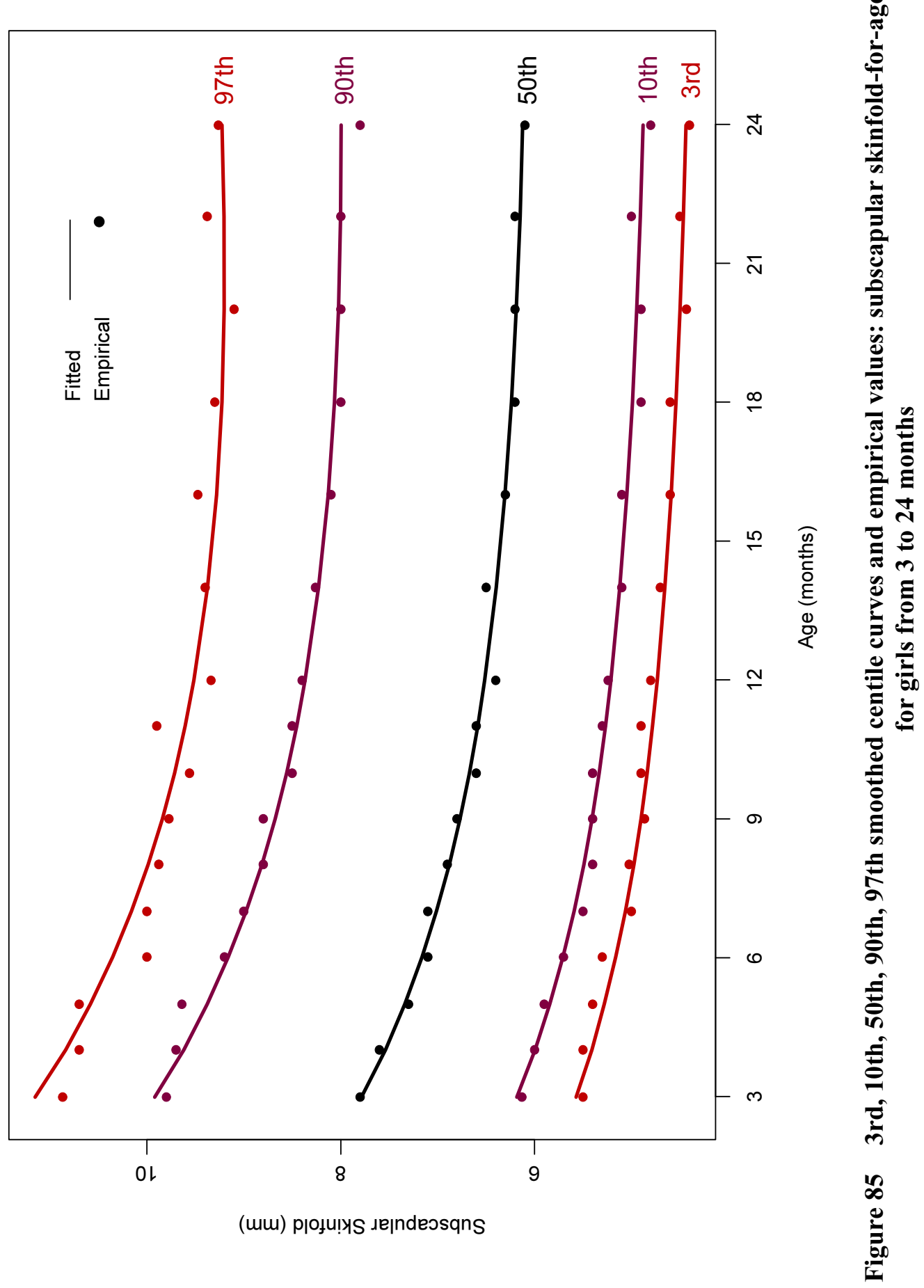




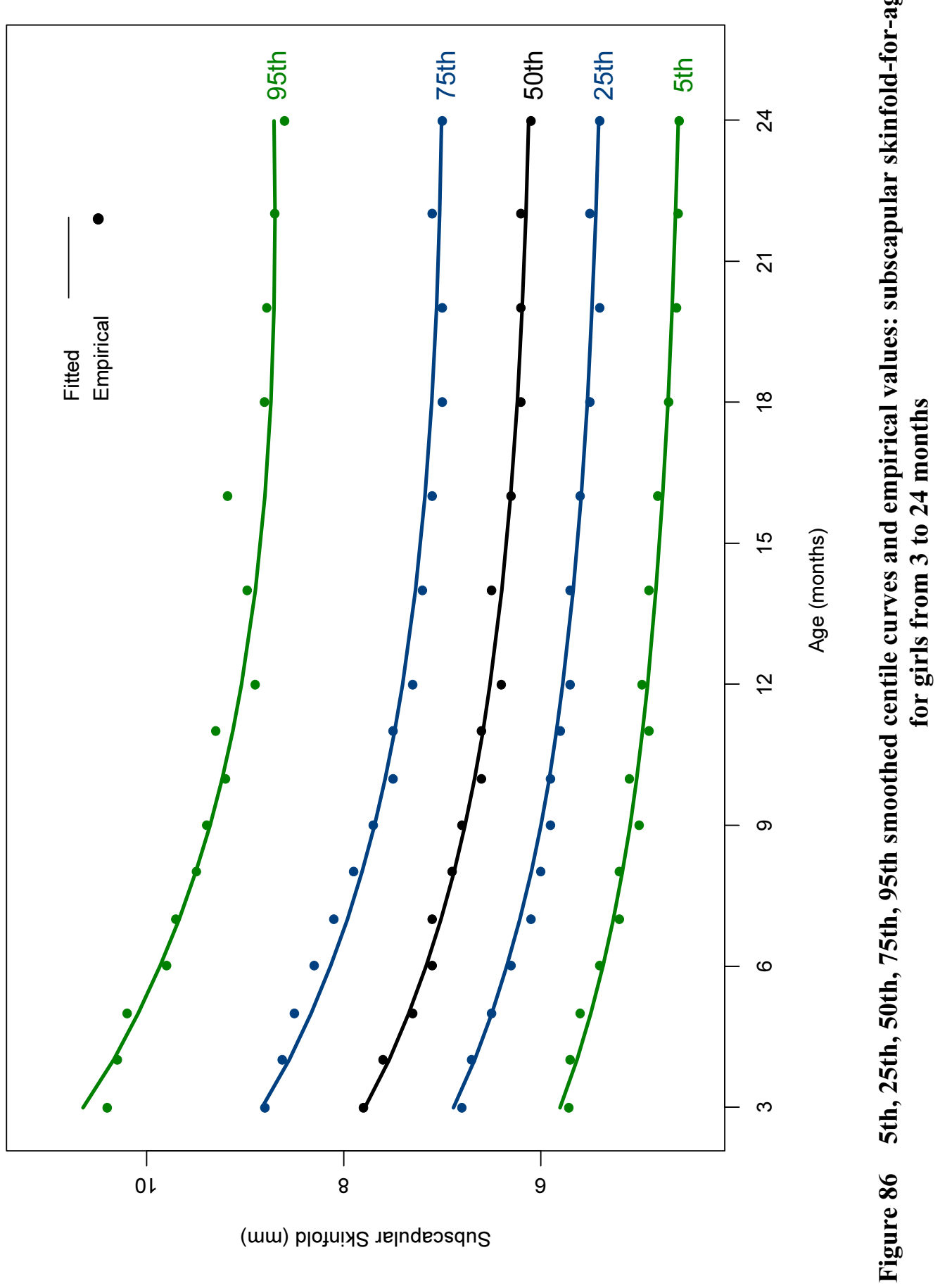




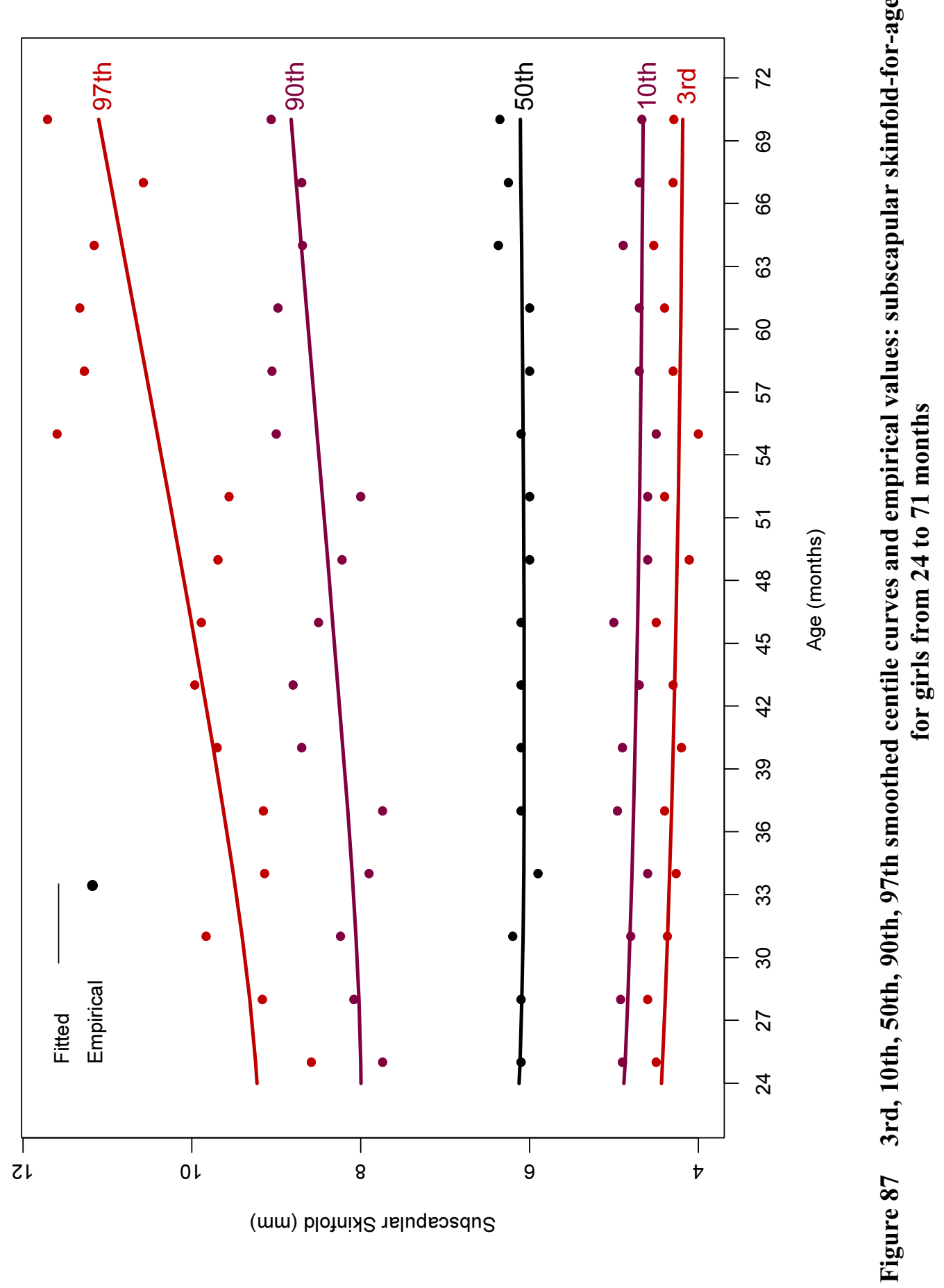




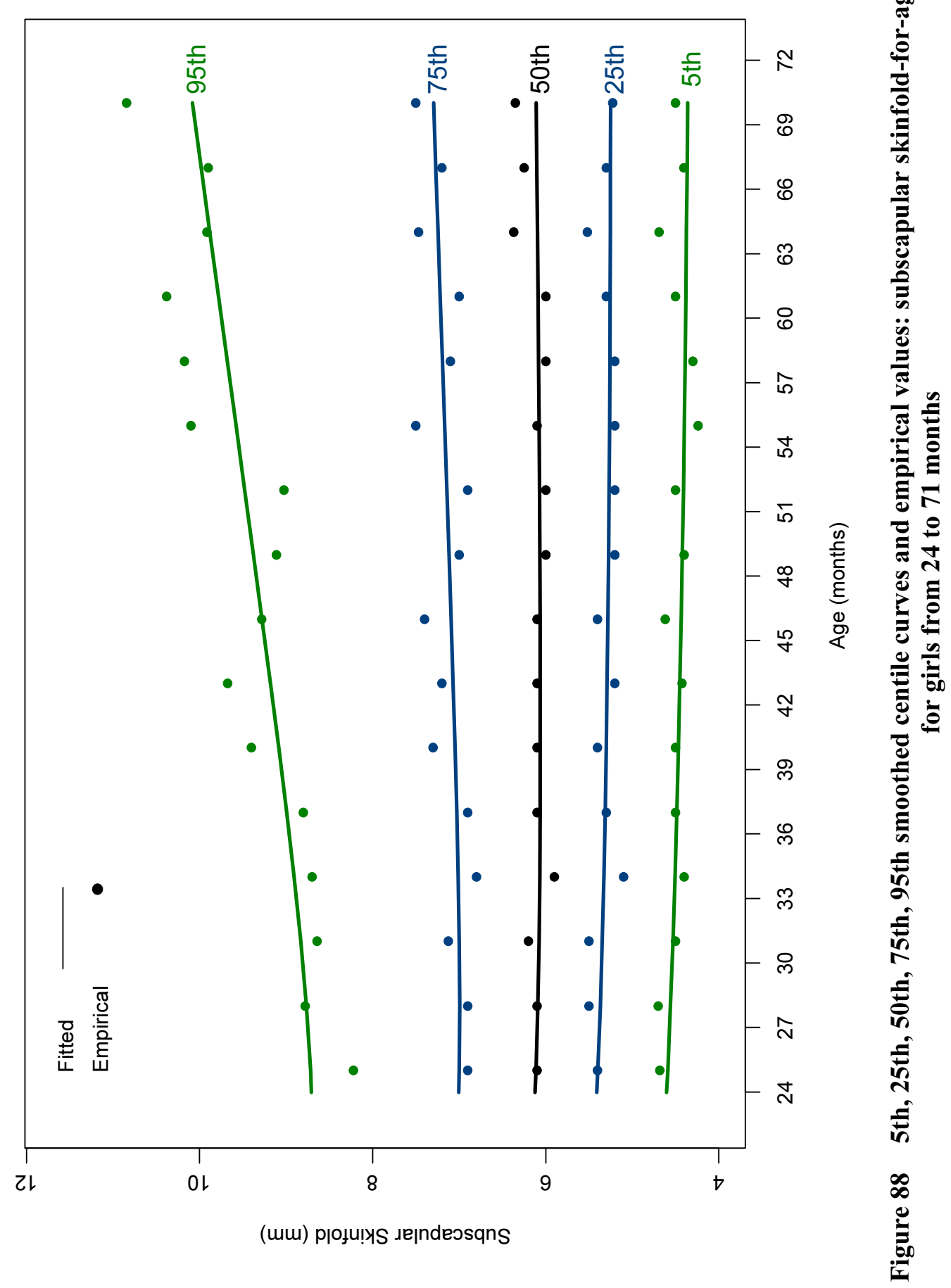




\subsubsection{WHO standards}

This section presents the final WHO subscapular skinfold-for-age z-score and percentile charts (Figures 89 and 90) and table (Table 74) for girls. 


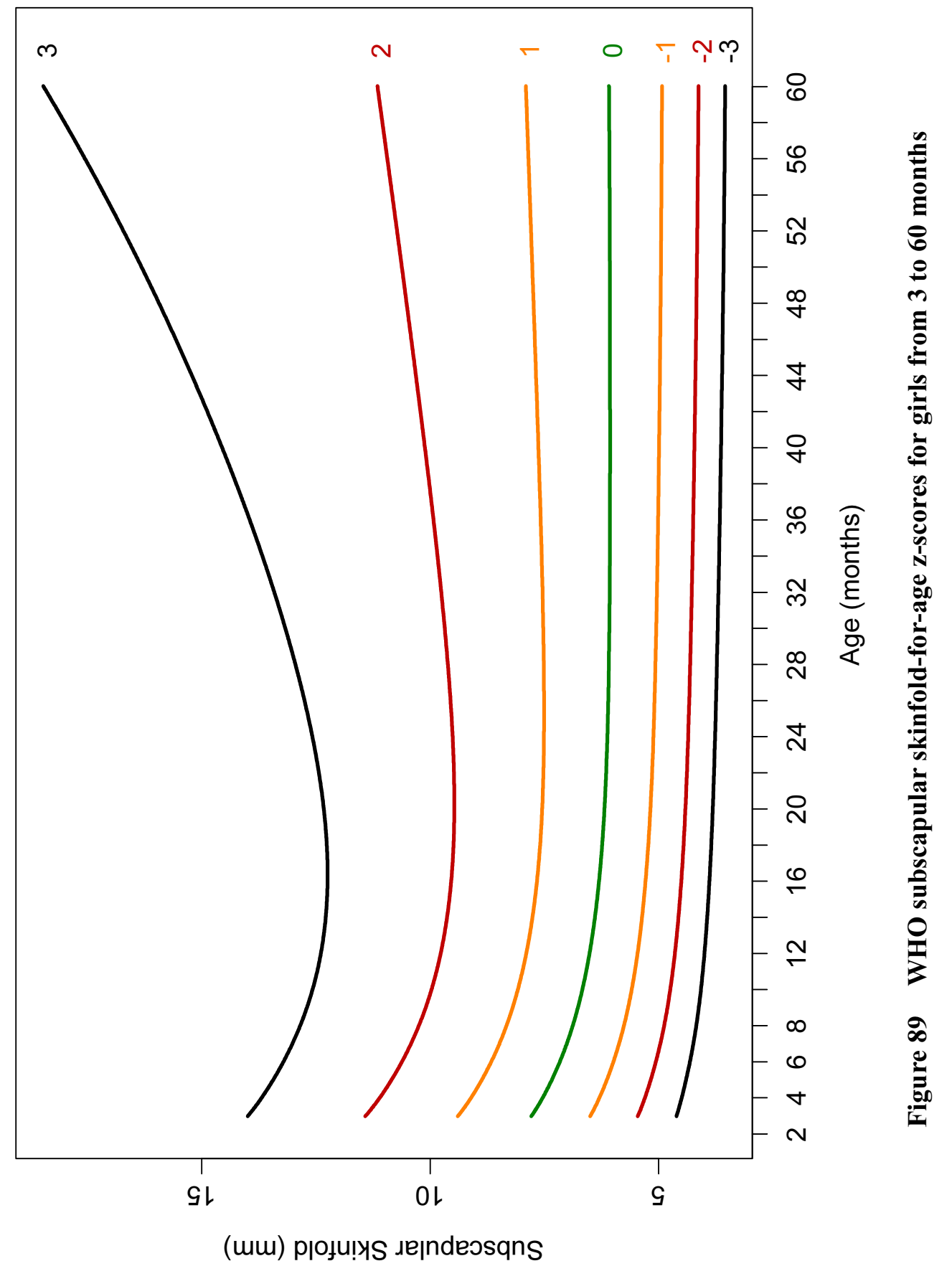




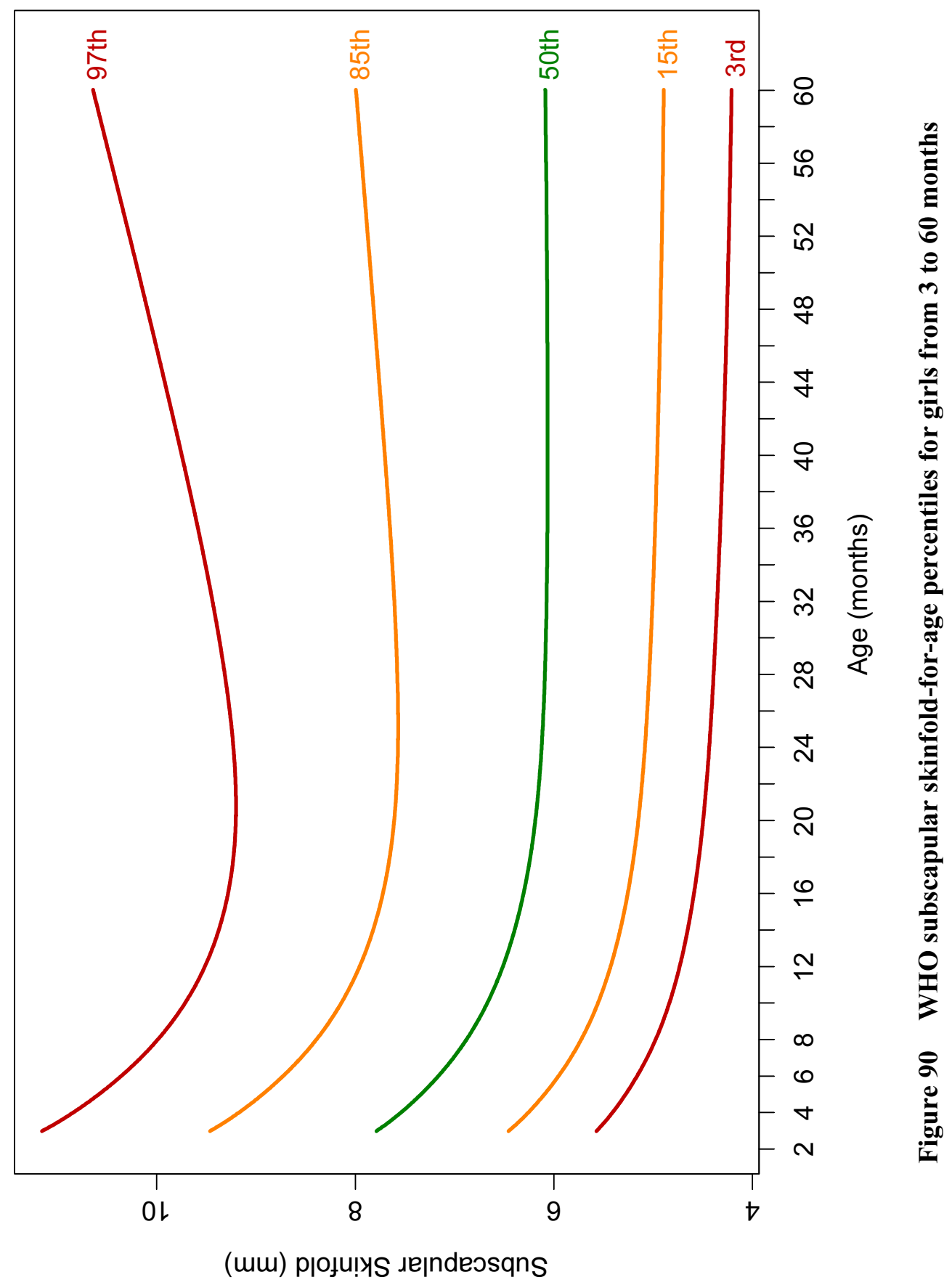




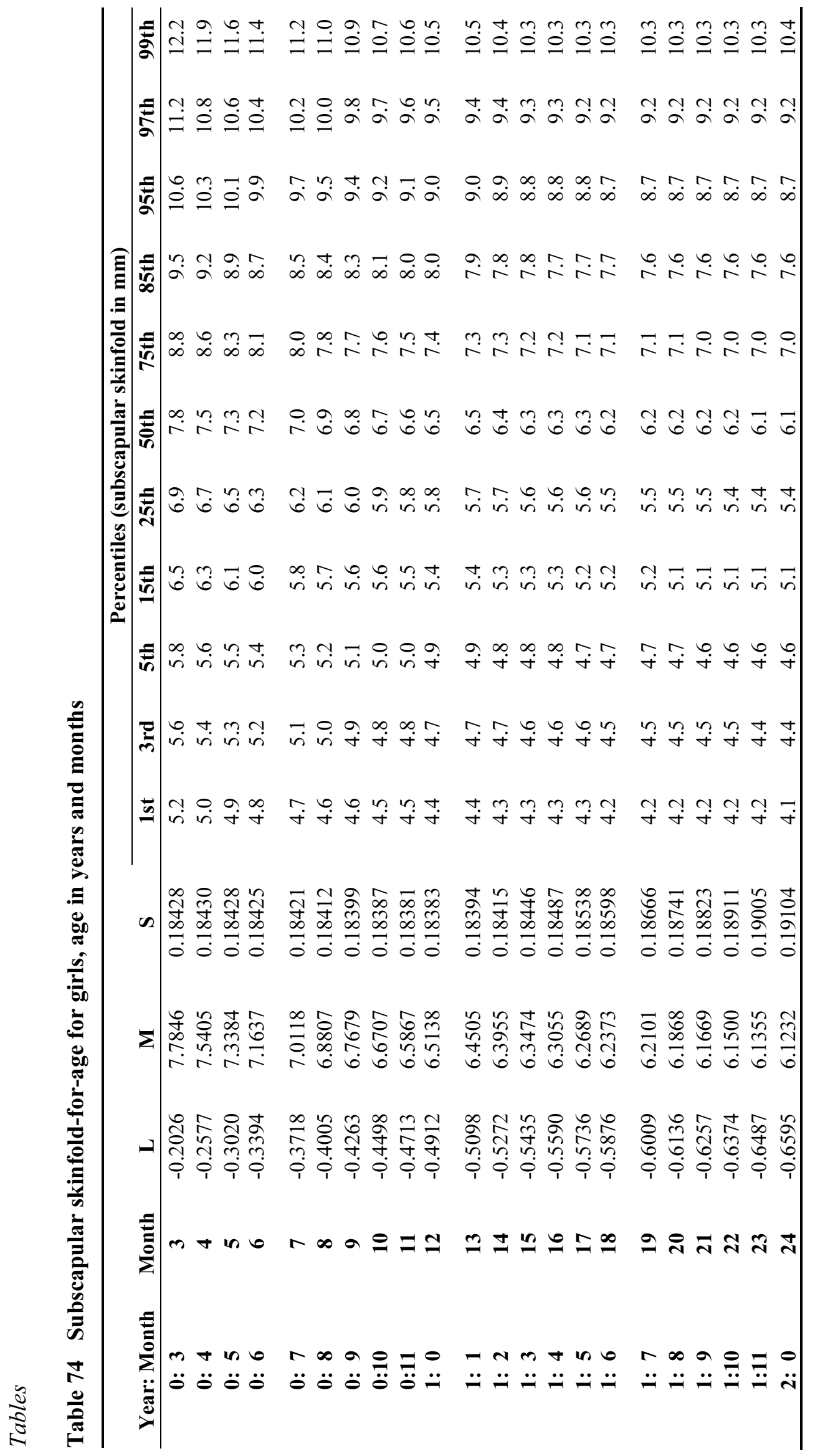




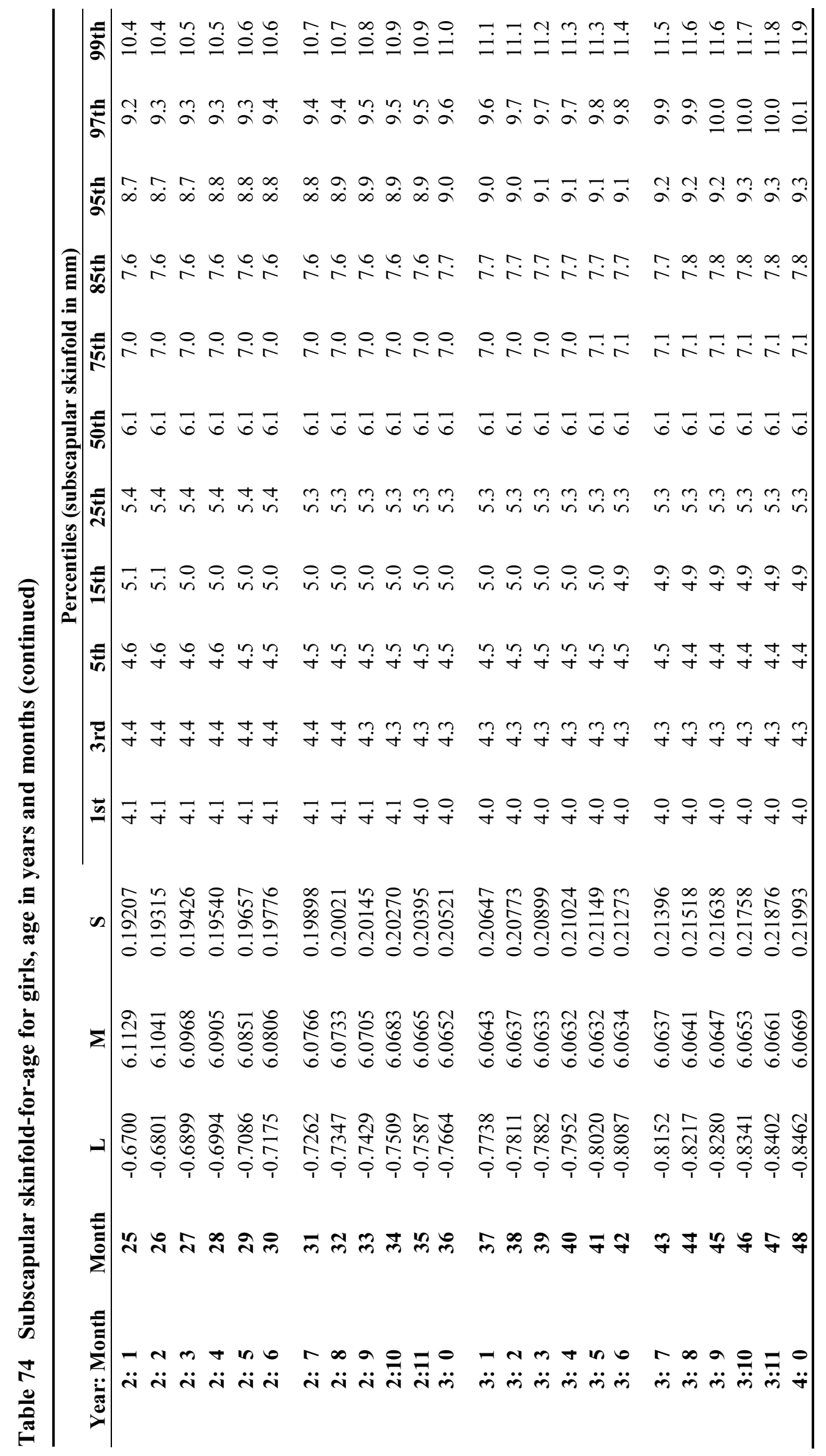




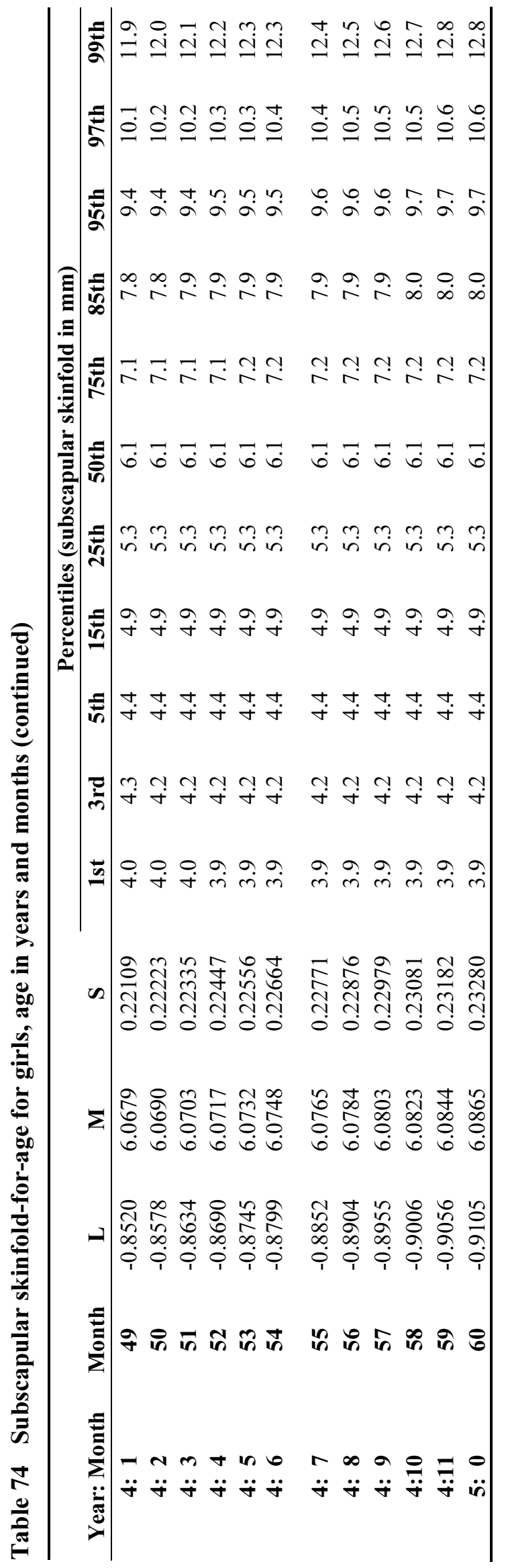




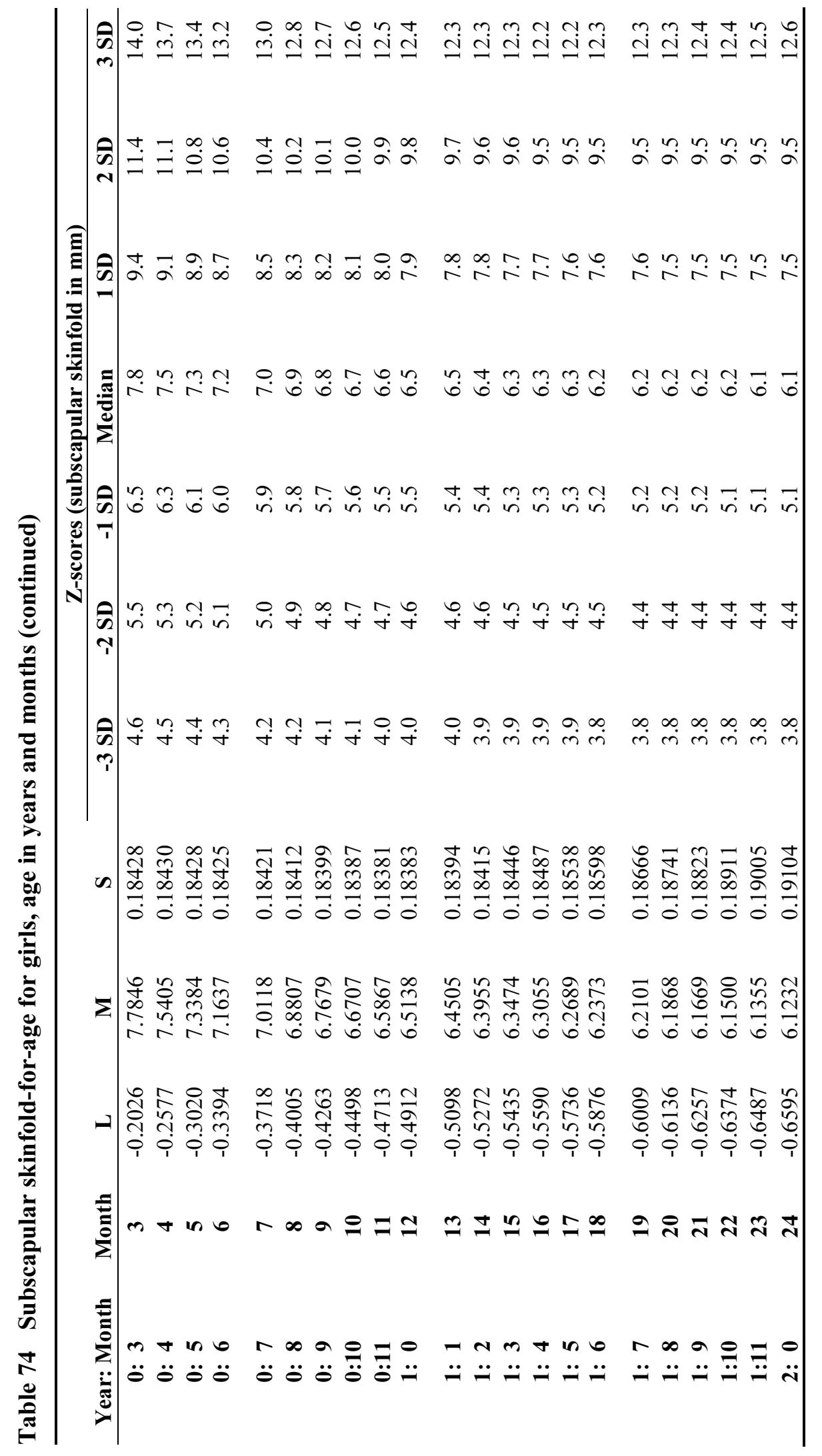




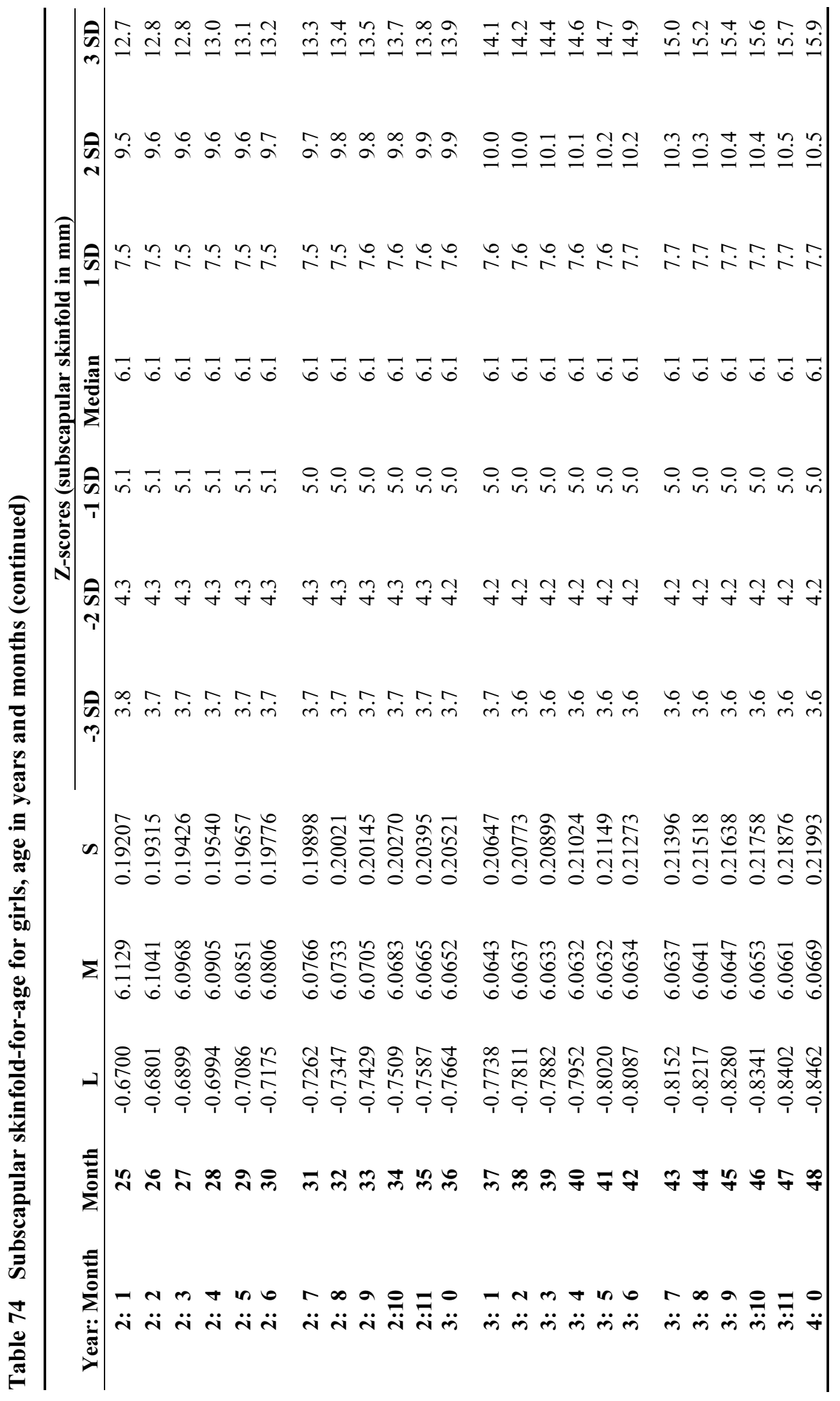




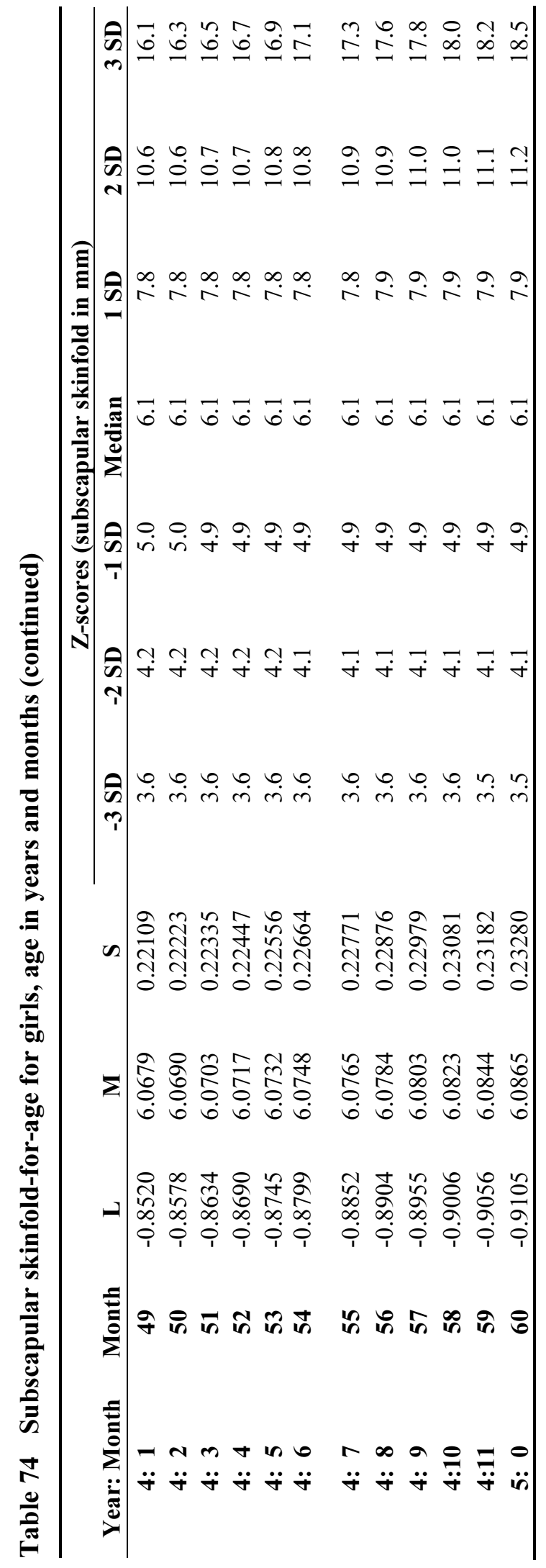




\subsection{Comparisons between boys and girls}

This section presents the subscapular skinfold-for-age z-score comparisons between boys and girls for the WHO standards (Figure 91). 


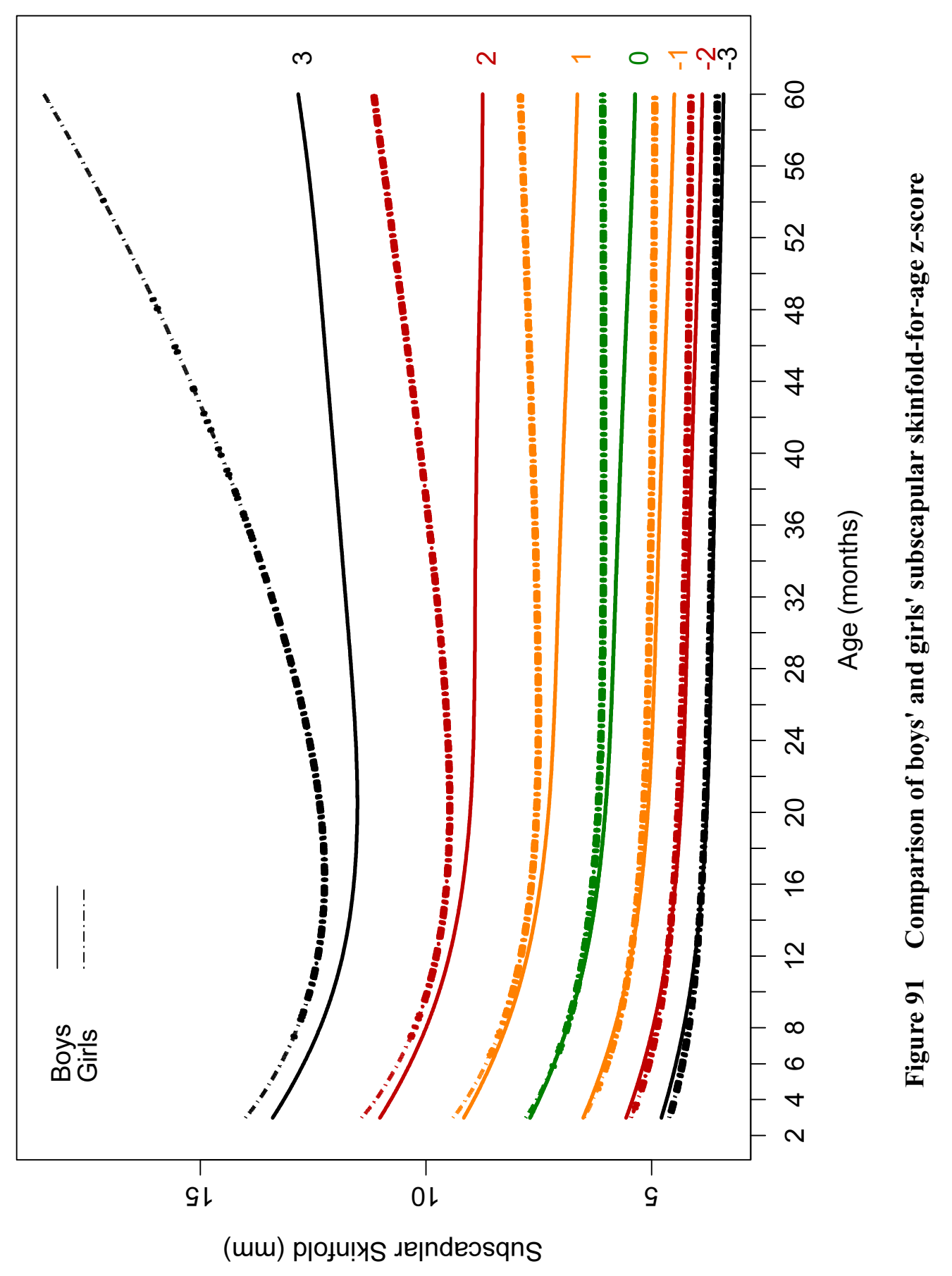

$\frac{0}{3}$ 


\section{COMPUTATION OF CENTILES AND Z-SCORES FOR HEAD CIRCUMFE- RENCE-FOR-AGE, ARM CIRCUMFERENCE-FOR-AGE, TRICEPS SKIN- FOLD-FOR-AGE, SUBSCAPULAR SKINFOLD-FOR-AGE}

The method used to construct the standards based on head circumference, arm circumference, skinfold thicknesses and age, generally relied on GAMLSS with the Box-Cox power exponential distribution (Rigby and Stasinopoulos, 2004). However, the final selected models simplified to the LMS model (Cole and Green, 1992) since none of the standards required adjustment for kurtosis. As a result, the computation of percentiles and z-scores for these standards uses formulae based on the LMS method. However, a restriction was imposed on all indicators to enable the derivation of percentiles only within the interval corresponding to $\mathrm{z}$-scores between -3 and 3 . The underlying reasoning is that percentiles beyond $\pm 3 \mathrm{SD}$ are invariant to changes in equivalent $\mathrm{z}$-scores. The loss accruing to this restriction is small since the inclusion range corresponds to the 0.135 th to 99.865 th percentiles.

For all indicators, the tabulated fitted values of Box-Cox power, median and coefficient of variation corresponding to age $t$ are denoted by $L(t), M(t)$ and $S(t)$, respectively.

\section{Centiles and z-scores for head circumference-for-age}

For this indicator, $L(t)$ is equal to 1, simplifying the Box-Cox normal distribution used in the LMS method (Cole and Green, 1992) to the normal distribution. Therefore, differences between adjacent standard deviations (e.g. between 2 SD and 3 SD) were constant for a specific age but varied at different ages.

In this case, the centiles at age $t$ can be estimated from:

$$
\begin{array}{r}
C_{100 \alpha}(t)=M(t)\left[1+L(t) S(t) Z_{\alpha}\right]^{1 / L(t)}=M(t)\left[1+S(t) Z_{\alpha}\right] \\
=M(t)+S t \operatorname{Dev}(t) Z_{\alpha}, \quad-3 \leq Z_{\alpha} \leq 3
\end{array}
$$

where $\mathrm{Z}_{\alpha}$ is the normal equivalent deviate for tail area $\alpha, C_{100 \alpha}(t)$ is the $100 \alpha$-th centile, and $S t \operatorname{Dev}(t)$ is the standard deviation at age $t$ (derived from multiplying $S(t)$ by $M(t)$ ).

The z-score for a measurement $y$ at age $t$ is computed as:

$$
z_{\text {ind }}=\frac{[y / M(t)]^{L(t)}-1}{S(t) L(t)}=\frac{y-M(t)}{S t \operatorname{Dev}(t)}
$$


Centiles and z-scores for arm circumference-for-age, triceps skinfold-for-age and subscapular skinfold-for-age

These indicators presented right-skewed distributions as did the weight-related indicators (WHO Multicentre Growth Reference Study Group, 2006d). When modelled correctly, right skewness in data has the effect of making distances between positive z-scores increase progressively the farther away they are from the median, while distances between negative z-scores decrease progressively. The LMS method fits skewed data adequately by using a Box-Cox normal distribution, which follows the empirical data closely. The drawback, however, is that the outer tails of the distribution are highly affected by extreme data points even if only very few (e.g. less than 1\%). A restricted application of the LMS method as the one used for the construction of the WHO weight-related indicators (WHO Multicentre Growth Reference Study Group, 2006d) was used, limiting the Box-Cox normal distribution to the interval corresponding to z-scores where empirical data were available (i.e. between $-3 \mathrm{SD}$ and $3 \mathrm{SD}$ ). Beyond these limits, the standard deviation at each age was fixed to the distance between $\pm 2 \mathrm{SD}$ and $\pm 3 \mathrm{SD}$, respectively. This approach avoids making assumptions about the distribution of data beyond the limits of the observed values.

As a result of this adjustment, the z-score distribution can depart slightly from normality in the extreme tails (beyond $\pm 3 \mathrm{SD}$ ), although the expected practical impact is minimal.

The centiles were calculated as follows:

$$
C_{100 \alpha}(t)=M(t)\left[1+L(t) S(t) Z_{\alpha}\right]^{1 / L(t)}, \quad-3 \leq Z_{\alpha} \leq 3
$$

The following procedure is recommended to calculate a z-score for an individual child with measurement $y$ at age $t$ :

1. Calculate

$$
z_{\text {ind }}=\frac{[y / M(t)]^{L(t)}-1}{S(t) L(t)}
$$

2. Compute the final z-score $\left(z_{\text {ind }}^{*}\right)$ of the child for that indicator as:

$$
z_{\text {ind }}^{*}=\left\{\begin{array}{ccc}
z_{\text {ind }} & \text { if } & \left|z_{\text {ind }}\right| \leq 3 \\
3+\left(\frac{y-S D 3 p o s}{S D 23 p o s}\right) & \text { if } & z_{\text {ind }}>3 \\
-3+\left(\frac{y-S D 3 n e g}{S D 23 n e g}\right) & \text { if } & z_{\text {ind }}<-3
\end{array}\right.
$$

where 
$S D 3$ pos is the cut-off $3 \mathrm{SD}$ calculated at $t$ by the LMS method:

$$
S D 3 p o s=M(t)[1+L(t) * S(t) *(3)]^{1 / L(t)}
$$

SD3neg is the cut-off -3 SD calculated at $t$ by the LMS method:

$$
\text { SD3neg }=M(t)[1+L(t) * S(t) *(-3)]^{1 / L(t)}
$$

SD23 pos is the difference between the cut-offs $3 \mathrm{SD}$ and $2 \mathrm{SD}$ calculated at $t$ by the LMS method:

$$
S D 23 \text { pos }=M(t)[1+L(t) * S(t) *(3)]^{1 / L(t)}-M(t)[1+L(t) * S(t) *(2)]^{1 / L(t)}
$$

and SD23neg is the difference between the cut-offs -2 SD and -3 SD calculated at $t$ by the LMS method:

$$
S D 23 n e g=M(t)[1+L(t) * S(t) *(-2)]^{1 / L(t)}-M(t)[1+L(t) * S(t) *(-3)]^{1 / L(t)}
$$

To illustrate the procedure, an example with arm circumference-for-age for boys is provided below and displayed in Figure 92.

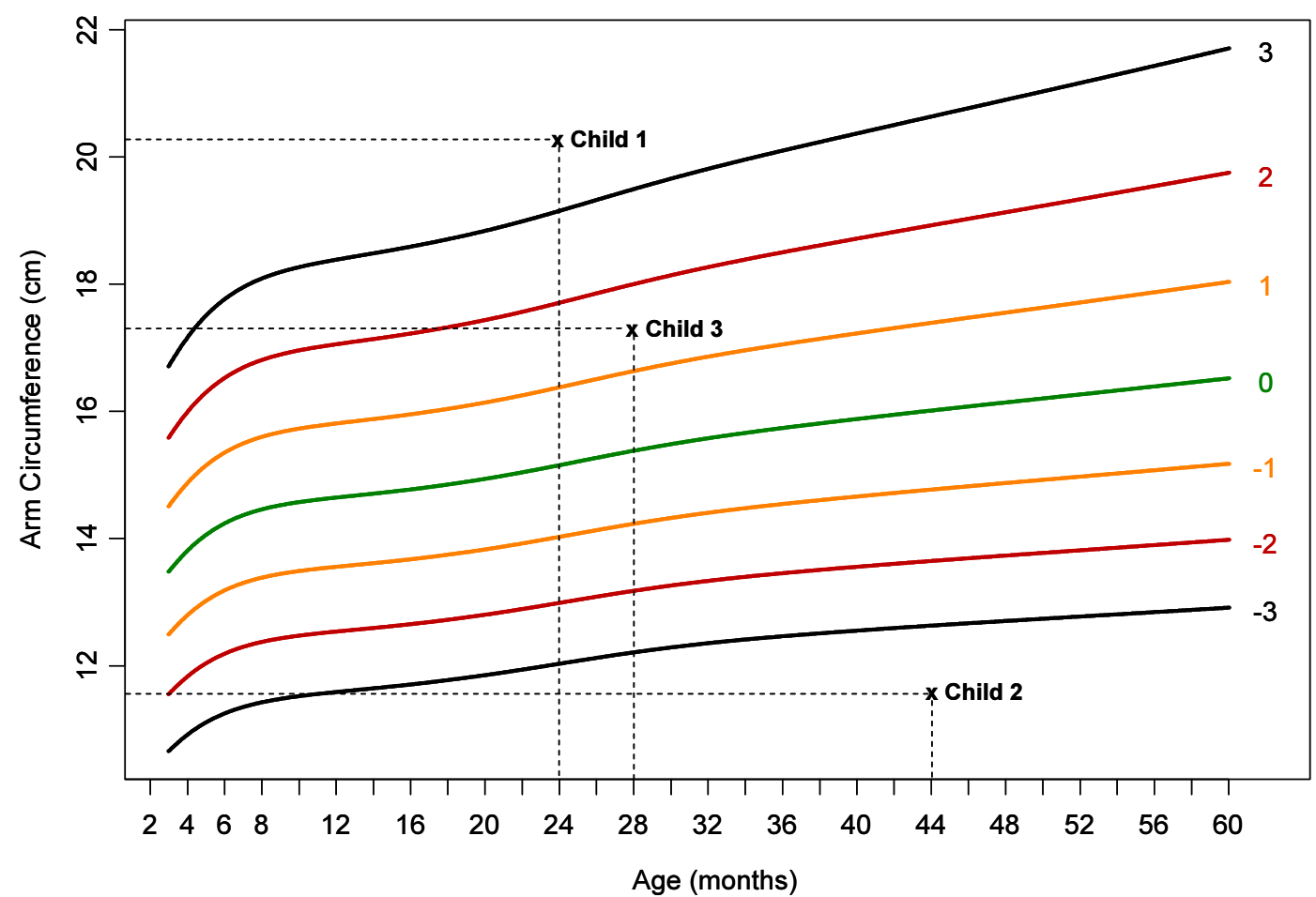

Figure 92 Examples of children ranked according to the WHO arm circumference-for-age standards 
Child 1: 24 month-old boy with arm circumference $=20.3 \mathrm{~cm}$.

$\mathrm{L}=-0.0643 ; \quad \mathrm{M}=15.1536 ; \quad \mathrm{S}=0.07746 ;$

$z_{\text {ind }}=\frac{[20.3 / 15.1536]^{(-0.0643)}-1}{0.07746 *(-0.0643)}=3.74>3$

$S D 3$ pos $=15.1536 *[1+(-0.0643) * 0.07746 *(3)]^{1 /(-0.0643)}=19.15$

$S D 2$ pos $=15.1536 *[1+(-0.0643) * 0.07746 *(2)]^{1 /(-0.0643)}=17.71$

$S D 23$ pos $=19.15-17.71=1.44$

$\Rightarrow z_{\text {ind }}^{*}=3+\left(\frac{20.3-19.15}{1.44}\right)=3.80$

Child 2: 44 month-old boy with arm circumference $=11.5 \mathrm{~cm}$.

$\mathrm{L}=-0.2730 ; \quad \mathrm{M}=16.0124 ; \quad \mathrm{S}=0.08166 ;$

$z_{\text {ind }}=\frac{[11.5 / 16.0124]^{(-0.2730)}-1}{0.08166 *(-0.2730)}=-4.24<-3$

SD2neg $=16.0124 *[1+(-0.2730) * 0.08166 *(-2)]^{1 /(-0.2730)}=13.65$

SD3neg $=16.0124 *[1+(-0.2730) * 0.08166 *(-3)]^{1 /(-0.2730)}=12.63$

SD23neg $=13.65-12.63=1.02$

$\Rightarrow z_{\text {ind }}^{*}=-3+\left(\frac{11.5-12.63}{1.02}\right)=-4.11$

Child 3: 28 month-old boy with arm circumference $=17.4 \mathrm{~cm}$.

$\mathrm{L}=-0.1132 ; \quad \mathrm{M}=15.3808 ; \quad \mathrm{S}=0.07794 ;$

$z_{\text {ind }}=\frac{[17.4 / 15.3808]^{(-0.1132)}-1}{0.07794 *(-0.1132)}=1.57 \geq-3$ and $\leq 3 \quad$ (LMS z-score) 


\section{CONCLUSION}

The goal of the MGRS was to describe the growth of healthy children. Well-defined criteria were applied in the study design to achieve this aim (de Onis et al., 2004b). The sample used for the construction of the growth standards for the head and arm circumferences and the skinfold thicknesses was the same one used for the construction of the standards based on length or height, weight and age (WHO Multicentre Growth Reference Study Group, 2006d, 2006g).

The construction of the child growth curves followed a careful, methodical process. Rigorous methods of data collection, standardized across sites, were followed during the entire study. Sound procedures for data management and cleaning were applied (Onyango et al., 2004). As a result, the anthropometric data available for analysis were of the highest possible quality. The selection of the best statistical approach to construct the standards followed a broad consultative process that included a thorough review of 30 available methods. State-of-the-art statistical methodologies were then employed to generate the standards (Borghi et al., 2006).

The Box-Cox-power-exponential (BCPE) method (Rigby and Stasinopoulos, 2004), with curve smoothing by cubic splines, was selected as the approach for constructing the growth curves. The $\mathrm{BCPE}$ accommodates various kinds of distributions, from normal to skewed or kurtotic, as necessary. There was wide variability in the degrees of freedom required for the cubic splines to achieve the best models. Except for head circumference-for-age, which followed a normal distribution, all other standards required the modelling of skewness but not kurtosis. A set of diagnostic tools was used to detect possible biases in estimated percentile or z-score curves. These included examining patterns of differences between empirical and fitted centiles, and comparing observed and expected proportions of children with measurements below selected percentile curves. Percentile and z-score curves for boys and girls were generated for head circumference-for-age (0-60 months), arm circumference-for-age (3-60 months), triceps skinfold-for-age and subscapular skinfold-for-age (3-60 months). Appendix A summarizes the specifications of the BCPE models for each of the standards.

Overall, concordance between smoothed curves and empirical centiles was free of bias in both the median range and the tails, indicating that the resulting curves are an adequate description of the true growth of healthy children. The average absolute difference between smoothed and empirical centiles was $0.091 \mathrm{~cm}$ for boys' and girls' head circumference-for-age (Figures 5-8 and 15-18); $0.102 \mathrm{~cm}$ and $0.098 \mathrm{~cm}$ for boys' and girls' arm circumference-for-age, respectively (Figures 27-30 and 38-41); $0.152 \mathrm{~mm}$ and $0.146 \mathrm{~mm}$ for boys' and girls' triceps skinfold-for-age, respectively (Figures 50-53 and 61-64); and $0.101 \mathrm{~mm}$ and $0.136 \mathrm{~mm}$ for boys' and girls' subscapular skinfold-for-age, respectively (Figures 73-76 and 85-88). Taking the sign into account, the average differences are close to zero: $0.031 \mathrm{~cm}$ and $0.022 \mathrm{~cm}$ for boys' and girls' head circumference-for-age, respectively; $0.004 \mathrm{~cm}$ and $-0.003 \mathrm{~cm}$ for boys' and girls' arm circumference-for-age, respectively; $0.024 \mathrm{~mm}$ and $0.028 \mathrm{~mm}$ for boys' and girls' triceps skinfold-for-age, respectively; and $0.018 \mathrm{~mm}$ and $0.024 \mathrm{~mm}$ for boys' and girls' subscapular skinfold-for-age, respectively. These results indicate lack of bias in the fit between smoothed and empirical percentiles.

As was done for the construction of the first set of standards (WHO Multicentre Growth Reference Study Group, 2006d), a restricted application of the LMS method was used for the construction of the indicators arm circumference-for-age and skinfold thicknesses, limiting the Box-Cox normal distribution to the interval corresponding to $z$-scores where empirical data were available (i.e. between $-3 \mathrm{SD}$ and $3 \mathrm{SD}$ ). Beyond these limits, the standard deviation was fixed to the distance between $\pm 2 \mathrm{SD}$ and $\pm 3 \mathrm{SD}$, respectively. This approach avoids making assumptions about the distribution of the data beyond the limits of the actual data (e.g. the 3 SD corresponds to the 99.9th percentile). 
All four indicators presented in this report are a new addition to the previously available set of indicators in the NCHS/WHO reference. Head circumference-for-age is often used in clinical settings as part of health screening for potential developmental or neurological disabilities in infants and young children (WHO, 1995). Very small and very large circumferences are both indicative of health or developmental risk. Arm circumference-for-age is used as an alternative indicator of nutritional status when the collection of length/height and weight measurements is difficult, as happens in emergency humanitarian situations due to famine or refugee crises (WHO, 1995). Triceps and subscapular skinfold measurements assess the thickness of subcutaneous tissue and reflect fatness primarily. The skinfold indicators are thus a useful addition to the battery of growth standards for assessing childhood obesity.

The WHO Child Growth Standards provide a technically robust set of tool for assessing the well-being of infants and young children. They were derived from children who were raised in environments that minimized constraints to growth such as poor diets and infection. In addition, their mothers followed healthy practices such as breastfeeding their children and not smoking during and after pregnancy. The standards depict normal early childhood growth under optimal environmental conditions and can be used to assess children everywhere, regardless of ethnicity, socioeconomic status and type of feeding. Together, three new elements - a prescriptive approach that moves beyond the development of growth references towards a standard, inclusion of children from around the world, and links between physical growth and motor development - provide a solid instrument for helping to assess the health and nutritional needs of the world's children. 


\section{BIBLIOGRAPHY}

Bhandari N, Bahl R, Taneja S, de Onis M, Bhan MK (2002). Growth performance of affluent Indian children is similar to that in developed countries. Bulletin of the World Health Organization, 80:189-195.

Borghi E, de Onis M, Garza C, Van den Broeck J, Frongillo EA, Grummer-Strawn L, van Buuren S, Pan H, Molinari L, Martorell R, Onyango AW, Martines JC for the WHO Multicentre Growth Reference Study Group (2006). Construction of the World Health Organization child growth standards: selection of methods for attained growth curves. Statistics in Medicine, 25:247-265.

Cole TJ, Green PJ (1992). Smoothing reference centile curves: the LMS method and penalized likelihood. Statistics in Medicine, 11:1305-1319.

de Onis M, Habicht JP (1996). Anthropometric reference data for international use: recommendations from a World Health Organization Expert Committee. American Journal of Clinical Nutrition, 64:650-658.

de Onis M, Yip R (1996). The WHO growth chart: historical considerations and current scientific issues. Bibliotheca Nutritio et Dieta, 53:74-89.

de Onis M, Garza C, Victora CG, Bhan MK, Norum KR, eds (2004a). WHO Multicentre Growth Reference Study (MGRS): Rationale, planning and implementation. Food and Nutrition Bulletin, 25(Suppl 1):S1-S89.

de Onis M, Garza C, Victora CG, Onyango AW, Frongillo EA, Martines J, for the WHO Multicentre Growth Reference Study Group (2004b). The WHO Multicentre Growth Reference Study: planning, study design and methodology. Food and Nutrition Bulletin, 25(Suppl 1):S15-S26.

de Onis M, Onyango AW, Van den Broeck J, Chumlea WC, Martorell R, for the WHO Multicentre Growth Reference Study Group (2004c). Measurement and standardization protocols for anthropometry used in the construction of a new international growth reference. Food and Nutrition Bulletin, 25(Suppl 1):S27-S36.

Garza C, de Onis M, for the WHO Multicentre Growth Reference Study Group (2004). Rationale for developing a new international growth reference. Food and Nutrition Bulletin, 25(Suppl 1):S5-S14.

Mohamed AJ, Onyango AW, de Onis M, Prakash N, Mabry RM, Alasfoor DH (2004). Socioeconomic predictors of unconstrained child growth in Muscat, Oman. Eastern Mediterranean Health Journal, 10:295-302.

Onyango AW, Pinol AJ, de Onis M, for the WHO Multicentre Growth Reference Study Group (2004). Managing data for a multicountry longitudinal study: Experience from the WHO Multicentre Growth Reference Study. Food and Nutrition Bulletin, 25 Suppl 1:S46-S52.

Owusu WB, Lartey A, de Onis M, Onyango AW, Frongillo EA (2004). Factors associated with unconstrained growth among affluent Ghanaian children. Acta Paediatrica, 93:1115-1119.

Rigby RA, Stasinopoulos DM (2004). Smooth centile curves for skew and kurtotic data modelled using the Box-Cox power exponential distribution. Statistics in Medicine, 23:3053-3076.

Royston P, Wright EM (2000). Goodness-of-fit statistics for age-specific reference intervals. Statistics in Medicine, 19:2943-2962. 
Stasinopoulos DM, Rigby RA, Akantziliotou C (2004). Instructions on how to use the GAMLSS package in R. Technical Report 02/04. London: STORM Research Centre, London Metropolitan University.

van Buuren S, Fredriks M (2001). Worm plot. A simple diagnostic device for modelling growth reference curves. Statistics in Medicine, 20:1259-1277.

WHO. Physical status: the use and interpretation of anthropometry (1995). Report of a WHO Expert Committee. World Health Organ Tech Rep Ser; 854:161-262.

WHO Multicentre Growth Reference Study Group (2006a). Assessment of differences in linear growth among populations in the WHO Multicentre Growth Reference Study. Acta Paediatric, Suppl 450:56-65.

WHO Multicentre Growth Reference Study Group (2006b). Breastfeeding in the WHO Multicentre Growth Reference Study. Acta Paediatrica, Suppl 450:16-26.

WHO Multicentre Growth Reference Study Group (2006c). WHO Motor Development Study: Windows of achievement for six gross motor development milestones. Acta Paediatrica, Suppl 450:86-95.

WHO Multicentre Growth Reference Study Group (2006d). WHO Child Growth Standards: Length/height-for-age, weight-for-age, weight-for-length, weight-for-height and body mass index-forage: Methods and development. Geneva: World Health Organization; pp 312.

WHO Multicentre Growth Reference Study Group (2006e). Enrolment and baseline characteristics in the WHO Multicentre Growth Reference Study. Acta Paediatrica, Suppl 450:7-15.

WHO Multicentre Growth Reference Study Group (2006f). Reliability of anthropometric measurements in the WHO Multicentre Growth Reference Study. Acta Paediatrica, Suppl 450:38-46.

WHO Multicentre Growth Reference Study Group (2006g). WHO Child Growth Standards based on length/height, weight and age. Acta Paediatrica, Suppl 450:76-85.

WHO Working Group on Infant Growth (1994). An evaluation of infant growth. Geneva: World Health Organization. 


\section{Appendix A. Model specifications of the WHO child growth standards}

Table A1 Degrees of freedom for fitting the parameters of the Box-Cox-power exponential (BCPE) distribution for the models with the best fit to generate standards based on age, head circumference, arm circumference, triceps skinfold and subscapular skinfold in children 0-60 months of age

\begin{tabular}{llccccc}
\hline Standards & Sex & $\lambda^{\mathbf{a}}$ & $\mathbf{d f}(\boldsymbol{\mu})^{\mathbf{b}}$ & $\mathbf{d f}(\boldsymbol{\sigma})^{\mathbf{c}}$ & $\mathbf{d f}(\mathbf{v})^{\mathbf{d}}$ & $\boldsymbol{\tau}^{\mathbf{e}}$ \\
\hline Head circumference, 0-60 mo & Boys & 0.20 & 9 & 5 & $0^{\mathrm{f}}$ & 2 \\
& Girls & 0.20 & 9 & 2 & $0^{\mathrm{f}}$ & 2 \\
Arm circumference, 3-60 mo & Boys & 0.35 & 7 & 4 & 2 & 2 \\
& Girls & 0.35 & 8 & 4 & 1 & 2 \\
Triceps skinfold, 3-60 mo & Boys & 0.30 & 7 & 5 & 2 & 2 \\
\multirow{3}{*}{ Subscapular skinfold, 3-60 mo } & Girls & 0.15 & 7 & 5 & 3 & 2 \\
& Boys & 0.65 & 6 & 2 & 2 & 2 \\
& Girls & 0.15 & 5 & 4 & 2 & 2 \\
\hline
\end{tabular}

${ }^{a}$ Age transformation power.

${ }^{b}$ Degrees of freedom for the cubic splines fitting the median $(\mu)$.

${ }^{\mathrm{c}}$ Degrees of freedom for the cubic splines fitting the coefficient of variation $(\sigma)$.

${ }^{\mathrm{d}}$ Degrees of freedom for the cubic splines fitting the Box-Cox transformation power $(v)$.

${ }^{\mathrm{e}}$ Parameter related to the kurtosis fixed $(\tau=2)$.

${ }^{\mathrm{f}} v=1$ : Normal distribution. 
